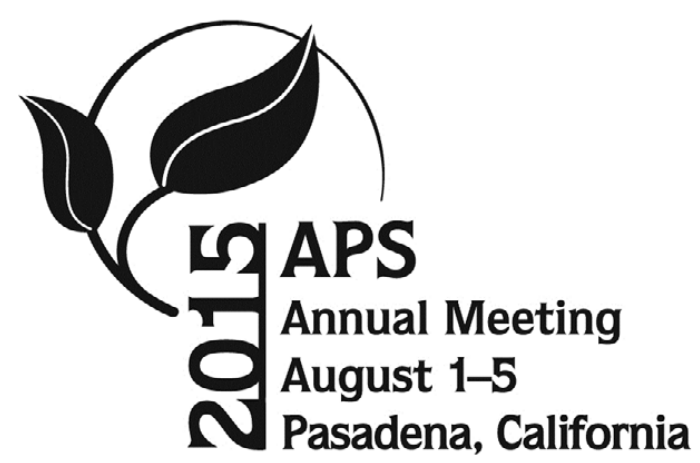

\title{
2015 APS Annual Meeting Abstracts of Presentations
}

\begin{abstract}
APS Annual Meeting in Pasadena, California, U.S.A., August 1-5, 2015. The abstracts are arranged alphabetically by the first author's name. Recommended format for citing joint meeting abstracts, using the first abstract below as an example, is as follows: Abbas, H. K., and Accinelli, C. 2015. Application of bioplastic materials in the biocontrol of agricultural pests. (Abstr.) Phytopathology 105(Suppl. 4):S4.1. http://dx.doi.org/10.1094/PHYTO-105-11-S4.1
\end{abstract}

\begin{abstract}
Application of bioplastic materials in the biocontrol of agricultural pests H. K. ABBAS (1), C. Accinelli (2)

(1) USDA ARS BCPRU, Stoneville, MS, U.S.A.; (2) University of Bologna, Dept of Agricultural Sciences, Bologna, Italy

Phytopathology 105(Suppl. 4):S4.1

A versatile corn starch-based bioplastic delivery system was developed for use in multiple formulations and agents. This technology was used as carriers of non-toxigenic Aspergillus flavus (NTAA) strains and can be adapted to current farm machinery. Granules applied to soil and plants were shown to increase the population of NTAA in soil by $88 \%$ and in corn by $93 \%$, thereby reducing aflatoxin in corn. In field trials in the USA and Italy from 2009-12, aflatoxin levels were reduced by $70 \& 80 \%$, respectively. This technology was also effective for applying other biological control agents (e.g., Trichoderma virens and T. harzianum) to control damping off caused by Rhizoctonia solani and Pythium ultimum in horticultural crops. Damping off was suppressed $50 \%$ to $91 \%$ in these crops using bioplastic granule formulations of these two Trichoderma species, demonstrating that this technology has the potential to extend biocontrol of plant disease to horticultural plants as well as in controlling aflatoxin in agronomic crops. A sprayable bioplastic-based formulation was also developed. Application of this liquid formulation containing Beauveria bassiana significantly reduced damage caused by the European corn borer in maize and the tarnished plant bug in cotton. Applying a formulation of spores of a NTAA strain resulted in a $97 \%$ reduction of aflatoxin contamination of maize. These results suggest that this formulation has potential for use in improving several biocontrol agents.
\end{abstract}

The effect of adjuvants on apple disease management

C. P. ABBOTT (1), J. L. Beckerman (1)

(1) Purdue University, West Lafayette, IN, U.S.A.

Phytopathology 105(Suppl. 4):S4.1

Apple growers rely on the fungicide captan to manage apple scab (Venturia inaequalis) and bitter rot (Colletotrichum spp.). There is a low risk of either fungus developing resistance to captan, increasing the importance of this

The abstracts are published as submitted. They were formatted but not edited at the APS headquarters office.

http://dx.doi.org/10.1094/PHYTO-105-11-S4.1

(C) 2015 The American Phytopathological Society fungicide in disease management. Label restrictions limit growers to $40 \mathrm{lbs}$ of captan per season, which may not provide sufficient control of both apple scab and bitter rot in wet years. Adjuvants are tank additives that may reduce the rate of captan needed per season by improving disease management. One method is to use adjuvants to increase the coverage and retention of captan treatments. Another is to use adjuvants to improve urea-driven-leaf litter decomposition. In 2013 and 2014, we examined the role of adjuvants combined with a low rate of captan in field applications. In 2013, adjuvants did not improve captan performance due to low disease pressure. In 2014, Li700, Bond, and LatronB enhanced control of apple scab on Golden Delicious. In 2013, a preliminary study found adjuvants had the potential to improve urea-driven decomposition and inoculum reduction of scab infected leaves. A larger study is underway to further evaluate this. Overall, the addition of adjuvants can improve disease management by increasing fungicide efficacy and decreasing overwintering inoculum, which may reduce overall fungicide input during the growing season.

Ethylene elicits soybean defense responses and reduces symptoms of sudden death syndrome

N. ABDELSAMAD (1), G. MacIntosh (1), L. Leandro (1)

(1) Iowa State Univ, Ames, IA, U.S.A.

Phytopathology 105(Suppl. 4):S4.1

The role of ethylene on soybean response to Fusarium virguliforme $(F v)$, the causal agent of soybean sudden death syndrome (SDS), was investigated in a resistant and a susceptible cultivar. Seedlings were drenched at the first unifoliate stage with either ethephon, an ethylene inducer, or cobalt chloride, an ethylene suppressor. To block ethylene perception, seedlings were sprayed with 1-MCP until runoff. All treatments were applied twice, $24 \mathrm{~h}$ before and after inoculation with an $F v$ infested sand-cornmeal mix. Defense response genes were quantified 0,2 , and 4 days after inoculation (DAI) using qPCR. Foliar and root symptoms, and $F v$ density in soil were assessed 21 DAI. In both cultivars, SDS foliar symptoms at $21 \mathrm{DAI}$ were lower in ethephon treated seedlings compared to water controls $(P<0.05)$. Ethephon activated expression of the ethylene biosynthesis genes ethylene oxidase (ACO) and ethylene synthase (ACS), while cobalt chloride and 1-MCP treatments had no effect on ethylene levels. Treatment with ethephon increased induction of soybean defense response genes such as pathogenesis related proteins (PR), basic peroxidase (IPER) and chalcone synthase (CHS), compared to the other treatments at all time points $(P<0.05)$. Treatment with ethephon or cobalt chloride had no effect on $F v$ density in soil. This study suggests that ethephon application induced resistance against SDS, possibly by induction of soybean ethylene levels and defense response genes. 
Characterization of Pyrenophora tritici-repentis isolates from rye in South Dakota

S. Abdullah (1), S. K. Sehgal (2), K. Glover (2), S. ALI (2)

(1) South Dakota State University, Brookings, SD, U.S.A.; (2) South Dakota State University, Brookings, SD, U.S.A.

Phytopathology 105(Suppl. 4):S4.2

Tan spot, caused by Pyrenophora tritici-repentis (Died.) Drechs., is an important foliar disease of wheat. The fungus has a broad host range including rye (Secale cerale). Eight races have been identified in the fungal populations based on their ability to induce necrosis and chlorosis symptoms. This fungus produces three host selective toxins Ptr ToxA, ToxB, and ToxC, which are associated with necrosis and chlorosis symptoms. Rye is cultivated as a forage crop in South Dakota and could serve as a source of virulence variation in the fungal population. In this study, we recovered 107 P. tritici-repentis isolates from rye and characterized them for their race structure. All 107 isolates were inoculated individually on wheat differentials, Glenlea, 6B365, 6B662, and Salamouni. The isolates were grouped into appropriate races based on their ability to produce necrosis and/chlorosis on the differentials. Twenty-one of 107 isolates were grouped as race 1 as they induced necrosis and chlorosis on appropriate wheat differential genotypes. The rest of the isolates were grouped as race 4 as they did not induce either necrosis or chlorosis symptoms. All 107 isolates tested on the differential set were genotyped by using Ptr ToxA specific primer. Those 21 isolates that were grouped as race 1 based on differentials amplified ToxA gene validating the presence of race 1 . The results indicate that rye harbors a diverse $P$. tritici-repentis population.

Reaction of global collection of rye germplasm to tan spot Pyrenophora tritici-repentis race 1,5 and Ptr ToxA

S. Abdullah (1), S. ALI (2), K. Glover (3), S. Sehgal (3)

(1) South Dakota State Univ, Brookings, SD, U.S.A.; (2) South Dakota State University, Brookings, SD, U.S.A.; (3) South Dakota State University, Brookings, SD, U.S.A.

Phytopathology 105(Suppl. 4):S4.2

Rye, Secale cerale, is grown as a grain and forage crop and has been utilized in improving wheat for productivity and disease resistance. Rye is alternative host of $P$. tritici-repentis, cause of Tan spot of wheat. In this study, 166 rye genotypes with diverse genetic background from various countries were evaluated for their reaction to tan spot using $P$. tritici-repentis race 1 , race 5, and Ptr ToxA. Two weeks old-seedling of all 166 rye lines were raised in containers in the greenhouse. The seedlings were inoculated individually with race 1 and race 5 using their spore suspension and placed in a humidity chamber for 24 hours and then moved to the greenhouse for seven days until rated for their reaction. Three leaves from each of 166 genotypes were infiltrated with Ptr ToxA and rated for their reaction. All 166-rye genotypes exhibited resistance to race 1 and insensitivity to Ptr ToxA. Sixty-five of 166 genotypes exhibited susceptibility to race 5 as they developed chlorosis. The results indicate that rye is more prone race 5 and could serve a reservoir of $P$. tritici-repentis race 5 population.

Fungicide sensitivity characterization of novel ectotrophic root-infecting fungi associated with bermudagrass roots

G. W. ABLES (1), M. Tomaso-Peterson (1), P. Vines (1), J. R. Standish (1)

(1) Mississippi State University, Mississippi State, MS, U.S.A.

Phytopathology 105(Suppl. 4):S4.2

Ectotrophic root-infecting (ERI) fungi are known for causing root-rot diseases in turfgrass. Previous research at Mississippi State University identified six novel ERI pathogens associated with root-rot of bermudagrass. ERI fungi colonize the root surface with thick, dark strands of runner hyphae. Dark brittle roots, yellowing, and necrotic patches can be observed in affected bermudagrass. Infection of the root system occurs prior to the onset of symptoms, making control of root-rot diseases difficult. Penetrant fungicides applied for root-rot control include but are not limited to strobilurins, methyl benzimidazoles carbamates, and demethylation inhibitors. The objective of this study was to determine fungicide sensitivity of novel ERI pathogens to azoxystrobin (AZ) by determining the effective concentration to inhibit fifty percent of growth $\left(\mathrm{EC}_{50}\right)$. Hyphal plugs of Gaeumannomyces paulograminis nov. sp. (DR1-4) were transferred to various concentrations of AZ-amended PDA. Relative growth was converted to percent inhibition and the $\mathrm{EC}_{50}$ was determined using linear regression of the probit-transformed data. The resultant $\mathrm{EC}_{50}$ value was determined to be greater than $10 \mathrm{ppm}$, indicating there may be an amino acid substitution in the target site conferring resistance to $\mathrm{AZ}$.
Endornaviruses in Rhizoctonia spp.

N. ABOUGHANEM-SABANADZOVIC (1), A. Lawrence (2), M. TomasoPeterson (3), S. Sabanadzovic (4)

(1) Institute for Genomics, Biocomputing and Biotechnology, Mississippi State University, Mississippi State, MS, U.S.A.; (2) Institute for Imaging and Analytical Technologies, Mississippi State University, Mississippi State, MS, U.S.A.; (3) Department of Biochemnistry, Molecular Biology, Entomology and Plant Pathology, Mississippi State University, Mississippi State, MS, U.S.A.; (4) Department of Biochemistry, Molecular Biology, Entomology and Plant Pathology, Mississippi State University, Mississippi State, MS, U.S.A. Phytopathology 105(Suppl. 4):S4.2

Endornaviruses are capsid-less viruses reported from plants, fungi and oomycetes and characterized by a persistent lifestyle. Their monocistronic RNA genome, ranging from 9.8 to $17.6 \mathrm{~kb}$ in size, code for a large polyprotein characterized by several functional domains, of which only RdRp is the only universally encoded by genomes of all characterized endornaviruses. During the study of mycoviruses of Rhizoctonia spp. isolated from various hosts in Mississippi, among other viruses, we detected the presence of endornaviruslike dsRNAs in few fungal isolates. Characterization of these dsRNAs showed that they are different from recently characterized endornaviruses reported from $R$. solani and $R$. cerealis isolated from potato and wheat in New Zealand and China, respectively.

Experimental transmission and improved detection of blackberry vein banding associated virus

N. ABOUGHANEM-SABANADZOVIC (1), T. Thekke-Veetil (2), I. E. Tzanetakis (3), S. Sabanadzovic (4)

(1) Institute for Genomics, Biocomputing and Biotechnology, Mississippi State University, Mississippi State, MS, U.S.A.; (2) Department of Plant Pathology, Division of Agriculture, University of Arkansas System, Fayetteville, AZ, U.S.A.; (3) Department of Plant Pathology, Division of Agriculture, University of Arkansas System, Fayetteville, AR, U.S.A.; (4) Department of Biochemistry, Molecular Biology, Entomology and Plant Pathology, Mississippi State University, Mississippi State, MS, U.S.A.

Phytopathology 105(Suppl. 4):S4.2

Blackberry vein banding associated virus $(\mathrm{BVBaV})$ is a recently characterized virus associated with yellow vein disease in the southern USA and closely related to grapevine leafroll associated virus-3 (GLRaV-3), the type species of the genus Ampelovirus. As a continuation of the study on its characterization, experiments were conducted to identify a possible vector and to develop improved detection tools. A mealybug species, preliminarily identified as Pseudococcus maritimus (American grape mealybug), found to naturally feed on a daughter plant of the original BVBaV source, transmitted this virus under experimental greenhouse conditions. A universal $\mathrm{BVBaV}$ detection primer set was designed, based on conserved sequences of the ORF coding for coat protein minor $(\mathrm{CPm})$ shared by 25 isolates. The new test considerably improved detection, as ascertained in a survey on a high number of blackberry samples collected from a large geographic area. Results of this study further the knowledge on $\mathrm{BVBaV}$ epidemiology and contribute in the design of better management strategies based on reliable detection and vector control.

\section{Characterization of three new amalgaviruses from different hosts}

N. Aboughanem-Sabanadzovic (1), S. SABANADZOVIC (2)

(1) Institute for Genomics, Biocomputing and Biotechnology, Mississippi State University, Mississippi State, MS, U.S.A.; (2) Department of Biochemistry, Molecular Biology, Entomology and Plant Pathology, Mississippi State University, Mississippi State University, MS, U.S.A.

Phytopathology 105(Suppl. 4):S4.2

A number of closely related viruses with dsRNA genomes of c $3.5 \mathrm{kbp}$ in size, containing two partially overlapping ORFs reminiscent of totiviruses, have been recently identified in plants belonging to different botanical species. However, phylogenetic analyses of the viral RNA-dependent RNA polymerase revealed closer evolutionary relationships of these viruses to the extant partitiviruses than to totiviruses. Therefore, unique properties of these viruses led to the establishment of a new virus family Amalgaviridae that accommodates a single genus Amalgavirus typified by Southern tomato virus (STV). During two independent studies on seed-borne viruses in cucurbits and viruses in Great Smoky Mountains National Park, we observed the presence of dsRNA bands resembling amalgavirus-infections in several samples of watermelon of different varieties and geographic origin and in samples of creeping buttercup (Ranunculus repens) and great rhododendron (Rhododendron maximum) collected in GSMNP. Complete sequencing showed that studied dsRNAs represent genomes of 3 new species in the genus Amalgavirus. The results of this study contribute to better understand the range of hosts for these newly recognized taxa of viruses with nonsegmented dsRNA genomes. 
Soybean vein necrosis virus and other viruses in of soybeans in Mississippi

N. Aboughanem-Sabanadzovic (1), W. F. Moore (2), T. W. Allen (3), R. C. Stephenson (4), S. SABANADZOVIC (2)

(1) Institute for Genomics, Biocomputing and Biotechnology, Mississippi State University, Mississippi State, MS, U.S.A.; (2) Department of Biochemistry, Molecular Biology, Entomology and Plant Pathology, Mississippi State University, Mississippi State, MS, U.S.A.; (3) Delta Research and Extensions Center, Mississippi State University, Stoneville, MS, U.S.A.; (4) Coastal Research and Extension Center, Mississippi State University, Biloxi, MS, U.S.A.

Phytopathology 105(Suppl. 4):S4.3

Soybean vein necrosis virus (SVNV) is a new tospovirus, the causal agent of an emerging disease recently reported from all major soybean-producing states in the USA. A study, designed to better understand SVNV incidence, symptomatology and population structure, was carried out in 2013 and 2014 in the framework of the project funded by the Mississippi Soybean Promotion Board. For this purpose, we carried out extensive field scouting in both years aimed at visual observations of symptoms and sample collection for further laboratory analyses. In order to investigate possible alternative hosts for this virus in MS, samples of several weeds associated with soybean production were collected and tested. All collected samples were also tested for the presence of an additional 3 viruses (bean pod mottle virus, soybean mosaic virus and tobacco ringspot virus). Partial characterization of several symptomatic samples that resulted negative for the target species revealed the presence of several additional viruses in soybean production fields in Mississippi.

Exploring de novo specificity: The Pyrenophora tritici-repentis-barley interaction

R. ABOUKHADDOUR (1)

(1) Univ of Alberta, Edmonton, AB, Canada

Phytopathology 105(Suppl. 4):S4.3

Pyrenophora tritici-repentis (Ptr), the causal agent of tan spot of wheat, produces several host-selective toxins. These toxins interact with compatible host sensitivity genes in an inverse gene-for-gene manner to cause foliar necrosis and/or chlorosis on wheat. Ptr ToxB, a chlorosis inducing toxin, is produced by different Ptr isolates, and affects only certain wheat genotypes. In this study, we explored the Ptr-barley interaction and provide evidence for the ability of Ptr ToxB-producing isolates to cause significant damage and induce chlorosis specifically on particular barley genotypes. Ptr ToxB was able to induce chlorosis in a highly specific manner when infiltrated into barley genotypes that are susceptible to Ptr race 5 (Ptr ToxB-producing). Analysis of the infection process in barley using light, fluorescence, scanning electron and confocal laser microscopy, showed that the invading pathogen penetrates through the epidermal cells and advances further to colonize the mesophyll layer intercellularly. Significantly different amounts of pathogen biomass were detected by quantitative PCR analysis of susceptible and resistant barley genotypes starting at $12 \mathrm{~h}$ after inoculation with Ptr. These results reveal the ability of Ptr ToxB and its producing fungus to cross the species boundary and cause disease in two different genera, with the cytology of infection on barley closely resembling that on wheat.

Using a cucumber mosaic virus vector to investigate the roles of plant microRNAs in symptom induction and host-vector interactions

D. D. S. ABREU (1), W. Chen (2), T. Tungadi (1), A. M. Murphy (1), Z. Du (2), J. P. Carr (1)

(1) University of Cambridge, Cambridge, United Kingdom; (2) Zhejiang SciTech University, Hangzhou, China

Phytopathology 105(Suppl. 4):S4.3

In Arabidopsis thaliana (Col-0) transgenic expression of the Cucumber mosaic virus (CMV) 2b silencing suppressor protein from the severe subgroup IA strain Fny disrupted microRNA (miRNA)-regulated development but $2 \mathrm{~b}$ protein from the mild subgroup II strain LS did not. To identify which miRNA affected by Fny $2 \mathrm{~b}$ was important in the triggering of Fny-CMV symptoms we developed a LS-CMV-based vector to express sequences mimicking miRNA targets (a target mimic or 'decoy' strategy). Expressing a miR159 target mimic sequence using LS-CMV depleted miR159 and induced symptoms recapitulating those of Fny-CMV. Suppression of Fny-CMV-induced symptoms in plants harboring mutant alleles for the miR159ab targets MYB33 and MYB65 confirmed the importance of this miRNA in pathogenesis. Aphids transmit CMV. We found that the Fny2b expression in transgenic Arabidopsis induces toxicity (antibiosis) against aphids (Myzus persicae). This appears to be mediated by interference with the activity of ARGONAUTE1 (AGO1) and consequent deregulation of a miRNA-regulated insect resistance mechanism. Currently, we are using the LS-CMV-based target mimic vector system to determine which miRNA(s) regulate antibiosis against aphids as a first step in identification of the plant genes and gene products conditioning this resistance. Funded by grants from the BBSRC (U.K.), CAPES (Brazil) and National Natural Science Foundation of China.

Dormant treatments with chlorothalonil-oil delay the production of primary inoculum of almond scab caused by Fusicladium carpophilum

J. Adaskaveg (1), H. FORSTER (1)

(1) University of California, Riverside, CA, U.S.A.

Phytopathology 105(Suppl. 4):S4.3

Almond scab has gained increased importance in California almond production due to QoI and SDHI resistance in populations of the pathogen Fusicladium carpophilum. The pathogen overwinters in lesions on $<1$ yearold twigs. These lesions provide primary inoculum in the spring when the fungus produces abundant wind- and rain-borne conidia that infect young leaves that may lead to premature tree defoliation. Chemical management strategies for scab currently include dormant copper-oil applications that delay the sporulation of twig lesions, and protective in-season treatments that prevent new infections in the spring. Field trials were conducted to evaluate the efficacy of alternative dormant treatments on the production of primary inoculum. Chlorothalonil and other fungicides were compared to copper, all in mixture with an agricultural oil. A single treatment was applied in mid- to late-January and sporulation of twig lesions was evaluated periodically between March and May. Chlorothalonil-oil delayed and reduced the amount of sporulation of twig lesions until late May and was significantly more effective than the other treatments. The delay of sporulation allows a more effective use of subsequent in-season protective treatments and aligns the application of in-season treatments for scab with those for other summer diseases such as Alternaria leaf spot, rust, and hull rot and thus, reduces the total amount of fungicide applications per season.

Identification of the CaMV gene that overcomes resistance in Arabidopsis thaliana ecotype Enkheim (En-2)

M. A. ADHAB (1), C. Angel (2), S. Leisner (3), J. E. Schoelz (1)

(1) Division of Plant Sciences, University of Missouri - Columbia, Columbia, MO, U.S.A.; (2) Colombian Sugarcane Research Center - CENICAÑA, Cali, Colombia; (3) Department of Biological Sciences, the University of Toledo, Toledo, OH, U.S.A.

Phytopathology 105(Suppl. 4):S4.3

Arabidopsis thaliana ecotype En-2 is resistant to several strains of Cauliflower mosaic virus (CaMV), including strain W260, but is susceptible to NY8153. NY8153 infects En-2 plants by producing small chlorotic primary lesions on the inoculated leaves 4-7 days after inoculation, whereas systemic symptoms developed 11-14 days after inoculation. The temporal appearance for both local and systemic symptoms was affected by environmental conditions. In contrast to previous studies, we found that W260 infected En-2 systemically with a 5-10 day delay relative to NY8153. To identify which gene of NY8153 is responsible for overcoming the En-2 resistance, we constructed several recombinant genomes between W260 and NY8153 by exploiting restriction enzyme sites common to both viruses. The portion of NY8153 responsible for breaking resistance was localized to a DNA segment delimited by BstEII and XhoI sites, containing portions of proteins P7 and P2 as well as all of P1. When comparing the amino acid sequence of P1 in NY8153 with P1 of W260, we found seven amino acid differences. Moreover, we found only one difference between P7 in NY8153 and W260 and no differences between the two strains P2. Previous studies have shown a key role of P1 in virus cell-to-cell movement. By contrast, P7 has no known function and the protein has not been detected in infected plants. We are now focused on identifying which mutations specifically are responsible for breaking En-2 resistance.

Population genetic analysis of early blight pathogen, Alternaria solani from tomato and potato

T. ADHIKARI (1), V. Rumsch (1), S. Gurung (2), D. Halterman (3), F. J. Louws (4)

(1) North Carolina State University, Raleigh, NC, U.S.A.; (2) Sakata Seed America, Inc., Salinas, CA, U.S.A.; (3) USDA/ARS Vegetable Crops Research Unit, Madison, WI, U.S.A.; (4) North Carolina State University, Raleigh, CA, U.S.A.

Phytopathology 105(Suppl. 4):S4.3

Early blight, caused by Alternaria solani is a devastating disease of tomato and potato. The main objective of this research was to determine the genetic diversity among and within isolates of $A$. solani. Tomato and potato fields in North Carolina and Wisconsin were sampled between 2012 and 2014. Genomic DNA was extracted from 120 isolates of $A$. solani from tomato $(n=$ $62)$ and potato $(n=58)$. PCR-based diagnostic primers were used to confirm 
the isolates of $A$. solani. The genetic structure of sampled populations was determined by sequencing of three gene regions (gpd, ITS4/ITS5 and RPB2); analyzing mutations in succinate dehydrogenase $(s d h)$ genes $s d h \mathrm{~B}, s d h \mathrm{C}$, and $s d h \mathrm{D}$ that confer resistance to SDHI fungicides (e. g., boscalid), and for genetic variations for mating type frequency and at 10 simple-sequence repeat (SSR) loci. All isolates were sensitive to SDHI fungicides, suggesting no evidence for point-mutations. Multi-locus genes and SSR analyses both indicated that the isolates from tomato and potato were genetically distinct, supporting the hypothesis that the population was structured by host of origin or geographic source. Clone-corrected data showed an equal frequency of both mating types, high genotypic and gene diversity, and significant differentiation between populations from tomato and potato. These results suggest that $A$. solani populations are highly diverse and genetically differentiated over tomato and potato-producing regions of the United States.

Oxathiapiprolin: A new fungicide for control of potato late blight (Phytophthora infestans)

J. I. ADKINS (1), P. Kuhn (2), A. Tally (2), B. Druebbisch (2)

(1) Syngenta, Richland, WA, U.S.A.; (2) Syngenta Crop Protection, LLC, Greensboro, NC, U.S.A.

Phytopathology 105(Suppl. 4):S4.4

Oxathiapiprolin is a new fungicide active ingredient with high intrinsic activity on Oomycete pathogens. This is the first member of the piperidinylthiazole-isoxazoline class of fungicide chemistry. Assigned FRAC Code U 15, oxathiapiprolin has a novel mode of action which is proposed to inhibit a vital oxysterol binding protein with a currently uncharacterized function. Potato will be among the crops included in the initial product launch in 2016. Excellent control of late blight (Phytophthora infestans) and yield protection have been observed following treatment with oxathiapiprolin at extremely low use rates compared to other potato fungicides. Oxathiapiprolin has excellent rainfastness, and provides disease control to expanding leaves along with translaminar activity. Due to the single site mode of action exhibited by oxathiapiprolin, the mitigation of potential for development of reduced sensitivity will be a high priority in directions for use. Strict stewardship guidelines will be included on the label regarding application régimes and the required use of mixture products containing two modes of action. Additional research is under way for potential future uses on pink rot (Phytophthora erythroseptica) and Pythium leak (Pythium ultimum).

Inhibitory effects of organic and inorganic salts on the growth of Pseudomonas cichorii, causative agent of lettuce varnish spot

H. Affia (1), V. Toussaint (2), R. TWEDDELL (1)

(1) Centre de recherche en horticulture, Université Laval, Quebec, QC, Canada; (2) Agriculture and Agri-Food Canada, Saint-Jean-sur Richelieu, QC, Canada

Phytopathology 105(Suppl. 4):S4.4

Varnish spot, caused by Pseudomonas cichorii, is an important disease affecting lettuce (Lactuca sativa). In Canada, no chemicals are registered to control bacterial diseases in lettuce. Consequently, control of varnish spot relies on cultural practices including good soil drainage and air movement through crop canopy. However, cultural practices are not always sufficient to effectively control the disease. Exploitation of antimicrobial properties of organic and inorganic salts widely used in food industry is an interesting approach for the control of varnish spot. The objective of the study was to determine in vitro the minimal inhibitory concentrations (MICs) of different salts and combinations of salts against $P$. cichorii. Sodium metabisulfite, sodium carbonate, sodium benzoate, potassium sorbate, calcium chloride and sodium bicarbonate MICs were $4.11 \mathrm{mM}, 35 \mathrm{mM}, 45 \mathrm{mM}, 75.6 \mathrm{mM}, 115 \mathrm{mM}$ and $137.8 \mathrm{mM}$, respectively. MICs for mixtures of sodium metabisulfite + calcium chloride, sodium benzoate + sodium carbonate, sodium metabisulfite + sodium carbonate, sodium benzoate + potassium sorbate, sodium carbonate + sodium bicarbonate, sodium carbonate + potassium sorbate, sodium bicarbonate + potassium sorbate and sodium benzoate + sodium bicarbonate were also determined. Future work will be undertaken to evaluate the efficacy of each salt / salt mixture to control the disease on lettuce plant when applied at concentrations corresponding to the MICs.

Performance of some lines of pepper (Capsicum) species under natural foliar infection in Nigeria

C. G. AFOLABI (1), O. A. Oladipupo (1), E. I. Ayo-John (1), A. W. Salau (1), A. R. Afolabi (1), I. A. Kehinde (1)

(1) Federal University of Agriculture, Abeokuta, Nigeria

Phytopathology 105(Suppl. 4):S4.4

Pepper, Capsicum spp, is an annual herbaceous vegetable crop grown in many countries of the world. Diseases pose major constraints to pepper production in Nigeria. The current study was undertaken to assess responses to foliar infection under natural infection in 14 lines of the genus Capsicum with a view to identifying species resistance to the foliar diseases that are currently prevalent in Nigeria. Leaf samples were randomly excised from each field plot and assessed by laboratory cultural and serological methods for fungal and viral presence. Symptoms of leaf spot, wilt, anthracnose, Phytophthora blight and viral infection were observed on the field: leaf spots and viral diseases had the highest frequency and greatest severity. Furthermore, species of Cercospora, Colletotrichum, and Phytophthora, were found associated with disease symptoms on leaf samples, while most of the virus-infected samples tested positive for Cucumber Mosaic Virus, Potato Virus Y and Pepper Veinal Mottle Virus. Viral infections and leaf spot differed significantly $(\mathrm{P}<0.05)$ among the pepper species in both disease incidence and severity. Severity scores ranged between 1 to 7 and 3 to 5 for leaf spot and viral infections respectively. This study has revealed leaf spot and viral diseases as two very important classes of foliar disease problems of pepper in Nigeria. None of the 14 lines of pepper species evaluated possessed any appreciably high level of resistance to the foliar infections.

\section{Management of bull's-eye rot of apple using pre- and postharvest fungicides}

C. G. AGUILAR (1), C. L. Xiao (2), M. Mazzola (3)

(1) Washington State University, Wenatchee, WA, U.S.A.; (2) USDA-ARS, Parlier, CA, U.S.A.; (3) USDA-ARS, Wenatchee, WA, U.S.A.

Phytopathology 105(Suppl. 4):S4.4

Bull's-eye rot caused by Cryptosporiopsis kienholzii, Neofabraea alba, $N$. malicorticis and $N$. perennans is a common postharvest disease of apple and pear in the US Pacific Northwest. Fruit infection by these causal fungi occurs in the orchard and is latent at harvest. A primary practice for control of this disease is the pre-harvest application of chemical sprays in the orchard, and drenching of fruit with postharvest fungicides prior to cold storage. In this study, the efficacy of pre- or post-harvest fungicide applications for disease control on fruit inoculated at two different time-points with either $C$. kienholzii or $N$. perennans was monitored. Fruit were treated with either one of three pre-harvest fungicides, (Zinc dimethyldithiocarbamate, pyraclostrobin + boscalid, and thiophanate-methyl) or one of three postharvest fungicide drenches, (fludioxonil, pyrimethanil, and thiabendazole) to determine an effective chemical control strategy for management of this disease. In general, thiophanate-methyl, pyrimethanil or thiabendazole significantly $(P<0.05)$ reduced incidence of fruit decay caused by either $C$. kienholzii or $N$. perennans relative to the no treatment control. Although both thiophanatemethyl and thiabendazole provided effective disease control, integration of both chemistries into a disease management program is discouraged as both fungicides share the same mode of action.

Characterization and pathogenicity of Rhizoctonia spp. associated with soybean in Illinois

O. O. AJAYI (1), C. A. Bradley (2)

(1) Department of Crop Sciences, University of Illinois, Urbana, IL, U.S.A.; (2) Univ of Illinois, Urbana, IL, U.S.A.

Phytopathology 105(Suppl. 4):S4.4

In an effort to identify and characterize the important species of Rhizoctonia associated with seedling diseases of soybean in Illinois, isolates of Rhizoctonia spp. were recovered from soybean seedlings with damping off and root and hypocotyl rot symptoms from 16 counties in 2012 and 2013. Using conventional and molecular techniques, 57 isolates were confirmed to be $R$. solani, 20 were binucleate Rhizoctonia spp., and nine were $R$. zeae. Anastomosis groups of $R$. solani isolates were determined by RFLP-PCR analysis, sequencing of the ITS region, and by anastomosis reactions with known testers. Of the $57 R$. solani isolates, 35 were AG-2-2IIIB, seven were AG-4 HGIII, three were AG-4 HGI, six were AG-11, five were AG-3 PT, and one was AG-B1. On soybean genotype "Williams 82", isolates of AG-2-2IIIB were the most aggressive, followed by AG-4, AG-11, and AG-3. On corn, AG-2-2IIIB and AG-4 isolates caused significant stunting and root damage, while other AGs did not appear to be pathogenic. R. zeae and the binucleate Rhizoctonia spp. were not pathogenic on soybean. Our results indicate that soybean and corn are hosts to the predominant and aggressive AGs of $R$. solani in Illinois, implying that rotation between these two crops may not be an effective management practice.

Botryosphaeriaceae associated with macadamia branch die-back is becoming a significant pathogen in Australia

F. AKINSANMI (1), C. Searle (2), A. Drenth (1)

(1) The University of Queensland, QAAFI, Centre of Plant Science, Brisbane, Australia; (2) Suncoast Gold Macadamias, Drummond Drive, Gympie, Australia

Phytopathology 105(Suppl. 4):S4.4 
Members of Botryosphaeriaceae are commonly observed as endophytes and are among the most destructive fungal pathogens of perennial tree crops. Macadamia branch die-back caused by species of Botryosphaeriaceae is usually only important in the first 8-10 years of tree growth, however, in Australia, the disease is increasing in prevalence and severity in $>15$ years old trees and a significant threat to production. Surveys of macadamia orchards between 2009 and 2014 revealed wide occurrence of branch die-back, mostly in cvs. A203, A268, HAES 741, HAES 842, Daddow and A16, respectively in decreasing order of severity. Branch die-back was more evident from late summer to early autumn following prolonged warm humid or unusually hot weather. Macadamia seedlings inoculated through wounding with Botryosphaeriaceae isolates obtained from diseased trees showed die-back symptoms within 2-3 months after inoculation. Expression of die-back symptoms was accelerated only in inoculated seedlings following water stress events for 3 weeks compared with seedlings that received regular watering in the glasshouse. In $60 \%$ of the inoculated seedlings, die-back severity progressed irreversibly until total seedling death even after regular watering resumed. The study provides information on the identity of the species of Botryosphaeriaceae involved. Further research is in progress to elucidate the disease cycle that will underpin development of effective disease management strategies.

\section{Next Generation Sequencing for the optimal detection of viral pathogens in grapevine}

M. AL RWAHNIH (1), S. Daubert (2), D. Golino (1), A. Rowhani (1)

(1) University of California-Davis. Department of Plant Pathology, Davis, CA, U.S.A.; (2) University of California, Department of Plant Pathology, Davis, CA, U.S.A.

Phytopathology 105(Suppl. 4):S4.5

A bioassay is the regulatory standard used to determine the viral phytosanitary status of commercial grapevine propagation material in the U. S. and many other countries around the world. That test is based on the symptoms developed in the field by specific indicator host plants that are graft inoculated from the vines being tested. We compared the bioassay against Next Generation Sequencing (NGS) analysis of grapevine material. NGS is a laboratory procedure that catalogs the genomic sequences of the viruses and other pathogens extracted as DNA and RNA from infected vines. NGS analysis was found to be superior to the standard bioassay in detection of viruses of agronomic significance, including virus infections at low titers. Unlike the bioassay, NGS was not effected by environmental conditions, and was effective in the detection of asymptomatic viral strains. NGS was also found to be superior to the bioassay in its accuracy and comprehensiveness, and in the cost of its analysis. Because the analysis can be completed in a number of weeks, as opposed to years for the bioassay, NGS would also be preferred for the discovery and characterization of novel, uncharacterized viruses. Grapevine reovirus $(\mathrm{GReV})$ has been discovered in grapevine from California by deep sequencing analysis. The source plant (Vitis vinifera) was a Cabernet Sauvignon grapevine (accession CS-Tok3). The GReV genome was characterized from a total nucleic acid extract of bark scrapings that was enriched for double-stranded RNA. The viral genome was found to make up $18 \%$ of the deep sequencing reads from this extract. The sequence homologies of its genomic components with those of other phytoreoviruses ranged from 66 to $30 \%$ for Raspberry latent virus, and from 57 to $22 \%$ for Rice ragged stunt virus, indicating that the novel grapevine reovirus is a distinct viral species.

\section{Description and detection of a novel Reovirus species in Cabernet} grapevines in California

M. AL RWAHNIH (1), S. Daubert (1), D. Golino (1), A. Durvasula (2), A. Rowhani (2)

(1) University of California-Davis. Department of Plant Pathology, Davis, CA, U.S.A.; (2) University of California-Davis. Department of Plant Pathology, Davis, CA, U.S.A.

Phytopathology 105(Suppl. 4):S4.5

The disease symptoms in the original source plant were those of severe grapevine leafroll disease; co-infecting Grapevine leafroll associated viruses -2 and -3 , and Grapevine viruses $A$ and $-B$ were identified in the deep sequencing analysis. Infectivity of the novel reovirus was demonstrated by its detection in a Cabernet franc host after graft-transmission from the source plant by bud chip inoculation. A specific PCR assay was developed from the viral genomic sequence, and used for its detection. The PCR product produced in this assay was sequenced and found to share $100 \%$ sequence identity with viral genomic segment number four, against which the primers were designed.

First report of Pepper vein yellows virus infecting pepper (Capsicum spp.) in the United States

O. J. ALABI (1), M. Al Rwahnih (2), J. L. Jifon (3), L. Gregg (4), K. M. Crosby (5), E. T. Mirkov (1)
(1) Department of Plant Pathology \& Microbiology, Texas A\&M AgriLife Research and Extension Center, Weslaco, TX, U.S.A.; (2) University of California-Davis, Davis, CA, U.S.A.; (3) Department of Horticultural Sciences, Texas A\&M AgriLife Research and Extension Center, Weslaco, TX, U.S.A.; (4) Texas A\&M AgriLife Research and Extension Center, Weslaco, TX, U.S.A.; (5) Department of Horticultural Sciences, Texas A\&M University, College Station, TX, U.S.A.

Phytopathology 105(Suppl. 4):S4.5

The virome of a hot and sweet pepper (Capsicum spp.) field in South Texas showing virus-like symptoms was explored by subjecting cDNA library constructed from total RNA extract of pooled leaf tissue samples to next generation sequencing (NGS) using the Illumina NextSeq 500 platform. The analysis generated $\approx 23.5$ million 76 nt reads of which 5,328 reads mapped to Pepper vein yellows virus (PeVYV; genus Polerovirus). The NGS data was validated by RT-PCR analysis of 18 plants showing symptoms of interveinal yellowing and vein clearing on the same field. Two sets of PeVYV-specific primers amplified $\approx 870 \mathrm{bp}$ of the $\mathrm{P} 0 / \mathrm{P} 1$ proteins and $\approx 580 \mathrm{bp}$ of the RdRP and intergenic noncoding region (NCR) from all samples. BLASTN analysis of gene-specific sequences derived from two representative samples showed that they were specific to PeVYV sharing 93.1-93.6\% and 91-91.5\% nt identities with corresponding $\mathrm{P} 0 / \mathrm{P} 1$-specific nt sequences of isolates from Japan (AB594828) and Spain (HM439608), respectively. Similarly, the RdRP/NCRspecific nt sequences derived in the study shared $99.4-100 \%$ identity, $93.6-$ 93.7\% with corresponding sequences of the isolate from Japan (AB594828) and $94.4-94.8 \%$ with isolate Is (HM439608). This is the first report of PeVYV in the United States and indicates an expanded geographical spread of the virus further highlighting its potential as a worldwide threat to pepper crops.

Microbiota of wild chickpea Cicer reticulatum: The examination of plantmicrobe interactions

B. ALFORD (1), A. Greenspan (1), D. Cook (1)

(1) UC Davis, Davis, CA, U.S.A.

Phytopathology 105(Suppl. 4):S4.5

Soil is among the most complex and understudied of microbial habitats, with 10,000 to 50,000 different species identified in a single gram of soil. Plants depend on soil and the constituent microorganisms for a range of services, including nutrient acquisition. Most crops are cultivated in areas distant from their center of origin. Domestication typically involves reduction in plant genetic diversity, and it is vital to understand the extent to which the structure, diversity and functions of the microbiome have been conserved through domestication. Wild chickpea communities of Cicer reticulatum are the ideal system to elucidate plant microbe interactions associated with domestication. The distribution of C. reticulatum in Turkey has been well characterized, with complete host genomic, soil and climate data. The rhizopheric microbiota was examined utilizing NGS techniques, such as $16 \mathrm{~S}$, on soil close to and associated with different compartments of individual plants, to determine whether the plant enriches microbial communities in the natural environment in repeatable ways. In this study, samples and data were collected from 21 distinct sites containing 9 different populations of C. reticulatum. The $16 \mathrm{~S}$ microbiome data was combined with plant genotype, microclimate data, and soil chemistry measurements to elucidate factors in microbiome selection. This work provides a foundation for understanding the ways in which the plant microbiome contributes to plant health.

Determination of the pathovar status of bacterial strains known to belong to Pseudomonas cannabina

E. I. ALGER (1), I. Rubio (2), P. Goldman (3), J. Elphinstone (4), D. Stead (4), C. T. Bull (5)

(1) Undergraduate Research Opportunities Center, California State University Monterey Bay, Seaside, CA, U.S.A.; (2) Department of Plant Pathology, University of Wisconsin, Madison, WI, U.S.A.; (3) USDA, ARS, Salinas, CA, U.S.A.; (4) The Food and Environment Research Agency, York, United Kingdom; (5) USDA ARS, Salinas, CA, U.S.A.

Phytopathology 105(Suppl. 4):S4.5

Multilocus sequence analysis of Pseudomonas syringae sensu lato indicates that pathogens $P$. syringae pv. coriandricola and $P$. syringae pv. philadelphi, and strains from diseased broad bean (Vicia faba) should be transferred to $P$. cannabina. Comparisons of the host ranges of these pathogens to those of $P$. cannabina pv. cannabina and $P$. cannabina pv. alisalensis are needed to determine whether these represent distinct pathovars. Pathogenicity on broad bean, mock orange (Philadephus coronarius), cilantro (Coriandrum sativum), and rapini (Brassica rapa subsp. rapa) were evaluated to correctly classify these strains. Pathotypes, representative strains of each pathovar, broad bean isolates and a negative buffer control were used to inoculate each plant of interest. Plants were spray-inoculated with bacterial suspensions adjusted to 
$0.6 \mathrm{OD}$ at $600 \mathrm{~nm}$ in sterile $0.01 \mathrm{M}$ potassium phosphate buffer ( $\mathrm{pH} 7.0$ ) and held for 48 hours at $100 \%$ humidity. Bacteria were re-isolated from symptomatic leaf tissue 14 days after treatment. Single colonies were identified by comparison of BOX-PCR DNA fragment-banding patterns to patterns from strains used as inoculum. Results indicated that pathovars were highly virulent on their original hosts and occasionally caused minor to moderate symptoms on reciprocal hosts. These and other data indicated that $P$. syringae pv. coriandricola, P. syringae pv. philadelphi and the broad bean strains should be considered as new pathovars of $P$. cannabina.

\section{The effect of nuclear-cytoplasmic interactions on resistance}

to Parastagonospora nodorum in wheat

A. F. ALHASHEL (1), S. Meinhardt (1), S. F. Kianian (2)

(1) North Dakota State University, Fargo, ND, U.S.A.; (2) Cereal Disease Laboratory, Saint Paul, MN, U.S.A.

Phytopathology 105(Suppl. 4):S4.6

Septoria nodorum blotch (SNB), caused by Parastagonospora nodorum, is a foliar pathogen of wheat and can cause yield losses of up to $50 \%$. So far the most economical and safe way to eliminate these losses has been the discovery of nuclear resistance or sensitivity genes to the host selective toxins which contribute to disease development. Genetic variation is the genome of the cytoplasmic organelles, which modify the nuclear-cytoplasmic (NC) interactions, can affect the phenotypic characteristics of plants including the disease response. In alloplasmic wheat, the wheat mitochondria and chloroplasts has been substituted by those of wild grasses such as Triticum spp. and Aegilops spp, while maintaining the nuclear genome. Alloplasmic wheat line studies have shown the effect of $\mathrm{NC}$ interactions on vigor, fertility, and disease resistance. The scope of this study was to investigate if NC interactions can give rise to increased resistance against two $P$. nodorum isolates (Sn2000, Sn4). Thirty fertile alloplasmic wheat lines were screened on a 5 point scale for their disease response and compared to their euplasmic parental varieties, Chris and Selkirk. Disease ratings were collected at 5, 7, and $10 \mathrm{DAI}$, were analyzed by estimating the relative effect value of each line and the confidence intervals at $\rho=0.05$. Two lines, one with each nucleus and the Ae. bicornis cytoplasm, showed statistically significant reduction in disease seen when compared to euplasmic parents.

Inhibition of Phytophthora spp. by silver nanoparticles synthesized using aqueous extract of Artemisia absinthium

G. S. ALI (1), M. Ali (2), D. Norman (2), M. Brennan (2)

(1) Univ of Florida, Apopka, FL, U.S.A.; (2) University of Florida, Apopka, FL, U.S.A.

Phytopathology 105(Suppl. 4):S4.6

Application of nanoparticles for controlling plant pathogens is a rapidly emerging area in plant disease management, and nanoparticles synthesis methods that are economical and eco-friendly are extensively investigated. In this project, we investigated the potential of silver nanoparticles (AgNPs) synthesized with aqueous extract of Artemisia absinthium L. against several Phytophthora spp., which cause many economically important crop diseases. In in vitro dose-response tests conducted in microtiter plates, $10 \mu \mathrm{g} \mathrm{mL}$ AgNPs inhibited mycelial growth of $P$. parasitica, $P$. infestans, $P$. palmivora, P. cinnamomi, P. tropicalis, $P$. capsici, and $P$. katsurae. Detailed in vitro dose-response analyses conducted with $P$. parasitica and $P$. capsici revealed that AgNPs synthesized with $A$. absinthium extract were highly potent (IC50: 2.1 to $8.3 \mu \mathrm{g} \mathrm{mL}^{-1}$ ) and efficacious $(100 \%)$ in inhibiting mycelial growth, zoospore germination, germ tube elongation, and zoospore production. Interestingly, AgNP treatment accelerated encystment of zoospores. Consistent with in vitro results, in planta experiments conducted in a greenhouse revealed that AgNP treatments prevented Phytophthora infection and improved plant survival. Moreover, AgNP in in vivo experiments did not produce any adverse effects on plant growth. These investigations provide a simple and economical method for controlling Phytophthora with AgNP without affecting normal plant physiology.

Variability of Watermelon mosaic virus isolates collected from cucurbits in Southern United States

A. ALI (1)

(1) Univ of Tulsa, Tulsa, OK, U.S.A

Phytopathology 105(Suppl. 4):S4.6

Cucurbits are economically important cash crops worldwide. In the United States, cucurbits are mostly grown in southern United States. Approximately 60 viruses are known to infect cucurbits and cause significant losses to cucurbit production annually. Watermelon mosaic virus (WMV) is one of the important Potyvirus that infects cucurbits in the US. The purpose of this study was to determine the incidence and variability among WMV isolates collected from field in some parts of southern states. Therefore, more than 700 cucurbit samples showing virus-like symptoms were collected from nine southern States during 2008-2012 growing seasons. All samples were screened against the antisera of WMV by dot-immunobinding assay (DIBA). The results showed that $30 \%$ of the samples were positive to WMV. Total RNA was extracted from randomly selected 57 DIBA positive WMV samples. Coat protein genes of WMV were amplified using high fidelity reverse transcription-polymerase chain reaction (RT-PCR) followed by cloning and sequencing. Phylogenetic analysis of both nucleotide and amino acid sequences of these 57 isolates and previously WMV isolate reported from Florida reported in 1990 showed high degree of variation in nucleotide sequences of $\mathrm{CP}$ genes. When compared with available sequences of WMV isolates in the NCBI database isolates reported, the US isolates showed distinct clusters. These interesting results will be presented and discussed.

Spring and fall structure and dynamics of nematode trophic groups among organically and conventionally managed golf courses

E. ALLAN (1), D. Manter (2), G. Jung (3)

(1) Univ of Massachusetts, Amherst, MA, U.S.A.; (2) USDA ARS, Fort Collins, CO, U.S.A.; (3) University of Massachusetts Amherst, Amherst, MA, U.S.A.

Phytopathology 105(Suppl. 4):S4.6

Nematode communities play important roles in plant health and in predicting the state of the soil food web. On golf courses, plant pathogenic nematodes are often studied because they can cause severe damage to turf stands, especially putting greens, and many of the control products have either been recently banned due to environmental concerns or have low efficacy. Although less studied, the free-living nematodes may be an untapped resource for improving plant health by transferring nutrients, bacteria, and fungi and by consuming or competing with possible plant pathogens. We studied the nematode communities on an organic (non-synesthetic fertilizers and pesticides) and two conventional golf courses in Massachusetts in the spring and fall of 2013 and 2014. We sampled all three management areas: the roughs (lowest management inputs), the fairways, and the putting greens (highest management inputs). Season significantly affected the bacterivores and plant pathogens (highest in spring and fall, respectively). The bacterivores and predators were greatest on the organic course and the plant pathogens were highest on the conventional course. Soil texture did not have a significant effect on nematode community structure and putting greens did not have larger nematode populations than the fairways or roughs as previously speculated.

\section{Colonization of wild potato plants by Streptomyces scabies}

C. ALLEN (1), S. H. Jansky (2), A. O. Charkowski (3)

(1) Univ of Wisconsin, Madison, WI, U.S.A.; (2) University of WisconsinMadison/USDA-ARS, Madison, WI, U.S.A.; (3) University of WisconsinMadison, Madison, WI, U.S.A.

Phytopathology 105(Suppl. 4):S4.6

Common scab, caused mainly by Streptomyces scabies, produces lesions on potato tubers reducing their marketability and profitability. M6 and 524-8 are two closely related inbred diploid lines of the wild potato species Solanum chacoense. After testing in both field and greenhouse assays, it was found that M6 was highly susceptible to common scab while 524-8 was highly resistant. Little is known about how $S$. scabies grows on potato plants. EGFP-labeled $S$. scabies was used to examine the areas of the potato plant that were colonized, whether S. scabies colonization differs on resistant and susceptible plants, and how colonization changes over time. To accomplish, this tubers of M6 and 524-8 were planted in potting mix inoculated with EGFP-labeled S. scabies. Plants were destructively harvested and fluorescence microscopy was used to study colonization of plant tissues by $S$. scabies.

A comprehensive field survey of the sweetpotato potyvirus complex in North Carolina

C. V. ALMEYDA (1), C. L. Ulibarri (1), T. Abernethy (1), J. A. Abad (2), Z. Pesic-VanEsbroeck (1)

(1) North Carolina State University, Raleigh, NC, U.S.A.; (2) USDA-APHIS PPQ FO PGQP, Beltsville, MD, U.S.A.

Phytopathology 105(Suppl. 4):S4.6

Due to limited knowledge about the status of sweetpotato viruses in North Carolina, we conducted virus surveys in sweetpotato plantings at two research stations and in seed production fields during 2012, 2013 and 2014 growing seasons. Susceptible indicator plants (Ipomoea setosa) were placed and replaced weekly in sweetpotato fields and tested for the presence of viruses by multiplex PCR, multiplex and quantitative RT-PCR. Additionally, naturally infected sweetpotato plants (Ipomoea batatas) were collected and virus tested using the same procedures. Results obtained for three consecutive years showed that potyviruses were the most prevalent in sweetpotato fields in 
North Carolina. Single and mixed potyvirus infections were detected in $34 \%$ and $66 \%$ of the samples, respectively. The North Carolina potyvirus complex includes Sweet potato feathery mottle virus (SPFMV), the most prevalent virus of the complex; followed by Sweet potato virus $G$ (SPVG), Sweet potato virus $C$ (SPVC) and Sweet potato virus 2 (SPV2). SPFMV seems to be ubiquitous and is the most predominant in single infections whereas SPV2 is rare. SPFMV, SPVG and SPVC were widespread in double and triple combinations. SPFMV has often masked the presence of these closely related potyviruses and therefore further research is needed to evaluate the role each component plays in the potyvirus complex and its impact on crop production.

Unusually large alphasatellite associated with Cotton leaf curl Gezira virus infecting okra in Jazan, Saudi Arabia

M. A. AL-SALEH (1), A. M. Zakri (2), J. K. Brown (3), A. M. Idris (4)

(1) Department of Plant Protection, College of Food and Agricultural Sciences, King Saudi University, Riyadh, Saudi Arabia; (2) Department of Plant Production, College of Food and Agricultural Sciences, King Saudi University, Riyadh, Saudi Arabia; (3) The University of Arizona, Tucson, AZ, U.S.A.; (4) King Abdullah Univ of Science and Technology, Thuwal, Saudi Arabia

Phytopathology 105(Suppl. 4):S4.7

Cotton leaf curl Gezira Virus (CLCuGeV) is a monopartite begomovirus (Geminiviridae) widespread in the Nile Basin, Sub-Saharan Africa and the Arabian Peninsula. The genome size of $\mathrm{CLCuGeV}$ is $\sim 2.7 \mathrm{~kb}$. This monopartite virus has been typically associated with alphasatellite and betasatellite, each having a genome size ranging between 1.3 and $1.4 \mathrm{~kb}$. Leaves (sample KSA23\} exhibiting curling and vein thickening were collected from okra plants in Jazan, Saudi Arabia during Spring 2011. Total DNA was extracted from leaves and used as template to amplify circular DNA using rolling circle amplification (RCA). Products were digested with Pst to linearize the helper viral genome, and associated alphasatellites, yielding a $2.8 \mathrm{kbp}$ and $1.4 \mathrm{kbp}$ fragment, respectively. The linearized fragments were cloned and sequenced. The $2.8 \mathrm{~kb}$ fragment was identified as the complete $\mathrm{CLCuGeV}$ genome, at $2780 \mathrm{bp}$. It shared $99 \%$ nucleotide (nt) identity with previously studied isolates from the region, including KSA27 [HG530540]. Also, the $1.4 \mathrm{~kb}$ cloned fragments represented three alphasatellites, KSA23-1, KSA23-5, KSA23-65, and one betasatellite, KSA23-8. The genome of alphasatellite KSA23-65 was 1630-bp in size. It was found to be a recombinant containing one fragment (1244-bp) that shared $99 \%$ nt identity with KSA27-3 alphasatellite [HG530556], and another fragment (386-bp) that shared $89 \%$ identity with betasatellite KSA27DI [HG530542], making it the largest alphasatellite reported to date.

First detection of Lucerne transient streak virus infecting alfalfa in the Kingdom of Saudi Arabia (KSA)

I. M. ALSHAHWAN (1), O. A. Abdalla (1), A. Raza (1), M. A. Al-Saleh (1), M. A. Amer (1)

(1) King Saud University, Riyadh, Saudi Arabia

Phytopathology 105(Suppl. 4):S4.7

Alfalfa, the major fodder crop in KSA, is cultivated on around 125,683 hectares with a production of 2,634,603 tons. The objective of this study was to detect Lucerne transient streak virus (LTSV) and characterize it. Alfalfa samples with variable symptoms were collected from plants in the five highly producing regions during 2012-2013 and in three regions during 2014 surveys. DAS-ELISA kits and protocol from AC Diagnostics, Inc. revealed positive results for LTSV in 34 out of 1166 and 55 out of 308 in these alfalfa samples, respectively. Eleven out of twenty three plant species used in the host range studies were positive both by symptoms and ELISA. Chlorotic spots were visible on three indicator plants (Chenopodoium glaucum, C. amaranticolor, and C. album) 15 days postinoculation with LTSV. Later on, these spots were changed into necrotic ones. RT - PCR was used for results confirmation and sequence analysis. Total RNA extraction and RT-PCR confirmation were performed for selected positive alfalfa samples as instructed in their commercial kits. The specific primers employed for LTSV detection were: (5'-GGATCGTTGCTATCTCCAAT-3') and (5'CGAAAACCATCATACGGCTA-3'). Fragments with 1033 base pairs were amplified from all selected samples confirming their infection with LTSV. Purified DNA products are in the process of sequencing for analysing their phylogenetic relationship. This report is the first ever for LTSV as a new emerging disease in KSA.

Sub-lethal doses of fungicide induce resistance emergence in Sclerotinia sclerotiorum

B. S. AMARADASA (1), S. Everhart (1)

(1) University of Nebraska-Lincoln, Lincoln, NE, U.S.A.

Phytopathology 105(Suppl. 4):S4.7
Fungicide resistance has been reported for 203 plant pathogens worldwide, yet mechanisms of fungicide resistance emergence remain relatively unstudied. One potential mechanism is that long-term, sub-lethal fungicide exposure increases mutation rates and subsequent resistance emergence. To test this hypothesis, we used Sclerotinia sclerotiorum as a model organism. Nine susceptible isolates were exposed to long-term, sub-lethal doses of fungicides with different modes of action: Boscalid (respiration inhibitor), iprodione (unclear mode of action), azoxystrobin and pyraclostrobin (quinone outside inhibitors). Fungicides were plated in a logarithmic gradient and mycelia were exposed independently to each fungicide for 12 generations. Effective concentration for $50 \%$ growth inhibition (EC50) was calculated for the first (g1) and twelfth (g12) generations. Results varied for each isolate and fungicide tested. Most isolates exposed to pyraclostrobin did not show a difference in sensitivity, whereas azoxystrobin induced an upward shift in EC50 for four isolates. Eight isolates exposed to Boscalid had higher EC50 values, while two showed increased sensitivity to iprodione, which may be due to measuring error resulting from narrow growth to non-growth interface. Currently underway is assessment of non-coding mutational changes from $\mathrm{g} 1$ to g12 using microsatellite and AFLP genotyping.

Genetic characterization of the Botrytis population from multiple hosts in the Americas

A. AMIRI (1), N. A. Peres (2)

(1) Univ of Florida, Wimauma, FL, U.S.A.; (2) University of Florida, Wimauma, FL, U.S.A.

Phytopathology 105(Suppl. 4):S4.7

Botrytis spp. and especially $B$. cinerea are known to have high genetic diversity because of their wide host range and their mode of reproduction. In this study, 470 isolates from strawberry (330), blueberry (80), tomato (30), grape (20) and eucalyptus (10) collected from flowers, leaves, and fruits from different locations in USA, Canada, and Brazil were investigated. The presence of the transposable elements Flipper and Boty, Botrytis group $\mathrm{S}, B$. caroliniana and $B$. pseudocinerea was determined using markers developed, previously. Neither $B$. caroliniana nor B. pseudocinerea were detected in our population. Isolates of $B$. group $S$, reported previously to be predominant in German strawberry fields, were detected in all countries and on all hosts except grape. However, their frequency was lower than $20 \%$ versus 80 to $100 \%$ of $B$. cinerea on different hosts. Transposa isolates containing Flipper and Boty, simultaneously, were predominant $(>80 \%)$ in all hosts except blueberry $(47 \%)$. Interestingly, Boty isolates were more frequent $(62 \%)$ in isolates from Canada versus only 27 and $25 \%$ in Brazil and USA, respectively. Vacuma isolates without Flipper and Boty were detected only in strawberry from Canada and USA and at a low frequency $(<1.5 \%)$. These results indicate that isolates with a different genetic background are being introduced every year from nurseries and potentially from other hosts to commercial strawberry fields.

Postharvest quarantine treatments for Diaphorina citri on infested curry leaves

D. ANCO (1), G. Poole (2), T. Gottwald (3)

(1) NCSU/USDA ARS, Fort Pierce, FL, U.S.A.; (2) UF, Fort Pierce, FL, U.S.A.; (3) USDA ARS, Fort Pierce, FL, U.S.A.

Phytopathology 105(Suppl. 4):S4.7

To reduce spread of huanglongbing, studies were conducted to evaluate treatments that reduce survival and attachment of nymphs of the vector Diaphorina citri on infested curry leaves (Bergera koenigii). Decontamination of curry leaves infested with $D$. citri in relation to disinfectant (none or ProSan), temperature $\left(0,40\right.$, and $\left.50^{\circ} \mathrm{C}\right)$, and treatment duration $(0,5,10$, and 20 min) was examined using a split-split plot design. Experiments were performed three times. Treatment duration did not significantly affect $D$. citri nymph survival or removal $(P>0.2)$. Temperature and disinfectant each significantly affected $D$. citri nymph survival and removal $(P<0.031)$. The interaction of temperature and disinfectant was significant with respect to nymph survival $(P<0.0001)$ but did not significantly affect removal $(P=$ $0.4589)$. Tissue damage was significantly affected by temperature $(P=$ $0.0056)$, duration $(P=0.0023)$, the interaction of temperature and duration $(P$ $=0.0320)$, and the interaction of disinfectant, temperature, and duration $(P=$ 0.0410 ). Of the treatments resulting in $100 \% D$. citri nymph mortality on infested curry leaves, $40^{\circ} \mathrm{C}$ for $5 \mathrm{~min}$ with Pro-San was accompanied with the least proportion curry leaf tissue damage ( 0.14 greater than untreated control, $P=0.25$ ). Results from these studies may be useful in formulation of future regulatory policies regarding trade of citrus foliage, especially those used as condiments, to reduce spread of huanglongbing. 
Current status and integrated management of Sugarcane brown rust (Puccinia melanocephala), and orange rust (Puccinia kuehnii) in Colombia

J. C. Angel (1), J. I. Victoria (1), M. Cadavid (1), C. A. ANGEL (1)

(1) Colombian Sugarcane Research Center - CENICAÑA, Cali, Colombia Phytopathology 105(Suppl. 4):S4.8

Sugarcane is intensively grown at the Colombia's Cauca river valley in 230,000 hectares, producing 24 million tons of cane, 2.4 million tons of sugar, 406 million liters of ethanol, 6 million tons of bagasse, and cogenerated 215 MWH of power. Brown rust (BR) (P. melanocephala) arrived in 1978 causing epidemics in susceptible varieties CP 57-603, MZC 74-275, and Mex 52-29. Orange rust (OR) (P. kuehnii) was found in 2010 on experimental clones, and infects few varieties. This work illustrates the integrated management used for $\mathrm{BR}$ and $\mathrm{OR}$, mainly based on resistant varieties developed by Cenicaña Colombia (CC). Early varieties were replaced by CC 85-92, CC 84-75, reaching almost $90 \%$ of the area; however, since 2007 BR infections have increased, breaking the resistance in some environments. A substitution plan is in progress with more than 30 varieties being planted according to sitespecific agriculture criteria. CC $85-92$ is down to $52 \%$ of the area and CC $84-$ 75 to $4.5 \%$, replaced by CC $93-4418$ (12.5\%), CC 01-1940 (11.8\%), and CC 93-4181 (1.4\%), among others. Experiments on OR and BR effects, applying fungicides, biologicals, and fertilizers did not show statistical differences in cane and sugar produced. Most new varieties planted in the region are BR and OR resistant and no economic impact has been found yet. Continue evaluations of OR and BR on breeding, nursery, and commercial fields indicates disease advance, and those varieties to propagate, relocate, and replace.

\section{Mapping of unique and broad spectrum resistance genes to Zymoseptoria tritici in durum wheat}

L. AOUINI (1), M. Maccaferri (2), R. Tuberosa (2), A. Prodi (2), S. Stefanelli (2), S. Hamza (3), G. H. Kema (4)

(1) Plant Research International- Wageningen University, Wageningen, Netherlands; (2) DISTA, Viale Fanin 44, University of Bologna, 40127 Bologna, Italy, Bologna, Italy; (3) National Institute of Agronomy of Tunisia, INAT, 43 Avenue Charles Nicolle, 1082 Tunis, Tunisia., Tunis, Tunisia; (4) Plant Research International B.V., 6700 AA Wageningen, The Netherlands, Wageningen, Netherlands

Phytopathology 105(Suppl. 4):S4.8

Very little progress was made in the understanding of the genetic basis of resistance in durum wheat to Zymoseptoria Tritici (Z. tritici). In our study, we explored the durum wheat resistance to $Z$. tritici in three recombinant mapping populations developed in Italy and Tunisia. All seedling experiments were performed under controlled conditions at Wageningen University, while adult test were either performed in Italy for Kofa/Svevo and Simeto/Levante, or in Tunisia for Khiar/Agili 39. In all populations we identified major QTLs conferring either partial or complete resistance to Z. tritici. New locations were identified on chromosome $4 \mathrm{~B}$ in Kofa/Svevo conferring partial resistance in the adult stage, and on chromosome $6 \mathrm{~B}$ for partial seedling resistance in Simeto/Levante. Other QTLs reported in bread wheat were also identified in both populations. Interestingly, we also identified a major QTL in Khiar/Agili 39 on chromosome 2B, which was effective in the adult stage in field tests across F6-F9 generations as well as against eight $Z$. tritici isolates from various origins in the seedling stage. Another unknown major QTL was mapped on chromosome $1 \mathrm{~A}$, which provided resistance to three $Z$. tritici isolates in the seedling stage. Hence, our study showed the existence of novel and wide spectrum resistance alleles to $Z$. tritici in durum wheat that can be used to mitigate the impact of $Z$. tritici in this important food crop.

\section{Root rot-like symptoms caused by grain-based inoculum substrates}

G. ARALDI DA SILVA (1), L. F. S. Leandro (1), D. S. Mueller (1)

(1) Iowa State University, Ames, IA, U.S.A.

Phytopathology 105(Suppl. 4):S4.8

Non-infested, grain-based inoculum substrates can cause soybean (Glycine max) root discoloration that can be confounded with rot caused by pathogens. Fusarium oxysporum, Pythium sylvaticum and Rhizoctonia solani were grown on perlite-cornmeal, millet grain, white rice, and sorghum grain. Soybean seeds were planted in a sand-soil mix amended with non-infested $(3 \%$ and $5 \%, \mathrm{v}: \mathrm{v})$ or infested ( $3 \% \mathrm{v}: \mathrm{v})$ substrates. Root discoloration and plant growth were evaluated 30 days after planting. All non-infested substrates caused root discoloration at both rates, with millet showing less discoloration $(P=0.0004)$ than the other substrates at the $5 \%$ rate. Plant growth was not affected by substrates. For substrates infested with $F$. oxysporum, millet, rice and sorghum caused the most severe root discoloration $(P<0.0001)$, and rice caused the smallest root length $(P<0.0001)$ and weight $(P=0.0034)$. No difference was observed for shoot height and weight. For $P$. sylvaticum, millet grain caused the most severe root discoloration $(P<0.0001)$, and substrates did not affect plant growth. For $R$. solani, there was no difference in root discoloration among infested substrates, but plant growth was smallest $(P<0.0038)$ with millet, perlite-cornmeal and rice. Our findings confirm that non-infested substrates cause root rot-like symptoms that can be mistaken for disease. Millet grain was less likely to cause root discoloration and was an effective inoculum carrier for the three pathogens studied.

Genome-informed diagnostics to discriminate geographically isolated populations of the select agent Rathayibacter toxicus

M. ARIF (1), G. Y. Busot (1), R. Mann (2), B. Rodoni (2), S. Liu (3), J. P. Stack (1)

(1) Department of Plant Pathology, Kansas State University, Manhattan, KS, U.S.A.; (2) Department of Primary Industries, La Trobe University, Bundoora, Australia; (3) Department of Plant Pathology, Kansas State University, Manhattan, Australia

Phytopathology 105(Suppl. 4):S4.8

Rathayibacter toxicus is a toxin-producing, gram-positive bacterial pathogen of annual ryegrass (Lolium rigidum) and a select agent in the United States. Livestock deaths in Australia due to consumption of contaminated ryegrass have raised concerns about the global spread of $R$. toxicus in ryegrass hay and seed. A method for early and accurate detection of $R$. toxicus is needed. Whole genome sequences (Illumina; MiSeq) were generated for representative isolates from different geographic areas. The genome assemblies of different isolates were used to design target-specific primers and probes. A sensitive qPCR assay based on an rpoD region was developed to detect all $R$. toxicus isolates. A multiplex PCR based on genome-informed strategically identified genes was developed to discriminate among three genetically-distinct populations of $R$. toxicus. Each amplification assay included a multi-target artificial internal control to enhance reliability and accuracy. The detection assays were validated in silico and in vitro with 64 DNAs of $R$. toxicus, other Rathayibacter species and Dietzia cinnamea for specificity; they accurately detected $R$. toxicus from infected annual ryegrass and successfully identified the $R$. toxicus population type. These sensitive detection methods are easy to implement and will be appropriate for routine diagnostics, pathogen and population specific surveys, disease management, and biosecurity decisions to support export/import of annual ryegrass.

Assessing the benefits of disease management by interpreting an ecological survey of grapevine viruses when contrasted with "planting booms"

K. ARNOLD (1), D. Golino (1), M. Cooper (2), R. Smith (3), V. Klaassen (4), N. McRoberts (1)

(1) University of California-Davis. Department of Plant Pathology, Davis, CA, U.S.A.; (2) University of California Cooperative Extension, Napa, CA, U.S.A.; (3) University of California Cooperative Extension, Santa Rosa, CA, U.S.A.; (4) University of California-Davis. Foundation Plant Services, Davis, CA, U.S.A.

Phytopathology 105(Suppl. 4):S4.8

Grape virus diseases are economically caustic to industry. Readily spreading vector transmitted viruses like GLRaV-3 can make the use of certified planting material seem futile when planting certified material near infected material. We conducted a multi-virus survey in 2014 in the north coast wine growing region of California in order to assess the benefits of disease virus management. We surveyed 112 vineyard blocks stratified in age to capture historical "planting booms". Age classes were: 1880-1980 (old material); 1981-1995, replants due to AXR\#1 rootstock removal due to phylloxera failure; 1996-2010 representing blocks culled in response to virus infected field selections grafted onto rootstocks not tolerant of viruses present in the material, and 2011-2014 representing a group of "new" material. 27-29 blocks were randomly selected in each age class and sampled using a simple random sampling strategy, in a "W" pattern to ensure subsample separation. Subsample intensity was dependent upon block acreage, and subsamples were tested using qRT-PCR for eight different viruses. The resulting disease incidence data are interpreted in relation to changes in virus distributions over time and the positive impact of grape certification and clean plant programs. Shifts in distributions of virus incidence are modeled by fitting disease incidence data to Beta distributions and plotting the trajectory of disease incidence in the Beta distribution parameter space.

The fate of aflatoxins associated with contaminated crops in soil

L. R. L. ARONE (1), R. Jaime (2), R. Ranajit Bandyopadhyay (3), P. J. Cotty (4) (1) University of Arizona, Tucson, AZ, U.S.A.; (2) School of Plant Sciences, University of Arizona, Tucson, AZ, U.S.A.; (3) International Institute of Tropical Agriculture (IITA), Ibadan, Nigeria; (4) Agricultural Research Service, USDA / School of Plant Sciences, University of Arizona, Tucson, AZ, U.S.A.

Phytopathology 105(Suppl. 4):S4.8 
Aflatoxins are potent mycotoxins that contaminate foods and feeds worldwide causing immune system suppression, stunting, cancer, and death in humans and domestic animals. Frequently, crops with severe aflatoxin contamination are incorporated into soil because such crops have no available markets. The current study was undertaken to establish the extent to which the process of plowing contaminated crops under influences both the long-term residence of aflatoxin-producers in agricultural soils and the rate of aflatoxin degradation. Improved understanding of the speed of degradation of aflatoxin contaminated crops may facilitate aflatoxin management. Elucidation of influences of soil incorporation on long-term survival of aflatoxin producers may clarify the value of agronomic practices for aflatoxin management and provide practices to optimize biocontrol with atoxigenic strains of Aspergillus flavus. Naturally contaminated cottonseed meal containing over $50 \mathrm{mg} / \mathrm{kg}$ aflatoxin $\mathrm{B} 1$ was sandwiched between layers of soil from an Arizona cotton field and incubated for 14 days at $15^{\circ} \mathrm{C}$. Both aflatoxins and A. flavus propagules were influenced by incubation with soil while the overall quantity of bacteria and fungi in the soil increased. The results suggest that the practice of incorporation of contaminated crops into agricultural soils can be manipulated to optimize long-term aflatoxin management.

Ultraviolet action spectrum against Oidium neolycopersici, the cause of powdery mildew in tomato

S. ARUPPILLAI (1), S. Arne (2), S. A. Knut (3), G. R. Hans (1)

(1) Department of Plant Sciences, Norwegian University of Life Sciences, Aas, Norway; (2) Bioforsk, Norwegian Institute for Agricultural and Environmental Research, Aas, Norway; (3) Department of Ecology and Natural Resource Management, Norwegian University of Life Sciences, Aas, Norway

Phytopathology 105(Suppl. 4):S4.9

Previous experiments with broad spectrum ultraviolet (UV) sources indicated that UV radiation above approximately $290 \mathrm{~nm}$ are inefficient against rose powdery mildew, whereas detailed action spectra for various developmental responses of the fungus are missing. Conidial germination, hyphal length, penetration attempt, and successful infection of tomato powdery mildew, Oidium neolycopersici, were examined at the Petri dish level with and without leaf disks of tomato cv. Espero. Inoculated samples were treated with UV $\left(1.04 \pm 0.05 \mu \mathrm{mol} / \mathrm{m}^{2} / \mathrm{s}\right)$ radiation at wavelengths of $250,260,270,280,290$, $300,310,320,350$ or $400 \mathrm{~nm}$, with exposure duration of 1, 12, or $24 \mathrm{~min}$. The effective wavelength range of UV radiation was $\leq 280 \mathrm{~nm}$ and this significantly reduced conidial germination, hyphal expansion, penetration attempt and successful infection at all exposure times. There was no effect of $\mathrm{UV} \geq 310 \mathrm{~nm}$, while there was a transition phase between 280 and $310 \mathrm{~nm}$, where the effect depended on duration of exposure. The effective range of UV was extended up to $290 \mathrm{~nm}$ if samples were exposed to 12 or $24 \mathrm{~min}$. Precise action spectra for UV efficiency against $O$. neolycopersici provides the knowledge for efficient disease control in practice.

Relationship of hydrophobicity of powdery mildew conidia to selfcleaning properties of strawberry leaves and management of the pathogen with water

B. ASALF (1), D. M. Gadoury (2), A. Stensvand (3)

(1) Norwegian Institute for Agricultural and Environmental Research (Bioforsk), Aas, Norway; (2) Cornell University, Geneva, NY, U.S.A.; (3) Bioforsk Norwegian Inst of Agric \& Env Res, Aas, Norway

Phytopathology 105(Suppl. 4):S4.9

Powdery mildew (Podosphaera aphanis) is a destructive pathogen of strawberry, particularly in glasshouse and high tunnel production systems. Spraying plants with water has been reported to decrease severity of various powdery mildews, but the mode of action of such treatments has not been conclusively demonstrated. We conducted experiments on the mode of action of water sprays in the laboratory wherein we determined the effect of free water on the attachment and retention of conidia on strawberry leaves, plastic and glass surfaces. In addition, we conducted experiments in high plastic tunnel to determine the effect of water sprays on powdery mildew development. Conidia were not only highly hydrophobic, but exhibited a high affinity with each other and form an aggregation or network on water droplets, which tended to fall off the leaf surface during showering events. Overhead sprinkling for 1 minute 4 times per day produced a degree of disease suppression comparable to that achieved through use of several commercial fungicides. Frequent overhead sprinkler resulted in a self-cleaning effect that prevented conidia from attaching to leaf surfaces and reduced conidia dispersal on already established colonies. Systematic overhead application of water to strawberry plants could help to manage the pathogen in glasshouse, high tunnel, and possibly in field production systems and nurseries.
Simultaneous detection of two viruses and one viroid infecting dahlias by multiplex RT-PCR and their distribution in Japanese dahlias

S. ASANO (1), Y. Matsushita (2), Y. Hirayama (1), T. Naka (3)

(1) Nara Prefectural Agricultural Research and Development Center, Kashihara, Japan; (2) NARO Institute of Floricultural Science, Tsukuba, Japan; (3) Nara Prefectural Agricultural Research and Development Center, Nara, Japan

Phytopathology 105(Suppl. 4):S4.9

In Japan Tomato spotted wilt virus (TSWV), Dahlia mosaic virus (DMV), and Chrysanthemum stunt viroid (CSVd) are prevalent and thought to decrease productivity of commercially cultivated dahlia crops. It is important to develop a sensitive and rapid method to detect viruses and viroids in order to ensure the selection and propagation of healthy dahlias. Thus, we selected the reliable sets of primer and developed a multiplex RT-PCR that simultaneously detects TSWV, DMV, and CSVd infections in dahlias. The sensitivity of multiplex RT-PCR for TSWV, DMV and CSVd was the same as that obtained using single RT-PCR. In addition, a simple RT-PCR method that does not require RNA extraction microtissue direct RT-PCR (Hosokawa et al. 2006), could be used to prepare samples for analysis by this multiplex RT-PCR. This method could detect latent infection of each virus and viroid. A field survey validated our results, indicating that TSWV was the dominant virus found in the Kansai region, DMV in the Tohoku and Kyushu regions, and CSVd in the Hokkaido region. This method represents a rapid, sensitive, and cost effective approach to diagnose viral infections in dahlias.

Survey of virus diseases of taro, Colocasia esculenta, in American Samoa N. ATIBALENTJA (1), E. M. Ilaoa (1), S. T. Fiafia (1), I. B. Gurr (1)

(1) American Samoa Community College, Division of Community and Natural Resources, Pago Pago, U.S.A.

Phytopathology 105(Suppl. 4):S4.9

Taro, Colocasia esculenta, is the most important staple crop in the U.S. territory of American Samoa, where it also serves in many cultural functions. In this South Pacific archipelago, taro diseases caused by viruses come second only to taro leaf blight (Phytophthora colocasiae), which devastated taro production in the early 1990s. This study investigated the incidence of taroinfecting viruses in the two major taro growing islands of American Samoa: Tutuila and Aunu'u. Leaf samples were collected from each of 164 symptomatic taro plants in both islands. Total genomic DNA and RNA were extracted from each sample, and the extracts were assayed by PCR and RTPCR for each of five viruses known to infect taro worldwide. Dasheen Mosaic Virus (DsMV) and Taro Bacilliform Virus (TaBV) were the most prevalent viruses, infecting $80 \%$ and $6 \%$, respectively, of 96 plants examined in Tutuila, compared to $76 \%$ and $28 \%$, respectively, of 68 plants in Aunu'u. Many of the plants that tested positive for DsMV also were co-infected with TaBV. Cultivar 'Palau 10' was particularly susceptible to DsMV in Tutuila, with $100 \%$ of 22 plants testing positive. In Aunu'u, 'Talo Manua' was the most susceptible to both DsMV and TaBV, with $100 \%$ and $38 \%$ of 13 plants, respectively, testing positive. This is the first report on the incidence of taro diseases caused by viruses in the Aunu'u Island.

Effect of temperature and water potential on the hyphal growth rate of Fusarium and Rhizoctonia pathogens of wheat

I. S. AUJLA (1), T. C. Paulitz (2)

(1) Washington State University, Pullman, WA, U.S.A.; (2) USDA ARS, Pullman, WA, U.S.A.

Phytopathology 105(Suppl. 4):S4.9

Temperature and moisture have profound influences on the activity of Fusarium culmorum, F. pseudograminearum, Rhizoctonia solani AG-8 and $R$. oryzae causing crown and root rots of wheat respectively, in the dryland wheat production area of the Pacific Northwest region of the United States. This study determined the effects of temperature and water potential on the mycelial growth of these wheat pathogens on potato dextrose agar adjusted to different osmotic and matric potentials $(-0.13$ to $-10 \mathrm{MPa})$ with sodium chloride, potassium chloride, and polyethylene glycol (PEG-8000), and incubated at temperatures ranging from 4 to $35^{\circ} \mathrm{C}$. Fusarium spp. grew optimally at $20-25^{\circ} \mathrm{C}$ and -1 to $-3 \mathrm{MPa}$. A decline in growth rate was observed at lower water potentials, but growth rates were $0.07-3.34 \mathrm{~mm} /$ day even at $-9 \mathrm{MPa}$. Rhizoctonia solani AG-8 was more restricted for optimal growth at $20-25^{\circ} \mathrm{C}$ and $-0.13 \mathrm{MPa}$. The optimal growth of $R$. oryzae occurred at $30^{\circ} \mathrm{C}$ and $-0.13 \mathrm{MPa}$, but the growth rate declined less compared to AG-8 with lower water potential and temperature. $R$. oryzae was the only pathogen to grow at $35^{\circ} \mathrm{C}$ where the optimum water potential was $-2 \mathrm{MPa}$, compared to $-0.13 \mathrm{MPa}$ at temperatures lower than $35^{\circ} \mathrm{C}$. The effect of water potential was independent of salt composition. This study contributes to the knowledge of the biology and epidemiology of these pathogens, and may be useful in predicting their potential distribution under future climate scenarios. 
Effects of inoculum concentration, temperature, and interrupted wetness periods on infection of boxwood by Calonectria pseudonaviculata H. F. AVENOT (1), T. P. Edwards (1), A. Baudoin (1), C. Hong (2)

(1) Virginia Tech, Blacksburg, VA, U.S.A.; (2) Virginia Polytechnic Institute and State University, Virginia Beach, VA, U.S.A.

Phytopathology 105(Suppl. 4):S4.10

Boxwood blight caused by Calonectria pseudonaviculata causes lesions on twigs and leaves, with defoliation occurring from the bottom branches upward. In growth chamber experiments, boxwood blight incidence (\% of infected leaves) on the susceptible cv. Suffruticosa increased with increasing inoculum dose from $1.25 \times 10^{3}$ to $2 \times 10^{4}$ conidia $/ \mathrm{ml}$ at 18,23 , and $25^{\circ} \mathrm{C}$, with most lesions formed at $25^{\circ} \mathrm{C}$ and least at $18^{\circ} \mathrm{C}$. No disease developed at $29^{\circ} \mathrm{C}$ regardless of inoculum dose. When infection periods composed of an initial 8 $\mathrm{h}$ of wetness were followed by a dry interruption of 3-20 $\mathrm{h}$ followed by a final $12-\mathrm{h}$ wetness period, a dry interruption of as little as $3 \mathrm{~h}$ reduced disease incidence on Suffruticosa plants from $50-82 \%$ (continuous wetness) to $10 \%$. The relationships between the development of boxwood blight on susceptible trap plants and the occurrence of infection criteria were also investigated in a naturally infected field during the 2014 season. Prolonged wetness duration caused modest disease development, but heavy rain followed by prolonged wetness produced a large outbreak, indicating that rainfall plays a major role in determining disease intensity in the field. These results are crucial in developing a reliable disease forecasting model for boxwood blight.

Pathogenicity and virulence of Zymoseptoria tritici with mutations conferring resistance to multiple fungicide modes of action

C. AVILA-ADAME (1), P. Gandra (2), J. Cao (2), R. Ponnala (2), T. Slanec (2) (1) Dow AgroSciences LLC, Indianapolis, IN, U.S.A.; (2) Dow AgroSciences, Indianapolis, IN, U.S.A.

Phytopathology 105(Suppl. 4):S4.10

Septoria leaf blotch (SLB) caused by the fungus Zymoseptoria tritici is the most aggressive disease of winter wheat in various regions of the world. Over the years, SLB has been managed with fungicides differing in mode of action (MOA), including beta-tubulin, cytochrome b-Qo, and 14-alpha demethylase inhibitors. Concurrently, the fungus developed some level of resistance to all of these fungicides. To determine if accumulation of mutations conferring resistance to multiple MOA impairs the fungus in its ability to cause disease, 11 isolates from 5 countries were subjected to 4 cycles of infection on wheat seedlings. Next generation sequencing analysis showed that 5 isolates carried the E198A mutation that confers resistance to beta-tubulin inhibitors as well as the G143A mutation associated with resistance to Qo fungicides. In addition, they also carried multiple mutations in the CYP51 gene decreasing sensitivity to 14-alpha demethylase inhibitors. Four other isolates differed because they had mutations associated with resistance to one or two fungicide classes but not to all three. The remaining 2 isolates were sensitive to all three fungicide classes. The resistant isolates infected wheat and caused the typical disease symptoms produced by the sensitive isolates. Two isolates resistant to all three fungicide classes were less virulent than the sensitive ones but overall the relationship between resistance phenotype and virulence was unclear.

Genomic characterisation and development of TaqMan ${ }^{\circledR}$ Real-Time RTPCR assay for Rose rosette virus

B. BABU (1), B. K. Washburn (2), K. Poduch (2), T. S. Schubert (3), C. Baker (3), J. Ayyemperumal (3), D. Jones (3), G. W. Knox (1), M. L. Paret (1) (1) North Florida Research \& Education Center, University of Florida, Quincy, FL, U.S.A.; (2) Department of Biological Science, Florida State University, Tallahasse, FL, U.S.A.; (3) Florida Department of Agriculture and Consumer Service, Department of Plant Industry, Gainesville, FL, U.S.A. Phytopathology 105(Suppl. 4):S4.10

Roses are one of the most important ornamental flowering shrubs grown worldwide. Among the major diseases of roses, the Rose rosette disease caused by Rose rosette virus (RRV) (genus Emaravirus) has been reported to be the most destructive. Genomic characterisation of RRV using RT-PCR primers corresponding to the conserved inverted 13 nucleotides found at the 5 ' and 3' termini, revealed two additional novel RNAs- RNA5 and RNA6, as compared to the previously described four RNA segments. RACE analysis showed that the novel RNAs share a high similarity in structural organisation to other RRV RNA segments. Northern blot as well as RT-PCR using specific primers also indicated the presence of novel RNA segments in infected rose and absent in non-symptomatic plants. Bioinformatic analysis of RNA5 and RNA6 did not reveal any potential function. Genomic characterisation of these novel RNAs would provide a better understanding of RRV biology, and to devise suitable management strategies. For the purpose of RRV indexing of roses, a specific and sensitive qRT-PCR assay, using primers/probes based on multiple genomic regions has been developed. Testing of the primers/probes against several rose infecting viruses indicated high specificity. Plasmid dilution assay indicated that the primer/probe could detect up to a template concentration of $1 \mathrm{fg} / \mu \mathrm{L}$. Thus the qRT-PCR assay could be used in the detection of the virus in both symptomatic and non-symptomatic infected samples.

Sharpshooters may be "flying syringes:" First direct evidence of mixed egestion and salivation in inoculation of Xylella fastidiosa

E. A. BACKUS (1), E. E. Rogers (1)

(1) USDA-ARS, SJVASC, Parlier, CA, U.S.A.

Phytopathology 105(Suppl. 4):S4.10

Despite nearly 70 years of research, the inoculation mechanism of Xylella fastidiosa $(\mathrm{Xf})$ by its sharpshooter vectors remains unproven. $\mathrm{Xf}$ is unique among insect-transmitted plant pathogens because it is propagative but noncirculative, adhering to and multiplying on the cuticular lining of the anterior foregut. A noncirculative mechanism for inoculation of Xf must explain how bacterial cells exit the vector's stylets via the food canal and directly enter the plant. A combined egestion-salivation mechanism has been proposed to explain these unique features. Egestion is the putative outward flow of fluid from the foregut via hypothesized bidirectional pumping of the cibarium (part of the foregut). The present study traced green fluorescent protein-expressing $\mathrm{Xf}$ or fluorescent nanoparticles acquired from artificial diets by glassy-winged sharpshooters as they were egested into simultaneously secreted saliva. Xf or nanoparticles were shown to mix with gelling saliva to form fluorescent deposits and salivary sheaths on artificial diets, providing the first direct, conclusive evidence of egestion by any hemipteran insect. Therefore, the present results strongly support an egestion-salivation mechanism of $\mathrm{Xf}$ inoculation. Also, a column of fluid, potentially containing suspended bacteria, may be held in the foregut during the vector's transit from plant to plant. Thus, sharpshooters could be true "flying syringes," a new paradigm for $\mathrm{Xf}$ inoculation

Bacillus mojavensis biofilm formation and biosurfactant production using a laser ablation electrospray ionization system

C. W. BACON (1), D. M. Hinton (2), T. R. Mitchell (3)

(1) USDA ARS, US National Poultry Research Center, Athens, GA, U.S.A.;

(2) USDA, ARS, US National Poultry Research Center, Russell Research Center, Athens, GA, U.S.A.; (3) USDA, ARS, US National Poultry Research Center, Athens, GA, U.S.A.

Phytopathology 105(Suppl. 4):S4.10

Biofilms are important extracellular polymeric compounds produced by bacteria that are useful for developmental phases including motility, swarming, signaling processes, and for hydrophobic nutrient utilization, all of which are important attributes for endophytic bacteria with biocontrol potential. Therefore, it is important to determine the ability of strains used for biocontrol purposes to produce biofilms. Laser ablation electrospray ionization (LAESI) MS technology is a modern method that allows the in situ molecular cell imaging of tissues, resulting in a fast, accurate, noninvasive measurement and chemical analyses of microbes and hosts parasitic interactions. An endophytic biocontrol bacterium, Bacillus mojavensis, was analyzed by LAESI MS to determine biofilm production relative to biosurfactants, and antagonisms to the maize pathogen Fusarium verticillioides. Nine strains were grown in stationary cultures to determine biofilm development and correlated with biosurfactants (surfactin and fengycin). The results indicated that all strains were motile and developed biofilms at the air-liquid interface of the culture medium. The data indicated that the in vitro production and biofilms could not be correlated to antagonism since all strains are antagonistical to $F$. verticillioides. However, it may be possible that homologues and concentrations of surfactin and fengycin and other unknown biosurfactants are interactive in the control of this pathogen.

Role of organic amendments and microbial antagonists on the growth and flowering of Zinnia elegans

R. Badar (1), V. SULTANA (2), J. Ara (3), S. Ehteshamul-Haque (4)

(1) Department of Botany, Jinnah University for Women, Karachi, Karachi, Pakistan; (2) Department of Biochemistry, University of Karachi, Karachi, Karachi, Pakistan; (3) Department of Food Science \& Technology, University of Karachi, Karachi, Pakistan; (4) Department of Botany, University of Karachi, Karachi, Pakistan

Phytopathology 105(Suppl. 4):S4.10

Zinnia elegans, a wonderful cut flower is gaining popularity in Pakistan because of its economic value. In this study, effect of soil amendment with Stokeyia indica a brown seaweed and coconut coir was evaluated on the growth and flowering of Zinnia alone or with Pseudomonas aeruginosa or Trichoderma viride in screen house. Greater shoot length and fresh weight was produced by $P$. aeruginos $a$ and $T$. viride used in coir amended soil. Seaweed also produced taller plants with significantly greater fresh weight as 
compared to untreated control. Highest number of flowers and leaves were found in plants grown in seaweed amended soil. Total amount of chlorophyl and carbohydrate in leaves were also found significantly higher in coir or seaweed treatment alone or with $P$. aeruginosa or $T$. viride as compared to untreated control and diammonium phosphate (DAP) treatment used as positive control. Role of coconut coir and seaweed alone or with microbial antagonists seems promising for better growth and flowering of Zinnia elegans.

\section{Emerging tomato viruses in Florida}

I. E. Badillo-Vargas (1), C. A. Baker (2), G. Frantz (3), H. C. Mellinger (3), J. E. Funderburk (1), S. ADKINS (4)

(1) UF-NFREC, Quincy, FL, U.S.A.; (2) FDACS-DPI, Gainesville, FL, U.S.A.; (3) Glades Crop Care, Inc., Jupiter, FL, U.S.A.; (4) USDA ARS USHRL, Fort Pierce, FL, U.S.A.

Phytopathology 105(Suppl. 4):S4.11

Tomato spotted wilt virus (TSWV) causes crop losses worldwide. This tospovirus is well-known for disease epidemics in vegetable, ornamental and peanut crops in the southeastern U.S. Two other tospoviruses have recently emerged in south Florida. Groundnut ringspot virus (GRSV) was first detected in tomato in late 2009 and subsequently in pepper, tomatillo and eggplant. Tomato chlorotic spot virus (TCSV) was detected in tomato in 2012, and subsequently in pepper. The host and geographic ranges of GRSV and TCSV have continued to expand. During surveys for tospoviruses in 2013 and 2014, fresh market tomato plants were observed with necrosis of leaves, petioles and stems, and necrotic rings or spots on fruits in south Florida. No TSWV, GRSV or TCSV was detected in these symptomatic tomato plants. Mechanical inoculation of tomato plants with symptomatic field tissue reproduced symptoms originally observed. Sequence analysis of replicase protein 1a, movement protein and coat protein genes indicated the presence of a virus that was most closely related to, but distinct from, Tulare apple mosaic virus and other subgroup 2 ilarviruses. Less identity was observed with subgroup 1 ilarviruses previously reported to infect tomato. These results indicate that virus-like symptoms observed in tomato in Florida since fall 2013 are caused by a novel subgroup 2 ilarvirus, for which the name Tomato necrotic streak virus (TomNSV) is proposed.

Detection of a new luteovirus in imported nectarine trees: A case study to propose adoption of metagenomics in post-entry quarantine

S. BAG (1), M. Al Rwahnih (1), A. Li (2), A. Gonzalez (2), A. Rowhani (1), J. K. Uyemoto (2), M. R. Sudarshana (2)

(1) University of California-Davis, Davis, CA, U.S.A.; (2) USDA-ARS, Davis, CA, U.S.A.

Phytopathology 105(Suppl. 4):S4.11

In spring 2013, five year-old nectarine (Prunus persica) trees, grafted on Nemaguard rootstock were found stunted in an orchard in California. These trees had been propagated from budwood of three nectarine varieties imported from France cleared through post-entry quarantine procedure. Examination of the canopy failed to reveal any obvious symptoms, but the examination of the trunks, after stripping the bark, revealed extensive pitting on the woody cylinder. To investigate the etiological agent, a cDNA library was prepared from dsRNA extracted from bark scrapings from the scion and rootstock portions, and sequenced using Illumina platform. BLAST analysis of the contigs generated by the de novo assembly of sequence reads indicated the presence of a novel luteovirus. Complete sequence of the viral genome was obtained by sequencing of three overlapping cDNA clones generated by RTPCR and amplification of 5'- and 3'- termini. The virus genome was 4,991 nucleotides long with a gene organization similar to members of the genus Luteovirus (family Luteoviridae). The presence of the virus, named 'Nectarine stem pitting-associated virus', was confirmed in symptomatic trees by RTPCR. Discovery of a new virus in nectarine trees after post-entry quarantine indicates the importance of including (i) metagenomic analysis as an essential tool to assess the plant health status, and (ii) examination of the woody cylinders as part of the indexing process.

\section{Viruses present in ornamental Allium in the United States}

D. Bampi (1), M. D. Reinsel (2), J. HAMMOND (2)

(1) Universidade Estadual Paulista Julia de Mesquita Filho, Botucatu, Brazil; (2) USDA ARS FNPRU, Beltsville, MD, U.S.A.

Phytopathology 105(Suppl. 4):S4.11

The genus Allium includes important species used for human consumption and many other species grown as ornamentals. Plants of the genus Allium can be infected by several viruses of the genera Allexivirus, Carlavirus and Potyvirus, often in mixed infections. Bulbs of fifteen different species of Allium were purchased from retail nurseries and planted in the field in the fall of 2013. Leaf tissue from the flowering symptomatic plants were collected in spring 2014, and tested by PCR using generic primers for the genus
Allexivirus, Carlavirus and Potyvirus. PCR-positive samples were re-tested using specific primers for Leek yellow stripe virus (LYSV) Onion yellow dwarf virus (OYDV), Shallot latent virus (SLV), Garlic common latent virus (GarCLV), Garlic virus A (GarVA), Garlic virus B (GarVB), Garlic virus C (GarVC), Garlic virus D (GarVD), Garlic virus E (GarVE), Garlic mite-borne filamentous virus (GarMbFV) and Shallot virus $X$ (ShVX). After flowering and foliage dieback, the bulbs were harvested and stored at $4^{\circ} \mathrm{C}$ for 60 days, before being planted in pots and maintained in a cooler until leaves emerged, and then transferred to a greenhouse. The sprouted plants were tested by ELISA using antibodies specific for the viral species described above. ELISA confirmed PCR detection of GarVB, GarVC, GarVD, GarVE and ShVX in Allium sphaerocephalon and Allium caeruleum, LYSV in Allium bulgaricum and Allium atropurpureum, and SLV in Allium moly.

Biofuel traits of sweet sorghum are affected by Fusarium stalk rot and charcoal rot diseases

A. Y. BANDARA (1), D. K. Weerasooriya (1), T. T. Tesso (1), C. R. Little (1)

(1) Kansas State University, Manhattan, KS, U.S.A.

Phytopathology 105(Suppl. 4):S4.11

Sweet sorghum [Sorghum bicolor (L.) Moench] is a prospective source of biofuel feedstock. It produces sugar-rich stalks and high biomass. Although the effects of stalk rots on grain sorghum are known, their potential impacts on sweet sorghum remain unclear. The objective of this study was to test the effects of Fusarium stalk rot and charcoal rot on sweet sorghum biofuel traits. Nineteen genotypes were tested in the field, against Fusarium thapsinum (FT) and Macrophomina phaseolina (MP). Experimental design was split-plot in RCBD with three replications. At $14 \mathrm{~d}$ after flowering, plants were stalkinjected with FT and MP. $35 \mathrm{~d}$ after inoculation plants were harvested and measured for disease severity using stem lesion length (LL), juice weight (JW), brix, and biomass (BM). Total soluble solids was determined per plant basis (TSS) using JW and BRIX. ANOVA revealed significant genotype and pathogen main effects on all traits measured. Both pathogens significantly increased LLs (FT, 12.5; MP, $8.4 \mathrm{~cm}$ ) in comparison to the control treatment $(1.9 \mathrm{~cm})$. Pathogens significantly reduced JW (22\%), BRIX (1.3\%), BM $(16 \%)$, and TSS $(27 \%)$ compared to control. LL had significant and positive correlations with BM (0.55) and TSS (0.46) indicating the vulnerability of high-biomass, high-sugar genotypes for stalk rot diseases. Findings revealed the negative effects of stalk rot diseases on key biofuel traits and the importance of breeding for resistance.

RNA-Seq reveals sorghum candidate genes associated with charcoal rot resistance

A. Y. BANDARA (1), D. K. Weerasooriya (2), S. Liu (2), C. R. Little (2)

(1) Kansas State Univ, Manhattan, KS, U.S.A.; (2) Kansas State University, Manhattan, KS, U.S.A.

Phytopathology 105(Suppl. 4):S4.11

Macrophomina phaseolina (MP) is the most important stalk rot pathogen causing charcoal rot of sorghum. Identification of resistant lines through field screening is tedious. The objectives of this study were to: i) discover differentially expressed genes (DEG) and ii) understand their contribution to resistance in order to discovered genes to be used as molecular tags. SC599 (resistant) and TX7000 (susceptible) sorghum lines were grown in the greenhouse and inoculated with MP. Control plants were mock-inoculated with phosphate buffered saline. RNA was extracted from 3 biological replicates at 2, 7, and $30 \mathrm{~d}$ (3 stages) post-inoculation (d.p.i.) from stem tissues and subjected to RNA-Seq. Analysis for DEG was performed with DESeq2 and pathway analysis was performed to explore DEG. 8530 annotated genes were identified across 3 stages as significantly differentially expressed between 2 genotypes, out of which 2053 were components of 200 known pathways. 1722 DEG were associated with 7 d.p.i. suggesting the impact of molecular changes occur by 7 d.p.i. in determining the resistance phenotype. Genes involved in isoflavonoid, chorismate, ethylene and jasmonic acid biosynthesis and triacylglycerol and glycerol degradation pathways were found to be significantly upregulated in TX7000, while those of SC599 showed non-significant differential expression. Findings suggest the contribution of non-upregulated susceptible genes in manifesting the resistance phenotype.

Genotype specific effects of Fusarium co-infection on sorghum stalk rot symptom development

A. Y. BANDARA (1), T. T. Tesso (2), C. R. Little (2)

(1) Kansas State Univ, Manhattan, KS, U.S.A.; (2) Kansas State University, Manhattan, KS, U.S.A.

Phytopathology 105(Suppl. 4):S4.11

Various Fusarium spp. have the ability to cause sorghum stalk rot. Although co-infection occurs in nature, experimental evidence for its impact on 
symptomatology is currently lacking. The objective of this study was to explore the potential additive, synergistic, or antagonistic effects of Fusarium co-infection on symptom development. Six genotypes (SC599, SC35, TX7000, BTX3042, DKS37-07, and 84G62) were inoculated with spore suspensions of 3 Fusarium spp. (F. thapsinum (FT), F. proliferatum (FP), $F$. andiyazi (FA) individually and in all possible combinations at $14 \mathrm{~d}$ after flowering. At $42 \mathrm{~d}$ after inoculation, symptom severity was measured by splitting stems and measuring lesion length. ANOVA revealed significant genotype*pathogen interaction, indicating the genotype dependency of coinfection on symptom development. Mean comparisons were made between additive values of individual pathogen inoculations and their counterpart coinfections. Bonferroni adjusted $\left(\alpha_{\mathrm{CER}}=0.006\right)$ mean separation within each genotype revealed the synergism of FT/FP co-infection on BTx3042 (6.7 vs. $3.1 \mathrm{~cm})$ and FT/FA/FP on SC35 (10.7 vs. $5.5 \mathrm{~cm})$ and the antagonism of FT/FA on DKS37-07 (5.3 vs. $7.3 \mathrm{~cm}$ ), demonstrating the implications of coinfection on resistance durability. For most comparisons, co-infection means did not significantly differ from their counterpart additive means. Hence the prominent effect of Fusarium spp. co-infection on symptom development was identified to be additive.

Use of isolate mixtures for screening sorghum germplasm against stalk rot diseases

A. Y. BANDARA (1), T. T. Tesso (2), C. R. Little (2)

(1) Kansas State Univ, Manhattan, KS, U.S.A.; (2) Kansas State University, Manhattan, KS, U.S.A.

Phytopathology 105(Suppl. 4):S4.12

Fusarium stalk rot (predominantly caused by Fusarium thapsinum (FT) and charcoal rot (Macrophomina phaseolina: MP) are high priority fungal diseases of sorghum. Resistance is the key control strategy. Conventionally, resistance screening is single isolate dependent. The objective of this study was to test the effects of isolate mixtures on disease severity and to explore it's appropriateness for resistance screening. Six genotypes (SC599, SC35, TX7000, BTx3042, DKS37-07, and 84G62) were inoculated through injection with 3 FT isolates individually and in all possible combinations (8 treatments with mock-inoculated control) at $14 \mathrm{~d}$ after flowering. The inoculum concentration was kept constant across treatments. The same process was repeated with $3 \mathrm{MP}$ isolates. The experiment was conducted in a split-plot design with 3 replications. At $42 \mathrm{~d}$ after inoculation, symptom severity was measured by splitting stems and measuring lesion length. ANOVA revealed non-significant treatment, genotype and interaction effects for both pathogens. Consequently, the mean separation did not reveal significant treatment differences across genotypes for both pathogens. Results suggested that mixtures do not aggravate the symptoms and behave similarly to their individual constituents in terms of their ability to cause the disease. Hence, a single virulent isolate from each pathogen would be adequate for a successful resistance screening. Effect of mixtures on tolerance is under investigation.

\section{Characterization of the Arabidopsis thaliana gene PBL13}

J. BANDERAS (1), D. Lin (1), G. Coaker (1)

(1) University of California Davis, Davis, CA, U.S.A.

Phytopathology 105(Suppl. 4):S4.12

Plants are attacked by various pathogens, which can cause huge losses in agriculture. In response, plants have evolved to recognize pathogen associated molecules. This event triggers a defense response by reprogramming gene expression. Kinases help relay signals by attaching phosphate groups to proteins, thereby changing their structure and function to influence their biological activities. The Arabidopsis gene PBL13 has been identified as a receptor like cytoplasmic kinase because of its homology with other known kinase coding genes. Additionally, $P B L 13$ knockout mutants display enhanced disease resistance, which indicates that $P B L 13$ is a negative regulator of plant immunity. To better understand the function of $P B L 13$, its promoter, which controls gene expression, was fused to the marker gene $\beta$-glucuronidase $(G U S)$. GUS codes for an enzyme that reacts with glucuronosides to produce a visible color in a process known as GUS staining. Next, Agrobacterium was used to transform Arabidopsis plants with the aforementioned promoter-GUS fusion. After obtaining stable transgenic lines from these plants, GUS staining will be performed to reveal the location and timing of PBL13 expression. By revealing this aspect of $P B L 13$ 's function, this project will provide a better understanding of plant defense signaling, which can help in implementing better control strategies for pathogens.

Effects of the $\operatorname{Lr} 34$ and $L r 46$ rust-resistance genes on different diseases of wheat

A. BANSAL (1), J. Brown (1)

(1) John Innes Centre, Norwich, United Kingdom

Phytopathology 105(Suppl. 4):S4.12
Lr34 and Lr46 are adult plant resistance genes providing durable resistance against biotrophic pathogens like rusts and powdery mildews in wheat. In seedlings, however, these genes increase susceptibility to Septoria tritici blotch (STB), caused by the fungus Zymoseptoria tritici. Lr34 and Lr46 also favoured the necrotrophic pathogens Magnoporthe grisea and Ramularia collo-cygnii in seedlings. A similar effect was observed in adult plants tested in polytunnels. The hypothesis that leaf age has a role in Lr34 and Lr46 resistance to mildew and susceptibility to $Z$. tritici was tested. In particular, it was hypothesised that enhanced senescence in plant leaves can make them resistant to biotrophs while making them more susceptible to necrotrophic pathogens. Leaf age altered significantly the effect of $L r 34$ and $L r 46$ on STB. The plant material tested included the Indian spring wheat Lal Bahadur (LB) and near-isogenic and mutant lines developed from LB. In young leaves, LB was less susceptible to STB than near-isogenic lines carrying Lr34 or Lr46. The opposite pattern was observed, however, in older leaves. Gene expression analysis of $L r 34$ lines revealed a higher expression of senescence and celldeath associated genes indicating a possible role of Lr34 in enhancing senescence. The results indicate that there may be significant consequences for the use of Lr34 and Lr46 to control rust and mildew in areas where necrotrophic diseases are prevalent.

Analysis of the virus-insect vector interactome of Maize mosaic rhabdovirus glycoprotein

K. BARANDOC-ALVIAR (1), D. Rotenberg (1), A. Whitfield (1)

(1) Kansas State University, Manhattan, KS, U.S.A.

Phytopathology 105(Suppl. 4):S4.12

Maize mosaic rhabdovirus (MMV) is a neurotropic negative-strand RNA virus of the Rhabdoviridae family in the genus Nucleorhabdovirus. It is transmitted by the corn planthopper, Peregrinus maidis, in a persistent propagative manner and causes disease in important crops like corn. Entry of rhabdoviruses into host cells requires binding and fusion activity of the viral glycoprotein $(\mathrm{G})$. For rhabdoviruses, $\mathrm{G}$ is a multifunctional protein that plays a role in entry, assembly and exit from cells. We hypothesize that MMV G is required for invasion, infection, and spread in the planthopper vector. In this study, we used a split ubiquitin membrane-based yeast two hybrid system (MbYTH) to identify vector proteins which interact with MMV G where a $P$. maidis cDNA library was used as prey and MMV G as bait. We confirmed and sequenced 423 putative MMV G interactors. Of these proteins, $77 \%$ have conserved domains and significant hits in non-redundant and $P$. maidis EST NCBI databases, while $23 \%$ had no significant match. Proteins implicated in membrane fusion events and vesicle trafficking were identified in our MbYTH screen like Rab small GTPases, SNARE, actin, E3 ubiquitin ligase, cyclophilin, HSP90 and F-ATPases. The identification of MMV G interactions with well characterized proteins in the endocytic pathway provides support for the hypothesis that MMV utilizes this conserved pathway to enter the insect host analogous to entry of other Rhabdoviruses into animal host cells.

Oxytetracycline sensitivity in Xanthomonas arboricola pv. pruni, the causal agent of bacterial spot of stone fruit

S. BARDSLEY (1), M. Jiménez-Gasco (2)

(1) Penn State Univ, University Park, PA, U.S.A.; (2) Penn State University, University Park, PA, U.S.A.

Phytopathology 105(Suppl. 4):S4.12

Bacterial spot of stone fruit (caused by Xanthomonas arboricola pv. pruni $[X a p])$ is the most important bacterial disease of peach in Pennsylvania where the antibiotic oxytetracycline is used for management. Extensive use of this antibiotic exerts a strong selective pressure on bacteria to support the growth of antibiotic resistant bacteria. In 2011, 2012, and 2013, 1,337 Xap isolates were collected from 10 PA stone fruit orchards in Adams, Lancaster, Chester, Franklin, and Delaware Counties as well as 2 research plots at the Penn State Fruit Research and Extension Center (FREC) in order to assess the sensitivity of these isolates to oxytetracycline. To date, 834 isolates have been screened for oxytetracycline sensitivity which varied greatly among orchards. The sensitivity was statistically related to the number of oxytetracycline applications made by the grower during and prior to the year of sample collection. In order to account for this variable sensitivity, molecular mechanisms for resistance have been evaluated, including screening isolates for tetracycline resistance genes. Isolates have been screened for the presence of tet $\mathrm{B}$ and tet $\mathrm{C}$ using PCR but none of these genes have thus far been found. However, over 40 tetracycline resistance genes have been described so continued investigation will help establish the molecular mechanism, either novel or not, behind this variability in sensitivity. 
Differential effects on nematode development of two QTLs for resistance to Meloidogyne incognita in cotton

M. BATISTA DA SILVA (1), P. Kumar (1), P. Ji (1), P. W. Chee (1), R. Davis (2)

(1) University of Georgia, Tifton, GA, U.S.A.; (2) USDA-ARS, Tifton, GA, U.S.A.

Phytopathology 105(Suppl. 4):S4.13

QTLs qMi-C11 and qMi-C14 impart resistance to Meloidogyne incognita in cotton. Breeders had backcrossed both QTLs into Coker 201 (C201; susceptible) to create M-120 RNR (M-120; highly resistant), and we crossed $\mathrm{C} 201$ and M-120 to create near isogenic lines with either $q M i-C 11$ or $q M i-$ C14. Previous work suggests different modes of action for $q M i-C 11$ and $q M i-$ C14. To document individual and combined effects of the QTLs, C201 (neither QTL), M-120 (both QTLs), CH11 isoline (qMi-C11), and CH14 isoline ( $q M i-C 14)$ were inoculated with $M$. incognita (one plant/pot, 4000 J2s/pot, 7 replications, completely randomized design). After 38 days, roots were weighed, $M$. incognita eggs were harvested, the number of galls was counted, and roots were stained to observed nematode developmental stages (J2, swollen $\mathrm{J} 2$ [SJ2], J3, J4, and Female [F] as percentage of total nematodes). Root weight did not differ among genotypes. QTLs were effective alone or combined: $\mathrm{C} 201$ had more nematodes becoming F than M120 , $\mathrm{CH} 11$ or $\mathrm{CH} 14$ ( $51 \%$ vs. $9 \%, 3 \%$, and $12 \%$ ). More nematodes remained $\mathrm{J} 2$ in M-120 (41\%), CH11 (58\%) and CH14 (27\%) than in C201 (9\%). CH14 had more J3 than $\mathrm{CH} 11$ (26\% vs. $10 \%$ ) and $\mathrm{CH} 14$ and $\mathrm{C} 201$ had more total nematodes and larger galls than $\mathrm{CH} 11$ and $\mathrm{M}-120$, suggesting that $\mathrm{CH} 11$ affects nematode development earlier than $\mathrm{CH} 14$. This study confirms that both $q \mathrm{Mi}-\mathrm{Cl} 1 \mathrm{\text {and }} q \mathrm{Mi}-\mathrm{Cl} 4 \mathrm{impart}$ resistance to $\mathrm{M}$. incognita and that $q \mathrm{Mi}$ C11 and qMi-C14 have different effects on nematode development.

First report of an ipomovirus in California: Identification of a divergent strain of Squash vein yellowing virus in the Imperial Valley of California O. BATUMAN (1), E. T. Natwick (2), W. M. Wintermantel (3), T. Tian (4), J. D. McCreight (3), L. L. Hladky (3), R. L. Gilbertson (1)

(1) University of California, Davis, CA, U.S.A.; (2) University of California ANR Cooperative Extension Imperial County, Holtville, CA, U.S.A.; (3) United States Department of Agriculture-Agricultural Research Service, Salinas, CA, U.S.A.; (4) California Dept of Food \& Agric, Sacramento, CA, U.S.A.

Phytopathology 105(Suppl. 4):S4.13

In fall 2014, unusual virus-like symptoms and Bemisia tabaci were observed on pumpkin (Cucurbita pepo L.) and melon (Cucumis melo L.) in Imperial Valley, California. Infected plants were stunted and leaves showed mottling, yellowing, epinasty and crumpling. RT-PCR and PCR tests of symptomatic plants revealed mixed infection with Cucurbit yellow stunting disorder virus and Squash leaf curl virus; and RT-PCR with degenerate potyvirus primers revealed a positive result with the cylindrical inclusion (CI) but not with helper component proteinase primer pair. Comparisons performed with CI sequences revealed the highest identity, $83-85 \%$, with Squash vein yellowing virus (SqVYV), a B. tabaci-transmitted ipomovirus (family Potyviridae) that occurs in Florida. RT-PCR performed with SqVYV capsid protein (CP) primers directed the amplification of the expected size $\sim 1.1 \mathrm{~kb}$ fragment and sequence analysis revealed $89-99 \%$ identity with SqVYV CP sequences. Sequence analysis of a $\sim 5.5 \mathrm{~kb}$ fragment amplified with CI specific and poly (T) primers, confirmed the $\mathrm{CI}$ and $\mathrm{CP}$ sequences were from a single ipomovirus isolate. In sap inoculation studies, pumpkin and squash plants developed mild mottling, vein clearing and yellowing symptoms whereas watermelon (Citrullus lanatus) plants were initially stunted and yellowed and eventually collapsed, similar to those reported for SqVYV. Thus, this is a divergent strain of SqVYV, and it is the first report of an ipomovirus in California.

Polyketide synthase gene clusters in the swainsonine producing Undifilum oxytropis, fungal endophyte of the locoweed plant, Oxytropis sericea

D. BAUCOM (1), D. Cook (2), C. Schardl (3), R. Creamer (4)

(1) New Mexico State Univ, Las Cruces, NM, U.S.A.; (2) USDA, Logan, UT, U.S.A.; (3) University of Kentucky, Lexington, KY, U.S.A.; (4) New Mexico State University, Las Cruces, NM, U.S.A.

Phytopathology 105(Suppl. 4):S4.13

Rangeland plants termed locoweeds have negatively impacted production of grazing animals both in the past and present. Of the genera Oxytropis and Astragalus, locoweeds host a fungal endophyte responsible for production of the toxic compound swainsonine. Ingestion of swainsonine by animals causes neurological problems resulting in financial losses to production. Swainsonine is an alpha-mannosidase inhibitor produced by some fungi, including the fungal endophytes associated with locoweeds, Undifilum spp. The biosynthetic pathway of producing swainsonine has not been completely illuminated in any fungal species. Because of the significant association between polyketide synthases and secondary metabolite (SM) production in fungi, we chose to identify and characterize these gene clusters in the toxic fungus $U$. oxytropis. A partially assembled genome sequence was obtained. Genome mining using reference gene clusters from other SM producing fungi revealed 23 polyketide synthase gene clusters. Complete nucleotide and amino acid sequences for the domains of each cluster were obtained and a selection of these were confirmed through primer design, PCR, and sequencing. Each gene cluster was analyzed for homology and predicted function, if available.

The role of pest control advisors in managing grapevine trunk diseases: A survey of perceptions of practice efficacy and trends in recommendations K. BAUMGARTNER (1), D. Doll (2), V. Hillis (3), J. Kaplan (4), M. Lubell (3)

(1) USDA-Agricultural Research Service, Davis, CA, U.S.A.; (2) University of California Cooperative Extension, Merced, CA, U.S.A.; (3) Department of Environmental Science and Policy, University of California, Davis, CA, U.S.A.; (4) Department of Economics, California State University, Sacramento, CA, U.S.A.

Phytopathology 105(Suppl. 4):S4.13

Trunk diseases (Botryosphaeria dieback, Esca, Eutypa dieback, Phomopsis dieback) significantly limit vineyard productivity. The causal fungi establish chronic wood infections, which accumulate over time. Symptoms do not become obvious until the vineyard is $6-8$ years old. Post-infection practices (vine surgery, replanting vines) are labor intensive. Effective preventative practices (delayed/double pruning, fungicide applications to pruning wounds) are less costly in the long run if started when the vineyard is young (and healthy), but convincing growers to adopt these practices in the absence of symptoms is difficult. To help guide a new outreach plan, we surveyed California pest control advisors (PCAs). Our online survey revealed that PCAs recommend post-infection practices as frequently as preventative practices. High disease incidence was correlated with a higher frequency of recommendation for all practices, suggesting no preferences for recommending certain practices in young vs. mature vineyards. Instead, PCAs recommended more frequently practices (both preventative and postinfection) they perceived as more effective and less costly. PCAs rated other PCAs, university publications, and field trials as their most frequently-used sources of disease management information. Our findings underscore the importance of communicating to PCAs the widespread prevalence of trunk diseases and the fact that preventative practices are effective, if adopted in young vineyards.

Evaluation of fungicides and production methods for the control of downy mildew of cucumber

F. BAYSAL-GUREL (1), S. A. Miller (1)

(1) The Ohio State University, Wooster, OH, U.S.A.

Phytopathology 105(Suppl. 4):S4.13

The effects of trellis vs. ground cucumber production, fungicide program (Bravo Weather Stik 6SC alone and Presidio 4SC+Bravo alternated with Ranman 400SC+Bravo) and application schedule (7 or 10 days) on downy mildew (Pseudoperonospora cubensis) severity were evaluated in 2013 and 2014. The method of production did not affect downy mildew severity. While all treatments significantly reduced downy mildew severity compared to the control, the disease was more severe in plants treated with Bravo alone than in those treated with Presidio+Bravo alt. Ranman+Bravo. There were no differences in disease severity in plants treated on 7- or 10-day application schedules with either fungicide program. The effects of fungicide treatment and production method on marketable yield were significant; the interaction between treatment and production method was also significant in 2013. Trellised plants treated with Presidio+Bravo alt. Ranman+Bravo on either a 7or 10-day schedule or with Bravo alone on a 10-day schedule yielded significantly more marketable fruits than plants produced on the ground and treated with Bravo on a 7-day schedule, or not treated. Trellised plants yielded significantly more marketable fruits and more culls than plants produced on the ground in 2014. However, the effect of treatment on marketable yield was not significant. While trellising cucumbers has apparently no effect on downy mildew severity, this production method significantly improves yield.

Effective disease management strategies for organic cucumber production

F. BAYSAL-GUREL (1), S. A. Miller (1)

(1) The Ohio State University, Wooster, OH, U.S.A.

Phytopathology 105(Suppl. 4):S4.13

Certified organic growers are required to use host resistance and cultural practices to disrupt pathogen life cycles before resorting to "rescue" treatments such as biorational products to manage diseases such as downy 
mildew (Pseudoperonospora cubensis) and bacterial wilt (Erwinia tracheiphila). The effects of season-long row covers and products approved for use in organic farming systems on the occurrence of downy mildew and bacterial wilt in parthenocarpic cucumber were tested. Across all treatments, downy mildew severity was lower and the disease progressed more slowly on covered than non-covered plants. Champ WG, OxiDate and neem oil significantly reduced downy mildew disease progress during the growing season compared to the other treatments and the non-treated control. Bacterial wilt incidence was low in 2013, but all covered plots had significantly fewer wilted plants than non-covered plots. Bacterial wilt was not observed in 2014. There were no significant differences in marketable fruit yield among treatments and between covered and non-covered plots in 2013, but all covered plots produced significantly more marketable fruit than non-covered plots in 2014. There were no significant differences between product treatments in marketable or total fruit yield. Combining season-long row covers over parthenocarpic cucumbers with biorational products may provide an effective approach to both downy mildew and bacterial wilt management in organic systems.

Reliable and sensitive detection of Clavibacter michiganensis subsp. michiganensis and Salmonella enterica in greenhouse irrigation water F. BAYSAL-GUREL (1), N. Khatri (1), A. Arciniega (1), J. T. LeJeune (1), S. A. Miller (1)

(1) The Ohio State University, Wooster, OH, U.S.A.

Phytopathology 105(Suppl. 4):S4.14

Reliable and sensitive detection of Clavibacter michiganensis subsp. michiganensis $(\mathrm{Cmm})$ and Salmonella enterica in source and production water could reduce the risk of serious losses in the greenhouse tomato production industry. Bioluminescent strains of $\mathrm{Cmm}$ and Salmonella enterica serovar Typhimurium were used to spike $5 \mathrm{~L}$ water samples with $10^{2}, 10^{4}$ or $10^{6} \mathrm{CFU}$. Samples were filtered through a disposable inline filter using a peristaltic pump. Cmm was eluted from the filters and tested directly with serological and molecular detection assays and culturing on modified CMM1 semiselective medium. Samples were also enriched in NBY broth for $24 \mathrm{hrs}$ in the filter housing. The lower limit of $\mathrm{Cmm}$ detection was $10^{4} \mathrm{CFU} / 5 \mathrm{~L}$ using the Cmm ImmunoStrip, DNAble and Smart-DART isothermal DNA amplification assays and end-point PCR, and $10^{2} \mathrm{CFU} / 5 \mathrm{~L}$ using real-time qPCR. Similar results were observed for source (well), fertigation and recirculated production water. The lower limit of detection of bioluminescent Salmonella, without enrichment in LB medium, from well, fertigation and recirculated water samples was $10^{2} \mathrm{CFU} / 5 \mathrm{~L}$ by culturing. Large-volume filtration followed by the use of sensitive detection methods will allow quick detection of low levels of bacterial pathogen contamination and prevention of possibly severe consequences to greenhouse operations.

Genetic diversity of Grapevine vein clearing virus ORF II indicates a complex viral population in wild and cultivated grapevines

S. BEACH (1)

(1) Missouri State University, Springfield, MO, U.S.A.

Phytopathology 105(Suppl. 4):S4.14

Grapevine vein clearing virus (GVCV) associated disease poses a threat to the grape production in the Midwest region of the United States. The GVCV genome is a circular, double-stranded DNA with the plus-stand encoding three open reading frames (ORFs), ORFI, ORFII, and ORFIII. Two isolates, GVCV-CHA with a genome of 7,753 bp and GVCV-VRU of 7,755 bp, have been identified in a grape cultivar 'Chardonnay' and a wild Vitis rupestris, respectively. The most variable ORFII regions share $83.3 \%$ identity at nucleotide levels with a characteristic $9 \mathrm{bp}$ insert in GVCV-VRU. To investigate the genetic diversity of GVCV, we cloned and sequenced the ORFII regions of GVCV isolates that were collected from grapevines in commercial vineyards and native habitats. We found that the ORFII of a GVCV isolate from grape cultivar 'Chardonel' shared $88.5 \%$ nucleotide identity with that of GVCV-VRU and contained the $9 \mathrm{bp}$ insert. The ORFII of a GVCV isolate from another wild $V$. rupestris accession does not contain the 9 bp insert and only shared $88 \%$ identity with both GVCV-VRU and GVCVCHA. Furthermore, we found minor changes of ORFII after GVCV-CHA were grafted onto different grape varieties. These results suggest that GVCV is present as genetically complex populations in cultivated and wild grapevines. The complexity of GVCV populations implies dynamic evolution of GVCV and imposes a challenge of managing GVCV-associated disease.

Effects of ILeVO and VOTiVO seed treatments on hatching, motility and root penetration of the soybean cyst nematode

A. BEEMAN (1), G. L. Tylka (1)

(1) Iowa State University, Ames, IA, U.S.A.

Phytopathology 105(Suppl. 4):S4.14
The soybean cyst nematode ( $\mathrm{SCN})$, Heterodera glycines, is the most damaging pathogen of soybean in the United States. Seed treatments recently have been introduced to provide nematode protection and strengthen the performance of SCN-resistant soybean varieties. The objective of this research was to understand how the nematode-protectant seed treatments ILeVO $^{\circledR}$ (fluopyram, Bayer CropSciences) and VOTiVO ${ }^{\circledR}$ (Bacillus firmus I-1582, Bayer CropSciences) affect SCN egg hatching and motility and root penetration of second-stage SCN juveniles (J2s). Exudates were collected by incubating treated seeds or radicles that emanated from treated seeds in sterile water and agitating for one hour. Hatching of SCN eggs in seed and radicle exudates from ILeVO-treated seeds in vitro was reduced by $98 \%$ and $35 \%$, respectively, compared to exudates from untreated controls. Motility of SCN $\mathrm{J} 2 \mathrm{~s}$ incubated in exudates from ILeVO-treated seeds in vitro also was decreased by $97 \%$ relative to $J 2 \mathrm{~s}$ in the untreated control. In a separate experiment, roots of seedlings grown from treated and untreated seeds were inoculated with SCN J2s and stained, and then the numbers of SCN J2s in the roots were counted. There were fewer penetrated SCN J2s in roots grown from ILeVO-treated seeds at one and three days after inoculation compared to the untreated control. These results indicate ILeVO may affect SCN eggs and juveniles in the seed and root zones, and may also reduce SCN root penetration.

Chemotaxis and pathogenicity tests of Phytophthora sojae in Lupinus spp. G. U. BELIGALA (1), V. Phuntumart (1)

(1) Bowling Green State University, Bowling Green, OH, U.S.A. Phytopathology 105(Suppl. 4):S4.14

Phytophthora is a genus of destructive soil-borne phytophathogens belonging to the class oomycetes. Members of this class produce motile spores called zoospores that can swim towards plant roots and subsequently attack causing root-rot diseases. Lupinus which is commonly known as lupines have been reported as one of the host plants of Phytophthora sojae. This study focuses on determining the chemotactic behavior of zoospores and pathogenicity of Phytophthora sojae Race 2 (P6497) towards different lupine accessions. Chemotaxis assays were performed in assay chambers using excised lupine roots incubated in a zoospore suspension. The root sections were then photographed under microscope and number of encysted zoospores was counted along a fixed distance from the tip of the root. Zoospore encystment on roots of different lupine accessions was then compared. Hypocotyl inoculation technique was used for pathogenicity assay. Symptoms were analyzed and rated on a visual scale taking susceptible (Williams) and resistant (Williams 82) soybean seedlings as controls. Susceptible plants usually die or develop soft and water-soaked lesions on the site of infection 3 to 5 days after inoculation. Plants that are resistant normally develop hypersensitive reactions. Disease resistance was scored as follows: $25 \%$ plant death is resistant; 26 to $75 \%$ plant death is intermediate; and more than $75 \%$ plant death is susceptible.

Spatial and temporal model for epidemics of oil palm bud rot in the Middle Magdalena River Valley in Colombia

E. R. BENITEZ (1), C. Garcia (1)

(1) Univ Nacl de Colombia, Bogota, Colombia

Phytopathology 105(Suppl. 4):S4.14

Epidemics of bud rot of oil palm in commercial crops in Colombia were evaluated at a regional scale during the years 2006-2009. Various methods of statistical analysis were used to identify spatial and temporal patterns of the epidemics of the disease. Classical epidemiological models were adjusted for temporal descriptions of the epidemics. For the spatial aspects, geostatistical techniques were applied. Development rates of the disease and intercept of the Gompertz model were used. Rates were correlated within 1 and $4 \mathrm{~km}$, associated with two directions $60^{\circ}$ and $165^{\circ}$, respectively. Higher ranks coincide with higher prevalence of daytime winds. The kriging predictions of the intercepts of Gompertz models were associated with the pattern of onset of the epidemic in the swampy areas of the Magdalena River. For the association of agroecological variables and disease, Exploratory Analysis Factor (AEF) techniques, using oblique rotations Promax and Varimax were implemented. Orthogonal factor loadings were used to estimate forecast models, via linear least squares, which identified the following variables: evidence of ponding, level of clays and deficiency of copper and zinc in the plant as explanatory variables of disease; calcium, magnesium and sulfur in the soil also appear related in the final model.

TaqMan assays designed on the coding sequence of the movement protein of Cucumber green mottle mosaic virus for its detection in cucurbit seeds S. BERENDSEN (1), J. Oosterhof (1)

(1) Rijk Zwaan Breeding B.V., De Lier, Netherlands

Phytopathology 105(Suppl. 4):S4.14 
The seed-borne pathogen Cucumber green mottle mosaic virus (CGMMV) causes great economic losses to commercial cucurbit crops and seed productions worldwide. The current method described for screening cucurbit seeds for CGMMV by ISTA is ELISA. A molecular confirmation of positive ELISA results is useful. Due to large amount of samples that are tested, a Real-Time based RT-PCR is prefered. A conserved region within the coding sequence of the movement protein was found by comparing the nucleotide sequences of different CGMMV accesions numbers from NCBI GenBank. This region was used to design two specific RZ CGMMVmp MGB based $\mathrm{TaqMan}^{\circledR}$ assays. The developed assays were tested and compared with a published assay targeting 3' noncoding region on a collection of isolates. Total RNA was isolated from CGMMV natural infected seed lots. All three assay were able to detect our available CGMMV isolates. The sensitivity of the three assays was compared with ELISA results. A dilution serie was made with a natural low CGMMV contaminated seed lot in a background of uninfected seeds beyond the ELISA detection limit. All three TaqMan ${ }^{\circledR}$ assays are at least a twentyfold more sensitive than the ELISA's minimum detection limit. Based on these results the RZ_CGMMVmp TaqMan ${ }^{\circledR}$ assays are suitable for confirmation of ELISA positive seed samples.

Developing a new approach to study the distribution of different genotypes of Citrus tristeza virus within citrus plants

M. BERGUA (1), D. M. Phelan (1), A. Bak (1), D. C. Bloom (1), S. Y. Folimonova (1)

(1) University of Florida, Gainesville, FL, U.S.A.

Phytopathology 105(Suppl. 4):S4.15

The RNAscope in situ hybridization (ISH) assay is a novel method for localizing RNA molecules, which shows outstanding specificity and sensitivity, compared to conventional ISH methods. The method relies on a unique probe design strategy and has been actively used in cancer-related research to analyze expression of endogenous mRNAs in situ in routine clinical specimens. However, no reports have been published on the use of this labeling method with viruses of plants. In order to study the distribution of different genotypes of Citrus tristeza virus (CTV) within citrus hosts, we optimized the multiplex RNAscope assay, in which two independent signal amplification systems, each carrying a different chromogenic enzyme, are used for detection of two different RNA targets. The method allowed us to specifically detect and visualize RNA molecules produced by two CTV isolates, T36 and T68-1, in the infected plants. Remarkably, no cross-reaction between a probe designed based on genomic sequences of a particular virus isolate and RNA molecules produced by a heterologous virus isolate was detected. Viral RNA of both strains was localized mainly in phloemassociated cells, however, some signal was also found outside of the vascular tissue. Our results confirmed that this multiplex ISH protocol exhibits high sensitivity and specificity for detection of different genotypes of CTV and shows a potential for simultaneous visualization of multiple virus variants in mixed infections.

Systemic movement of Raffaelea lauricola through the rhizomes of pondberry

G. S. Best (1), S. W. FRAEDRICH (1)

(1) USDA Forest Service, Athens, GA, U.S.A.

Phytopathology 105(Suppl. 4):S4.15

Pondberry (Lindera melissifolia) is a federally endangered, clonal shrub that occurs as isolated populations in forests of the southeastern United States. Pondberry is highly susceptible to laurel wilt caused by Raffaelea lauricola, and the disease was previously detected in a Georgia population. A study was conducted to determine if $R$. lauricola could move systemically through rhizomes of infected plants to adjacent ramets. A pondberry seedling was established in each of six 15 gallon pots, and after three years, there was an average of 17 ramets per pot. The stems of the original plants in four pots were inoculated with $R$. lauricola; the original stems in two pots were mockinoculated with sterile water. Foliage of plants inoculated with $R$. lauricola developed wilt symptoms within two weeks, and symptoms in adjacent ramets soon followed. After 10 weeks, an average of 8.5 ramets per pot had wilted, and a brown discoloration was observed in the xylem of rhizomes connected to wilted stems. Xylem tissues from symptomatic stems and rhizomes were plated on agar media, and $R$. lauricola was consistently isolated. Control plants remained healthy. The experiment was repeated once with similar results. Our study indicates that $R$. lauricola can move rapidly through rhizomes of infected plants. Although infections by R. lauricola are thought to occur rarely in pondberry, when infections do occur, many ramets within a population could be at risk of wilt.
Genomics and effector characterization of the novel sugar beet pathogen Fusarium secorum

Z. BIAN (1), R. De Jonge (2), G. Secor (1), M. Bolton (3)

(1) North Dakota State University, Fargo, ND, U.S.A.; (2) Ghent University, Ghent, Belgium; (3) USDA ARS, Fargo, ND, U.S.A.

Phytopathology 105(Suppl. 4):S4.15

Sugar beet is an important source of sucrose for human consumption throughout the world. A new disease of sugar beet, named Fusarium yellowing decline, was recently found in in the Red River Valley of MN and ND. This disease is caused by a novel pathogen named Fusarium secorum. To investigate the evolutionary origin of $F$. secorum, we conducted phylogenetic analyses of the concatenated alignment of approximately 7,000 conserved single-copy core orthologs across 20 Fusarium species for which the entire genome is sequenced. These analyses resulted in the generation of a robust species topology that clusters $F$. secorum closely to $F$. acutatum in the $F$. fujikuroi species complex. Pathogens such as $F$. secorum secrete proteins during infection called 'effectors' that help establish disease. Here, candidate effectors were identified by screening the set of 15,872 predicted proteins with several bioinformatics tools combined with RNA-seq data. To characterize putative effectors, we developed a transformation system using polyethylene glycol-mediated transformation. Several mutant lines have been made with an effector deleted from the genome using a split-marker knock-out strategy. To explore their role in pathogenicity, mutant strains have been inoculated to sugarbeet and compared to WT $F$. secorum. These effector characterization results and a detailed analysis of the F. secorum genome will be presented.

Development and evaluation of molecular methods for specific detection of Phytophthora alni subsp. alni and $P$. alni subsp. multiformis

J. C. Bienapfl (1), Z. G. ABAD (1), L. M. Knight (1), M. J. Sullivan (2), M. K. Nakhla (1)

(1) USDA APHIS PPQ S\&T, Beltsville, MD, U.S.A.; (2) USDA APHIS PPQ S\&T, Fort Collins, CO, U.S.A.

Phytopathology 105(Suppl. 4):S4.15

Phytophthora alni is listed as a priority pest in the Pests of Economic and Environmental Importance of (2013-2015). It is ranked \#6 out of 29 Phytophthora spp. exotic to the USA (CPHST Plant Epidemiology and Risk Analysis Laboratory) and of concern due to the potential damage they can cause if introduced. The pathogen is reported to cause collar and root rot of multiple alder spp. in several Europe countries. However, P. alni is composed of three subspecies: P. alni subsp. alni (Paa), P. alni subsp. multiformis (Pam), and $P$. alni subsp. uniformis (Pau). Pau has been reported in the USA while Paa and Pam have not been found in the USA. To enhance efforts in detection of Phytophthora spp. of concern, we developed a multiplex conventional PCR assay that combines primers that target the ras-like related ypt 1 gene with SCAR primers that allow for differentiation among the three subspecies. We also developed a real-time PCR assay that also targets the yptl gene of Paa and Pam. Genomic DNA extracted from the ex-type of each subspecies, as well as from other Phytophthora spp., has been used to test the specificity of both assays. Preliminary results indicate both assays allow detection of Paa and Pam. Additional tests are underway using DNA from Phytophthora spp. commonly reported from agricultural crops and forests in the USA, and close relatives to $P$. alni, to further evaluate the specificity of the assays and results for validation will be presented.

Development and evaluation of molecular methods for species-specific detection of Phytophthora quercina

J. C. BIENAPFL (1), Z. G. Abad (1), L. M. Knight (1), M. J. Sullivan (2), M. K. Nakhla (1)

(1) USDA APHIS PPQ S\&T, Beltsville, MD, U.S.A.; (2) USDA APHIS PPQ S\&T, Fort Collins, CO, U.S.A.

Phytopathology 105(Suppl. 4):S4.15

Phytophthora quercina is considered a priority pest in the commodity-based survey listing for the USDA Cooperative Agriculture Pest Survey. It is ranked \#1 out of 29 Phytophthora spp. exotic to the USA (CPHST Plant Epidemiology and Risk Analysis Laboratory) and of concern due to the potential damage they can cause if introduced. P. quercina has been reported as a prevalent root rot pathogen of multiple oak species in Austria, Belgium, France, Germany, Hungary, Italy, Netherlands, Russia, Serbia, Spain, Sweden, United Kingdom, and Turkey, but has not been reported in the USA. To enhance efforts in pest detection of Phytophthora spp. of concern, we enhanced a published conventional PCR assay that targets the ras-like related ypt1 gene of $P$. quercina by adding an internal control that targets Oomycetes and evaluated this test against Phytophthora spp. not evaluated in the original publication. In addition, we have developed a real-time PCR assay that also targets the ypt1 gene of $P$. quercina. Genomic DNA extracted from the extype of P. quercina, as well as from other Phytophthora spp., has been used to 
test the specificity of both assays. Preliminary results indicate both assays are specific for $P$. quercina. Additional tests are underway using DNA from Phytophthora spp. commonly reported from agricultural crops and forests in the USA, and close relatives to P. quercina, to further evaluate the specificity of the assays and results for validation will be presented.

Comparison of soils and their associated microbial communities as affected by sugarcane cultivation

A. F. BIGOTT (1), J. Hoy (1), L. Fultz (1)

(1) Louisiana State University Agricultural Center, Baton Rouge, LA, U.S.A. Phytopathology 105(Suppl. 4):S4.16

Reduced productivity of sugarcane has been associated with monoculture. In Louisiana, sugarcane has been cultivated on the same soils for more than 200 years. Higher yields can be obtained in soils newly converted to sugarcane and in sterilized monoculture soils, suggesting a biotic component to the yield decline. Three paired sites of adjacent fields with a long-term sugarcane cultivation history (continuous caneland) and newly converted to sugarcane (new caneland) are under study. Comparisons were made within and among paired sites for soil nutrients, soil enzymatic activity assays, a greenhouse experiment, and fatty acid methyl ester (FAME) profiles. Individual soil nutrients varied by site. However, differences in continuous and new caneland were not consistent among sites. Beta-glucosaminidase activity varied between sites and was different within two of three paired sites but was not consistently greater in new or continuous caneland. Greenhouse plant growth was not consistently affected by cropping history or sterilization. Relative abundance of FAME biomarkers distinguished continuous caneland microbial communities from those in new caneland. Old caneland was dominated by bacterial communities and new caneland by fungal communities for two of three paired sites. The results will be considered with a comparative metagenomic analysis to develop a comprehensive understanding of the effects of long-term sugarcane cultivation on soil microbial communities.

Fusarium graminearum mycotoxin accumulation in wheat straw after anthesis in wheat cultivars ranging in susceptibility to Fusarium Head Blight

K. M. BISSONNETTE (1), K. A. Ames (1), Y. Dong (2), F. L. Kolb (1), C. A. Bradley (3)

(1) University of Illinois, Urbana, IL, U.S.A.; (2) University of Minnesota, St. Paul, MN, U.S.A.; (3) Univ of Illinois, Urbana, IL, U.S.A.

Phytopathology 105(Suppl. 4):S4.16

Mycotoxins are commonly associated with wheat grain affected by Fusarium head blight (FHB; caused by Fusarium graminearum), but little is known about their presence in straw. Wheat straw is used as bedding material for swine, which are sensitive to mycotoxins and may eat up to $4 \mathrm{~kg}$ of bedding per day. A field trial in Urbana, Illinois in 2013 and 2014 tested for the accumulation of mycotoxins in straw tissue. Twelve soft red winter wheat cultivars ranging in susceptibility to FHB were planted, and whole plants were sampled from each plot 14 days after anthesis (daa), 21 daa, and 28 daa. After harvest, stem tissue also was collected. Samples were dried, ground, and sent to the University of Minnesota for mycotoxin analysis. Three mycotoxins were tested for in this study: deoxynivalenol (DON), 3-acetyl-deoxynivalenol $(3 \mathrm{ADON})$, and 15-acetyl-deoxynivalenol (15ADON). Toxin concentrations ranged from 0-39.6 ppm for DON, 0-3.8 ppm for 3ADON, and 0-16.3 ppm for $15 \mathrm{ADON}$. Portions of the stem were compared at each collection date and at 28 daa, the portions significantly $(P \leq 0.05)$ differed in susceptible cultivars. Positive, significant correlation for toxin concentrations in grain and stems was observed for DON $(P=0.0001 ; R=0.80)$, 3ADON $(P=0.0007 ; R=$ $0.34)$, and 15ADON $(P=0.0001 ; R=0.70)$. Results indicate that cultivars with resistance to FHB may also have lower mycotoxin accumulation in straw tissue compared to cultivars susceptible to FHB.

Nitrogen nutrition impact on incidence of Rhizoctonia infection of Agrostis stolonifera

B. BLACK (1), B. D. Geary (1), B. G. Hopkins (1)

(1) Brigham Young University, Provo, UT, U.S.A.

Phytopathology 105(Suppl. 4):S4.16

Creeping bentgrass (Agrostis stolonifera L.) is tolerant of short mowing and high traffic, but these conditions increase pathogen susceptibility. A prevalent disease on bentgrass golf course greens and tee boxes is Brown Patch (Rhizoctonia solani). One potential component of integrated pathogen management is correct nitrogen $(\mathrm{N})$ fertilization. Bentgrass was grown in a chamber hydroponically at deficient, optimum, and excessive levels of $\mathrm{N}(2.5$, 10 , and $80 \mathrm{mM}$ ) with or without Rhizoctonia inoculation and grown for $56 \mathrm{~d}$. Not surprisingly, increasing solution $\mathrm{N}$ resulted in increased shoot and decreasing root biomass. Rhizoctonia inoculation did not appear to dramatically impact biomass. However, infection resulted in near total necrosis of all tissues at the low rate of $\mathrm{N}$ for inoculated plants, but plants at higher $\mathrm{N}$ rates and those not inoculated appeared healthy. The degree of infection will be assessed by means of quantitative PCR by determining the ratio of DNA from R. solani and A. stolonifera.

Does this antibiotic make my hypha look fat? Phenotypes associated with Bacillus mojavensis antagonism of Fusarium verticillioides

A. BLACUTT (1), T. R. Mitchell (2), S. E. Gold (2)

(1) University of Georgia, Athens, GA, U.S.A.; (2) USDA ARS, Athens, GA, U.S.A.

Phytopathology 105(Suppl. 4):S4.16

Fusarium verticillioides, a mycotoxigenic fungus, alternates between pathogenic and asymptomatic endophytic lifestyles in maize. Due to the pathogen's ubiquity and intimate association with maize, chemical control is cost-prohibitive. Bacillus mojavensis strain RRC101 is a bacterial endophyte demonstrating both in vitro antagonism of $F$. verticillioides and in planta reductions of disease and mycotoxin accumulation. Genomic followed by biochemical characterization of RRC101 show that it produces the fengycin lipopeptides and that these are responsible for in vitro activity against $F$. verticillioides. Fengycin exposure results in increased vacuole size and frequency as well as the presence of swollen hyphae, resulting in formation of "bulbs." Fluorescent staining indicates that both the cell wall and membrane expand in these bulbs, often followed by violent lysis. These effects are in keeping with the hypothesized membrane destabilization mechanism of action of these antibiotics and consistent with observations in other fungi. Biochemical analysis of antagonized $F$. verticillioides cultures showed an increase in mycotoxin production upon exposure to cyclic lipopeptides. We predict that inhibitor exposure directly or indirectly influences gene expression and we will present preliminary results from planned RNAsequencing experiments using lipopeptide-challenged $F$. verticillioides.

Developing bioclimatic models to predict suitable habitat for Armillaria spp. in Wyoming

J. T. Blodgett (1), J. W. Hanna (2), E. W. Pitman (2), S. Ashiglar (2), J. E. Lundquist (3), M. S. Kim (4), A. Ross-Davis (2), N. KLOPFENSTEIN (2)

(1) USDA Forest Service, FHP Region 2, Rapid City, SD, U.S.A.; (2) USDA Forest Service - RMRS, Moscow, ID, U.S.A.; (3) USDA Forest Service, FHP Region 10/PNWRS, Anchorage, AK, U.S.A.; (4) Kookmin University - Dept. Forestry, Environment and Systems, Seoul, South Korea

Phytopathology 105(Suppl. 4):S4.16

Armillaria species range from virulent root pathogens of diverse woody hosts to beneficial saprophytes, depending on host, geographic location, and environment. In conifers, A. solidipes (= A. ostoyae) is a main cause of growth loss and/or tree mortality, while other Armillaria spp. are frequently less aggressive. In Wyoming, A. sinapina and A. gallica can cause root disease of hardwoods. The objective of this study was to identify areas in Wyoming that are at potential risk for disease caused by Armillaria spp., based on the relationships among climatic conditions and known occurrences. Forest surveys resulted in a collection of 181 Armillaria isolates, which were identified by pairing tests and DNA-sequencing. Maximum Entropy species distribution models were generated based on 19 location-specific climatic variables and known occurrences of $A$. solidipes (106 locations), A. sinapina (51 locations), A. gallica (17 locations), and A. cepistipes (7 locations). Resulting bioclimatic models predict substantial areas with climate space suitable for Armillaria spp.; however, suitable climate space differs among species. Identifying Armillaria species and understanding their potential distributions are critical to develop prescriptive, habitat-specific, management decisions aimed at reducing disease impacts.

Seasonal occurrence of thrips species prevalent in soybean and the economic impact of Soybean vein necrosis virus in Wisconsin

C. BLOOMINGDALE (1), R. L. Groves (1), D. Smith (1)

(1) Univ of Wisconsin, Madison, WI, U.S.A.

Phytopathology 105(Suppl. 4):S4.16

Soybean vein necrosis disease (SVND) is caused by Soybean vein necrosis virus (SVNV), which is a relatively new tospovirus first discovered in the southern U.S. in 2008. Very little is known about the impact and importance of SVNV on soybeans. To better understand SVND and the vector (soybean thrips), field experiments were established in 2013 and 2014 to investigate the seasonality of thrips populations in Wisconsin soybean fields and the impact of SVND on soybean yield and quality. Yellow sticky card traps were used to sample thrips present in soybean fields on a weekly basis in both years. Sampling was performed at five locations across the major soybean-growing regions of Wisconsin. Peak captures in 2013 suggest two major movements of thrips into sampled fields, the first of which occurred in mid-July and the second in late August. Similar trends were observed in 2014. The diversity of 
thrips species captured in 2013 was low. Eastern flower thrips represented $91 \%$ of total captures, while soybean thrips represented $2 \%$. The magnitude of captures did differ by week and location $(\mathrm{P}<0.01)$. Preliminary data from 2014 suggest similarities in the species composition to those in 2013. The yield studies conducted in both years revealed a weak negative correlation between disease severity and yield and quality. Symptoms of SVND were not observed until late August in either year, suggesting SVND may be more problematic in years or climates conducive to early vector arrival.

Development of microsatellite markers for Fusicladium effusum, the causal agent of pecan scab

C. H. BOCK (1), C. Chen (1), K. L. Stevenson (2), B. W. Wood (1)

(1) USDA-ARS-SEFTNRL, 21 Dunbar Rd., Byron, GA, U.S.A.; (2) University of Georgia, Natural Products Lab, 2360 Rainwater Road, Tifton, GA, U.S.A.

Phytopathology 105(Suppl. 4):S4.17

Pecan scab (caused by F. effusum) is the most important diseases of pecan in the southeastern U.S. Microsatellite (simple sequence repeat, SSR) motifs were mined from the genome of Fusicladium effusum assembled from 454 pyrosequencing and Illumina Miseq reads. A total of 278 SSR primers were designed. The lengths of the predicted amplicons ranged from 119 to $209 \mathrm{bp}$, and contained 2 to 6 repeat units of di- to septo-nucleotide sequences. The primers were used to screen $48 \mathrm{~F}$. effusum isolates sampled from 11 populations of the pathogen throughout the southeastern U.S., using a 3500 Genetic Analyzer. Only 33 of the SSR primers were found to be both reliable and polymorphic. The number of polymorphic alleles among these codominant markers ranged from 2 to 19 , and the 33 primers differentiated all the 48 isolates from the 11 populations. Polymorphic loci ranged from 69.7$97.0 \%$ depending on the populations of $F$. effusum that were screened. These SSR primers should be useful in studying the genetic diversity and population structure of the pathogen.

Components of yield in pecan depend on tree height due to the distribution of scab in the canopy

C. H. BOCK (1), M. W. Hotchkiss (1), B. W. Wood (1)

(1) USDA-ARS-SEFTNRL, 21 Dunbar Rd., Byron, GA, U.S.A.

Phytopathology 105(Suppl. 4):S4.17

Scab (caused by Fusicladium effusum) severity (SS) and sample height in a pecan canopy has been previously characterized. In 3 field experiment in GA, SS at different sample heights and consequent effect on components of yield were characterized in fungicide treated (FT) and control (C) trees. FT trees were sprayed using an airblast sprayer. In August 2010 on cv Desirable mean SS on fruit was $39.6 \%$ on C and $22.4 \%$ on FT trees. Due to dry conditions in 2011 , SS was $1.1 \%$ on C and $0.12 \%$ on FT trees on cv Desirable, but was $10.4 \%$ and $4.0 \%$, respectively on $\mathrm{cv}$ Wichita. ANOVA showed significant effects of height in August on SS on fruit, fruit weight, and in October on fruit weight, nut fresh and dry weight, depending on $\mathrm{cv}$ and season $(\mathrm{P}=<0.0001$ $0.4)$. Whereas SS decreased with height on $\mathrm{C}$ trees, it remained similar or increased with height on FT trees. However, yield components tended to increase with height on $\mathrm{C}$, but remained similar or decrease with height on FT trees. SS on C trees decreased with height $\left(\mathrm{R}^{2}=0.57-0.98\right)$, but there was little effect on FT trees $\left(\mathrm{R}^{2}=0.01-26\right)$. On $\mathrm{C}$ trees components of yield (fruit, nut fresh and dry weight) most often increased with height $\left(\mathrm{R}^{2}=0.08-0.94\right)$, while on FT trees components of yield most often decreased with height $\left(\mathrm{R}^{2}=0.04\right.$ 0.89). SS (all heights) in August was most often negatively related to fruit weight in August $\left(\mathrm{R}^{2}=0.01-0.92\right)$ and nut dry weight $\left(\mathrm{R}^{2}=0.29-0.86\right)$ in October in both $\mathrm{C}$ and FR trees.

Genomic comparison of Ralstonia solanacearum strains which are pathogenic at low temperatures on tomato

A. M. B. BOCSANCZY (1), J. C. Huguet-Tapia (2), D. J. Norman (3)

(1) University of FLorida, Apopka, FL, U.S.A.; (2) University of Florida, Gainesville, FL, U.S.A.; (3) University of Florida, Apopka, FL, U.S.A. Phytopathology 105(Suppl. 4):S4.17

Ralstonia solanacearum is one of the most important bacterial plant pathogen in the world. It causes bacterial wilt on a very broad range of plant species, including mono and dicotyledons. In the U.S. it is most economically important in solanaceous crops, including potato, tomato, and tobacco. $R$. solanacearum is mostly adapted to tropical climates however members of phylotype IIB sequevar 1(also known as R3B2) and sequevar 4NPB can cause disease in tomato at low temperatures. In our lab we sequenced 4 strains from the phylotype IIB and IIA groups. The latter are non pathogenic at low temperature. A genome comparison of 3 strains (phylotype IIB) pathogenic and 3 (phylotype IIA and I) non pathogenic at low temperature reveals fewer differences than expected. We identified 74 genes that are present in the cold pathogenic group and absent in the non pathogenic one. Forty two (42)\% of the genes in the list are hypothetical proteins. The other two representative groups are transcriptional regulators (11\%) and transport related (16\%). The high number of transcriptional regulators suggests a possible role in the activation or deactivation of virulence genes at low temperature. Thirty eight (38) genes in the list are identical in all the R3B2 genomes publicly available. A full comparison, including metabolic pathways and SNP analysis, is in progress. The comparative analysis will provide new hypothesis and candidates for the explanation of virulence at low temperature.

Characterization of root associated and foliar fungal endophytes of winter wheat grass

D. BOKATI (1), K. Craven (1), B. Prithiviraj (1), M. H. Chi (1)

(1) The Samuel Roberts Noble Foundation, Ardmore, OK, U.S.A.

Phytopathology 105(Suppl. 4):S4.17

Symbiotic fungal endophytes are known to enhance plant fitness by conferring abiotic and biotic stress tolerance, especially under stressful conditions. Most symbiotic endophytes enhance the absorption of water and minerals and in return get direct access to host carbohydrates. Winter wheat is used as dual purpose crop i.e. for forage and grain, and usually planted in late summer and harvested early summer of next year. During extended summers, the excessive heat tends to severely affect growth and survival of these plants. We are aiming on characterizing the root-associated and foliar fungal endophytes of this important forage using culture-dependent and -independent methods. Both methods will utilize the Internal Transcriber Spacer region of fungal ribosomal DNA for identification purposes. Any symbiotic fungal endophytes associated with heat tolerance will be determined using a high throughput in vitro assay we have developed. Overall, our goal is to mitigate heat tolerance in winter wheat using beneficial fungal endophytes. These studies are currently underway, and our results to dates will be presented.

A Fusarium fujikuroi population isolated from grapes reveals the need to re-evaluate the species' fumonisin production potential

S. L. BOLTON (1), P. M. Brannen (1), A. E. Glenn (2)

(1) Univ of Georgia, Athens, GA, U.S.A.; (2) USDA - ARS -TMRU, Athens, GA, U.S.A.

Phytopathology 105(Suppl. 4):S4.17

Mycotoxins pose a documented threat to crops worldwide, making plant pathology and food science collaboration necessary for the safety and growth of our food supply. The genus Fusarium produces several mycotoxins, including fumonisins, associated with serious diseases of humans and animals. $F$. verticillioides and $F$. proliferatum are recognized as the main fumonisin producers in economically significant crops. To date, most $F$. fujikuroi strains tested in-vitro produce undetectable/low amounts of fumonisins. In 2013, a large population of Fusarium spp. was recovered from southeastern U.S. winegrapes, representing eight vineyards and ten grape varieties. This population includes 239 isolates of $F$. fujikuroi and 52 isolates of $F$. proliferatum, as identified via PCR amplification of the translation elongation factor 1-alpha gene followed by sequence comparisons to verified strains in GenBank. A phylogenetic analysis revealed genetic diversity within populations of both species, and an in-vitro mycotoxin assay quantified fumonisin B1-B3 production for representative isolates using HPLC-MS/MS. In contrast to previously published work, these F. fujikuroi isolates produced high levels of fumonisins comparable to $F$. verticillioides and $F$. proliferatum. Published strains of all three species were included in the assay for comparison. This research shows that $F$. fujikuroi needs to be reconsidered as both a fumonisin producer of concern and as a threat to food safety.

Insight into DMI-resistance - RNA-seq analysis of Cercospora beticola DMI-resistant and -sensitive strains in response to tetraconazole

M. BOLTON (1), L. Faino (2), B. Thomma (2), R. De Jonge (3), G. Secor (4)

(1) USDA ARS, Fargo, ND, U.S.A.; (2) Wageningen University, Wageningen, Netherlands; (3) Ghent University, Ghent, Belgium; (4) North Dakota State University, Fargo, ND, U.S.A.

Phytopathology 105(Suppl. 4):S4.17

The hemi-biotrophic fungus Cercospora beticola causes Cercospora leaf spot (CLS) of sugarbeet. CLS management measures rely on the application of sterol demethylation inhibitor (DMI) fungicides. Reduced sensitivity to DMIs has been reported recently in sugarbeet growing regions worldwide. We have shown previously that $C b C y p 51$, which encodes the DMI target enzyme sterol P450 14 $\alpha$-demethylase in C. beticola, is over-expressed in DMI-resistant isolates. To gain additional insight and identify other mechanisms involved with DMI-resistance, we used RNA-seq to identify genes differentially expressed in DMI-resistant and -sensitive C. beticola isolates upon exposure to tetraconazole or the control treatment in vitro. Interestingly, both the resistant and sensitive isolates responded with a marked induction of 16 of the 22 genes in the ergosterol biosynthesis pathway in response to tetraconazole. 
All 16 genes were induced to similar expression levels between the two isolates except for $C b C y p 51$ and $C b E r g 3$. In total, 110 genes were uniquely differentially-expressed in the DMI-resistant isolate. Genes previously implicated with fungicide resistance such as ABC and MFS transporters were identified. The most highly induced gene in the DMI-resistant isolate encodes a protein with multiple transmembrane regions known to be associated with DMI resistance in yeast. A detailed pathway analysis will be presented.

\section{Diversity of Phytophthora species detected from retail nursery plants in} Nevada

R. A. Bomberger (1), S. WANG (1)

(1) Nevada Department of Agriculture, Sparks, NV, U.S.A.

Phytopathology 105(Suppl. 4):S4.18

Nursery stock movement is one of most effective pathways for spreading regulated plant pathogens such as Phytophthora ramorum, the causal agent of the disease sudden oak death. To detect potential introductions of $P$. ramorum and other Phytophthora species via nursery stock, major retail nurseries in Washoe, Douglas, Carson City, and Clark counties were surveyed for plants showing leaf blight, dieback, stem canker, or root and crown rot. Plant samples were taken and then plated on PARP selective medium to isolate Phytophthora species. Phytophthora isolates were transferred to corn meal agar and V8 juice agar for further morphological identification. DNA sequences amplified from regions of rRNA, elongation factor and/or betatubulin genes were obtained and used to complement the morphological determination. During the 2013 and 2014 summer surveys, $P$. nicotianae was the most prevalent species found from 6 plant families; P. cactorum and P. citricola were frequently found on Rhododendron and other 2 plant families. Of 24 Phytophthora detections, 20 were from retail nurseries in southern Nevada, where nursery stock suffer both acute and chronic heat and drought stress due to the desert climate. The frequent detections of multiple species on a range of host plants suggest that Phytophthora pathogens carried by nursery stock survive well in hot and dry condition, and that Nevada's harsh environment does not prevent introduction of Phytophthora when it occurs.

\section{Morphological and molecular identification of Verticillium species causing vascular wilt of peppermint in Nevada}

R. A. BOMBERGER (1), S. Wang (1)

(1) Nevada Department of Agriculture, Sparks, NV, U.S.A.

Phytopathology 105(Suppl. 4):S4.18

In 2014, an outbreak of vascular wilt disease was noticed in peppermint (Mentha $\times$ piperita) fields with patches of affected plants exhibiting rapid death of foliage and darkened stems. To isolate the causative agent, sections of affected vascular tissues were placed onto potato dextrose agar (PDA) amended with streptomycin sulfate and incubated at $22 \circ \mathrm{C}$ in the dark. Verticillium was invariably isolated from all samples collected from 4 fields, confirming our initial diagnosis of Verticillium wilt. To further identify the species, 38 single spore cultures (SSCs) were obtained from 11 field isolates and compared morphologically on $2 \%$ water agar and PDA. All SSCs and field isolates produced abundant microsclerotia on both media and there was no significant morphological variation observed among them. Specific DNA primers for $V$. dahliae were used to amplify a fragment of $495 \mathrm{bp}$ from the ITS region of rRNA gene. Sequence analysis of amplicons revealed that 33 out of 38 SSCs had the same amplicon sequence (Accession No. KP822065), with $100 \%$ homology to the corresponding regions of $V$. dahliae JR2 strain (Accession No. CP009075.1). Five SSCs had minor mutations, deletion and/or insertions in their amplicon sequences. Our data suggest that the pathogen isolated from diseased peppermint plants belongs to $V$. dahliae, and that there is no other Verticillium species involved in the outbreak. To our knowledge, this is the first report of $V$. dahliae on peppermint in Nevada.

Effects of "extreme" diurnal temperature highs on initiation of infection and sporulation by Uromyces transversalis, causal agent of gladiolus rust M. R. BONDE (1), S. E. Nester (2), C. L. Palmer (3)

(1) USDA-ARS, Middletown, MD, U.S.A.; (2) USDA-ARS, Frederick, MD, U.S.A.; (3) IR-4, Rutgers University, Princeton, NJ, U.S.A.

Phytopathology 105(Suppl. 4):S4.18

Preliminary studies in our laboratory suggested the APHIS-regulated pathogen $U$. transversalis, causal agent of Gladiolus rust, might be sensitive to high temperatures. To determine where in the U.S. the disease might become established, a study was conducted to show effects of extreme diurnal temperature highs on the pathogen. Gladiolus plants cv. Border Mix were inoculated with a urediniospore suspension and distributed to four growth chambers. One operated with a continuous $23^{\circ} \mathrm{C}$ high $/ 12^{\circ} \mathrm{C}$ low for the duration of the experiment and served as the "no heat wave" control. The others operated with a $35^{\circ} \mathrm{C}$ high $/ 24^{\circ} \mathrm{C}$ low (termed "heat wave") for 3,6 , or 9 days, and then were reprogrammed to operate with the same temperature profile as the control. Urediniospores were collected weekly and percent leaf area covered by lesions determined. With no heat waves, urediniospore production began at 15 days and produced a total of $138 \times 10^{6}$ spores per plant in 66 days. Plants subjected to 3, 6, or 9 days heat waves produced $28 \%, 30 \%$, and $6.5 \%$ as many urediniospores as no heat waves. Percent pustule area per plant by 22 days was $15 \%, 3 \%, 0.5 \%$, and $0.0 \%$, respectively, for $0,3,6$, and 9 days heat waves. The experiment was conducted twice and showed that a diurnal temperature high of $35^{\circ} \mathrm{C}$ for 1 hour, for as few as three days, significantly reduced lesion areas and total urediniospores. Effects of diurnal temperature highs on later stages of disease are being studied.

Emergence of common bean seedlings from seeds infected by Sclerotinia sclerotiorum and treated with Clonostachys rosea

F. C. BOREL (1), L. A. Maffia (2), L. F. Soares (1)

(1) Univ Federal De Vicosa, Vicosa, Brazil; (2) Universidade Federal de Vicosa, Vicosa, Brazil

Phytopathology 105(Suppl. 4):S4.18

White mold, caused by Sclerotinia sclerotiorum, is an important disease in common bean. The fungus infects the cotyledons of seeds and reduces plant emergence in the field. We evaluated the use of Clonostachys rosea on bean seed treatment for management of white mold. Seeds were treated with alcohol $70 \%$ and sodium hypochlorite $2 \%$ and laid on either S. sclerotiorum culture on PDA or plain PDA. After $24 \mathrm{~h}$ at $20^{\circ} \mathrm{C}$, the seeds were removed, immersed in a suspension with $10^{7}$ C. rosea conidia. $\mathrm{mL}^{-1}$ or in sterile distilled water for 30 or $60 \mathrm{~min}$, and planted in an organic substrate in $0.5 \mathrm{~L}$ pots. After one week, the emergence of seeds infected with $S$. sclerotiorum and immersed in water for 30 or $60 \mathrm{~min}$ was 1 or $0 \%$, respectively, whereas the emergence of seeds treated with C. rosea for 30 or 60 min was $14 \%$ or $37 \%$, respectively. The emergence of healthy seeds treated with $C$. rosea or water was similar and higher than $60 \%$. After one month, root, stem, and leaf fragments were immersed in alcohol $70 \%$ and sodium hypochlorite $2 \%$, and transferred to paraquat-chloramphenicol-agar medium in Petri dishes. C. rosea grew and sporulated in all plant fragments. As $C$. rosea increased the emergence of bean seedlings infected with $S$. sclerotiorum and was endophytic in bean plants, it will be tested for use in the integrated management of white mold. Sponsored by FAPEMIG and CNPq.

Compound(s) produced by Clonostachys rosea are deleterious to Botrytis cinerea

A. V. BORGES (1), R. M. Saraiva (2), F. C. Borel (3), L. A. Maffia (3)

(1) Univ Federal De Vicosa, Vicosa, Brazil; (2) Universidade Federal de Vicosa, UFV, Brazil; (3) Universidade Federal de Vicosa, Vicosa, Brazil Phytopathology 105(Suppl. 4):S4.18

Enzyme and secondary metabolites produced by Clonostachys rosea are deleterious to some pathogens. Therefore, we evaluated the production of compound(s) as a mechanism of $C$. rosea antagonism to $B$. cinerea in in vitro and in vivo experiments. After 3 days of growing $C$. rosea over cellophane above potato-dextrose-agar medium (PDA) in Petri dishes, we removed the cellophane with the colony and set sclerotia or mycelial disc of $B$. cinerea onto the medium. No sclerotial germination nor mycelial growth took place. Both growth and germination resumed when the mycelium and sclerotia were transferred to sterile PDA. After 4 days of growing C. rosea in potato dextrose broth, we filtered the culture. The filtrate was applied 1 day before, simultaneously, 1 or 5 days after $B$. cinerea inoculation on the tops of cut tomato stem segments. Lesion length was reduced more than $90 \%$ when the filtrate was applied 1 day before or simultaneously with the inoculation. We repeated the procedures with whole tomato plants stem inoculated with $B$. cinerea. Lesion length was reduced by about $50 \%$ when the filtrate was applyied one day before inoculation. We concluded that the antagonism of $C$. rosea is also due to the production of compound(s) that is(are) deleterious to $B$. cinerea, most likely with a fungistatic effect. Sponsored by FAPEMIG and CNPq.

Identifying novel resistance sources for bacterial diseases of rice in West Africa

A. BOSSA-CASTRO (1), C. Raghavan (2), E. E. Delorean (3), C. M. Vera Cruz (4), H. Leung (2), G. Mosquera (5), V. Verdier (6), J. E. Leach (7)

(1) Colorado State Univ, Fort Collins, CO, U.S.A.; (2) International Rice Research Institute (IRRI), Los Baños, Philippines; (3) Colorado State University, Fort Collins, CO, U.S.A.; (4) Intl Rice Research Inst, Metro Manila, Philippines; (5) International Center for Tropical Agriculture (CIAT), Palmira, Colombia; (6) Institut de Recherche pour le Développement, Montpellier, France; (7) Colorado State University, Ft. Collins, CO, U.S.A. Phytopathology 105(Suppl. 4):S4.18

Two devastating diseases of rice, bacterial blight (BB) and bacterial leaf streak (BLS) caused by Xanthomonas oryzae pv. oryzae (Xoo) and 
Xanthomonas oryzae pv. oryzicola (Xoc), respectively, are widespread in Africa and Asia. So far, there is no known source of single gene resistance for African $\mathrm{Xoo}$ and $\mathrm{Xoc}$, and effective BB and BLS resistance is largely absent in the rice germplasm currently used in Africa. Our goal is to identify novel, broad-spectrum resistance sources to control BB and BLS in rice. We are using a mapping resource called Multi-parent Advanced Generation InterCross (MAGIC) population to facilitate identification and mapping of new resistance traits. MAGIC populations have an increased level of recombination and provide higher precision and resolution to detect quantitative trait loci (QTL). The indica MAGIC population was developed using eight elite indica cultivars with diverse genetic backgrounds as founders. Two subsets were randomly selected at the fourth (S4) and eighth (S8) stage of selfing for genotyping by sequencing and phenotyping using African Xoo and Xoc strains, along with founders. To map disease resistance QTL, we performed genome-wide association and interval mapping analyses and identified QTL that potentially confer broad-spectrum resistance effective against African Xoo and Xoc strains. Introduction of identified sources of resistance to breeding programs will be facilitated, since MAGIC lines are derived from elite cultivars.

\section{Phytophthora species infesting Californian forest soil during a three year drought \\ T. B. BOURRET (1), H. K. Mehl (1), D. M. Rizzo (1) \\ (1) University of California, Davis, Davis, CA, U.S.A \\ Phytopathology 105(Suppl. 4):S4.19}

In coastal Californian forests, Phytophthora ramorum causes the devastating disease sudden oak death (SOD). Following a 2008 fire in the Big Sur region, $P$. ramorum appeared to survive the disturbance only in living infested plants, while the co-occurring $P$. pseudosyringae was able to survive away from hosts, presumably in the soil. The stress caused by the 2012-2014 Californian drought may have had a similar effect on Phytophthora survival. During the second and third summers of the drought, $662 \sim 1 \mathrm{~kg}$ samples of soil were collected from 90 long-term forest plots established for the purposes of monitoring SOD. Plots containing coast redwood (Sequoia sempervirens) were sampled in 2013 while mixed evergreen forest plots were sampled in the more severe drought year of 2014. Soils were homogenized and $50 \mathrm{~g}$ was flooded with $200 \mathrm{ml} \mathrm{H} \mathrm{H}_{2} \mathrm{O}$. A Rhododendron leaf was floated as bait for four days. Discs were excised from surface sterilized leaves, and submerged in Phytophthora-specific media. Strains were isolated and identified using ITS rDNA sequences. Surprisingly, $P$. cactorum was the most commonly isolated species in 2013, followed by $P$. ramorum and P. pseudosyringae. In 2014, only $P$. pseudosyringae and $P$. syringae were isolated from soil, despite frequent isolations of $P$. ramorum from plant material. While P. ramorum appears to depend on living hosts to survive abiotic disturbance, other species produce durable propagules able to survive harsh conditions on their own. Weather conditions during time of corn silking affects aflatoxin
contamination

K. BOWEN (1)

(1) Auburn University, Auburn, AL, U.S.A.

Phytopathology 105(Suppl. 4):S4.19

High temperatures and dry conditions are known to be conducive for aflatoxin contamination of corn. While these conditions commonly occur in Alabama, aflatoxin contamination of corn is highly variable. We monitored aflatoxin concentrations in corn from 2010 through 2012 at research sites across Alabama, for a total of 17 site-yrs. At each site, several cultivars were planted; these cultivars had relative maturity ratings from 111 to 117 . No differences in aflatoxin contents were found among cultivars within site-yrs. Over all siteyrs, aflatoxin contamination ranged from $0 \mathrm{ppb}$ (at two sites in 2010) to greater than $140 \mathrm{ppb}$ (at two other sites in 2010 and 2011). Maximum, minimum and average temperatures, as well as rainfall, dry days and 2 and 3 day dry periods for varying intervals around silking for each site-yr were examined for their relationship to average aflatoxin content. Stepwise regression indicated that $73 \%$ of the variation in aflatoxin content was explained by (1) the number of dry days over a 10 -day period immediately following silking and (2) the average maximum daily temperature over the 20 day period prior to silking. Average aflatoxin contents $>20 \mathrm{ppb}$ were only noted with $>5$ dry days over 10 days after silk. Highest aflatoxin contents $(>$ $100 \mathrm{ppb}$ ) were found only when maximum daily temperatures for 20 days preceding silk averaged $>30.5 \mathrm{C}$.

Efficacy of late-dormant calcium polysulfide applications for control of Exobasidium leaf and fruit spot of blueberry

P. BRANNEN (1), H. Scherm (2), A. Savelle (2), B. Shirley (3), J. Jacobs (3),

J. Taylor (3), P. Edwards (3), R. Holland (3), S. Curry (3), T. Varnedore (3)
(1) Univ of Georgia, Athens, GA, U.S.A.; (2) University of Georgia, Athens, GA, U.S.A.; (3) University of Georgia Cooperative Extension Service, Athens, GA, U.S.A.

Phytopathology 105(Suppl. 4):S4.19

Calcium polysulfide products, lime sulfur and Sulforix, were evaluated for control of Exobasidium leaf and fruit spot, caused by Exobasidium maculosum, on the highly susceptible rabbiteye blueberry (Vaccinium virgatum) cultivar 'Premier' in the south Georgia counties of Appling, Bacon, Clinch, Irwin, and Jeff Davis. Treatments included an untreated control, lime sulfur (47.3 1/ha; $n=8)$, and Sulforix (19 1/ha; $n=7)$ applied at a late-dormant phenology with an airblast sprayer ( $\sim 663 \mathrm{l} / \mathrm{ha}$ total solution); no additional fungicides were applied for the remainder of the season. Despite the highly reduced application rate, the Sulforix formulation, containing calcium polysulfide plus unspecified additives, provided equivalent efficacy to that of lime sulfur; a single late-dormant application of either lime sulfur or Sulforix provided substantive management of Exobasidium leaf spots, 86.5 and $88.1 \%$ control, and fruit spots, 90.6 and $93.8 \%$ control, respectively. The epidemiology of Exobasidium maculosum has not been elucidated, but the high level of disease control afforded with both calcium polysulfide products at the late-dormant stage may indicate that overwintering inoculum and/or early-season infections are of primary importance to disease development.

Effect of waste lime on Aphanomyces damping-off of sugar beet in soils over a wide range of $\mathbf{p H}$

J. R. BRANTNER (1), E. A. Crane (2), A. K. Chanda (1)

(1) Department of Plant Pathology, University of Minnesota, Crookston, MN, U.S.A.; (2) Department of Plant Pathology, North Dakota State University, Fargo, ND, U.S.A.

Phytopathology 105(Suppl. 4):S4.19

Damping-off and root rot of sugarbeet, caused by the soilborne pathogen Aphanomyces cochlioides, are widespread problems in the sugar beet growing areas of Minnesota $(\mathrm{MN})$ and North Dakota (ND). Application of factory waste lime to fields is used to control A. cochlioides, but data is lacking on efficacy in fields with higher $\mathrm{pH}$ soils. Effect of waste lime amendment on Aphanomyces damping-off was evaluated in 12 field soils collected from six counties in $\mathrm{MN}$ and $\mathrm{ND}$, spanning a $\mathrm{pH}$ range of 5.3 to 8.0. Each field soil was split and amended with lime [22.5 metric ton wet weight/ha $(162 \mathrm{~g} / 5 \mathrm{~L}$ soil)] or not amended and incubated 4 weeks in a growth chamber at $25^{\circ} \mathrm{C}$ with a 14-hour photoperiod. Sugar beet seed was sown $(25 \mathrm{seed} / \mathrm{pot})$ and incubated 4 weeks under the same conditions with daily watering to provide high soil moisture to favor damping-off. Aphanomyces soil index values (SIV $=0-100$ scale where $0=$ no disease and $100=$ all plants died during the $4-$ week assay) were determined. In addition, soil samples were submitted for commercial $\mathrm{pH}$ and nutrient analyses. Aphanomyces damping-off was significantly $(P=0.05)$ reduced in 9 of 12 soils with lime amendment. Reduction in Aphanomyces SIV was not correlated with original soil $\mathrm{pH}$ or with change in soil $\mathrm{pH}$ following lime amendment. Reduction in Aphanomyces SIV was correlated with increase in soil $\mathrm{Ca}^{2+}(P=0.05)$. Waste lime has the potential to reduce infection of sugar beet by $A$. cochlioides over a range of soil $\mathrm{pH}$.

\section{Integrated management of Rhizoctonia on sugar beet: What's new?}

J. R. Brantner (1), A. K. CHANDA (1)

(1) Department of Plant Pathology, University of Minnesota, Crookston, MN, U.S.A.

Phytopathology 105(Suppl. 4):S4.19

Damping-off and crown and root rot of sugar beet, caused by Rhizoctonia solani AG 2-2, are prevalent in Minnesota and North Dakota. Past control options included early planting of partially resistant cultivars and the use of in-furrow (IF) or postemergence (PE) fungicides. In this study, newly available seed treatments were compared to azoxystrobin IF on a resistant and susceptible cultivar as stand-alone treatments and with PE application of azoxystrobin for control of Rhizoctonia. Seed treatments included metconazole + rizolex (Metlock), penthiopyrad (Kabina), Metlock + Kabina, sedaxane and an untreated control. There were significant $(P=0.05)$ cultivar by at-planting treatment interactions for plant stand and number of harvestable roots. On the resistant cultivar, all seed treatments except Metlock resulted in higher stand than azoxystrobin IF. On the susceptible cultivar, azoxystrobin IF resulted in stands as high as the most efficacious seed treatments. There were no significant interactions for root yield. Root yield for azoxystrobin IF, Kabina, sedaxane, Metlock + Kabina, and Metlock were significantly higher than the untreated control, averaging 55.0, 53.7, 51.9, 51.7, 48.1, and $41.1 \mathrm{Mg}$ per ha, respectively. Application of PE azoxystrobin did not affect stand or yield. New seed treatments provided similar control of damping-off and increased root yield compared to azoxystrobin IF with less cost and greater convenience at planting. 
Thiophanate-methyl sensitivity of the frogeye leaf spot pathogen in Mississippi

N. BROCHARD (1), M. Tomaso-Peterson (1), T. Allen (2), J. R. Standish (1) (1) Mississippi State University, Mississippi State, MS, U.S.A.; (2) Mississippi State University, Stoneville, MS, U.S.A.

Phytopathology 105(Suppl. 4):S4.20

Frogeye leaf spot (FLS) is a foliar disease of soybean caused by Cercospora sojina Hara. Quinone outside inhibitor (QoI) fungicides are widely used to manage FLS, but QoI resistance has been confirmed in Mississippi (MS). Based on this, alternative fungicide classes need to be considered for FLS control. Thiophanate-methyl (TM) is a methyl benzimidazole carbamate fungicide effective for controlling FLS. TM inhibits microtubule production in the beta tubulin 2 (TUB2) gene. The objective of this study was to determine the sensitivity of a subset of $C$. sojina isolates from MS to TM. QoI-resistant and-sensitive isolates were screened at various concentrations of TM in vitro. The effective concentration at which growth was inhibited by 50 percent $\left(\mathrm{EC}_{50}\right)$ was determined and a partial fragment of the $C$. sojina TUB2 gene was sequenced to determine the presence of an amino acid substitution at the binding site of TM. Substitutions at position 198 from glutamic acid (E) to alanine $(A)$, glycine $(\mathrm{G})$, or lysine $(\mathrm{K})$; or a substitution from phenylalanine (F) to tyrosine (Y) at position 200 are known to confer resistance to TM. As a result of this study, the isolates were found to have an $\mathrm{EC}_{50}$ range of 0.97 to $13.67 \mu \mathrm{g} / \mathrm{ml}$. The partial fragment of the TUB2 gene revealed no amino acid substitutions confirming that resistance to TM did not occur in this subset of C. sojina isolates. Based on these results additional screening needs to be performed on the remaining isolates from MS.

Phenylalanine ammonia lyase pathway components contribute to resistance of Arabidopsis thaliana seeds to Aspergillus infection

M. BRODHAGEN (1), J. C. Young (1), A. Batson (1), Z. Cranny (1), T. DeSitter (1), M. Lahman (1)

(1) Western Washington Univ, Bellingham, WA, U.S.A.

Phytopathology 105(Suppl. 4):S4.20

Aspergillus flavus and Aspergillus parasiticus are cosmopolitan, mycotoxigenic fungi that contaminate food and feeds with carcinogenic and potentially lethal aflatoxins. Identification of genes conferring plant resistance to Aspergillus would benefit from a model pathosystem. Seeds of the plant model Arabidopsis thaliana permit only mild infection by Aspergillus. However, we found that $A$. thaliana lines mutated in chalcone synthase, a gene at the head of the phenylalanine ammonia lyase (PAL) biosynthetic pathway, are very susceptible to infection by both Aspergillus flavus and the closely related model fungus, Aspergillus nidulans. Our objective was to develop a two-model seed pathosystem with which to identify $A$. thaliana PAL pathway genes responsible for seed resistance to infection by $A$. nidulans. Our studies revealed that $A$. thaliana mutants disrupted in biosynthesis of PAL pathway intermediates between chalcone and leucocyanidin failed to resist infection when directly inoculated with spores of A. nidulans, while mutants disrupted in branches from this pathway leading to kaempferol, quercetin, or cyanidin remained resistant. Furthermore, inhibition of $A$. nidulans growth on defined medium by aqueous extracts from WT but not chalcone synthase or dihydroflavonol reductase (leucocyanidin) mutant seeds provides evidence that the resistance factor(s) is/are water-soluble.

A previously undescribed badnavirus genome sequence determined by Illumina sequencing from cacao trees showing virus-like symptoms in Trinidad

J. K. BROWN (1), M. Zia-Ur-Rehman (2), N. Chingandu (3), T. N. Sreenivasan (4), S. Surujdeo-Maharaj (4), O. Gutierrez (5), H. W. Herrmann (1), P. Umaharan (4)

(1) University of Arizona/School of Plant Sciences, Tucson, AZ, U.S.A.; (2) University of Lahore, Punjab, Pakistan; (3) University of Arizona/School of Plant Sciences, TUCSON, AZ, U.S.A.; (4) Cocoa Research Center/The University of the West Indies, St. Augustine, Trinidad and Tobago; (5) USDA-ARS Subtropical Horticultural Research Station, Miami, FL, U.S.A. Phytopathology 105(Suppl. 4):S4.20

Cacao (Theobroma cacao) is a tropical crop, with origins in the Amazon Basin that dominates the economies of most West African countries where Cacao swollen shoot virus (CSSV) causes tree death 2-5 yrs post-infection. Cacao leaf samples showing symptoms like those previously associated with suspect virus 'strain-A' were collected from cacao trees in Trinidad and subjected to CTAB-DNA isolation. Circular DNA was enriched using rolling circle amplification followed by Illumina HiSeq. 2500 libraries were prepared for DNA sequencing per manufacturer's instructions. An apparent complete genome sequence of 7533 nucleotides (nt) was assembled de novo. Alignment and phylogenetic analysis (MUSCLE, SDT 1.2) of the RT/RNase-H region revealed that the Trinidad virus shared maximum nt identity at $67.1 \%$ with
Sweet potato badnavirus (SwPBV), thereby exceeding the $80 \%$ badnavirus species threshold, and suggesting it is a new species. However, the complete genome shared $63.8 \%$ nt identity with Dioscorea alata bacilliform virus $(\mathrm{DaBV})$. The genome contained four open reading frames: ORF1, ORF2, ORF3 and ORF Y. It differs from CSSV, which in addition to those ORFs, contains ORF-X. The viral genome was also badnavirus-like in having a tRNA $^{\text {met }}$ priming sequence, CAAT and TATA boxes, and a polyadenylation signal. Even though virus infection of cacao was suspected in 1944, this is the first association of a badnavirus with cacao in Trinidad, and in the Western Hemisphere.

Evaluation of dormant sprays for reducing pecan scab inoculum and implications for disease management

K. A. BROWN (1), T. B. Brenneman (2)

(1) Univ of Georgia, Baconton, GA, U.S.A.; (2) University of Georgia, Tifton, GA, U.S.A.

Phytopathology 105(Suppl. 4):S4.20

Field trials in 2014 in Tifton, Baconton and Albany, GA evaluated the effect of dormant applications of fungicides on pecan scab epidemics by reducing inoculum levels. All tests were RCBD with 4-5 replications. Treatments included: Sulforix $(14.0 \mathrm{~L} / \mathrm{ha})$, Elast $(3.6 \mathrm{~L} / \mathrm{ha})$, Sulforix + Elast $(14.0+3.6$ $\mathrm{L} / \mathrm{ha}$ ), and an untreated. All trees received a standard fungicide program after budbreak. True dormant sprays of Elast alone or with Sulforix showed some reductions in leaf scab, nut scab, and stem lesions on current shoots at 2 of 3 locations. An additional trial at Tifton evaluated a mid-season "dormant" fungicide applied in early June. Specific treatments included: Lime-sulfur $(37.3 \mathrm{~L} / \mathrm{ha})+$ Pentra-Bark, Sulforix $(14.0 \mathrm{~L} / \mathrm{ha})$ or nontreated. No fungicides were applied prior to initiation of the test, allowing scab to develop on the leaves. Paired trees were then either coversprayed with a standard commercial program the remainder of the season or left nontreated. Both sulforix and lime-sulfur reduced nut scab, but not nearly as much as commercial coversprays which reduced scab to $8 \%$ severity, versus $50 \%$ in the nontreated trees. Of the dormant sprays evaluated, Elast provided the most consistent disease reductions, but the benefits were relatively small. Early season use may provide additional disease control due to reductions in overwintering inoculum. Applications could be made after bud break to capitalize on the known protectant activity of the product.

Optimizing anaerobic soil disinfestation for control of Prunus replant disease

G. T. BROWNE (1), N. J. Blackburn (2)

(1) USDA ARS, Davis, CA, U.S.A.; (2) University of California, Davis, CA, U.S.A.

Phytopathology 105(Suppl. 4):S4.20

Prunus replant disease (PRD) is a soilborne complex that suppresses growth and productivity of replanted almond and stone fruit orchards. Preplant soil fumigation (SF) prevents PRD but is increasingly regulated and costly. We reported in 2014 that anaerobic soil disinfestation (ASD) implemented in 2013 with rice bran ( 20 metric tons/treated ha; in strips covering $50 \%$ plot area) was as effective as SF (Telone C35, $600 \mathrm{~kg} /$ treated ha; $50 \%$ plot area) for preplant control of Pythium ultimum (a PRD contributor) and early stimulation of replanted almond orchard growth, but cost of the ASD was more than double that of SF. Here, we report: i) full-season orchard responses to 2013 ASD treatments and ii) early responses to new 2014 treatments designed to reduce ASD costs. By end of 2014, the 2013 ASD and SF treatments had improved replanted almond orchard growth equally (by 85 to $92 \%$ ), compared to controls, indicating sustained ASD benefit. The 2014 ASD treatments were applied as in 2013 (bran incorporated to 15-cm depth, covered with clear plastic film, drip irrigated to maintain soil moisture at or above field capacity for $6 \mathrm{wk}$ ), except bran rates and treated areas were 100,60, and 36\% of those used in 2013. All 2014 ASD treatments $(100,60$, and 36\%) were as effective as SF in reducing $P$. ultimum inoculum density (to 0 to $95 \mathrm{cfu} / \mathrm{g}$ soil), compared to controls (2008 to $3663 \mathrm{cfu} / \mathrm{g}$ ). These results suggest that ASD offers a cost-effective, non-fumigant approach to PRD control.

Concentrated ethanol-derived switchgrass extractives inhibit plant and foodborne pathogens

A. BRUCE (1), H. B. Korotkin (1), B. H. Ownley (1), J. Tao (1), L. M. Kline (1), N. Labbe (1), K. D. Gwinn (1), D. H. D’Souza (1), N. Moustaid-Moussa (2)

(1) University of Tennessee, Knoxville, TN, U.S.A.; (2) Texas Tech University, Lubbock, TX, U.S.A.

Phytopathology 105(Suppl. 4):S4.20

Conversion efficiency of switchgrass lignocellulose to ethanol for biofuel production is reduced by a non-structural portion of the biomass known as extractives. This fraction inhibits enzymes and microorganisms in the biofuel 
conversion process and contains high concentrations of phenolic compounds. Our goal is to develop a value-added antimicrobial product from switchgrass extractives. Our specific aim was to test four concentrations $(30 \times, 45 \times, 60 \times$, $75 \times$ ) of ethanol extracts of switchgrass for inhibition of bacterial pathogens in disk diffusion assays with controls. Agrobacterium tumefaciens, Clavibacter michiganensis subsp. michiganensis, C. m. subsp. nebraskensis, Erwinia amylovora, Pseudomonas syringae pv. tabaci, P. s. pv. tomato, Xanthomonas axonopodis pv. malvacearum, X. perforans, Escherichia coli $\mathrm{O} 157$ (isolates O111, O26, O45, Cider, and Jack in the Box) or Salmonella enterica [serovars Enteritidis (SE) and Typhimurium (ST)] were spread onto tryptic soy agar. Sterile filter disks treated with extractives were dried to remove ethanol, rehydrated in water, and placed on bacterial spread plates. All concentrations significantly inhibited plant pathogenic bacteria; Xanthomonas was inhibited most and Agrobacterium was inhibited least. Inhibition of $S$. enterica and $E$. coli increased significantly as extractives concentration increased. Serovar ST was inhibited more than SE. E. coli isolates varied in sensitivity; Cider and O45 were most inhibited.

\section{Rubus armeniacus sensu stricto is not susceptible to Phragmidium violaceum in Oregon}

W. L. BRUCKART (1), J. L. Michael (1)

(1) USDA ARS FDWSRU, Fort Detrick, MD, U.S.A.

Phytopathology 105(Suppl. 4):S4.21

Five species of invasive blackberry have been identified in the United States (U.S.), based on molecular characteristics. In 2005, Phragmidium violaceum, a candidate rust disease for biological control of invasive blackberry, was discovered in Oregon. Anecdotal reports were that the disease was very damaging, but subsequently, the disease has not caused significant decline in blackberry populations. Investigation was made to clarify this and to determine if biological control by $P$. violaceum is viable. Artificial inoculations of blackberry were initiated in 2009 using an isolate from the Elk River, OR, increased on a blackberry accession from Lincoln City, OR. In 2010, additional living plant samples were collected from 30 field sites for greenhouse inoculations. Results enabled separation of clones on the basis of susceptibility. Sites that were source of these plants were visited in 2013 and 2014 when the blackberry was in flower, thus enabling morphological identification of the various plant accessions and correlation of susceptibility to $P$. violaceum by Rubus species. The most common species of invasive blackberry along the Pacific coast of the U.S., R. armeniacus, is not susceptible to the rust disease. Another species, " $R$. anglocandicans", was commonly diseased both in the field and in greenhouse tests. Proper identification of the latter species is needed. Options with $P$. violaceum for biological control of invasive Rubus are more clearly understood.

\section{Morphological, cultural, pathogenic and genetic characteristics of} Macrophomina phaseolina, causer of sugar beet charcoal root rot

D. BUDAKOV (1), N. Nagl (2), V. Stojšin (1), K. Taški-Ajduković (2), F. Bagi (1), O. T. Neher (3)

(1) University of Novi Sad, Faculty of Agriculture, Department for environmental and plant protection, Novi Sad, Serbia; (2) Institute for field and vegetable crops, Novi Sad, Serbia; (3) The Amalgamated Sugar Company LLC, Boise, ID, U.S.A.

Phytopathology 105(Suppl. 4):S4.21

Macrophomina phaseolina is currently the most important root pathogen of sugar beet in Serbia, which in extreme environmental conditions, such as warm summers and severe droughts, may cause damages up to $100 \%$. Although only one species is known within the genus Macrophomina, great variability exists among isolates from the same and different hosts in regard to chlorate resistance, morphology, cultural and genetic characteristics, as well as pathogenicity. Limited data on occurrence and significance of $M$. phaseolina in sugar beet defined the aims of this research, which were to (i) classify isolates by describing their morphological and cultural characteristics, (ii) determine their genetic variability, and (iii) test their pathogenicity on young sugar beet plants and to compare them to isolates from other hosts (sunflower, soybean and maize). For this research, 18 isolates of $M$. phaseolina, 15 from sugar beet and one isolate from sunflower, soybean and maize each, were tested for size of microsclerotia formed on PDA, chlorate resistance (Pearson et al., 1986), pathogenicity on young sugar beet plants (Omar et al., 2007; Stojšin et al., 2012) and genetic diversity using SSR markers. Based on correlation between all tested traits, isolated were clustered into groups. Results of this research will improve understanding of heterogeneous population of Macrophomina phaseolina.

\section{Alternaria species on carrot in Serbia}

A. R. BULAJIĆ (1), I. M. Stanković (1), A. B. Vučurović (1), K. N. Milojević (1), D. M. Nikolić (1), S. D. Teodorović (2), B. B. Krstić (1)
(1) Institute of Phytomedicine, Department of Phytopathology, University of Belgrade-Faculty of Agriculture, Belgrade, Zemun, Serbia; (2) Forensics Department, Academy for Criminalistic and Police Studies, Belgrade, Serbia Phytopathology 105(Suppl. 4):S4.21

Given that species within Alternaria genus are among the most destructive carrot (Daucus carota) pathogens, an extensive survey was conducted from 2003 to 2014, in order to establish their presence in Serbia. Over 60 isolates originating from commercial seed samples, as well as leaves, taproots and infested soil, were collected. Pathogenicity of all monosporic isolates was confirmed using spore suspension spraying of carrot seedlings and subsequent development of typical symptoms. Conidia and colony (on PDA and CLA) characteristics, including colony margins and pigment production, crystals and microsclerotia, as well as conidia measurements and catenulation, revealed the presence of previously reported Alternaria dauci, A. radicina and A. alternata, but also indicated the presence of a new species in Serbia, A. carotiincultae. For further confirmation, selected isolates were molecularly characterized by sequencing various genomic fragments, including ITS and mitochondrial small subunit (mtSSU) rDNA, and major allergen Alt a1, beta-tubulin, and translation elongation factor-1 alpha (EF1a) protein-coding genes. Closely related $A$. radicina and $A$. carotiincultae are similar, thus particular isolates share morphological and pathogenic features. Sequence analysis of Alt a1 gene proved to be a useful identification tool. To the best of our knowledge, this represents the first report of $A$. carotiincultae pathogenic to carrot in Serbia.

\section{The importance of cold shock proteins in Xylella fastidiosa virulence} L. BURBANK (1), D. C. Stenger (1)

(1) USDA Agricultural Research Service, Parlier, CA, U.S.A. Phytopathology 105(Suppl. 4):S4.21

Xylella fastidiosa $(X f)$, causal agent of Pierce's Disease (PD) of grapevine, is mainly prevalent in warmer climates. Subjecting $X f$ infected grapevines to cold temperatures can, in many cases, effectively eliminate the bacterial population, a phenomenon known as cold curing. However, very little is known regarding the physiological response of $X f$ to cold temperatures. Cold shock proteins (CSPs), a family of nucleic acid binding proteins, are known to be an important component in the response of bacteria to temperature downshift. Genes encoding CSPs are often present in multiple copies, and expression is strongly induced by cold temperature. Additionally, CSPs can contribute to cell survival under osmotic stress, and during stationary phase. A putative CSP homolog (Csp1) with conserved cold shock and nucleic acid binding domains was identified in $X f$ Stag's Leap. As compared with the wild type, a deletion mutant of Csp1 $(\Delta \operatorname{csp} 1)$ had a decreased rate of survival at $4^{\circ} \mathrm{C}$ in vitro. Notably, $\Delta \operatorname{csp} 1$ was significantly less virulent in grapevine, as compared with wild type, under normal summer growing conditions. Reduced virulence in the absence of cold stress suggests a broader function of Csp1 in $X f$ during plant colonization beyond cold tolerance. Further study of the role of CSPs in $X f$ survival and virulence in planta will lead to a better understanding of pathogen survival under changing environmental conditions, and interaction with the host plant.

\section{Genetic diversity in Fusarium andiyazi populations from sorghum}

V. BUSHULA (1), J. F. Leslie (2), C. R. Little (2)

(1) Kansas State Univ, Manhattan, KS, U.S.A.; (2) Kansas State University, Manhattan, KS, U.S.A.

Phytopathology 105(Suppl. 4):S4.21

Fusarium andiyazi is an important pathogen of grain sorghum (Sorghum bicolor) causing grain mold and stalk rot. There are numerous reports of " $F$. andiyazi-like" strains from sorghum around the world. However, little is understood about the genetic variation in $F$. andiyazi and these $F$. andiyazilike populations. The objective of this study was to evaluate genetic diversity in $F$. andiyazi and $F$. andiyazi-like populations. Eighty strains from sorghum in Kansas (USA), Cameroon, and Uganda were analyzed. An unweighted pair group method with arithmetic mean (UPGMA) analysis of 56 amplified fragment polymorphism (AFLP) markers separated the strains into three clusters. The clusters were not based on geographic origin of the strains. Cluster 1 had 53 strains that grouped together with $F$. andiyazi tester strains. Cluster 2 and 3 contained 13 and 14 strains, respectively. Furthermore, STRUCTURE differentiated the 80 strains into two subpopulations. Subpopulation 1 consisted of all the $53 \quad F$. andiyazi strains, while subpopulation 2 was represented by the 27 strains in clusters 2 and 3 . These results indicate the presence of at least one and possibly two groups that represent sister taxa of $F$. andiyazi that have not yet been adequately described and defined. More work is needed to further characterize these strains of $F$. andiyazi, their relationships to each other and other species in the genus, and to understand their role in pathogenicity of sorghum and other potential hosts. 
LAMP and multiplex endpoint PCR-based diagnostics for detection and discrimination among Pseudomonas syringae pv. actinidiae strains

G. Y. BUSOT (1), M. Arif (1), J. Rascoe (2), M. K. Nakhla (2), J. P. Stack (1) (1) Department of Plant Pathology, Kansas State University, Manhattan, KS, U.S.A.; (2) USDA-APHIS-PPQ CPHST Beltsville Lab, USA, Beltsville, MD, U.S.A.

Phytopathology 105(Suppl. 4):S4.22

Strain-specific detection of the kiwifruit pathogen, $P$. syringae pv. actinidiae (Psa), is essential to support plant biosecurity decisions regarding trade and production. To detect and discriminate among Psa strains (including the 2008 global outbreak strain, Psa-V), a multiplex assay using conventional endpoint PCR and a Loop Mediated Isothermal Amplification (LAMP) assay were developed for laboratory and field use, respectively. The primer sets were screened against isolates of Psa and closely related $P$. syringae pathovars. The expected amplicons were generated and no cross-reaction was observed with any primer set. hopZ3 and hopS2 amplicons were obtained in all Psa strains tested. HopS2 amplicon was also observed in PsD (ex Psa-LV; formerly considered Psa low virulent strain). hopO1 and hopZ5 amplicons were specific to PsD and Psa-V strains, respectively. The LAMP protocol targeting three effector genes (hopZ3, hopZ5 and hrpW) was highly specific, discriminating between Psa virulent strains (hopZ5 and hrpW primers) and other Psa strains (hopZ3 primers). The LAMP assay is a valuable tool for infield pathogen detection to support surveillance and at ports of entry to facilitate interception. Both assays can be used for the accurate detection and discrimination of Psa.

Genome analysis of nonpathogenic Pseudomonas syringae: Secretion systems, effectors and toxins

G. BUSOT (1), M. Arif (1), J. P. Stack (1)

(1) Department of Plant Pathology, Kansas State University, Manhattan, KS, U.S.A.

Phytopathology 105(Suppl. 4):S4.22

Pseudomonas syringae populations are global in distribution occupying a very wide array of habitats and comprised of pathogenic and nonpathogenic strains. To better understand the basic differences among pathogenic and nonpathogenic strains of $P$. syringae, comparative genomic analyses were performed targeting two major systems required for survival and reproduction: secretion and virulence. The complete genome of $P$ syringae pv. syringae (Psy B728a) was used to compare draft genomes of four nonpathogenic strains of $P$. syringae (ESC-10, ESC-11, Ps Cit7 and BRIP39023). Psy B728a, Cit7 and BRIP39023 genomic sequences were downloaded from the NCBI GenBank. Draft genomes of ESC-10 and ESC-11 were obtained after sequencing and de novo assembly of paired-end libraries (Illumina MiSeq). Genomic comparisons revealed large differences between pathogenic and nonpathogenic strains and among nonpathogenic types. All targeted gene clusters were found in the pathogenic strain used as a reference. The hrp-hrc cluster encoding structural and regulatory components of the type III secretion system was only absent in Ps ESC-11. Nonpathogenic strains had a relatively small number of effector genes compared to pathogenic strains and some of them appeared truncated. The gene cluster encoding for syringolin A, a peptide derivative that activates non-host plant defense related genes, was absent in ESC-11. Several genes within the syringopeptin gene cluster appeared to be truncated. In contrast, syringomycin gene cluster was present in all four nonpathogenic strains analyzed. Genome analyses suggest that pathogenic and nonpathogenic strains of $P$. syringae might best be considered a continuum rather than as discrete taxonomic entities.

Influence of application technology on foliar fungicide efficacy on Cercospora sojina infected soybean

S. BUTLER (1), H. Young-Kelly (2)

(1) Univ of Tennessee, Jackson, TN, U.S.A.; (2) University of Tennessee Extension, Jackson, TN, U.S.A.

Phytopathology 105(Suppl. 4):S4.22

Introduction of new herbicide tolerant crops in soybean production will require growers to use nozzle types that produce coarser droplets. Pesticides are less effective applied in larger droplets due to reductions in coverage. Soybean infected with Cercospora sojina, causal agent of frogeye leaf spot (FLS), could be impacted by reductions in coverage. Field studies were conducted in Jackson and Milan, Tennessee in 2014 to evaluate effects of droplet size on foliar fungicide efficacy against FLS. Quadris Top SB was applied through 3 nozzles with varying droplet sizes including: 247, 505, and $837 \mu \mathrm{m}$. All droplet sizes were analyzed using laser diffraction with fungicide. Disease ratings were taken at 14 and 28 days after application (DAA). Leaf samples were collected $0,2,4,7$ and 14 DAA for dosage quantification using HPLC-MS. Quantified results allow for in vitro fungicide assays to determine effects of droplet size on residual control of FLS. Soybean yield data was recorded. No significant differences were found amongst treatments based on FLS disease index at either location $(\alpha=.05)$. All treatments were significantly different than the non-treated. Also, no significant differences were found in yield between treatments and non-treated control in Jackson. However, in Milan, Quadris Top applied in largest droplets yielded greater than the nontreated control $(\mathrm{P}=.0509, \alpha=.10)$. Results suggest that coarser droplets could improve Quadris Top efficacy against FLS.

Full-length genome sequence of a pepper infecting Beet western yellows virus (BWYV) isolate in Turkey

N. BUZKAN (1), B. B. Arpaci (2), A. G. Tekik (1), B. Moury (3)

(1) Kahramanmaras Sutcu Imam University Agriculture Faculty Plant Protection Department, Kahramanmaras, Turkey; (2) Kilis Yedi Aralik University Agriculture Faculty Horticulture Department, Kilis, Turkey; (3) INRA, UR407 Pathologie Végétale, Montfavet, France

Phytopathology 105(Suppl. 4):S4.22

Beet western yellows virus (BWYV), a species of the genus Polerovirus (family Luteoviridae), is an agriculturally important virus infecting over 150 plant species in 23 dicotyledonous families worldwide. An isolate of this virus was first described in Turkey, the first polerovirus reported to infect cultivated pepper naturally and to cause a severe disease. This study presents a simple method to elucidate the first complete full-length genome sequence of pepperinfecting BWYV isolates directly from total RNA extracts of field samples, starting with reverse-transcription polymerase chain reaction (RT-PCR) amplification of a small polerovirus fragment using universal polerovirus PCR primers. Eleven virus isolates were selected to determine the full-length genome sequence from a set of 137 pepper field samples. Using four primer pairs, overlapping RT-PCR amplicons were synthesized at 1496, 1026, 1213, and $1321 \mathrm{bp}$ in size, respectively, corresponding to parts of the ORF0, RdRp, $\mathrm{CP}$, and ORF5. Then, they were sequenced directly in two directions with forward and reverse primers by GENOSCREEN (Lille, France). Sequences of the five clones were aligned, and assembled to the DNA sequence. New primer pair combinations are still being used to complete the missing parts of approx. $1440 \mathrm{bp}$ in size corresponding to the parts of the ORF1, ORF2, and $\mathrm{CP}$ regions. This research was granted by TUBITAK (Project number: $113 \mathrm{O}$ 423).

Role of vector mealybugs in spreading Grapevine leafroll associated virus5 (GLRaV-5) through the main grape growing areas in Turkey

N. BUZKAN (1), M. Gumus (2), A. A. Isikber (3), Y. Ben-Dov (4)

(1) Kahramanmaras Sutcu Imam Univ, Kahramanmaras, Turkey; (2) Ege University Agriculture Faculty Plant Protection Department, Izmir, Turkey; (3) Kahramanmaras Sututcu Imam Univ, Kahramanmaras, Turkey; (4) The Volcani Center Department of Entomology, Bet Dagan, Israel

Phytopathology 105(Suppl. 4):S4.22

A successful approach for the control of leafroll disease in most grapevine growing countries entails the use of certified plant material. Grapevine leafroll-associated virus-5 (GLRaV-5) is one of the definitive species of the Ampelovirus genus (Closteroviridae family) causing leafroll disease. The virus was recently found in mostly autochthonous table grapevine cultivars on their own roots and vector mealybugs from Southeast Anatolia regions in Turkey. In order to evaluate the role of this vector in virus dissemination, GLRaV-5 and its vectors were investigated in grapevines in the Eastern Mediterranean and Aegean regions. Both plant and insect samples were screened by RT-PCR with primers designed from the heat shock protein homolog gene (hHSP70). The virus was detected in several cultivars, including Sultani cekirdeksiz, Cal, Yalova incisi and insect samples Planococcus ficus Signoret, the same vector found in Southeast Anatolia region from where GLRaV-5 was previously reported. GLRaV-5 was also detected in vineyards where there was no mealybug infestation. Moreover, the virus was not found in the vines with $P$. ficus. Results showed that the main reason for virus dissemination in surveyed areas is the use of uncertified plant materials, and vector mealybugs have only to spread GLRaV-5 from infected material to healthy ones in the vineyard. This research was granted by TUBITAK (Project number: 109 O 391).

Determining Heterodera glycines HG types to improve soybean cyst nematode management in South Dakota

E. Byamukama (1), K. ACHARYA (1), G. L. Tylka (2)

(1) South Dakota State University, Brookings, SD, U.S.A.; (2) Iowa State University, Ames, IA, U.S.A.

Phytopathology 105(Suppl. 4):S4.22

Soybean cyst nematode (SCN; Heterodera glycines) is the most important yield-limiting factor of soybean in the United States. In South Dakota, SCN has been found in 29 counties so far and continues to spread. Determining the HG type of the SCN populations reveals the diversity of the SCN populations 
and the sources of resistance that would be most effective for management. To determine the HG types prevalent in South Dakota, 220 soil samples were collected from 7 to 8 arbitrarily selected fields in each of the 28 counties. SCN was detected in $69(31 \%)$ fields, and combined egg and juvenile counts ranged from 50 to 65,200 per $100 \mathrm{~cm}^{3}$ of soil. The SCN population in each soil sample was grown on the seven SCN HG type test indicator soybean lines and William 82 as a susceptible check. A female index (FI) was calculated based on the number of females found on each differential line relative to the susceptible check. Female indices equal to or greater than $10 \%$ in any line was assigned as that HG type. Out of $50 \mathrm{SCN}$ populations on which HG type tests were done, $66 \%$ had FI $\geq 10 \%$ on Cloud, $30 \%$ on PI $88788,24 \%$ on PI 209332 , $4 \%$ on Peking, $4 \%$ on PI 90736 , and $4 \%$ on PI89722. None of the SCN populations had FI $\geq 10 \%$ on PI 437654 . HG types with $>10 \%$ reproduction on indicator lines 2, 5 and 7 were most prevalent in these samples, and use of these sources of resistance should be integrated with nonhost crops and SCNresistant cultivars should be rotated for sustainable SCN management.

Potassium-modulated photosynthetic performance of mango plants infected by Ceratocystis fimbriata

I. S. Cacique (1), W. M. S. Bispo (1), L. Araujo (1), C. E. Aucique-Perez (1), J. A. Rios (1), L. C. Silva (1), F. A. RODRIGUES (2)

(1) UFV, Viçosa, Brazil; (2) Univ Federal De Vicosa, Vicosa, Brazil Phytopathology 105(Suppl. 4):S4.23

Mango wilt, caused by Ceratocystis fimbriata, is one of the most important diseases affecting mango production. This study aimed to evaluate how plants supplied with potassium $(\mathrm{K})$ respond physiologically to the infection caused by C. fimbriata. Plants were grown in plastic pots containing 58 or $240 \mathrm{mg}$ $\mathrm{K} / \mathrm{dm}$ of substrate. The disease symptoms were more pronounced in the inoculated plants that were grown at the lower $\mathrm{K}$ level $(58 \mathrm{mg} \mathrm{K} / \mathrm{dm})$. These plants showed higher values of upward relative lesion length (79\%), relative lesion length (48\%) and radial fungal colonization (40\%) compared with those grown at the higher $\mathrm{K}$ level $(240 \mathrm{mg} \mathrm{K} / \mathrm{dm})$. Substantial declines in stomatal conductance, in line with decreases in the internal-to-ambient $\mathrm{CO}_{2}$ concentration ratio and the absence of detectable changes in the chlorophyll $a$ fluorescence parameters, suggest that the decrease in the net carbon assimilation rate of the plants is due, at least initially, to stomatal limitations. High concentrations of potassium, calcium and manganese were found in the stem tissues of plants inoculated and supplied with the highest concentration of $\mathrm{K}$, most likely due to the involvement of these tissues in the local development of defense mechanisms.

Distribution, prevalence, and incidence of Sugarcane yellow leaf virus (SCYLV) in sugarcane in Colombia

M. Cadavid (1), J. C. Angel (1), M. L. Guzmán (1), F. A. Herrera (1), J. I. Victoria (1), C. A. ANGEL (1)

(1) Colombian Sugarcane Research Center - CENICAÑA, Cali, Colombia Phytopathology 105(Suppl. 4):S4.23

Sugarcane yellow leaf virus (SCYLV, Polerovirus) is one of the most important pathogens of sugarcane worldwide, is transmitted through infected seed setts and aphids, and cause variable effects and losses. SCYLV was detected in Colombia in 1998; since then Cenicaña has evaluated nurseries and commercial fields using tissue blot immunoassay and/or reversetranscription PCR. Out of Colombia's 550,000 hectares in sugarcane 230,000 are intensively grown at the Cauca river valley to produce sugar, ethanol, and to cogenerate power. This work analyzes SCYLV prevalence $(\%$ of infected samples), and incidence ( $\%$ of infected plants within a sample of 20 plants per field) for this valley from 2000 to 2014 , including location, variety, etc., to study virus distribution. The number of samples increased from 677 in 2000 to 2,260 in 2014. SCYLV prevalence had two major peaks; one peak in $2003-$ 2004 with $21.6 \%$ and $20 \%$, followed by the lowest values of $4.3 \%$ and $3.9 \%$ in 2005 and 2006, and a second peak in 2014 with $22.7 \%$. The incidence followed this pattern, the highest in $2003(8.7 \%)$, the lowest in $2006(0.6 \%)$, and $4.5 \%$ in 2014 . SCYLV is widely distributed along the valley with a higher prevalence at the southern regions, infecting almost all varieties planted through the years; for instance, in 2014 were RB 73-2223 (54.4\%), SP 716949 (50\%), CC 84-75 (40\%), and CC 01-1940 (39.3\%). These results support planting only tested healthy seed to keep SCYLV under potential economic impact.

Strategies for durable resistance to the grapevine powdery mildew fungus, Erysiphe necator

L. CADLE-DAVIDSON (1)

(1) USDA ARS GGRU, Geneva, NY, U.S.A.

Phytopathology 105(Suppl. 4):S4.23

Nearly all cultivars of Vitis vinifera are highly susceptible to the grapevine powdery mildew fungus, Erysiphe necator. Grape breeders around the world are working to introgress resistance from wild Vitis. Of the widely-used introgressions, most involve dominant, race-specific resistance phenotypes typified by $R$-genes. In an effort to identify resistance gene combinations that may result in improved durability, we developed a set of E. necator isolates based on their phenotype on the NB-LRR gene Runl, which is the best characterized and most widely used $R$-gene in grapevine. This $E$. necator set represents wild-type individuals unable to colonize Run 1 vines, individuals with long latent periods on Run 1 vines, and individuals that infect Runl vines as if no $R$-gene were present. Having tested this set on ten sources of resistance, the results generally suggest that dominant $R$-genes will not improve the efficacy nor spectrum of resistance. Weaker $R$-genes hold some promise for protecting Runl but will not prevent selection for virulence. The exceptions to these generalizations include the Ren 4 resistance gene, for which no virulent isolates have yet been identified, and two recessive resistance genes. Because grape breeders are actively stacking $R$-genes, plant pathology-based research and guidance are urgently needed to increase the likelihood that new powdery mildew resistant varieties will be durable.

Characterization of Ophiosphaerella species causing spring dead spot disease of bermudagrass in South Carolina and Buenos Aires, Argentina A. CANEGALlO (1), S. B. Martin (1), W. J. Park (1), P. Agudelo (2), L. McCarty (2), J. Kerrigan (2), L. Tredway (3), R. Cantoro (4)

(1) Pee Dee Research \& Education Ctr, Florence, SC, U.S.A.; (2) Clemson University, Clemson, SC, U.S.A.; (3) Syngenta Lawn and Garden, Zebulon, NC, U.S.A.; (4) Universidad Catolica Argentina, Buenos Aires, Argentina Phytopathology 105(Suppl. 4):S4.23

Spring dead spot (SDS) disease is an important root disease of bermudagrass (Cynodon spp.) in the transition zone habitats around the world. Causal agents have been identified in the U.S. as Ophiosphaerella korrae (OK), $O$. herpotricha $(\mathrm{OH})$, and $O$. narmari, but not in Argentina. Pure cultures were obtained from symptomatic roots from six different bermudagrass cultivars from 24 different golf courses in SC, and 21 different golf courses, polo fields and sod farms in Argentina. The ITS region of genomic ribosomal DNA extracted from mycelial tissue was amplified using the primers specific for OK: OKITS1 and OKITS2 and OH: OHITS1 and OHITS2, to identify the causal agents of SDS of bermudagrass in South Carolina and Argentina. In SC, OKITS primers amplified a 454-bp fragment from 207 cultures out of 216 while in Argentina OHITS primers amplified a 454-bp fragment on all of 145 cultures. PCR products were purified and fragments sequenced for phylogenetic analysis among locations within SC and Argentina. This comparative study of the nucleotide sequences of the ITS region does not show significant differences within a geographical region. But, there were significant genetic differences corresponding to location when isolates of $\mathrm{OH}$ and $\mathrm{OK}$ from Oklahoma and Missouri were compared with the Argentine $(\mathrm{OH})$ and $\mathrm{SC}(\mathrm{OK})$ isolates. Also there was no evidence of correlations between Ophiosphaerella spp. with cultivars.

Effect of necrosis and crown gall caused by Agrobacterium vitis on graft take and root development of grapevines

D. CANIK OREL (1), T. J. Burr (2)

(1) Ankara University, Ankara, Turkey; (2) Cornell University NYSAES, Geneva, NY, U.S.A.

Phytopathology 105(Suppl. 4):S4.23

In this study investigated the effects of Agrobacterium vitis caused necrosis and crown gall on graft take of grapevines. Grafts were inoculated with tumorogenic and non-tumorogenic strains that differed according to their necrosis capacity on young grapevine shoots and tumorigenic capacity on grapevines in the greenhouse. Cabernet sauvignon grafts were inoculated with three different cell concentrations of the strains of. In addition the base of the cuttings were also treated with the bacterial suspensions prior to rooting in perlite. Graft take and the presence of crown gall and the degree of root formation and necrosis were determined eight weeks after inoculation. We determined significant effects of the different strains on graft take, the degree of crown gall and on root development and necrosis.

Effector discovery in the necrotrophic fungal plant pathogen Rhizopus stolonifer

L. M. CANO (1), A. Scruggs (1), L. M. Quesada-Ocampo (2)

(1) North Carolina State University, Raleigh, NC, U.S.A.; (2) North Carolina University, Raleigh, NC, U.S.A.

Phytopathology 105(Suppl. 4):S4.23

Rhizopus stolonifer is a fungal necrotrophic plant pathogen and the causal agent of a major postharvest disease in roots of sweetpotato (Ipomoea batatas) known as soft rot. Broad host range necrotrophs, such $R$. stolonifer, secrete a diverse set of effector proteins that are actively used to destroy plant tissues. Effectors can interfere with host recognition-mediated defense against 
necrotrophs increasing host susceptibility. The objective in this study was to identify and annotate the sequences corresponding to the predicted secreted effector repertoire of $R$. stolonifer that will be useful for functional characterization of these proteins in the future. We performed RNAsequencing from $R$. stolonifer mycelia and generated a transcriptome assembly that resulted in 40,312 transcripts equivalent to $45.6 \mathrm{Mb}$. From the assembly we identified 2,628 secreted proteins including effectors annotated with domains for chitin-binding and hydrolase activities. This study provides insights into the mechanisms of pathogen virulence and host immune responses for future management of necrotrophic pathogens.

\section{Discovery of effectors in the hop downy mildew pathogen Pseudoperono-} spora humuli

L. M. CANO (1), S. Withers (1), D. Gent (2), N. Noel (1), L. M. QuesadaOcampo (1)

(1) North Carolina State University, Raleigh, NC, U.S.A.; (2) Oregon State University, Corvallis, OR, U.S.A.

Phytopathology 105(Suppl. 4):S4.24

Oomycete plant pathogens secrete effector molecules that manipulate host physiology to achieve colonization. Effector proteins have been identified in various oomycete species but little is known about the sequence, function, and evolution of effectors of downy mildew plant pathogens. Pseudoperonospora humuli is the causal agent of downy mildew of hop, an economically important disease that occurs worldwide and has gained importance in recent years in the United States due to an increase of hop production. The main goal of our study was to identify and make available to the oomycete community a set of candidate pathogen effectors from $P$. humuli using transcriptomics. Identified effector candidates can be employed in high throughput functional genomics screens, commonly referred to as effectoromics, to test hop germplasm for specific pathogen recognition by resistance $R$ proteins. Here we report the identification of approximately 500 sequences annotated as cytoplasmic and apoplastic effectors in $P$. humuli and discuss their potential roles in disease. Our findings are a first step toward effector-assisted breeding in hop for resistance to downy mildew.

Genome characterization and genetic diversity of Sweet potato symptomless virus 1: A mastrevirus with an unusual nonanucleotide

M. CAO (1), P. Lan (2), J. Abad (3), F. Li (4), C. Zhou (5), R. Li (6)

(1) USDA-ARS, Beltsville, MD, U.S.A.; (2) Yunnan Agricultural University, Kunming, China; (3) USDA-APHIS, Beltsville, MD, U.S.A.; (4) Yunnan Agricultural University, Kunming, U.S.A.; (5) Southwest University, Chongqing, China; (6) USDA ARS, Beltsville, MD, U.S.A.

Phytopathology 105(Suppl. 4):S4.24

Next generation sequencing of small interfering RNAs (siRNAs) revealed the presence of Sweet potato symptomless virus 1 (SPSMV-1), a recently described virus in the genus Mastrevirus of the family Geminiviridae, in both a diseased and a symptomless sweet potato plant. Its full-length genome of 2602 nucleotides (nt) was determined from PCR products using back-to-back primers. The genome organization of SPSMV-1 is typical of dicot-infecting mastreviruses, containing 5 open reading frames. The virus has an unusual nonanucleotide (TAAGATTCC) in its large intergenic region reported from only a few viruses in the genera Becurtovirus and Eragrovirus. Blast search showed that it had the highest nucleotide sequence identity $(64.1 \%)$ with Chickpea chlorotic dwarf virus. Subgenomic sequences of 3 distinct sizes were also obtained from the two plants, and analysis showed they were defective DNAs. Analysis of a larger-than-genome sequence (2886 nt) revealed an insertion of a partial host gene of 287-nt in the small intergenic region (SIR), which is prone to recombination. Full-length SPSMV-1 genomes of 2599-2602 nt were also obtained from seven other sweet potato accessions from several countries. The nine isolates shared nucleotide sequence identities of $97-99 \%$, indicating low genetic divergence among them. The distribution of siRNA size frequencies indicated that SPSMV-1 was likely subject to both transcriptional and post-transcriptional gene silencing.

\section{An analysis of the Magnaporthe oryzae infection process in wheat blast} disease

R. D. CAPOUYA (1), T. K. Mitchell (2)

(1) Ohio State University, Columbus, OH, U.S.A.; (2) Department of Plant Pathology, The Ohio State University, Columbus, OH, U.S.A.

Phytopathology 105(Suppl. 4):S4.24

Wheat blast is a devastating disease of wheat caused by the fungal pathogen Magnaporthe oryzae, Triticum pathotype. Discovered in 1985, the spread of wheat blast in South America in recent years poses a threat to the United States wheat industry and has fostered a need for better understanding of the pathogen's interactions with its host. While much is known about the biology of other Magnaporthe varieties, namely those that cause rice blast disease, there remains a dearth of information about the intricacies of the wheat blast pathosystem. We have transformed the closest known native relative of the wheat blast pathogen, the causative agent of grey left spot disease on turfgrass, with two fluorescent proteins, GFP and tdTomato, driven by the strong ToxA promoter. Using these fluorescing fungi, we infected susceptible wheat plants and observed the process of infection at multiple stages in the fungal and wheat life cycles. By examining the pathogen's method of penetration, infection, and reproduction of a grass host, we are able to elucidate the histology and etiology of $M$. oryzae and make strides towards discovering the basis of resistance and control. Presented will be a detailed view into the pathogen's infection process over time, including an analysis of the physical infection structures of the pathogen and points of interaction of the pathogen and host, and the resulting outlook for future progress with this pathosystem.

Population genomics of an overwintering bi-parental Phytophthora capsici population

M. O. CARLSON (1), C. D. Smart (1)

(1) Cornell University NYSAES, Geneva, NY, U.S.A.

Phytopathology 105(Suppl. 4):S4.24

A recent increase in flooding events in the northeast has introduced Phytophthora capsici, the cause of Phytophthora Blight, into fields with no prior history of this pathogen. While asexual reproduction is responsible for the rapid escalation of disease within a season, sexual reproduction via oospores allows $P$. capsici to survive in the soil for many years. To understand the evolutionary dynamics of novel populations of $P$. capsici, an isolated research farm with no history of $P$. capsici in Geneva, NY was inoculated with two isolates of opposite mating type (A1 and A2). Approximately, 50 isolates were sampled each year from 2009-2014 from a variety of susceptible plant species. Isolates were genotyped using genotyping-bysequencing (GBS), which generates single nucleotide polymorphism (SNP) markers from a reduced representation genome, resulting in approximately 100,000 high-quality markers distributed throughout the genome. Shifts in allele and genotype frequencies, and estimates of within year genetic diversity, were used to assess changes in population structure across years. Genome-wide analysis of linkage disequilibrium provided insight into the role of recombination in the population genetics of $P$. capsici. In addition, these data were used to perform a genome-wide association study, which identified SNPs associated with mating type. Results from the research population will be compared to field populations analyzed using an analogous GBS approach.

Delivering site-specific applications to farmers in a knowledge network via cloud integration of weather mesonets and proven predictive models J. CARROLL (1), P. Oudemans (2), D. Cooley (3), J. Clements (4), D. Robinson (5), T. Bradshaw (6), R. Crassweller (7), M. Concklin (8), K. Peter (9), A. DeGaetano (10)

(1) Cornell University, Geneva, NY, U.S.A.; (2) Rutgers The State University of New Jersey, Chatsworth, NJ, U.S.A.; (3) University of Massachusetts, Amherst, MA, U.S.A.; (4) University of Massachusetts, Belchertown, MA, U.S.A.; (5) Rutgers The State University of New Jersey, Piscataway, NJ, U.S.A.; (6) University of Vermont, Burlington, VT, U.S.A.; (7) Pennsylvania State University, University Park, PA, U.S.A.; (8) University of Connecticut, Storrs, CT, U.S.A.; (9) Pennsylvania State University, Biglerville, PA, U.S.A.; (10) Cornell University, Ithaca, NY, U.S.A.

Phytopathology 105(Suppl. 4):S4.24

Cloud computing integration of existing weather networks with predictive phenological models provides site specific agriculture tools for farmers and field professionals. The collection, analysis and application of data to increase agricultural efficiency are made possible through interactive, web-based apps that forecast plant disease, arthropod and crop development. Our goal is to advance farmer access to site specific fruit and vegetable applications for crop protection. In New York, the NYS IPM Program and The Northeast Regional Climate Center collect weather data from a mesonet of farmer-owned Rainwise Inc. instruments and generate IPM tools online in the Network for Environment and Weather Applications (NEWA). In New Jersey, the office of the State Climatologist maintains three mesonets. Expansion of NEWA into Connecticut, Massachusetts, Pennsylvania, and Vermont, and inclusion of the New Jersey mesonets, now delivers site specific applications across six states. National Weather Service data from airport locations are also applied in the network. Climate and weather forecast data are implemented in over 20 IPM tools, available and useful to our grower communities via newa.cornell.edu. Individual farmers are connecting to NEWA in Minnesota, New Hampshire, Virginia, and Wisconsin. Researchers at land grant universities utilize the NEWA knowledge network to validate and implement phenological models into food security applications for farmers. 
Optimizing extraction to detect plant pathogenic and non-pathogenic nematodes of regulated palm weevils

L. CARTA (1), S. Li (2)

(1) USDA ARS, Beltsville, MD, U.S.A.; (2) USDA-ARS Nematology Laboratory, Beltsville, MD, U.S.A.

Phytopathology 105(Suppl. 4):S4.25

A potential introduction of the regulated red ring nematode Bursaphelenchus cocophilus into the United States would have a high impact on the landscape and tourism industries. B. cocophilus and its palm weevil vector Rhynchophorus palmarum, the latter detected in California and Texas recently, are listed on a U. S. survey target priority list. Surveys employ a moderately large volume of sugar solution that may contain target and other nematodes separate from weevils. To facilitate nematode detection, we generated accurate specific gravity data needed for their efficient extraction. The nonpathogenic Bursaphelenchus cf. gonzalezi from Mexican parlor palm was a substitute for targeted B. cocophilus, and cultured on fungus. Pristionchus entomophagus, similar to other diplogastrids found in weevils, was cultured on E. coli. Mixed stages of both cultures were used for specific gravity profiles of the relative numbers of nematodes that sank or floated in a range of sugar solutions under the standard, concentrated 1.13 specific gravity extraction solution of $1 \mathrm{M}$ sucrose. Bursaphelenchus had a profile similar to Caenorhabditis elegans, and required a relatively high sugar concentration to float compared to tylenchid plant parasites. Pristionchus did not float due to apparently rapid equilibration of sugar within its body. This information will reduce the time needed to find small numbers of tiny nematodes in trap liquid, sawdust slurry or other extraction solutions.

In vitro fungicide efficacy against the emerging pathogens Macrophomina phaseolina and Fusarium oxysporum f. sp. fragariae of strawberry

M. CARTER (1), G. Holmes (2), K. Ivors (1)

(1) Department of Horticulture and Crop Science, California Polytechnic State University, San Luis Obispo, CA, U.S.A.; (2) Strawberry Sustainability Research and Education Center, California Polytechnic State University, San Luis Obispo, CA, U.S.A.

Phytopathology 105(Suppl. 4):S4.25

Macrophomina phaseolina (Mp) and Fusarium oxysporum f. sp. fragariae (Fof) are emerging soilborne pathogens causing crown rot and Fusarium wilt (respectively) in commercial strawberry production in California. The objective of this study was to evaluate seven fungicides for their ability to inhibit mycelial growth of both pathogens using in vitro assays. Half-strength potato dextrose agar was amended with 6 different concentrations $(0.01,0.1$, 1.0, 5.0, 10, $50 \mu \mathrm{g}$ a.i./ml) of seven fungicides in FRAC groups 3, 7 and 12. Concentrations that suppressed fungal growth by $75 \%\left(\mathrm{EC}_{75}\right)$ compared to unamended media were determined for two different isolates each of $\mathrm{Mp}$ and Fof. Tebuconazole strongly inhibited the mycelial growth of both pathogens (average $\mathrm{EC}_{75}$ for $\mathrm{Mp}=2.4 \mu \mathrm{g}$ a.i. $/ \mathrm{ml}$; average $\mathrm{EC}_{74}$ for $\mathrm{Fof}=7.48 \mu \mathrm{g}$ a.i. $/ \mathrm{ml}$ ), as did metconazole (average $\mathrm{EC}_{75}$ for $\mathrm{Mp}=2.53 \mu \mathrm{g}$ a.i. $/ \mathrm{ml}$; average $\mathrm{EC}_{75}$ for Fof $=1.28 \mu \mathrm{g}$ a.i. $/ \mathrm{ml})$. Fludioxonil strongly inhibited mycelial growth of $\mathrm{Mp}$, but had no significant impact on the growth of Fof. Penthiopyrad, fluopyram, flutriafol, and flutolanil were less effective at suppressing fungal growth of either fungus. Fungicides with the lowest $\mathrm{EC}_{75} \mathrm{~S}$ will be evaluated in planta in greenhouse and field studies.

Cellulose is a major component of biofilms formed by Erwinia amylovora L. F. CASTIBLANCO (1), G. W. Sundin (2)

(1) Michigan State Univ, East Lansing, MI, U.S.A.; (2) Michigan State University, East Lansing, MI, U.S.A.

Phytopathology 105(Suppl. 4):S4.25

The formation of biofilms inside the xylem vessels of host plants is a major virulence trait in vascular pathogens. After colonization and population establishment, the fire blight pathogen Erwinia amylovora forms a biofilm inside the vascular tissue of apple leaves. The exopolysaccharides amylovoran and levan and attachment structures such as fimbriae, type IV pili and curli, have been demonstrated to be critical for the formation of a mature biofilm. In addition, the secondary messenger cyclic di-GMP has been reported to be an activator of biofilm formation in E. amylovora, when the intracellular levels of this molecule are high. In this study we demonstrated that cyclic di-GMP binds to BcsA, the cellulose synthase subunit, and activates the biosynthesis of cellulose in E. amylovora. In addition, using SEM and confocal microscopy, we demonstrated that cellulose is a main component of the biofilms formed by E. amylovora in vitro and in the host. Using gene overexpression and site-directed mutagenesis analyses, we demonstrated the BcsZ, an endoglucanase, is also required for cyclic di-GMP activation of cellulose biosynthesis and biofilm formation. Our results provide evidence of the molecular mechanisms for activation of cellulose biosynthesis and its implications in the expression of a critical virulence phenotype of $E$. amylovora in late stage infections.

qRT-PCR as a tool for regulatory network reconstruction in Phytophthora infestans

J. C. Castro (1), I. Valdés (2), G. Danies (3), L. N. Gonzalez (1), S. Cañas (4), C. E (5), S. RESTREPO (6), D. M. Riaño-Pachón (7)

(1) Universidad de los Andes, Bogotá, Colombia; (2) Utrecht University, Utrecht, Netherlands; (3) Cornell University, Ithaca, NY, U.S.A.; (4) Harvard University, Cambridge, MA, U.S.A.; (5) Universidad Nacional de Colombia, Bogotá, Colombia; (6) Universidad de los Andes, Bogota, Colombia; (7) Laboratório Nacional de Ciência e Tecnologia do Bioetanol (CTBE), Campinas, Brazil, São Paulo, Brazil

Phytopathology 105(Suppl. 4):S4.25

The advent of novel methods for the inference and evaluation of regulatory functions, coupled with the generation of massive transcriptomic and proteomic datasets, calls for the development of a common framework to integrate both data and theoretical background in order to gain significant insight into biological problems. Given that transcriptional regulation plays a central role in decision-making processes, especially in complex cellular systems as such as host-pathogen interactions, we attempted to link expression data with mathematical models in order to infer gene regulatory networks assembled by transcription factor interactions. Using qRT-PCR we evaluated the expression levels of 48 Phytophthora infestans transcription factors during its interaction with Solanum tuberosum group Phureja. We also reconstructed the regulatory network of $P$. infestans when faced with two different varieties of the host, one tolerant and one susceptible. Our preliminary results showed rewiring of the network according to the resistance level of the host, which as expected, indicates that different regulatory processes are activated in response to different environmental cues.

Detection of Ceratocystis fimbriata in mango trees and soil

G. P. CAVALCANTE (1), R. A. Brito (2), L. A. Maffia (1)

(1) Univ Federal De Vicosa, Vicosa, Brazil; (2) Universidade Federal de Vicosa, Vicosa, Brazil

Phytopathology 105(Suppl. 4):S4.25

Mango wilt, caused by Ceratocystis fimbriata (Cf), is a problem in Brazil and Oman. There is a lack of knowledge regarding the disease, including pathogen field detection. We studied the detection of $\mathrm{Cf}$ through carrot baits, semiselective medium, and nested PCR, in 15 year-old mango trees from a naturally diseased orchard (trees), 1 year-old mango plants that were stem inoculated (plants), and artificially infested soil (soil). With the baits, Cf was detected at the inoculation point in some plants, but neither from the trees nor the soil. With the medium, $\mathrm{Cf}$ was detected up to $16 \mathrm{~cm}$ beyond the lesion site in the plants; mostly at the lesion site in the trees; and colonies could be counted by pouring suspension with $10^{2}, 10^{4}$ and $10^{5}$ conidia in $0.3 \mathrm{~g}$ of soil samples. With PCR, Cf was detected up to $16 \mathrm{~cm}$ beyond the inoculation point in the plants; up to $120 \mathrm{~cm}$ beyond the lesion site in the trees; and in all soil samples with as little as 10 conidia/0.3g soil. From ten asymptomatic trees sampled in the orchard, Cf was detected with the medium (one tree) and PCR (seven trees), but not with the baits. Therefore, with the nested PCR the pathogen can be detected with more confidence, even beyond the point where the symptoms are noticed. A rapid and accurate detection is demanded for epidemiological studies and, most importantly, to manage mango wilt. Sponsored by Vale.

Characterization of Xanthomonas spp. that cause bacterial spot of peppers in Oklahoma

F. Cevallos (1), J. DAMICONE (2), C. Diaz (2)

(1) Universidad de las Fuerzas Armadas, Sangolqui, Ecuador; (2) Oklahoma State University, Stillwater, OK, U.S.A.

Phytopathology 105(Suppl. 4):S4.25

Outbreaks of bacterial spot on chili peppers grown for capsaicin production in Oklahoma have been severe recently. Isolates of Xanthomonas spp. from 2013 and 2014 were compared with isolates from 1999 to 2008 to determine if changes in species, avirulence $(A v r)$ alleles, or copper sensitivity have occurred. PCR assays with species-specific primers were performed on 32 representative isolates. Most $(\mathrm{n}=20)$ were positive for $X$. euvesicatoria, but none of the remaining isolates were positive using primers for $X$. gardneri, $X$. vesicatoria, or $X$. perforans even though $16 \mathrm{~S}$ ribosomal sequences were at least $99 \%$ homologous with Xanthomonas spp. Bacteria were infiltrated into leaves of 'Early Cal Wonder' isolines containing no resistance genes or $B s 1$, $B s 2, B s 3$; and PI235047 containing Bs4. Leaves were monitored for hypersensitive or compatible reactions. None of the $X$. euvesicatoria isolates had AvrBs 1, 16 had AvrBs2, 5 had AvrBs3, and 14 had AvrBs4. Of the four isolates virulent on $B s 2$, two were from 2014 and one each was from 1999 and 
2000. However, there was one isolate from 2014 virulent on all resistance genes. The locally grown cultivars 'Okala' and 'Jolox' were susceptible to all isolates. All isolates were all sensitive to $450 \mu \mathrm{g} / \mathrm{ml}$ or less metallic copper as copper sulfate. Obvious changes in $A v r$ alleles or copper sensitivity do not explain the recent severe outbreaks. There is a need for bacterial spot resistance in locally grown cultivars.

\section{The effect of Dominus ${ }^{\mathrm{TM}}$ and chitin treatments on actinomycete soil populations}

N. CHALASANI (1), J. Hong (2), N. Kokalis-Burelle (3), E. N. Rosskopf (4) (1) St. Edward's School, Vero Beach, FL, U.S.A.; (2) USDA ARS, Fort Pierce, FL, U.S.A.; (3) USDA, ARS, Fort Pierce, FL, U.S.A.; (4) USDA ARS, Ft Pierce, FL, U.S.A.

Phytopathology 105(Suppl. 4):S4.26

Dominus $^{\circledR}$ (aka IRF-135, active ingredient allylisothiocyanate) is a newly registered commercial soil fumigant. Although there are few options available for fumigation, particularly for cut flower producers, who are in highly urbanized areas, this biofumigant shows promise. Previous experiments have shown that chitin has an effect on soil populations of actinomycetes and can improve management of plant nematode populations. A field experiment was designed to compare the application of chitin before and after fumigation to no application of chitin. Each treatment was replicated three times. Soil was sampled repeatedly: prior to any treatment application; after application of chitin and before fumigation; after fumigation; after application of chitin following fumigation; mid-season of the flower crop; and at the initiation of flower harvest. Soil samples were taken using $30-\mathrm{cm}$ soil probes and samples were split into $0-15 \mathrm{~cm}$ and $15-30 \mathrm{~cm}$. Total genomic DNA was extracted from soil and DNA was amplified using actinomycete-specific primers using the technique of length heterogeneity polymerase chain reaction (lh-PCR) to analyze changes in the actinomycete populations under the various treatments. IRF-135 had the greatest effect on the actinomycete population, yet the effect was not as long lasting as chitin.

Efficacy of drip tape and shank applications of Dominus ${ }^{\text {TM }}$ against Macrophomina phaseolina, the cause of charcoal rot in strawberry

M. CHAMORRO RODRIGUEZ (1)

(1) University of Florida, Wimauma, FL, U.S.A.

Phytopathology 105(Suppl. 4):S4.26

Macrophomina phaseolina, the cause of charcoal rot on strawberry. The efficacy of Dominus (allyl isothiocyanate) and standard fumigation alternatives: chloropicrin and 1,3 dichloropropene (PicClor60), metam potassium (K-pam) and dimethyl disulfide (Inline) were tested. Macrophomina-infested corn-cob litter was buried in the middle of the strawberry bed at different depths $(7.6$ and $20 \mathrm{~cm})$ or the side of the bed at 7.6 $\mathrm{cm}$ (7.6s) to test the efficacy of the fumigants. The drip applications of Dominus were carried out at 567, 284, 236, 189 and $1421 / \mathrm{ha}$ and $253 \mathrm{~kg} / \mathrm{ha}$. For shank applications, baggies were buried immediately after treatment applications ( $284 \mathrm{l} / \mathrm{ha}$ or $253 \mathrm{~kg} / \mathrm{ha}$ ) with 3 or 4 knives. A non-treated control was included in both trials. Inoculum was removed from the beds 14 days after treatment applications and the number of surviving microsclerotia of $M$. phaseolina on each treatment was quantified by counting colonies on the semi-selective media. All the treatments of Dominus applied by shank reduced the number of microsclerotia of $M$. phaseolina but PicClor60 was not different from the non-treated control. Highest M. phaseolina population was obtained in baggies buried at 7.6s. For the drip applications, all treatments reduced $M$. phaseolina when the inoculum was buried in the middle of the strawberry bed. However, IRF 135 (284 1/ha), IRF 235 (142 1/ha), and Inline were not significantly different than the non-treated control when the inoculum was buried at $7.6 \mathrm{~s}$.

Effect of a single application of factory waste lime on Aphanomyces root rot of sugar beet after ten years

A. K. CHANDA (1), C. E. Windels (1), A. L. Sims (2), J. R. Brantner (1)

(1) Department of Plant Pathology, University of Minnesota, Crookston, MN, U.S.A.; (2) University of Minnesota, Northwest Research and Outreach Center, Crookston, MN, U.S.A.

Phytopathology 105(Suppl. 4):S4.26

Aphanomyces cochlioides, a major pathogen of sugar beet in the Red River Valley (RRV) of Minnesota (MN) and North Dakota (ND) and southern MN, causes significant yield reduction. Lime (calcium carbonate) is used in the extraction of sucrose from beet juice to remove impurities. The resulting byproduct is called "waste lime". In 2004, waste lime was applied to soil at rates of $0,6,12,18$ and $24 \mathrm{Mg}$ dry wt per ha at Breckenridge (MN) to study longterm effects on Aphanomyces root rot and sugar beet yield. Since 2006 sugar beet was grown in a four-year rotation with other crops (wheat, corn and soybean). In 2014, an Aphanomyces-susceptible cultivar (S) and a moderately resistant cultivar (MR) treated with $45 \mathrm{~g}$ of hymexazol per 100,000 seed were planted. There were significant $(P=0.05)$ lime rate by cultivar interactions for plant stand, root rot, yield and recoverable sucrose. At 5 and 7 weeks after planting, the $\mathrm{S}$ cultivar without hymexazol had increasing stands as lime rate increased, while the MR cultivar with hymexazol had higher stands regardless of the lime rate. The $\mathrm{S}$ cultivar showed a very strong linear response to rate of lime for all harvest parameters, whereas the MR cultivar also had a significant, but not as steep response as the $\mathrm{S}$ cultivar. Aphanomyces soil index values were high in all plots, but were significantly $(P=0.05)$ reduced with high rates of lime. Thus, waste lime was effective on $A$. cochlioides even after ten years.

Disease severity and colonization of Fusarium oxysporum f. sp. niveum transformants on watermelon

P. F. CHANG (1), P. H. Chan (2), Y. H. Lin (3), C. C. Chen (2), K. S. Chen (4), J. W. Huang (2)

(1) Department of Plant Pathology, National Chung Hsing University; Agricultural Biotechnology Center, National Chung Hsing University, Taichung City, Taiwan; (2) Department of Plant Pathology, National Chung Hsing University, Taichung City, Taiwan; (3) Department of Plant Medicine, National Pingtung University of Science and Technology, Taichung City, Taiwan; (4) Fengshan Tropical Horticultural Experiment Branch, Agricultural Research Institute, Council of Agriculture, Kaohsiung City, Taiwan Phytopathology 105(Suppl. 4):S4.26

Fusarium oxysporum f. sp. niveum (Fon) is one of the limiting factors that affect watermelon production. In order to understand the factors affecting disease progress, we transformed Fon with a $p$ PTGFPH binary vector, carrying a stable green fluorescent protein (sGFP), via Agrobacteria tumefaciens. Seven transformants stably expressed green fluorescent protein gene $(g f p)$ were identified. Pathogenicity assays on watermelon seedlings inoculated with spore suspension revealed that two transformants, named Fongfp-PH1 and Fongfp-PH2, induced disease severity at levels similar to that of the wild type Fon H0103 isolate. Three transformants, Fongfp-PH3, Fongfp-PH4 and Fongfp-PH7, displayed a reduced virulence on watermelon compared with wild-type. Fongfp-PH3 and Fongfp-PH4 showed medium disease progress; whereas transformant Fongfp-PH7 showed the mildest symptom. Confocal laser scanning microscopy observation of watermelon inoculated with the Fongfp- $\mathrm{PH} 2$ isolate revealed that this isolate produced chlamydospores at 9 days after inoculation (DAI), similar to the wild-type. Transformants Fongfp-PH3 and Fongfp-PH7 produced chlamydospores at 11 and 14 DAI, respectively. Identification of the Fon genes affected by plasmid insertion is currently underway. The flanking genes of the $g f p$ insertion will be further analyzed to understand Fon genes affecting Fusarium wilt disease severity in watermelon.

Effects of salinity on root rot of soybean caused by Fusarium avenaceum K. F. CHANG (1), S. F. Hwang (2), H. U. Ahmed (2), Q. Zhou (2), S. E. Strelkov (3), R. L. Conner (4), D. L. McLaren (5), B. D. Gossen (6), G. D. Turnbull (1), M. W. Harding (7)

(1) Alberta Agriculture \& Rural Development, Edmonton, AB, Canada; (2) Alberta Agriculture and Rural Development, Edmonton, AB, Canada; (3) University of Alberta, Edmonton, AB, Canada; (4) Agriculture and Agri-Food Canada, Morden, MB, Canada; (5) Agriculture and Agri-Food Canada, Brandon, MB, Canada; (6) Agriculture and Agri-Food Canada, Saskatoon, SK, Canada; (7) Alberta Agriculture \& Rural Development, Brooks, AB, Canada Phytopathology 105(Suppl. 4):S4.26

Soybean production has expanded into the semiarid regions of Alberta, Canada, where salts often accumulate in the soil and reduce plant germination, growth and crop productivity. Fusarium root rot (FRR), a major constraint to soybean production, is difficult to control and resistant cultivars are not available. To determine the effect of soil salinity on FRR severity and soybean growth, soil from a highly saline field was mixed with a soilless potting medium at four concentrations ranging from pure field soil to pure potting medium. Electrical conductivity (EC) was $0.5 \mathrm{dS} / \mathrm{m}$ for the pure potting medium, and 1.63, 2.01, 2.43 and $2.62 \mathrm{dS} / \mathrm{m}$ for the various mixtures of field soil with potting medium. Soybean 'TH29002R' was planted into the respective mixtures and inoculated with three levels of a virulent strain of $F$. avenaceum or without inoculum. Inoculum concentration served as the main plots and salinity treatments as the sub-plots. Root rot severity was assessed at 4 weeks, and dry weight was measured. In inoculated plots, seedling emergence, plant height, and total dry weight decreased and disease severity increased with increasing EC value, while without inoculation, seedling emergence and plant growth were reduced only at the highest EC value. Disease was noted, but was significantly higher at the highest EC value. High EC values in the soil reduced plant growth parameters and increased root rot disease severity caused by $F$. avenaceum. 
Identification of a toxic protein, FvTox6, produced by Fusarium virguliforme that causes foliar symptoms typical of Soybean sudden death syndrome

H. X. CHANG (1), L. L. Domier (2), O. Radwan (3), C. Yendrek (4), M. Hudson (3), G. L. Hartman (5)

(1) Univ of Illinois, Urbana, IL, U.S.A.; (2) USDA-Agricultural Research Service, University of Illinois, Urbna, IL, U.S.A.; (3) University of Illinois, Urbana, IL, U.S.A.; (4) Institute for Genomic Biology, Urbana, IL, U.S.A.; (5) USDA-Agricultural Research Service, University of Illinois, Urbana, IL, U.S.A.

Phytopathology 105(Suppl. 4):S4.27

Soybean sudden death syndrome (SDS) is caused by Fusarium virguliforme, a soilborne pathogen that rarely infects above ground parts of soybean but induces severe foliar symptoms including intervienal chlorosis and necrosis, leaf marginal curling, and defoliation. These foliar symptoms are well known for its sudden appearance that generally exhibits around flowering to maturity stages. Translocation of toxins that produced by $F$. virguliforme has been regarded as the cause of foliar symptoms; however, none of the toxins found to date is able to reproduce complete SDS foliar symptoms typical to the symptoms observed in the field. We hypothesized that multiple toxins together induce the complicated foliar symptoms. In this study, we tried to identify additional toxins produced by $F$. virguliforme through transcriptomic analysis under a toxin-producing condition, and to test their contribution individually on causing SDS foliar symptoms. Two secondary metabolites and 11 toxic proteins were identified, but in contrast to our hypothesis, one toxic protein FvTox6 individually was sufficient to complete SDS foliar symptoms through viral expression system. Instead, neither application of secondary metabolites nor overexpression of other toxic proteins induced foliar symptoms similar to SDS. Our study identified FvTox6 as a major toxin for inducing SDS foliar symptoms.

\section{Phytophthora nicotianae and huanglongbing cause different nutritional} imbalances in grapefruit trees

S. CHAUDHARY (1), M. Setamou (1), O. J. Alabi (2), J. L. Jifon (2), M. Kunta (1), K. Crosby (3), J. V. DaGraca (1), V. Ancona (1)

(1) Texas A\&M University-Kingsville Citrus Center, Weslaco, TX, U.S.A.; (2) Texas A\&M University, Weslaco, TX, U.S.A.; (3) Texas A\&M University, College Station, TX, U.S.A.

Phytopathology 105(Suppl. 4):S4.27

The recent introduction of the citrus disease HLB in Texas has raised concerns about the effect of HLB on trees already infected with Phytophthora nicotianae foot rot. We conducted a field study to evaluate the effect of HLB on foot rot infected grapefruit trees. Soil propagules of $P$. nicotianae, fibrous root density (FRD) and root length density (RLD) were recorded to assess root health of each tree. In addition, fibrous roots and leaves were analyzed for starch, soluble sugar, macro and micro nutrients. Results indicate that FRD and RLD are not significantly different within HLB positive and negative trees. However, FRD in HLB positive trees was significantly affected by higher $P$. nicotianae propagule counts. Leaf analysis revealed that boron is lower in foot rot infected trees compared to healthy or HLB positive trees. In addition, HLB positive trees with or without foot rot accumulate lower manganese than HLB negative trees. Furthermore, calcium levels were lower in HLB positive trees with foot rot compared to healthy trees or trees infected with only one disease. Although no significant differences were found in starch or soluble sugars between HLB positive and negative trees, higher glucose in root and leaf tissue were observed in foot rot infected trees. Altogether, our results indicate that foot rot and HLB infections affect the balance of nutrients differently and that dual infections have a combined effect to decrease calcium levels in grapefruit trees.

Molecular characterization and genetic diversity of Mycospharella fijiensis, in Costa Rica using sequence based nuclear markers

S. CHAVAN (1), M. Wyatt (1), L. G. Alpizar (2), M. E. Munoz (3), J. B. Ristaino (1)

(1) North Carolina State University, Raleigh, NC, U.S.A.; (2) University of Costa Rica, Costa Rica, Costa rica; (3) DOLE - Standard Fruit Co., Costa Rica, Costa rica

Phytopathology 105(Suppl. 4):S4.27

Mycospharella fijiensis (M. fijiensis), causes Black Sigatoka disease on banana that can cause $20-80 \%$ yield loss in absence of fungicides in banana plantations worldwide. One hundred and thirteen isolates of M. fijiensis, collected from Costa Rica between 2010 and 2013 were analyzed using multilocus genotyping of seven loci. The isolates were collected from sprayed or unsprayed bananas grown in commercial plantations or backyards, and in full sun or shade grown bananas. All isolates were identified as $M$. fijiensis from PCR of ITS DNA. Five to six multilocus haplotypes, were observed with haplotypic diversity, $\left(\mathrm{H}_{\mathrm{d}}\right)$ ranging 0.45 to 0.52 for three of the 7 loci examined. Coalescent-based genealogies of all seven loci documented six lineages leading to present day haplotypes of M. fijiensis on banana in Costa Rica. The Costa Rica populations shared haplotype diversity with haplotypes from South-East Asia and the Americas for five of the seven loci studied. The remaining two loci showed association with isolates from only South-East Asia, which is the center of origin and source of initial inoculum for the global invasion of $M$. fijiensis. This data indicates that the haplotypic diversity observed in Costa Rica could be derived from admixtures between genetically differentiated sources from South-East Asia and Americas.

Temporal progress and interaction of irrigation with phosphorus levels in Brown eye spot of coffee

E. Chaves (1), E. A. POZZA (1), G. A. Dornelas (1), A. A. A. Pozza (1), M. S. Scalco (2), R. J. Guimarães (1)

(1) Federal University of Lavras, Lavras, Brazil; (2) EMBRAPA/UFLA, Lavras, Brazil

Phytopathology 105(Suppl. 4):S4.27

Brown eye spot (BES - Cercospora coffeicola) is an important disease of coffee, and its occurrence is affected by water supply and nutritional balance. Water can be supplied by drip irrigation, with the advantage of providing fertigation. Thus, this study evaluated the interaction of different water application levels and phosphorus doses in the incidence of BES in coffee leaves at Brazil. Assessments of disease were performed at 30-day intervals for 26 months, from November 2011 to January 2014. The experiment used a randomized block design at factorial scheme to analysis of variance (5 irrigation levels $\mathrm{x} 4$ phosphorus doses) with four replications. The area under incidence progress curve (AUIPC) was calculated. Adjust to non-linear models was calculated for two years. The progress curve of the average incidence of BES varied in both years. In year 1 (November 2011 to December 2012) the incidence peaked on Aug 12, 2012 (22.45\%) while in Year 2 (January 2013 to January 2014), the incidence reached its highest level on Sep 12, 2013 (16.29\%). The best model adjusted was exponential, because the leaves fall easily at harvest due to ethylene production associated to BES. There was interaction between irrigation and phosphorus levels on Oct 07, 2012, after harvest. In this date, increase of phosphorus levels in smaller irrigation levels and absence of phosphate fertilizer in larger irrigation levels caused higher incidences. AUIPC was not significant among treatments.

Nucleotide heterogeneity at the genomic 5'- and 3'-termini of California (CA) isolates of Citrus tristeza virus (CTV)

A. Y. CHEN (1), S. Watanabe (1), R. K. Yokomi (2), J. C. Ng (1)

(1) Department of Plant Pathology and Microbiology, University of California, Riverside, Riverside, CA, U.S.A.; (2) United States Department of Agriculture-Agricultural Research Service, Parlier, CA, U.S.A.

Phytopathology 105(Suppl. 4):S4.27

Nucleotide (nt) sequences in the genomic ends of (+)-sense RNA viruses serve essential biological functions and are important considerations in the construction of infectious clones. We have determined the terminal RNA sequences of CTV-T30 and -T36 infecting mexican lime and madame vinous plants, respectively. The 5' and 3' ends of viral double stranded (ds)RNA were amplified by Rapid Amplification of cDNA-PCRs and sequenced. Compared with the $5^{\prime}$ end nts in the most related sequence (GenBank KC517491), most of the T30-CA sequences had an extra A or G upstream of the first nt in the (+)-RNA and an extra C downstream of the last nt in the (-)RNA. Most of the 3' end sequences of T30-CA were conserved, with a small number having one or two heterogenous nts at the extreme ends. The 5' end sequences of T36-CA were heterogenous compared to that of a Florida (FL) T36 isolate (GenBank AY170468). More than 15 variations of nts were seen at the extreme 5' end of the T36-CA sequences, most of which had an extra A upstream of the first nt in the (+)-RNA and an extra $\mathrm{C}$ downstream of the last nt in the (-)-RNA. Nucleotide heterogeneity was also observed in the $3^{\prime}$ ' end sequences of T36-CA $-45 \%$ of the 5 ' end sequences in the (-)-RNA were identical to those of T36-FL, while $47.6 \%$ of the 3 ' end sequences in the (+)RNA had an extra $\mathrm{T}$ downstream of the last nt. These results were consistent with published reports of nt variations in CTV isolates from different hosts and locations.

Observation and identification of wood-decay fungi from the heartwood of peach tree limbs in central Georgia, USA

C. Chen (1), C. H. BOCK (1), M. W. Hotchkiss (1), M. M. Garbelotto (2), T. E. Cottrell (1)

(1) USDA-ARS-SEFTNRL, 21 Dunbar Rd., Byron, GA, U.S.A.; (2) University of California, Department of Environmental Science, 130 Mulford Hall, Berkeley, CA, U.S.A.

Phytopathology 105(Suppl. 4):S4.27 
Discoloration in the heartwood of broken peach scaffold limbs was observed consistently on otherwise apparently healthy limbs in peach orchards in central Georgia, USA. A dissection of broken scaffolds from 6 trees showed symptoms of colonization along the entire limbs, with $41 \%$ of primary branches having symptoms of decay, but no secondary branches with symptoms. Only limb sections of peach scion cultivars, but not rootstock cultivars were colonized. A Chi-square analysis in 2 separate experiments indicated no difference in the incidence of colonization among scion cultivars $\left(1^{\text {st }}\right.$ expt $\mathrm{P} \geq \chi^{2}=0.81(95 \% \quad C I s=0.808-0.814), \quad 2^{\text {nd }}$ expt $\mathrm{P} \geq \chi^{2}=0.96 \quad(95 \%$ $\mathrm{CIs}=0.963-0.966$ ). Metagenomic analysis of next generation sequencing reads revealed the contigs were aligned to genomic DNAs and ESTs of Trametes versicolor and/or Schizophyllum commune, suggesting these two white-rot fungi were present in the sample tested. Subsequent multiplexing PCR diagnostics of 10 samples from scion wood confirmed the dominance of Trametes spp. (all 10 samples), although Stereum spp. (5 samples), Schizophyllum spp. (3 samples) and Hericium spp, (1 sample) were sometimes found coexisting with Trametes spp. Pruning of scion wood may provide an infection court for colonization by wood decay fungi, and also increase availability of air in the wood, allowing growth of wood decay fungi which are normally inhibited in anoxic wood environments. Future research needs on this peach-fungus association are discussed.

Phosphorylation of C-terminal threonine on PFLP is required for enhancing resistance to bacterial wilt in Arabidopsis thaliana

T. Y. Chen (1), L. Y. Shiu (1), Y. J. Chen (1), Y. H. LIN (1)

(1) National Pingtung University of Science and Technology, Pingtung, Taiwan

Phytopathology 105(Suppl. 4):S4.28

PFLF is a protein that can enhance disease resistance against bacterial wilt in transgenic plants. In these plants, PFLP intensifies responses triggered by pathogen-associated molecular patterns to further enhance disease resistance. Moreover, the improvement of disease resistance requires the casein kinase II phosphorylation (CK2P) site of PFLP. Whether the phosphorylation of threonine in CK2P site is also required for enhancing defense responses was still unknown. In this study, we used single amino acid substitution to generate mutants of truncated PFLP, PEC, which still intensifies disease resistance. One is the un-phosphorylated PECT90A; the other is the mimic phosphorylated PECT90D. These two mutants were used to evaluate their efficacy on PEC-intensified HR and disease resistance. Results revealed that the PEC-intensified HR was abolished in the treatment of PECT90A. However, these responses were also reduced in the treatment of PECT90D. Results on inoculation assay also exhibited that the disease resistance to bacterial wilt was abolished in transgenic plants with PECT90A and partial reduced in that with PECT90D. Furthermore, the CK2 mutant of Arabidopsis plants was used to demonstrate that the extracellular CK2 was required for PFLP to intensify HR. Taken together, we concluded that the phosphorylation of C-terminal threonine by CK2 on PFLP was required to enhance resistance to bacterial wilt in Arabidopsis plants.

Virulence determination of Phytophthora infestans effector IPI-O1 and IPI-O4

Y. CHEN (1), J. Jiang (1), D. Halterman (2)

(1) University of Wisconsin-Madison, Madison, WI, U.S.A.; (2) United States Department of Agriculture, Madison, WI, U.S.A.

Phytopathology 105(Suppl. 4):S4.28

Potato late blight, caused by the oomycete pathogen Phytophthora infestans, is one of the most destructive plant diseases. The $R B$ gene, cloned from the diploid wild potato species Solanum bulbocastanum, confers partial resistance to most $P$. infestans isolates through its recognition of the corresponding pathogen effector family IPI-O. While the majority of IPI-O proteins are recognized by RB to elicit host resistance (e.g. IPI-O1, IPI-O2), some family members are able to elude detection (e.g. IPI-O4). In addition, we have previously shown that the presence of IPI-O4 in planta is able to suppress the HR elicited by IPI-O1 in the presence of RB. The goal of the present study is to determine the effect of IPI-O1 and IPI-O4 on P. infestans virulence. In order to test the effect, we have stably transformed the susceptible potato cultivar 'Katahdin' with IPI-O1 and IPI-O4 separately. We also transformed the potato line $\mathrm{K} 41$, which contains the $R B$ gene and was developed from the potato-S. bulbocastanum hybrid by backcrossing with 'Katahdin' three times, with IPI-O4. The effect of IPI-O on the ability of $P$. infestans to cause disease symptoms in potato is being assayed using whole plant and detached leaf assays. Results from this study may provide conclusive evidence whether IPI$\mathrm{O} 4$ is involved in the overcoming resistance of RB by $P$. infestans.
Transmission of Tomato spotted wilt virus by two thrips populations and evidence that adult thrips emerging from soil are an inoculum source L. F. CHEN (1), D. C. Mackie (1), B. A. Bazor (1), O. Batuman (1), D. E. Ullman (1), R. L. Gilbertson (1)

(1) Univ of California, Davis, CA, U.S.A.

Phytopathology 105(Suppl. 4):S4.28

Transmission efficiency of Tomato spotted wilt virus (TSWV) by the western flower thrips (Frankliniella occidentalis) populations, collected from Fresno and Yolo counties in California, was compared using two virus isolates from these counties (TSWV-F and TSWV-Y). Individual viruliferous adult male or female thrips were placed on healthy datura leaves, maintained for 6 days at room temperature in the dark and virus infection determined with ELISA. The overall mean transmission rate of the Fresno population was greater (41\%) than that of the Yolo population (28\%), regardless of the virus isolate. For both populations, male thrips transmitted TSWV more efficiently (39\%) than female thrips (31\%). To test if thrips pupae can overwinter in soil and emerging adult thrips can serve as a primary source of inoculum for TSWV, rates of emergence of adult thrips were tested by maintaining thrips pupae at $4^{\circ} \mathrm{C}$ over an 8 week period. The rates of emergence for nonviruliferous and viruliferous adult thrips were similar, and declined over time from $50 \%$ (1 wk) to $4 \%$ ( $8 \mathrm{wks}$ ). For all time points, adult thrips emerging from viruliferous pupae transmitted TSWV. Viruliferous thrips pupae were then incubated at $10^{\circ} \mathrm{C}$ (a typical winter soil temperature in California) over an 8 week period. Adult thrips emerged at an average rate of $45 \%$, with peak emergence at the third and fourth weeks. Importantly, emerging adult thrips transmitted TSWV over the course of the entire experiment.

Etiological and genetic characteristics of fruit spot disease in apple caused by Colletotrichum gloeosporioides based on morphology and pathogenicity W. CHEON (1), Y. Jeon (1)

(1) Andong National University, Andong, South Korea

Phytopathology 105(Suppl. 4):S4.28

Colletotrichum are considered to be major plant pathogens worldwide. Apple (Malus pumila Mill.) is an important economic crop in Korea. From 2013 to 2014, disease occurrences in apple cultivars were investigated in the apple orchards of northern Gyeongbuk Province in Korea. As static anthracnose develops, a small lesion is observed at the beginning of the growth period. The spot remains static until the harvesting season. Static symptoms were observed on apples together with typical symptoms. We observed that the conidia shape, size, and appressorium shape were similar for the two types of fungi (typical symptoms and static symptoms). Although the nucleotide sequences of the actin, chitin synthase, B-tubulin and internal transcribed spacer regions were $100 \%$ consistent, PCR analysis by the specific primers was observed two types isolates consistent $C$. gloeosporioides. The conidia were incubated in ethephon suspension and started to germinate within $4 \mathrm{~h}$. After $8 \mathrm{~h}$ of incubation, the germ tubes began to swell and subsequently differentiated into appressorium with dark thick walls. In the advanced symptom, fungus conidia underwent germination of penetration hyphae after appressorium formation within $16 \mathrm{~h}$. In the static symptom, fungus conidia, there was no germination of the penetration hypha within $72 \mathrm{~h}$. These findings indicate that ethephon has an important role in the germination of penetration hyphae of the two types of C. gloeosporioides.

How rater bias and assessment method used to estimate disease severity affect hypothesis testing in different experimental designs

K. S. CHIANG (1), C. H. Bock (2), I. H. Lee (1), M. El Jarroudi (3), P. Delfosse (4)

(1) National Chung Hsing Univ, Taichung, Taiwan; (2) USDA-ARSSEFTNRL, Byron, GA, U.S.A.; (3) Univ of Liege, Arlon, Belgium; (4) Luxembourg Institute of Science and Technology (LIST), Belvaux, Luxembourg

Phytopathology 105(Suppl. 4):S4.28

The impact of rater bias and assessment method on hypothesis testing was studied for different experimental designs (balanced and unbalanced data sets). The assessment methods were nearest percent estimates (NPEs), the Horsfall-Barratt (H-B) scale, and an amended 10\% incremental scale. Estimates of severity of Septoria leaf blotch on leaves of winter wheat were used to develop distributions for a simulation model. Experimental designs are presented through simulation experiments which consider the optimal distribution between the number of specimens (individual units sampled) and the number of replicates per specimen for a fixed total number of measures (total sample size for that treatment). The criterion used to gauge the method was the power of hypothesis test. As expected, at a given fixed number of measures, the balanced experimental design invariably resulted in a higher power compared with the unbalanced design at different disease severity means, mean differences, and variances, respectively. The number of 
replicates taken per specimen can be small - no more than 2 or 3 replicates are required. Furthermore, a difference in probability to reject the null hypothesis was observed between both assessment and experimental design methods for biased estimates. The study suggests that knowledge of replication and balanced experimental design are valuable to optimize results from experiments using disease severity estimates for comparing treatments.

\section{Molecular characterization of recombinant strains of Potato virus $Y$ from Saudi Arabia}

M. CHIKH-ALI (1), H. Alruwaili (1), D. Vander Pol (1), A. V. Karasev (2)

(1) University of Idaho, Moscow, ID, U.S.A.; (2) Univ of Idaho, Moscow, ID, U.S.A.

Phytopathology 105(Suppl. 4):S4.29

The practical importance of PVY recombinant strains has increased due to their wide distribution and the ability of most of these recombinants to induce potato tuber necrotic ring spot disease (PTNRD) that seriously affects the quality of tubers. In Saudi Arabia, potato production has increased five-fold during the last three decades reaching 450,000 tons per year. PVY has been reported as one of the main viruses infecting potatoes in the country, but no information is available on PVY strains circulating in the country. In August 2014, a small survey was conducted in a seed potato field in Al Jouf, Saudi Arabia. Nine PVY-positive samples selected based on visual symptoms and serological reactivity were subjected to two multiplex RT-PCR assays and all nine determined to represent recombinant PVY strains, NE-11 and SYR-III. Whole genome sequences were determined for two isolates, S2 (SYR-III) and S9 (NE-11). Both recombinants, PVY-NE11 and SYR-III, were previously found associated with PTNRD but thought to be rare. However, the current identification of PVY-NE11 and SYR-III in a new geographic region suggests that these strains may not be as rare as believed previously. This is the first report on the occurrence of recombinant strains of PVY in Saudi Arabia.

\section{A novel strain of Potato virus $\boldsymbol{Y}$ from tomato}

M. CHIKH-ALI (1), D. Vander Pol (1), O. V. Nikolaeva (1), M. J. Melzer (2), A. V. Karasev (3)

(1) University of Idaho, Moscow, ID, U.S.A.; (2) University of Hawaii, Honolulu, HI, U.S.A.; (3) Univ of Idaho, Moscow, ID, U.S.A.

Phytopathology 105(Suppl. 4):S4.29

Potato virus $Y$ (PVY) exists as a complex of strains that display a range of hypersensitive resistance (HR) responses in potato cultivars carrying $N$ genes. The $N c_{t b r}$ gene, which has been mapped to chromosome IV of potato, confers $\mathrm{HR}$ against the $\mathrm{PVY}^{\mathrm{C}}$ strain. Isolates from the $\mathrm{PVY}^{\mathrm{C}}$ strain have become very rare in potato, while they still remain common in pepper and tomato, and the reasons for this are not fully understood. Here, an isolate of PVY, PVY-H, collected from tomato plants exhibiting mosaic, leaf deformations, and stunting, was subjected to biological and molecular characterization to gain insight into the epidemiology of PVY and its interactions with the $N$ genes in potato. Host range studies indicated that PVY-H triggered the $N c_{t b r}$ gene in potato cultivars 'King Edward' and 'Maris Bard' inducing HR, typical of $\mathrm{PVY}^{\mathrm{C}}$ strain, and was unable to systemically infect potato. The whole genome of PVY-H was sequenced, and found to be only $91 \%$ identical to the genome of isolate PRI-509 (strain PVYC), with identities to other strains of PVY, e.g. $\mathrm{PVY}^{\mathrm{N}}, \mathrm{PVY}^{\mathrm{NA}-\mathrm{N}}, \mathrm{PVY}^{\mathrm{O}}, \mathrm{PVY}^{\mathrm{O}}-\mathrm{O}$, and Chile3, ranging between $82-87 \%$. Based on these host range and sequence data, PVY-H may represent a novel strain of PVY.

\section{Association of genotype with phenotype in Fusarium solani from soybean} P. CHITRAMPALAM (1), B. Nelson (2)

(1) North Dakota State Univ., Fargo, ND, U.S.A.; (2) North Dakota State Univ, Fargo, ND, U.S.A.

Phytopathology 105(Suppl. 4):S4.29

Fusarium solani is one of the primary root rotting pathogens of soybean in North Dakota (ND) and Minnesota (MN), but the genetic diversity in this pathogen is unknown. The genetic and phenotypic diversity in this pathogen and an association between genotype and phenotype was studied. A multilocus phylogeny based on ITS $+28 \mathrm{~S}$ of rDNA, $E F 1-\alpha$, and $R P B 2$ was used for genetic diversity, and pathogenicity on four different hosts, growth at $23^{\circ} \mathrm{C}$ and $37^{\circ} \mathrm{C}$ and a fungicide sensitivity test to fludioxonil were used for phenotypic diversity. Two genetic clusters, C-I and C-II, were observed among 93 isolates from both $\mathrm{ND}$ and $\mathrm{MN}$. Isolates from $\mathrm{C}$-II were pathogenic on all four hosts tested, soybean, dry bean, pea and lentil, and grew only at $23^{\circ} \mathrm{C}$. However, isolates from C-I were non-pathogenic to weakly pathogenic on those hosts, but grew at both $23^{\circ} \mathrm{C}$ and $37^{\circ} \mathrm{C}$, a required temperature for clinical isolates. An in-vitro fungicide sensitivity test with fludioxonil revealed that nine out of ten tested isolates representative of both genetic groups, were highly resistant to fludioxonil. This is the first report of genetic groups and fludioxonil resistance in $F$. solani from the northern soybean production area of the US. Furthermore, this study revealed the first association of soybean isolates with clinically important members of the Fusarium solani species complex.

Streptomyces griseus S4-7 sporulation factor, whi, involves in antifungal activity

H. CHO (1), J. Kim (2), Y. S. Kwak (3)

(1) Gyeongsang National University, Jinju, Gyeongnam, Korea; (2) Gyeongsang National University, Jinu, Korea; (3) Gyeongsang National University, Jinju, Korea

Phytopathology 105(Suppl. 4):S4.29

Streptomyces have been known ability to produce bioactive secondary metabolites, such as antifungals, antitumorals, anti-hypertensives, and immunosuppressants. Streptomyces griseus S4-7 was isolated from rhizosphere of strawberry as a responsible microbial agent in strawberry Fusarium wilt suppressive soil. S. griseus S4-7 showed specific and mighty antifungal ability against Fusarium oxysporum f. sp. fragariae. In Streptomyces genus, the sporulation systems lead to differentiation, septation and control antibiotic production. The ability of the spores stimulates cell development and secondary metabolite production. It was reported that whiG, whiH, whil, whiA, whiB, and whiD are essential for the sporulation of aerial hyphae. In this study, whiH and whiB-like genes of S. griseus S4-7 are revealed their biological function by knock-out mutagenesis. S. griseus S4-7 mutants (whiH and whiB-like) were generated by intergeneric conjugation protocol because the species has a methyl-specific restriction mechanism. Sporulation of S4-7 mutants (whiH and whiB-like) was later than the wild type strain and the mutants dramatically lost antifungal activity in laboratory condition. In general, biocontrol agents secrete antibiotics that have been related to pathogens control mechanism. The present results indicated that a novel biocontrol strain, S4-7, produces effective antifungal secondary metabolites and whi transcription factors have critical role in antifungal production.

Production of transgenic chrysanthemum events resistant to Chrysanthemum stunt viroid

S. K. CHOI (1), G. S. Choi (1), J. Y. Yoon (1)

(1) Virology Unit, Department of Horticultural Environment, National Insititute of Horticultural and Herbal Science, RDA, Wan-Ju, South Korea Phytopathology 105(Suppl. 4):S4.29

The chrysanthemum stunt disease caused by Chrysanthemum stunt viroid (CSVd), a small non-protein-coding circular RNA is widespread in Korea as well as countries in which chrysanthemum is being cultivated. In spite of the economic losses due to CSVd disease, there are no distinct CSVd resistant chrysanthemum cultivars or in-bred chrysanthemum lines in Korea. To examine whether resistance against CSVd could be achieved through RNA silencing, transgenic chrysanthemum plants expressing a hairpin RNA (hpRNA) construct containing inverted-repeat and double-stranded RNAs derived from CSVd genome were produced in this study. Transgenic chrysanthemum plants were engineered to produce 189 to $354 \mathrm{bp}$ inverted hairpin transcripts with an intron, in two orientations each, which were processed to hpRNA-derived short interfering RNAs (siRNAs). Interestingly, fewer lines were regenerated from the transformants with 354 bp-inverted hairpin transcripts. Resistance in the transgenic chrysanthemum plants is correlated with high-level accumulation of hpRNA-derived siRNAs in the plants, showing that hpRNA-derived siRNAs appear to effectively target the mature CSVd genomic RNA. These data suggest that chrysanthemum highly resistant to CSVd can be produced through RNA silencing, as well shown for Potato spindle tuber viroid and many viruses.

Applications of Bacillus amyloliquefaciens PMB01 for managing tomato bacterial wilt caused by Ralstonia solanacearum

H. P. Chou (1), Y. H. Lin (2), T. C. Huang (3), W. L. DENG (4)

(1) Natl Chung Hsing University, Department of Plant Pathology, Taichung, Taiwan; (2) National Pingtung Universityof Science and Technology, Department of Plant Medicine, Pingtung, Taiwan; (3) Kaohsiung District Agricultural Research and Extension Station, Kaohsiung, Taiwan; (4) National Chung Hsing University, Department of Plant Pathology, Taichung, Taiwan Phytopathology 105(Suppl. 4):S4.29

Bacterial wilt, caused by Ralstonia solanacearum (Rs), is one of the most devastating diseases on solanaceous plants. Until now, there are no effective chemicals for controlling the disease; however, several greenhouse studies demonstrated that the severity of the disease can be reduced by the applications of antagonistic rhizobacteria, suggesting that biocontrol agents might be potential solutions for the management of bacterial wilt disease. In this study, several bacterial strains with inhibitory effects on $R s$ growth were isolated from soils. Among them, Bacillus amyloliquefaciens PMB01 showed 
good antagonistic activity against $R S$ in confrontation assays. To further evaluate the efficacy of PMB01 on controlling bacterial wilt disease of tomato (BWT), studies in greenhouses and fields were conducted for 3 years, from 2012 to 2014, in Taiwan. Our results revealed that the applications of formulated B. amyloliquefaciens PMB01 significantly reduced the disease severity of BWT up to $70 \%$ in both the greenhouse and field trials. Whole genome shotgun sequencing showed that B. amyloliquefaciens PMB01 harbors genes involved in the biosynthesis of antibiotics, such as iturins, fengycins and surfactins, which might contribute to its biocontrol activity. Toxicological testing results revealed that $B$. amyloliquefaciens PMB01 was non-toxic to mammals. Taken together, we proposed that $B$. amyloliquefaciens PMB01 is a safe and effective biocontrol agent for managing BWT.

Screening and application of the nonpathogenic Fusarium oxysporum in Taiwan

W. H. CHUNG (1), C. J. Wang (1)

(1) Natl Chung Hsing Univ, Taichung, Taiwan

Phytopathology 105(Suppl. 4):S4.30

Nonpathogenic Fusarium oxysporum ( $F o$ ) has been demonstrated to delay the symptom expression of Fusarium wilt of cucumber caused by $F$. oxysporum $\mathrm{f}$. sp. cucumerinum in greenhouse. Previous studies indicated that the modified primers (FIGS11/NPIGS-R) showed high specificity on detecting the nonpathogenic Fo with activity of biocontrol. In this study, 10 nonpathogenic Fo isolates detected by FIGS11/NPIGS-R primers were examined its efficacy on controlling Fusarium wilt of cucumber in greenhouse and field. The results showed that these nonpathogenic Fo isolates had high capability to delay the symptom development and reduce the disease severity from 41 to $65 \%$ of Fusarium wilt on cucumber in the greenhouse. For field experiment, three isolates, Fo276, Fo95022 and Fo95024, were choose to evaluate the efficacy on controlling the Fusarium wilt of cucumber at Nantou County, Taiwan. The result also indicated that the three isolates could delay the disease progressing and reduce the disease severity from 29 to $54 \%$. In addition, the phylogenetic analysis demonstrated that these 10 nonpathogenic $F o$ isolates were grouped into a unique clade and differentiated from other reference $F_{O}$ isolates of human and plant pathogens.

\section{Pathogenicity of Cylindrosporium concentricum on cultivated and weedy} Brassica hosts

B. J. CLAASSEN (1), M. Serdani (2), C. Mallory-Smith (1), W. J. Thomas (1), M. L. Putnam (2), C. M. Ocamb (1)

(1) Oregon State University, Corvallis, OR, U.S.A.; (2) Oregon State University - Plant Clinic, Corvallis, OR, U.S.A.

Phytopathology 105(Suppl. 4):S4.30

Cylindrosporium concentricum, the causal agent of light leaf spot disease of Brassica species, was detected in numerous fields in the Willamette Valley of Oregon during 2014. To our knowledge, C. concentricum is new to North America; therefore, it is imperative to examine its host range as this pathogen is capable of infecting and causing significant losses in a wide range of cultivated Brassica species. Numerous isolates were single spored from cultivated crucifers including turnip, canola, forage turnip, and related weed plants. Various crucifers, including cabbage, canola, turnip, radish, kale, broccoli, mustard and several weed species, were inoculated at the cotyledon to two-leaf stage with spore suspensions $\left(1 \times 10^{-5}\right.$ spores $\left./ \mathrm{mL}\right)$. Plants were incubated at $20 \mathrm{C}$ with $12-\mathrm{hr}$ photoperiods for 3-4 weeks. After symptom development, diseased leaf tissue was detached and incubated in moist chambers $(5 \mathrm{C})$ to induce sporulation. Conidia arising from diseased tissue were used for re-isolating the fungus as well as for DNA extraction to confirm the presence of $C$. concentricum by PCR. Canola, cabbage and turnip were susceptible in preliminary pathogenicity tests. The results from a wider testing of weed and crop crucifers will be presented. Examination of the host range for $C$. concentricum will aid in determining the disease potential of light leaf spot in the Willamette Valley and the capability of weeds to act as reservoirs for this new pathogen in western Oregon.

New Zealand's "Emerging Risks System" for biosecurity

S. CLARK (1), M. Newfield (1), C. Reed (2)

(1) Ministry for Primary Industries, Wellington, New Zealand; (2) Ministry for Primary Industries, Wellington, New Zealand

Phytopathology 105(Suppl. 4):S4.30

Identifying potential and emerging threats is an important component of biosecurity risk management. While staff from across New Zealand's Ministry for Primary Industries (MPI) have historically kept watch on new and emerging pests and diseases for some time, MPI renewed efforts in 2012 to centralize the identification and management of emerging risks by implementing an "Emerging Risks System" focusing specifically on biosecurity threats. Implementation of the Emerging Risks System was a key deliverable in MPI's response to an independent review of import requirements and border processes following the inadvertent introduction of Pseudomonas syringae pv. actinidiae (Psa) into New Zealand in 2010. The MPI Emerging Risks System links with existing early warning and reporting systems for pest information, creates "alerts", applies systematic screening and analysis of information, and reports on any risk management actions taken, including implementation of specific control measures where needed. Since the system has been operating, over 800 alerts for plant pests or diseases have been received and assessed for biosecurity risk to New Zealand. Although only $1 \%$ of alerts resulted in amendments to biosecurity measures, all of the biosecurity amendments have been for plant pathogens on nursery stock or cut flower importation pathways. The pathogens requiring biosecurity amendments included five bacterium species, a virus, a viroid, a fungus and an oomycete.

\section{Frogeye leaf spot response to solo and combination fungicides}

A. COCHRAN (1), H. M. Kelly (2), K. Lamour (3), C. A. Bradley (4)

(1) Univ of Tennessee, Jackson, TN, U.S.A.; (2) University of Tennessee, Jackson, TN, U.S.A.; (3) University of Tennessee, Knoxville, TN, U.S.A.; (4) University of Illinois, Urbana, IL, U.S.A.

Phytopathology 105(Suppl. 4):S4.30

Cercospora sojina, the causal agent of frogeye leaf spot (FLS) disease in soybean, has demonstrated resistance to the quinone outside inhibitor (QoI) fungicides. In an effort to understand the efficacy of different fungicides in light of QoI fungicide resistance, field trials were conducted in a randomized complete block design in four locations in Tennessee and one in Illinois in 2013 and 2014. Foliar fungicides comprising DMI+QoI, SDHI+QoI, QoI, DMI, MBC, and chlorothalonil chemical groups were evaluated on a FLSsusceptible soybean variety using a R3 application time. Additionally $\mathrm{SDHI}+\mathrm{QoI}+\mathrm{DMI}$ and MBC+DMI fungicides were also evaluated, but only in 2014. FLS disease severity and soybean yield were obtained. The negative correlation between yield and increasing FLS disease severity was demonstrated during both growing seasons. In general, combination fungicides were within the top three highest-yielding treatments and conferred the greatest disease control; however, solo-DMI and solo-MBC fungicides also demonstrated adequate FLS disease control and yield protection, while QoI and chlorothalonil treatments did not significantly differ from the nontreated. Data analyzing FLS lesion DNA from the various locations for the G143A mutation (conferring QoI-fungicide resistance) using qPCR will also be presented, and may indicate how selection for resistance has been influenced by fungicide treatment.

Fusarium species reduce plant stand and biomass yield of switchgrass S. B. COLLINS (1), M. M. Dee (1), K. D. Gwinn (1), B. H. Ownley (1) (1) University of Tennessee, Knoxville, TN, U.S.A. Phytopathology 105(Suppl. 4):S4.30

Fusarium species can cause diseases of switchgrass (Panicum virgatum L.). Based on detached leaf assays, we have shown that $F$. graminearum, $F$. armeniacum, $F$. acuminatum, $F$. equiseti, and $F$. tricinctum are pathogens of switchgrass. The aims of this study were to determine the impact of virulent isolates of five Fusarium species on plant stand, growth, and health. To determine the impact on plant stand, 100 'Alamo' switchgrass seeds were inoculated with Fusarium at low ( $5 \mathrm{ml}$ of $10^{1}$ conidia/ml) or high $\left(5 \mathrm{ml}\right.$ of $10^{5}$ conidia $/ \mathrm{ml}$ ) rates. Plant stand was recorded 14 days after planting. To determine the impact of infection on established plants, pots of 6-week-old plants were sprayed with Fusarium conidia ( $7 \mathrm{ml}$ of $10^{5}$ conidia/ml). After 10 weeks, height, foliar disease severity, and fresh weight of plants were determined. Replicated trials were arranged in a completely randomized design and data were analyzed with Proc Glimmix (PC-SAS 9.3). Within a species, isolates varied in virulence; however, at least one isolate of each Fusarium species significantly reduced plant stand compared with the untreated control; losses ranged from $5 \%$ to $49 \%$. For whole plants treated with Fusarium isolates, there were significant reductions in plant height and fresh weight, and increased foliar disease compared with untreated controls, especially with $F$. graminearum and $F$. armeniacum isolates. This suggests that Fusarium species can reduce stand establishment and biomass yield of switchgrass.

Phytophthora species recovered from irrigation reservoirs in Mississippi and Alabama nurseries and pathogenicity of three new species

W. COPES (1), X. Yang (2), C. Hong (2)

(1) USDA ARS, Poplarville, MS, U.S.A.; (2) Virginia Polytechnic Institute and State University, Virginia Beach, VA, U.S.A.

Phytopathology 105(Suppl. 4):S4.30

From a survey for Phytophthora spp. in containment basins at one nursery each in Alabama and Mississippi, eight species and one taxon were recovered with $P$. gonapodyides dominant in cooler months and $P$. hydropathica in 
warmer months, accounting for $39.6 \%$ and $46.6 \%$ overall recovery, respectively. Among the recoveries were P. macilentosa, P. mississippiae and $P$. stricta, three new species recently described from a small lake (labeled M4) that serves as a primary water source for irrigation and to feed another irrigation pond (M5) at the Mississippi nursery. Neither of ponds M4 and M5 directly receives runoff from any production area. The three new species were tested for pathogenicity with Catharanthus roseus, Gardenia jasminoides 'August Beauty', Hydrangea quercifolia 'Semmes Beauty', Ilex magland 'Oakland', Pieris japonica 'Mountain Snow', and Rhododendron $\times$ 'Brandi Michele Raley'. None of the three species infected any of the test plants or became established in peat or pine bark growing media. Based on the result of pathogenicity trials as well as the observation that none of the Phytophthora species recovered from irrigation reservoirs have caused episodic disease in the nurseries, the eight Phytophthora spp. and one taxon appear to present a low risk to ornamental plants at those nurseries.

Low-volume spray technology for control of major diseases of strawberry in Florida

L. G. CORDOVA (1), B. W. Hammons (1), N. A. Peres (1)

(1) University of Florida, Wimauma, FL, U.S.A.

Phytopathology 105(Suppl. 4):S4.31

The two main diseases that affect strawberry production in Florida are Botrytis and anthracnose fruit rots, caused by Botrytis cinerea and Colletotrichum acutatum, respectively. Fungicide applications are the most effective method for control of both diseases. Traditionally, Florida strawberry growers use sprayers equipped with hydraulic nozzles with a flow rate of about $9351 /$ ha. The objectives of this study were to investigate the effects of reduced flow rates on the control of both diseases and to compare the efficiency of the standard hydraulic nozzle sprayer with a low-volume atomizer. Moreover, the reduction of fungicide rate by $25 \%$ was also evaluated. The experiment consisted of four treatments: 1) and 2) hydraulic nozzle technology with a flow rate of 935 and $561 \mathrm{l} / \mathrm{ha}$, respectively, and 3) and 4) atomizer with a flow rate of $234 \mathrm{l} / \mathrm{ha}$, but in treatment 4 , the fungicide rate was reduced by $25 \%$. Fungicides were sprayed 10 times over the season (November to March) when weather conditions were favorable for disease. Water sensitive papers were placed during spray operations to evaluate penetration and coverage, and plots were harvested twice weekly to evaluate yield and disease incidence. No significant differences in either yield or disease incidence was observed among the treatments. Botrytis isolates have been collected to evaluate the effect of reduced rate on fungicide resistance development.

Optimization of sample processing for qPCR detection of silver scurf and black dot on potatoes

J. CRANE (1), A. Gevens (2)

(1) Univ of Wisconsin, Madison, WI, U.S.A.; (2) University of Wisconsin, Madison, WI, U.S.A.

Phytopathology 105(Suppl. 4):S4.31

Helminthosporium solani, causal agent of silver scurf, and Colletotrichum coccodes, causal agent of black dot, are increasingly problematic in potato production and storage in Wisconsin. The two blemish diseases are not easily distinguished, and standard microscope diagnostic approaches are limited by the long incubation period needed for $H$. solani sporulation on tubers. An important first step in the process of refining a molecular diagnostic protocol for the pathogens is the optimization of sample processing. We compared 8 methods of DNA extraction from tuber periderm tissue. All methods produced DNA that could be PCR-amplified. The highest quality DNA was produced using the Qiagen DNeasy Plant Mini kit, the Omega E.Z.N.A. HP Plant DNA Mini kit, and the phenol-chloroform method. We selected the Qiagen kit for future work, based on its additional advantage of not using hazardous reagents. We also compared 4 methods of periderm homogenization. Grinding in liquid nitrogen generated the highest quality DNA, but this approach was also the most time intensive. Similar qPCR results were obtained when tissue was ground using liquid nitrogen and in a FastPrep Instrument. We are optimizing sample size for accurate pathogen detection. Our optimized tuber processing protocol, in combination with $H$. solani and $C$. coccodes qPCR primers, is being used for rapid pathogen detection from diseased tubers.

First study on the genetic diversity of Clavibacter michiganensis subsp. michiganensis strains isolated from tomato crops in Uruguay

V. Croce (1), M. González (2), M. A. Jacques (3), M. J. PIANZZOLA (1), M. I. Siri (1)

(1) Universidad de la República, Montevideo, Uruguay; (2) Instituto Nacional de Investigaciones Agropecuarias, Salto, Uruguay; (3) Institut de Recherches en Agriculture et Semences, Angers, France

Phytopathology 105(Suppl. 4):S4.31
Bacterial canker caused by Clavibacter michiganensis subspecies michiganensis $(\mathrm{Cmm})$ is considered one of the most important diseases of tomatoes both in tropical and temperate regions. In this study, $39 \mathrm{Cmm}$ strains were collected from several outbreaks in Uruguay where the disease is a constraint for tomato production. In order to identify the pathogen, molecular assays and pathogenicity tests on a tomato susceptible cultivar were performed showing positive results. A TaqMan system based on Ptssk primers and probe demonstrated good specificity with all strains tested, giving no false negatives. In addition, the first epidemiological study of $\mathrm{Cmm}$ in Uruguay was carried out to elucidate the origin of outbreaks and sources of infection and dissemination of the pathogen. Multi Locus Sequence Typing (MLST) based on five housekeeping genes revealed high diversity among Cmm strains present in Uruguay, expanding the known diversity within this particular subspecies when compared with a worldwide collection. This approach reveals 36 haplotypes or sequence types (STs) within a $108 \mathrm{Cmm}$ strains. Ten STs were assigned to strains isolated in Uruguay, including 8 novel STs first identified for the subspecie michiganensis. These results highlight the introduction of the pathogen by seeds from different origins as a result of seeds importation. The MLST analysis is an efficient tool for popultation genetics and epidemiological studies of this pathogen.

Evaluation of methods to detect the cotton pathogen Fusarium oxysporum f. sp. vasinfectum race 4

F. K. CRUTCHER (1), H. Doan (2), A. A. Bell (3), R. M. Davis (2), R. L. Nichols (4), J. Liu (3)

(1) USDA - ARS, College Station, TX, U.S.A.; (2) University of California, Davis, CA, U.S.A.; (3) USDA-ARS, College Station, TX, U.S.A.; (4) Cotton Incorporated, Cary, NC, U.S.A.

Phytopathology 105(Suppl. 4):S4.31

Fusarium wilt caused by Fusarium oxysporum f. sp. vasinfectum (Fov) is an important disease of cotton. Fov race 4, identified in the San Joaquin Valley of California, has caused serious losses and is a potential threat to US cotton production. Tests have been developed to rapidly identify race 4 in the laboratory and the field. These tests were evaluated on a panel of 35 Fov isolates from a USDA Insect Control and Cotton Disease Research Unit collection that represented distinct races, genotypes, and origins. Two different PCR protocols have been published. Screening the isolate panel with one of the protocols resulted in correct identifications, with the exception of one isolate that has not been found in US cotton. The second PCR protocol had several false positives that may have resulted from using a low annealing temperature. Another method, a commercial kit, was also tested on genomic DNA, fungal tissue, and infected plant tissue. The use of genomic DNA resulted in 12 false positives with the kit. The strain that gave the exceptional reading in the PCR screen also produced a false positive when tested on fungal and infected plant tissues using the kit. The presentation will clarify limitations of current protocols for identification of Fov race 4. Such diagnostic technology is essential for the prevention of further spread of Fov 4 within California and preventing its movement across US cotton production areas.

Effects of temperature and $\mathrm{pH}$ on Fusarium oxysporum and soybean seedling disease

D. R. CRUZ (1), L. F. Leandro (1), G. P. Munkvold (1)

(1) Iowa State University, Ames, IA, U.S.A.

Phytopathology 105(Suppl. 4):S4.31

Root rot of soybeans caused by Fusarium oxysporum is an important disease that reduces soybean yield in the Midwest. Information on the effects of abiotic factors on the disease is important for improving management strategies. This study assessed the effect of $\mathrm{pH}$ and temperature on $F$. oxysporum in vitro growth and seedling disease. In an in vitro assay, $14 F$. oxysporum strains were grown on petri dishes with basal medium at $4 \mathrm{pH}$ levels $(4,5,6,7)$, incubated in the dark at 4 temperatures $\left(1520,25\right.$, or $\left.30^{\circ} \mathrm{C}\right)$. Radial growth was measured after 5 days. In a rolled-towel assay, seeds were individually inoculated with $200 \mu 1$ of a $1 \times 10^{6}$ conidia/ml suspension of a pathogenic or a non-pathogenic strain. Rolls were placed in buffer solutions at $4 \mathrm{pH}$ levels $(4,5,6,7)$, and incubated at $15,20,25$, or $30^{\circ} \mathrm{C}$ for 8 days. Seedling root rot was rated on a percent scale. Strains showed optimal in vitro radial growth at $25^{\circ} \mathrm{C}$ and $\mathrm{pH} 6(5.1 \mathrm{~mm})$, and the lowest growth at $15^{\circ} \mathrm{C}$ and $\mathrm{pH} 5(0.9 \mathrm{~mm})$. For the rolled-towel assay, there was significant $\mathrm{pH} \times$ temperature interaction; the pathogenic strain caused the most severe root rot at $\mathrm{pH} 6$ at 25 and $30^{\circ} \mathrm{C}$ ( $28 \%$ and $37 \%$ severity, respectively), whereas no disease was observed at $\mathrm{pH}$ levels 4 and 5 at $15^{\circ} \mathrm{C}$. These results indicate that optimal $\mathrm{pH}$ and temperature conditions for $F$. oxysporum growth are similar to optimal conditions for infection and disease in soybean seedlings. 
Magnaporthe oryzae conidia on basal wheat leaves as a potential source of wheat blast inoculum

C. CRUZ (1), J. Kiyuna (2), W. Bockus (1), T. Todd (1), B. Valent (1)

(1) Kansas State University, Manhattan, KS, U.S.A.; (2) CETABOL, Santa Cruz de la Sierra, Bolivia

Phytopathology 105(Suppl. 4):S4.32

Wheat blast caused by the fungus Magnaporthe oryzae Triticum continues to cause significant losses on wheat in South America during outbreak years. Despite various reports of wheat blast leaf lesions in certain cultivars, the prevalence of inoculum originating from leaves on severely affected commercial fields is currently disputed. The purpose of this study was to i) report the widespread detection of wheat blast on basal leaves, ii) estimate the inoculum potential from these leaves, and iii) determine the impact of current fungicide application practices on the inoculum produced from sporulating lesions on basal wheat leaves. Artificial inoculations at the three-leaf stage showed that certain cultivar and isolate combinations caused more disease on old wheat leaves compared to young expanding leaves. Under optimum conditions $M$. oryzae had the potential to produce tens to hundreds of thousands of conidia on small amounts of wheat basal leaves. A mean of 500,741 conidia were produced on 0.3 -g dry basal leaves of a highly susceptible cultivar under optimum conditions for sporulation. Moreover, conidia production on leaves was timed with heading under both greenhouse and field conditions. In addition, foliar fungicide applications reduced the amount of M. oryzae conidia on basal leaves by $62-77 \%$ compared to the two non-sprayed checks. The information presented has the potential to contribute to wheat blast disease management in the future.

Profiling virus infection using small RNA sequencing data: A case study of virome from a small-holder farm

B. Cui (1), Y. Zheng (1), B. Xiang (1), Z. XIONG (2)

(1) Shihezi University, Shihezi, China; (2) University of Arizona, Tucson, AZ, U.S.A.

Phytopathology 105(Suppl. 4):S4.32

Mixed viral infections occur frequently in nature, especially in small-holder farms where multiple crops are planted in a small, confined space. To assess the extent of mixed virus infections, we characterized tomato virome in a small-holder farm in Xinjiang Province, China. Small RNAs from tomato exhibiting various types of viral symptoms were sequenced. We used a custom small RNA processing pipeline to identify known viruses and discover novel viruses. Cucumber mosaic virus (CMV) alone was present in tomatoes exhibiting leaf curling but CMV and its satellite RNA were found in tomatoes displaying leaf curling and stem and fruit necrosis. Both CMV and Zucchini yellow mosaic virus were identified in tomatoes with yellow mottling on leaves. In tomato plants exhibiting extreme leaf distortion and shoestring symptoms, we identified CMV and discovered a novel virus that is distantly related to Cycas necrotic stunt virus (CyNSV), a member of Nepovirus. The nearly full-length RNA genome of this virus has been obtained by sequencing overlapping PCR fragments. The two assembled genomic segments shared only about $80 \%$ sequence identity with those of two CyNSV isolates. This novel virus is tentatively named Tomato shoestring virus. This study illustrates the power of small RNA sequencing in virus identification and discovery, and demonstrates the extent of mixed viral infections in a closely confined space and the consequences of mixed infections on viral disease symptoms.

\section{Detection of a $\mathrm{Ca}$. Liberibacter americanus variant in Asian citrus psyllids, Diaphorina citri, in Texas \\ J. V. DA GRAÇA (1), M. Kunta (1) \\ (1) Texas A\&M University Kingsville, Weslaco, TX, U.S.A. \\ Phytopathology 105(Suppl. 4):S4.32}

Since 2008, intensive surveys and $\mathrm{qPCR}$ analysis in Texas for $\mathrm{Ca}$. Liberibacter spp. of citrus leaves with symptoms resembling huanglongbing (HLB), and Diaphorina citri have been conducted. In 2012, the first confirmed detection of $\mathrm{Ca}$. L. asiaticus in a citrus tree was reported. In April 2013, one psyllid sample produced a positive qPCR result for $\mathrm{Ca}$. L. americanus (CLam), which was confirmed by conventional PCR and sequence analysis. Its sequence data had $98 \%$ homology to CLam in Brazil. The psyllid sample was collected in a residential site, and citrus trees in the neighborhood were sampled and tested for CLam, but none tested positive. In February 2014, and again in February 2015, a single psyllid sample produced a qPCR positive result for this CLam variant. No citrus or orange jasmine samples have tested positive. Since the start of the HLB survey, over 73,000 psyllid samples have been tested, but only three have been positive for this CLam variant, all during the cooler time of the year. CLam in Brazil is reported to be sensitive to high temperatures. It is possible that this CLam variant is native to
North America, and occasionally infects $D$. citri; a host range study showed that the psyllid can feed on several native rutaceae. There are also native psyllid species which could serve as vectors of this bacterium.

\section{QTL mapping for Charcoal Rot resistance in soybean}

M. P. DA SILVA (1), P. Chen (1)

(1) University of Arkansas, Fayetteville, AR, U.S.A.

Phytopathology 105(Suppl. 4):S4.32

Charcoal rot (Macrophomina phaseolina) of soybean (Glycine max (L.) is a disease of economic significance in the United States. The objectives of this research are to map quantitative trait loci (QTL) for disease resistance and find single nucleotide polymorphism (SNPs) markers for charcoal rot. Two soybeans accessions were chosen based on disease severity, PI 567562A resistant and PI 567437 susceptible. Manual crosses were made between them and, $F_{1}$ seeds were planted under greenhouse conditions in Fayetteville, AR, and allowed to set seeds. Parental polymorphism and purity of the $F_{1}$ hybrids was established using forty SSR markers covering the entire genome of soybean (two per chromosome). SSR marker Satt531 was chosen to validate polymorphism between parents. Following, $200 \mathrm{~F}_{2}$ plants derived from the cross will be used for DNA extraction and genotyping by Illumina's SNP60K. Phenotype will be performed in $\mathrm{F}_{2-3}$ derived lines using 16 plants per family. Plants will be screened in the greenhouse using cut-stem inoculation technique to evaluate resistance to charcoal rot. Phenotypic and genotypic data will be combined to create a linkage map by JoinMap and WinQTLCart to detect putative QTLs. QTLs and linked SNP markers will be used to facilitate screening of breeding material for Charcoal rot resistance in soybeans.

\section{Changes in the population structure of PVY during systemic movement in a potato plant}

W. L. DA SILVA (1), S. M. Gray (2)

(1) Cornell University, Ithaca, NY, U.S.A.; (2) USDA-Agricultural Research Service; Department of Plant Pathology and Plant-Microbe Biology, Cornell University, Ithaca, NY, U.S.A.

Phytopathology 105(Suppl. 4):S4.32

Potato virus Y (PVY) is a major virus pathogen of potato worldwide. Surveys indicate that recombinant strains of PVY have emerged in recent years to predominate in the U.S. potato crop. To test the hypothesis that PVY populations evolve quickly and that vegetative propagation can be a driver of the selection of more fit variants; Illumina NGS was used to study the genetic diversity of PVY populations moving systemically within a potato plant. A potato plant was mechanically inoculated with the WI3 isolate of PVY ${ }^{\mathrm{O}}$. Four months post inoculation total RNA was extracted from systemically infected leaves at different positions on the plant, from a tuber, and from the tuber's sprout. A SNV at the position 7379 was present in the NIB cistron of viral RNA samples from systemically infected leaves and sprout, but was absent in RNA from the tuber. In contrast, RNA from the tuber contained eight additional SNVs, which induced alterations in two alpha helices of the CP protein as predicted by Phyre-2. The results suggest that vegetative propagation through tubers is a mechanism to generate additional diversity in the PVY genome, but that bottlenecks likely exist in the movement and survival of PVY variants in foliar portions of the plant. Understanding how rapidly PVY can evolve and how vegetation propagation can influence the genetic diversity will assist in the development of appropriate management strategies for PVY in the potato crop.

Elevated $\mathrm{CO}_{2}$ impacts bell pepper growth with consequences in the feeding behaviour and performance of the green peach aphid, Myzus persicae

B. Dader (1), A. Fereres (1), A. Moreno (2), P. TREBICKI (3)

(1) Institute of Agricultural Sciences, Spanish Council for Scientific Research, Madrid, Spain; (2) Institute of Agricultural Sciences, Spanish Council for Scientific Research, Madrid, Spain; (3) Department of Economic Development, Horsham, Australia

Phytopathology 105(Suppl. 4):S4.32

Current carbon dioxide concentration has reached $400 \mathrm{ppm}$ and future predictions estimate an increase up to $550 \mathrm{ppm}$ within only few decades away. Among the observed effects on plants, increasing $\mathrm{CO}_{2}$ concentrations stimulate plant growth, reduction in stomatal conductance and transpiration, improved water-use efficiency and higher rates of photosynthesis. At the same time, these changes have an indirect impact on pest biology and behaviour, e.g. altering their population growth or feeding habits, as well as on the symptomatology and incidence of associated pathogens such as plant viruses. Our first aim was to study the effect of ambient ( $400 \mathrm{ppm})$ and elevated $\mathrm{CO}_{2}$ $(650 \mathrm{ppm})$ on pepper growth (Capsicum annuum L.). Height, leaf area, dry weight, plant temperature by thermal imaging and carbon, nitrogen and 
chlorophyll content via SPAD were measured. Exposure to elevated $\mathrm{CO}_{2}$ caused an increase in height and above-plant dry weight. Elevated $\mathrm{CO}_{2}$ also caused a decrease in specific leaf area and chlorophyll content in pepper, which is an indirect indicator of decreased nitrogen levels in elevated $\mathrm{CO}_{2}$ leaves. Besides, elevated $\mathrm{CO}_{2}$ plants had a higher leaf temperature than those grown under ambient concentration. Secondly, pepper plants were exposed to the green peach aphid, Myzus persicae Sulzer (Hemiptera: Aphididae) for development and fecundity assessment. Aphids grew slower and produced fewer nymphs under elevated $\mathrm{CO}_{2}$. Lastly, aphid feeding behaviour was studied using the Electrical Penetration Graph (EPG) technique, which provides a live visualization and recording of plant penetration by aphid mouthparts. Results of EPG experiments will be presented and discussed.

\section{Meta-analytical synthesis of ten years of DMI and DMI + QoI fungicide trials for managing soybean rust in Brazil}

F. DALla LANA (1), C. Vieira Godoy (2), P. Anderson Paul (1), E. Medeiros Del Ponte (3)

(1) The Ohio State University, Wooster, OH, U.S.A.; (2) Embrapa Soja, Londrina, Brazil; (3) Universidade Federal de Viçosa, Viçosa, Brazil Phytopathology 105(Suppl. 4):S4.33

Soybean rust (SBR), caused by Phakopsora pachyrhizi, is the most damaging disease of soybean in Brazil. Effective management of this disease depends on the use of fungicides. Uniform field trials have been conducted since 2003/04 to evaluate the performance of several fungicides against SBR, including the DMIs tebuconazole (TEB) and cyproconazole (CYP), and the premix of QoI and DMI azoxystrobin + cyproconazole $(\mathrm{AZ}+\mathrm{CY})$, with results suggesting that over time some fungicides have become less effective. Multivariate random-effects meta-analyses were used to estimate mean log response ratios (LR) for SBR severity and yield for TEB, CYP, and AZ+CY, using year as a categorical moderator variable. LRs for both SBR and yield were significantly different from zero for the three fungicides. The overall mean percent SBR control and yield increase were 56.4 and $28.7 \%$ (TEB), 55.2 and $27.6 \%$ (CYP), and 77.6 and $48 \%(\mathrm{AZ}+\mathrm{CY})$. Mean percent control decreased over time from 84.7 to $28.6 \%$ (TEB), 68.5 to 34.1 (CYP), and 84.1 to $60 \%$ $(\mathrm{AZ}+\mathrm{CY})$. The highest mean yield responses were estimated in 2007, 2005, and 2013 for TEB (54.6\%), CYP (49.5\%), and AZ+CY (83\%), whereas the lowest responses were estimated in 2014, 2011 and 2013 for TEB (9\%), CYP $(14.7 \%)$ and $\mathrm{AZ}+\mathrm{CY}(26 \%)$. These results suggest that declines in fungicide efficacy may be associated with continuous use of the same fungicides. Further analyses will be conducted to estimate the rate of reduction in efficacy for TEB, CYP and AZ+CY.

\section{Aspergillus flavus biomass in inoculated corn kernels as a function of mating type}

K. DAMANN (1), S. Chalivendra (2), C. DeRobertis (2)

(1) Louisiana State Univ, Baton Rouge, LA, U.S.A.; (2) LSU AgCenter, Baton Rouge, LA, U.S.A.

Phytopathology 105(Suppl. 4):S4.33

Previous evidence from Louisiana indicated a highly skewed incidence (95\%) of Mat1-2 isolates from naturally infected corn kernels while soil isolates approximated a 1:1 ratio. Four isolates from soil (two Mat1-1 and two Mat12) with no history of corn infection were pin inoculated alone and in all paired combinations into individual kernels in a $4 \times 5$ array on sweet corn ears and allowed to develop for four days. Kernels were excised, frozen, and ground to a powder. Nucleic acids were extracted and RT-qPCR was performed using primers for histone His4 and for both mating type idiomorphs which would indicate copy number of these genes and by extension biomass. When inoculated alone, mean His 4 copy number for the Mat1-2 isolates represented a 78\% increase over Mat1-1 strains. Mat1-2 strains mean Mat1-2 copy numbers represented a $139 \%$ increase over Mat1-1 strains mean Mat1-1 copy number. Clearly, these 4 isolates indicate an enhanced ability of the Mat $1-2$ strains to grow in inoculated kernels. Also in the four cases where paired Mat1-1 and Mat1-2 isolates were co-inoculated the Mat1-2 copy number was always higher. The kernel powders were also extracted for aflatoxin $\mathrm{B}_{1}$ and 3 of the 4 isolates produced aflatoxin when inoculated alone. The six possible paired combinations of the four isolates were all greatly reduced in aflatoxin production indicating intraspecific aflatoxin inhibition (biocontrol) even among paired toxigenic isolates.

\section{Non-PCR high throughput multiplex detection of citrus pathogens}

T. DANG (1), B. Ramirez (1), F. Osman (1), S. Bodaghi (1), G. Vidalakis (1) (1) University of California, Riverside, CA, U.S.A.

Phytopathology 105(Suppl. 4):S4.33

High throughput multiplex diagnostics are critical for the early detection and eradication of pathogens. PCR based assays are limited in their multiplex capacity (3-5 targets). A QuantiGene Plex-Luminex based assay was developed for the robust, sensitive, and simultaneous detection, identification and quantification of multiple citrus pathogens. The developed assay is the first to successfully detect 14 targets in one multiplex reaction consisting of 1 housekeeping citrus gene (control), 2 universal citrus viroid probes (detection of seven citrus viroid species and their variants) and 11 citrus pathogen probes for: Citrus tristeza virus, Citrus psorosis virus, Citrus tatter leaf virus, Citrus leaf blotch virus, Citrus leprosis virus, Citrus vein enation virus, Citrus variegation virus, Citrus yellow vein clearing virus, Satsuma dwarf virus, Citrus exocortis viroid, and Hop stunt viroid. The QuantiGene Plex assay is performed in 96-well format and can be utilized to detect and quantify up to 80 RNA or 33 DNA targets per well in one assay. There is no need for reverse transcription reactions or DNA amplification because the QuantiGene Plex assay is based on sequential hybridization using magnetic beads and subsequent branched DNA signal amplification. The QuantiGene Plex is paired with a high throughput, semi-automated, robotic nucleic acid extraction procedure optimized for citrus tissues for increased results uniformity and cost effectiveness.

Genetic architecture of complex traits of Phytophthora infestans determined through genome-wide association mapping

G. DANIES (1), J. A. Romero-Navarro (1), L. N. Gonzalez-Garcia (2), K. Myers (1), E. Bevels (1), M. Bond (3), Y. Wu (1), S. Restrepo (2), W. E. Fry (1)

(1) Cornell University, Ithaca, NY, U.S.A.; (2) Universidad de los Andes, Bogotá, Colombia; (3) University of Hawaii at Manoa, Manoa, HI, U.S.A.

Phytopathology 105(Suppl. 4):S4.33

Phytophthora infestans, the causal agent of late blight of potatoes and tomatoes has been a major threat to global food security ever since the Irish famine of the 1800 's. The use of resistant cultivars has proven to be challenging due to the absence of durable resistance genes. Furthermore, despite the usefulness of fungicides, the pathogen has proven capable of evolving resistance to certain highly effective fungicides. Phenotypic analysis may take weeks to months. Therefore, an understanding of the genetic basis of complex traits of value in managing late blight is critical to rapidly predict the pathogen's phenotype using molecular markers. In this study, we systematically phenotyped the mating type, host preference, sensitivity to mefenoxam and the effect of temperature on germination and mycelia growth of three diverse populations of $P$. infestans: i) a diverse clonal population ii) a recombinant population detected in northeastern US in 2010 and 2011, and iii) a natural sexual population from Mexico. For these isolates we conducted a genome-wide association study to identify genetic markers associated with mating type and with sensitivity to mefenoxam at $100 \mu \mathrm{g} \mathrm{ml}^{-1}$ of mefenoxam.

Complete genome sequence of Spiroplasma kunkelii: New insights into spiroplasma evolution

R. E. DAVIS (1), J. Shao (1), E. L. Dally (1), Y. Zhao (1), G. E. Gasparich

(2), B. J. Gaynor (2), J. Athey (1), N. A. Harrison (3), N. Donofrio (4)

(1) Molecular Plant Pathology Laboratory, USDA-Agricultural Research Service, Beltsville, MD, U.S.A.; (2) Department of Biological Sciences, Towson University, Towson, MD, U.S.A.; (3) University of Florida, Ft. Lauderdale Research and Education Center, Davie, FL, U.S.A.; (4) College of Agriculture and Natural Resources, Plant and Soil Sciences, University of Delaware, Newark, DE, U.S.A.

Phytopathology 105(Suppl. 4):S4.33

The helical, motile, cell wall-less prokaryote infecting corn stunt diseased maize (Zea mays L.) plants and the insect vector Dalbulus maidis was the first spiroplasma discovered and is the microbe for which the term 'spiroplasma' was originally coined. The genome of this plant pathogen, Spiroplasma kunkelii, like that of the plant pathogen S. citri, has long resisted complete nucleotide sequence assembly because of numerous, homologous plectroviral genes that are integrated in the chromosome. In the present work, genomic DNA was extracted and purified from a culture of S. kunkelii strain CR2-3x grown in liquid medium. Nucleotide sequencing of the DNA followed by assembly of the sequence reads yielded a single circular chromosome and four plasmids. Comparison of the assembled chromosome with a previously constructed, detailed physical and genetic map of the S. kunkelii chromosome made it possible to verify that the sequence reads were correctly assembled to yield the first completely sequenced chromosome of a plant pathogenic spiroplasma. Comparative analysis of the S. kunkelii genome with the completely sequenced genomes of non-plant pathogenic members of class Mollicutes has opened new insights into the evolutionary emergence of plant pathogenic spiroplasmas. 
It's good to be green: A link between photosynthesis and polerovirus infection revealed by high-resolution mass spectrometry

S. L. DEBLASIO (1), R. Johnson (2), J. Chavez (2), M. Alexander (3), K. Parks (4), J. Ramsey (4), A. Karasev (5), S. M. Gray (6), J. E. Bruce (2), M. J. MacCoss (2), M. Cilia (7)

(1) USDA-Agricultural Research Service; Boyce Thompson Institute for Plant Research, Ithaca, NY, U.S.A.; (2) Department of Genome Sciences, University of Washington, Seattle, WA, U.S.A.; (3) Department of Plant Pathology and Plant-Microbe Biology, Cornell University, Ithaca, NY, U.S.A.; (4) Boyce Thompson Institute for Plant Research, Ithaca, NY, U.S.A.; (5) University of Idaho, Moscow, ID, U.S.A.; (6) USDA-Agricultural Research Service; Department of Plant Pathology and Plant-Microbe Biology, Cornell University, Ithaca, NY, U.S.A.; (7) USDA-Agricultural Research Service; Boyce Thompson Institute for Plant Research; Department of Plant Pathology and Plant-Microbe Biology, Cornell University, Ithaca, NY, U.S.A. Phytopathology 105(Suppl. 4):S4.34

Repression of photosynthesis is a common signature of virus infection. Poleroviruses are among the best-studied, phloem-restricted viruses. Symptoms are characterized by general leaf or interveinal chlorosis associated with a reallocation of carbohydrates and starch deposition in chloroplasts. However, the direct effects of infection on the host photosynthetic machinery are not well described. Using a combination of chemical cross-linking and affinity purification coupled to high-resolution mass spectrometry, we identified over 300 plant proteins with plastid-related functions including 126 involved in photosynthesis pathways, to directly or indirectly bind with the major and/or minor polerovirus capsid proteins or the P1 replicationassociated protein. Virus-induced gene-silencing (VIGS) of phytoene desaturase, a key enzyme in chlorophyll biosynthesis, resulted in an increase in virus titer during plant infection whereas silencing of PSBQ2, a component of the oxygen-evolving complex identified as directly binding to virus, had a negative effect on virus titer indicating that poleroviruses may use different strategies to manipulate photosynthesis to ensure successful propagation in plants. Understanding the mechanisms involved in photosynthetic regulation of polerovirus infection could be useful in developing strategies to prevent infection and/or viral transmission.

\section{Physiological aspects of Bipolaris oryzae-infected rice plants mediated by} a strobilurin fungicide

D. DEBONA (1), K. J. Nascimento (2), J. G. Gomes (3), C. E. Aucique-Pérez (1), F. A. Rodrigues (1)

(1) Universidade Federal de Viçosa, Viçosa, Brazil; (2) Instituto Federal de Goiás, Rio Verde, Brazil; (3) Universidade Federal de Viçosa, Rio Paranaíba, Brazil

Phytopathology 105(Suppl. 4):S4.34

Although strobilurins are among the most important fungicides used for plant disease control, they can also improve crop physiology. Nonetheless, the impact of azoxystrobin (Az), the main marketed strobilurin, on rice physiology is still unknown. Detailed gas exchange measurements and chlorophyll $a$ fluorescence analysis were used to examine the Az effects on the photosynthetic performance of rice plants (cultivar Metica-1) either challenged or not with Bipolaris oryzae, the causal agent of brown spot. Az impaired carbon (C) fixation in the non-inoculated plants mainly due to diffusive constraints. The photosynthesis of rice plants that were not sprayed with Az dramatically decreased upon $B$. oryzae infection, which was chiefly governed by photochemical and biochemical limitations. The energy surplus caused by limited $\mathrm{C}$ fixation in the rice plants that were treated with $\mathrm{Az}$ and inoculated with $B$. oryzae was thermally and effectively dissipated until 72 hours after inoculation. In Az absence, however, this mechanism was not sufficient to prevent chronic photoinhibition to photosynthesis. The inoculated plants were not able to fully capture and exploit the collected light energy, but these constraints were greatly limited in the presence of Az. In conclusion, Az impaired the photosynthetic performance of non-infected plants by diffusive constraints but prevented, to a greater extent, the damage to the photosynthetic apparatus during the infection process of B. oryzae.

\section{Development of a novel PCR Macroarray for multiplex detection of main} potato viruses

C. DEBONNEVILLE (1), D. Altenbach (1)

(1) BIOREBA AG, Reinach, Switzerland

Phytopathology 105(Suppl. 4):S4.34

Because of low virus titer in dormant potato tubers molecular methods are required for the detection of viruses from this material. Here we present a novel diagnostic tool allowing multiplex detection of 7 potato viruses and 1 viroid (PVA, PVM, PVS, PVX, PVY, PLRV, PMTV, PSTVd) from dormant tuber. This PCR-based technology has been developed and validated in parallel with a rapid nucleic acid extraction method. The PCR macroarray analysis presents the advantages to be fast, reliable, very sensitive (as sensitive as quantitative PCR), and cost effective. It does not require to buy expensive equipment making it a method of choice for large scale testing. Results will be presented showing the comparison of different methods (PCR macroarray, qPCR, and ELISA) for the detection of PVY and PLRV on a large number of tubers issued from the Swiss potato seed certification program (in collaboration with the Swiss Federal Agronomic Station Agroscope).

Wheat streak mosaic virus risk assessment models in the Central Great Plains

D. DEISHER (1), E. DeWolf (1)

(1) Kansas State University, Manhattan, KS, U.S.A.

Phytopathology 105(Suppl. 4):S4.34

Wheat steak mosaic virus (WSMV) epidemics are a major concern for wheat growers throughout the Great Plains. In Kansas specifically, 18\% yield losses in an agriculture-reporting district are possible. At a local level, complete crop failures are common. Such losses can be catastrophic for agriculture dependent economies and thus it is important to understand the epidemiology and risk of such outbreaks. The objective of this research was to develop disease forecasting and risk assessment models, which could provide vital information for growers most at risk. Historical records of WSMV in Kansas and Nebraska $(\mathrm{n}=129)$ were combined with monthly summaries of temperature, relative humidity and rainfall. Variables correlated with WSMV epidemics were identified using a combination of recursive partitioning and regression analysis. Preliminary results indicate that WSMV epidemics were associated with dry conditions in Oct, Feb and May. Temperature in Aug and Jan also influenced the risk of severe WSMV within a region. In the future these models may help wheat producers evaluate the potential risk of yield losses due to WSMV.

Population structure of Pythium ultimum from greenhouse floral crops in Michigan

J. DEL CASTILlO MUNERA (1), L. Quesada-Ocampo (2), A. Rojas (3), M. Chilvers (3), M. K. Hausbeck (3)

(1) Michigan State Univ, East Lansing, MI, U.S.A.; (2) North Carolina State University, Raleigh, NC, U.S.A.; (3) Michigan State University, East Lansing, MI, U.S.A.

Phytopathology 105(Suppl. 4):S4.34

Pythium ultimum causes seedling damping-off, and root and crown rot in greenhouse ornamental plants. To understand the population dynamics and structure of $P$. ultimum in Michigan floriculture crops, simple sequence repeats (SSRs) were developed using P. ultimum transcriptome. After screening in-silico SSR markers, six SSRs were selected based on their polymorphism after evaluation with bulk-segregant analysis of pool of $P$. ultimum isolates. A total of 166 isolates sampled from 2011 to 2013 from greenhouses in Kalamazoo, Kent and Wayne counties were analyzed using six fluorescent-labeled SSRs. The average genotypic diversity (0.938), evenness (0.56), and the recovery of 12 major clones, out of the 64 multilocus genotypes obtained, may suggest that $P$. ultimum is not a recent introduction into Michigan greenhouses. Analysis revealed a clonal population, with limited differentiation among seasons, hosts and counties sampled. Despite of the sampling effort, sample size was limited for some locations; therefore monitoring program using the markers developed in this study will help to understand further the dynamics of this population. Results also indicate that sanitation could be enhanced in order to more completely eradicate resident $P$. ultimum populations. Finally, the presence of common genotypes among counties suggest that there is an exchange of infected plant material among greenhouses, or that there is a common source of inoculum coming to the region.

Assessing the reliability of fungicide in vitro assays to predict whole-plant control of Zymoseptoria tritici on wheat

J. A. DELGADO (1), C. J. Klittich (1)

(1) Dow AgroSciences, Indianapolis, IN, U.S.A.

Phytopathology 105(Suppl. 4):S4.34

Leaf blotch caused by Zymoseptoria tritici is the most damaging wheat disease in Europe and commercial wheat production requires foliar application of fungicides. European populations of $Z$. tritici historically evolve rapidly and fungicides with novel modes of action are needed. Most fungicide invention exploits the growth of target fungi in vitro (IV) to screen compounds for fungitoxicity, but translation of IV toxicity to disease control in a whole plant (WP) pathosystem can be affected by compound residuality, cuticular penetration, and compound translocation and redistribution. Curative activity is a desirable fungicide attribute that is difficult to predict from IV screens. Compounds with curative properties must accumulate sufficiently in 
the apoplast to control mycelial growth of the pathogen post-infection. A comparison between a spore-based Z. tritici IV assay and a mycelial-based IV assay was carried out to determine if the mycelial assay was a better predictor of curative and protectant WP disease control. The evaluation of 45 commercial fungicides showed that inhibition of spore germination was more predictive of WP fungicidal activity than the mycelial IV assay, predicting both protectant and curative activity. Predictability of the spore-based IV assay was influenced by mode of action and physical properties of the active ingredients. Furthermore, the WP protectant assay was a better predictor of curative activity than either of the IV assays.

Determination of phytotoxic concentrations of different salts for potential use as antibacterial agents on lettuce

M. Delisle-Houde (1), V. Toussaint (2), R. TWEDDELL (1)

(1) Centre de recherche en horticulture, Université Laval, Quebec, QC, Canada; (2) Agriculture and Agri-Food Canada, Saint-Jean-sur Richelieu, QC, Canada

Phytopathology 105(Suppl. 4):S4.35

Bacterial leaf spot (Xanthomonas campestris pv. vitians) and varnish spot (also called bacterial midrib rot, Pseudomonas cichorii) are economically important diseases of commercial lettuce (Lactuca sativa) production. In Canada, no chemicals are registered to control bacterial diseases in lettuce. Consequently, control of bacterial leaf spot and varnish spot relies on cultural practices including crop rotations, good soil drainage and air movement through crop canopy. However, cultural practices do not provide effective control of the diseases. Exploitation of antimicrobial activity of salts used in food industry is an interesting approach. As part of ongoing research aimed to evaluate the efficacy of potassium sorbate, sodium benzoate, sodium bicarbonate, sodium carbonate and sodium metabisulfite to control bacterial diseases of lettuce, the objective of the study was to determine phytotoxic concentrations of each salt. Phytotoxic concentrations were determined on lettuce leaf tissue discs in vitro and on plants in greenhouse. The results obtained show that salts are phytotoxic at relatively low concentrations. In addition, it was observed that leaf apex and outer leaves are more sensitive to salts. Future work will be undertaken to evaluate the efficacy of salts to control bacterial leaf spot and varnish spot when applied at non-phytotoxic doses.

Screening of plant extracts for antibacterial activity against Xanthomonas campestris pv. vitians and Pseudomonas cichorii

M. Delisle-Houde (1), V. Toussaint (2), A. Gosselin (1), R. TWEDDELL (1)

(1) Centre de recherche en horticulture, Université Laval, Quebec, QC, Canada; (2) Agriculture and Agri-Food Canada, Saint-Jean-sur-Richelieu, QC, Canada

Phytopathology 105(Suppl. 4):S4.35

Over the past decades, increasing number of studies have reported that many compounds extracted from wild and cultivated plants show biocidal activity against fungi, bacteria, and insects. Moreover, the efficacy of plant extracts to control plant diseases was demonstrated using different test plant / pathogen models. The objective of the study was to identify plant extracts showing antibacterial activity against Xanthomonas campestris pv. vitians and Pseudomonas cichorii, causative agents of bacterial leaf spot and varnish spot (also called bacterial midrib rot) of lettuce (Lactuca sativa), respectively. Determination of antibacterial activity of the extracts was carried out using standard procedures in agar and liquid medium. Among the extracts tested, extracts of blueberry fruits, blueberry leaves, broccoli, cranberry fruits, garlic, onion, raspberry fruits, raspberry leaves, spinach, strawberry fruits, strawberry leaves, and sugar maple leaves showed antibacterial activity against both $X$. campestris pv. vitians and $P$. cichorii in agar or liquid medium. Future work will be undertaken to evaluate the efficacy of plant extracts to control bacterial leaf spot and varnish spot on lettuce plants grown in greenhouse. Plant extracts could eventually find application for the control of bacterial leaf spot and varnish spot of lettuce.

Mining the Uromyces transversalis genome for molecular markers to investigate genetic diversity of the quarantine significant Gladiolus rust fungus

J. DELONG (1), J. Buck (2), K. Pedley (3), J. Stewart (1), M. Brewer (1)

(1) University of Georgia, Athens, GA, U.S.A.; (2) University of Georgia, Griffin, GA, U.S.A.; (3) United States Department of Agriculture, Fort Detrick, MD, U.S.A.

Phytopathology 105(Suppl. 4):S4.35

Globalization, leading to world-wide movement of plant material, has resulted in recurring introductions of plant pathogens to the USA and elsewhere. Uromyces transversalis, the causal agent of Gladiolus rust, is quarantined in the EU and USA because it can be devastating to Gladiolus production and is difficult to eradicate once established. Our overall goal is to develop molecular markers to: (i) determine the origin of introductions to the USA, (ii) track the movement of genotypes, and (iii) understand the world-wide genetic diversity of the rust fungus $\mathrm{U}$. transversalis. Whole genome sequencing of three isolates collected in the USA was performed using the Illumina MiSeq platform. Genomes were assembled de novo and mined for microsatellite regions. Primers flanking putative polymorphic microsatellite regions were developed and tested for PCR-amplification on a panel of ten isolates from the USA resulting in nine robust polymorphic markers with more in development. Markers showed polymorphism within each isolate with no diversity detected among isolates. The microsatellite loci and nine housekeeping genes showed high diversity and two divergent genomes within dikaryotic individuals, yet no diversity among individuals, suggesting that $U$. transversalis populations are strictly clonal. Future studies will be conducted to assess genetic diversity of $U$. transversalis populations from around the world, including regions where the fungus likely originated.

Unexpected diversity of rust fungi on Panicum: Identification of four species infecting switchgrass

J. DEMERS (1), L. Castlebury (2)

(1) USDA ARS, Beltsville, MD, U.S.A.; (2) USDA-ARS, Beltsville, MD, U.S.A.

Phytopathology 105(Suppl. 4):S4.35

Switchgrass (Panicum virgatum) is an important biofuel crop, as well as a common forage and ornamental grass. Rust is one of the most important diseases of switchgrass and can severely limit biomass production. Puccinia emaculata has been presumed to cause the majority of rust disease in switchgrass. The objective of this study was to determine the diversity of rust fungi infecting switchgrass and relatives. Phylogenetic analyses were performed using 28S and IGS rDNA regions for 20 specimens from switchgrass and 48 from related grasses. Specimens were also morphologically characterized. Results indicate that at least four distinct rust fungi infect switchgrass and its close relative bitter panicgrass in North America, namely Puccinia panici, Uromyces graminicola, Puccinia panici var. robusta and an apparently undescribed Puccinia sp. The most commonly observed switchgrass rust pathogen was identified as $P$. panici. $P$. panici, $P$. panici var. robusta, and the undescribed species are difficult to distinguish morphologically and have been previously conflated. P. emaculata, originally described from witchgrass (Panicum capillare), was not found on switchgrass. Only $P$. panici var. robusta had an identified alternate host, Euphorbia. None were found on any of the related grasses examined, suggesting these rust fungi have a narrow host range. Accurate identification of these rust pathogens is necessary to study their epidemiology and to breed resistant varieties.

Functional genomics of biocontrol agents Bacillus mycoides BM02 and $\boldsymbol{B}$. pumilus PMB102 against Fusarium oxysporum f. sp. lycopersici

W. L. DENG (1), J. J. Wu (2), J. Y. Tzeng (1), J. Leveau (3), J. W. Huang (1) (1) National Chung Hsing University, Department of Plant Pathology, Taichung, Taiwan; (2) National Chung Hsing University and Academia Sinica, Ph.D. Program in Microbial Genomics, Taichung, Taiwan; (3) University of California at Davis, Department of Plant Pathology, Davis, CA, U.S.A.

Phytopathology 105(Suppl. 4):S4.35

Fusarium oxysporum f. sp. lycopersici (FOL) elicits devastating Fusarium wilt disease on tomatoes that leads to decreased productivity and yield inconsistency in warm vegetable production areas. In greenhouse experiments, B. mycoides BM02 and Collinade (a mixture of B. subtilis and Collimonas arenae Ca135) were found to reduce FOL-elicited disease symptoms on tomato plants, which warrant further investigation on the efficacy of Bacillus spp. as biocontrol agents and the biocontrol synergism between Collimonas sp. and Bacillus spp. for future development of the combinatorial crop protection. Comparative genomics of $B$. mycoides BM02, B. pumilus PMB102, and $C$. arenae Cal35 predicted the three biocontrol-active strains harbored various genes for their disease control capabilities. Among the predicted gene clusters, PMB102 encodes several NRPS/PKS genes for the biosynthesis of lipopeptides, BM02 harbors a gene cluster for lantipeptide synthesis and secretion, and Cal35 codes for multiple chitinases, which are likely candidates for their biological activities and environmental adaptation to the rhizosphere. A pilot study using in-vitro culturing systems demonstrated that the bacterial strains differentially inhibit fungal growth or the development of disease symptom, which is positively associated with the presence of the bio-active gene products. Underway characterization of the bioactive compounds will help clarify their roles in reducing Fusarium wilt disease. 
Epidemiology of the Rose rosette virus

P. L. DI BELLO (1), I. E. Tzanetakis (1)

(1) University of Arkansas, Fayetteville, AR, U.S.A.

Phytopathology 105(Suppl. 4):S4.36

Rose rosette disease (RRD), the most important issue for rosarians in North America, is manifested by a number of symptoms including excessive thorniness, mosaic, mottling, reddening on young shoots, malformed flowers, and an overall decline in vigor leading to plant death. The causal agent of the disease is the emaravirus Rose rosette virus (RRV). Given the viral nature of RRV, resistant material is the only viable option to combat the disease. To determine whether resistance exists in commercially available material two approaches were used: varieties were grafted with RRV-infected material and, in another set of experiments, plants were infested with viruliferous Phyllocoptes fructiphilus mites, the vector of RRV. The inoculation and acquisition access periods for the eriophyid vector were determined as well as the ability of RRV to move systemic to the roots within a three-month period. The majority of the roots tested positive for RRV; suggesting that pruning is not an appropriate management strategy as suggested by some rosarians.

\section{Current status of kiwifruit arm dieback in Chile}

G. A. DIAZ (1), M. Lolas (1), B. A. Latorre (2), J. P. Zoffoli (2)

(1) Universidad de Talca, Talca, Chile; (2) Pontificia Universidad Catolica de Chile, Santiago, Chile

Phytopathology 105(Suppl. 4):S4.36

Kiwifruits (Actinidia deliciosa) is one of the most important crops in Chile. Chilean kiwifruit industry comprises over 9,000 ha representing an important economic impact for the country. Kiwifruit arm dieback has become a major problem for Chilean kiwifruit growers. However, a systematic study of the importance of kiwifruit trunk diseases and identification of agent causals in Chile has not yet been completed. Therefore, field surveys were conducted throughout the main kiwifruit-growing regions to determine the prevalence and to identify the major pathogens associated with arm dieback. A total of 170 arm samples obtained during surveys of south-central of Chile between 2013 and 2014 were plated on PDA plus antibiotics and Igepal. Cultures were identified morphologically and molecularly using the ITS region of rDNA, $\beta$ tubulin and/or EF1- $\alpha$ genes. The results allowed to determine a prevalence between 5 and $75 \%$ of arm dieback in kiwifruit orchards with 8 and 25 years old, respectively. Based on morphologic and phylogenetic analyses, we identified several species in the families Botryosphaeriaceae, Diaporthaceae, as well as species in the genera Cadophora and Phaeoacremonium associated with wood canker symptoms. This study reveals that kiwifruit arm dieback appears to be associated with a fungi complex in Chile. Species of Phaeoacremonium, Cadophora, Diaporthe and Neofusicoccum are apparently the most frequently fungal pathogen obtained from kiwifruit with arm dieback.

\section{Detection of Speck rot on Pink Lady apple fruit in the Maule Region in Chile}

G. A. DIAZ (1), B. A. Latorre (2), J. P. Zoffoli (2), M. Caceres (1), R. Mendez (1), M. Lolas (1)

(1) Universidad de Talca, Talca, Chile; (2) Pontificia Universidad Catolica de Chile, Santiago, Chile

Phytopathology 105(Suppl. 4):S4.36

Chile has a production area of 37,207 Ha of apples. The fungal diseases is one of the major problems for Chilean industry of apple. Recently in Washington, USA, it has been described speck rot or rubbery rot, a new fungal disease that affect to apples in the orchard and in the cold storage caused by Phacidiopycnis washingtonensis. Speck rot affecting of Pink Lady apples were observed during cold storage and in apples on the floor of orchards in Region of Maule in Chile during 2014 and 2015. Speck rot was characterized by an initial light brown skin discoloration that progressed to a black skin discoloration, with a firm rubbery textured. Small fragments were collected from diseased tissues and aseptically placed on PDA. Gray colonies with aerial mycelia and showing alternating growth rings were observed. The conidia were smooth, unicellular, hyaline, ellipsoid to lacriform with flattened ends, and measured $6.6 \times 3.6 \mu \mathrm{m}$. The ITS region of rDNA was amplified using primer ITS4/ITS5. Based on morphology and phylogenetic analyses, this fungus was identified as $P$. washingtonensis. All inoculated apple fruits developed necrotic lesions and the re-isolations were $100 \%$ positive to $P$. washingtonensis. Virulence was tested on different cultivars of apples. The isolates were sensitive to the three commonly used fungides. To our knowledge, this study is the first description and partial characterization of isolates of $P$. washingtonensis causing speck rot in Chile.
A new virus isolated from wild raspberry exhibiting leaf curl symptoms A. DIAZ-LARA (1), J. Dittrich (2), K. E. Keller (2), R. R. Martin (3)

(1) Oregon State University, Corvallis, OR, U.S.A.; (2) USDA-ARS Horticultural Crops Research Unit, Corvallis, OR, U.S.A.; (3) USDA ARS, Corvallis, OR, U.S.A.

Phytopathology 105(Suppl. 4):S4.36

Raspberry leaf curl disease was first reported in the 1920s and reported only in North America. Previous studies suggested an aphid transmitted virus as the causal agent of the disease. During a field survey in the state of Wisconsin a wild black raspberry (Rubus sp.) showing leaf curl and mosaic symptoms was collected and analyzed by means of PCR and ELISA tests, with negative results for all the known viruses affecting Rubus species except for Rubus yellow net virus. DsRNA analysis suggested the presence of a virus with a genome size $\sim 10 \mathrm{~Kb}$. The dsRNA virus was subjected to shotgun cloning, sequencing and analysis using BLAST. The two most closely related viruses identified were two carlaviruses (Elderberry carlavirus $C$ and $D$ ), but whose genomes are smaller and are not reported in Rubus sp. Universal Carlavirus primers amplified a $200 \mathrm{bp}$ amplicon confirming the presence of the viruses in the collected and grafted plants. Additionally, a R. idaeus cv. Munger grafted with the wild raspberry developed similar symptoms and the presence of Carlavirus nucleic acids was confirmed with the above mentioned primers. Together these results suggest the existence of a new Carlavirus affecting raspberry, which is graft-transmissible and may be involved in the raspberry leaf curl disease. Currently NGS is being used to further analyze the nucleic acids extracted from the wild black raspberry.

Investigating Alternaria species composition and role in the potato early blight complex in Wisconsin

S. DING (1), K. Meinholz (1), A. J. Gevens (1)

(1) UW-Madison, Madison, WI, U.S.A.

Phytopathology 105(Suppl. 4):S4.36

The potato early blight complex (EBC), caused by Alternaria solani and $A$. alternata, is a perennial and destructive potato disease. Infected leaves develop necrotic lesions which can cause loss in tuber quality and quantity. Currently no commercial cultivars are resistant to the $\mathrm{EBC}$, so control is maintained through fungicide application. An improved understanding of the ecology and epidemiology of the EBC pathogens would enhance fungicide program design and overall EBC management. Our initial study focused on the pathogen population assemblage in the field in 2014. Results indicated an earlier emergence of $A$. alternata and a possible interaction between the pathogens. There was an inverse relationship between disease severity and quantity of $A$. alternata present at sampled field sites. A greenhouse experiment with conidial inoculations of the two pathogens revealed that $A$. solani was the major pathogen causing symptoms on potato plants. Greater disease was observed when $A$. alternata and $A$. solani were inoculated simultaneously, and less disease was observed when A. alternata was inoculated two days prior $A$. solani. Because $A$. alternata is a weak pathogen, it causes less severe disease symptoms but may trigger plant defenses thereby limiting severity of subsequent $A$. solani infection. Further studies will be conducted to better understand role of A. alternata in EBC and the interactions between the two pathogens.

Detection of 'Candidatus Liberibacter asiaticus' in citrus using tissue prints and anti-OmpA polyclonal antibodies

F. DING (1), Y. Duan (2), J. Hartung (1)

(1) USDA ARS MPPL, Beltsville, MD, U.S.A.; (2) USDA ARS USHRL, Fort Pierce, FL, U.S.A.

Phytopathology 105(Suppl. 4):S4.36

We have made rabbit polyclonal antibodies against a fragment of the major outer membrane protein OmpA, and used them in tissue print assays to detect 'Ca. Liberibacter asiaticus' (CaLas) in citrus. Our primary rabbit antibodies were used with secondary goat anti-rabbit polyclonal antibodies (Pab) conjugated with alkaline phosphatase, and produced very strong purple color in individual phloem cells when CaLas was present. The reactivity of the antibody was specifically blocked by the antigen used to immunize the rabbit and the antibody did not identify plants infected with two genotypes of Citrus tristeza virus, Citrus chlorotic dwarf virus and Xylella fastidiosa. Thus, the assay was entirely specific for CaLas. This assay also preserved the anatomical distribution of this pathogen. The extent of colonization of phloem cells varied greatly among serial sections from individual petioles and midribs. The pathogen was also easily detected in cross sections of green stems, peduncle, seed coats and roots of sweet orange. The assay was also used to demonstrate systemic colonization of periwinkle leaves by CaLas, and the sensitivity was at least as good as qPCR. Our assay provides low cost detection and a level of spatial and anatomical detail not possible with other methods like qPCR. The simplicity, cost and ability to scale the tissue print 
assay with the polyclonal antibody makes this an attractive assay to complement PCR-based assays currently in use.

\section{Investigating fungicide resistance in potato early blight complex} pathogens in Wisconsin

S. DING (1), S. Jordan (1), K. Cleveland (1), K. Fairchild (2), P. Wharton (3), A. Gevens (4)

(1) University of Wisconsin, Madison, WI, U.S.A.; (2) University of Idaho, Aberdeen, ID, U.S.A.; (3) University of Idaho, Aberdeen, ID, U.S.A.; (4) Univ of Wisconsin, Madison, WI, U.S.A.

Phytopathology 105(Suppl. 4):S4.37

The potato early blight complex (EBC) is an important potato disease in Wisconsin caused by Alternaria solani and A. alternata. Primary management of EBC is by fungicide application. However, all currently used fungicides have at risk of fungicide resistance. One such group is the quinone outside inhibitors (QoIs) which target the cytochrome $b$ complex. In this study, a PCR-based method was developed to detect fungicide resistance caused by single nucleotide mutations on cytochrome $b$. Isolates were obtained from potato leaves collected in the 2012 growing season in Wisconsin. Primers were designed to detect resistance mutations F129L and G143A in cytochrome $b$ of both A. solani and A. alternata. The spiral gradient dilution method was also used to screen the isolates for resistance to the QoI fungicide azoxystrobin. Of the $30 \mathrm{~A}$. alternata isolates tested, no F129L was detected but G143A was detected in 22 isolates (73\%). For the $27 \mathrm{~A}$. solani isolates, no G143A mutations were detected, but 26 isolates had the F129L substitution (96\%). Spiral gradient dilution results correlated with molecular results and showed that out of 57 isolates, $23 \mathrm{~A}$. alternata and $22 \mathrm{~A}$. solani isolates were resistant to azoxystrobin. These methods provided an efficient way of screening a large number of isolates for fungicide resistance. Enhanced understanding of fungicide resistance should allow us to develop more effective EBC control programs for Wisconsin growers.

\section{Antifungal and antioomycete properties of California Collimonas isolates and their use in synergy-based biocontrol of Fusarium wilt of tomato} H. K. DOAN (1), N. Maharaj (1), E. Miyao (2), R. M. Davis (3), J. H. Leveau (3) (1) Univ of California, Davis, CA, U.S.A.; (2) University of California, Woodland, CA, U.S.A.; (3) University of California, Davis, CA, U.S.A. Phytopathology 105(Suppl. 4):S4.37

The bacterial genus Collimonas comprises three species: C. fungivorans, $C$. pratensis, and $C$. arenae. Collimonads are best known for their mycophagous, weathering, and antifungal properties. A total of five Collimonas isolates from Northern California forest soils were tested along five strains from the Netherlands and one from Japan for their ability to inhibit the growth of phytopathogenic fungi and oomycetes in vitro. Of the collimonads tested, $C$. arenae $\mathrm{Cal} 35$, was by far the most antagonistic in these confrontation assays. Collimonas arenae Cal35 showed the greatest antagonism against Fusarium oxysporum f. sp. lycopersici (FOL), the causal agent of Fusarium wilt of tomato, and Verticillium dahliae, the causal agent of Verticillium wilt of tomato. In greenhouse trials, Fusarium wilt of tomato was effectively controlled by a mixture of $C$. arenae Cal35 and a commercially available, Bacillus-based biofungicide, but not by either one alone. Tomato plants had reduced vascular discoloration and increased shoot dry weight with soil drenches of the mixture of $C$. arenae Cal35 and the Bacillus-based biofungicide. In field experiments, application of this mixture reduced the incidence of FOL in tomato plants and increased fruit yield. In addition, this mixture reduced fruit sunburn and prevented FOL-induced loss of plant foliage.

Young almond orchard performance as affected by preplant soil fumigation or steam in a replanted site with the presence of plant parasitic nematodes

D. DOLL (1), A. Johnson (2), G. T. Browne (3), B. Hanson (2), S. Fennimore (4)

(1) Univ of California, Merced, CA, U.S.A.; (2) Univ of California, Davis, CA, U.S.A.; (3) USDA ARS, Davis, CA, U.S.A.; (4) Univ of California, Salinas, CA, CA, U.S.A.

Phytopathology 105(Suppl. 4):S4.37

Successive generations of almond orchards are suppressed by replant problems, e.g. Prunus replant disease (PRD), a soilborne complex, plant parasitic nematodes (PPN), and other factors. Preplant soil fumigation can control PRD and NP, but due to methyl bromide (MB) phase-out and various regulatory mandates, optimized alternative treatments are needed. In 2 replanted almond orchards, efficacy of pre-plant treatments was evaluated. Treatments included: steam (applied with tractor-mounted soil augers $90 \mathrm{~cm}$ diameter to a $60 \mathrm{~cm}$ depth, centered at tree planting sites [TPS]); and soil fumigation (MB, 1,3-dichloropropene [1,3-D], chloropicrin [PIC], or 1,3-D plus PIC [C35], applied using a commercial rig to row strips [RS], $2.1 \times 2.1 \mathrm{~m}$ centered at TPS, or broadcasted areas [BC]). Treatment efficacy was assessed as yield and trunk diameter increase for trial 1 and 2, respectively. Sampling confirmed the presence of PRD and PPN. In trial 1, 1,3-D RS and BC, and C35 RS cumulative yields were 172,202 , and $146 \%$ of controls, respectively. In trial 2, trunk diameters for fumigated treatments were 110 through $117 \%$ of controls. Steam had no measurable effect in both trials. PPN were detected in all treatments 3 years post-treatment and not correlated with performance in both trials. Pre-plant soil fumigation with 1,3-D, C35, or PIC increased young orchard performance without suppressing PPN, suggesting that PRD is the primary factor in reducing young almond tree growth and yield.

Sequence analysis of Grapevine leafroll-associated virus 1 from Washington vineyards

B. DONDA (1), N. Rayapati (1)

(1) Washington State University, Prosser, WA, U.S.A.

Phytopathology 105(Suppl. 4):S4.37

Grapevine leafroll-associated virus 1 (GLRaV-1, genus: Ampelovirus, family: Closteroviridae) has been reported in many grapevine-growing regions worldwide. However, only limited information is available on the molecular biology of GLRaV-1. In this study, we have isolated the genomic-length, double-stranded RNA (dsRNA) of GLRaV-1 from two wine grape (Vitis vinifera) cultivars that tested positive for the virus in reverse transcriptionPCR (RT-PCR). The dsRNA was subsequently used as a template to amplify overlapping portions of the genome by RT-PCR using virus-specific primers. The 5'-end sequence was determined using a commercially available 5'RACE system. The resulting amplicons were cloned and nucleotide sequence determined. The genome sequences of two isolates of GLRaV-1 were determined to be $\sim 18,730$ (isolate WA-CH) and $\sim 18,945$ (isolate WA-PN) nucleotides (nt). WA-CH and WA-PN isolates encode nine open reading frames and the overall genome organization is similar with previously reported GLRaV-1 isolates. WA-CH and WA-PN isolates have $\sim 857 \mathrm{nt}$ and 922 nt 5'nontranslated region (NTR), respectively, and contain a $130 \mathrm{nt}$ tandem duplication. An additional $65 \mathrm{nt}$ sequence was present only in the 5'NTR of the WA-PN isolate. The size of 3'NTR of WA-CH and WA-PN isolates is being determined. Using unique sites of a restriction enzyme in the 5'NTR, an RT-PCR-based RFLP assay was developed for molecular typing of GLRaV-1 isolates present in Washington vineyards.

Effectiveness of rice bacterial blight $R$ gene $\mathrm{Xa} 4$ decreased with increase of drought and high temperature stresses

G. S. C. Dossa (1), I. Quibod (1), A. Henry (1), R. Torres (1), A. Kumar (2), R. Oliva (1), E. Maiss (3), K. Wydra (4), J. E. Leach (5), C. M. VERA CRUZ (1) (1) Intl Rice Research Inst, Metro Manila, Philippines; (2) Intl Rice Research Inst, Hyderabad, India; (3) Leibniz Universität Hannover, Hannover, Germany; (4) Erfurt University of Applied Sciences, Erfurt, Germany; (5) Colorado State University, Ft. Collins, CO, U.S.A.

Phytopathology 105(Suppl. 4):S4.37

Drought and high temperature will influence rice resistance $(R)$ gene response to bacterial blight $(\mathrm{BB})$ caused by Xanthomonas oryzae pv. oryzae $(X o o)$. To understand the interaction among drought, high temperature and $\mathrm{BB}$ resistance, comparative analysis of single and double stress responses by greenhouse, field study and time-course transcriptome analysis were performed. Seventeen rice genotypes, screened for combined drought/BB stresses and transcriptome profiling of IR24 (no effective $R$ gene) and IRBB67 (Xa4+Xa7) inoculated with PXO145 (avrXa4,avrXa7) under high $\left(35 / 31^{\circ} \mathrm{C}\right)$ and low $\left(29 / 21^{\circ} \mathrm{C}\right)$ temperature at three time points were compared. Disease severity was less under drought stress compared to non-stress while disease increased at high temperature on IR24 and IRBB4 (Xa4). Genotypes with Xa-gene+drought-QTLs showed more effective disease resistance to PXO61(avrXa4) compared to drought-QTLs genotypes alone. BB NILs were resistant under drought, except when only Xa4 was present. Xoo multiplication and spread in planta increased in genotypes carrying Xa4 but restricted in IRBB7 (Xa7) under water limitation. Transcriptome profiling analysis showed temperature effects on induction of OsSweet14 in IR24 and jasmonic acid-related gene in IRBB67. Our results suggest that high temperature and drought affect $\mathrm{Xa} 4$ response and combination of $\mathrm{Xa} 4+\mathrm{Xa}$ was most resistant to both $\mathrm{BB} /$ high temperature and $\mathrm{BB} /$ drought stresses.

Engineering broad resistance tailored against rice bacterial blight in Mali H. Doucoure (1), F. Auguy (1), C. Tekete (2), M. Hutin (1), S. Baufume (1), R. Koebnik (1), B. Szurek (1), O. Koita (2), V. VERDIER (3), S. Cunnac (1)

(1) Institut de Recherche pour le Developpement, Montpellier, France; (2) Laboratoire de Biologie Moléculaire Appliquée, Université des Sciences Techniques et Technologiques de Bamako, Bamako, Mali; (3) Institut de Recherche Pour Le Développement, 34394 Montpellier Cedex 5, France Phytopathology 105(Suppl. 4):S4.37 
Bacterial blight caused by Xanthomonas oryzae pv. oryzae (Xoo) is an important disease of rice and is prevalent in irrigated and rainfed lowland rice growing areas in Mali. Our project aims at implementing a new breeding strategy to create tailored resistances against many of the BB-causing Xoo strains from Mali. Our strategy relies on the identification of the DNA regulatory boxes required for the induction of SWEET susceptibility genes that are targeted by most Malian Xoo strains and their subsequent modification using the TALEN and CRISPR/Cas technologies. Ideally, genome editing and breeding will be performed using a Malian elite breeding rice line. Ultimately, pyramiding of TAL type III effectors-unresponsive alleles should provide broad spectrum resistance against virtually all Malian Xoo strains. We will report on (i) the characterization of a set of widely grown rice varieties in Mali for their susceptibility against a representative collection of Malian Xoo strains ; (ii) the repertoire of Malian Xoo TAL effectors targeting SWEET genes and (iii) the current status of our efforts to edit the corresponding DNA-binding boxes in the rice genome.

\section{Gamma irradiation for management of post-harvest fruit rot of papaya} caused by Phytophthora palmivora

M. A. DRAGICH (1), J. Uchida (1), L. Wong (2), C. Kadooka (1), K. Kamiya (3)

(1) University of Hawaii at Manoa, Honolulu, HI, U.S.A.; (2) Pa'ina Hawaii LLC, Kunia, HI, U.S.A.; (3) Kamiya Gold Inc., Laie, HI, U.S.A.

Phytopathology 105(Suppl. 4):S4.38

Phytophthora palmivora is an Oomycete pathogen that causes fruit rot of papaya both in the field and during post-harvest. A post-harvest treatment that retains fruit qualities for looks, taste and texture that controls fruit rot would reduce post-harvest losses and risk at market. Two types of irradiation experiments were conducted: one measuring spore germination and mycelial growth in perti dishes, and another measuring pathogen growth on mature green papaya fruits. Doses of 400 gry, 500 gry, 700 gry, 1 kgry, and 4 kgry were used to treat $P$. palmivora in petri dishes. Doses of 400 gry and 700 gry were used to treat the pathogen on papaya fruit. The results for each treatment were compared to untreated controls. In petri dishes, 700 gry was shown to be the minimum dose which arrested spore germination and eliminated mycelial growth. This rate on papaya fruit also prevented lesion development. 400 gry doses on papaya fruit also drastically reduced development, and we were unable to re-isolate $P$. palmivora from fruit treated at 400 gry. For the purpose of extended shelf life, 400 gry treatments have eliminated lesion development for 6 days post treatment. Currently 400 gry is the appropriate dose for purposes of complete elimination of papaya fruit rot caused by P. palmivora, based on three repeated tests, with ten fruit per treatment.

Development of a detection method for the survey of the oak pathogens Diplodia corticola and D. quercivora in Florida

T. DREADEN (1), J. A. Smith (2)

(1) University of Florida, Gainesville, FL, U.S.A.; (2) University of Florida, Gainesville, FL, U.S.A.

Phytopathology 105(Suppl. 4):S4.38

Diplodia corticola and D. quercivora cause cankers on oak (Quercus spp.) and were recently found in Florida in 2010 and 2014, respectively, on live oak (Q. virginiana). Both taxa cause similar symptoms on the same hosts making diagnosis to species level difficult. An accurate, rapid and cost-effective method to identify both taxa is needed to support detection and survey efforts. The detection method presented here utilizes a PCR specific to both taxa then an $M s e$ I restriction enzyme digestion to differentiate $D$. corticola and $D$. quercivora. A survey of symptomatic oak samples from Florida and resulting detections of D. corticola and D. quercivora are presented.

\section{Assessing the pathogenicity of Raffaelea spp. isolated from Xyleborus} glabratus to swamp bay, Persea palustris

T. J. DREADEN (1), A. Campbell (1), R. C. Ploetz (2), J. A. Smith (1)

(1) University of Florida, Gainesville, FL, U.S.A.; (2) University of FloridaTropical Research Education Center, Homestead, FL, U.S.A.

Phytopathology 105(Suppl. 4):S4.38

Laurel wilt is a devastating disease of members of the Lauraceae family in the southeastern United States. It is caused by the fungus Raffaelea lauricola, which is a nutritional symbiont of its vector, the Asian redbay ambrosia beetle, Xyleborus glabratus. A total of six additional Raffaelea spp. have been recovered from $X$. glabratus adults in the United States. This study investigated the pathogenicity of five of these species and an additional Raffaelea spp. on swamp bay (Persea palustris), which is highly susceptible to laurel wilt. Six weeks after inoculation, only $R$. lauricola caused significantly greater symptoms than the mock-inoculated control. The ability of the inoculated fungi to move systemically and be recovered from the host at the end of the experiment varied by taxa. Although the roles these mycangial fungi play in the life cycle of the redbay ambrosia beetle are not known, they do not appear to be involved in the development of laurel wilt on this host tree.

Characterization of targets of PthA4 and their involvement in citrus canker development

S. DUAN (1), H. Jia (2), G. Siddrame (1), J. B. Jones (3), N. Wang (4)

(1) Citrus Research and Education Station, Lake Alfred, FL, U.S.A.; (2) University of Florida, Lake Alfred, FL, U.S.A.; (3) Department of Plant Pathology, University of Florida, Gainesville, FL, U.S.A.; (4) University of Florida - CREC, Lake Alfred, FL, U.S.A.

Phytopathology 105(Suppl. 4):S4.38

Citrus bacterial canker, caused by Xanthomonas citri subsp. citri (Xac), is a severe disease affecting all commercial citrus cultivars worldwide. The development of canker symptom requires injection of type III effectors (T3Es) into host cytosol via type III secretion system (T3SS). Previous studies reveal that activation of a citrus susceptible gene CsLOB1 by a transcription activator-like (TAL) effector PthA4 is crucial for pustule development. CsLOB1 is a member of the Lateral Organ Boundaries (LOB) gene family of transcription factors and $\mathrm{LOB}$ domain proteins are involved in the regulation of lateral organ development, anthocyanin and nitrogen metabolism, and are responsive to phytohormones and environmental stimuli. We aimed to characterize the function of CsLOB1. For this purpose, the CsLOB1 gene was silenced using a CTV vector. We will present data on RNA-seq analysis of CsLOB1 silenced and wild type plants. The targets of CsLOB1 will be identified. In addition, PthA4 specifically binds to effector binding elements (EBE) in the promotor region and transcriptionally regulate the expression of its downstream targets. A plethora of EBEs containing genes are identified in citrus genome using microarray analysis, suggesting that multiple downstream targets might be regulated in PthA4-dependent manner. We will confirm whether those genes containing putative EBE are targets of PthA4 and their involvement in citrus canker development.

Genetic diversity and pathogenesis of Candidatus Liberibacter asiaticus isolates

Y. DUAN (1), B. WU (2), L. Zhou (2), E. Stover (2), R. Shatters (1)

(1) USDA ARS USHRL, Fort Pierce, FL, U.S.A.; (2) USDA-ARS-USHRL,

Fort Pierce, FL, U.S.A.

Phytopathology 105(Suppl. 4):S4.38

Candidatus Liberibacter asiaticus (Las) is the prevalent species of the citrus huanglongbing bacteria. We describe here the molecular characterization of Las bacterial populations and their association with disease progression in host plants. By sequencing 35 Las isolates collected from different geographic origins, we found that most surveyed Las isolates contained at least two prophages/phages. In addition, all but one of the 26 isolates from Florida clustered into two major phylogenetic groups. Variation within the chromosome was less than $1 \%$ overall but could be as high as $3 \%$ in the prophage regions. Based on prophage sequences, we identified nine Las variants. Types A, B, C, and D are the most abundant types in Las-infected plants, while only A, B and C are abundant in Las-infected psyllids. The Type D Las population does not appear to exist in the psyllids but is dominant in mealybugs, and the Las bacteria transmitted by the mealybugs do not cause disease. However, Type D can be detected in infected citrus plants with leaf blotchy mottle. Using severe and mild Las isolates, we found that the movement and titers of Las bacteria varied greatly in different genotypes of root stocks and commercial varieties, and most Las infections were detectable in the shoots before the roots of inoculated plants, with the titer of Las being significantly higher in the shoots.

Novel reports of Penicillium spp. causing blue mold on onion and garlic in the Pacific Northwest U.S.A.

F. M. DUGAN (1), S. L. Lupien (2), C. Vahling-Armstrong (3), G. A. Chastagner (4), B. K. Schroeder (5)

(1) USDA ARS, Pullman, WA, U.S.A.; (2) USDA-ARS, Pullman, WA, U.S.A.; (3) USDA-ARS, Fort Pierce, FL, U.S.A.; (4) Washington State University, Puyallup, WA, U.S.A.; (5) University of Idaho, Moscow, ID, U.S.A.

Phytopathology 105(Suppl. 4):S4.38

Single isolates of Penicillium albocoremium, P. crustosum, P. expansum, $P$. glabrum, and $P$. paraherquei, identified by morphology and DNA betatubulin sequences, were inoculated to garlic (Allium sativum) and two varieties of onion (A. cepa, Gold Pearl and Forum) in a design of sample size $\mathrm{n}=3$ for each of 3 replications with identical mock-inoculated controls and a single repeat. Rates of lesion expansion were compared to controls. Pencillia were re-isolated from treatments but not mock-inoculated controls. Inoculations with $P$. polonicum served as positive controls. All isolates 
originated from bulb crops in Washington State, except for P. glabrum from an onion in Idaho. In paired T-tests or GLM pair-wise comparisons, $P$. albocoremium, $P$. crustosum, $P$. glabrum, and $P$. polonicum were pathogenic to both varieties of onion $(\mathrm{P} \leq 0.05)$. Penicillium albocoremium, $P$. glabrum, $P$. paraherquei and $P$. polonicum were pathogenic on garlic $(\mathrm{P} \leq 0.03)$. Tests failed to indicate consistent pathogenicity to garlic for $P$. crustosum and $P$. expansum. On onion, $P$. expansum and $P$. paraherquei were consistently pathogenic only on Gold Pearl $(\mathrm{P} \leq 0.04)$ or Forum $(\mathrm{P} \leq 0.03)$, respectively. Penicillium expansum has been reported on onion, anecdotally, in the Pacific Northwest and $P$. polonicum has also been previously reported there; otherwise these reports appear geographically novel.

Presence of Salmonella enterica in the alimentary canal of Macrosteles quadrilineatus, and role of $\mathrm{prgH}$ in persistence of the ingested bacteria J. P. DUNDORE-ARIAS (1), R. L. Groves (2), J. D. Barak (2)

(1) Univ of Wisconsin, Minneapolis, MN, U.S.A.; (2) Univ of Wisconsin, Madison, WI, U.S.A.

Phytopathology 105(Suppl. 4):S4.39

Phytophagous hemipterans can serve as vectors of Salmonella enterica, transmitting externally attached and ingested bacteria within or among plants. Previous studies have demonstrated that $S$. enterica can adhere to various structures of the exoskeleton and mouthparts of Macrosteles quadrilineatus, however, the fate of ingested $S$. enterica in their alimentary canal remain uncharacterized. To observe the presence and persistence of ingested bacteria, M. quadrilineatus were fed an artificial liquid diet inoculated with fluorescent-S. enterica for a $12 \mathrm{~h}$ acquisition access period (AAP), and then moved to two consecutive non-inoculated liquid diets for $48 \mathrm{~h}$ inoculation access period (IAP). Confocal imaging of dissected insects showed the presence of ingested $S$. enterica predominantly in the middle and posterior parts of the alimentary canal of $M$. quadrilinetus, and the persistence of the bacteria inside of the insect for up to $48 \mathrm{~h}$ IAP. We also investigated the presence and persistence of two $S$. enterica mutants lacking Type III Secretion System (T3SS) SPI-1 $(\mathrm{prgH})$ and SPI-2 (ssaK) genes. It was demonstrated that $\operatorname{prgH}$ is important for persistence of $S$. enterica inside of $M$. quadrilineatus, but lack of this gene does not affect the ability of the insect to excrete ingested bacteria. This study is the first to visualize the presence of $S$. enterica inside of a phytophagous insect, and demonstrate that SPI-1 T3SS influences the persistence in the gut of a potential vector.

Effects of secondary contamination of pepper seeds by Xanthomonas euvesicatoria on pathogen distribution, incubation period and seedling transmission

B. DUTTA (1), R. Gitaitis (2), S. Smith (2), D. Langston (3)

(1) University of Georgia, Tifton, GA, U.S.A.; (2) University of Georgia, Tifton, GA, U.S.A.; (3) Virginia Tech, Suffolk, VA, U.S.A.

Phytopathology 105(Suppl. 4):S4.39

The role of externally contaminated pepper seed with Xanthomonas euvesicatoria on secondary contamination of healthy seedlots and its significance on pathogen distribution, incubation period and seedling transmission of pathogen was investigated. In independent experiments, a contaminated seed was either retained or removed following thorough mixing with healthy seedlot ( $\mathrm{n}=100$ seeds/lot). Further, distribution of bacterial populations in individual seed within a seedlot was determined by dilution plating on a semi-selective medium. Additionally, replicates of similar treated lots were planted under greenhouse conditions. One hundred percent of the seedlots were contaminated with X. euvesicatoria, irrespective of the retention or removal of a contaminated seed. The distribution of bacterial populations in the seedlots, where a contaminated seed was either retained or removed, respectively followed inverse gaussian class. Seedlots where a contaminated seed was retained, developed symptoms in 12-21 days after planting (DAP) and seed-to seedling transmission ranged from $10-62 \%$. In contrast, seedlots where a contaminated seed was removed, seedlings developed symptoms in 36-42 DAP and seed-to-seedling transmission ranged from $8-64 \%$. These results suggest that secondary contamination of healthy seedlots can occur from an externally contaminated infested seed which may have implications on epidemiology of seedborne pathogen.

Adaptation and validation of Eprobe Diagnostic Nucleic Acid analysis for
screening viruses in metagenomic data using Universal virus microarray
probes M. DUTTA (1), A. Stobbe (2), W. Schneider (3), C. M. Malmstrom (4), U. K. Melcher (1)

(1) Oklahoma State University, Stillwater, OK, U.S.A.; (2) Pennsylvania State University, University Park, PA, U.S.A.; (3) USDA-ARS, FORT DETRICK, MD, U.S.A.; (4) Michigan State University, East Lansing, MI, U.S.A. Phytopathology 105(Suppl. 4):S4.39
Next generation sequencing (NGS) is becoming an increasingly common method in diagnostics but it suffers from certain disadvantages such as the large amount of time and computational power needed. To address these problems, Eprobe Diagnostic Nucleic acid Analysis (EDNA), a tool for rapid, effective identification and characterization of multiple pathogens was adopted and validated. In EDNA unassembled NGS datasets are queried using pathogen specific sequences, called electronic probes (eprobes) to enable detection of specific viruses or organisms in plant sample metagenomes. In this study, EDNA was tested with NGS datasets generated from soybean plants exhibiting symptoms of viral infection, herbarium plant samples infected with Barley yellow dwarf virus (BYDV) and plant samples from the Tall Grass Prairie Preserve (TGPP). Datasets available in Genbank SRA database served as blind control. The eprobes that were used were Universal Plant Virus Microarray (UPVM) probes and probes designed by the DeRisi laboratory for a microarray study named the DeRisi Viral Microarray (DVM) probes. In all queries the eprobes were able to detect the pathogen sequences present in the NGS unassembled datasets and yielded numbers of matches that were significantly different from those obtained using random decoy probes. This study supports the ability of EDNA for viral detection using UPVM and DMS eprobes for rapid response methodology and for detecting evidence of novel viruses.

\section{Detection, discrimination and discovery of a new Tobacco streak virus strain}

M. DUTTA (1), A. Ali (2), U. K. Melcher (1)

(1) Oklahoma State University, Stillwater, OK, U.S.A.; (2) Univ of Tulsa, Tulsa, OK, U.S.A.

Phytopathology 105(Suppl. 4):S4.39

Soybean plants that exhibited symptoms of virus infection were sampled from different counties of Oklahoma. These plants were tested serologically for 15 viruses known to infect soybean plants. Fifty-seven samples that exhibited typical virus-like symptoms did not test positive for any of the 15 viruses used in a dot-immunobinding assay (DIBA). Four samples were pooled and used for next generation sequencing using the 454- Roche protocol. Sequence and phylogenetic analysis of the sequences obtained revealed infection with a distinct strain of Tobacco streak virus (TSV). TSV was one of the 15 viruses initially tested for using DIBA and had tested negative. TSV belongs to the genus Ilarvirus and has been reported as a causal agent of bud blight in soybean crops in Brazil and the United States. Out of 10 reported primer pairs for TSV reverse transcription-polymerase chain reaction (RT-PCR), only two had the potential, based on sequence similarity, to amplify part of the genome of the distinct strain of TSV found in Oklahoma and only one was actually able to amplify the region. In this study, a new primer pair, specific to the Oklahoma strain (TSV-OK), was designed and end-point PCR and quantitative RT-PCR detection methods were developed and their sensitivity assayed. Twenty-three of the 57 DIBA soybean samples that initially tested negative were retested with the new specific end-point PCR method and found positive for TSV infection.

\section{Rhizoctonia solani suppressive soil from safflower field soil}

G. EBADZAD (1), S. Turner (2), S. D. Soby (3)

(1) Midwestern University, Glendale, AZ, U.S.A.; (2) Eastern Agricultural Research Center, Montana State University, Sidney, MT, U.S.A.; (3) Biomedical Sciences, College of Health Sciences and College of Veterinary Medicine, Midwestern University, Glendale, AZ, U.S.A.

Phytopathology 105(Suppl. 4):S4.39

Rhizoctonia solani causes damping-off of seedlings, and root and crown rot of older sugarbeet plants. Rhizoctonia-suppressive soils can occur in sugarbeets, and suppressiveness can be evaluated with high sensitivity using seeding bioassays. We detected Rhizoctonia suppressive soil using a greenhouse experiment as a first step in deciphering the potential of the rhizosphere microbiome for controlling root diseases. Candidate soil samples were collected from safflower and sugarbeet fields located in Sidney and Huntley, MT. Half of each soil sample was autoclaved to destroy microbial life. Autoclaved and non-autoclaved soil samples were artificially infested with barley seed inoculum containing $R$. solani anastomosis group 2-2IIB, then sown with sugarbeet seeds. The soil obtained from a safflower field in Sidney produced lower damping off rates at 21 days than soils from sugarbeet fields in either Sidney or Huntley, and than autoclaved soil. A likely explanation for this observation is that the safflower soil harbors active microbial antagonists against Rhizoctonia. This soil is suppressive to $R$. solani. It is essential to use an integrated approach to explore key groups of microbial communities involved in Rhizoctonia suppression, therefore soils with different levels of suppressiveness will be analyzed using denaturing gradient gel electrophoresis (DGGE) or pyrosequencing methods to identify antagonistic microorganisms involved in soil suppressiveness. 
Contribution of type III-secreted effectors to virulence and host preferential association of Acidovorax citrulli

N. Eckshtain-Levi (1), M. Gershovits (2), B. Zhao (3), G. Welbaum (3), T. Pupko (2), R. Walcott (4), S. BURDMAN (1)

(1) Hebrew University of Jerusalem, Rehovot, Israel; (2) Tel Aviv University, Tel Aviv, Israel; (3) Virginia Tech, Blacksburg, VA, U.S.A.; (4) University of Georgia, Athens, GA, U.S.A.

Phytopathology 105(Suppl. 4):S4.40

Acidovorax citrulli (Ac) causes bacterial fruit blotch (BFB) of cucurbits, a disease that represents a serious threat to melon and watermelon production worldwide. Despite the economic importance of BFB, there is a significant gap in the understanding of basic aspects of Ac-host interactions. Based on biochemical traits, two main groups of strains exist within Ac. The groups also differ in their association with cucurbits: while group I strains have been mainly isolated from melon and other non-watermelon cucurbits, most group II strains were isolated from watermelon. Ac requires a functional type III secretion system (T3SS) for pathogenicity. We hypothesize that differences in the repertoire of type III-secreted effectors (T3Es) between group I and II strains contribute to their distinguished host preferential association. Based on similarity to T3E genes of other phytopathogenic bacteria, we identified eleven putative T3E genes in the sequenced strain AAC00-1. The ORFs of these genes were sequenced from a set of 21 additional strains isolated from different geographic locations. Comparative analyses revealed that T3E genes cluster according to the group I/II classification. We are using marker exchange mutagenesis, virulence assays and heterologous expression to assess the role of individual effectors in virulence and host range determination in Ac. Bioinformatics approaches are also being employed to identify novel putative effectors.

Suppression of root diseases and enhancement of VA mycorrhizal population in sunflower and tomato by the mycorrhizospheric fluorescent Pseudomonas

S. EHTESHAMUL-HAQUE (1), S. S. Bokhari (2), S. A. Ali (3), V. Sultana (4), J. Ara (5)

(1) Department of Botany, University of Karachi, Karachi, Pakistan; (2) Department of Botany, University of Karachi, Karachi, Pakistan; (3) HEJ Research Institute of Chemistry, University of Karachi, Karachi, Pakistan; (4) Department of Biochemistry, University of Karachi, Karachi, Pakistan; (5) Department of Food Science \& Technology, University of Karachi, Karachi, Pakistan

Phytopathology 105(Suppl. 4):S4.40

The vesicular arbuscular mycorrhizal (VAM) fungi, are obligate symbionts, improved uptake of nutrients by plants especially phosphorus. Several investigations have indicated that mycorrhizosphere bacteria may affect VAM fungi and their host plant. In this study, efficacy of some isolates of mycorrhizospheric fluorescent Pseudomonas (MRFP) that have shown significant activity against root rotting fungi (Macrophomina phaseolina, Rhizoctonia solani, Fusarium solani, F. oxysporum) and root knot nematode (Meloidogyne javanica) in vitro was evaluated in pots and in field plot experiments using sunflower and tomato as test plants. Most of the test MRFP showed significant biocontrol potential against soilborne pathogens by reducing the infection of root rotting fungi and gall formation by root knot nematode. The application of mycorrhizospheric fluorescent Pseudomonas significantly increased VAM population around the roots and in most of the cases showed better uptake of phosphorus by the plants. However, mixed application of VAM and fluorescent Pseudomonas did not show any added advantage. MRFP caused pronounced effect on sunflower by producing taller plants with greater fresh shoot weight both in screen house and field plot experiments. Fruit weight of tomato and flower weight of sunflower were also found significantly higher in most of the bacterized treatments as compared to control plants.

Weather conditions conducive to infection of winter wheat by Puccinia striiformis sp. tritici race 'warrior'

M. EL JARROUDI (1), L. Kouadio (2), M. El Jarroudi (3), C. H. Bock (4), B. Tychon (5), J. Junk (6), P. Delfosse (6)

(1) Univ of Liege, Arlon, Belgium; (2) ICACS, University of Southern Queensland, Toowoomba, Australia; (3) LMA, FST Tanger, Université Abdelmalek Essaâdi, Tangier, Morocco; (4) USDA-ARS-SEFTNRL, Byron, GA, U.S.A.; (5) Department of Environmental Sciences and Management, Université de Liège, Arlon, Belgium; (6) Luxembourg Institute of Science and Technology (LIST), Belvaux, Luxembourg

Phytopathology 105(Suppl. 4):S4.40
Wheat stripe rust (WSR) (caused by Puccinia striiformis sp. tritici) i continues to be a major threat in most wheat growing regions of the world, with potential to inflict regular yield losses where susceptible cultivars are grown and when weather conditions are favourable. A recently isolated strain of $P$. striiformis $s p$. tritici, 'warrior', first identified in 2011 in Europe, is now virulent on adult plants of susceptible wheat cultivars throughout most wheat growing regions, including Luxembourg. Daily weather conditions were monitored and related to development of WSR during the 2012-2014 seasons in Luxembourg. Favourable weather conditions were determined by (i) analysing Dennis model outputs generated through a Monte Carlo method, and (ii) identifying the best correlation between the frequencies of weather condition classes and the area under the disease progress curve on the uppermost three leaves (L1, L2, and L3; L1 being the flag leaf). Our results showed that a combination of weather conditions, including relative humidity $>92 \%$ for $\geq 4$ hours and air temperatures of $4^{\circ} \mathrm{C}$ to $16^{\circ} \mathrm{C}$ for $\geq 36$ hours are necessary for WSR development, assuming inoculum is available. Furthermore, comparisons with reported WSR outbreaks in previous years showed that in recent years the disease is occurring at earlier stages in the growing season, suggesting a likely effect of climate change and/or climate variability.

Control of two decays of grape and strawberry during cold storage by continuous treatment with reactive oxygen species

M. T. Elkahky (1), J. A. BARTZ (2), M. Elsheshtawi (3), S. Elafifi (3), M. Elmazaty (3)

(1) University of Florida, Gainesville, FL, U.S.A.; (2) Univ of Florida, Gainesville, FL, U.S.A.; (3) Mansoura University, Mansoura, Egypt Phytopathology 105(Suppl. 4):S4.40

Continuous exposure of strawberries and table grapes to reactive oxygen species (ROS) emitted from the reaction chamber of an Ingersoll Rand Environmental Management System inhibited postharvest decays caused by Botrytis cinerea and Rhizopus stolonifer. Quantification of ROS $\left(\mathrm{O}_{3}\right.$ and $\mathrm{H}_{2} \mathrm{O}_{2}$ ) exiting the chamber was based on $\mathrm{O}_{3}$ measurement. Continuous exposure of fungal colonies on potato dextrose agar to ROS at $0.1 \mathrm{ppm} \mathrm{v} / \mathrm{v}$ ozone did not affect radial growth or fresh or dry weight of the colonies. However, sporulation and spore germination were inhibited. With freshly inoculated fruits stored at $5^{\circ} \mathrm{C}$, continuous ROS $\left(0.1 \mathrm{ppm} \mathrm{O} \mathrm{O}_{3}\right)$ and a chlorine dioxide oxidation control $(1 \mathrm{gm} / 2.3 \mathrm{~kg})$ treatments inhibited fungal outgrowth and sporulation as well as delayed lesion development. At 3 wks post treatment, strawberry fruit treated with ROS in cold storage were free of fungal nesting as well as visible mycelia of $B$. cinerea or $R$. stolonifer, whereas both the control and chlorine dioxide treated fruit had visible nesting. At 4 wks post-treatment, grapes similarly treated with ROS were free of visible pathogen structures, whereas both the chlorine dioxide and control treatments had signs of decay. Neither oxidative gas treatment caused phytotoxicity. Low levels of ROS continuously applied to strawberry and table grape fruits show promise for controlling postharvest fungal decays in cold storage rooms as well as marine transport containers.

Association of fungal effectors with pathogenicity of Fusarium oxysporum isolates on soybean

M. L. ELLIS (1), A. Lanubile (2), C. Garcia (1), G. P. Munkvold (3)

(1) California State University, Fresno, Fresno, CA, U.S.A.; (2) Università Cattolica del Sacro Cuore, Piacenza, Italy; (3) Iowa State Univ, Ames, IA, U.S.A.

Phytopathology 105(Suppl. 4):S4.40

Fungi within the Fusarium oxysporum species complex (FOSC) can cause root rot, seedling blight, and wilt of soybean. Isolates recovered from soybean vary in aggressiveness and symptoms produced. The goal of this study was to identify genetic markers to detect aggressive soybean wilt isolates. FOSC isolates from soybean and other hosts were screened for the presence of previously described fungal effectors and for their ability to produce wilt symptoms on soybean. Eighty isolates from soybean roots were screened for the fungal effectors Fmk1, Fowl, Pdal, PelA, PelD, Pepl, Prt1, Rhol, Sgel, Sixl, Six6, and Snfl. Additionally, 27 isolates from other hosts, primarily common bean, were screened for Six6. The 107 isolates used in this study were tested in the greenhouse for their ability to produce wilt symptoms using susceptible cultivars Jack and/or MN1805. Fmk1, Fowl, PelA, Rhol, Sgel, and Snfl were present in all isolates screened and phylogenies corresponded with previous clades based on the translation elongation factor $($ tefl $\alpha)$ gene and mitochodrial small subunit (mtSSU). Pdal, PelD, Pep1, Prt1, Sixl, and Six 6 were dispersed among isolates. Symptoms of vascular discoloration were observed with 42 isolates. All isolates with Six6 caused wilt symptoms, suggesting that this gene may be involved in the ability of some isolates to cause wilt in soybean. 
Botrytis cinerea grows symptomlessly in host plants: How?

C. J. EMMANUEL (1), J. A. van Kan (2), M. W. Shaw (3)

(1) Department of Botany, University of Jaffna, Jaffna, Sri Lanka; (2) Laboratory of Phytopathology, Wageningen University, Wageningen, Netherlands; (3) School of Agriculture, Policy and Development, University of Reading, Reading, United Kingdom

Phytopathology 105(Suppl. 4):S4.41

Botrytis cinerea also causes symptomless infection: the fungus has been isolated from the apparently healthy plants. We wished to understand the nature of this symptomless infection. A. thaliana and lettuce were inoculated with spore dust at rosette growth stage and four leaves stage respectively. Plants were sampled at different time interval and sections plated out on selective medium. The amount of fungal DNA and the relative mRNA concentrations of selected virulence genes were compared between symptomless and necrotic samples. Symptomless samples had 100 to 1000 times less $B$. cinerea DNA than necrotic samples. In both host species, the mRNA concentration of signalling genes $B c g l, B m p l$ and calcineurin, and the pathogenicity genes $B c s o d l$ and $B c p g l$ were similar or slightly greater in symptomless samples than in necrotic samples. The mRNA of signalling gene $B a c$ and pathogenicity genes Bcbot1 and Bcnepl, were not detected or detected in lower abundance in symptomless $A$. thaliana. In lettuce, the leaves developing distant from the site of inoculation showed similar results to $A$. thaliana, but in healthy leaves close to the site of inoculation mRNA concentrations of $B a c$ and Bcnepl were similar to necrotic samples. Thus, in both host species, the fungus grew along with the plant and moved to newly growing plant parts without producing symptoms; during this growth some pathogenicity genes were less expressed than in necrotic infection.

\section{Metagenome data mining for eukaryotic plant pathogens}

A. S. ESPINDOLA (1), W. L. Schneider (2), S. Marek (1), P. Hoyt (1), C. D. Garzon (1)

(1) Oklahoma State University, Stillwater, OK, U.S.A.; (2) USDA ARS, Frederick, MD, U.S.A.

Phytopathology 105(Suppl. 4):S4.41

Proper microorganism detection is crucial for diagnosing plant pathogens, developing and optimizing management strategies, and preventing the spread of inoculum. Multiplexed PCR can detect multiple eukaryotic pathogens simultaneously, but requires prior sequence information from the potential pathogens. To detect unexpected pathogens, we are developing the E-probe Diagnostic for Nucleic Acid (EDNA) bioinformatic tool which analyzes Next Generation Sequencing (NGS) data using pathogen-specific electronic probe databases. EDNA is applicable to metagenomic samples from many organisms. Four plant pathogens (Pythium ultimum, Phytophthora ramorum, Puccinia graminis and Phakopsora pachyrhizi) were inoculated onto susceptible hosts, and DNA from infected plants was extracted and sequenced using Roche 454 pyrosequencing. The objective of this study was to compare the pathogen detection efficiency of EDNA to MEGAN (MEtaGenome ANalyzer, traditional metagenome analysis software) for fungal-infected plant metagenomes. EDNA surpassed MEGAN in specificity when assigning taxons to the four eukaryotic pathogen metagenomes. The curated, pathogenspecific e-probe database used by EDNA likely improves the accuracy of taxa identification compared to the less curated databases (e.g. NCBI-nt) used by MEGAN. Current efforts focus on building a comprehensive e-probe database that will allow detection of most economically relevant plant pathogens in metagenomes.

\section{The draft genome of Sclerotinia minor}

A. S. ESPINDOLA (1), W. L. Schneider (2), H. Melouk (1), S. Marek (1), C. D. Garzon (1)

(1) Oklahoma State University, Stillwater, OK, U.S.A.; (2) USDA ARS, Frederick, MD, U.S.A.

Phytopathology 105(Suppl. 4):S4.41

Sclerotinia minor is a necrotrophic soilborne ascomycete with a wide host range. Sclerotinia minor rarely reproduces sexually. Asexual reproduction as sclerotia is common and considered clonal. The infection is initiated by mycelia from germinating sclerotia when environmental conditions are favorable. In this study, genomic DNA from S. minor isolate Sm120 mycelia grown on PDB was sequenced using Roche 454 pyrosequencing. Three sequencing runs generated a total of 423,186 quality reads. The draft genome was assembled using Newbler and the genome size was estimated at $43.4 \mathrm{Mb}$. The number of bases contributing to the genome assembly was $33.98 \mathrm{Mb}$, with an average contig size of 4,245 and an N50 of 6,378; and the largest contig size had 39,554 nt. The average sequencing depth was $6 \mathrm{x}$ with a peak depth of 850x. Genome completeness was assessed by using CEGMA and mapping the genome to 248 Core Eukaryotic Genes (CEG), with 218 complete proteins mapped onto the $S$. minor genome. Comparative genomics was performed by using the largest contig $(39,554)$ with the nearest taxonomical species genome (S. sclerotiorum) using Mummer (nucmer). Genome annotation is currently underway using a combination of ab initio gene predictions and RNA sequencing.

Worldwide population structure of Sclerotinia sclerotiorum from cultivated common bean

S. E. EVERHART (1), R. Jhala (1), B. S. Amaradasa (2), R. Higgins (1), J. R. Steadman (1)

(1) University of Nebraska, Lincoln, NE, U.S.A.; (2) University of NebraskaLincoln, Lincoln, NE, U.S.A.

Phytopathology 105(Suppl. 4):S4.41

Characterization of pathogen population structure can yield important insight into pathogen biology and epidemiology. A worldwide collection of $366 S$. sclerotiorum isolates from 19 dry and snap bean cultivars in Australia, France, Mexico, and 10 bean production states in the U.S. collected from 2003 to 2012 were genotyped at 16 microsatellite loci. A total of 165 multilocus genotypes were identified using a subset of 11 loci that followed an expected repeat motif. Discriminate analysis of principal components using clone corrected data showed state/country of origin was the best separator (AMOVA $p=0.037$ ). The Mexican population was highly divergent, showing closest association with the California population. There was no significant difference ( $p=0.057$ ) between populations once the Mexican population was removed. Despite overall genetic linkage, none was observed for populations from Washington in 2008, Oregon in 2003, California in 2004, Nebraska in 2005, Tasmania in 2004, or France in 2004 and 2005. These preliminary results suggest that despite large physical separation, divergence of populations is low, which is not surprising given the low rate of homothallic recombination thought to occur. Additional analyses are underway to investigate population structure within individual sites from year-to-year and association of genotypes with aggressiveness and fungicide sensitivity.

Pathogen response to altering levels of soil fertility in soybean fields across Ohio

M. EYRE (1), C. Martin (2), S. Culman (2), A. Dorrance (2)

(1) The Ohio State University, Westlake, OH, U.S.A.; (2) The Ohio State University, Wooster, OH, U.S.A.

Phytopathology 105(Suppl. 4):S4.41

Soil fertility has the potential to directly affect soybean productivity and profitability. Growers must balance fertilizer inputs to maximize yield while minimizing cost and environmental impacts. Despite its importance, fertility recommendations for soybean in Ohio are 20 years old. In an effort to evaluate these recommendations and fertility needs of modern cultivars, a more holistic understanding of how soil fertility may or may not influence soybean pathogens is also necessary. Therefore, the objective in this ongoing study is to evaluate the impact of higher rates of $\mathrm{P}$ and $\mathrm{K}$ on soil borne pathogens and soybean cyst nematode populations. On three Ohio farms in 2014, P, K, and P+K treatments were added to plots at planting. Treatments were arranged in a randomized complete block design and the same cultivar was used at all sites. Final stand counts, leaf area affected by foliar pathogens, disease severity of Sudden Death Syndrome (SDS), and yield were collected. At 1 of the 3 locations, SDS severity was significantly reduced $(\mathrm{p}<0.05)$ by the addition of both $\mathrm{K}$ and $\mathrm{P}$ separately, but not $\mathrm{P}+\mathrm{K}$ applied together. At this same location, plots treated with $\mathrm{P}$ had a significantly higher yield. Preliminary results suggest an interaction between soil fertility and soil borne pathogens may play an important role in disease development.

Influences of temperature and host on atoxigenic Aspergillus flavus isolates

P. FALKENBERG (1), P. J. Cotty (1)

(1) United States Department of Agriculture - ARS, Tucson, AZ, U.S.A.

Phytopathology 105(Suppl. 4):S4.41

The extent to which crops become contaminated with aflatoxins depends on the composition of Aspergillus populations. In the current study, influences of temperature on production of conidia by A. flavus on Czapek agar, maize, soybean, and peanuts were quantified. Three isolates of A. flavus, one aflatoxin producer and two atoxigenic isolates, were examined. Media composition influenced the temperature of maximum sporulation. However, during individual growth both atoxigenics produced more conidia at $35 \mathrm{C}$ than at $25 \mathrm{C}$ on all three crops. More aflatoxins were produced on maize at $35 \mathrm{C}$ than $25 \mathrm{C}$. The atoxigenic isolates reduced contamination at both temperatures but the greatest reductions occurred at $25 \mathrm{C}$. Results suggest increased production of conidia during host invasion is not associated with increased reductions in contamination during co-infection of hosts. However, conidia production is likely a key characteristic dictating successful displacement of aflatoxin producers during production of commercial crops. Hosts 
differentially influenced growth and sporulation of isolates, suggesting isolates have some level of host specialization. Host adaptation may be an important factor influencing compositions of A. flavus populations in agroecosystems and therefore should be taken into consideration when designing biocontrol products.

Fitness and competitive ability of Alternaria alternata field isolates with resistance to SDHI, QoI, and MBC fungicides

Z. FAN (1), G. Schnabel (2)

(1) Clemson University, Clemson, SC, U.S.A.

Phytopathology 105(Suppl. 4):S4.42

Alternaria rot, caused by Alternaria alternata, was observed in well managed, commercial peach orchards in South Carolina. In total, 64 isolates were collected in 2013 and 2012 from fruit of problem orchards. Most isolates were resistant to boscalid, pyraclostrobin, and thiophanate-methyl. Resistance to boscalid was due to point mutations in the SDH subunits, resistance to pyraclostrobin was due to the G143A mutation in the cytb gene, and resistance to thiophanate-methyl was due to $167 \mathrm{Y}$ in the $\beta$-tubulin gene. The four most commonly found genotypes based on mutations in the SDH subunits $(\mathrm{H} 277 \mathrm{Y}$ in $s d h \mathrm{~B}, \mathrm{H} 134 \mathrm{R}$ in $s d h \mathrm{C}, \mathrm{D} 123 \mathrm{E}$ in $s d h \mathrm{D})$ were selected for fitness evaluations. Genotypes H277Y and H134R didn't suffer fitness penalties based on mycelia growth on PDA, spore production in vitro, osmotic sensitivity, oxidative sensitivity, germination ability, and ability to cause disease. Hypersensitivity to oxidative stress and weak sporulation capacity was observed in genotype D123E. No competitive advantage was detected for sensitive isolates over the course of five generations when mixed with genotypes H277Y or H134R. The results suggest that in the absence of fungicide pressure, isolates with resistance to the three active ingredients may still effectively compete with the wildtype population.

Western Region IR-4: Protecting specialty crops, practicing IPM, promoting global trade

J. FARRAR (1), R. Hirnyck (2), S. O’Neal (3), R. Sisco (4)

(1) Western IPM Center, Davis, CA, U.S.A.; (2) Integrated Pest Management Center, University of Idaho, Boise, ID, U.S.A.; (3) Irrigated Agriculture Research and Extension Center, Washington State University, Prosser, WA, U.S.A.; (4) Western Region IR-4 Center, Davis, CA, U.S.A.

Phytopathology 105(Suppl. 4):S4.42

The purpose of the Interregional Research \#4 (IR-4) Program is to insure that producers of specialty crops and ornamental plants have adequate tools to conduct sound, sustainable integrated pest management (IPM). The Western Region IR-4 Program oversees field trials, laboratory analyses, and quality assurance protocols that result in the registration of crop protection tools for specialty crops in Alaska, Arizona, California, Colorado, Hawaii, Idaho, Montana, Nevada, New Mexico, Oregon, Utah, Washington, and Wyoming. This results in production of a greater variety of crops and sets the stage for Western agricultural producers to compete successfully in the global marketplace by practicing IPM. IR-4's evolving mission includes consideration of international maximum residue levels (MRLs) in support of U.S. exports, attention to invasive pests, service to organic producers, and an increased focus on biopesticide products. With support from our partners at the Western IPM Center, USDA-ARS, USDA-NIFA, US EPA, the crop protection industry, and the land grant universities of the West, the Western Region IR-4 program serves specialty crop producers and food processors in the West, enabling them to serve food consumers of the U.S. and the world.

\section{Adoption of IPM practices for disease management in the Western U.S \\ J. FARRAR (1), M. Baur (1), S. Elliott (1) \\ (1) Western IPM Center, Davis, CA, U.S.A. \\ Phytopathology 105(Suppl. 4):S4.42}

Integrated Pest Management, or IPM, is a scientific approach to pest management that integrates biological, cultural, mechanical and chemical options to control pest problems. When applied specifically to plant disease management, IPM is sometimes termed Integrated Disease Management, or IDM. The goal of IPM is to reduce risks to people and the environment by using pest biology, environmental information and all available technology to reduce pest damage to acceptable levels by the most economical means. Adoption of IPM practices for disease management in agriculture crops in the thirteen Western states was assessed through a survey of peer-reviewed, federal and state government, Extension service and consulting service publications. The data show that many disease prevention, avoidance, monitoring and suppression techniques are broadly adopted in the West and can be considered conventional disease management. For example, equipment sanitation, crop debris management, crop rotation, use of diagnostic laboratory services, scouting for diseases, use of weather data to assist pest management decisions and rotation of pesticide modes of action are employed on more than
$50 \%$ of vegetable crop acreage in Arizona, California, Colorado, Oregon and Washington.

Biological and molecular characterization of Bean common mosaic virus isolates from different pathogenicity groups

X. FENG (1), J. R. Myers (2), A. V. Karasev (3)

(1) University of Idaho, Moscow, ID, U.S.A.; (2) Oregon State University, Corvallis, OR, U.S.A.; (3) Univ of Idaho, Moscow, ID, U.S.A.

Phytopathology 105(Suppl. 4):S4.42

Bean common mosaic virus (BCMV) exists as a complex of strains that can be distinguished biologically, on a set of bean differentials, into pathogenicity groups (PG) numbered I to VII. In order to establish molecular signatures characteristic of different pathogenicity groups of BCMV, we performed a complete biological and partial molecular study of 12 field BCMV isolates collected in Oregon, and also biological and molecular characterization of two control isolates from PG-I (US1) and PG-V (NY15P). All but one field isolates were found to have B-serotype, with four of them belonging to PG-I, five to PG-III, two to PG-IV, and one to a new PG-VIII. One isolate had Aserotype and was classified as Bean common mosaic necrosis virus. Partial sequences of the HC-Pro region were determined for eight out of 12 field isolates of BCMV, and no correlation was found between the PG assignment of a BCMV isolate and the HC-Pro sequence similarities. The complete genomes of US1 and NY15P isolates were sequenced and found closest to the BCMV strain NL1 (PG-I, 94\% identity to US1 and 97\% identity to NY15P). Sequence analysis revealed that strain US1 might be a product of recombination between strains US10 and NY15P. The data obtained suggest that the comparative genomics of BCMV isolates may be useful to identify genetic determinants of BCMV pathogenicity in common bean.

Insights of the genome of spinach downy mildew pathogen Peronospora farinosa f. sp. spinaciae

C. FENG (1), B. Bluhm (2), K. Lamour (3), J. Correll (2)

(1) Univ of Arkansas, Fayetteville, AR, U.S.A.; (2) University of Arkansas, Fayetteville, AR, U.S.A.; (3) University of Tennessee, Knoxville, TN, U.S.A. Phytopathology 105(Suppl. 4):S4.42

Downy mildew disease, caused by the obligate pathogen Peronospora farinosa f. sp. spinaciae (Pfs), is the most economically important disease of spinach. The fast emergence of new races of Pfs continue to be a threat to the spinach industry. Fifteen races of Pfs have been identified, 12 of which were found in the past 25 years. The emergence of new races in recent years may be the result of a more favorable environment for the pathogen in the current spinach production system, the selection pressure of modern resistant hybrids, and either point mutations or sexual reproduction of the pathogen. Sequencing the genome of this pathogen could provide valuable insights into the race diversity of the pathogen as well as allow for the development of molecular markers that can be used to genotype strains of the pathogen to evaluate population diversity of Pfs. The genomes of three new races of Pfs, race 12 (UA2209), race 13 (UA0510C), and race 14 (UA4410) have been sequenced using the Illumina HiSeq 2000 platform. The raw sequence data was assembled into contigs for sequence totals of 142,427 , and $268 \mathrm{Mb}$ for races 12,13 , and 14 , respectively. Virulence factors, secreted effector peptides and sporulation proteins were identified among the sequences. Thousands of simple sequence repeats (SSRs) and single nucleotide polymorphisms (SNPs) were found in the three sequenced genomes of Pfs, which can be used for comparing genetic diversity within and between races and for analyzing the global population structure of this important pathogen.

Characterization of a sunflower (Helianthus annuus L.) germplasm collection for resistance to Phomopsis stem canker and adaptation in South Dakota

J. Feng (1), K. Grady (1), X. Gu (1), F. MATHEW (1)

(1) South Dakota State University, Brookings, SD, U.S.A.

Phytopathology 105(Suppl. 4):S4.42

South Dakota leads the United States in sunflower (Helianthus annuus L.) production. According to Kandel (2010-2014), the most yield-limiting factor in sunflower production was uneven plant stand among many others including disease or insect related issues, lodging and drought. This is likely because seed emergence and vigor can be affected by the type of sunflower varieties and seed biology in a range of field conditions. The aim of this project was to evaluate seed vigor from diverse sunflower collections and integrate the major genes underlying seed vigor into the local cultivars to improve early seedling stand under optimal and adverse conditions. Ten plants of 288 plant introduction lines (USDA North Central Regional Plant Introduction Station, Ames, Iowa) representing nearly $90 \%$ of the allelic diversity present within the cultivated sunflower gene pool were planted in a field trial arranged in a completely randomized design. A number of agriculturally important traits 
were recorded for local adaptation such as flowering time, plant architecture (i.e., branching), and resistance to Phomopsis stem canker. Self-pollinated seeds were harvested after physiological maturity for germination test. Preliminary results suggest that RHA367, HA1, HA383, and HA433 may be considered as resources for sunflower breeding given that they have early flowering time, low temperature germinability $\left(12^{\circ} \mathrm{C}\right)$ and resistance to Phomopsis stem canker.

Protein expression profiling of Rathayibacter toxicus using mass spectrometry

C. M. FENNESSEY (1), J. Blanc (1), A. E. Sechler (1), J. G. King (1), M. McMahon (1), W. Garrett (2), D. G. Luster (1), W. L. Schneider (1)

(1) USDA ARS, Frederick, MD, U.S.A.; (2) USDA ARS, Beltsville, MD, U.S.A.

Phytopathology 105(Suppl. 4):S4.43

Annual ryegrass toxicity (ARGT) is a frequently fatal disease that primarily affects livestock in southern and western Australia. The causative agent of the disease is Rathayibacter toxicus, a bacterium that often contaminates the indigenous grasses used for grazing and hay-making. $R$. toxicus produces a tunicamycin-like toxin under certain conditions, causing extreme hepatic and neurological damage in livestock that consume contaminated grass. Despite the significant toll exacted on infected livestock and its ever-increasing geographical prevalence, little is known about this pathogen. Mass spectrometry (MS) proteomic analysis was performed on bacterial lysates and supernatants under various growth conditions in an effort to elucidate protein expression patterns. This approach provides insight into the basic expression profile of Rathayibacter under benign vs. toxin-producing conditions, as well as facilitating the identification of proteins which may be utilized to generate diagnostic assays, allowing for rapid detection of $R$. toxicus prior to livestock ingestion. The initial MS analysis of cultured R. toxicus identified 290 expressed proteins, representing the typical biological categories, with the largest number of proteins related to protein synthesis. Bioinformatic analysis identified 9 unique proteins that were viable candidates for antibody production. These results are a significant first step toward a comprehensive evaluation of the $R$. toxicus proteome.

Metagenomic analysis of the sugarcane virome in Florida reveals prevalent and potential novel viruses

E. Fernandez (1), D. Filloux (1), J. C. Comstock (2), P. Roumagnac (1), P. ROTT (3)

(1) Centre de coopération internationale en recherche agronomique pour le développement (CIRAD), Montpellier, France; (2) United States Department of Agriculture, Agricultural Research Service, Canal Point, FL, U.S.A.; (3) University of Florida, Belle Glade, FL, U.S.A.

Phytopathology 105(Suppl. 4):S4.43

Viral metagenomics has revolutionized the way pathologists decipher viral diseases, and has already produced key advances in viral ecology. It has also the potential to become a central approach for viral surveillance at the ecosystem scale. A viral metagenomics study of the sugarcane virome in Florida was carried out in 2013/2014 to analyze occurence of known and potentially new viruses. One hundred and eighty sugarcane leaf samples were collected from different commercial sugarcane (Saccharum interspecific hybrids) fields in Florida and from other Saccharum and related species taken from two local germplasm collections. Virion-associated nucleic acids (VANA) metagenomics was used for detection and identification of viruses present within the collected leaf samples. All four previously reported sugarcane viruses occuring in Florida were detected: Sugarcane yellow leaf virus (149 infected samples out of 180), Sugarcane mosaic virus $(2 / 180)$, Sugarcane mild mosaic virus (10/180) and Sugarcane bacilliform virus (51/180). Interestingly, this viral metagenomics approach also resulted in the detection of potential new viruses of sugarcane, including Chrysovirus, Mastrevirus, and Umbravirus. This study provided a snapshot of the prevalence and genetic diversity of Sugarcane yellow leaf virus in 2013/2014 in Florida where at least two genotypes of this virus (BRA and CUB) are present. Impact of this diversity on sugarcane production needs to be investigated.

Temperature effects on lesion development and on R-genes when infected by Leptosphaeria maculans causing blackleg disease of canola

D. FERNANDO (1), L. Rong (2), H. Borhan (3)

(1) Univ of Manitoba, Winnipeg, MB, Canada; (2) Uni of Manitoba, Winnipeg, MB, Canada; (3) Agriculture and Agri-Food Canada, Saskatoon, SK, Canada

Phytopathology 105(Suppl. 4):S4.43

Blackleg caused by Leptosphaeria maculans is the most serious canola disease worldwide. To determine whether resistance genes specific for $L$. maculans in Brassica napus cultivars are temperature sensitive and whether the resistance would be overcome at higher temperatures, inoculations were carried using $L$. maculans pycnidiospores on 7-d old cotyledons of 4 B. napus isogenic lines of cultivar Topaz carrying single R-genes against blackleg (Topaz Rlm1, Topaz Rlm2, Topaz Rlm3 and Topaz Rlm4). The plants were grown under 4 temperature regimes $\left(15 / 10,20 / 15,25 / 20\right.$ and $\left.30 / 25^{\circ} \mathrm{C}\right)$. The lesion size was measured and resistance was rated on the $10^{\text {th }} \mathrm{dpi}$. Results across the 4 cultivars showed that there was a significant effect of lesion size for each of the isogenic lines $(P<0.05)$ and temperature regimes $(P<0.05)$. The lesion size increased as the temperature increased, where Topaz R $1 \mathrm{~m} 4$ illustrated the largest lesion followed by Topaz Rlm1, Topaz Rlm2, and Topaz Rlm3. There were no pycnidiospores produced on the lesions 10dpi, indicating that there was no resistance breakdown in any of the Brassica napus isolines at any temperature treatment. Lesion size is highly dependent on temperature; however, the influence of temperature on Rlm mediated resistance appears to depend on the specific cultivar, as we did not find similar temperature sensitivities for Rlm mediated resistance as was shown for DarmorMX and Quinta cultivars (Huang et al. 2006).

A comparative analysis of sequence variations in the TRIS gene among different species in the Fusarium graminearum species complex

D. FERNANDO (1), C. Amarasinghe (2)

(1) Univ of Manitoba, Winnipeg, MB, Canada; (2) Uni of Manitoba, Winnipeg, MB, Canada

Phytopathology 105(Suppl. 4):S4.43

Species within the Fusarium graminearum species complex (FGSC) are the dominant and most widespread pathogen causing Fusarium Head Blight (FHB) in the world. Trichothecene chemotype variation is one of the key factors that used to analyse the populations of Fusarium species that cause FHB. A better understanding of the DNA sequence variation in trichothecenes biosynthesis genes and quantitative variation of pathogenicity and DON production in FGSC is important to predict the Fusarium population dynamics. It has been reported that the differential activity of TRI8 gene is a key determinant of the $3 \mathrm{ADON}$ and 15ADON chemotypes in Fusarium. Therefore in this study we have analysed the sequence variation of TRI8 gene in different species in the FGSC. The Bayesian phylogenetic tree represented 3 monophyletic clades each representing 3ADON, 15ADON and NIV trichothecene chemotypes. TRI8 haplotypes did group according to chemotype rather than by species, indicating that NIV, 3ADON and 15ADON chemotypes have a single evolutionary origins. Also the unique DNA sequence polymorphisms in TRI8 gene suggests the potential use of TRI8 gene as a marker to identify chemotypes within the FGSC. All examined species in the FGSC showed considerable disease in spikelets both on highly susceptible (HS) and resistant (R) wheat cultivars. The highest mean disease severity was shown by $F$. graminearum s.s. 3ADON strains and $F$. asiaticum 3ADON strains both on HS and R cultivars.

Management of root rot caused by Phytopythium helicoides on pistachio rootstock

E. J. Fichtner (1), G. T. Browne (2), C. L. BLOMQUIST (3)

(1) Univ of California Coop Ext, Visalia, CA, U.S.A.; (2) USDA ARS, Davis, CA, U.S.A.; (3) Plant Pest Diagnostics Center, California Department of Food and Agriculture, Sacramento, CA, U.S.A.

Phytopathology 105(Suppl. 4):S4.43

Phytopythium helicoides has recently been associated with root rot of pistachio in California. Currently, there are no recommended strategies for management of the pathogen on pistachio. Objectives of this study include: i) determining the influence of soil saturation on disease severity, and ii) assessing the potential for foliar applications of Ascophyllum nodosum extract (Acadian Sea Plants) and phosphite (Fungi-Phite) to mitigate disease symptoms. Potted pistachio rootstocks of clonally propagated UCB-1 received a foliar spray application of $2.5 \%$ Fungi-phite 7 days prior to soil infestation, and $0.8 \%$ Acadian $24 \mathrm{~h}$ prior to soil infestation. Soil was infested with V8/vermiculite/oat medium colonized with $P$. helicoides; uncolonized medium was incorporated in soil of control plants. Plants were submerged in water for $6 \mathrm{~h}$ to stimulate zoospore release. Wilting and mortality was observed within 7 days of soil infestation; plants in uninfested soil remained asymptomatic. Plants treated with either Acadian or Fungi-Phite exhibited higher root mass and survival rates than non-sprayed plants; however, the pathogen was isolated from surface-sterilized roots of both Acadian- and Fungi-Phite-treated plants. Saturation was not required for disease development, but enhanced disease severity. The results suggest the benefit of irrigation management and foliar application of Acadian or Fungi-phite for reducing root rot disease on pistachio caused by P. helicoides. 
Developing a risk assessment model for wheat blast in the US based on weather conditions that favor rice blast epidemics

T. FISCHER (1), E. D. De Wolf (1)

(1) Kansas State University, Manhattan, KS, U.S.A.

Phytopathology 105(Suppl. 4):S4.44

Wheat blast (Magnaporthe oryzae Triticum) has emerged as a serious problem for wheat production in South America. To prepare for the possible introduction of wheat blast in the US, it would be helpful to identify areas of the country most at risk for blast epidemics. Therefore, the objectives of this research are to identify environmental conditions that could be conducive for wheat blast epidemics by examining historical epidemics of rice blast and to develop predictive models for rice blast in the US. The dataset used in this analysis consisted of 60 site-years of historical observations of rice blast levels from Arkansas, Louisiana and Texas. These observations were coupled with monthly summaries of hourly weather variables based on temperature, relative humidity, precipitation, and regional moisture indices. Recursive partitioning was used to identify variables associated with rice blast epidemics. The results indicate that rice blast epidemics were favored by cooler April temperatures and above normal precipitation in June. Preliminary models for rice blast based on these variables were able to correctly classify epidemic years with $>75 \%$ accuracy. The results of this project will be used as part of a risk assessment for wheat blast and a rice blast forecast system in the Southern US.

Identification of a previously uncharacterized global regulator in Pseudomonas syringae pv. tomato DC3000

M. FISHMAN (1), J. Zhang (1), P. A. Bronstein (2), P. Stoghill (3), M. J. Filiatrault (3)

(1) Cornell University, Ithaca, NY, U.S.A.; (2) USDA FSIS, Washington, DC, U.S.A.; (3) USDA ARS, Ithaca, NY, U.S.A.

Phytopathology 105(Suppl. 4):S4.44

Pseudomonas syringae pv. tomato DC3000 (Pst) is used as a model system to understand the dynamics behind molecular plant-microbe interactions. Identification of conserved genes necessary for survival of bacterial plant pathogens in the apoplast could lead to new management methods. We have identified a conserved two-component system (TCS) in Pst, PSPTO 3380 and PSPTO_3381 (3380/3381), that is involved in virulence on tomato. $\bar{\Delta} 3380$ and $\Delta 3381 \bar{P}$ st strains still caused a hypersensitive response on tobacco, therefore they produce the type three secretion system. TCSs are signaling relays bacteria use to sense changes in their environment and adapt accordingly. To determine the environmental stimuli of 3380/3381, we designed a reporter gene construct and found that $3380 / 3381$ is stimulated by $\mathrm{Ca}^{2+}, \mathrm{Fe}^{2+}, \mathrm{Zn}^{2+}$, and $\mathrm{Cd}^{2+} . \mathrm{Ca}^{2+}$ was also found to inhibit swarming motility and cause abnormal exopolysaccharide and calcium precipitation in $\Delta 3380$ and $\Delta 3381$ strains, but not wild-type Pst. Due to the phenotypic changes associated with $\mathrm{Ca}^{2+}$, we performed an RNA-seq experiment comparing WT Pst to $\Delta 3380$ and $\Delta 3381$ Pst grown in the presence of $\mathrm{Ca}^{2+}$. Analysis of the RNA-seq data showed differential expression of alginate and Psl biosynthesis genes, and genes related to motility, carbohydrate utilization, and lipopolysaccharide production. This research shows that $3380 / 3381$ is an important global regulator involved in the regulation of several virulence related phenotypes. Liberibacter and psyllid derived proteins bind Candidatus $\mathrm{L}$. asiaticus
bacteriophage late gene promoter

L. A. FLEITES (1), M. Jain (1), D. W. Gabriel (1)

(1) University of Florida, Gainesville, FL, U.S.A.

Phytopathology 105(Suppl. 4):S4.44

The majority of Candidatus Liberibacter asiaticus (Las) strains described carry bacteriophage similar to SC1 and SC2 of Las UF506. The SC1 lytic cycle, marked by upregulation of several late genes including a functional holin (SC1 gp110) and an active peroxidase, is activated in planta, but not in infected psyllids. The holin promoter was strongly active in $L$. crescens (Lcr), a culturable proxy for Las, but nearly silent in E. coli. Activity of the promoter in Lcr was suppressed by aqueous extracts from psyllids applied outside of the cells, indicating cell penetration and also potentially explaining why Las has not been cultured (Fleites et al. 2014. AEM 80:6023). The suppressor activity was sensitive to heat and proteinase treatment, indicating direct repression by a protein, and size fractionation demonstrated the the size was $10-50 \mathrm{kDa}$. To further explore promoter activation in L. crescens and suppression by psyllid extracts, electrophoretic mobility shift assays were performed. The mobility of the holin promoter fragment was decreased in the presence of both the psyllid and Lcr extracts, and was outcompeted by analogous unlabeled promoter DNA, indicating sequence-specific DNA binding. Using small, overlapping holin promoter fragments as competitor DNA, the binding sites of the proteins were further delineated. The DNA-binding proteins were purified by DNA affinity capture. Identification of the repressor and activator proteins and target sequences is underway.

Red light stimulates apothecial development of Monilinia vacciniicorymbosi

J. FLORENCE (1), J. W. Pscheidt (1)

(1) Oregon State University, Corvallis, OR, U.S.A.

Phytopathology 105(Suppl. 4):S4.44

Pseudosclerotia of Monilinia vaccinii-corymbosi, the fungus that causes mummy berry disease of blueberry, overwinter on the soil surface producing apothecia in early spring. Although light is necessary, it is unknown what quality or intensity is needed for apothecium development. Pseudosclerotia, collected from beneath bushes of cv. 'Berkeley' in Corvallis, OR, were placed on $10 \mathrm{~g}$ of sterile sand inside black painted polyethylene scintillator vials and watered. Vials were capped with a clear, red, or blue light filter, placed into one of two photosynthetrons in a completely randomized design, and incubated at $9-10^{\circ} \mathrm{C}$. Photosynthetrons were positioned $60 \mathrm{~cm}$ from two compact fluorescent bulbs on a 12-hr photoperiod with $2.1 \mu \mathrm{mol} / \mathrm{m}^{2} / \mathrm{s}$ intensity. Pseudosclerotia were monitored for 14 days for apothecia development. The mean proportion of apothecia for the blue filtered light treatment, averaged across 3 trials, was 0.27 (standard deviation $[\mathrm{SD}]=0.46$ ), while the mean proportion of apothecia for clear and red filtered light were $0.45(\mathrm{SD}=0.51)$ and $0.41(\mathrm{SD}=0.50)$, respectively. The blue filtered light treatment had the lowest mean but was not statically significant. An increase in sample size by combining the results from 2014 and current 2015 trials may provide enough power to detect a significant difference. Results may help determine depth of mulch used to inhibit apothecial emergence.

Interactions between Fusarium virguliforme and other common fungal pathogens in soybean root rot complexes

C. M. Floyd (1), D. M. MALVICK (2)

(1) University of Minnesota, St. Paul, MN, U.S.A.; (2) University of Minnesota, St. Paul, MN, U.S.A.

Phytopathology 105(Suppl. 4):S4.44

Many species of Fusarium and other root-rotting fungi commonly infect soybean roots with and without visible symptoms of infection. This includes $F$. virguliforme $(\mathrm{FV})$, the cause of sudden death syndrome (SDS). Roots of soybean plants with SDS symptoms in MN typically also contain other fungal pathogens. Little is known about interactions between fungal species in soybean roots. The objective of this study was to determine how soybean growth and SDS symptoms are affected by co-inoculations with FV and $F$. oxysporum (FO), F. solani (FS), and Clonostachys rosea (CR). Greenhouse trials were conducted with soybean cvs. susceptible and partially resistant to SDS. FV and one of the other fungi were mixed at equal ratios into potting soil and seeds were planted. Foliar SDS severity, root rot, and biomass were assessed $\sim 30$ days after planting. Across two trials of co-inoculations with FV and FO or FS, root rot and foliar symptoms were significantly $(\alpha=0.05)$ reduced and biomass significantly increased for one or more of the pairings, compared to $\mathrm{FV}$ alone (varieties pooled). In one of two $\mathrm{FV}+\mathrm{CR}$ trials, root rot was significantly $(\alpha=0.05)$ lower with both pathogens compared to FV alone (varieties pooled). In other FV co-inoculation studies with Fusarium or Clonostachys, no significant treatment differences were detected. These studies suggest that significant interactions can occur between root-infecting fungi and they indicate challenges of working with interactions.

Erysiphe australiana causing powdery mildew on Lagerstroemia speciosa in Brazil

N. R. FONSECA (1), L. M. Guimaraes (1), R. P. Pires (1), A. C. Alfenas (1)

(1) Universidade Federal de Vicosa, Vicosa, Brazil

Phytopathology 105(Suppl. 4):S4.44

Powdery mildew symptoms and signs were observed on seedlings of Lagerstroemia speciosa 'Resedá gigante' in an ornamental plant nursery in Viçosa, MG, Brazil during September, 2014. Signs were most prominent as intense mycelial growth and sporulation on the shoots, new branches and leaves, which led to tissue necrosis. Morphological and phylogenetic analyses of the regions (ITS and 28S rRNA) were performed to identity the pathogen. Total cellular DNA was extracted from fungal mycelia and conidia by the Chelex method. The sequences obtained were aligned with homologous sequences from a powdery mildew pathogens of other $L$. indica collected in Viçosa and other sequences obtained from GenBank. Hyphae were septate, branched and hyaline; conidiophores were epiphytic, $51.7-64 \times 6.6-8 \mu \mathrm{m}$, septate, hyaline; foot-cells were cylindrical, $26-39 \times 6.6-8 \mu \mathrm{m}$; conidia were produced singly at the apex of the conidiophores, cylindrical, doliiform or 
ellipsoid, $24.5-35.5 \times 10-13 \mu \mathrm{m}$, aseptate, hyaline. The teleomorph was absent. The anamorph morphology was identical to that described for Erysiphe australiana, a common powdery mildew species infecting Lagerstroemia spp. worldwide. Through phylogenetic analyses, the powdery mildew pathogen isolated from $L$. speciosa and $L$. indica were grouped with E. australiana, confirming the morphological identification. To our knowledge, this is the first report of $E$. australiana causing powdery mildew on L. speciosa in Brazil.

\section{Resistance in strawberry isolates of Colletotrichum acutatum from Florida} to Quinone-outside inhibitor fungicides

B. B. FORCELINI (1), N. A. Peres (2), A. Amiri (2), T. E. Seijo (2)

(1) Univ of Florida, Wimauma, FL, U.S.A.; (2) University of Florida, Wimauma, FL, U.S.A.

Phytopathology 105(Suppl. 4):S4.45

Anthracnose Fruit Rot (AFR) of strawberry, caused by Colletotrichum acutatum, is a major disease in Florida and frequent fungicide applications are needed for disease control. Quinone outside inhibitor (QoI) fungicides such as azoxystrobin and pyraclostrobin are commonly used to control AFR. From 1994 to 2014, 177 C. acutatum isolates were collected from multiple strawberry fields in Florida with or without a QoI spray history. Isolate sensitivity to both fungicides was tested based upon mycelial growth, germ tube elongation and fruit inoculation assays. Mean $\mathrm{EC}_{50}$ values for isolates collected prior to 2013 based upon mycelial growth and germ tube elongation were 0.22 and $0.013 \mu \mathrm{g} / \mathrm{ml}$ for azoxystrobin and pyraclostrobin, respectively. Mycelial growth and germ tube elongation of 43 isolates collected from 2013 to 2014 were not inhibited at the highest concentrations of azoxystrobin (3 $\mu \mathrm{g} / \mathrm{ml})$ and pyraclostrobin $(0.110 \mu \mathrm{g} / \mathrm{ml})$. Azoxystrobin and pyraclostrobin sprayed preventively on detached strawberry fruit inoculated with $C$. acutatum failed to control resistant isolates. Isolates were characterized by sequencing of the cytochrome $b$ gene and resistant isolates had the G143A amino acid substitution at the cytb locus. To our knowledge this is the first report of resistance to QoI fungicides in C. acutatum. Our findings indicate that development of AFR management practices is essential to limit selection and spread of QoI resistant populations of C. acutatum.

Laurel wilt in Louisiana: The disease continues to spread on sassafras (Sassafras albidum)

S. W. FRAEDRICH (1), W. Johnson (2), R. D. Menard (2), G. S. Best (1), T. C. Harrington (3)

(1) USDA Forest Service, Athens, GA, U.S.A.; (2) USDA Forest Service, Pineville, LA, U.S.A.; (3) Department of Plant Pathology and Microbiology, Ames, IA, U.S.A.

Phytopathology 105(Suppl. 4):S4.45

Laurel wilt, caused by Raffaelea lauricola, has spread rapidly through the coastal plains of the southeastern United States (US), affecting primarily redbay (Persea borbonia). Other members of the Lauraceae such as sassafras are also susceptible, but these species are less common in areas where wilt has decimated redbay populations and they may not support high populations of Xyleborus glabratus, the ambrosia beetle that carries $R$. lauricola. In September 2014, three sassafras trees with wilt-like symptoms were examined near Bernice, Louisiana. The trees had wilted foliage and black discolored sapwood with beetle holes similar to those produced by X. glabratus. Chips of discolored sapwood were plated on malt agar amended with cycloheximide and streptomycin, and a fungus morphologically identical to $R$. lauricola was consistently recovered. The identification was confirmed using sequences of $28 \mathrm{~S}$ rDNA. Follow-up surveys found additional sassafras trees with laurel wilt in a three parish area of northern Louisiana, representing the first report of laurel wilt west of the Mississippi. This is not the first instance of laurel wilt in areas where redbay is absent. In 2010, laurel wilt was discovered in sassafras in Marengo Co., Alabama, and the disease has since spread to three adjacent counties. Sassafras occurs in various interior forest types over much of the eastern half of the US, and laurel wilt may spread northward where sassafras is generally more common.

Chondrostereum purpureum from apple and blueberry: Temperatures for spore release and germination, and virulence of mono and dikaryotic mycelia

R. A. FRANCE (1), D. E. Grinbergs (1)

(1) Inst de Investigaciones Agropecuarias, Chillan, Chile

Phytopathology 105(Suppl. 4):S4.45

Chondrostereum purpureum is a Basidiomycete that produces Silverleaf disease in numerous plants, including important fruit crops such as apple, cherry, kiwi, peach, pear and plum. In 2005 this fungus was determined in blueberry causing severe damage to commercial orchards. Although this fungus is already present in the major blueberry production areas of the world, only in Chile is reported as a disease. Chondrostereum purpureum reproduce by basidiospores, necessary for infection of recently pruned stems. The objective was to compare the performance of spores and mono or dikaryotic mycelia of C. purpureum isolated from apple and blueberry. The effect of the temperature $\left(10\right.$ to $\left.35^{\circ} \mathrm{C}\right)$ was evaluated in the spore release from basidiocap, germination, and mycelia growth of mono and dikaryotic colonies. The results showed that both type of colonies from blueberry grew better than those from apple. Later, these colonies were used for cuttings inoculations of apple and blueberry, indicating in both cases that the cuttings were more susceptible $(90 \%)$ to mono or dikaryotic blueberry isolates. The monokaryotic isolates of apple were unable to colonize, and the dikaryotic ones colonized the stems but at lower rate. The genetic variability of $C$. purpureum may explain the differences in pathogenicty and virulence. Because of the better performance, it is expected that the blueberry strain will be the dominant one on fruit trees affected by silverleaf in the coming years.

\section{Identification and characterization of a novel fungal PAMP recognised in} diocots

B. FRANCO (1), P. Birch (2), A. Berepiki (1), A. Avrova (1)

(1) James Hutton Institute, Dundee, United Kingdom; (2) University of Dundee - James Hutton Institute, Dundee, United Kingdom

Phytopathology 105(Suppl. 4):S4.45

$R$. commune is the causal agent of scald, one of the most destructive diseases of barley. Grain quality can also be affected leading to significant yield losses. Little is known about $R$. commune pathogenicity factors. At the moment is recognized the action of secreted effector proteins released to suppress host immune system. Recently sequenced $R$. commune germinating conidia transcriptome has been a big resource for identification of candidate pathogenicity genes. Analysis of transcripts produced by $R$. commune germinated conidia during infection of barley plants has been a valuable strategy for identification of pathogenicity factors less variable in $R$. commune populations. These transcripts are used to understand plant immune responses and novel sources of resistance to $R$. commune. The principal objective is the understanding of molecules released by pathogen during the interaction. Analysis of transcripts produced by $R$. commune during infection of barley plants is a valuable resource for identification of pathogenicity factors. At the same time the use of Pichia pastoris expression system to produce proteins of the genes in evaluation. P. pastoris culture filtrate was used to infiltrate different species of monocots and dicots to analyse their ability to recognise one of $R$. commune candidate pathogenicity genes, $R c l$. In the same way the analysis of several N. benthamiana genes, Sgtl, Bakl and CMPGl in HR development upon recognition of Rc1.

Cultural, morphological and molecular diversity among Stemphylium lycopersici isolates causing Tomato gray leaf spot in Argentina

M. E. Franco (1), J. Vera Bahima (2), B. L. Ronco (2), M. C. Saparrat (3), P. BALATTI (4)

(1) Centro de Investigaciones de Fitopatología Facultad de Ciencias Agrarias y Forestales Universidad Nacional de La Plata, LaPlata, Argentina; (2) Centro de Investigaciones de Fitopatología Facultad de Ciencias Agrarias y Forestales Universidad Nacional de La Plata, La Plata, Argentina; (3) Instituto Carlos Spegazzini, Facultad de Ciencias Naturales y Museo. La Plata, Buenos Aires, Argentina, La Plata, Argentina; (4) Univ Nacl de la Plata, La Plata, Argentina

Phytopathology 105(Suppl. 4):S4.45

Twenty eight Stemphylium lycopersici strains were isolated from a similar number of tomato plants growing in several regions of Argentina with typical symptoms of gray leaf spot disease between 2010 and 2013. Cultural and morphological characteristics were studied on both homemade and commercial Potato Dextrose Agar (PDA) after culturing them for 7 days at $25^{\circ} \mathrm{C}$ in the dark. Features such as growth rate, colony color, elevation, margin, zonation, media pigmentation and sporulation were examined. Furthermore, fifty mature conidia from each isolate were measured at $\times 100$ magnification and their morphology was assessed. Also variability was analyzed by using six inter-simple sequence repeat markers (ISSR-PCR). High levels of variability were observed at cultural and morphological characteristics. Using genomic fingerprints isolates were clustered at similarity coefficient values between 0.83 and 1.00 and no relationship was found between phenotypic and genotypic characters as well as the place of origin. The high rate of cultural and morphological variability as well as their sensitivity to environmental conditions, suggesting that the cultural conditions for diagnostic purposes must be carefully standardized, which should be accompanied by markers developed based on conserved gene sequences. 
Draft genome sequence of the plant-pathogenic fungus Stemphylium lycopersici strain CIDEFI-216

M. E. Franco (1), S. Lopez (2), R. Medina (3), M. C. Saparrat (4), P. BALATTI (5)

(1) Centro de Investigaciones de Fitopatología Facultad de Ciencias Agrarias y Forestales Universidad Nacional de La Plata, La Plata, Argentina; (2) Instituto de Fisiología Vegetal (INFIVE), UNLP-CONICET. La Plata, Buenos Aires, Argentina., La Plata, Argentina; (3) Centro de Investigación y Desarrollo en Fermentaciones Industriales (CINDEFI), UNLP-CONICET. La Plata, Buenos Aires, Argentina, La Plata, Argentina; (4) Instituto Carlos Spegazzini, Facultad de Ciencias Naturales y Museo. La Plata, Buenos Aires, Argentina, La Plata, Argentina; (5) Univ Nacl de la Plata, La Plata, Argentina Phytopathology 105(Suppl. 4):S4.46

Stemphylium lycopersici is a pathogenic fungus that provokes the leaf spot disease on over 30 host genera worldwide, among them Tomato. Here we report the draft genome sequence of $S$. lycopersici strain CIDEFI-216. Total genomic DNA was isolated from a monosporic culture using the DNeasy Plant Mini Kit (Qiagen) and was used to construct $100 \mathrm{bp}$ paired-end libraries, which were then sequenced by Illumina HiSeq 2000 technology. Reads were assembled using SOAPdenovo2 software at Macrogen (Korea). Gene prediction was performed by Fgenesh software (Softerry), functional annotation was carried out with Blast2GO software (BioBam) and tRNAs and rRNAs were predicted using tRNAscan-SE and HMM-rRNA tools from WebMGA server. The paired-end libraries produced 31117554 reads with a total of $3142872954 \mathrm{bp}$, representing an average coverage of $77.39 \mathrm{X}$. The genome was assembled into 419 scaffolds with a total length of $35.18 \mathrm{Mbp}$ $(1000 \mathrm{bp} ; \mathrm{N} 50=498048 \mathrm{bp})$ and an overall $\mathrm{G}+\mathrm{C}$ content of $50.5 \%$. A total of 8998 protein-coding genes were predicted, whose functional annotation is discussed. Additionally, 94 tRNAs and 44 rRNAs were found. This draft genome sequence, the first available for $S$. lycopersici, represents a new resource for further research into the taxonomy, biology and phytopathology of this plant pathogen.

\section{Susceptibility of weed hosts and crops to host-adapted pathotypes of} Verticillium dahliae

Z. FREDERICK (1), T. Cummings (1), D. Johnson (1)

(1) Washington State University, Pullman, WA, U.S.A.

Phytopathology 105(Suppl. 4):S4.46

Verticillium dahliae is a vascular pathogen of potato and 200 other dicotyledonous species. Some isolates of $V$. dahliae have been separated into host-adapted pathotypes but the susceptibility of weed species to these pathotypes is not known. Twelve weed and five crop species were evaluated for susceptibility to eight isolates of $V$. dahliae by dipping plant roots in a conidial suspension at $1.0 \times 10^{6}$ conidia/ml followed by planting into potting mix in a greenhouse. Aggressiveness of $V$. dahliae host-adapted pathoypes was confirmed by a previous study. Significant plant host species or family $\mathrm{x}$ $V$. dahliae isolate or pathotype interactions were observed. As examples, weed host species Poa апnиa was susceptible to a $V$. dahliae isolate from tomato, whereas Amaranthus powellii was resistant to that isolate. Significant $(P=0.05)$ differences in individual host-pathogen interactions were observed in nine out of 17 crop and weed species. For example, Solanum nigrum was observed to be resistant to an isolate belonging to the mint pathotype and susceptible to isolates belonging to the potato pathotype. These findings show differential susceptibility to $V$. dahliae pathotypes within individual plant host species, suggesting that the crop and weed species growing in fields between potato crop rotations may influence the presence of potato host-adapted pathotypes of $V$. dahliae.

\section{Manipulation of amino acid gradients reduces root-knot nematode penetration of roots}

T. S. FREY (1), K. Navarro (2), G. Valero (3), C. G. Taylor (2)

(1) Ohio State Univ, Wooster, OH, U.S.A.; (2) Ohio State University, Wooster, OH, U.S.A.; (3) University of Puerto Rico, Mayaguez, Mayaguez, U.S.A. Phytopathology 105(Suppl. 4):S4.46

Plant-parasitic nematodes represent a significant threat to world agricultural production. Nematodes parasitize plants and draw immense amounts of energy away from their plant hosts and in turn significantly reduce vigor and yield in important crops such as soybean and tomato. After hatching from environmentally resilient eggs, nematodes must navigate through the soil environment in order to find a plant root. Previous studies have shown that nematodes employ a chemotaxic mechanism to move non-randomly through the soil toward roots following a root exudate gradient. Few studies have looked at which specific compounds in these exudates are attractive to nematodes and whether root-exudate composition can be manipulated to control nematode behavior. We hypothesized that root-knot nematode (Meloidogyne incognita, $\mathrm{RKN}$ ) would be preferentially attracted to certain amino acids in root exudates and that these "attractive" compounds could be used to distract and/or reduce nematode entry into roots when applied exogenously. Using an experimental agar-based system we found that some amino acids were highly attractive to nematodes while others were repellent. Using a combination of exogenously applied attractive and repellent amino acids we were able to significantly reduce the number or juvenile nematodes that could enter a root system. These findings represent demonstrate a novel means for control of plant-parasitic nematodes utilizing a ubiquitous biological molecule.

Impact of antimicrobial lipopeptides iturin and surfactin for Fusarium wilt of lettuce

S. Fujita (1), K. YOKOTA (1)

(1) Tokyo Univ of Agriculture, Tokyo, Japan

Phytopathology 105(Suppl. 4):S4.46

Iturin and surfactin are antimicrobial lipopeptides produced by antagonistic Bacillus spp., and are deduced to play key roles in biological control by Bacillus spp. Previously, we reported that both lipopeptides suppress a Fusarium disease on Taasai, Brassica rapa subsp. narinosa by soil amendments, and the excess amount of lipopeptide amendments caused loss of disease suppressions. Otherwise, there are optimal concentrations to show the disease suppressions for both lipopeptides. In this study, we report that the impacts for disease suppressions by iturin and surfactin against Fusarium wilt of lettuce, Lactuca sativa. Both lipopeptides showed disease suppressions by soil amendments. The disease suppression by iturin amendment were observed at the range from 0.47 to $1.88 \mathrm{mg} \mathrm{L}^{-1}$ soil; whereas loss of disease suppression more than $3.75 \mathrm{mg} \mathrm{L}^{-1}$ soil. On the other hand, the disease suppression by surfactin-amendment were observed from $1.88 \mathrm{mg} \mathrm{L}^{-1}$ soil up to $7.5 \mathrm{mg} \mathrm{L}^{-1}$ soil. Same observations were confirmed among four-kinds of cultivars of lettuce. Our results suggested that chemical characteristics of lipopeptide effect for the existence of the optimal concentrations to show disease suppression by combination with host plants.

Identification and evaluation of SNP and SCAR markers linked to the $\boldsymbol{R} y_{\text {chc }}$ gene for resistance to Potato virus $\boldsymbol{Y}$ in diploid potato populations

A. C. FUlladolsA (1), S. H. Jansky (2), D. A. Halterman (3), A. O. Charkowski (1)

(1) University of Wisconsin-Madison, Madison, WI, U.S.A.; (2) University of Wisconsin-Madison/USDA-ARS, Madison, WI, U.S.A.; (3) USDA-ARS, Madison, WI, U.S.A.

Phytopathology 105(Suppl. 4):S4.46

Potato virus $Y$ (PVY) is the most significant cause of economic loss due to plant pathogens in seed potato production. With the emergence of various PVY strains and infection phenotypes in the last decade, the need for varieties with broad resistance has increased in North America. Extreme resistance to PVY has been attributed to single-dominant genes found in wild relatives of potato. Two accessions of Solanum chacoense, carrying the PVY resistance gene $R y_{c h c}$, were used to develop segregating diploid populations. The phenotypes of 175 individuals of a population derived from an accession heterozygous for $R y_{c h c}$ showed a 1:1 resistance segregation ratio. We used the SolCAP 8303 SNP Infinium array to genotype the population and identified five SNPs correlating with resistance, located on the distal end of chromosome IX. One SNP was selected to design a TaqMan ${ }^{\circledR}$ SNP Genotyping Assay. We also sequenced the genome of the other $S$. chacoense accession, homozygous for $R y_{\text {chc }}$, from which a useful breeding clone, XD3, was selected and selfed. We used the genome sequence of the parental $S$. chacoense to develop a co-dominant SCAR marker. Initial screening of our populations with the $\operatorname{TaqMan}^{\circledR}$ assay shows that it is effective for SNP identification, but the SNPs associated with resistance vary between populations derived from different parents. Similarly, the SCAR marker is useful for genotyping the XD3-selfed population, but not for others.

Development of a predictive model to estimate conditions lethal to soilborne inoculum of Phytophthora ramorum and pini during soil solarization

F. FUNAHASHI (1), J. Parke (1)

(1) Oregon State University, Corvallis, OR, U.S.A.

Phytopathology 105(Suppl. 4):S4.46

Soil solarization has been used to manage soilborne plant pathogens. Research on solarization efficacy has focused on the minimum temperature and exposure time required to kill pathogens, and most mathematical models of the effects are based on cumulative temperature over time. However, two additional factors, soil water potential and diurnal temperature fluctuation, may influence pathogen survival. Our objective was to develop a more accurate predictive model based on results from controlled lab experiments. We assessed temperature, water potential, and intermittent heat effects on 
survival of Phytophthora ramorum and P. pini in infested leaf inoculum that contained chlamydospores or oospores, respectively. For both pathogens, survival frequency at high temperature was greater at lower water potentials. Survival was also greater when exposure to high temperature was interrupted by a cooler temperature. Results indicate that heat effects on pathogen survival increase gradually during heat treatment, suggesting that the temperature effect is not simply cumulative. The mathematical model was tested in solarization field trials conducted in 2013 by comparing calculated heat units to recovery of $P$. ramorum and $P$. pini subjected to various heat regimes. The model was improved significantly by adding the factors water potential and temperature fluctuation, allowing for greater accuracy in predicting soil solarization efficacy.

Effect of waxy (low amylose) on fungal infection of sorghum grain D. L. FUNNELL-HARRIS (1), S. E. Sattler (2), P. M. O'Neill (2), K. M. Eskridge (3)

(1) USDA ARS, Lincoln, NE, U.S.A.; (2) USDA-ARS; Grain, Forage and Bioenergy Research Unit, Lincoln, NE, U.S.A.; (3) Department of Statistics; University of Nebraska, Lincoln, NE, U.S.A.

Phytopathology 105(Suppl. 4):S4.47

Loss of function mutations in the Waxy $(W x)$ gene, encoding Granule Bound Starch Synthase (GBSS) that synthesizes amylose, results in starch granules containing mostly amylopectin. Grain with this trait has increased usability for feed, food and grain-based ethanol, due to altered starch properties. In sorghum, two classes of waxy $(w x)$ alleles had been previously characterized for absence or presence of GBSS: $w x^{a}\left(\mathrm{GBSS}^{-}\right)$and $w x^{b}\left(\mathrm{GBSS}^{+}\right.$, with reduced activity). Field-grown grain of wild-type; waxy, GBSS; and waxy, $\mathrm{GBSS}^{+}$ Plant Introduction accessions, were screened for fungal infection. Overall, results showed that waxy grains were not more susceptible than wild-type. GBSS $^{-}$and wild-type grain had similar infection levels. However, height was a factor with waxy, GBSS ${ }^{+}$lines: $w x^{b}$ accessions, which were short, were more susceptible than tall waxy accessions, with an undescribed allele. In greenhouse experiments, grain from accessions and near-isogenic $w x^{a}, w x^{b}$ and wild-type lines, were inoculated with Alternaria sp., Fusarium thapsinum and Curvularia sorghina, to analyze germination and seedling fitness. As a group, waxy lines were not more susceptible to grain pathogens than wildtype, supporting field evaluations. Amongst most waxy and wild-type lines, reduced emergence, survival and seedling weights were observed after $C$. sorghina and $F$. thapsinum inoculations. These results are valuable for developing waxy hybrids with resistance to grain-infecting fungi.

Wood-decay abilities of grapevine trunk pathogens Diaporthe ampelina, Diplodia seriata, Eutypa lata, and Neofusicoccum parvum

E. GALARNEAU (1), C. Wallis (2), A. Morales-Cruz (3), D. Cantu (3), K. Baumgartner (4)

(1) USDA ARS CPGRU, Davis, CA, U.S.A.; (2) USDA ARS, Parlier, CA, U.S.A.; (3) University of California Davis, Davis, CA, U.S.A.; (4) USDAAgricultural Research Service, Davis, CA, U.S.A.

Phytopathology 105(Suppl. 4):S4.47

Trunk pathogens are fungi that infect grapevine wood through pruning wounds and destroy fruiting positions, thereby impacting grape production. Neofusicoccum parvum (causal fungus of Botryosphaeria dieback) and Eutypa lata (causal fungus of Eutypa dieback) cause chronic infections (cankers) of the trunk/cordons, but vary in their utilization of woody substrate. We evaluated the wood-decay abilities of $N$. parvum (highly virulent), E. lata (known soft-rot fungus), Diplodia seriata (weakly virulent causal fungus of Botryosphaeria dieback), and Diaporthe ampelina (attacks both green and woody tissue, causal fungus of Phomopsis dieback/cane and leaf spot). The ability of these fungi to degrade cell wall components (e.g. phenolics, lignin, and cellulose) was evaluated by amending fungal cultures with these compounds and observing changes to compound levels over time using HPLC and colorimetric methods. Observed differences between these species on amended media were likely from differences in the preferred tissues each attacks. Findings were somewhat consistent with previous findings of similarities in the predicted secretomes of each fungus, which were seen between E. lata and $N$. parvum (e.g. enzymes that target cellulose and hemicellulose). Additional studies will examine levels of tested compounds in grapevine tissues surrounding wood lesions, in addition to fungal gene expression in-planta. Our findings may allow some of these fungi to be classified as soft-rot fungi.

\section{Mapping stem rust resistance genes in 'Kingbird'}

K. GAMBONE (1), I. Fuentes-Bueno (2), R. Bowden (2)

(1) Kansas State University, Manhattan, KS, U.S.A.; (2) USDA-ARS Hard Winter Wheat Genetics Research Unit, Manhattan, KS, U.S.A.

Phytopathology 105(Suppl. 4):S4.47
The spring wheat variety 'Kingbird' is thought to contain multiple quantitative trait loci (QTLs) that provide resistance against wheat stem rust (Puccinia gminiras). Susceptible Kansas winter wheat line 'KS05HW14' was backcrossed to Kingbird and 379 lines were advanced to BC1F5 and then increased for testing. The lines were screened for stem rust resistance in the greenhouse and field in Kansas and in the field in Kenya over multiple years. We identified 16,237 single nucleotide polymorphisms (SNPs) with the Wheat 90K iSelect SNP Chip assay. After filtering for marker quality, chromosomal locations of a subset were obtained from the Wheat 90K Consensus Map. QTLs were consistently detected by single marker analysis on chromosomes 2BL, 7BL and two on 3B. Furthermore, the presence of resistance genes Lr34 and $\mathrm{Sr} 2$ were confirmed in Kingbird using diagnostic markers. These results confirm that there are multiple QTLs present in Kingbird. Ultimately, the identification of the SNPs that are closely linked to QTLs associated with stem rust resistance will aid in development of markers for marker assisted selection.

Using the relationship between foliar emission rate and disease progress over time as a tool for managing black sigatoka in plantain

L. GAÑÁN (1), E. Alvarez (1)

(1) International Center for Tropical Agriculture, Palmira, Colombia Phytopathology 105(Suppl. 4):S4.47

Black sigatoka (BS), caused by the pathogenic fungus Mycosphaerella fijiensis, is the most important leaf disease of banana and plantain. It is managed mainly by periodic fungicide applications. However, this control method is often too costly for most small-scale farmers in developing countries. To design an eco-efficient approach to disease management, we evaluated the relationship between BS temporal dynamics and the physiological variable of foliar emission rate (FER) in plantain (Musa AAB). A 1.5-hectare plot of plantain was established in an area with high BS inoculum pressure. The variables, evaluated weekly, were youngest leaf with streaks (YLSt), youngest leaf with spots (YLS), disease severity index (DSI), and, to calculate FER, number of developed leaves. No cultural or chemical practices for disease management were performed during the crop's cycle. The first symptoms appeared 10 to 20 days after the first leaf had fully opened. Values for FER correlated positively with those for variables YLSt and YLS, and negatively with DSI. The highest correlations were observed during a 50-day period between pre-bloom and fruit formation, near the time when leaf emission is stopped. Thus, farmers can wait until they observe that foliar emission is about to cease before applying fungicides, thereby saving on costs.

Bio-control agent Bacillus subtilis SMS combined with silver nanoparticles against Botrytis squamosa

S. GAO (1), X. Yue (1), L. Luo (1), J. Li (1)

(1) China Agricultural University, Beijing, China

Phytopathology 105(Suppl. 4):S4.47

Chinese chives (Allium tuberosum Rottler) is one kind of traditional condiment and essential vegetable in China. Gray mold disease, caused by Botrytis squamosa, has resulted in substantial economic losses in Chinese chives production. Bio-control strategy is highly recommended for environmental benefit and potential possibilities of being integrated with other methods of disease management. In this study, Bacillus subtilis strain SMS, HL29 and H6, B. licheniformis strain JM-3, and B. amyloliquefaciens strain D2 were tested for their effects on the growth of Botrytis squamosa by dual culture plate assays. The results showed that the mycelium growth of Botrytis squamosa were significantly decreased by strain SMS, HL29, JM-3, H6, and $\mathrm{D} 2$, and the inhibition rate were $87.5 \pm 2.7 \%, 62.3 \pm 4.1 \%, 65.0 \pm 3.8 \%$, $74.2 \pm 5.1 \%$, and $57.9 \pm 6.3 \%$, respectively. It was also found that strain SMS possesses extracellular synthesis of silver nanoparticles (AgNPs), which was achieved by addition of cell free culture supernatant of strain SMS with aqueous silver nitrate solution. Furthermore, the synthesized nanoparticles were characterized by UV-Vis spectrophotometer, Fourier Transform Infrared Spectroscopy (FTIR), and Scanning electron microscopy (SEM). The result showed that the synthesized nanoparticle was a promising agent against Botrytis squamosa. It is indicated that the B. subtilis strain SMS could be integrated with nanoparticles to control gray mold disease on Chinese Chives.

Genome wide association study (GWAS) mapping identifies genomic regions associated with Parastagonospora nodorum virulence on wheat Y. GAO (1), J. Richards (1), R. S. Brueggeman (1), T. L. Friesen (2)

(1) Department of Plant Pathology, North Dakota State University, Fargo, ND, U.S.A.; (2) Department of Plant Pathology, North Dakota State University; USDA-ARS, Norhern Crop Science Lab, Cereal Crops Research Unit, Fargo, ND, U.S.A.

Phytopathology 105(Suppl. 4):S4.47 
Parastagonospora nodorum (formerly Stagonospora nodorum) is a necrotrophic fungal pathogen and the causal agent of Septoria nodorum blotch (formerly Stagonospora nodorum blotch) on wheat. We found that $P$. nodorum produces necrotrophic effectors (NE) that are recognized directly or indirectly by wheat dominant sensitivity genes that confer susceptibility. Nine NE-host dominant sensitivity gene interactions have been identified and three NE genes have been cloned including SnToxA, SnTox1 and SnTox3. To identify more genomic regions harboring NE genes, we used genome wide association study (GWAS) mapping using a global collection of $200 P$. nodorum isolates (13 US states and 12 countries). DNA of the 200 isolates was extracted and genotyping by sequencing (GBS) was performed using Ion Torrent sequencing technology. Reference sequence tags were assembled and sequencing reads were aligned to the reference tags using the BurrowsWheeler Aligner 'mem' algorithm. A total of 2,046 SNP markers were identified using SAMtools for an average SNP density of one marker every $18 \mathrm{~kb}$ throughout the $37 \mathrm{MB} P$. nodorum genome. Inoculation and infiltration data of the 200 isolates on several wheat lines was completed and utilized for association mapping (AM). The AM was performed using four models including marker trait association, PCA, PCA + kinship and structure + kinship using JMP Genomics 7.0. Many genomic regions were associated with NE sensitivity and pathogen susceptibility.

\section{Molecular exploration of beta-lactamases in Fusarium verticillioides}

M. GAO (1), A. E. Glenn (2), S. E. Gold (2)

(1) The University of Georgia, Athens, GA, U.S.A.; (2) USDA ARS, Richard B. Russell Res Center, Toxicology \& Mycotoxin Res Unit, Athens, GA, U.S.A.

Phytopathology 105(Suppl. 4):S4.48

The mycotoxigenic fungus Fusarium verticillioides $(F v)$ is one of the most prevalent maize fungal pathogens. $F v$ mycotoxins are a significant food safety issue and have given rise to exposure concerns worldwide. The $F D B 1$ locus, a beta-lactamase-containing $F v$ gene cluster, was previously shown to be induced by and responsible for the breakdown of and resistance to the maize benzoxazolinones phytoanticipins. Examination of the $F v$ genome indicates a large expansion of genes with beta-lactamase domains. Of 46 identified $F v$ lactamase domain containing proteins, 38 were predicted to be associated with lactam hydrolysis and fall into two families, the metallo-beta-lactamases and cephalosporinases. We hypothesize that many of these beta-lactamases contribute to tolerance to additional unidentified lactam xenobiotics. Based on the results at the $F D B 1$ locus, in order to better understand the function of beta-lactamase genes and their putative clusters, we will assess the induction of particular subsets of beta-lactamase genes upon exposure to select lactams. Mutants in induced beta-lactamase genes will be tested for altered sensitivity to the gene inducing lactams. Amongst our current lactam collection we will initially focus on pyrrocidine $\mathrm{A}$, isolated from Acremonium zeae, a coinhabitant of maize seed with $F v$. We will present current results on bioinformatic analysis of the $F v$ beta-lactamase gene family and on global gene expression in response to specific lactam molecules.

Horizontal gene transfer confers adaptive advantages to phytopathogenic fungi: A case study of catalase-peroxidase in Fusarium verticillioides S. GAO (1), S. E. Gold (2), A. E. Glenn (2)

(1) University of Georgia, Athens, GA, U.S.A.; (2) USDA ARS, Richard B. Russell Res Center, Toxicology \& Mycotoxin Res Unit, Athens, GA, U.S.A. Phytopathology 105(Suppl. 4):S4.48

Horizontal gene transfer (HGT), the exchange and stable integration of genetic material between different evolutionary lineages, is widely observed in fungi. We hypothesize that successful stabilization of HGT elements provides adaptive advantages (e.g., virulence). Catalase/peroxidases (KatGs) are a superfamily of fungal reactive oxygen species (ROS)-degrading enzymes previously revealed via phylogenetic analysis to be horizontally acquired by ancient Ascomycota from bacteria. A subsequent gene duplication resulted in two KatGs paralogues: the widely distributed, intracellular group (KatG1), and the Sordariomycete-unique (mainly phytopathogens), extracellular group (KatG2). We functionally characterized $F v C P O 1$ (KatG1) and $\mathrm{FvCPO} 2$ (KatG2) in the maize pathogen, Fusarium verticillioides. Single deletion mutants were generated by the OSCAR method, and double mutants were obtained from sexual crosses between two single mutants. Both $\triangle \mathrm{FvCPO} 1$ and $\triangle \mathrm{FvCPO} 2$ mutants, when compared to the wild type, showed moderate impaired tolerance to $\mathrm{H}_{2} \mathrm{O}_{2}$ in vitro, while the double mutant was dramatically sensitive to $\mathrm{H}_{2} \mathrm{O}_{2}$. The dual function encoded by these two genes appears to confer protection from exogenous oxidative stress, such as may occur during plant infection. Subsequent experiments will evaluate effects on virulence and endophytic infection of maize. Further genomic analyses are underway to identify additional HGTs in $F$. verticillioides given its plantassociated niches.
Diversity of Stagonosporopsis species causing gummy stem blight of gherkin in Karnataka region, India

R. H. GARAMPALLI (1), M. K. G. (2), H. X. Li (3), M. T. Brewer (3)

(1) University of Mysore, Mysore, Karnataka, India, Mysore, India; (2) University of Mysore, Mysore, India; (3) University of Georgia, Department of Plant Pathology, Athens, GA, U.S.A.

Phytopathology 105(Suppl. 4):S4.48

Gummy stem blight (GSB), caused by Stagonosporopsis cucurbitacearum (syn. Didymella bryoniae), S. citrulli, and S. caricae, is a destructive disease that is a major threat to cucurbit production worldwide. In India, a variety of cucurbits are cultivated, which contribute to approximately $5.6 \%$ of the total vegetable production. Gherkin (Cucumis sativus), a cultivar of pickling cucumber, was introduced into India in the early 1990's and is grown predominantly in the states of Karnataka and Andra Pradesh. Karnataka accounts for $90 \%$ of gherkin production in India. In 2008, severe disease epidemics with symptoms resembling GSB emerged on gherkin in Karnataka. The causal agent was tentatively identified as $D$. bryoniae based on morphology, original host, and disease symptoms upon inoculation. Our objective was to use molecular techniques to positively identify the causal organism. Nine isolates were obtained from two outbreaks on gherkin in late 2014 in Karnatka. The internal transcribed spacer region (ITS) was sequenced and a previously developed PCR-based marker for distinguishing species causing GSB was used. Based on both methods we determined that seven of the isolates were $S$. caricae and two were $S$. citrulli with both species occurring within the same field. Additional field outbreaks will be surveyed in spring 2015 to study population dynamics and fungicide resistance profiles since these species vary in sensitivity to different fungicides in the US.

Identification and management alternatives of powdery mildew in rosebushes (Rosa sp.)

R. GARCÍA-VELASCO (1), D. Dominguez-Serrano (1), M. E. Mora-Herrera (1), J. G. Gonzalez-Diaz (1), M. L. Salgado-Siclan (2), D. Nieto-Angel (3) (1) Autonomous University of the State of Mexico, Tenancingo, Mexico; (2) Autonomous University of the State of Mexico, Toluca, Mexico; (3) Postgraduate College, Texcoco, Mexico

Phytopathology 105(Suppl. 4):S4.48

Podosphaera pannosa is one of the main phytosanitary problems in rose cultivation, because its management is based on continuous fungicide applications which tends to generate resistant populations. Thus, search for alternatives is crucial. The aims of this work were to morphologically and molecularly confirm the identity of the causal agent of powdery mildew as well as to evaluate the effect of potassium phosphite $\left(\mathrm{K}_{3} \mathrm{PO}_{3}\right)$, chitosan and silicon for its handling in Rosa spp. Morphological characterization was done with a compound microscope. For molecular characterization studies, the DNA ribosomal of the ITS region was amplified with ITS1f and ITS4 primers. Treatments were potassium phosphite, chitosan, silicon, dodemorph acetate and a control. The experimental design was completely randomized. The variables evaluated were incidence and severity; and the variables data were transformed with the AUDPC. Means comparison was carried out by Tukey $(\alpha=0.05)$. Morphological and molecular data corresponded to $P$. pannosa (KP902716). As for disease incidence, all treatments were significantly different; silicon and $\mathrm{K}_{3} \mathrm{PO}_{3}$ reduced the incidence with an AUDPC of 568.8 and 1207.5, respectively, compared to the control (3517.5). As for severity, the control showed the highest damage with an AUDPC of 1286862.3, but the silicon (140968.04) and $\mathrm{K}_{3} \mathrm{PO}_{3}(281982.86)$ treatments reported the lowest damage levels. Silicon and $\mathrm{K}_{3} \mathrm{PO}_{3}$ are alternatives for the rosebush powdery mildew management.

Not just Botrytis: Multiple fungal pathogens cause leaf spots on peony in the United States

A. R. GARFINKEL (1), G. A. Chastagner (1)

(1) Washington State University, Puyallup, WA, U.S.A.

Phytopathology 105(Suppl. 4):S4.48

The herbaceous peony (Paeonia lactiflora) is grown as a high-value cut flower crop and landscape plant worldwide. Through interactions with peony growers, we found that many perceive Botrytis gray mold as a significant production and post-harvest issue at their farms. In a survey to determine the cause of leaf spots on peony, we acquired infected foliage from commercial growers and through visual scouting of peony plantings throughout the United States with an emphasis on Washington, Oregon, and Alaska. Using morphological and molecular methods, we identified multiple fungal pathogens never before reported on peony in the United States in addition to the commonly-reported pathogens, Botrytis, Cladosporium, and Alternaria. The discoveries of one of these pathogens, Mycocentrospora acerina, in Washington and Alaska represent only the second and third reports of this pathogen infecting peony in the world. The detection of Pilidium concavum in 
North Carolina is the first report of this pathogen on peonies outside of East Asia. We also identified a complex of Botrytis species infecting peony, of which at least one species is undescribed and appears to be unique to Alaska peony production. Clearly, a wider range of fungal pathogens, including multiple species of Botrytis, infect peony than is indicated in the common literature, such as extension bulletins and growers' guides. These diseases are going misdiagnosed by growers, potentially hindering disease management.

Whole-genome sequencing of Botrytis paeoniae using the Ion Proton platform for microsatellite discovery

A. R. GARFINKEL (1), K. P. Coats (1), M. R. Wildung (2), G. A. Chastagner (1)

(1) Washington State University, Puyallup, WA, U.S.A.; (2) Washington State University, Pullman, WA, U.S.A

Phytopathology 105(Suppl. 4):S4.49

Botrytis paeoniae is a putatively host-specific pathogen that causes Botrytis gray mold on peony (Paeonia spp.). The decreasing cost of high-throughput next-generation sequencing technologies makes whole-genome sequencing of non-model organisms a cost-efficient option for aid in microsatellite marker development. For this purpose, 250 nanograms of high quality genomic DNA of B. paeoniae was used to construct an Ion Proton sequencing library. DNA fragments were sequenced on a single Ion Proton P1 chip to produce 10.4 gigabases of raw reads. Reads were assembled into 11,756 contigs, the longest of which was 165 kilobases. The sum of contigs totaled 44.4 megabases, similar in size to published assembled $B$. cinerea genomes. 4,746 repetitive regions were identified in the assembled genome using the default parameters in QDD v3.1.2, of which 3,614 were unique. Primer3, executed within QDD, was used to identify 3,336 microsatellite sequences for which primers could be developed. Primers will be designed for selected loci and tested on several populations to identify a panel of informative genetic markers for future analysis of $B$. paeoniae. To our knowledge, this represents one of the first fungal organisms sequenced using the Ion Proton technology for the purpose of microsatellite repeat discovery.

Secondary metabolite production and biological activity of endophytic microbes of Mahonia aquifolium

B. Geary (1), T. Smart (1), P. Hatch (1), R. SWEENEY (1)

(1) Brigham Young University, Provo, UT, U.S.A.

Phytopathology 105(Suppl. 4):S4.49

Mahonia aquafolium is a plant species more commonly known as the Oregon grape. Mahonia aquifolium is known to produce an antibacterial compound, berberine, which may a play a role in the survival. The object of this research was to determine if an endophytic fungus from $M$. aquifolium is responsible for producing a portion of the berberine. Plant material was gathered from the Wasatch front in Utah. Roots, stems and leaves were washed in $50 \%$ bleach and then flame sterilized before culturing on 1/10 PDA. After 3 weeks, multiple fungi were isolated and cultured on 1/10 PDA. Based on berberine's distinct yellow color, one fungus was identified as possibly producing berberine. This fungus was placed in M1D broth and cultured for 2 weeks. The broth was filtered, and then berberine was extracted with dichloromethane. The sample was rotary evaporated to dryness and then further purified using a silica column with DCM and methanol. The thin layer chromotography $\mathrm{Rf}$ value of 0.55 of the sample matched the berberine standard using three different mobile phases. A more purified sample was collected using preparative TLC. The sample was then subjected to mass spectrometry and nuclear magnetic resonance. The results of MS, NMR and TLC all showed that berberine was present in the fungal sample. Sequencing data and scanning electron microscopy was used to identify the genus and species of the fungus.

Identification of Candidate genes in a Major QTL for resistance to Fusarium graminearum

C. R. GEDLING (1), B. Acharya (2), B. J. Cassone (3), S. Lee (4), R. Mian (3), L. K. McHale (5), A. P. Michel (1), A. E. Dorrance (1)

(1) The Ohio State University, Wooster, OH, U.S.A.; (2) Virginia Tech University, Blacksburg, VA, U.S.A.; (3) The Ohio State University, Wooster, OH, U.S.A.; (4) North Carolina State University, Raleigh, NC, U.S.A.; (5) The Ohio State University, Columbus, OH, U.S.A.

Phytopathology 105(Suppl. 4):S4.49

Fusarium graminearum is primarily a fungal pathogen of wheat and related cereal crops, but it can also infect corn and soybean. Currently, fungicide seed treatments are used to manage this pathogen. Host resistance is often the best management strategy for field crops. Thus, our overall objective is to identify and characterize sources of resistance that are effective towards seed, seedling, and root rots caused by $F$. graminearum in soybean. Using QTL mapping in an F7:10 population derived from a cross between Wyandot (susceptible) and PI 567301B (resistance), two regions of the soybean genome were identified, with one QTL mapping to chromosome 8, contributing 38.5\% of the phenotypic variation. Annotation of this region in Williams 82 indicated that there are 38 genes including the rhg4 locus for soybean cyst nematode (SCN). Based on previous screens, PI 567301B is susceptible to SCN. DNA sequencing of the genes within this QTL region using the Illumina MiSeq platform was carried out to identify key sequence variation among the resistant and susceptible lines in both the coding sequence and upstream promoter regions. Protein domain annotations of the genes within these QTLs point to several intriguing candidates, including Leucine rich repeats and heat shock transcription factors. Future functional genomics approaches (e.g. VIGS, hairy roots) will be used to knock down expression of candidate genes to better assess their contribution to $F$. graminearum resistance.

Risk factors for perennation of Podosphaera macularis and landscape level development of hop powdery mildew

D. GENT (1), M. Twomey (2), S. Wolfenbarger (3), J. Woods (2)

(1) USDA ARS NFSPRC, Corvallis, OR, U.S.A.; (2) USDA-ARS FSCRU, Corvallis, OR, U.S.A.; (3) Oregon State University, Corvallis, OR, U.S.A. Phytopathology 105(Suppl. 4):S4.49

Disease spread by pathogens capable of long distance dispersal often can be described by power law dispersal models. The epidemic expansion front for such epidemics is multiplicatively related to the size of the initial disease focus, which has fundamental implications for management of overwintering inoculum density. The hop powdery mildew fungus, Podosphaera macularis, overwinters exclusively in association with host tissue by means of bud perennation (termed flag shoots) in the Pacific Northwestern U.S. Therefore, understanding factors associated with perennation is potentially important for understanding epidemic development at the landscape level. Historical data from commercial hop yards collected during 2000 to 2015 was analyzed to identify risk factors linked to flag shoot occurrence and frequency. Preliminary analyses indicated that prior occurrence of flag shoots, pruning method, and winter temperature were associated with flag shoot development. Extensive surveys of hop yards in Oregon in 2014 and 2015 identified that landscape-level outbreaks of powdery mildew were associated with survival of the fungus in a small number of hop yards. In many instances, perennation of $P$. macularis was associated with chronic infection of highly susceptible cultivars when specific pruning practices were utilized in spring. Thus, focused management efforts in a subset of yards where overwintering is most probable may moderate disease severity area-wide.

Differential host specificity of the two related pathovars Pantoea agglomerans pvs. gypsophilae and betae as expressed by type III effectors M. Gershovitz (1), G. Nissan (2), L. Chalupowicz (3), M. Morozov (4), S. Manulis-Sasson (5), G. Sessa (2), T. Pupko (2), I. BARASH (6)

(1) Tel Aviv University, Trl Aviv, Israel; (2) Tel Aviv University, Tel Aviv, Israel; (3) ARO, The Volcani Center, Bet Dagan, Israel; (4) Tel Aviv University, Tel Aviv, Israel; (5) ARO, The Volcani, Center, Bet Dagan, Israel; (6) Tel Aviv Univ, Tel Aviv, Israel

Phytopathology 105(Suppl. 4):S4.49

Pantoea agglomerans $(\mathrm{Pa})$, a widespread epiphyte and commensal bacterium, has evolved into a host-specific tumorigenic pathogen by acquiring a plasmid containing a pathogenicity island. Two related pathovars have been investigated: $P a$ pv. gypsophilae (Pag), which elicits galls on gypsophila and hypersensitive response (HR) on beet and $P a$ pv. betae (Pab), which elicits galls on both beet and gypsophila. Pathogenicity of Pag and Pab depends on a type III secretion system (T3SS), which resides on the pathogenicity plasmids

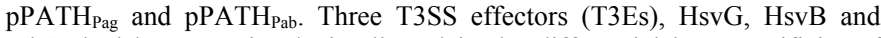
PthG, had been previously implicated in the differential host specificity of $P a g$ and Pab. Here we aimed to identify all the T3Es encoded by the two pathovars and to investigate their potential role in host specificity. To this end, we first obtained draft genome sequences of both Pag (824-1) and Pab (4188). Then a machine-learning approach was applied for predicting encoded T3Es. A tanslocation assay into beet roots, based on features of $p t h G$, was constructed and applied for validation of predicted T3Es. It was found that $P a g$ and Pab have a genome size of 5.01and 5.02 Mb, 4598 and 4599 ORFs and a GC content of $54.8 \%$ and $54.9 \%$, respectively. Eleven plasmid-borne T3Es are common to both pathovars, whereas 2 and 3 effectors are specific to $P a g$ and $P a b$, respectively. The possible contribution of specific T3Es to host specificity will be presented.

Identification and functional characterization of bacterial community of tropical forage grasses Brachiaria spp. grown in their natural habitat

S. GHIMIRE (1), C. Mutai (1), J. Njuguna (1), M. Ahonsi (1)

(1) International Livestock Research Institute, Nairobi, Kenya

Phytopathology 105(Suppl. 4):S4.49 
Endophytic and plant associated bacteria were isolated from plant and rhizosplane soils of Brachiaria plants grown in their natural habitat. A total of 110 bacterial strains were isolated from aerial tissues, root tissues and rhizosplane soils on two different media. These strains were identified to the lowest possible taxonomic unit using $16 \mathrm{~S}$ rDNA primers, and were characterized for phosphate solubilization; production of IAA, siderophore and hydrogen cyanide; AAC deaminase activities; antifungal properties; and plant growth promotion. Based on the $16 \mathrm{~S}$ rDNA sequence analysis, these 110 strains were grouped into three phyla, five classes, eight orders, ten families and thirteen genera with frequent occurrence of genus Pantoea $(26 \%)$, Pseudomonas (22\%), Enterobacter (17\%) and Acinetobacter (8\%). The functional characterization showed at least 29 strains $(26 \%)$ with more than three plant beneficial properties. Plant growth promotion tests with Pantoea spp., Shingomonas sp. and Bacillus sp. showed an increase biomass in wheat seedlings. This study demonstrates that Brachiaria hosts diverse bacteria with several desirable functional properties including those that might play key roles in the unique adaptation potential of Brachiaria grasses to drought and marginal soils, and their resistance to pests and diseases. The phylogenetic relationships among the test bacterial strains and their potential for wider agricultural applications are the focus of this presentation.

Spatial pattern analysis of the incidence of strawberry angular leaf spot under outdoor growing conditions in California

C. GIGOT (1), N. McRoberts (1), W. Turechek (2)

(1) Plant Pathology Department, University of California, Davis, CA, U.S.A.; (2) Horticulture Research Laboratory, USDA-ARS, Fort Pierce, FL, U.S.A. Phytopathology 105(Suppl. 4):S4.50

Angular leaf spot (ALS), caused by Xanthomonas fragariae, is a serious disease in California strawberry nursery production because of the quarantine status of the pathogen outside the U.S.A. In addition, substantial yield losses in fruit production fields are possible anywhere strawberry is grown if environmental conditions are conducive. To begin to understand the epidemiology of this disease, we worked in close collaboration with nurseries to characterize the spatial patterns of ALS in different locations in Northern California. At each location, 10 evenly-spaced rows consisting of 20 sampling units spaced 5 $\mathrm{m}$ apart were sampled. Within each sampling unit, 9 leaflets were randomly collected within a $1 \mathrm{~m}^{2}$ quadrat and assessed for disease incidence. The binomial power law regression gave a good description of all 55 data sets collected in $2014\left(\mathrm{R}^{2}=0.94\right)$. The slope of the regression between the $\log _{10}$ of observed variance and the $\log _{10}$ of binomial variance was computed for the first time for ALS in nursery field conditions, and was found to have a quite high value of 1.23 . This is consistent with what is usually observed for plant pathogens with steep dispersal gradients, typically spread during rainfall or overhead irrigation events. Such quantitative characterizations of ALS epidemiology may us allow to unravel and rank pathogen dispersal mechanisms, with the final aim to lead to optimization of disease control.

\section{Application of phosphite inhibits growth of plant fungal pathogens and primes plant defense response \\ U. GILL (1), K. Mysore (1) \\ (1) The Samuel Roberts Noble Foundation, Ardmore, OK, U.S.A. \\ Phytopathology 105(Suppl. 4):S4.50}

Phosphite (Phi) is used as fertilizer or fungicide specifically against plant diseases caused by oomycetes such as Phytophthora spp. The effect of Phi on fungal pathogens is not known. We therefore studied the effect of Phi on rust pathogens such as Puccinia emaculata and Phakopsora pachyrhizi, the casual agents of switchgrass rust and Asian soybean rust, respectively. Application of Phi at different concentrations inhibited spores germination and germ-tubes growth of $P$. emaculata and $P$. pachyrhizi at varying degrees under both in vitro and in vivo conditions. Switchgrass and soybean plants sprayed with Phi exhibited reduced disease symptoms when inoculated with their respective rust pathogens. However, washing off Phi from leaves prior to rust inoculation partially recovered disease susceptibility. Our results indicated roles of Phi in both, priming plant defense and inhibiting fungal growth. A microarray experiment was conducted to understand the molecular mechanism of Phiinduced switchgrass defense. Microarray data revealed a dramatic increase in transcript levels of phenyl propanoid pathway genes, several chitinase encoding genes, and some jasmonic acid induced genes. In addition, reactive oxygen species pathway gene were also induced. We also initiated an RNAseq experiment to identify $P$. emaculata genes whose expression may be affected by Phi treatment. These results will be presented at the meeting.

Management of Phomopsis stem canker in sunflowers

M. GILLEY (1), R. Harveson (2), J. Caroline (3), J. Schaefer (4), S. Meyer (1), J. Nehring (4), M. Kirsch (3), F. Mathew (5), S. Markell (1)
(1) North Dakota State University, Fargo, ND, U.S.A.; (2) University of Nebraska, Scottsbluff, NE, U.S.A.; (3) DAS/ Mycogen Seed, Breckenridge, MN, U.S.A.; (4) CHS Sunflower, Grandin, ND, U.S.A.; (5) South Dakota State University, Brookings, SD, U.S.A.

Phytopathology 105(Suppl. 4):S4.50

Phomopsis stem canker, caused by Diaporthe helianthi, D. gulyae and other $D$. spp. (anamorph - Phomopsis spp.), is one of the major yield-limiting diseases of sunflowers worldwide. Phomopsis stem canker re-emerged as a threat to sunflower production in the United States in 2008, and by 2013, 47\% to $90 \%$ percent of sunflower fields in North Dakota, South Dakota, Minnesota and Nebraska had some level of Phomopsis stem canker. Fungicide recommendations for Phomopsis stem canker in the United States have not been developed. The objective of this study was to evaluate fungicide efficacy and timing for management of Phomopsis stem canker. In 2014, fungicide trials were conducted in Mapleton, ND, Rothsay, MN and Scottsbluff, NE. Trials were arranged in a randomized complete block design with four replications. Boscalid (FRAC 7), tebuconazole (FRAC 3) and pyraclostrobin (FRAC 11) were applied singly and in combination at R1 and R5. Plots were rated for disease severity, incidence, and/or percent wilted plants. Statistical separation of disease ratings occurred between some treatments and the nontreated control at all locations. The most severe disease pressure occurred at Rothsay, MN, where yield of the most efficacious treatment was approximately $70 \%$ higher than that of the non-treated control. Although differences were observed, treatments including the R1 timing and the fungicide pyraclostrobin were among the most efficacious.

Unraveling the Citrus phytobiome: Profiling endophytic microbes with potential to improve tolerance to plant diseases

N. A. GINNAN (1), T. Dang (1), P. Ruegger (1), J. Borneman (1), P. E. Rolshausen (1), G. Vidalakis (1), C. Roper (1)

(1) University of California, Riverside, Riverside, CA, U.S.A.

Phytopathology 105(Suppl. 4):S4.50

There is compelling evidence that the health of an animal or plant is linked to the structure and activities of its associated microbiome. The goal of this project is to characterize the endophytic phytobiome in citrus grown across the United States. We have, thus far, isolated and identified the culturable microbes primarily from the phloem of citrus trees collected from two distinct citrus production areas in southern and central California. In addition, our study included the 142 year-old Parent Washington Navel Orange tree in Riverside, California. We isolated both fungi and bacteria and sequenced the amplified 16S rRNA gene from bacteria and the ITS region of the ribosomal operon from fungi for microbial identification. A culture-independent analysis of the endophytic citrus phytobiome using an Illumina MiSeq platform is also underway. We hypothesize that the citrus entophytes, particularly phloeminhabiting microorganisms, and their associated metabolomes can be used to reduce or prevent colonization of citrus pathogens, such as Candidatus Liberibacter asiaticus, due to their shared ecological niche of the phloem. We are currently experimenting with techniques designed to transfer the endophytic citrus phytobiome from a donor tree to a recipient host tree. We envision that such methods can be used to transfer beneficial phytobiomes from a donor tolerant or disease resistant tree to a recipient host tree to combat citrus diseases.

Characterization of the ITS region of isolates of race 65 of Colletotrichum lindemuthianum collected in different states of Brazil

M. C. Gonçalves-Vidigal (1), M. Coelho (2), R. F. AZEVEDO (1), L. L. Sousa (1), M. Z. Galván (3)

(1) Universidade Estadual de Maringá, Maringá, Brazil; (2) Universidade Estadual de Maringá, Maringa, Brazil; (3) CONICET, Biotecnología, INTA EEA Salta, Salta, Argentina

Phytopathology 105(Suppl. 4):S4.50

Anthracnose, caused by Colletotrichum lindemuthianum, is one of the most destructive diseases of common bean in the world. The populations of $C$. lindemuthianum are comprised of many virulent strains or races. Race 65 of is widespread and it has been reported from various regions of Brazil as well as from other countries of the world. The objective of this study was to characterize different isolates of race 65 by sequencing their ITS region. Seventeen isolates of race 65 collected in the states of Mato Grosso (MT), Minas Gerais (MG), Paraná (PR), Santa Catarina (SC) and São Paulo (SP) states were analyzed and compared with the sequence of race 23 from GenBank. Nucleotide sequence alignment of the internal transcribed spacer (ITS) regions 1 and 2 were amplified by PCR using the primers ITS1F and ITS4. Sequence analyzes from isolates of the race 65 revealed the presence of eleven SNPs in the ITS regions. The phylogenetic tree obtained from sequences of the ITS1 and ITS2 regions revealed the formation of two groups. One group contained most isolates from Santa Catarina and a subgroup 
included another isolate also from Santa Catarina. Five isolates from MT, MG, PR, SC and SP states were included in the second group. The greatest genetic divergence was observed between two isolates from Santa Catarina with a magnitude of 0.772 . Two isolates, one from Mato Grosso and the other from Santa Catarina were the most similar with a genetic distance of 0.0002 .

Reaction of detached leaves of different varieties of sweet orange (Citrus sinensis L. Osbeck) to inoculation with Xanthomonas citri subsp. citri (ex Hasse) Gabriel et al.

A. M. Goncalves-Zuliani (1), K. A. Kern (2), J. Belasque Jr (3), C. A. Zanutto (1), H. T. Hashiguti (2), C. V. Nakamura (2), C. H. Bock (4), W. M. C. NUNES (5) (1) State University of Maringa, Maringa, Brazil; (2) State University of Maringa, Maringa, Brazil; (3) State University of Sao Paulo, Piracicaba, Brazil; (4) USDA, Byron, GA, U.S.A.; (5) Univ Estadual de Maringa, Maringa, Brazil Phytopathology 105(Suppl. 4):S4.51

Asiatic citrus canker (ACC) (caused by Xanthomonas citri subsp citri, Xcc) is a major disease of citrus in wet tropical and subtropical production regions. Screening for resistance is important to breeding programs. The objective of this study was to evaluate a detached leaf method to compare nine different citrus genotypes that exhibit a range of resistance to ACC, and to contrast infection, colonization and symptom development of the most resistant and most susceptible varieties using Scanning Electron Microscopy (SEM). Inoculation was by needle wounding with $\mathrm{Xcc}\left(10^{8} \mathrm{CFU} / \mathrm{ml}\right)$. At 7,10 and 13 days after inoculation (DAI), lesion diameters were measured. The variety Pera IAC was the most resistant to the pathogen (mean lesion diameter in two experiments $=1.213$ and 1.317 , respectively). The variety Washington navel was the most susceptible (mean lesion diameter in two experiments $=1.815$ and 1.838 , respectively), indicating low resistance to Xcc. However, there was variability in lesion size on each cultivar that highlights potential issues with using the pin-prick method compared to other methods used in canker screening. SEM images were taken of lesion development after wounding of the most resistant variety Pera IAC and the most susceptible variety Washington navel at 3,7 and 14 DAI. Pera IAC had smaller stomatal openings and produced a greater quantity of foliar exudates compared with Washington navel. It is possible that the exudates may contain compounds toxic to Xcc, but further research is required to establish any antibiotic effects of the exudates against citrus canker.

Resistance of sweet orange Pera (Citrus sinensis) genotypes to Xanthomonas citri subsp. citri under field conditions

A. M. Goncalves-Zuliani (1), D. S. NANAMI (1), P. T. Nocchi (1), A. A. Frias (1), J. G. Franco (1), C. A. Zanutto (1), C. H. Bock (2), W. M. Nunes (1) (1) Univ Estadual de Maringa, Maringa, Brazil; (2) USDA, Byron, GA, U.S.A. Phytopathology 105(Suppl. 4):S4.51

Citrus canker control is based on protection measures and eradication of plants infected with Xanthomonas citri subsp. citri. Although these measures show satisfactory results, the use of resistant genotypes is an important alternative for citrus canker control. The aim of this study was to evaluate resistance to citrus canker of genotypes of Pera sweet orange under field conditions at three commercial orchards in Congonhinhas, Cornélio Procópio and Paranavaí located in Paraná state, in 2010, 2011 and 2012. Canker incidence and severity was evaluated on 10 plants of each genotype. Four branches were sampled per plant. On each branch the total leaves, diseased leaves, and lesions per leaf were counted, and the severity estimated using using a diagrammatic scale. The results from the experiment at Congonhinhas showed a larger number of resistant genotypes in relation to other locations. EEL and Ovale Siracusa genotypes had greater resistance to citrus canker on leaves with an incidence $<1 \%$ and a severity of 0.00 to $0.40 \%$ on fruit. There were small variations in productivity of the genotypes, indicating the most important factor affecting choice of Pera genotypes to plant in Paraná State may be resistance to X.c. subsp. citri.

Resistance evaluation of Pera (Citrus sinensis) genotypes to citrus canker in greenhouse conditions

A. M. Goncalves-Zuliani (1), P. T. NOCCHI (1), A. A. Frias (1), D. S. Nanami (1), L. S. Soares (1), H. S. Souza (1), C. H. Bock (2), W. M. Nunes (1) (1) Univ Estadual de Maringa, Maringa, Brazil; (2) USDA, Byron, GA, U.S.A. Phytopathology 105(Suppl. 4):S4.51

Citrus canker, caused by the bacterium Xanthomonas citri subsp. citri results in serious yield losses and phytoregulation penalties. The use of resistant genotypes is recognized as an important tool to facilitate control of the pathogen. Studies have show that artificial inoculation results in typical symptoms of citrus canker (using injury, followed by spraying or infiltration of the inoculum into the plant tissue). This study evaluated the resistance of genotypes of the sweet orange variety Pera to $X$. citri under partially controlled conditions (in a greenhouse). A total of 25 Pera genotypes were evaluated in two trials. The leaves were inoculated by needle punching $(0.55 \times$ $0.20 \mathrm{~mm}$ ), infiltration of inoculum and maintaining material uder constant wetness. The inoculum was adjusted to a concentration of $10^{8} \mathrm{CFU} / \mathrm{mL}$ and evaluations were made measuring lesions diameter. Bacterial quantification was performed by counting the Colony Forming Unit (CFU) isolated from each lesion. The results showed that the genotypes EEL Pera and IAC2000/1 had the smallest lesion diameters both assays, moreover, Bianchi/CC Pera and IAC obtained the smallest diameters in the second assay and the lowest bacterial populations, suggesting that these genotypes have a greater level of resistance to the pathogen.

Civitas affects in planta populations of bacterial endophytes, some of which can induce systemic resistance

P. GOODWIN (1), W. Gao (2)

(1) Univ of Guelph, Guelph, ON, Canada; (2) University of Guelph, Guelph, ON, Canada

Phytopathology 105(Suppl. 4):S4.51

Eight bacterial endophyte colony types were cultured from Nicotiana benthamaiana roots, stem+petioles and/or leaves and were identified as six species of Bacillus and two species of Pseudomonas. Populations of the colony types were examined after the addition of water, the commercial mixture of branched-chain alkanes known as Civitas, or the emulsifier that is used to make a solution of Civitas in water. Populations of the B. simplex strain LW4 colony type in roots and stem+petioles, and populations of the Pseudomonas sp. strain LW3 colony type in roots were significantly increased compared to the water and emulsifier controls. Those colony types also grew more in minimal media containing Civitas compared to minimal media alone or minimal media with emulsifier, indicative of alkane catabolism. In contrast, populations of the other six colony types were generally not higher in $N$. benthamiana treated with Civitas or in minimal media containing Civitas compared to the controls. Application of Civitas, B. simplex strain LW4 or Pseudomonas sp. strain LW3 to $N$. benthamiana roots induced systemic resistance against Colletotrichum orbiculare on leaves of seedlings. Civitas applied to roots through the soil appeared to act as a selective endophyte growth promoter, increasing the populations of certain bacterial endophytes that can induce systemic resistance and may have the ability to catabolise alkanes.

Characterization of foliar diseases on processed lima bean in New York State

A. M. GORNY (1), J. R. Kikkert (2), A. R. Dunn (1), H. R. Dillard (3), C. D. Smart (1), S. J. Pethybridge (1)

(1) Cornell University - NYSAES, Geneva, NY, U.S.A.; (2) Cornell Cooperative Extension, Canandaigua, NY, U.S.A.; (3) University of California, Davis, Davis, CA, U.S.A.

Phytopathology 105(Suppl. 4):S4.51

In 2013 and 2014, a new foliar disease was observed affecting processed lima bean (Phaseolus lunatus L.) plants in fields across western and central New York State. Isolations conducted in 2013 from lesions resulted in the fungus Boeremia exigua var. exigua (Desm.) Aveskamp, Gruyter \& Verkley. Additional isolations conducted in 2014 resulted in a Peyronellaea americana-like fungus. The fungi were identified by characterization of morphological characteristics and multiple loci (internal transcribed spacer, partial actin, $\beta$-tubulin, and translation elongation factor $1-\alpha$ ) informative for speciation within the Didymellaceae. Pathogenicity of B. exigua var. exigua was confirmed through repeated inoculations in greenhouse pathogenicity experiments. Inoculations were also conducted to other beans commonly encountered in the cropping rotation demonstrating pathogenicity of $B$. exigua var. exigua to several varieties of snap bean and soybean. To the best of our knowledge, this is the first report of Boeremia exigua var. exigua causing foliar disease on lima bean. The disease is likely to contribute to a foliar disease complex affecting lima bean productivity in New York State. Work to confirm the identity and pathogenicity of the $P$. americana-like species is continuing. Further work will also assess the economic impact of foliar disease on processed lima bean production.

Spread of clubroot on canola in North America, 2003-2014

B. D. GOSSEN (1), S. Strelkov (2), V. P. Manolii (3), T. Cao (3), S. F. Hwang (4), G. Peng (5), M. R. McDonald (6)

(1) Agric \& Agri-Food Canada, Saskatoon, SK, Canada; (2) Department of Agricultural, Food and Nutritional Science, University of Alberta, Edmonton, AB, Canada; (3) Department of Agriculture, Food and Nutrition, University of Alberta, Edmonton, AB, Canada; (4) Alberta Agriculture, Edmonton, AB, Canada; (5) Agriculture and Agri-Food Canada, Saskatoon, SK, Canada; (6) Univ of Guelph, Guelph, ON, Canada

Phytopathology 105(Suppl. 4):S4.51 
Clubroot (Plasmodiophora brassicae) was first reported on canola (Brassica napus) on the northern Great Plains from a cluster of 12 infested fields in the province of Alberta, Canada in 2003. Surveys assessed 41 fields in Alberta for clubroot in 2004, 112 in 2005, and 250 in 2006. Since 2008, more than 400 commercial canola crops in Alberta were examined each year. Also, more than 100 canola crops in both Saskatchewan and Manitoba were conducted in each year since 2008. P. brassicae has spread across large areas of Alberta, with more than 1800 fields infested, and infested fields have been identified in Saskatchewan, Manitoba, and recently North Dakota. Clearly, clubroot is spreading rapidly on the northern Great Plains. In contrast, clubroot is not a problem on canola in Ontario; and is spreading slowly, if at all. Severity at a site only $50 \mathrm{~m}$ from a heavily infested block (severity $~ 100 \%$ ) at the Muck Crops Research Station (MCRS) in Ontario was $<50 \%$. Similarly, severity at nearby sites accessed routinely with equipment from the MCRS were $<20 \% 1$ $\mathrm{km}$ away and $0 \%$ only $4 \mathrm{~km}$ away. Pathogen spread from the MCRS is similar to that reported from Germany, where there is little movement of inoculum from region to region. This difference is likely related, at least in part, to the size of the inoculum source (field size and proximity, resting spore quantity), the pattern of equipment movement, and perhaps even soil type.

\section{Vertical dispersal of resting spores complicates clubroot management} using fumigants

B. D. GOSSEN (1), T. Cramner (2), J. Robson (2), A. Deora (3), F. Al-Douad (2), S. F. Hwang (4), G. Peng (5), M. R. McDonald (2)

(1) Agric \& Agri-Food Canada, Saskatoon, SK, Canada; (2) University of Guelph, Guelph, ON, Canada; (3) Syngenta, Guelph, ON, Canada; (4) Alberta Agriculture and Rural Development, Edmonton, AB, Canada; (5) Agriculture and Agri-Food Canada, Saskatoon, SK, Canada

Phytopathology 105(Suppl. 4):S4.52

Replicated field trials were conducted in Ontario in 2014 to assess the efficacy of the fumigants metam sodium and chloropicrin against clubroot (Plasmodiophora brassicae) in naturally infested fields. The trials were treated, then seeded to a susceptible host that was assessed (0-3) at $6 \mathrm{wk}$ after planting. Label rates of metam sodium provided excellent reduction of clubroot under controlled conditions. However, metam sodium had no effect at a field site where disease pressure was high. In contrast, chloropicrin substantially reduced severity and increased plant growth at that site, where the plots treated with chloropicrin had been covered after application with a totally impermeable film (TIF) for 14 days. At a second site where disease pressure was low, moderate rates of either metam sodium or chloropicrin eliminated clubroot and increased plant growth. In addition, soil cores were collected from four naturally infested sites to assess the vertical distribution of resting spores. Spore concentration was assessed using a multiplex qPCR assay. Concentration generally declined with depth, but spores were present to the bottom of each $50-\mathrm{cm}$ core. We conclude that reductions in severity will be more consistent where spore concentrations are low and treatment includes a TIF cover. The presence of localized high concentrations of spores, and spores dispersed in the soil profile, complicates both fumigation and removal of infested topsoil to minimize spore dispersal.

\section{A new lethal canker disease affecting Paradox walnut rootstock in California}

H. GOURAN (1), R. Bhat (2), R. Beede (3), E. Fichtner (4), J. Hasey (5), R. Buchner (6), G. Browne (1)

(1) USDA-ARS, Davis, CA, U.S.A.; (2) None, Davis, CA, U.S.A.; (3) Division of Agriculture and Natural Resources, Hanford, CA, U.S.A.; (4) UC cooperative extension, Tulare, CA, U.S.A.; (5) Division of Agriculture and Natural Resources, Yuba City, CA, U.S.A.; (6) UC cooperative extension, Red Bluff, CA, U.S.A.

Phytopathology 105(Suppl. 4):S4.52

Virtually all US Persian (English) walnut production occurs in California, and $>70 \%$ of the trees are on Paradox seedling rootstock (mainly Juglans hindsii $\times$ J. regia). Compared to other stocks, Paradox offers greater tree vigor and resistance to some soilborne plant pathogens. Nevertheless, Paradox is subject to crown gall disease incited by Agrobacterium tumefaciens, root rot incited by Armillaria mellea, nematode parasitism, and crown and root rot incited by Phytophthora. Over a 7-yr period, we recognized a new canker disease associated with death of trees on Paradox rootstock that we refer to as lethal Paradox canker (LPC). Symptoms of LPC superficially resemble those of Phytophthora crown rot (continuous dark necrosis and bleeding of bark extending up from the soil into the lower tree trunk), except LPC exhibits with more regular, rounded canker margins. Also, unlike crown rot, LPC has emergent light-brown colored, rounded lobes of new canker activity extending from the periphery of older, darker portions of the canker. Based on our surveys in affected orchards of 8 California counties, LPC has typically killed trees within 2 years of symptom onset, resulting in substantial tree mortality.
Among numerous samples from LPC-affected trees, no recognized pathogens were consistently associated with LPC. We will report on culture-based and culture-independent methods (i.e., shotgun sequencing using an illumina platform) being used to identify an LPC pathogen.

Comparison of weather conditions influencing stripe rust epidemics in the Great Plains, Northwestern and Southern United States

B. S. GRABOW (1), E. D. De Wolf (2)

(1) Kansas State University, Manhattan, KS, U.S.A.; (2) Kansas State Univ, Manhattan, KS, U.S.A.

Phytopathology 105(Suppl. 4):S4.52

Predictive models for stripe rust (Puccinia striiformis f. sp. tritici) were developed for the Great Plains based on historical records of disease in Kansas. These models use regional moisture conditions in the fall and winter to predict severe rust epidemics and are refined with local weather conditions during the growing season. The objective of this study was to determine if variables correlated with stripe rust epidemics in Kansas were also important in the other areas of US. Historical estimates of yield losses caused by stripe rust from four states in the Northwest (NW) and two states in the Southern US were used to evaluate the relationship between weather and stripe rust epidemics. The data set included 60 and 30 cases for the NW and Southern regions, respectively. Correlation and recursive partition analyses were used to identify monthly weather variables important to stripe rust epidemics in the two regions. Results indicate stripe rust epidemics are strongly influenced by fall moisture conditions in the Great Plains, NW and Southern regions of the US. Temperature and relative humidity in January were associated with epidemics in the South. Whereas moisture conditions in February were associated with epidemics in Kansas. May temperature and relative humidity were associated with epidemics in both the NW and Great Plains regions. These results suggest that similar variables are associated with epidemics between these geographically diverse regions.

Effect of fungicides on chlorophyll content and percent green leaf area of winter wheat subjected to high disease pressure in a controlled environment

N. GRAF GRACHET (1), R. Hunger (1), M. Payton (1), J. Edwards (1)

(1) Oklahoma State University, Stillwater, OK, U.S.A.

Phytopathology 105(Suppl. 4):S4.52

Resistant wheat varieties lose green leaf tissue under high foliar disease pressure as a result of the hypersensitive reaction. The objective of this study was to evaluate if foliar fungicide application prevents the reduction of green leaf area (GLA) and relative chlorophyll content (RCC) in resistant varieties when foliar disease pressure is high. In growth chamber experiments, 'Duster' (resistant) and 'OK Bullet' (susceptible) hard red winter wheat varieties were sprayed with the commercial fungicides pyraclostrobin, metconazole, and a pre-mixture of pyraclostrobin plus metconazole. Two days after fungicide application, plants were inoculated with urediniospores of Puccinia triticina (wheat leaf rust). RCC of leaves was determined before and after fungicide application, and percent GLA was determined at the experiment's end. Fungicide sprayed and inoculated plants of both cultivars had similar percent GLA compared to not sprayed and not inoculated plants. By contrast, not sprayed and inoculated plants had significantly less GLA. RCC of sprayed and inoculated OK Bullet plants did not differ from not sprayed and not inoculated plants. Pyraclostrobin- and mixture-sprayed Duster plants were the fungicides that had an RCC similar to not sprayed and not inoculated plants. These results indicate that pyraclostrobin maintained RCC in Duster, and may explain why resistant varieties sometimes show a yield response to a fungicide applied in the presence of foliar disease.

Baseline sensitivities of new fungicides against Phytophthora spp. causing brown rot and root rot of citrus

M. A. GRAY (1), W. Hao (2), H. Forster (3), J. E. Adaskaveg (2)

(1) University of California, Riverside, Chatsworth, CA, U.S.A.; (2) University of California, Riverside, Riverside, CA, U.S.A.; (3) University of California, Riverside, Riverside, CA, U.S.A.

Phytopathology 105(Suppl. 4):S4.52

Phytophthora brown rot and root rot are diseases of citrus worldwide that frequently require cultural and chemical management. Among the main species of Phytophthora on citrus in California (e.g., P. parasitica, P. citrophthora, $P$. syringae, $P$. hibernalis), $P$. syringae is a quarantine pathogen in some countries that restricts trade of citrus with California. We are evaluating three new oomycete-specific fungicides with different modes of action to more effectively manage Phytophthora diseases. Baseline sensitivities for inhibition of mycelial growth were determined using the spiral gradient dilution method and compared to mefenoxam. $\mathrm{EC}_{50}$ values for fluopicolide, mandipropamid, oxathiapiprolin, and mefenoxam were 0.040 to 
$0.080,0.003$ to $0.008,<0.001$, and 0.080 to $0.280 \mu \mathrm{g} / \mathrm{ml}$ for 16 isolates of $P$. parasitica, 0.027 to $0.080,0.002$ to $0.005,<0.0004$, and 0.012 to $0.058 \mu \mathrm{g} / \mathrm{ml}$ for 54 isolates of $P$. citrophthora, and 0.019 to $0.507,0.002$ to $0.011, \leq 0.0002$, and 0.003 to $0.075 \mu \mathrm{g} / \mathrm{ml}$ for 70 isolates of $P$. syringae, respectively. Sensitivities to these fungicides were also determined for other growth stages of selected isolates such as germination of zoospore cysts, as well as sporangium and chlamydospore formation. The high activity of these new fungicides supports their development as treatments on citrus. Additionally, three new modes of action will allow for resistance management and potentially high levels of disease control to meet export standards.

Bioinformatic and regulatory analysis of the iac gene cluster coding for bacterial biodegradation of IAA

I. V. GREENHUT (1), N. N. Maharaj (1), J. C. Scott (2), J. H. J. Leveau (1) (1) University of California-Davis, Davis, CA, U.S.A.; (2) Oregon State University, Madras, OR, U.S.A.

Phytopathology 105(Suppl. 4):S4.53

The indole-3-acetic acid catabolic (iac) bacterial gene cluster confers the ability to degrade the plant hormone indole-3-acetic acid (IAA) into catechol. To understand the evolutionary origins and ecological roles of this gene cluster, we investigated patterns of phylogenetic distribution and divergence of the cluster, as well as regulation of iac gene expression in response to the presence of IAA. We constructed a Maximum Likelihood phylogenetic distance matrix using publicly available IacD amino acid sequences from a representative set of iac-carrying bacterial genera. Multi-Dimensional Scaling analysis of this matrix showed clustering patterns similar to that of RecA protein, indicating a long association of the iac cluster with members of the Alpha-, Beta-, Gammaproteobacteria and Gram (+) Actinobacteria, and with some exceptions to this pattern suggesting horizontal transfer between clades. To further elucidate the regulation of $i a c$ gene expression, we cloned the promoter region of $i a c H$ from an Enterobacter strain upstream of a promoterless $g f p$ gene and demonstrated that the $i a c H$ gene is expressed in a dose-dependent manner in response to IAA. This construct will be used as a GFP-based bioreporter for the bioavailability of IAA in natural environments.

Plant virus biology for advancing STEM teaching

B. Grimberg (1), C. I. POL (1), K. Ehlert (1), F. Menalled (1)

(1) Montana State University, Bozeman, MT, U.S.A.

Phytopathology 105(Suppl. 4):S4.53

Agro-ecology, or the ecology of food systems, encompasses a systems approach to agriculture based on multiple perspectives to solve complex, wicked-defined problems. This approach provides a fertile ground for science teaching that addresses the 21 st Century skills including: i) adaptability, ii) communication, iii) non-routine problem solving, iv) self management, and v) system thinking. A team of scientists, and educators developed an educational module focused on plant virus biology, epidemiology, ecology, and management. The module includes science content, simulations, and hands-on activities. Content is delivered in a multimodal way, using text and audio/video. The module was tested in the context of a teacher professional development program. Survey data attest to the high potential of the module to implement the suggestions of the New Framework for K-12 Science Education such as: the notion of cross-cutting concepts through multiple science disciplines, problem solving, and student engagement in STEM learning using real-world scenarios. Teachers reported that: the module increased their understanding of science content and practice, its multimodal aspect contributed to their understanding, the module can impact the way they teach, hands-on activities and animations can engage students in science learning, and the topic of the module is relevant to G6-12 science teaching. These findings demonstrate that instruction on systemic problems advances STEM teaching.

Susceptibility of apple germoplasm to Silverleaf disease (Chondrostereum purpureum) and effects in fruit yield and quality

D. E. GRINBERGS (1), R. A. France (1), R. J. Chilian (1)

(1) Inst de Investigaciones Agropecuarias, Chillan, Chile

Phytopathology 105(Suppl. 4):S4.53

Chondrostereum purpureum, causing agent of Silverleaf disease, is an important pathogen in fruit trees. In apple, it has been increasing due to the changes in orchards management, such as new varieties and intensive tree training. Curative control and preventive methods are not sufficiently effective; therefore, an option is the use of resistant cultivars. The objective of this research was to determine the susceptibility of different varieties and rootstocks to the fungus, and the effect of the disease in fruit yield and quality. Cuttings of Royal Gala cultivar were inoculated with 18 fungal strains and later, the most virulent, in 35 varieties. Internal necrosis was measured and the presence of the fungus confirmed by molecular techniques. In addition, size, weight, $\mathrm{pH}$, titratable acidity, starch, hardness and micronutrients were measured in fruit from three commercial orchards of Royal Gala and Fuji, from healthy and sick trees. The study was performed for two years. Strains were diverse in their virulence, where necrosis ranged from 2 to $65 \%$ of the cuttings. Royal Gala, Pink Lady and Fuji Raku Raku were among the most susceptible varieties, whereas the most resistant were Brookfield, Granny Smith and Northern Spy. Fruit yield decreased significantly in diseased trees, as well as their maturation was delayed. Likewise, nutrient contents were lesser, leading to physiological disorders in postharvest.

Performance of plant virus nanoparticle formulated abamectin in a range of soil types and application conditions against nematodes

R. H. GUENTHER (1), C. H. Opperman (2), S. A. Lommel (3), D. L. Lindbo (4), J. P. Kerns (2), T. L. Sit (2)

(1) North Carolina State University, Raleigh, NC, U.S.A.; (2) North Carolina State University Department of Plant Pathology, Raleigh, NC, U.S.A.; (3) North Carolina State University College of Agriculture and Life Sciences, Raleigh, NC, U.S.A.; (4) North Carolina State University Department of Soil Science, Raleigh, NC, U.S.A.

Phytopathology 105(Suppl. 4):S4.53

Nanoparticle formulations of agrochemicals may enhance their performance while mitigating adverse environmental effects. Red clover necrotic mosaic virus is a soil-transmitted plant virus that can function as a plant viral nanoparticle (PVN) when loaded with various compounds such as the nematicide abamectin (Abm). During a feasibility study, it was found that PVNs formulated with $\mathrm{Abm}\left(\mathrm{PVN}^{\mathrm{Abm}}\right)$ were able to reduce galling on tomato seedlings from a root-knot nematode challenge by mobilizing and making Abm bio-available in the soil. To assess the potential applications for this technology, the behavior of various PVN formulations was assayed in a range of characterized soils. PVNs were quickly adsorbed to silt and clay rich soils while remaining suspended in the interstitial liquid for weeks when applied to sandy and organic rich soils. PVNs were found to maintain structural integrity for at least two weeks in both sterile and biologically active soils. The mobility of Abm (in three formulations) through $20 \mathrm{~cm}$ soil columns and 10 $\mathrm{cm}$ turf plugs was evaluated over a range of applied concentrations above and below the approved rate. When eluted fractions were tested in a nematode acute toxicity assay, the PVN formulation was bioactive at $1 / 10$ th the approved rate. The commercial formulation (AVID ${ }^{\mathrm{TM}}$ ) was effective but only at 10X the approved rate. These results suggest that PVNs may usher in a new generation of safe and effective formulations.

Seed treatments with fungicides, resistance inducer and hot air to control downy mildew of basil

M. L. GULLINO (1), G. Gilardi (2), A. Garibaldi (2)

(1) University of Torino, Agroinnova, DISAFA, Grugliasco Torino, Italy; (2) University of Torino, Agroinnova, Grugliasco, Italy

Phytopathology 105(Suppl. 4):S4.53

Downy mildew of basil, incited by Peronospora belbahrii, causes severe losses in several countries. By considering that the pathogen is seed transmitted, one possible management strategy is represented by seed treatment. Trials were carried out in order to evaluate the efficacy of different products (chemicals, resistance inducers and natural products) when applied to basil seeds naturally infested by the pathogen. The different products were tested alone and combined with hot air treatment at $60^{\circ} \mathrm{C}$ for 10 minutes. Results showed that many of the seed treatments had a positive effect on the reduction of disease incidence and on plant biomass when disease incidence in the untreated control was lower than $10 \%$ applied by fumigation. Among registered chemicals, thiram and mancozeb reduced disease incidence of $62-$ $91 \%$ and $62-84 \%$, respectively. The efficacy shown by hot air at $65^{\circ} \mathrm{C}$ for 10 $\min (50-87 \%$ efficacy), thyme oil (36-79\% efficacy) and potassium phosphite (47-81\% efficacy) is of special interest for organic farming. Such treatments in general provided significant improvement also in vigour and germinability of seeds. Any treatment completely reduced seed contamination, with the exception of thiram combined with hot air in one out of four trials.

Penicillium expansum and Penicillium griseofulvum are agents of blue mould and produce patulin on pome and stone fruit

M. L. GULLINO (1), D. Spadaro (2), A. Garibaldi (3)

(1) University of Torino, Agroinnova, DISAFA, Grugliasco Torino, Italy; (2) University of Torino, DISAFA, Agroinnova, Grugliasco, Italy; (3) University of Torino, Agroinnova, Grugliasco, Italy

Phytopathology 105(Suppl. 4):S4.53

Different species of Penicillium can cause blue mould on fruit, including Penicillium expansum and $P$. griseofulvum. The virulence and patulin production of eight strains of $P$. expansum and two strains of $P$. griseofulvum were studied on 17 cultivars of pome and stone fruit. The strains tested 
showed a great variability, both in virulence and in patulin accumulation. Virulent strains of $P$. expansum produced larger rots on all the species and cultivars. The strains of $P$. griseofulvum showed a higher virulence on apples, while their virulence was significantly lower on pear, peach, plum, and apricot. 'Golden Delicious' and 'Royal Gala' apples were the most susceptible to $P$. expansum and $P$. griseofulvum. Accumulation of patulin in infected apple tissue and blue mould of eight strains of $P$. expansum tested on four cultivars of apple were negatively correlated. Very high values of patulin contamination corresponded to very low virulence on apples. Moreover, the most virulent strains of $P$. expansum produced lower levels of patulin. Though not as strongly pathogenic as $P$. expansum, the strains of $P$. griseofulvum were high patulin producers, particularly on apples and on apricots 'Aurora'. 'Golden Delicious' and 'Gala' apples were more conducive to patulin contamination. $P$. griseofulvum, besides $P$. expansum, can be a significant patulin producer on apple and that $P$. expansum can contaminate other fruit species, and particularly stone fruit.

EMPHASIS, a European-funded project to provide integrated solutions for the effective management of pests and harmful alien species M. L. GULLINO (1)

(1) University of Torino, Agroinnova, DISAFA, Grugliasco Torino, Italy Phytopathology 105(Suppl. 4):S4.54

EMPHASIS is an international project funded by the European Commission under Horizon2020 Program addressing native and alien pests threats (insect pests, pathogens, weeds) for a range of both natural ecosystems and farming systems. The project consortium gathers 22 partners from 10 countries and it includes research institutes, enterprises, SME's and international organizations bringing cross-sectorial and complementary expertise. The project aims to ensure a European food security system and the protection of biodiversity and of ecosystems services while developing integrated mechanisms of response measures (practical solutions) to predict, to prevent and to protect agriculture and forestry systems from native and alien pests threats. A cross-cutting approach to participatory research and technology transfer is adopted, in order to strengthen the connectivity between agricultural research and other system actors. Thus, on-farm testing and participatory learning activities are being developed since the beginning of the project, in order to facilitate co-design, co-development and coimplementation. The project is not focused on a single management systems but the plant/pest ecosystems dealt with are treated with a multi-method approach to design true IPM methodology that will be developed for key systems with portability to other similar systems, thereby having a large impact.

\section{The population dynamics of coexistence between Cochliobolus sativus and} Fusarium pseudograminearum in wheat

E. GUNNINK TROTH (1), J. Johnston (1), A. Dyer (1)

(1) Montana State University, Bozeman, MT, U.S.A.

Phytopathology 105(Suppl. 4):S4.54

Both Cochliobolus sativus $(C S)$ and Fusarium pseudograminearum $(F p)$ cause substantial losses worldwide through seedling blight and crown rots of wheat. Based on reports of antagonism between the fungi, a study was conducted to examine their coexistence within wheat crowns. For this, co-inoculations of field plots were conducted at three locations using the spring wheat cultivar 'McNeal,' two isolates of $C s$, and four isolates of $F p$. Emergence, plant vigor, yield, disease severity, and pathogen populations in crown tissues as determined by quantitative polymerase chain reaction were measured. The effects the two fungi had on one another's populations varied based on isolate. While inoculation with either $C s$ isolate significantly increased $C s$ populations in crown tissues (both $p<0.001$ ), only $F p$ isolate 2228 significantly reduced $C s$ populations $(p=0.007)$. Conversely, all $F p$ isolates significantly increased Fusarium populations within crowns, while only $C s$ isolate 2344 decreased Fusarium populations $(p=0.003)$. There was no strong correlation between populations of the two fungi $\left(\mathrm{R}^{2}=0.018, p=0.075\right)$. For emergence, disease severity scores, and yield, the effects of inoculations were variable. Both $C s$ isolates significantly reduced emergence (both $p<0.001$ ), while only $F p$ isolate 2228 affected disease severity scores $(p=0.006)$. Yield reductions were only associated with $C s$ populations $(p=0.032)$. These dynamics provide new insight into the interactions of these pathogens.

Understanding pathogenesis of Fusarium oxysporum on Arabidopsis thaliana using comparative transcritptomics

L. GUO (1), K. Vescio (1), P. Travers (1), L. J. Ma (1)

(1) University of Massachusetts Amherst, Amherst, MA, U.S.A.

Phytopathology 105(Suppl. 4):S4.54

Filamentous fungus Fusarium oxysporum (Fox) is the causal agent of vascular wilt diseases on a broad range of crop and forest plants including tomato, cotton, watermelon and banana etc. Fox pathogenicity is host-specific and outcome of Fox-host interaction depends on combination of particular Fox formae speciales and plant species. To understand the molecular basis of Foxhost interaction, we studied infection process of model plant Arabidopsis thaliana (At) and two Fox isolates Fox5176 (pathogenic) and Fox47 (nonpathogenic). In order to identify pathogenicity and host defense related genes involved in Fox infection, we sequenced full transcriptome of $A t$ infected with each of the two isolates at multiple stages during infection: 12hpi (hours post inoculation), $24 \mathrm{hpi}, 48 \mathrm{hpi}$ and 96 hpi using Illumina Hiseq. RNA-seq reads were de novo assembled into transcripts using Trinity and transcript abundance were estimated by RSEM, followed by differential gene expression analysis using DESeq. The fungal and plant genes differentially expressed will help us comprehend the molecular mechanisms underlying fungal pathogenesis and plant response to the pathogen and nonpathogen. In addition, comparative analysis of two Fox transcriptome will reveal critical information on fungal gene expression difference during interaction of pathogenic vs. nonpathogenic Fox with At. We will report and discuss the findings and their significance in this study.

A simple and convenient detached leaf assay using mycelium as inoculum for assessing genotype resistance to boxwood blight

H. GUO (1), R. T. Olsen (2), M. R. Pooler (2)

(1) USDA/ARS U.S. National Arboretum / Rutgers Univ., Beltsville, MD, U.S.A.; (2) USDA/ARS U.S. National Arboretum, Beltsville, MD, U.S.A.

Phytopathology 105(Suppl. 4):S4.54

Boxwood blight is a newly emergent disease of boxwood (Buxus L.) which causes severe defoliation and dieback. Recently published screening assays have employed fungal conidia as inoculum. However, the preparation of conidial inoculum is a time-consuming process, especially when large quantities of inocula are needed and sporulation capacity varies across isolates. In the present study, we used mycelium as inoculum to develop a simple detached leaf assay (DLA). The mycelia inocula were prepared directly from 2-week-old culture plates with vigorously growing mycelia. Ten-mm-diameter agar disks were taken from the growing colony and ground with $1 \mathrm{ml}$ water per disk. The resultant suspension was diluted to the desired concentration for testing optimal dilution rates for both drop and spray inoculation methods. Individual detached leaves on water-agar plates were then inoculated by dropping $5 \mathrm{ul}$ of the suspension on the abaxial leaf surface. Eight boxwood cultivars belonging to 6 species were tested in both inoculum and assay comparisons. In both the DLA and the whole plant assay (WPA), mycelium and conidia resulted in similar susceptibility rankings among the cultivars. The DLA was validated by similar susceptibility rankings with those from the WPA. Therefore, fungal culture mycelium can replace conidia for use as inoculum in screening assays, and our DLA was reliable for assessing genotype resistance to boxwood blight and may be applicable to other fungal pathogens.

Viral suppressors of RNA silencing in Wheat mosaic virus (WMoV)

A. GUPTA K (1), G. L. Hein (2), R. A. Graybosch (3), S. Tatineni (1)

(1) United States Department of Agriculture-Agricultural Research Services (USDA-ARS) and Department of Plant Pathology, University of NebraskaLincoln, Lincoln, NE, U.S.A.; (2) Department of Entomology, University of Nebraska-Lincoln, Lincoln, NE, U.S.A.; (3) United States Department of Agriculture-Agricultural Research Services (USDA-ARS) and Department of Agronomy and Horticulture, University of Nebraska-Lincoln, Lincoln, NE, U.S.A.

Phytopathology 105(Suppl. 4):S4.54

RNA silencing is the most effective antiviral adaptive defense mechanism mounted in higher plants to combat viral infection and proliferation. However, viruses have developed a variety of efficient counter-defense mechanisms by suppression of RNA silencing (VSR) in order to successfully impede the host defense. Wheat mosaic virus (WMoV), the causative of High Plains disease, an economically important disease in wheat (Triticum aestivum L.) and maize (Zea mays L.), is an eriophyid-mite (Aceria tosichella Keifer) transmitted Emaravirus. It harbors an octapartite negative-sense single-stranded RNA genome with monocistronic RNA segments. In this study, we screened the WMoV genome for potential candidates with VSR activity through GFPreporter assays by agroinfiltration. Each open reading frame of WMoV RNAs 2 to 8 was cloned into a $35 \mathrm{~S}$ driven expression vector pCASS4 and transformed into Agrobacterium tumefaciens. Equal amounts of agrosuspensions harboring WMoV ORFs and 35S-GFP were coinfiltrated into Nicotiana benthamiana wild-type and GFP-transgenic $16 \mathrm{c}$ leaves. Agroinfiltrated leaves showed prolonged, albeit weak, green fluorescence in the case of ORFs 5, 7 and 8 and substantially reduced accumulation of GFPspecific siRNAs in the presence of ORFs 7 and 8. Our data suggest that WMoV encodes multiple proteins with weak VSR activity. 
Undergraduate research as a bridge for College of Agricultural Sciences and Natural Resources recruiting

K. GWINN (1), J. Logan (2), A. Vanderpool (3), S. J. Domingo (2), C. A. Beyl (2)

(1) Univ of Tennessee, Knoxville, TN, U.S.A.; (2) University of Tennessee, Knoxville, TN, U.S.A.; (3) Lincoln Memorial University, Harrogate, TN, U.S.A.

Phytopathology 105(Suppl. 4):S4.55

Students who participate in undergraduate research programs become part of a learning community and often make career choices based on that experience. The aim of the BRIDGE program at The University of Tennessee (UT) and Lincoln Memorial University (LMU) was to provide opportunities for undergraduate research based in the USDA-NIFA Priority Areas Faculty in the College of Agricultural Sciences and Natural Resources (CASNR) supervised research projects for six interns from LMU. The two students who have completed the undergraduate program at LMU have pursued MS degrees in Entomology or Plant Pathology. Three students will graduate in May 2015; at least one intends to go to graduate school. Seventeen projects were sponsored for UT students. Most projects were categorized as Sustainable Energy (42\%); fewest projects focused on Childhood Obesity $(10 \%)$. The remaining projects were equally divided among the remaining three priorities (16\% each). Most students were majors from colleges of Arts and Sciences (A\&S) $(58 \%)$ or Engineering $(30 \%)$. The majority of mentors have appointments in CASNR $(53 \%)$ or A\&S (35\%). Of the students who responded to interview requests, all who have graduated are working or are in graduate school in an agriculture or natural resource research area and indicated that undergraduate research shaped their career choices. Career plans for recent graduates were split among careers in sustainable energy, professional schools, and science education.

Epidemiology and management of bacterial spot of almond, caused by Xanthomonas arboricola pv. pruni

S. HAACK (1), L. Wade (2), H. Forster (1), J. E. Adaskaveg (1)

(1) University of California, Riverside, Riverside, CA, U.S.A.; (2) Arysta LifeScience, Cary, NC, U.S.A.

Phytopathology 105(Suppl. 4):S4.55

Bacterial spot of almond, caused by Xanthomonas arboricola pv. pruni (Xap), was first observed at high incidence in California's Central Valley in the spring of 2013. Fruit symptoms are the most common and include amber gumming with internal brown corky lesions. Leaf spots are less common and cankers have not been observed to date. Studies were done to increase understanding and develop management strategies for this new disease. Viable Xap was consistently isolated from symptomatic mummified fruit collected through February, indicating their role in overwintering the pathogen and producing primary inoculum in the spring. In field trials, delayed-dormant (late January) treatments of copper-mancozeb significantly reduced disease incidence, whereas early dormant (November/December) treatments did not. Although copper-mancozeb is a standard in-season treatment for bacterial diseases, copper can be phytotoxic to almond, while mancozeb has label use restrictions. In evaluating alternative in-season treatments, the antibiotic kasugamycin significantly reduced disease incidence, and based on this work, kasugamycin has been accepted into the federal IR-4 program for registration on almonds for bacterial spot management. This research represents the first studies conducted to better understand the epidemiology and management of bacterial spot in California almond orchards.

Population structure of the obligate biotroph Peronospora tabacina, the cause of blue mold of tobacco

D. HADZIABDIC (1), P. Wadl (2), F. Runge (3), J. Ristaino (4), O. Spring (5), R. Trigiano (2)

(1) Univ of Tennessee, Knoxville, TN, U.S.A.; (2) University of Tennessee, Knoxville, TN, U.S.A.; (3) University of Hohenheim, Institute of Botany, Stuttgart, Germany; (4) North Carolina State University, Raleigh, NC, U.S.A.; (5) University of Hohenheim, Institute of Botany, Stuttgart, Georgia Phytopathology 105(Suppl. 4):S4.55

Peronospora tabacina is an obligate parasite that causes blue mold of tobacco. The pathogen rarely reproduces sexually by oospores, hence asexual sporangia are commonly observed form of propagation. Forty-two isolates were genotyped with nine microsatellites to assess population structure of individuals from Europe, the Middle East, and America. Clone corrected data revealed 34 alleles or three-to-five alleles per locus. Genetic variation among five subpopulations accounted for $7 \%$ of total variation indicating low-tomoderate levels of genetic differentiation, high gene flow among these subpopulations, and positive correlation between geographic and genetic distance $(P=0.041)$. Evidence of linkage disequilibrium $(P<0.001)$, indicated that populations contained partially clonal subpopulations. When linkage disequilibrium was tested across five subpopulations, significant deviation from random mating was indicated in the central European subpopulation $(P<0.001)$. The indices of association were not significant for the remaining subpopulations, supporting the hypothesis of mixed sexual/asexual reproduction among individuals. Low-to-moderate genetic variation, and lack of population structure among old and new world isolates could be due to continual gene flow across continents via infected transplant exchange. Despite the limited number of isolates in this study, the nine microsatellites demonstrated usefulness in assessing population structure of $P$. tabacina.

\section{Differential response of sugar maple cultivars to anthracnose}

A. K. HAGAN (1), K. Conner (2)

(1) Auburn Univ, Auburn, AL, U.S.A.; (2) Auburn University, Auburn, AL, U.S.A.

Phytopathology 105(Suppl. 4):S4.55

Sugar maple (Acer saccharum) is a superior shade tree and numerous cultivars are available for landscape use. Anthracnose caused by Discula sp. (teleomorph: Apiognommonia acerina) can reduce the vigor and aesthetics of sugar maple. Sugar maple cultivars were planted in 2001 in north central Alabama site and maintained in park-like setting with limited management inputs. From 2012 through 2014, anthracnose intensity was visually rated at monthly intervals from May through October on a 1 to 10 scale where $1=$ no disease, $2=$ light leaf spotting in lower canopy to $10=$ trees defoliated. In all study years, minimal leaf spotting and no premature defoliation was noted prior to mid-July, while rapid disease intensification occurred between midAugust and mid-September when defoliation levels reached a high of $90 \%$ on Sugar Queen. By October in all study years, symptom severity ranged from light to moderate leaf spotting with a low level of premature defoliation to nearly $100 \%$ defoliation. Autumn Faith, Fall Fiesta, and Legacy sugar maples displayed the least leaf spotting and defoliation along with good fall color. Elevated but still moderate defoliation was seen on Autumn Blush and Steeple. In contrast, defoliation levels in excess of $90 \%$ with no fall color were recorded in all study years for Fairview, Gold Spire, Flax Mill Majesty, Sugar Queen, and Sweet Shadow. Apollo, Bonfire, Endowment, Morton, and Seneca Chief also suffered consistently high defoliation levels.

Yield response and cotton root knot nematode control with seed treatment and granular nematicides on corn

A. K. HAGAN (1), L. Campbell (2)

(1) Auburn Univ, Auburn, AL, U.S.A.; (2) Auburn University, Auburn, AL, U.S.A.

Phytopathology 105(Suppl. 4):S4.55

In the South, cotton root knot nematode (Meloidogyne incognita race 3) causes significant yield loss in corn. In 2013 and 2014, efficacy of Avicta Duo Corn and Poncho/VOTiVO seed treatment nematicides along with the granular nematicide Counter 20G@6.5 lb/A was assessed for cotton root knot suppression and yield response of Armour 704 corn at two irrigated Alabama sites. Cruiser 5FS and Poncho 600 insecticide seed treatments served as negative controls. All seed was treated with Maxim Quattro fungicide. A factorial with the seed treatment as the main plot and granular nematicide as the subplot treatment was used. In 2013, the seed treatments and Counter 20G failed to reduce the root knot reproduction. Higher seedling fresh weights and yields were obtained with Counter $20 \mathrm{G}$ but not the nematicide and insecticide seed treatments. At the South Alabama site in 2014, root knot reproduction ratio was reduced and seeding fresh weight increased with Counter 20G but not the insecticide or nematicide seed treatments. While no yield gains were seen with any seed treatment alone, higher yields were recorded with Poncho 600 or Poncho/VOTiVO but not Cruiser 5FS or Avicta Duo Corn when combined with Counter 20G. For the Central Alabama site, the seed treatments did not impact root knot reproduction, seedling fresh weight, or yield. Higher yields were noted for the Counter 20G-treated than the non-Counter treated corn. Yield gains with Counter 20G ranged from 12 to $22 \mathrm{bu} / \mathrm{A}$.

QTL analysis of minor gene resistance to leaf rust in the CIMMYT spring wheat cultivar, 'Roelfs F2007'

J. P. HAHN (1), I. Fuentes-Bueno (2), R. L. Bowden (2)

(1) Kansas State University, Manhattan, KS, U.S.A.; (2) USDA-ARS Hard Winter Wheat Genetics Research Unit, Manhattan, KS, U.S.A.

Phytopathology 105(Suppl. 4):S4.55

Leaf rust of wheat caused by the fungus, Puccinia triticina, is a major disease of wheat in North America. Breeding efforts to introgress sources of resistance have historically been plagued by a boom-and-bust cycle in which a major, race-specific gene is discovered, deployed, and defeated within a short time of release, effectively wasting genetic gains. An optimal stack of minor, race-nonspecific genes, however, could potentially confer near-immunity to the pathogen via additive and epistatic effects. The CIMMYT spring wheat, 
'Roelfs F2007', is thought to possess multiple minor genes for durable slowrusting resistance to leaf rust. However, the number and locations of the loci are unknown. In this study, Roelfs F2007 was backcrossed with the susceptible Kansas winter wheat cultivar, 'Lakin'. A population of $\mathrm{BC}_{1} \mathrm{~F}_{6}$-derived progeny of this cross was screened for adult-plant resistance to leaf rust over the past three years at nurseries in Kansas and Texas, as well as in greenhouse studies. Genotyping-by-sequencing was used to generate 12,245 markers which were filtered to 4,154 good quality markers. Single marker analysis indicated the presence of resistance QTLs on chromosomes 1BL, 3B, and 7BL. Markers identified in this study may be useful for marker-assisted breeding.

\section{Etiology and management strategies for sour rot on grapes}

M. E. HALL (1), G. M. Loeb (2), W. F. Wilcox (2)

(1) Cornell Univ, Geneva, NY, U.S.A.; (2) Cornell University, Geneva, NY, U.S.A.

Phytopathology 105(Suppl. 4):S4.56

Sour rot, a disease affecting grapes near harvest, has been observed for years with only limited research into its biology or control. It is characterized by the discoloration and rotting of grapes on the vine accompanied by the smell of acetic acid and presence of Drosophila (fruit fly) species. Sixteen samples of grape clusters with sour rot were collected from 12 vineyards in the Finger Lakes region of NY and analyzed for acetic acid and ethanol content. These concentrations were inversely proportional among individual samples, suggesting that alcoholic fermentation of the pulp followed by oxidation of ethanol to acetic acid was occurring within berries. The rinsate from a composite sample of rotting berries revealed large populations of Saccharomyces spp. We successfully reproduced the field symptoms of sour rot in the lab by wounding a healthy berry, inoculating it with $S$. cerivisiae and Acetobacter aceti and simultaneously exposing the berry to D. melanogaster, without which typical symptoms did not develop. Symptoms were notably less pronounced when axenic flies were produced and used comparatively, suggesting a causal role for the insects' normal microflora. In a spray trial, integrating antimicrobials and insecticides provided $50 \%$ control of disease severity, with negligible control from either component alone. In a commercial vineyard, disease incidence and severity were reduced significantly on vines with a VSP versus top wire cordon training system.

Oxathiapiprolin, a new fungicide active ingredient for control of foliar diseases caused by Oomycetes on leafy vegetables

J. E. HAMILL (1), R. Bounds (2), P. Kuhn (3), A. Tally (3), J. Haskell (4), B. Druebbisch (3)

(1) Syngenta Crop Protection, Santa Maria, CA, U.S.A.; (2) Syngenta Crop Protection, Visalia, CA, U.S.A.; (3) Syngenta Crop Protection, Greensboro, NC, U.S.A.; (4) Syngenta Crop Protection, Vero Beach, FL, U.S.A. Phytopathology 105(Suppl. 4):S4.56

Oxathiapiprolin is a new fungicide active ingredient (a.i.) that provides excellent control of downy mildew, Bremia lactucae and Peronospora effusa, on lettuce and spinach, respectively. Currently, California and Arizona combine to produce more than $90 \%$ of the fresh market lettuce and spinach grown in the United States. The estimated cash value of the lettuce and spinach crops in the US currently exceeds $\$ 2.5$ billion. Downy mildew is the most important pathogen in both crops, and multiple sprays are required to manage the disease. Oxathiapiprolin has shown to provide outstanding preventative and residual control of both pathogens when applied at rates as low as $12 \mathrm{GA} / \mathrm{ha}$ on a 7 - to 10-day interval. In field trials, superior disease control has been observed in rotation and, or tank-mix with Actigard ${ }^{\circledR}$, Revus $^{\circledR}$, Ridomil Gold $\mathrm{SL}^{\circledR}$, and phosphorus acid products. Since oxathiapiprolin has high potency and a single site mode of action, there is potential for selection of strains with reduced sensitivity. In order to mitigate this possibility, label directions will incorporate stewardship guidelines on application régimes (use patterns), as well as use of mixtures containing products with different modes of action. EPA registration is anticipated towards the end of 2015, product launch in 2016. Leafy vegetables will be included in the first wave of crops registered.

Detection of Helenium virus $S$ and two distinct isolates of Butterbur mosaic virus in a single plant of Veronica

J. HAMMOND (1), M. D. Reinsel (1), B. E. Lockhart (2), D. Mollov (3)

(1) USDA ARS FNPRU, Beltsville, MD, U.S.A.; (2) University of Minnesota, Minneapolis, MN, U.S.A.; (3) USDA ARS NGRL, Beltsville, MD, U.S.A. Phytopathology 105(Suppl. 4):S4.56

A Veronica plant showing mosaic symptoms was examined by electron microscopy, which revealed particles typical of carlaviruses. RNA extracted from virions partially purified by high speed centrifugation through a $30 \%$ sucrose cushion was used as template for random PCR to produce a viral cDNA library; six distinct cloned amplicons were sequenced. Sequences revealed similarity to Butterbur mosaic virus (ButMV) coat protein (CP) and replicase (RdRp), and Helenium virus $S$ (HelVS) RdRp, while another RdRp fragment showed distant relationships to different carlaviruses. When cDNA was produced using a tagged oligo-dT primer, and the tag primer paired for PCR with a degenerate potexvirus/carlavirus primer, products of a size consistent with carlaviruses were cloned; sequence analysis identified HelVS and ButMV. Use of a ButMV-specific TGB2 forward primer (based on the genome of the Japanese isolate, ButMV-J) yielded an additional ButMV clone distinct from the first. Veronica ButMV-A and ButMV-B have $78 \%$ nt identity to each other, and $82 \%$ and $78 \%$ nt identity to the full genome of ButMV-J (AB517596). Veronica HelVS has 98\% identity to HelVS from the UK (D10474, S71594). The recovery of two distinct clones each of the RdRp and 3'-terminal regions of ButMV; RdRp and 3'-terminal regions of HelVS; and an RdRp clone of as yet unidentified carlavirus from Veronica indicate the presence of a complex mixed infection in this plant. ButMV has previously been reported only from Japan.

Isolates of Turnip mosaic virus collected in Korea showed differences in biological function of certain ORFs

J. HAN (1), J. Chung (1), J. Kim (1), N. Kim (2), C. Jang (2), I. Hwang (2), J. Hammond (3), H. S. Lim (1)

(1) Chungnam National University, Daejeon, South Korea; (2) Central Research Institute of Kyung Nong Corporation, Gyeongju, South Korea; (3) United States Department of Agriculture-Agricultural Research Service, Beltsville, MD, U.S.A.

Phytopathology 105(Suppl. 4):S4.56

In 2014, we performed a nationwide survey in Korea radish fields to investigate the distribution of Turnip mosaic virus (TuMV). In order to investigate variation among Korean TuMV isolates, we determined the full sequence of selected ORFs. Sequence identities between isolates were 91.899.54\% (nucleotide) and $95.55-100 \%$ (amino acid). The helper component protease (HC-Pro) proteins of different isolates were tested for their RNA silencing suppressor activity and subcellular localization in Nicotiana benthamiana. An RNA silencing suppression assay by co-agroinfiltration of HC-Pro with soluble-modified GFP (smGFP) showed that six of nine isolates have reduced RNA silencing suppression activity. The nine isolates showed $>95.5 \%$ amino acid identity, and all six weak silencing suppressor isolates have variable $\mathrm{C}$-terminal domain compared to isolates with strong silencing suppression activity. Formation of large subcellular aggregates of GFP:HCPro fusion proteins in N. benthamiana was only observed for HC-Pro from isolates with strong silencing suppression activity. We also demonstrated that TuMV replication and HC-Pro function in infected plants was reduced by the commercial biocontrol agent 'FarmWorld'.

Characterization of the structure and function of the microbiome in citrus rhizosphere

U. HANDIQUE (1), Y. Zhang (2), N. Wang (2), J. W. Grosser (2)

(1) University of Florida, Lake Alfred, FL, U.S.A.; (2) University of Florida, Lake Alfred, FL, U.S.A.

Phytopathology 105(Suppl. 4):S4.56

Soil microbiomes represent the greatest reservoir of biological diversity known in the world. Microorganisms in the soil are critical to the productivity and sustainability of agricultural ecosystems by affecting plant health, nutrient availability and many other physiological processes. The rhizosphere, the neighboring zone of soil that is influenced by root secretions, can harbor up to $10^{11}$ microbial cells per gram root and thousands of prokaryotic species. The next generation sequencing technology renders it feasible to further understand the microbiomes in soil. The rhizosphere microbiome is known to be affected by the plant type, soil variety, and other environmental factors. Currently, the citrus rhizosphere microbiome and how plant diseases affect citrus rhizosphere microbiome are poorly understood. The goal of my study is to attain a better understanding of the microbiome of citrus rhizosphere. Rhizosphere microbiome is affected by the health status of the plants, e.g., plant diseases. Our current effort is focused on understanding the effect of Candidatus Liberibacter asiaticus (Las), the causal agent of citrus greening disease (or Huanglongbing, HLB), on the citrus rhizosphere microbiome. We will present the metatranscriptomic analysis of the microbial community in the citrus rhizosphere in HLB diseased and healthy Hamlin sweet orange on Cleo. This study will greatly advance our understanding of the microbial community associated with citrus ecosystems.

A Colletotrichum sp. causing root rot in sugar beet (Beta vulgaris)

L. E. HANSON (1), O. T. Neher (2)

(1) USDA ARS, East Lansing, MI, U.S.A.; (2) The Amalgamated Sugar Company, LLC, Boise, ID, Canada

Phytopathology 105(Suppl. 4):S4.56 
In fall of 2014 sugar beets were observed in a field in Washington State with shallow, dark, firm lesions on the surface. When examined under magnification, minute black "dots" were observed on the surface of the lesions. Isolations were made from the lesions and a Colletotrichum species was consistently isolated from all lesions sampled. The Colletotrichum produced black sclerotia on and adjacent to which were produced conidiomata with setae and hyaline, straight spores when grown on Malt extract agar or water agar. When sugar beet stecklings of four different USDA germplasm were inoculated with $10 \mathrm{ml}$ of a spore suspension of $10^{4}$ spores per $\mathrm{ml}$ as a soil drench, all germplasm developed symptoms by 5 weeks after inoculation. One germplasm had less than $10 \%$ of the root surface with lesions while the other three germplasm all had $40-50 \%$ of the root surface with shallow, dark firm lesions with minute black "dots". A Colletotrichum sp. that was morphologically identical to the initial isolate was re-isolated from inoculated plants. Control plants (a soil drench with sterile water) did not show symptoms and did not yield Colletotrichum. Colletotrichum has not previously been reported on sugar beet in the United States and the Colletotrichum isolates are not $C$. dematium, the species reported on beet in other countries. An additional field in Idaho was subsequently identified with similar symptoms and research is ongoing to determine the species involved.

\section{Xylella fastidiosa chemosensory-like PilG protein involvement in Pierce's} disease

L. Hao (1), L. Cursino (2), K. Johnson (3), T. J. Burr (3), P. MOWERY (2)

(1) Cornell University, Geneva, NY, U.S.A.; (2) Hobart \& William Smith Colleges, Geneva, NY, U.S.A.; (3) Cornell University / New York State Agricultural Experiment Station, Geneva, NY, U.S.A.

Phytopathology 105(Suppl. 4):S4.57

Xylella fastidiosa is a Gram-negative, xylem-limited bacterium that causes Pierce's disease of grapevines. X. fastidiosa is transmitted to plants by insect vectors and once in the xylem, $X$. fastidiosa is postulated to migrate, aggregate, and form biofilm that clogs the vessels leading to Pierece's disease. $X$. fastidiosa performs flagella-independent twitching motility along surfaces using a polar localized type IV pili. Movement of bacteria is often regulated by a chemotaxis system, which allows the organism to sense stimuli in the environment and move towards the desired location. We previously began characterization of a chemotaxis-like operon, pil-chp, which is required for $X$. fastidiosa movement and Pierce's disease development. Based on analogy to other chemotaxis systems, it is proposed that xylem chemical stimuli bind the chemoreceptor, which leads to autophosphorylation of a kinase that phosphorylates the Pil-Chp response regulator protein, PilG. Phosphorylated PilG is proposed to stimulate type IV pili motors and motility. We deleted the $X$. fasitiodsa pilG and found that multiple key virulence factors involved in disease development were reduced. Therefore the $X$. fastidiosa PilG has multiple regulatory functions and is a pivotal protein in Pierce's disease.

\section{Characterization the role of a virulence factor PD1311 in Xylella fastidiosa}

L. HAO (1), P. Mowery (2), L. C. Parent (2), K. Johnson (3), T. Burr (1)

(1) Cornell University, Geneva, NY, U.S.A.; (2) Hobart and William Smith Colleges, Geneva, NY, U.S.A.; (3) none, Washington DC, WA, U.S.A. Phytopathology 105(Suppl. 4):S4.57

Xylella fastidiosa $(X f)$ induces Pierce's Disease (PD) of grapevines, which leads to significant economic losses, and there are limited management options for PD. We deleted PD1311 in Xf Temecula ( $\triangle$ PD1311) and found the mutant is avirulent on grapevines. Additionally, when grapevines were pretreated with $\triangle \mathrm{PD} 1311$, wild-type (WT)-induced PD was suppressed suggesting that $\triangle \mathrm{PD} 1311$ has promise as a biocontrol. The PD1311 protein belongs to the fatty acyl-coA synthetase family, which is involved in metabolic pathways such as phospholipid biosynthesis of membrane structures. Genes upstream of PD1311 are annotated as lipopolysaccharide (LPS) associated enzymes, and therefore we hypothesize that deletion of PD1311 alters $X f$ LPS structure. On PD3 plates, $\triangle$ PD1311 is more sensitive to $100 \mathrm{mM}$ and $500 \mathrm{mM}$ hydrogen peroxide compared to the WT cells. In addition, $\triangle \mathrm{PD} 1311$ is at least 8 -fold more sensitive to polymyxin $\mathrm{B}(\mathrm{PB})$ compared to the WT strain; addition of $2 \mu \mathrm{g} / \mathrm{ml}$ of PB to PD3 plate completely suppressed $\triangle \mathrm{PD} 1311$ growth, while no suppression was observed for WT $X f$ with PB as high as $16 \mu \mathrm{g} / \mathrm{ml}$. These data suggest that $\Delta \mathrm{PD} 1311$ has compromised LPS integrity and may have a defective lipid A structure, which is the target of PB. Characterization of $\triangle \mathrm{PD} 1311 \mathrm{LPS}$ and whether this change results in a different innate immune response in grapevines is ongoing. Further understanding of PD1311 function will help to advance the mutant strain as a biocontrol agent for PD.
Distribution and frequency of Phytophthora species causing brown rot of citrus in the Central Valley of California

W. HAO (1), T. D. Miles (2), F. N. Martin (2), H. Förster (1), J. E. Adaskaveg (1)

(1) University of California, Riverside, CA, U.S.A.; (2) USDA-ARS, Salinas, CA, U.S.A.

Phytopathology 105(Suppl. 4):S4.57

Citrus brown rot, caused by several species of Phytophthora, is a serious concern for the California citrus industry because one of the species, $P$. syringae, is a quarantine pathogen for the Chinese export market. To maintain trade, brown rot management strategies have to be re-evaluated; and to estimate the risk of exporting a quarantine pathogen, the distribution and frequency of $P$. syringae in the main citrus growing areas in the Central Valley of California need to be investigated. In orchard surveys, fruit $(>20$ per orchard) with brown rot symptoms were collected from 28 Navel (winter samplings) and 20 Valencia (summer samplings) orange orchards from 2013 to 2015. For isolation of Phytophthora spp., a selective medium (PARPH-V8) was used. Species were initially identified by morphology, ITS sequencing, and species-specific banding patterns using three RAPD primers. Subsequently, a TaqMan PCR assay targeting a mitochondrial locus with species-specific probes was validated to detect Phytophthora spp. in DNA extracts from fruit tissue. In winter samplings, ca. $75 \%$ of isolates were identified as $P$. syringae, and ca. $25 \%$ were $P$. citrophthora. In summer samplings, only $P$. citrophthora was recovered. These results demonstrate that $P$. syringae is currently a major pathogen causing brown rot of citrus in California during the winter months, emphasizing the importance of an integrated management program to keep the incidence of brown rot at low levels.

Seasonal phytobiome alterations associated with wild and cultivated cranberry fruit

A. HARRISON (1), M. Mohabbatizadeh (1), S. Soby (1)

(1) Midwestern University, Glendale, AZ, U.S.A.

Phytopathology 105(Suppl. 4):S4.57

Cranberries are an important North American crop. While genetically similar and grown in the same geographic regions as native cranberry plants, cultivated cranberries have a much higher incidence and severity of disease. We hypothesize that bacterial populations associated with wild cranberry plants play some role in suppressing fruit disease in native cranberry ecosystems. As part of a large survey of the bacteria associated with native and cultivated cranberry plants, bacteria were aseptically cultured from cranberries growing in wild and cultivated bogs in eastern Massachusetts. Berries were collected over several years to determine the composition of the phytobiome as the growing season progressed. 16S rRNA sequences were determined from berry surface isolates and compared with the NCBI nucleotide database to suggest taxonomic relationships among isolates. Early season (June) flower and berry isolates included the genera Aquitalea, Lysinibacillus, Bacillus, Pseudomonas and Paenibacillus; pre-harvest (October) isolates included Bacillus, Paenibacillus, Leifsonia, Xylophilus, Pseudomonas, Ewingella, Erwinia and Curtobacterium. Overwintered berry surface bacteria included Methylobacterium, Bacillus, Neisseria, Sphingomonas, Burkholderia and Frondihabitans. The cultivated berry phytobiome is more constant throughout the seasons, and include Bacillus, Delftia, Stenotrophomonas and Pantoea.

Degradome sequencing reveals history and diversity of Citrus leprosis viruses in preserved specimens

J. S. HARTUNG (1), A. Roy (2), S. Fu (3), J. Shao (1), W. L. Schneider (2), R. H. Brlansky (4)

(1) USDA ARS MPPL, Beltsville, MD, U.S.A.; (2) USDA-ARS, Frederick, MD, U.S.A.; (3) USDA ARS, Beltsville, MD, U.S.A.; (4) Univ of Florida, Lake Alfred, FL, U.S.A.

Phytopathology 105(Suppl. 4):S4.57

historical disease problem. Two distinct viral genera are associated with ringspot symptoms on fruit, stems and leaves of citrus commonly referred to as leprosis: CiLV-N (Dichorhavirus) and CiLV-C (Cilevirus). CiLV-C is widespread in the new world but the distribution of CiLV-N is more restricted. Neither virus occurs in the USA, although leprosis disease was originally described from Florida and was a major problem for Florida in the first half of the $20^{\text {th }}$ century after which time the disease disappeared. The US National Fungus Collection at Beltsville, MD, maintains samples of diseased citrus that document the interception of restricted pathogens at border inspection stations. This includes samples identified at the inspection stations 50 or more years ago as citrus leprosis. We have applied RNA degradome sequencing to selected samples. Sufficient RNA was obtained from 3-4 leprosis lesions from orange peels. Although the RNA quality was very poor 
$(\mathrm{RIN}<2)$, it was sufficient for RNA sequencing with Illumina technology. We have obtained near full length genome sequences of both a typical CiLV-C isolate intercepted from Argentina in 1967 (positive sense ssRNA genome) and a novel CiLV-N isolate obtained in Florida in 1948 (negative sense ssRNA genome). We have also assembled significant genome sequence data and documented the presence of CiLV-N in Mexico in the mid $20^{\text {th }}$ century.

\section{Dry rot canker disease of sugar beet in Nebraska}

R. M. HARVESON (1), M. D. Bolton (2)

(1) Univ of Nebraska, Scottsbluff, NE, U.S.A.; (2) USDA-ARS, Fargo, ND, U.S.A.

Phytopathology 105(Suppl. 4):S4.58

Dry rot canker (DRC) is a root disease of sugar beet that was first identified from Utah in 1921, and since that time has been identified from most sugar beet-growing areas of the western U.S. The disease was traditionally assumed to be caused by a strain of Rhizoctonia solani, the pathogen causing the wellknown Rhizoctonia root and crown rot (RRCR) disease. DRC has now been proven to be induced by s binucleate specie of Rhizoctonia (AG-F) after sequence analysis of the internal transcribed spacer region Foliar symptoms of DRC are similar to those of RRCR, consisting of yellowing and wilting. However, root symptoms are distinct and serve as the major method for distinguishing between the two diseases. Lesions on roots are dry, sunken, and circular to oblong in shape, additionally producing a recognizable series of concentric circles. Beneath surface lesions is a brown spongy material that penetrates deeply into taproots and is sharply demarcated from healthy tissue. Continued analyses of isolates found infesting more than a dozen fields throughout western Nebraska indicate that DRC is a separate disease caused by a unmistakably different pathogen from $R$. solani. Historically, this disease has been very rare in occurrence, however our ongoing investigations are revealing a much greater incidence and distribution of the disease than previously thought.

\section{Assessment of Calonectria pseudonaviculata microsclerotia survival in} compost over varying times and temperatures

R. HARVEY (1), J. Pecchia (2), D. Davis (2)

(1) Penn State University, State College, PA, U.S.A.; (2) Penn State University, University Park, PA, U.S.A.

Phytopathology 105(Suppl. 4):S4.58

Boxwood blight is a new and emerging disease caused by Calonectria pseudonaviculata. First observed in Connecticut, the disease has now been confirmed in at least 15 U.S. states and 3 Canadian provinces. Boxwood blight is of serious concern to nurseries due to the devastating monetary losses associated with this disease. In addition to boxwood, other species in the Buxaceae family are affected, including Pachysandra spp. and Sarcocca spp. Recently, concern has been raised that if diseased plants were composted, microsclerotia might survive the process. If the compost was then applied near susceptible species, they could become infected with the pathogen. Microsclerotia survival was investigated utilizing three composting bioreactors that were housed inside high temperature incubators, allowing for precise control of compost temperatures and oxygen levels. Cellophane placed on top of glucose yeast extract tyrosine agar in petri dishes was used to harvest large numbers of microsclerotia. Microsclerotia were sealed in a 38 $\mu \mathrm{m}$ stainless steel mesh pouches and placed in compost at various temperatures and for various times. Survival was determined based on the percent mortality of microsclerotia in each pouch.

\section{An ecological study of endophytes from Mahonia aquafolium \\ P. HATCH (1), B. Geary (1), C. R. Sweeney (1), T. Smart (1) \\ (1) Brigham Young University, Provo, UT, U.S.A. \\ Phytopathology 105(Suppl. 4):S4.58}

Mahonia aquifolium, commonly known as the Oregon Grape, is known to produce berberine, an antimicrobial which may a play a role in the survival of M. aquifolium. The object of this research was to determine the ecological diversity of endophytes contained in M. aquifolium compared to endophytes in other plants of the Mahonia genus. Plant samples were collected from multiple sites along Utah's Wasatch Front. Roots, stems and leaves were washed in $50 \%$ bleach and then sterilized aseptically before culturing all extracts on water agar. Over the course of 3 weeks, all endophytes were isolated and cultured on 1/10 PDA and water agar. Twenty-two isolates were characterized based on hyphae structure using light microscopy and scanning electron microscopy. Spore formation, rate of growth, color, setpa, and other characteristics were measured. After identifying the isolates morphologically, bioactivity was measured by using assay-guided procedures, including plating the various endophytes of $M$. aquifolium with known pathogenic fungi (fusarium, pythium, rhizoctonia, etc) and screening M. aquifolium endophytes for zones of inhibition.
Fitness of Microdochium majus and M. nivale field isolates with resistance to QoI fungicides

Y. HAYASHI (1), S. Akino (1), N. Kondo (1)

(1) Hokkaido University, Sapporo, Japan

Phytopathology 105(Suppl. 4):S4.58

Microdochium spp. are one of several pathogens that cause head blight in cereals. Recently, resistant strains to the strobilurins were reported in northern Japan. It is needed the estimate of fungicide resistant frequency in populations to evaluate the risk of development of fungicide resistance. We therefore investigated the fitness of strobilurin-resistant strains of $M$. majus and $M$. nivale. The mycelial growth, conidia germination, sporulation ability and stability of resistance were measured in vitro. A significant reduction in conidial germination of resistant strains was observed compared to that of the sensitive isolate, whereas there was little difference in the mycelial growth and sporulation ability on a strobilurin-free medium between resistant and sensitive strains. However, resistant strains of M. majus produced a great deal of spores compared to that of $M$. nivale on medium containing strobilurin at $1 \mathrm{ppm}$. This ability might have led to the situation that the predominance of $M$. majus in Microdochium populations in northern Japan. Furthermore, strobilurin resistance was not significantly reduced from the first generation to the fifth generation that were grown and preserved on a strobilurin-free medium. The results showed that there is no significantly competitive disadvantage of resistant strains compared to sensitive ones, suggesting that the strobilurin resistance of Microdochium spp. is stable in the absence of the fungicide.

Using rapid-cycling Brassica species and Albugo candida to teach concepts of plant disease resistance

M. HAYSLETT (1), V. Kartanos (2), D. I. Rouse (3), P. Williams (2)

(1) Univ of Wisconsin, Madison, WI, U.S.A.; (2) University of Wisconsin, Madison, WI, U.S.A.; (3) University of Wisconsin-Madison, Madison, WI, U.S.A.

Phytopathology 105(Suppl. 4):S4.58

Resistance is generally considered in plant pathology to be the best way to manage plant diseases and we focus on this concept in our introductory plant pathology course. Lab exercises on this topic concentrate on single gene, complete resistance of a single plant genotype to a single pathotype. We created a lab activity to allow students to see a diversity of interaction phenotypes based on a genetically diverse population of hosts. The activity involved inoculating populations of two species of rapid-cycling Brassica (developed by the Wisconsin Fast Plants ${ }^{\circledR}$ program) with one isolate of Albugo candida. One species population (B. juncea) had no resistance and the other (B. rapa) had both single gene and partial resistance. Our learning objectives were for students to (1) identify differences in interaction phenotype, (2) use interaction phenotype to make predictions about the molecular mechanisms responsible, (3) use terms related to resistance correctly, and (4) analyze and explain experimental results. We assessed the effectiveness of the activity by rating student lab manuals and conducting a student survey. The results of the lab manuals and survey show that the students understood the purpose of the activity. The activity also aided student learning on the specified objectives. The activity could be improved by spending more time discussing the purpose and the results. We will also make improvements to the disease rating scale to make it easier to use.

The population structure of Fusarium oxysporum f sp. fragariae, cause of Fusarium wilt of strawberry, in California

P. HENRY (1), T. R. Gordon (2), S. Kirkpatrick (2)

(1) Univ of California, Davis, CA, U.S.A.; (2) University of California, Davis, Davis, CA, U.S.A.

Phytopathology 105(Suppl. 4):S4.58

Fusarium wilt of strawberry, caused by Fusarium oxysporum $\mathrm{f}$ sp. fragariae (FOF), was first detected in California in 2006 in fields where flat fumigation with methyl bromide and chloropicrin was no longer used. Although initially limited to Ventura Co., the disease is now present in all major production areas in California. The objectives of this study were to investigate the structure of the FOF population in California and to evaluate methods for its detection. Fifty-nine FOF isolates were collected from diseased strawberry plants and their identity was confirmed by pathogenicity testing. The full intergenic spacer (IGS) and elongation factor 1-alpha (EF1a) region were amplified by PCR and sequenced to elucidate the phylogenetic relationships among isolates. IGS and EF sequences revealed three main lineages and isolates within lineages were all somatically compatible. Primers designed to detect FOF in Japan amplified a 239bp product for 55 out of 59 isolates and did not amplify a product for any non-pathogenic isolates obtained from infested fields. The sequence of this PCR product is identical to the sequence obtained in Japan and its presence in isolates associated with two of the three 
lineages defined by IGS and EF sequences. Our findings show the $\mathrm{CA}$ population of FOF to be polyphyletic. It was most likely was established through multiple introductions, at least one of which may have come from Japan.

Effect of wetness duration on the spread of three species in the sooty blotch and flyspeck complex in an Iowa apple orchard

E. HERNANDEZ (1), J. C. Batzer (2), H. Rolsi (1), M. L. Gleason (1)

(1) Iowa State University, Ames, IA, U.S.A.; (2) Iowa State Univ, Ames, IA, U.S.A.

Phytopathology 105(Suppl. 4):S4.59

To test the hypothesis that cumulative leaf wetness duration (CLWD) affects spread of sooty blotch and flyspeck (SBFS), an Iowa apple orchard was equipped with overhead sprinkler irrigation. Eight 3-tree plots received 3 nightly wetness levels: $11 \mathrm{~h}, 6 \mathrm{~h}$, or $0 \mathrm{~h}$. CLWD was monitored from inoculation to harvest. Two weeks after petal fall, 45 apples per tree were covered with fruit bags. On 21 July, 5 non-inoculated apples per tree were left in bags and 10 were inoculated with either Peltaster sp. P2 (P2), Microcyclosporella mali (RH), Stomiopeltis sp. RS2 (RS), or water after debagging. At harvest, colonies per apple (CPA) were counted on all inoculated fruit and on 30 non-inoculated, non-bagged apples per plot. No SBFS colonies appeared on apples that were bagged all season. CPA of inoculated apples was not affected by CLWD. On non-inoculated trees, CLWD did not affect CPA. For water inoculated apples on inoculated trees, however, isolate $\mathrm{P} 2$ increased by 0.085 CPA per hour increase in CLWD $\left(\mathrm{R}^{2}\right.$ $=0.52$ ). Similarly, for non-inoculated non-bagged apples on inoculated trees, P2 increased 0.67 CPA per hour increase in CLWD $\left(\mathrm{R}^{2}=0.7\right)$. CPA of P2 from $11 \mathrm{~h} /$ day irrigation plots was 10 -fold higher than in the other irrigation treatments. CPA of RH and RS2 was not affected by CLWD. The study will be repeated in 2015 .

Identification and pathogenicity of Fusarium sp. associated with yellowing and wilting of blackberry (Rubus sp.) in Michoacan, Mexico

A. Hernandez-Cruz (1), A. REBOLLAR-ALVITER (2), H. V. Silva-Rojas (3), T. B. Urbina-Cortes (4)

(1) Instituto Tecnológico del Valle de Morelia, Morelia, Mich, Mexico; (2) Univ Autonoma Chapingo, Morelia, Mich, Mexico; (3) Lab. de Biotecnología de Semillas, Colegio de Posgraduados, Montecillo, Texcoco, Mexico, Mexico; (4) Lab. de Biotecnología de Semillas. Colegio de Posgraduados, Montecillo, Mexico, Mexico

Phytopathology 105(Suppl. 4):S4.59

Yellowing and wilting of blackberry (Rubus sp.) is a recent disease causing considerable losses in production. The objective of this research was to determine the causal agent of yellowing and wilting of blackberry in Michoacan, Mexico. Samples of tissue from the vascular system of symptomatic plants were obtained and placed in Petri dishes containing PDA medium. Pure isolates were used for phylogenetic and morphological identification, and pathogenicity tests. Complete ITS region including the 5, end of 28S rDNA, and translation elongation factor alpha 1 gene were PCRamplified using primers ITS5 / NL4, and EF-446F / EF1-1251R, respectively. The amplified fragments were sequenced and compared with those deposited in Genbank by performing a BLASTN. Pathogenicity tests were completed on 2-months old plants (cv tupy), by two methods: a) pruning the plants at ground level, then a applying $1 \mathrm{ml}$ of a suspension of $1 \times 10^{6}$ conidia $/ \mathrm{mL}$, and b) excising the lower part of plant roots and immersing in a conidial suspension. Sequences comparison indicated that all isolates corresponded to Fusarium oxysporum with $100 \%$ identity with accessions JX244011, EU124522 HQ130713 from USA, Austria and Mexico respectively. Plants inoculated as in the first method, showed the typical symptoms 8 months after inoculation, including the black streak on the base of the cane. Inoculated plants, as in the second method, died 2 months after inoculation.

Invasion and saturation risk of pathogens and pests based on global cropland connectivity: The case of banana, cassava, potato, sweetpotato, and yam

J. F. HERNANDEZ NOPSA (1), Y. Xing (2), J. Andrade-Piedra (3), F. Beed (4), G. Blomme (5), M. Carvajal Yepes (6), D. Coyne (7), G. A. Forbes (8), J. Kreuze (9), J. Kroschel (10), L. Kumar (11), J. Legg (12), M. Parker (13), E. Schulte-Geldermann (13), K. A. Garrett (14)

(1) University of Florida and Kansas State Univ, Manhattan, KS, U.S.A.; (2) University of Florida and Kansas State University, Gainesville, FL, U.S.A.; (3) International Potato Center (CIP), Quito, Ecuador; (4) AVRDC - The World Vegetable Center, Bangkok, Thailand; (5) Bioversity, Kampala, Uganda; (6) International Center for Tropical Agriculture (CIAT), Cali, Colombia; (7) International Institute of Tropical Agriculture (IITA), Nairobi, Kenya; (8) International Potato Center (CIP), Beijing, China; (9) International
Potato Center (CIP), Lima, Peru; (10) International Potato Center (CIP) Global Crop Diversity Trust, Filderstadt, Germany; (11) International Institute of Tropical Agriculture (IITA), Ibadan, Nigeria; (12) International Institute of Tropical Agriculture (IITA), Dar es Salaam, Tanzania; (13) International Potato Center (CIP), Nairobi, Kenya; (14) University of Florida (UF) and Kansas State University (KSU), Gainesville, FL, U.S.A.

Phytopathology 105(Suppl. 4):S4.59

The geographic connectedness of croplands plays a major role as a risk factor for the invasion of crop-specific pathogens, and the risk of saturation by endemic pathogens. Understanding these network structures supports sampling and management strategies. We evaluated global networks of five vegetatively-propagated crops (banana/plantain, cassava, potato, sweetpotato, and yam) that are key to food security. Diseases transmitted through vegetative propagation are notoriously damaging. We analyzed the structure of the global crop networks, where the potential link between geographic location pairs was evaluated using a gravity model, as a function of the distance between the pair of locations and the product of the harvested crop area in the two locations. Networks were evaluated using a novel index of pathogen or pest invasion and saturation risk, based on the role of locations in bridging cropland areas and the degree of connectedness of a location and its neighbors. For example, in addition to high risk locations with high cropping density, locations with high risk because of their role as bridges for cassava include South-Central Nigeria, Central Ghana, and Southwestern Democratic Republic of Congo. For potato, bridges include Central and Southern Poland and Northern Ukraine. The highly-linked hub and bridge locations we identified are likely priorities for surveillance and management, and for tracing intra-region movement of diseases.

Impact Network Analysis of potato seed systems: The CONPAPA case in Ecuador

J. F. HERNANDEZ NOPSA (1), J. Andrade-Piedra (2), G. A. Forbes (3), L. Montesdeoca (4), F. Montesdeoca (5), K. A. Garrett (6)

(1) University of Florida and Kansas State Univ, Manhattan, KS, U.S.A.; (2) International Potato Center (CIP), Quito, Ecuador; (3) International Potato Center (CIP), Beijing, China; (4) CONPAPA, Ambato, Ecuador; (5) Universidad Central del Ecuador, Quito, Ecuador; (6) University of Florida (UF) and Kansas State University (KSU), Gainesville, FL, U.S.A.

Phytopathology 105(Suppl. 4):S4.59

The potato seed system-a multilayer network-includes social, economic, and agroecological components (e.g., market prices, disease outbreaks, IPM, and seed production). Seed and ware potato producers, breeding institutions, technical support, pests, and diseases are part of this complex system. An "impact network" is the linked socioeconomic and biophysical networks through which new technologies can impact system outcomes. An "Impact Network Analysis" (INA) is an evaluation of how impact networks function in a particular setting such as seed systems, and where the control points for improvement are. We apply INA to understand interconnectivity and impacts on the potato seed system of the Consortium of Small Potato Producers (CONPAPA) in Ecuador, evaluating the implications of the CONPAPA structure for sampling, IPM, and farmer decision making. Identification of key nodes and control points that influence the success of seed systems (e.g., farmers, farms, information sources) supports enhancement of the system (e.g., maximizing the distribution of new seed varieties using fewer distribution channels, managing disease outbreaks, and targeting improvement of communication and infrastructure). Resources can be invested in particular nodes to improve practices and to control pest and disease outbreaks, leading to improvements in the seed system. We present results for CONPAPA along with general recommendations for improving seed system structure.

Anaerobic soil disinfestation disease control performance in strawberry as influenced by environmental variables

S. S. HEWAVITHARANA (1), C. Shennan (2), J. Muramoto (2), M. Mazzola (3)

(1) Washington State University, Wenatchee, WA, U.S.A.; (2) University of California-Santa Cruz, Santa Cruz, CA, U.S.A.; (3) USDA ARS, Wenatchee, WA, U.S.A.

Phytopathology 105(Suppl. 4):S4.59

Sustainability of the California strawberry industry is challenged by soil-borne diseases caused by Fusarium oxysporum (Fo), Macrophomina phaseolina (Mp) and Verticillium dahliae (Vd). Anaerobic soil disinfestation (ASD) has been studied as a non-fumigant measure for control of these diseases. This study examined relationships between ASD temperature and duration, and ensuing levels of disease control and plant growth performance. Soils naturally infested with one of the three pathogens were subjected to ASD using rice bran under four temperature regimes of 3 or 6 week duration. Soils were planted with strawberry and grown in the greenhouse for 3-5 months. 
Wilt scores, cumulative yield, fresh total/shoot/root biomass (TB/SB/RB), and percent crown infection (PCI) were obtained at harvest. ASD reduced soil density and wilt score of Fo, Mp and Vd. Significantly higher cumulative yields were obtained in Fo and Vd soils in response to ASD, with duration of the ASD treatment affecting suppression of Vd. ASD treatment resulted in significantly higher TB in Fo infested soil. ASD significantly improved SB and $\mathrm{RB}$ in $\mathrm{Mp}$ and $\mathrm{Vd}$ infested soils. Incubation temperature only influenced SB in Vd soil. Efficacy of ASD, as assessed by PCI, was influenced by incubation temperature for $\mathrm{Fo}$ and $\mathrm{Mp}$, and by duration for $\mathrm{Mp}$ and $\mathrm{Vd}$. Knowledge of how these environmental variables alter ASD efficacy is required to optimize its implementation for control of soil-borne strawberry diseases.

Detached-leaf assay for assessing variation in pathogenicity of the sugarcane orange rust pathogen (Puccinia kuehnii) in Florida

M. HINCAPIE (1), C. Lopez Ramos (2), A. Jameson (1), R. N. Raid (1), S. Sood (3), J. C. Comstock (3), P. Rott (1)

(1) University of Florida, Belle Glade, FL, U.S.A.; (2) Escuela agricola panamericana Zamorano, Tegucigalpa, Honduras; (3) United States Department of Agriculture, Agricultural Research Service, Canal Point, FL, U.S.A.

Phytopathology 105(Suppl. 4):S4.60

Sugarcane orange rust caused by Puccinia kuehnii was first identified in Florida in 2007. Since then, shifts from resistance to susceptibility have been reported for several sugarcane cultivars, suggesting changes in pathogenicity within the $P$. kuehnii population. In order to study this pathogenic specialization, we developed a detached-leaf assay using disease-free sugarcane plants. Urediniospores were collected by vacuum from cultivars CP89-2143 and CL85-1040 grown in the field, and were brush inoculated onto $10 \mathrm{~cm}$ leaf pieces of susceptible CP80-1743, CP89-2143, and CL851040. Leaf pieces were first kept overnight in the dark in a humidity box at $21^{\circ} \mathrm{C}$. They were then individually transferred to magenta boxes containing 40 $\mathrm{ml}$ deionized water, and placed in an incubator $\left(8 \mathrm{hr}\right.$ light at $25^{\circ} \mathrm{C}$ and $16 \mathrm{hr}$ dark at $21^{\circ} \mathrm{C}$ ). Fourteen days post inoculation, leaves of CP80-1743 and CL85-1040 inoculated with spores from CP89-2143 and CL85-1040 exhibited up to 240-300 sporulating rust pustules. However, leaves of CP89-2143 inoculated with spores from the same cultivar showed 10 times more rust pustules than leaves of CP89-2143 inoculated with spores from CL85-1040. This is first evidence of pathogenic specialization within P. kuehnii in Florida, and supports the shift from resistance to susceptibility of CP89-2143 in 20102012. Assessing variation in pathogenicity of $P$. kuehnii populations using this method could help to improve the control of sugarcane orange rust.

Discovery and evolutionary studies on five carlaviruses infecting elderberry

T. HO (1), K. E. Keller (2), R. R. Martin (2), J. D. Postman (2), I. E. Tzanetakis (1)

(1) University of Arkansas, Fayetteville, AR, U.S.A.; (2) USDA ARS, Corvallis, OR, U.S.A.

Phytopathology 105(Suppl. 4):S4.60

The genomes of five viruses infecting elderberries at the National Clonal Germplasm Repository were elucidated and tentatively named as elderberry virus A-E (EVA-EVE). Genome organization of the new viruses are typical of the carlavirus genus coding for six open reading frames and ranging in size between 8,540-8,628 nucleotides. Phylogenetic analysis indicated that the elderberry carlaviruses could have originated from two distinct lineages. EVA, EVB and EVD cluster alongside poplar mosaic virus, whereas EVC and EVE cluster with American hop latent virus. The speciation events have probably taken place in elderberry as viruses in group A (EVA, EVB \& EVD) and group B (EVC \& EVE) are most closely related to each other than any other virus in the databases. Degenerate and specific PCR assays were designed to detect the individual viruses. Preliminary results indicated that the novel carlaviruses are widespread in the germplasm and may pose a threat to the nascent industry in the United States.

\section{Discovery of five virus species in currant}

T. HO (1), J. D. Postman (2), I. E. Tzanetakis (1)

(1) University of Arkansas, Fayetteville, AR, U.S.A.; (2) USDA ARS, Corvallis, OR, U.S.A.

Phytopathology 105(Suppl. 4):S4.60

Five virus species closest to the genera of Closterovirus, Idaeovirus, Phytoreovirus, Trichovirus, and Waikavirus were discovered in the National Clonal Germplasm Repository Ribes collection. Currant latent virus (family Secoviridae, only reported in Europe) was also detected in the tested plants. PCR assays were conducted successfully to detect individual viruses and confirm the results of large scale sequencing. The discovery of several virus species belonging to diverse virus families and collected from different parts of the world emphasizes the importance of implementation of new technologies for virus detection and discovery. As we move towards open boarders for movement of propagation material it is important to be proactive and initiate efforts so as all material is tested using the new technologies to minimize the possibility of introduction of exotic pathogens of economic importance.

Differences in in vitro fungicide sensitivity among multiple snow mold species

K. HOCKEMEYER (1), A. Orshinsky (1)

(1) University of Minnesota, St. Paul, MN, U.S.A.

Phytopathology 105(Suppl. 4):S4.60

Myriosclerotinia borealis causes snow scald, an under-studied winter disease occurring in areas with harsh winters. Studies on the efficacy of fungicides against $M$. borealis are lacking, and fungicides effective against other snow molds may not be effective against $M$. borealis. Fungicides from nine fungicide groups were tested against five snow mold fungi: $M$. borealis, Michrodochium nivale, Typhula incarnata, T. ishikariensis, and $T$. phacorrhiza. Fungicides were added to potato dextrose agar in $90 \mathrm{~mm}$ diameter Petri dishes at concentrations of $0,0.01,0.1,1$, and $10 \mathrm{ppm}$ of active ingredient. Cultures were inoculated with $5 \mathrm{~mm}$ plugs from the edge of active colonies. M. borealis and M. nivale were incubated at $15^{\circ} \mathrm{C}$ for 3 days, $T$. phacorrhiza at $15^{\circ} \mathrm{C}$ for 10 days, and T. incarnata and T. ishikariensis at $10^{\circ} \mathrm{C}$ for 14 days, after which colony diameters were measured. Relative growth was determined by dividing colony diameter on fungicide-amended media by the diameter of colonies grown without fungicide. Relative growth was plotted against the logarithm of fungicide concentration to define the concentration reducing growth by $50 \%\left(\mathrm{EC}_{50}\right)$. Tebuconazole and fluazinam were among the most potent fungicides against all five fungi. Boscalid and thiophanate-methyl were able to reduce $M$. borealis growth, but they did not reduce growth of the other snow mold fungi. These results highlight the importance of proper pathogen identification for strategic fungicide application.

A reassessment of grapevine (Vitis vinifera) trunk diseases in Washington state vineyards

L. A. HOLLAND (1), G. G. Grove (2), D. A. Glawe (1)

(1) Washington State University, Pullman, WA, U.S.A.; (2) Washington State University, Irrigated Agriculture Research and Extension Center, Prosser, WA, U.S.A.

Phytopathology 105(Suppl. 4):S4.60

Grapevine trunk diseases (GTD) are destructive grapevine diseases worldwide. The present study characterized fungi associated with GTD in Washington State. During the summer of 2014, eight vineyards in the American Viticultural Areas "Yakima Valley" and "Horse Heaven Hills" were surveyed. GTD incidence in 14- to 65-year-old vineyards ranged from 2.8 to $33 \%$. A total of 235 strains of fungi were isolated from symptomatic grapevines. Isolates were characterized using morphological features and sequences of internal transcribed spacer (ITS) region and $\beta$-tubulin genes. Eutypa lata and E. laevata were the most common species $(\sim 73 \%)$ isolated. The other grape-pathogenic diatrypaceous fungi Cryptosphaeria pullmanensis and Diatrype whitmanensis also were isolated, as well as Coniolariella limonispora, Xylaria cubensis, and Discostroma fuscellum (12\%). Species of Botryosphaeriaceae represented about $8 \%$ of the implicated GTD fungi isolated. The remaining $7 \%$ of fungi isolated included Pleosporales (Phoma sp., Pleosporales sp., and Camarosporium brabeji) and Diaporthales (Cytospora rhodophila, C. chrysosperma, and Diaporthe eres). There was a positive correlation between vineyard age and symptom incidence. Results of this study suggest that the diversity of GTD pathogens in Washington State is greater than established previously, and that differences in the biology of these fungi should be taken into account when developing management strategies for GTD in the region.

Changes in microbial communities associated with anaerobic soil disinfestation

J. C. HONG (1), K. Martin (2), N. Kokalis-Burelle (3), D. Butler (4), P. Serrano-Perez (5), E. Rosskopf (3)

(1) USDA ARS, Fort Pierce, FL, U.S.A.; (2) William Paterson University, Wayne, NJ, U.S.A.; (3) USDA, ARS, Fort Pierce, FL, U.S.A.; (4) University of Tennessee, Knoxville, TN, U.S.A.; (5) Centro de Investigaciones Científicas y Tecnológicas de Extremadura (CICYTEX), Badajoz, Spain Phytopathology 105(Suppl. 4):S4.60

Anaerobic Soil Disinfestation (ASD) is a potential alternative to chemical soil fumigation for managing soil-borne plant pathogens and weeds. This technique has been applied in diverse cropping systems. ASD in Florida 
includes incorporating composted broiler litter (CBL) and molasses into the soil, covering the planting bed with polyethylene tarp, and saturating the soil. The soil becomes anaerobic, conditions favorable for anaerobic and facultative anaerobic organisms. ASD was applied to five different fields in Florida, and soil sampled every 2-3 days during the 3-week treatment. Total microbial DNA was extracted from soil samples, and bacterial communities were characterized using length-heterogeneity PCR (LH-PCR). Based on the bacterial population profile, it could be determined if the ASD treatment was successfully applied and the environment became anaerobic. Bacterial populations important to ASD were identified by comparing the results derived from the five fields. Based on LH-PCR, a population with amplicons of $445 \mathrm{bp}$ increased significantly after $24 \mathrm{hrs}$ post-ASD application, and remained the dominate population throughout the treatment. This population was identified using next generation sequencing. Amplicons of $445 \mathrm{bp}$ were identified as Firmicutes. LH-PCR performed on CBL revealed that over $60 \%$ of the bacterial community was the 445 population. Thus, CBL could serve as a microbial starter culture, analogous to foods created by fermentation.

In vitro testing of antimicrobial compounds for citrus HLB management J. HU (1), A. Nagaraju (2), N. Wang (3)

(1) Univ of Florida, Lake Alfred, FL, U.S.A.; (2) CREC-University of Florida, Lake Alfred, FL, U.S.A.; (3) University of Florida, Lake Alfred, FL, U.S.A. Phytopathology 105(Suppl. 4):S4.61

Huanglongbing (HLB) or citrus greening is a destructive disease worldwide and drastically affects citrus production in Florida. Our previous studies have identified over a dozen of antimicrobial compounds targeting SecA protein of Candidatus Liberibacter asiaticus (CLas), the causal agent of HLB. The identified small molecules had demonstrated high inhibitory activity against SecA protein of $C$ Las. This study was undertaken to determine the minimum inhibitory concentration (MIC) and minimum bactericidal concentration (MBC) of these compounds with streptomycin as a positive control against eight phylogenetically closely-related bacterial species. The MIC and MBC assays were conducted in tetra-replicates in accordance with a standardized method. MIC and MBC for Liberibacter crescens ranged from 16 to 128 $\mu \mathrm{g} / \mathrm{mL}$ and from 32 to $128 \mu \mathrm{g} / \mathrm{mL}$; MIC and MBC for $X$. citri subsp. citri ranged from 16 to $64 \mu \mathrm{g} / \mathrm{mL}$ and from 32 to $64 \mu \mathrm{g} / \mathrm{mL}$; MIC and MBC for four Rhizobium bacteria ranged from 8 to $32 \mu \mathrm{g} / \mathrm{mL}$ and from 6 to $64 \mu \mathrm{g} / \mathrm{mL}$; MIC and MBC for Escherichia coli ranged from 256 to $512 \mu \mathrm{g} / \mathrm{mL}$ and $>512$ $\mu \mathrm{g} / \mathrm{mL}$; MIC and MBC for Agrobacterium tumefaciens ranged from 32 to 512 $\mu \mathrm{g} / \mathrm{mL}$ and from 128 to $>512 \mu \mathrm{g} / \mathrm{mL}$; MIC and MBC of streptomycin against all bacterial species but $X$. citri subsp. citri were in the ranges from 32 to $512 \mu \mathrm{g} / \mathrm{mL}$ and from 64 to $>512 \mu \mathrm{g} / \mathrm{mL}$. The results showed that the antimicrobial compounds had antimicrobial activity comparable to that of streptomycin

\section{Identification and characterization of resistance related genes in soybean to Phakopsora pachyrhizi infection}

D. HU (1), D. R. Walker (2), Z. Chen (1)

(1) Department of Plant Pathology and Crop Physiology, Louisiana State University Agricultural Center, Baton Rouge, LA, U.S.A.; (2) USDA-ARS Soybean/Maize Germplasm, Pathology, and Genetics Research Unit, Urbana, IL, U.S.A.

Phytopathology 105(Suppl. 4):S4.61

Asian soybean rust (ASR), caused by Phakopsora pachyrhizi, was first reported in Louisiana in 2004. Currently, the only available method to manage this disease is through fungicide application due to that all U.S. commercial soybean cultivars are susceptible to $P$. pachyrhizi. Recently, a pair of recombinant inbred soybean lines segregating for rust disease resistance was developed. An RNA-seq approach was employed (in our recent effort) to identify genes differentially expressed between the two lines with and without $P$. pachyrhizi infection. A list of differentially expressed genes were identified, including the genes that encode ankyrin repeat family protein (GmARFP), peroxidase superfamily protein (GmPSP), cytochrome P450 (GmP450), L-ascorbate oxidase (GmAO), L-ascorbate peroxidase (GmAPX) as well as several unknown genes (GmUN_1, GmUN_2, GmUN_3). Realtime RT-PCR confirmed that these genes were significantly induced by $P$. pachyrhizi. Virus-induced gene silencing (VIGS) was used to further evaluate the roles of these genes in soybean resistance to rust disease. The expression level of GmAPX in silenced plant was reduced by $50 \%$. These silenced plants are being inoculated with $P$. pachyrhizi to determine the change in soybean resistance to rust infection.

Quantification of Puccinia striiformis f. sp. tritici in urediniospores and infected wheat leaves using real-time quantitative PCR of DNA and RNA X. P. HU (1), L. J. Ma (2), B. T. Wang (3), Z. S. Kang (3), X. M. Xu (4)
(1) Northwest A\&F University, Yangling, China; (2) Northwest A\&F University, YanglingY, China; (3) Northwest A\&F University, Yangling, Shaanxi, China; (4) East Malling Research, East Malling, United Kingdom Phytopathology 105(Suppl. 4):S4.61

Wheat stripe rust, caused by Puccinia striiformis f. sp. tritici (PST), is one of the most important diseases of wheat worldwide. The amount of overwintered inoculum, together with spring conditions, determine the epidemic level of wheat stripe rust and finally yield loss of wheat. This study investigated whether Real-time quantitative PCR (qPCR) of both DNA and RNA (cDNA) could accurately estimate viable PST inoculum, with DNA and RNA extracted from PST spores and from infected wheat leaves. Quantified RNA decreased sharply within 4 days and then remained at a very low level irrespective of spore viability or storage conditions (ambient or $-20^{\circ} \mathrm{C}$ ). In contrast, quantified DNA generally increased over time for dead spores at ambient conditions or viable spores at $-20^{\circ} \mathrm{C}$ but varied greatly with time for viable spores maintained under ambient conditions. On inoculated wheat leaves, the temporal dynamics of quantified DNA and RNA was similar within 11 days after inoculation. However, in the late stage of sporulation, quantified DNA remained at a high level whereas quantified RNA was close to zero. These results suggested that both qPCR of DNA and RNA could provide reliable measurement of viable PST inoculum in plant tissues prior to the late sporulation stage. However, both methods cannot distinguish viable spores from dead spores. Further research is needed to assess whether using different cDNA primers could determine spore viability.

Fungicide resistance profiles of blossom-derived Botrytis isolates from strawberry fields

M. J. HU (1), D. Fernández-Ortuño (2), P. K. Bryson (1), G. Schnabel (1)

(1) School of Agricultural, Forest \& Environmental Sciences, Clemson University, Clemson, SC, U.S.A.; (2) Instituto de Hortofruticultura Subtropical y Mediterránea "La Mayora"-Universidad de Málaga-Consejo Superior de Investigaciones Científicas (IHSM-UMA-CSIC), Dept. de Microbiología, Campus de Teatinos, Málaga, Spain

Phytopathology 105(Suppl. 4):S4.61

From 2012 to 2014, a total of 1484 Botrytis isolates collected from strawberry blossoms from ten states in the United States were subject to the fungicide sensitivity assays described in our previous publication. The overall frequencies of resistant isolates collected in 2012 for thiophanate-methyl, pyraclostrobin, fenhexamid, cyprodinil, iprodione, boscalid, and fludioxonil were $68.6,20.5,10.5,12.7,2.3,13.2$, and $0.5 \%$, respectively. Frequencies of isolates collected in 2013 were $88.2,51.8,13.8,19.1,1.8,9.8$, and $0.9 \%$, respectively. Frequencies of isolates collected in 2014 were 86.6, 70.7, 30.9, $28.7,24.0,7.4$, and $2.8 \%$, respectively. Resistance to thiophanate-methyl was found in virtually every location in all three years, whereas resistance to fludioxonil was rarely found. Frequencies of isolates with resistance to pyraclostrobin, fenhexamid, cyprodinil, and fludioxonil were increasing, whereas resistance frequency of isolates for boscalid was decreasing during 2012 to 2014. Resistant isolates were resistant to either one (1SR; 41.4\%), two (2SR; $16.4 \%$ ), three (3SR; $8.2 \%$ ), four (4SR; $6.8 \%$ ), five (5SR; $0.5 \%$ ), or six (6SR; 0) in 2012. In 2013, this distribution was 37.1, 24.2, 16.0, 9.6, 2.4, and $0.2 \%$ whilst it was $17.2,23.8,19.4,15.5,8.7$, and $2.1 \%$ in 2014 . The data showed a shift from 1SR to 6SR in the frequencies of multifungicide resistant isolates from 2012 to 2014, indicating an increase of multifungicide resistance.

\section{Evaluation of laurel wilt tolerance in redbay (Persea borbonia)}

M. A. HUGHES (1), J. A. Smith (1)

(1) University of Florida, Gainesville, FL, U.S.A.

Phytopathology 105(Suppl. 4):S4.61

The evergreen hardwood tree, redbay (Persea borbonia) has experienced immense mortality rates to its populations since the emergence of laurel wilt in the southeastern United States around 2002. Forest stands and residential areas infested with the redbay ambrosia beetle (Xyleborus glabratus) and its pathogenic fungal symbiont (Raffaelea lauricola) are quickly succumbing to this vascular disease, with few management options available. However, certain asymptomatic individuals persist in areas of high mortality. In an effort to assess disease tolerance among individuals from within these regions, asymptomatic $(>3$ " $+\mathrm{DBH})$ trees were selected for long-term monitoring and vegetatively propagated using a variety of methods. To optimize a screening protocol, experiments were conducted to determine the role of conidial concentration upon disease progression and the genetic variability of $R$. lauricola using Amplified Fragment Length Polymorphism (AFLP) markers. Three disease screening trials using clones were conducted via artificial inoculation experiments at a research farm near Gainesville FL, with results suggesting a durable tolerance among a small subset of the challenged clones. Future plans include out-plating of these clones in areas of laurel wilt 
mortality to assess their long term survival under natural conditions. These results will improve future efforts to manage the disease and restore affected ecosystems.

Genome analysis reveals a complex modular evolution of the biosynthetic pathway for the phytotoxin thaxtomin in plant pathogenic Streptomyces species

J. HUGUET-TAPIA (1), G. Pettis (2), R. Loria (1)

(1) Plant Pathology Department, University of Florida, Gainesville, FL, U.S.A.; (2) Department of Biological Sciences, Louisiana State University, Baton Rouge, LA, U.S.A.

Phytopathology 105(Suppl. 4):S4.62

Streptomyces spp. are highly differentiated actinomycetes with large, linear chromosomes that encode an arsenal of biologically active molecules and catabolic enzymes. Out of the hundreds of species in the genus Streptomyces, a small group has evolved the ability to infect plants. Production of the nonribosomal dipeptide, pyhtotoxin thaxtomin is a hallmark of pathogenesis in plant pathogenic Streptomyces. The recent availability of Streptomyces genome sequences, including four genomes of pathogenic species, provides an opportunity to investigate the gene content and evolutionary relations of the pathogenic group. Topology comparisons of phylogenetic trees based on the core genome and trees based on the thaxtomin biosynthetic pathway reveal processes of lateral gene transfer (LGT) within pathogenic species. Gene content analysis also reveals that the current structure of the thaxtomin cluster is the result of multiple recombination processes. The presence of insertion sequences within the thaxtomin pathway of all the pathogens suggests that transposition events have been critical to the evolution of some of the elements of the pathway. We postulate a mechanism of evolution of the thaxtomin biosynthetic pathway within pathogenic Streptomyces species.

Diversity of endophytic fungi from Cirsium kawakamii and the risk assessment

C. HUI-JUAN (1), C. Wen-Hsin (1)

(1) Dept. of Plant Pathology, National Chung Hsing University, Taichung, Taiwan

Phytopathology 105(Suppl. 4):S4.62

A total of 1836 fungal endophytes were obtained from Cirsium kawakamii, a Chinese herb. Among these fungal endophytes, Fusarium torulosum and Neofabraea sp., reportedly two pathogens to potato and Rosaceae fruits respectively, showed activities against $F$. oxysporum f. sp. lactuctum. The objective of this study is to investigate the pathogenicity of $F$. torulosum and Neofabraea sp. on various crops. Based on wounded inoculation, the $F$. torulosum isolates could cause dry rot on potato at $4^{\circ} \mathrm{C} 28$ days after inoculation with $20 \mu \mathrm{l}$ of $10^{6}$ conidia/ml inoculum. Moreover, these isolates could also cause Fusarium rot symptoms with circular and brown lesions on apple with wounded inoculation. For Neofabraea sp. isolate, it could cause brown and soaking symptom on apple and pear when mycelium disc was used for wounded inoculation. The symptom is similar to the bull's-eye rot of apple and pear caused by Neofaraea spp. in previous reports. This is the first report of Fusarium rot caused by $F$. torulosum on apple fruits. Based on the pathogenicity tests, the two fungal endophytes are capable of causing diseases on potato, apple or pear. Thus, certain fungal endophytes warrant further scrutiny before being released in nature for bio-control purpose.

Effect of blackleg (Leptosphaeria maculans) on yield of Brassica napus canola in Alberta, Canada

S. F. HWANG (1), S. E. Strelkov (2), H. U. Ahmed (3), Q. Zhou (3), G. D. Turnbull (3), G. Peng (4)

(1) Alberta Agriculture, Edmonton, AB, Canada; (2) University of Alberta, Edmonton, AB, Canada; (3) Alberta Agriculture and Rural Development, Edmonton, AB, Canada; (4) Agriculture and Agri-Food Canada, Saskatoon, SK, Canada

Phytopathology 105(Suppl. 4):S4.62

Blackleg, caused by Leptosphaeria maculans, is an important constraint on canola (Brassica napus) production. Knowledge of disease-yield relationships is a prerequisite for measuring the agronomic efficacy and economic benefits of control methods, and for making informed crop management decisions. To determine the relationship between blackleg severity and yield loss, blackleg susceptible ('Westar') and moderately resistant to resistant canola cultivars ('1950RR' and '46S5RR') were grown in L. maculans-infested plots in four site-years of field experiments. A $1 \mathrm{~m}^{2}$ area of each plot was harvested and all plants were sorted into disease categories on a $0-5$ scale. Pod number and seed yield were determined for each plant. Average disease severity per plot also was determined. Blackleg severity on the cultivars '46S653RR' and ' 1950 RR' was $48 \%$ and $63 \%$ lower than on 'Westar', respectively, while pod number and seed yield per plant were $60 \%$ and $36 \%$ greater. There were negative, linear relationships between disease severity and both pod number and seed yield. The regression coefficent was larger for the resistant to moderately resistant cultivars compared with the susceptible cultivar. Resistant to moderately resistant canola cultivars exhibited a lower blackleg severity and greater yield compared with the susceptible canola. Pod numbers and seed yield declined as disease severity increased.

An endoglucanase/expansin hybrid protein and the type II secretion system affect the virulence and systemic colonization of Xylella fastidiosa

B. M. INGEL (1), P. Wang (1), J. Labavitch (2), M. C. Roper (1)

(1) University of California Riverside, Riverside, CA, U.S.A.; (2) University of California Davis, Davis, CA, U.S.A.

Phytopathology 105(Suppl. 4):S4.62

$X y$ lella fastidiosa $(X f)$ is a xylem-limited, gram-negative bacterium that causes Pierce's Disease in grapevine. Movement of $X f$ within the xylem relies on the degradation of plant cell walls separating xylem vessels. This is facilitated by the cooperation of at least two types of cell wall-degrading enzymes (CWDEs), a polygalacturonase (PG) and an endoglucanase (EGase), each targeting different cell wall components. Plant cell wall disassembly by $X f$ is not fully understood, and characterization of CWDEs and the mechanisms by which they are secreted will yield further insights into how $X f$ systemically colonizes grapevines. As systemic colonization is highly correlated with Pierce's Disease development, understanding and preventing bacterial movement are critical to devising management strategies. In this study, we focus on characterizing an $X f$ EGase (EngXCA1) and the role of the Type II Secretion System (T2SS) in CWDE secretion. EngXCA1 contains a domain with homology to plant expansins, which non-enzymatically loosen cell walls during development. Expansins have been found in several plant-associated bacteria, and could play a role in pathogenicity. Our results indicate that a mutation in engXCA1 reduced the virulence of Xf. We also demonstrate that a mutation in $x p s E$, which encodes a putative ATPase of the T2SS, mimics the non-pathogenic phenotype of a PG-deficient $X f$ mutant, providing support for our hypothesis that the T2SS is involved in CWDE secretion.

In-furrow control of Rhizoctonia solani with a combination of starter fertilizer and azoxystrobin

C. IRWIN (1), D. Anderson (2), J. Dinglasan (2), H. Pham (2), R. Gong (2)

(1) Vive Crop Protection, Guelph, ON, Canada; (2) Vive Crop Protection, Toronto, ON, Canada

Phytopathology 105(Suppl. 4):S4.62

Soil applied azoxystrobin can be used to control rhizoctonia root and stalk rot in corn, rhizoctonia damping off and root rot in soybean, and other soil-borne seedling disease. In many cases, growers want to apply azoxystrobin in combination with a starter fertilizer, either banded or in-furrow at planting. In this study, a fertilizer-compatible liquid azoxystrobin $\mathrm{SC}$ was developed and evaluated. The liquid SC formulation is easy to handle, produces no dust at planting, and directly targets early season disease without having to do multiple passes. In order to demonstrate fertilizer compatibility, efficacy, and yield enhancement, field trials were conducted in 2014 and 2015. The fertilizer-compatible azoxystrobin SC was applied in-furrow as a spray band at planting, mixed with water or liquid fertilizer at two rates. The product had excellent fertilizer compatibility and in 2014 significantly increased yield as compared to the untreated control. Field and lab efficacy studies were also conducted on Rhizoctonia solani in 2015, and results from these experiments will also be presented.

Integrated Pest Information Platform for Extension and Education (iPiPE): A new USDA Cooperative Agricultural Program (CAP) S. A. ISARD (1), F. Louws (2)

(1) Penn State Univ, University Park, PA, U.S.A.; (2) North Carolina State University, Raleigh, NC, U.S.A.

Phytopathology 105(Suppl. 4):S4.62

Food security is best served by a national infrastructure of private and public professionals who routinely monitor crop health and pest incidence then translate this knowledge to a shared platform enabling rapid dissemination of mitigation measures to limit crop loss. The iPiPE CAP, funded by a 2015 USDA AFRI 5-yr \$7 million grant, provides such an infrastructure with cyberage tools, information products and expert commentary for detection and management of new, foreign, or emerging target pests and endemic pests that threaten U.S. crops. By categorizing pests, data, and users, it enables sharing observations while protecting privacy of individuals, companies, and government agencies. iPiPE Crop-Pest Programs (CPPs) will incentivize growers and consultants to submit observations on target and endemic pests by providing tools and information for timely management decisions. Coordinated by extension professionals from across the nation, programs address a variety of crops and pests and provide undergraduate students with 
hands-on extension and diagnostic experiences. Risk-based research will prioritize detection efforts for target pests and direct in-field scouting for endemic pests. Observations housed in a national pest observation depository will enable future research using geographically extensive, multi-year databases. iPiPE success can be measured by numbers of CPPs, participating stakeholders and trained students.

Inhibitory activity of the new SDHI fungicide benzovindiflupyr against Colletotrichum species and other plant pathogens

H. ISHII (1), F. Zhen (2), M. Hu (2), X. Li (3), G. Schnabel (2)

(1) Natl Inst for Agro-Environmental Sciences/Clemson University/Kibi International University, Tsukuba, Japan; (2) Clemson University, Clemson, SC, U.S.A.

Phytopathology 105(Suppl. 4):S4.63

Inhibitory activity of the new SDHI fungicide benzovindiflupyr was compared with boscalid, penthiopyrad, fluxapyroxad and fluopyram using Colletotrichum species as well as boscalid-sensitive and -resistant isolates of Botrytis cinerea, Alternaria alternata, and Monilinia fructicola. On YBA agar medium, boscalid, fluxapyroxad and fluopyram did not inhibit the mycelial growth of $C$. gloeosporioides, C. acutatum, C. cereale, and C. orbiculare, but penthiopyrad did inhibit the growth of the former three species. Most interestingly, benzovindiflupyr showed high inhibitory activity against mycelial growth and/or conidial germination and germ tube growth of all Colletotrichum species used. Benzovindiflupyr also effectively controlled anthracnose disease on cucumber plants inoculated with $C$. orbiculare. Sequence analysis of $s d h B, s d h C$ and $s d h D$ genes encoding the subunits of fungicide-targeted succinate dehydrogenase revealed no known resistance mutations in Colletotrichum species that could explain the weak performance of boscalid. Benzovindiflupyr also showed high inhibitory activity in vitro against boscalid-sensitive and -resistant isolates of B. cinerea, A. alternata and M. fructicola isolates. Differential activity of SDHI fungicides against Colletotrichum and other species might be exploited when designing disease management strategies.

Genetic diversity of L-strain isolates of Aspergillus flavus of potential use for aflatoxin biocontrol in sub-Saharan Africa

M. S. ISLAM (1), K. A. Callicott (1), J. Atehnkeng (2), J. Augusto (3), S. Bonkoungou (4), D. Agbetiameh (5), P. M. Diedhiou (6), H. Daudi (7), M. Mukanga (8), R. Bandyopadhyay (2), P. J. Cotty (1)

(1) USDA ARS, School of Plant Sciences, The University of Arizona, Tucson, AZ, U.S.A.; (2) International Institute of Tropical Agriculture, Ibadan, Nigeria; (3) International Institute of Tropical AgricultureMozambique, Nampula, Mozambique; (4) Institut de l'Environnement et de Recherches Agricoles (INERA), Farako Ba, Burkina faso; (5) Kwame Nkrumah University of Science and Technology, Kumasi, Ghana; (6) Université Gaston Berger, Saint-Louis, Senegal; (7) Agriculture Research Institute, Naliendele, Tanzania; (8) Zambia Agricultural Research Institute, Mount Makulu, Zambia

Phytopathology 105(Suppl. 4):S4.63

Biological control of crop aflatoxin contamination with atoxigenic Aspergillus flavus is very successful in US agriculture. Atoxigenic lineages of $A$. flavus are used to reshape fungal communities so that average aflatoxin-producing potentials are reduced. These community modifications are associated with large reductions in crop aflatoxin content. We have been adapting this technology to aflatoxin management in sub-Saharan Africa. Development of locally and/or regionally directed biocontrol products requires precise information about diversity, distribution and lineages of potential biocontrol isolates with differential adaptations. SSR analyses were performed on 1798 L-strain isolates of A. flavus obtained from Benin, Burkina Faso, Ghana, Kenya, Mozambique, Nigeria, Senegal, Tanzania and Zambia. Number of isolates analyzed differed among countries, but similar genetic diversity was observed in each country based on 17 SSR loci. Over 1000 atoxigenic haplotypes of potential value in biocontrol were detected. Many haplotypes were restricted locally, but certain haplotypes were widely distributed. Although some signals of local adaptation were detected, all major genetic lineages included isolates from several countries, suggesting substantial migration. While the SSR diversity in each country was high, significant linkage disequilibrium and minimum spanning network suggest clonal reproduction and mutation are primary forces shaping the observed population structure.

Fvstr1, a striatin ortholog in Fusarium virguliforme required for conidiation, conidiophore development, and virulence

K. T. ISLAM (1), J. P. Bond (1), A. M. Fakhoury (1)

(1) Southern Illinois Univ, Carbondale, IL, U.S.A.

Phytopathology 105(Suppl. 4):S4.63
Fusarium virguliforme (O’Donnell \& Aoki) is a soil-borne pathogenic fungus that causes Sudden Death Syndrome (SDS), one of the most devastating soilborne diseases of soybean (Glycine max [L.] Merrill) in North and South America. Despite the importance of SDS, a clear understanding of fungal genetic factors that affect the development of the disease is still lacking. We have identified Fvstrl, a $F$. virguliforme gene that encodes a protein similar to a family of striatin proteins previously reported to regulate cell differentiation, conidiation, sexual development, and virulence in filamentous fungi. To address the molecular function of a striatin homolog in F. virguliforme, Fvstr 1 was disrupted and functionally characterized using gene knock out strategies. The resulting Fv $\Delta$ strl mutants were largely impaired in conidiation and pigmentation and displayed defective conidia and conidiophore morphology compared to the wild type. The greenhouse pathogenicity assay results revealed that the disruption of Fvstrl resulted in complete loss of virulence in $F$. virguliforme. Microtome studies using fluorescence microscopy showed the mutant was defective in colonizing the vascular system. The Fvstrl mutant also showed a reduced transcript level of genes involved in asexual reproduction and secondary metabolite production. These results suggest that Fvstrl has a critical role in asexual sporulation, and virulence in $F$. virguliforme.

Impact of ILeVO ${ }^{\circledast}$ on the infection and colonization of soybean roots by Fusarium virguliforme

K. ISLAM (1), J. P. Bond (1), J. Riggs (2), A. M. Fakhoury (1)

(1) Southern Illinois Univ, Carbondale, IL, U.S.A.; (2) Bayer CropScience LP, Research Triangle Park, NC, U.S.A.

Phytopathology 105(Suppl. 4):S4.63

The soil-borne fungus Fusarium virguliforme is a destructive pathogen causing Sudden Death Syndrome (SDS) on soybean. From 2009 to 2011, average losses from SDS in the USA were estimated at 42 million bushels per year. Despite the importance of the disease, few agronomic practices have been used to manage SDS successfully. ILeVO, a recently registered seed treatment fluopyram product from Bayer CropScience, has been reported to affect SDS. We studied the impact of ILeVO on $F$. virguliforme infection and colonization of soybean roots through qPCR and microscopy. Soybean seeds treated with fluopyram were used in field trials at multiple locations. Root samples were collected 21 days after emergence, and $\mathrm{qPCR}$ was used to quantify the fungal biomass in roots. The qPCR results showed that ILeVOtreated plants had significant reduction in fungal colonization compared to the untreated control. A greenhouse experiment was also conducted using a $F$. virguliforme GFP strain to assess and visualize fungal infection and colonization of soybean roots. Confocal microscopy revealed that the epidermal, cortical and vascular tissues of the untreated roots were heavily infested with fungal mycelia whereas considerably less amount of fluorescent mycelia were observed in the epidermal and cortical tissues of the treated roots.

Diversity of Sclerotinia sclerotiorum isolates collected from Bangladesh and Ohio, USA

M. M. ISLAM (1), S. A. Miller (2)

(1) The Ohio State Univ, Wooster, OH, U.S.A.; (2) The Ohio State University OARDC, Wooster, OH, U.S.A.

Phytopathology 105(Suppl. 4):S4.63

Sclerotinia sclerotiorum causes white mold of more than 400 plant species. The disease is considered a problem of temperate climates, but was observed in oil-seed mustard for the first time in Bangladesh in 2007. A total of 124 isolates of S. sclerotiorum collected from mustard in 2015 in Jamalpur, Gazipur, Tangail and Shirajganj, Bangladesh were compared with eight isolates from temperate crops in Ohio. Radial growth of isolates on PDA ranged from $76.5-82.75 \mathrm{~mm}$ after 72 hours of culture. Radial growth of isolates from Ohio was significantly less than the growth of Bangladeshi isolates. No significant difference was observed in number of sclerotia formed by different isolates. However, sclerotia from Ohio were significantly heavier than sclerotia from Jamalpur. Based on the sequence data from the rRNA ITS region of 20 randomly selected isolates from five locations, a phylogenetic tree was constructed and four distinct groups were differentiated. Nine Bangladeshi isolates from Jamalpur, Tangail and Shirajgonj, the Ohio isolates and other Sclerotinia reference sequences formed a distinct group. Isolates from Tangail, Gazipur and Shirajganj clustered in a separate group. From the morphological and sequence data, it appears that diversified pathogen populations are present in Bangladesh. Some populations are very similar to the populations of temperate regions in the USA while others cluster in separate, distinct groups. 
Spots on pot: Comparative genomics of two cannabis pathogens reveals insight into the evolution of Xanthomonas pathogenicity

J. M. JACOBS (1), C. Pesce (2), P. Lefeuvre (3), R. Koebnik (1)

(1) Institut de recherche pour le développement - RPB, Montpellier, France; (2) Inst de Recherche Pour Le Dev - RPB, Montpellier, France; (3) CIRAD, St. Pierre, France

Phytopathology 105(Suppl. 4):S4.64

Pathogenic bacteria in the genus Xanthomonas collectively cause disease on many host plants, including Cannabis sativa (cannabis). We sequenced the genomes of two strains that were previously isolated from diseased cannabis in Japan and Romania. Comparative MLSA and whole genome sequence analyses based on average nucleotide identity (ANI) demonstrated that these strains belong to the same species. Genome analysis revealed that neither strain possesses a Type III secretion system (T3SS), a major virulence secretion system, or Type III-secreted virulence effectors found in many Xanthomonas spp. Most notably the genomes contained genes encoding the T3SS regulators HrpG and HrpX. Promoter prediction of HrpX-regulated genes suggests that HrpX regulates the expression of Type II-secreted hydrolytic enzymes, which are similarly found in other plant-pathogenic Xanthomonas strains. The genome of distantly related rice isolate, Xanthomonas strain 97M, similarly lacks the T3SS but encodes HrpG, HrpX and putatively HrpXregulated hydrolytic enzymes. Further comparative genome analysis using ANI suggest that Xanthomonas sp. strain Nyagatare is in the same species as the cannabis strains, but intriguingly $X$. sp. str. Nyagatare possesses a T3SS and associated effectors. We propose a stepwise evolution of pathogenicity in Xanthomonas spp. where strains acquire: 1) pathogenicity regulators HrpG and HrpX and then 2) T3SS and T3-secreted effector genes.

Deficient, adequate and excess growth curves ( $N$, P, and $K$ ) established in hydroponics for biotic and abiotic stress-interaction studies in lettuce D. K. JACOBSON (1), B. D. Geary (2), G. T. Myers (2), O. J. Taylor (2)

(1) Brigham Young University, Orem, UT, U.S.A.; (2) Brigham Young University, Provo, UT, U.S.A.

Phytopathology 105(Suppl. 4):S4.64

Mineral nutrients have marked effects on plant health by providing the building blocks for plant growth, as well as for mitigating abiotic and biotic stress factors, particularly disease development. Even if mineral nutrition field studies are conducted to study pest management, they are at the mercy of complex soil, water, and climatic conditions not amenable to strict experimental control. Therefore, a hydroponic method of growing lettuce was developed and growth curves were established for the macronutrients nitrogen $(\mathrm{N})$, phosphorus $(\mathrm{P})$, and potassium $(\mathrm{K})$. Lettuce plants were grown at varying levels of each nutrient: $2.5,5.0,10,20,40,80,160$, and $320 \mathrm{mg} \mathrm{N} \mathrm{mg/L} \mathrm{N}$; $0.5,1.0,2.0,4.0,8.0,16.0,32.0$ and $64.0 \mathrm{mg} / \mathrm{L} \mathrm{P}$; and $0,2.5,5.0,10.0,20.0$, $40.0,80.0$ and $160.0 \mathrm{mg} / \mathrm{L} \mathrm{K}$. Optimal levels of N, P, and K were $160 \mathrm{mg} / \mathrm{L}$, $4.0 \mathrm{mg} / \mathrm{L}$, and $160 \mathrm{mg} / \mathrm{L}$ respectively. Deficient and excess levels are significantly lower or higher than the optimum levels. These growth curves can be used to test lettuce resilience to various biotic and abiotic stresses. Currently, lettuce is being grown at varying concentrations (deficient, optimal, and excess) of each nutrient and being subjected to Verticillium dahliae. Verticillium dahliae causes significant root damage resulting in major crop loss. It is hypothesized that optimal N, P, and K levels will help to mitigate the effects of $V$ dahliae.

Spore yield and resource depletion by Aspergillus flavus AF36 on wheat grain R. JAIME (1), P. J. Cotty (2)

(1) University of Arizona, Tucson, AZ, U.S.A.; (2) USDA ARS, University of Arizona, Tucson, AZ, U.S.A.

Phytopathology 105(Suppl. 4):S4.64

Aflatoxins, toxic and carcinogenic metabolites produced by Aspergillus section Flavi, contaminate several crops, including cottonseed. Cottonseed contamination is a frequent problem in desert areas of Arizona. Use of atoxigenic $A$. flavus to competitively displace aflatoxin producers and reduce the average aflatoxin-producing potential of fungal communities associated with crops has been very successful. Displacement requires sporulation and spread of the biocontrol agent from the formulated product. The period over which the product produces spores influences success of the biocontrol. The present study used laboratory studies to determine the period over which a commercial biocontrol product produced spores prior to the biocontrol agent completely consuming the product's nutritional base (wheat grain). Both the quantity of spores produced and the weight of the product were determined weekly at $20^{\circ} \mathrm{C}$ to $40^{\circ} \mathrm{C}$. Results suggest product viability and spore production period are significantly affected by temperature but under all temperature regimes the product yielded spores for at least 2 weeks. Fastest sporulation occurred at $35^{\circ} \mathrm{C}$; fastest decay ( 2 weeks) occurred at $35^{\circ} \mathrm{C}$ to $40^{\circ} \mathrm{C}$. Incubation at the temperature extremes $\left(20^{\circ} \mathrm{C}\right.$ and $\left.40^{\circ} \mathrm{C}\right)$ resulted in the lowest observed sporulation. Results suggest biocontrol products produce sufficient spores for effective biocontrol activity for at least 14 days if applied when temperatures range from $20^{\circ} \mathrm{C}$ to $40^{\circ} \mathrm{C}$.

SC2 prophage encoded peroxidase (SC2_gp095) in Candidatus Liberibacter asiaticus is secreted and a likely virulence effector

M. JAIN (1), L. A. Fleites (1), D. W. Gabriel (1)

(1) University of Florida, Gainesville, FL, U.S.A.

Phytopathology 105(Suppl. 4):S4.64

Most pathogenic strains of $\mathrm{Ca}$. Liberibacter asiaticus (Las, causal agent of citrus Huanglongbing disease) carry two nearly identical prophages, named SC1 and SC2 in strain UF506. The highly reduced Las genome lacks the hallmark features of ROS defenses, indicating that the SC2 encoded peroxidase (SC2 gp095) and glutathione peroxidase (SC2 gp100) may represent important "lysogenic conversion" genes determining bacterial fitness and symptom development in the host plant. Both SC2 gp095 and SC2_gp100 were expressed at significantly higher levels in planta than in insects. SC2 gp095 alone, and in tandem with SC2 gp100 were separately cloned in a wide-host-range (repW) shuttle vector pUFR071 (under control of the lacZ promoter) and transformed into $E$. coli and into L. crescens (Lcr), a culturable proxy for Las. The transformed Lcr cells showed 20-25\% higher resistance to $\mathrm{H}_{2} \mathrm{O}_{2}$ in vitro, $47 \%$ higher enzymatic activity and faster growth rates in culture as compared to Lcr cells transformed with only pUFR071. A non-classical secretion potential was predicted for SC2_gp095 and confirmed by enzymatic and Western blot analyses in Lcr and suggesting such secretion from Las. We hypothesize that Las peroxidase, a) mitigates the direct antibacterial effect of host-generated ROS, and b) disrupts systemic propagation of $\mathrm{H}_{2} \mathrm{O}_{2}$-mediated signaling in the host plant. The latter idea may explain the surprisingly long incubation period before symptoms appear.

A novel approach to control gray mold, anthracnose, and powdery mildew on strawberry using low-dose UV-C irradiation

W. J. Janisiewicz (1), F. Takeda (1), W. JURICK II (2), B. Nichols (1), S. Wolford (1), D. M. Glenn (1)

(1) USDA-ARS, AFRS, Kearneysville, WV, U.S.A.; (2) USDA ARS - Food Quality Laboratory, Beltsville, MD, U.S.A.

Phytopathology 105(Suppl. 4):S4.64

Irradiation of plants with UV-C for protection against phytopathogens is an attractive approach that still awaits full commercial development. The cost and negative effect of UV-C on plants have been the main obstacles. A new approach combining UV-C irradiation at $12.36 \mathrm{~J} / \mathrm{m}^{2}$ followed by a dark period and the application of antagonists was used to control major pathogens of strawberry. The post-irradiation dark period prevented activation of the photoactivated fungal DNA repair mechanism which resulted in more effective biocidal effect on $B$. cinerea, $C$. acutatum and $P$. aphanis conidia in vitro and in situ tests. The UV-C irradiation did not impair pollen germination and tube growth, had no negative effect on fruit set and strawberry yield, did not cause loss of chlorophyll, or affect leaf photosynthesis. A UV-C delivery apparatus has been developed for application in high tunnel strawberry culture. Irradiation of strawberry plants twice a week in a tunnel culture resulted in high quality fruit that was comparable to fruit treated with fungicides. In greenhouse experiments, irradiation of powdery mildew infected plants twice a week for three weeks resulted in a reduction of disease symptoms and a four-fold reduction in conidia on irradiated surfaces. We envision combining the UV-C treatment with biocontrol agents to prevent recolonization by phtyopathogens, and expanding this technology to all protected and unprotected fruit, vegetable and ornamental cultures.

Limited host range of Sclerotiorum trifoliorum relative to $S$. sclerotiorum examined using comparative pathogenicity tests and transcriptomics T. M. JARDINI (1), D. Qiu (1), W. Chen (2)

(1) Department of Plant Pathology, Washington State University, Pullman, WA, U.S.A.; (2) USDA ARS Washington State University, Pullman, WA, U.S.A. Phytopathology 105(Suppl. 4):S4.64

Sclerotinia trifoliorum is a major pathogen of alfalfa, clover, and chickpea. Its host range is limited mainly to cool season legumes, unlike those of other Sclerotinia species, which have much broader host ranges. In order to understand the mechanisms underlying the difference in host range, we previously assessed responses of $S$. trifoliorum and $S$. sclerotiorum in their growth and ability to modulate environmental $\mathrm{pH}$ at different temperatures. Here we examine the two species using comparative pathogenicity tests and transcriptomics. Ten isolates each of S. sclerotiorum and S. trifoliorum were compared by lesion size and expressed transcripts. Lesion size was analyzed on cool season (pea, faba bean) and warm season (green bean, soybean) legumes on detached leaves and intact plants. RNA was extracted and sequenced with a 454 Titanium RNA sequencing kit. Although both species 
can readily colonize detached leaves of the four host plants, lesions caused by S. trifoliorum are generally $25 \%$ smaller than those caused by S. sclerotiorum. On intact plants, $S$. trifoliorum could form only limited lesions on green bean and soybean. Transcriptome data showed transcripts with $60 \%$ similarity between the two species and $43 \%$ of the transcripts were genes with known functions. The two species differed in levels of polygalacturonases, which may provide insight to the differences in host ranges between the two species. Other genes and transcripts are still under investigation.

Evaluation of Soil-borne wheat mosaic virus as a viral vector for monocots S. JARUGULA (1), S. R. Charlesworth (2), F. Qu (3), L. R. Stewart (4)

(1) Center for Applied Plant Sciences, Ohio State University, Wooster, OH, U.S.A.; (2) Queensland University of Technology, Brisbane, Australia; (3) Department of Plant Pathology, Ohio Agricultural Research and Development Center, Ohio State University, Wooster, OH, U.S.A.; (4) USDA/ARS, Ohio Agricultural Research and Development Center, Ohio State University, Wooster, OH, U.S.A.

Phytopathology 105(Suppl. 4):S4.65

Soil-borne wheat mosaic virus (SBWMV) is a Furovirus with bipartite, single stranded positive sense RNA genome, which causes yellow mosaic disease in wheat. The rod-shaped virion morphology and taxonomic proximity to other commonly used viral vectors like Tobacco rattle virus and Barley stripe mosaic virus in the family Virgaviridae, prompted study of the virus for its efficacy as a viral vector for monocots. In this study, a full-length infectious cDNA clone of an Ohio isolate of SBWMV was developed in an agro-binary vector. The virus was shown to move systemically and cause symptomatic infection in both the dicotyledonous experimental host Nicotiana benthamiana and in the natural monocotyledonous host wheat (Triticum aestivum L.). Green fluorescent protein (GFP) gene or partial phytoene desaturase (PDS) gene sequences from $N$. benthamiana or wheat were inserted in four different positions in the SBWMV genome, to analyze the potential utility of the virus as a gene expression or gene silencing vector for monocots. Insertions replacing part of the CP-readthrough region showed unique stability and moved systemically in both hosts, but induced gene silencing only in $N$. benthamiana. Expression of GFP gene through SBWMV was limited in both hosts. Identification of factors that limit VIGS in wheat could improve the utility of SBWMV as a gene silencing vector in monocots.

Development of new primers and TaqMan ${ }^{\circledR}$ probes for the detection of grapevine viruses associated with red blotch and leafroll by qPCR and digital PCR

M. JAYANTH (1), T. L. Lawler (2), D. A. Kluepfel (3), M. R. Sudarshana (3) (1) USDA ARS, Davis, CA, U.S.A.; (2) UC Davis, Davis, CA, U.S.A.; (3) USDA-ARS, Davis, CA, U.S.A.

Phytopathology 105(Suppl. 4):S4.65

$\operatorname{TaqMan}^{\circledR}$ assays are highly sensitive and specific in the detection of infectious agents. Previously, TaqMan ${ }^{\circledR}$ probes and primers were developed for the detection of several leafroll-associated grapevine viruses. However, since their development genetic variants of several leafroll-associated viruses have been reported in the region targeted for binding of the TaqMan ${ }^{\circledR}$ primers and probes. This precipitated a need for the design of new probes and primers. Additionally, a new DNA virus, Grapevine red blotch-associated virus (GRBaV), was found to be widespread in wine grape production areas in the US and Canada for which a TaqMan ${ }^{\circledR}$ assay had yet to be developed. Herein, we describe the design of TaqMan ${ }^{\circledR}$ primers and probes for GRBaV and grapevine leafroll-associated viruses 1 and 3 , three of the most widely distributed and economically important grapevine viruses. We have also optimized TaqMan ${ }^{\circledR}$ assays for the detection and quantitation of these three viruses in nucleic acid extracts prepared from virus-infected grapevines by qPCR and digital PCR.

\section{Modifying citrus genome using Cas9/sgRNA}

H. JIA (1), N. WANG (2)

(1) University of Florida, Lake Alfred, FL, U.S.A.; (2) Univ of Florida, Lake Alfred, FL, U.S.A.

Phytopathology 105(Suppl. 4):S4.65

Citrus is one of the most economically important and extensively grown fruit tree crops worldwide. Due to its narrow genetic diversity and long juvenile period, classical breeding is greatly challenged to timely improve citrus for disease resistance. Targeted genome engineering is expected to contribute significantly to future citrus varietal improvement. Here, we reported our progress in modifying citrus genome suing Cas9/sgRNA technology. We first developed a novel tool, Xanthomonas citri subsp. citri (Xcc)-facilitated agroinfiltration, for enhancing transient protein expression in sweet orange leaves. Our results showed Xcc-facilitated agroinfiltration could be used on other citrus varieties including Duncan grapefruit, Valencia sweet orange, Key lime, Carrizo citrange, Sour orange, and Meiwa kumquat. We then employed Xcc-facilitated agroinfiltration to deliver Cas9, along with a synthetic sgRNA targeting the $C_{s} P D S$ gene, into sweet orange. DNA sequencing confirmed that the CSPDS gene was mutated at the target site in treated sweet orange leaves. The mutation rate using the Cas9/sgRNA system was approximately 3.2 to $3.9 \%$. Off-target mutagenesis was not detected for CsPDS-related DNA sequences in our study. Furthermore, Cas $9 / \mathrm{sgRNA}$ system is being used to modify the PthA4 effector binding elements in the promoter of CsLOB1 $\left(\mathrm{EBE}_{\mathrm{pthA} 4}-\mathrm{CsLOBP}\right)$, a susceptibility gene for citrus canker, to generate canker resistant citrus.

Optimization of Bacillus amyloliquefaciens BAC03 application in controlling Streptomyces scabies

H. JIANG (1), Q. Meng (2), J. Hao (3)

(1) Univ of Maine, Orono, ME, U.S.A.; (2) Univ of Maine, Spencer, IA, U.S.A.; (3) Univ of Maine, orono, ME, U.S.A.

Phytopathology 105(Suppl. 4):S4.65

Bacillus amyloliquefaciens strain BAC03 is a novel bacterial strain that had previously been studied for biological control of Streptomyces scabies. In order to optimize its efficacy, various application strategies were investigated for their effects on scab in radish and potato. Assessments included application using foliar spray, seed treatment, or potting-mix treatment. Tests were conducted with different timing, frequency, and concentrations of BAC03 in potting mix that was infested with $S$. scabies. Results showed that foliar application and seed treatment with BAC03 did not affect the disease severity in either radish or potato. BAC03 applied 5 days before planting completely suppressed $(P<0.05)$ radish scab, but the efficacy decreased as BAC03 application was postponed $(P<0.05)$. BAC03 at $10^{5} \mathrm{CFU} \mathrm{\textrm {cm } ^ { - 3 }}$

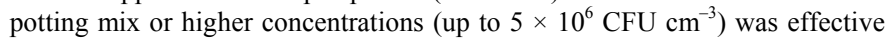
$(P<0.05)$ at reducing severity of radish scab. Increasing the frequency of BAC03 application did not increase efficacy for disease reduction. In addition, BAC03 increased $(P<0.05)$ the biomass of radish roots and leaves in the presence of the pathogen. The above results were confirmed by quantitative polymerase chain reaction that when BAC03 was applied before radish planting, the population of $S$. scabies was significantly lower $(P<0.5)$ than that in other BAC03 treatments at later stages.

Investigation of the genes involved in the viable but nonculturable state in Clavibacter michiganensis subsp. michiganensis

N. JIANG (1), S. Han (2), Q. Lv (2), R. Walcott (3), J. Li (2), L. Luo (2)

(1) China Agricultural University, Athens, GA, U.S.A.; (2) China Agricultural University, Beijing, China; (3) The University of Georgia, Athens, GA, U.S.A. Phytopathology 105(Suppl. 4):S4.65

The viable but nonculturable (VBNC) state in which bacteria fail to produce colonies on culture media but remain viable, is a unique survival strategy for some non-sporulating bacteria. Clavibacter michiganensis subsp. michiganensis (Cmm), the causal agent of bacterial canker of tomato, could be induced into the VBNC state by exposure to copper ions in an oligotrophic environment. To study the mechanisms involved in $\mathrm{Cmm}$ VBNC state, the transcriptional profiles of VBNC cells induced by copper sulfate for $5 \mathrm{~min}, 1$ $\mathrm{d}, 2 \mathrm{~d}$ and $10 \mathrm{~d}$ were analyzed using RNA-Seq. Gene expression in these cells was compared to that of $\mathrm{Cmm}$ cells that were not exposed to copper sulfate. Under the experimental conditions, approximately $95 \%$ of the total 3080 $\mathrm{Cmm}$ genes were expressed. Of these expressed genes 114 were up-regulated and 38 were down-regulated in bacterial cells exposed to copper ions. Seventy of the up-regulated genes were associated with catalytic processes. Other notable up-regulated genes included genes associated with copper resistance ( (relA and $\operatorname{clpC}$ ). The down-regulated genes included those that were involved in the biosynthesis of amino acids and $\mathrm{CoA}, \mathrm{ABC}$ transporters, electron carrier activity, transcriptional repressors, DNA repair and cell division. These findings will advanced our understanding of the mechanisms involved in the VBNC state in Cmm cells.

Phenotypic and genotypic characterization of isolates of the wheat stem rust pathogen collected from epidemic regions in East Africa in 2013 and 2014

Y. JIN (1), P. Olivera (2), M. Newcomb (2), G. Woldeab (3), E. Hailu (3), R. Wanyera (4), D. Hodson (5), M. Rouse (6), D. Luster (7), L. Szabo (6)

(1) USDA ARS, St. Paul, MN, U.S.A.; (2) University of Minnesota, Department of Plant Pathology, St. Paul, MN, U.S.A.; (3) Ethiopian Institute of Agricultural Research, Ambo Center, Ambo, Ethiopia; (4) Kenya Agricultural Research Institute, Njoro, Kenya; (5) CIMMYT-Ethiopia, Addis Ababa, Ethiopia; (6) USDA ARS, St. Paul, MN, U.S.A.; (7) USDA ARS, Fort Detrick, MD, U.S.A.

Phytopathology 105(Suppl. 4):S4.65 
Recent severe yield losses due to stem rust (caused by Puccinia graminis $\mathrm{f}$. sp. tritici-Pgt) have been documented in wheat crops in Ethiopia (2013-14) and Kenya (2013-14), in areas where newly detected virulences have caused high disease severities on previously resistant varieties. Early detection and characterization of $P g t$ races is critical for developing sound control strategies. Here we report on the identification and characterization of selected isolates of Pgt from field collections in 2013 and 2014, including from areas affected by epidemics in Ethiopia and Kenya. The races were identified using the North American stem rust differentials, and were further characterized phenotypically using a set of stem rust resistance gene stocks and genotypically using SNP markers. The Pgt race associated with the 2013-14 epidemic in southern Ethiopia was identified as TKTTF. Isolates of this same race was identified from field collections from a number of countries in the Middle East and Europe. Further phenotypic characterizations showed differences in virulences to 4 resistance genes, while genotypic data revealed isolate differences within and between countries. We identified two newly detected races associated with the 2014 epidemic in parts of Kenya. Combined phenotypic and genotypic analyses indicated that these Pgt isolates from Kenya are new variants within the Ug99 race group. These isolates have been used to evaluate North American and international breeding germplasm and cultivars.

\section{Defining the host range of the switchgrass rust fungus, Puccinia} emaculata

B. Johnson (1), G. ORQUERA (1), C. Garzón (1), S. Marek (1)

(1) Oklahoma State University, Stillwater, OK, U.S.A

Phytopathology 105(Suppl. 4):S4.66

Switchgrass (Panicum virgatum) is a perennial grass being developed as a potential feedstock for cellulosic biofuel production. Puccinia emaculata, the fungal pathogen causing yield limiting switchgrass rust, has an uncertain host range. The main objective of this study was to evaluate the susceptibility to $P$. emaculata of twenty-one accessions of native and introduced grasses, representing sixteen different species. Plant responses to urediniospore inoculations were recorded at 2, 3, and 5 weeks post-inoculation and spores were collected from symptomatic plant tissues at 3- and 5-week intervals. Identification of $P$. emaculata infection was confirmed by PCR using DNA from urediniospores and symptomatic plant tissues and three increasingly specific primers sets: PuccF2 and Rust1, ITS1rust-F10d and -R3c, and SGRSP1-FW and -RV. While, two accessions of P. amarum (bitter panicgrass) and three accessions of $P$. virgatum, were identified as hosts, fifteen accessions, including Andropogon gerardii, Schizachyrium scoparium, Sorghastrum nutans, $P$. antidotale, $P$. bergii, $P$. coloratum, $P$. deustum, $P$. hallii, $P$. miliaceum, and $P$. repens, showed no response to inoculations with $P$. emaculata and are likely nonhosts. Symptomatic accessions displayed responses ranging from chlorotic flecks to varying amounts of sporulation. In conclusion, $P$. amarum is a potential alternative host of $P$. emaculata that may act as a rust inoculum reservoir in bioenergy switchgrass fields.

\section{The use of endophytes to reduce growth of the soybean pathogen Sclerotinia sclerotiorum \\ A. JOHNSON (1), A. Impullitti (1) \\ (1) Augsburg College, Minneapolis, MN, U.S.A. \\ Phytopathology 105(Suppl. 4):S4.66}

Soybean diseases are managed by host resistance, agronomic techniques, or pesticides, but biological control is a rarely used tactic. Endophytes could be integrated into disease management since endophytes colonize plants asymptomatically and are known to reduce disease. The role of endophytes within soybean is unknown, and we investigated whether soybean fungal endophytes or their metabolites would inhibit growth of Sclerotinia sclerotiorum (Ss) in vitro and in planta. Antagonism assays were completed by plating one of thirty endophytic fungi and Ss onto PDA. Growth of Ss was reduced by $15 \%$ of the endophytes assayed, with antibiosis being the most common form of antagonism. Endophyte metabolites were also screened for antagonism to Ss. Ss was plated on PDA amended with metabolite concentrations of $0 \%, 0.05 \%, 0.5 \%, 5 \%$, and $10 \%(\mathrm{v} / \mathrm{v})$. Metabolites of nine endophytes reduced growth of SS by $40-60 \%$ when metabolite concentrations were 5 and $10 \%$. These nine endophytes were assayed in planta by planting soybean seeds with endophytes, inoculating plants with Ss, and ranking disease severity every three days. In a single experiment, there was no difference between inoculated soybeans planted with endophytes compared to soybeans planted without endophytes. This could suggest the endophyte application method was not effective, or that metabolites should be directly applied rather than the fungal cultures.
Differential response of citrus rootstock breeding lines to Huanglongbinginduced root loss

E. G. JOHNSON (1), J. H. Graham (1), J. W. Grosser (1)

(1) University of Florida, Lake Alfred, FL, U.S.A.

Phytopathology 105(Suppl. 4):S4.66

Commercial citrus rootstocks used in Florida are susceptible to Huanglongbing (HLB)-associated root loss caused by Candidatus Liberibacter asiaticus (Las). Las stimulates new fibrous root growth, while significantly reducing root lifespan, resulting in an overall loss of root mass. This dynamic of increased root growth and dieback shifts the carbohydrate balance in the tree, reducing the sugar available for fruit development and ripening and amplifying carbohydrate disruption caused by phloem plugging. New genetically complex rootstock breeding lines have slowed or delayed scion decline in one field trial even though their root systems were infected with Las. If these rootstocks are less susceptible to Las-induced root loss, they may reduce allocation of carbohydrate for root regeneration in favor of vegetative growth and fruit development. While most rootstock breeding lines had similar root loss compared to the commercial rootstocks, UFR-4 increased root mass as Las infection progressed through the tree and disease symptoms became more severe. UFR-4 was also the breeding line with the least symptomatic sweet orange scion after multiple years of infection. Las still stimulates root growth in UFR-4, but root lifespan is either not affected or less affected than other rootstocks evaluated. While natural sources of resistance remain elusive, reduced susceptibility to HLB root loss might sustain yields until trees become profitable.

Findings on the life cycle and biology of Botrytis cinerea causing cane botrytis on red raspberries in the Pacific Northwest

L. A. JONES (1), J. W. Pscheidt (1)

(1) Oregon State University, Corvallis, OR, U.S.A.

Phytopathology 105(Suppl. 4):S4.66

The Pacific Northwest (PNW) is the leading red raspberry growing region in the United States. Each year growers must manage gray mold and cane botrytis caused by Botrytis cinerea. Overwintering sclerotia produced on raspberry canes are one of the main sources of primary inoculum for flowers and fruits. In years where environmental conditions are very conducive for disease development large yield losses still occur even with current management practices. Much of what is known about cane botrytis has come from observations in regions other than the PNW. Specific regional information about a disease is important for management. Observations of $B$. cinerea on red raspberries in the PNW were made monthly during the growing season of 2014-2015. A number of these observations did not match details currently available in the literature including; many cane infections were not associated with petiole infections, some cane lesions did not produce sclerotia, and several isolates were not characteristic of $B$. cinerea when grown in the laboratory.

Amino terminal region of Phytophthora sojae cel12 endoglucanase confers tissue collapse function in Nicotiana

R. JONES (1)

(1) USDA ARS, Beltsville, MD, U.S.A.

Phytopathology 105(Suppl. 4):S4.66

Phytophthora encodes an unusually large number of glycosyl hydrolases $(\mathrm{GH})$, with many large gene families resulting from duplication events. There are ten copies of GH 12 (cel12) present in Phytophthora sojae. This is the only pathogen endoglucanase family to which plants produce an inhibitory protein termed xyloglucan-specific endoglucanase inhibitor protein (XEGIP). In an effort to genetically dissect the function of ten Phytophthora sojae cel12 endoglucanases, each gene was expressed in tobacco leaves using Agrobacterium infiltration. Expression of three Phytophthora cell2 endoglucanases, designated $12-\mathrm{G}, 12-\mathrm{A}$ and $12-\mathrm{I}$, caused water soaking and tissue collapse between 42-48 hours post infiltration of Nicotiana benthamiana leaves. None of the other Phytophthora cel12s caused macroscopic damage. Protein determinants were mapped using a series of domain swaps. Tissue collapse function was mapped to the exposed amino terminus of the protein and a signal peptide for extracellular transport was required for tissue damage to occur. Peptide infiltration did not elicit a response, and non-enzymatic fusion proteins also lacked tissue collapse functions. Expression levels of hypersensitivity marker genes PR2 and NADPH oxidase were not significantly different after infiltration of the tissue collapse constructs and other constructs. The cel12 amino terminus may confer unique activity on the cell wall substrate. 
Combating Rose Rosette Disease: Development of rapid, efficient, easyto-use virus diagnostic tools and studying virus-vector interactions R. JORDAN (1), J. Hammond (2), M. L. Paret (3), B. Babu (4), F. OchoaCorona (5), J. Olson (6), R. Ochoa (7), E. Roundey (8), D. Byrne (8) (1) US National Arboretum, Floral \& Nursery Plants Research, USDA-ARS, Beltsville, MD, U.S.A.; (2) USDA ARS FNPRU, Beltsville, MD, U.S.A.; (3) Department of Plant Pathology, North Florida Research and Education Center, University of Florida, Quincy, FL, U.S.A.; (4) North Florida Research \& Education Center, University of Florida, Quincy, FL, U.S.A.; (5) Oklahoma State University, Stillwater, OK, U.S.A.; (6) Department of Entomology \& Plant Pathology, Oklahoma State University, Stillwater, OK, U.S.A.; (7) Systematic Entomology Laboratory, Beltsville Agricultural Research Center, USDA-ARS, Beltsville, MD, U.S.A.; (8) Department of Horticultural Sciences, Texas A\&M University, College Station, TX, U.S.A.

Phytopathology 105(Suppl. 4):S4.67

Garden roses, which form the cornerstone of the multi-billion dollar landscape industry, annually generate wholesale US domestic production valued at $\sim \$ 400$ million. Over the past few decades Rose Rosette Disease has become very serious and threatens to decimate the US rose industry. The causal agent, Rose rosette virus (RRV), is transmitted by wind-blown eriophyid mites, and can kill a rose within 2-3 years of infection. The long term goal of a newlyfunded USDA-NIFA-SCRI Project (which includes 17 scientists in 6 states) is to develop roses resistant to this virus or mite vector. Key to this effort is the development of efficient diagnostic tools to enable rapid, user-friendly and accurate detection of the virus. RRV-specific primers, probes and monoclonal and $\mathrm{scFv}$ antibodies will be developed. Lateral flow devices (for serologicaland nucleic acid-based tests), for in-field detection, as well as ELISA, RTLAMP and self-quenched primer ( $\mathrm{SqP}$ ) technologies (for laboratory detection) will be developed, validated and transferred to plant diagnostic labs. Interactions between the mite vector and rose genotypes that are either resistant or susceptible to mite feeding, reproduction, or RRV transmission will be examined by low temperature SEM. Identified differences in leaf surface properties will be utilized for screening breeding lines. This project hopes to identify sources of resistance and develop genetic tools to move resistance into commercial cultivars.

Detection and characterization of Shamrock chlorotic ringspot virus and Beet ringspot virus in ornamental Oxalis

R. JORDAN (1), D. Mollov (2), M. A. Guaragna (1), A. Phibbs (3), B. E. Lockhart (4)

(1) US National Arboretum, Floral \& Nursery Plants Research, USDA-ARS, Beltsville, MD, U.S.A.; (2) USDA ARS NGRL, Beltsville, MD, U.S.A.; (3) Plant Industry Laboratory, Wisconsin Department of Agriculture, Trade and Consumer Protection, Madison, WI, U.S.A.; (4) University of Minnesota, Minneapolis, MN, U.S.A.

Phytopathology 105(Suppl. 4):S4.67

Ornamental Oxalis, commonly known as the Shamrock plant, is grown as a potted plant in the United States especially for marketing in the spring around St. Patrick's Day. A potyvirus causing chlorotic ringspot in ornamental Oxalis regnellii was first described in WA in 1981. Plants showing similar symptoms and having potyvirus particles were reported in NY in 2009 and in FL in 2012. The name Shamrock chlorotic ringspot virus (SCRV) was coined. Plants from WI (2012-2013) showing similar symptoms were submitted for analysis to the UM Plant Disease Clinic. Potyvirus-like filamentous virus particles were observed in symptomatic leaf samples by TEM. Total RNA extracted from a symptomatic plant tested positive for the presence of Potyvirus in RT-PCR using universal primers. The 3'-teminal $1690 \mathrm{bp}$ region was sequenced, found to be unique and deposited in GenBank as Shamrock chlorotic ringspot virus (Acc. \#KJ619376). The complete genome has since been fully sequenced. TEM analysis of partially purified virion preparations from symptomatic tissue revealed filamentous and spherical virus particles. RNA extracted from these preparations was used as template for a random PCR to produce a cDNA library. Cloned sequences revealed similarity to SCRV and Beet ringspot virus (BRSV). The complete nepovirus genome sequence (RNA 1 and 2) of BSRV has been determined. This is also the first report of BRSV in Oxalis. Inoculation of healthy Oxalis with the separate viruses is in progress.

\section{Metabolic and nutritional strategies of oomycete plant pathogens} H. JUDELSON (1), M. Kagda (1), M. Abrahamian (1), A. Ah Fong (1) (1) University of California, Riverside, CA, U.S.A.

Phytopathology 105(Suppl. 4):S4.67

In order to reproduce, pathogens must feed on their hosts. Understanding how pathogens acquire and metabolize host nutrients is therefore fundamental to plant-microbe interactions. We have reannotated the genome of the potato late blight pathogen, Phytophthora infestans, to help study its metabolic proteins.
Analyses of the results indicates that $P$. infestans is largely auxotrophic and carries out metabolism using an amalgam of genes obtained by normal evolutionary descent and by horizontal gene transfer from bacteria, fungi, and/or plants. Expression-profiling during infection has identified signatures of the biotrophic and necrotrophic stages of growth, and infection-regulated metabolic genes. These include both metabolic enzymes and transporters used to acquire nutrients. Gene silencing and subcellular localization studies using fluorescently-tagged proteins has identified genes involved in carbon and nitrogen assimilation that are specifically expressed in infection structures and essential for pathogenesis. Some of the genes may also be important for inactivating toxicants that the pathogen may experience in planta. Comparisons of expression in different oomycetes suggest that some specialization has occurred in their respective metabolomes, perhaps reflecting adaptation to different host species and life styles.

Does disease escape cause a trade-off between yield and Septoria in wheat?

C. JUDGE (1), J. Brown (1)

(1) John Innes Centre, Norwich, United Kingdom

Phytopathology 105(Suppl. 4):S4.67

Zymoseptoria tritici, the fungus that causes Septoria tritici blotch (STB) of wheat, is spread by splash borne transfer from the base of the plant to the flag leaf. Our studies into a QTL strongly associated with field levels of STB indicate that this region contains genes that cause variation in disease escape rather than disease resistance. Near isogenic lines generated for this region showed no significant differences in STB symptoms when leaves were directly inoculated with Z. tritici. However, there were clear differences in STB levels in trials that were basally inoculated or naturally infected. This QTL is also associated with yield traits, which led to the hypothesis that genes in this region may control a physiological trait that improves yield at the cost of aiding Z. tritici spore transmission. We considered candidate physiological and developmental traits that could influence this process, ruling out the difference being caused by known escape traits such as height and leaf spacing. Leaf area and senescence showed consistent differences in the NIL pairs in all glasshouse and field trials. Field levels of Septoria were lower in the lines which had smaller, earlier senescing leaves, but these also had lower grain filling potential and thus lower 1000 grain weights. This indicates that whilst breeding for larger, longer living leaves has increased crop yield, this ideotype also increases levels of STB by reducing the amount of disease escape.

Exploring the Penicillium expansum genome for virulence genes mediating blue mold infection on pome fruits

W. JURICK (1), J. Yu (2), V. L. Gasins (2), Y. Yan (3)

(1) USDA ARS - Food Quality Laboratory, Beltsville, MD, U.S.A.; (2) UDSA-ARS, Beltsville, MD, U.S.A.; (3) Department of Biological Sciences, Northern Illinois University, Dekalb, IL, U.S.A.

Phytopathology 105(Suppl. 4):S4.67

According to the US Apple Association, 248.6 million bushels of apples were produced with a farm-gate value of $\sim \$ 2.7$ billion dollars in 2013. Apples are stored for extended periods of time during which postharvest decays develop. The most economically significant and widespread disease is blue mold, caused by Penicillium expansum. The fungus is highly virulent and produces mycotoxins that contaminate processed apple products. Whole genome sequencing of a wild-type $P$. expansum isolate via the Illumina NGS platform was conducted to identify genes involved in virulence and to better understand regulatory mechanisms of infection. The sequences were assembled de novo and annotated using bioinformatics tools. The resulting $P$. expansum genome was estimated to be $31.4 \mathrm{Mb}$, which is similar to what has been reported for $P$. chrysogenum $(32.2 \mathrm{Mb})$. The fungus has 10,554 predicted genes, with a mean length of $1,599 \mathrm{bp}$. Total coding sequence comprises $53.70 \%$ of the fungal genome. Gene functional classification by KEGG indicated 664 categories of the 2,458 genes with predicted function. Genes potentially involved in virulence can now be identified and are the subject of further investigation. The knowledge obtained from $P$. expansum functional genomics studies represents a significant step toward understanding the mechanisms of infection to design control strategies against blue mold decay during storage.

Virulence of Fusarium oxysporum f.sp. cubense on selected banana cultivars in the Philippines

M. H. Juruena (1), A. B. MOLINA (2), T. U. Dalisay (3), M. P. Natural (3), C. M. Protacio (4)

(1) Office of the Provincial Agriculturist, Tagum City, Philippines; (2) Bioversity Intl, Laguna, Philippines; (3) Department of Plant Pathology, University of the Philippines Los Banos, Los Banos, Philippines; (4) Department of Horticulture, University of the Philippines Los Banos, Los Banos, Philippines Phytopathology 105(Suppl. 4):S4.67 
Fusarium oxysporum f.sp. cubense (Foc) VCG 1213/16, strain associated to Tropical Race 4 (TR4) and VCG 0126, a Race 1 strain, were evaluated for their pathogenicity and virulence to five banana cultivars under screen house and field conditions in Davao del Norte, Philippines. Virulence of the Foc strains was measured in terms of vascular discoloration and leaf yellowing indices, and disease incidence in the field. Foc strains caused varying levels of virulence depending on the cultivars. VCG 1213/16 caused more vascular discoloration and leaf yellowing indices on Lakatan (AAA), Gran Naine (AAA) and Latundan (AAB) as compared to Cardaba (BBB??) under screen house evaluation. In the field Fusarium wilt incidence in Lakatan, Gran Naine and Latundan was 100, 72, and 50\% respectively, and Cardaba at $11 \%$. VCG 0126 was more virulent on Latundan and Cardaba based on the Fusarium wilt incidence and severity rating resulting in susceptible reaction, while Lakatan and Gran Naine were resistant to VCG 0126. A somaclonal Cavendish variant selection GCTCV 119 (AAA) was resistant to both Foc strains in both screen house and field tests. Results indicate existence of VCG by variety interaction in terms of virulence and resistance, a concept relevant in cultivar deployment. While TR4 may be virulent in many cultivars, a Race 1 strain maybe more virulent on some varieties.

\section{Predisposition of maize and groundnut to aflatoxin contamination in} Zambia

P. KACHAPULULA (1), R. Bandyopadhyay (2), J. Akello (3), M. Mukanga (4), P. J. Cotty (5)

(1) Univ of Arizona, Tucson, AZ, U.S.A.; (2) International Institute of Tropical Agriculture, Ibadan, Nigeria; (3) International Institute of Tropical Agriculture, Lusaka, Zambia; (4) Zambia Agriculture Research Institute, Lusaka, Zambia; (5) University of Arizona, Tucson, AZ, U.S.A.

Phytopathology 105(Suppl. 4):S4.68

Aflatoxins are carcinogenic toxins that frequently contaminate maize and groundnut. Aspergillus flavus, A. parasiticus and two unnamed taxa have been implicated as causal agents of aflatoxin contamination in Africa. Improved understanding of etiology in Zambia may facilitate aflatoxin management. Maize $(n=225)$ and groundnut $(n=137)$ samples from three agroecologies of Zambia were examined from 2012 to 2014. Fungi were isolated and assigned to morphological species with $A$. parasiticus the most common followed by $A$. flavus L strain and fungi with S strain morphology. Crop aflatoxin content was quantified with lateral flow immunochromatographic assays. Agroecozone One had the highest proportion of unfit $(>10 \mathrm{ppb})$ groundnuts and maize ( $56 \% \& 20 \%$, respectively). To investigate potential for safe crops to become contaminated in storage, crop samples $(5 \mathrm{~g})$ were incubated without inoculation $\left(31^{\circ} \mathrm{C}, 100 \% \mathrm{RH}, 7\right.$ days $)$ and the aflatoxin content quantified with fluorescence densitometry after thin layer chromatography. Aflatoxins increased $(\mathrm{p}<0.05)$ in $70 \%(\mathrm{n}=61)$ of the crops; $60 \%(\mathrm{n}=37)$ increased to above $10,000 \mathrm{ppb}$. Greater increases occurred in groundnuts than maize. The most common species in groundnut was also the species that produced the highest average concentrations of aflatoxins in sterilized maize, $A$. parasiticus $(40 \%$ and $22 \%$ of section Flavi in groundnut and maize, respectively). Etiology of contamination in Zambia is complex with several causal agents.

Efficacy of seed-applied nematicides on the management of the reniform nematode (Rotylenchulus reniformis) in cotton

S. KAKAIRE (1), T. W. Allen (2), B. R. Golden (1)

(1) Mississippi State University, Stoneville, MS, U.S.A.; (2) Mississippi State Univ, Stoneville, MS, U.S.A.

Phytopathology 105(Suppl. 4):S4.68

The reniform nematode (Rotylenchulus reniformis) is a serious pest of cotton (Gossypium hirsutum) in the Mississippi (MS) Delta. A trial to determine the efficacy of three seed-applied nematicides on the management of the reniform nematode in cotton was conducted in Stoneville, MS during 2012. Three cotton cultivars, Deltapine 0912B2RF, Barbren and Lonren were treated with either Aeris, Avicta, or Votivo with a non-treated control of each cultivar included for comparison. Two silt-loam fields infested with the reniform nematode were utilized for the trial. Data on cotton stand counts and plant heights, as well as reniform nematode population densities pre-plant, 10weeks post-plant, and at harvest were collected in addition to lint yield. Data were analyzed by test location using PROC GLM in SAS (version 9.3), since significant differences between lint yield and nematode population densities at harvest occurred between fields. Seed treatment did not significantly affect lint yield; however, measureable mathematical differences were observed. Reniform nematode population densities increased throughout the season; however, no significant differences were observed between seed-applied nematicide treatments or the cotton cultivars investigated.
Detection of adult plant resistance (APR) to Puccinia triticina in native wheat species, transfer and mapping in wheat

B. KALIA (1), R. P. Singh (2), R. L. Bowden (3), E. Edae (1), J. Poland (1), B. S. Gill (1)

(1) Kansas State University, Manhattan, KS, U.S.A.; (2) CIMMYT, El Batan, U.S.A.; (3) USDA-ARS, Manhattan, KS, U.S.A.

Phytopathology 105(Suppl. 4):S4.68

Resistance to wheat rusts may be race-specific and subject to boom and bust cycles, or race-nonspecific, adult plant resistance (APR), which is associated with durability. We evaluated Aegilops tauschii, one of the diploid ancestors of wheat, for APR to leaf rust, caused by the fungus, Puccinia triticina. The Ae. tauschii populations in Caspian Iran and eastern Afghanistan commonly exhibited APR, suggesting that APR may be an important defense in nature against leaf rust. APR to leaf rust was transferred from Ae. tauschii (TA2474) to wheat through production of synthetic hexaploid wheat (SHW), but APR expression was suppressed in the progeny. To unlock the expression of APR, a population of 261 recombinant inbred lines (RILs) was developed from a cross of SHW with wheat cultivar WL711. The RILs were phenotyped for maximum disease severity (MDS) at Manhattan, Kansas in 2013-2014 and at CIMMYT, Mexico in 2013. Genotyping-by-sequencing (GBS) detected quantitative trait loci (QTLs) associated with APR and was contributed by both the parents. Two major QTLs from WL711 were mapped on chromosome $1 \mathrm{BL}$, explaining $11-24 \%$ of the phenotypic variance across environments and two additional QTLs were mapped on 5AL and 6BL. SHWderived QTLs for APR were mapped on 1AL, 1BS, 2DS, 2DL and 5DL. The results demonstrate complex genetic control and evolution of APR. The novel APR genes and their linked GBS-based SNP markers are potentially useful for durable control of leaf rust in wheat.

Characterization of the transcriptome and pathogen small RNA associated with stress memory in a commercial crop

M. L. KALISCHUK (1), D. L. Johnson (2), L. M. Kawchuk (3)

(1) Lethbridge College, Lethbridge, AB, Canada; (2) University of Lethbridge, Lethbridge, AB, Canada; (3) Agriculture and Agri-Food Canada, Lethbridge, $\mathrm{AB}$, Canada

Phytopathology 105(Suppl. 4):S4.68

Stress memory is a form of inherited resistance in plants. To explore stress memory in a commercial crop, Polish canola (Brassica rapa $c v$ R018) was exposed to 50,100 or $200 \mathrm{ng}$ purified Cauliflower mosaic caulimovirus isolate LRC2010 (CaMV) at two, three or four weeks following germination. Exposure of B. rapa to CaMV at $50 \mathrm{ng}$ purified virus particles at four weeks following germination produced plants with significantly large seeds and the progeny showed resistance to CaMV. The average seed sizes were $2.12+/-$ $0.08 \mathrm{~mm}, 1.99+/-0.08 \mathrm{~mm}$ and $2.09+/-0.09 \mathrm{~mm}$ for CaMV challenged resistant, susceptible and healthy treatment progeny, respectively. Differences in defense pathways involving fatty acids, primary and secondary metabolites were detected in the transcript of CaMV challenged progeny. To examine the interplay between host and pararetrovirus interactions, the Rubus yellow net caulimovirus (RYNV) genome and viral small RNA were sequenced. Numerous nucleic acid binding motifs, multiple zinc finger-like sequences and domains associated with cellular signalling were detected. Silencing as a mechanism to combat virus accumulation was indicated by an uneven genome-wide distribution of 22-nt length virus-derived small RNAs with strong clustering to small regions distributed over both strands of the RYNV genome. Results from these studies continue to elucidate the complex arms race between plant hosts and plant pararetrovirus pathogens.

Evidence for at least two introductions of the sudden oak death pathogen into Oregon forests

Z. N. KAMVAR (1), M. M. Larsen (2), A. M. Kanaskie (3), E. M. Hansen (1), N. J. Grünwald (2)

(1) Oregon State University, Corvallis, OR, U.S.A.; (2) USDA ARS HCRL, Corvallis, OR, U.S.A.; (3) Oregon Department of Forestry, Salem, OR, U.S.A.

Phytopathology 105(Suppl. 4):S4.68

In the mid 1990's, a disease known as sudden oak death was discovered in California forests. The causal agent was formally described as Phytophthora ramorum. After first detection in Oregon forests in 2001, the epidemic was immediately subjected to an aggressive eradication effort by the Oregon Department of Forestry. A total of 512 isolates from symptomatic tanoaks were sampled from 2001 to 2014 and genotyped using five microsatellite markers. Population genetic history was inferred using model-free methods appropriate for clonal populations. While three clonal lineages exist on the North American west coast, only the NA1 lineage has been discovered in OR forests. Since the initial introduction event into the Joe Hall area, the pathogen has spread North, West, and Southeast within Curry county. A discovery in 
the Hunter Creek area in 2011 appears to be a second introduction, as it does not cluster with the early introduction or subsequent infections. Including west coast nursery populations $(\mathrm{n}=216)$ in the analyses provides support for at least two distinct introduction events from Oregon or California nurseries into OR forests. Continued vigilance and eradication of nursery populations of $P$. ramorum are important to avoid further emergence and potential introduction of other clonal lineages.

Influence of planting date, seed treatment, and cultivar on plant establishment, sudden death syndrome, and yield of soybean

Y. R. KANDEL (1), K. A. Wise (2), C. A. Bradley (3), A. U. Tenuta (4), L. F. S. Leandro (1), D. S. Mueller (1)

(1) Iowa State University, Ames, IA, U.S.A.; (2) Purdue University, West Lafayette, IN, U.S.A.; (3) University of Illinois, Urbana, IL, U.S.A.; (4) Ontario Ministry of Agriculture Food and Rural Affairs, Ridgetown, ON, Canada

Phytopathology 105(Suppl. 4):S4.69

A two year study was conducted in Iowa, Illinois, Indiana, and Ontario in 2013 and 2014 to determine the effects of planting date, seed treatment, and cultivar on plant stand, sudden death syndrome (SDS), and grain yield of soybean. Soybeans were planted from late April to mid-June at around 15-day intervals, for a total of three to four plantings per site-year. For each planting date, two cultivars differing in SDS susceptibility were planted with and without fluopyram seed treatment. Effect of planting date on foliar SDS symptoms was inconclusive. Mid-May plantings resulted in higher disease compared to other planting dates in two site-years, early June plantings in three, and the remaining six site-years were unaffected by planting date. Root rot was higher in May plantings for most site-years. Resistant cultivars had lower disease than the susceptible variety in most site-years. Fluopyram reduced disease severity by 12.5 to $95.2 \%$ across the site-years, and increased yield nearly in all plantings and cultivars, with a maximum yield response of $1142 \mathrm{~kg} / \mathrm{ha}$. Plant population was reduced by fluopyram seed treatment and early plantings in some site-years; however grain yield was not affected by these reductions. Mid-June plantings yielded up to $30 \%$ lower grain than early May plantings. Based on this experiment, delayed planting was not useful for SDS management, but cultivar selection combined with fluopyram seed treatment can reduce SDS in timely planted soybeans.

Control efficacy of an extract of cultured Xylogone ganodermophthora on powdery mildew caused by Podosphaera xanthii on watermelon in Korea H. J. KANG (1), Y. Kim (1), T. Kim (1), C. U. Han (1), T. Jeong (1), S. Y. Nam (2)

(1) Watermelon Research Institute CBARES, Eumseong-gun Chungcheongbukdo, Korea; (2) Watermelon Research Institute CBARES, Eumseong-gun Chungcheongbuk-do, South Korea

Phytopathology 105(Suppl. 4):S4.69

Xylogone ganodermophthora $(\mathrm{Xg})$ is an ascomycetous fungus that causes yellow rot on cultivated Ganoderma lucidum. Previously, we reported the in vitro antifungal activities of an $\mathrm{Xg}$ culture extract against several watermelon pathogens, including Phytophthora capsici, Fusarium oxysporum, Sclerotium rolfsii, and Podosphaera xanthii (Px). In 2014, we carried out greenhouse experiments to evaluate the control efficacy of a water extract of cultured $\mathrm{Xg}$ on watermelon powdery mildew (WPM). The test material (stock solution) was prepared by autoclaving an $\mathrm{Xg}$ culture in water at a ratio $800 \mathrm{~g}$ of culture per $6 \mathrm{~L}$ of water and then filtering it through filter paper. In the first trial, application of the solution (diluted 100- and 1,000-fold) at 7-day intervals suppressed the formation of conidiophores and conidia significantly. In the second trial, the 100-fold diluted solution was applied to watermelon plants 14 times before harvest (no fungicide was used). Near harvest time, more than $90 \%$ leaves of the plants were infected with Px. However, the farmer harvested $93.5 \%$ marketable fruits. These results suggest that a water extract of cultured $\mathrm{Xg}$ could be used as an environmentally friendly agent for the control of WPM in greenhouses.

Fungicide sensitivity of the wheat stripe rust pathogen (Puccinia striiformis f. sp. tritici)

Z. Kang (1), X. Li (1), A. Wan (1), M. Wang (1), X. CHEN (2)

(1) Washington State University, Pullman, WA, U.S.A.; (2) USDA ARS, Pullman, WA, U.S.A.

Phytopathology 105(Suppl. 4):S4.69

Fungicides have been widely used to control wheat stripe rust caused by Puccinia striiformis f. sp. tritici (Pst) but it is not clear whether any tolerance has developed in the Pst population. In this study, Pst isolates were tested for sensitivity to Tilt (propiconazole) and Headline (pyraclostrobin) by germinating urediniospores and infecting wheat seedlings and adult-plants in a greenhouse at various concentrations. No significant difference was observed among isolates at the concentration rates of 1.00 (the full-labeled rate; 1.64 $\mathrm{mg} / \mathrm{ml})(100 \%$ dead $)$ and of 0.04 and lower; while significant differences were observed among the isolates at the rates of $0.06-0.20$ in the germination test with Tilt. With Headline, significant germination differences were observed among the isolates at the rates of $0.0015-0.0040$, but no significant differences in the rates below 0.0015 or at 0.0075 and 1.0 (the full-labeled rate, 3.7 $\mathrm{mg} / \mathrm{ml})(100 \%$ dead). No sporulation was observed at the rates of 1.0 and 2.0 of Tilt or 0.5 and 1.0 of Headline applied one day before; 0.25 and higher rates of Tilt or 0.5 and 1.0 of Headline applied at the same day; and at the rate of 1.0 of Headline applied three days after Pst inoculation, while significant differences were observed in the other rates in the seedling tests. Similar results were obtained in the adult-plant tests. Thus, the fungicides are still effective at the full-labeled rates, but different sensitivities exist among Pst isolates.

\section{Molecular detection of rust fungi on turfgrass}

B. KARAKKAT (1), V. Jackson (1), P. Koch (1)

(1) University of Wisconsin-Madison, Madison, WI, U.S.A.

Phytopathology 105(Suppl. 4):S4.69

Rust is one of the main diseases of turfgrass in Wisconsin and across the country. Historically, the two primary rust diseases on cool-season turfgrasses have been crown rust [CR] (Puccinia coronata) and stem rust [SR] (Puccinia graminis). Increasing rust severity has been documented in the past 10 years, and recent research indicates it may be due to increasing activity of CR on a wider array of turfgrass species. We are developing a Quantitative polymerase chain reaction (QPCR) detection method to boost this screening process so that the identification is more reliable and faster than only microscopic observation of urediniospores and teliospores. Initial attempts at extraction of genomic DNA from rust infected leaf samples gave only a few positive results in the traditional PCR mainly because genomic DNA from uredinia were only present in trace amounts. However, more consistent positive results were obtained when QPCR was employed with the same genomic DNA using sequence specific fluorescent probes of $P$. coronata and $P$. graminis. By conducting this study, we aim to test over 400 rust samples collected from different turf plots from around the Midwest in 2013 and 2014 and report the percentage of each Puccinia species observed.

Genetic relationships between race nonspecific and race specific interactions in the wheat-Pyrenophora tritici-repentis pathosystem

G. KARIYAWASAM (1), A. H. Carter (2), J. B. Rasmussen (1), J. D. Faris (3), S. S. Xu (3), M. Mergoum (1), Z. Liu (4)

(1) North Dakota State University, Fargo, ND, U.S.A.; (2) Washington State University, Pullman, WA, U.S.A.; (3) USDA-ARS, Northern Crop Science Laboratory, Fargo, ND, U.S.A.; (4) North Dakota State Univ, Fargo, ND, U.S.A.

Phytopathology 105(Suppl. 4):S4.69

Tan spot, caused by Pyrenophora tritici-repentis, is a destructive disease on wheat worldwide. The disease system is known to involve race-specific interactions that are determined by fungal-produced necrotrophic effectors (NEs) and corresponding host sensitivity genes. Recently, host genetic factors conferring race-nonspecific resistance have also been identified. In this work, we mapped a quantitative trait locus (QTL) conferring race-nonspecific resistance and characterized its relationships with the NE-host gene interactions in a recombinant inbred line (RIL) spring wheat population derived from Louise and Penawawa. We identified a major QTL on the chromosome 3BL for resistance to races 1, 2, 3 and 5 (race nonspecific), a QTL on 1AS for resistance to races 1 and 3 (race specific), which likely corresponded to the $T s c 1$ locus, and a few minor QTL. Although the entire population was sensitive to Ptr ToxA, the majority of RILs carrying the 3BL QTL from Penawawa were highly resistant to race 2, which produces Ptr ToxA. $F_{1}$ plants derived from Penawawa and Louise were highly resistant to race 2, but had an intermediate reaction to race 3 . These results suggest that the 3BL race-nonspecific QTL is epistatic to the Ptr ToxA-Tsn1 interaction, but is additive to the Ptr ToxC-Tscl interaction. This work provides further understanding of genetic resistance in wheat tan spot system as well as an important guidance for tan spot resistance breeding.

Efficacy of fungicides on mycelial growth and pigmentation, and sclerotia and oxalic acid production by Sclerotinia sclerotiorum

G. KAUR (1), P. Lujan (2), S. Sanogo (2), N. Puppala (2)

(1) New Mexico State University, Las Cruces, NM, U.S.A.

Phytopathology 105(Suppl. 4):S4.69

Sclerotinia blight caused by Sclerotinia sclerotiorum is a disease that affects several crops worldwide. This pathogen was reported on peanut and cabbage 
in New Mexico, and on peanut in West Texas; however, the extent to which the pathogen has spread is still unknown. The objective of this study was to evaluate the efficacy of four fungicides (fluopyram, penthiopyrad, fluazinam and boscalid) on mycelium growth and pigmentation, and sclerotia and oxalic production by $S$. sclerotiorum under lab conditions. A suspension of each fungicide was prepared at the recommended field rate, and spread and dried over the surface of solidified potato dextrose agar (PDA) medium. PDA plates with no fungicides were used as control. A 1-cm mycelium plug from one isolate of S. sclerotiorum from peanut and cabbage was placed centrally onto amended PDA and control PDA plates. Mycelial growth and pigmentation, and sclerotia and oxalic acid production were recorded daily for one week. The study was repeated four times with five replications for each treatment. Results showed that fluopyram and boscalid reduce mycelium growth compared to control, fluazinam, and penthiopyrad. Sclerotia (on both isolates) and mycelium pigmentation (only on peanut isolate) were noticed on control PDA, and PDA amended fluazinam and penthiopyrad. All fungicides did not inhibit oxalic acid production based on the presence of yellow halo on bromophenol blue plates.

\section{Apoplastic targeting of plant defensin MtDef4 confers strong resistance to leaf rust pathogen Puccinia triticina in transgenic wheat}

J. KAUR (1), J. Fellers (2), T. Clemente (3), D. Shah (1)

(1) Donald Danforth Plant Science Center, Saint Louis, MO, U.S.A.; (2) Dept. of Plant Pathology, Kansas State University, Manhattan, KS, U.S.A.; (3) Center for Biotechnology, University of Nebraska-Lincoln, Lincoln, NE, U.S.A.

Phytopathology 105(Suppl. 4):S4.70

Rust diseases caused by fungi of the order Pucciniales remain a major threat to global wheat production. Basidiomycete Puccinia triticina, an obligate biotroph causes leaf rust disease in wheat. Current disease management strategies involving the use of resistant wheat cultivars and fungicide application fail to provide complete resistance because of the rapid evolution of new virulent pathotypes. Genetic engineering of wheat using antifungal plant defensins can augment the current control strategies. MtDef4 is a 47 aa cysteine-rich defensin from Medicago truncatula with broad-spectrum antifungal activity. This plant defense effector protein contains an RxLR-like motif that is required for its entry into fungal cells. Expression of MtDef4 in the apoplast confers resistance to an obligate oomycete biotroph Hyaloperonospora arabidopsidis in transgenic Arabidopsis. We have generated transgenic wheat lines expressing apoplast-targeted MtDef4. Two of the four homozygous single-copy transgenic wheat lines showed strong resistance to $P$. triticina race MCPSS in the greenhouse, compared to nontransgenic controls. Histopathological observations and fungal gene expression profile data further support the resistance observed in transgenic wheat lines. This study demonstrates that expressing an apoplast-localized defensin is an effective strategy in providing resistance to a biotrophic basidiomycete fungal pathogen in transgenic wheat.

\section{Temporally altered gene expression in Bemisia tabaci during feeding on tomato infected with the semipersistent Tomato chlorosis virus}

N. KAUR (1), W. Chen (2), Y. Zheng (2), Z. Fei (2), W. M. Wintermantel (1) (1) USDA ARS, Salinas, CA, U.S.A.; (2) Boyce Thompson Institute, Ithaca, NY, U.S.A.

Phytopathology 105(Suppl. 4):S4.70

The intimate relationship between vector whiteflies, plant viruses, and host plants are well documented, but little information exists on how host plant infection by semipersistent viruses may influence the whitefly vector or its ability to acquire and retain a plant virus. In order to determine changes in global transcriptional gene regulation in whiteflies resulting from feeding on plants infected with a semipersistent virus, paired-end 151-bp RNA-Seq was performed on whitefly (Bemisia tabaci MEAM1) after 24, 48, and 72 hours of feeding on Tomato chlorosis virus (ToCV)-infected and healthy tomato in three biologically replicated tests. Among a total of 14,061 genes predicted in the whitefly genome, we identified significantly increased expression levels for 88,2 , and 15 genes in whiteflies fed on ToCV-infected tomato during the time course of $24 \mathrm{~h}, 48 \mathrm{~h}$, and $72 \mathrm{~h}$, respectively, compared with whiteflies fed on healthy plants. This indicates temporally influenced gene expression in whiteflies due to the presence of $\mathrm{ToCV}$ in phloem, and that gene expression is influenced within the first 24 hours, with fewer and different genes activated at 72 hours, corresponding with the time at which ToCV retention in the vector subsides approximately 72 hours following virus acquisition. Further characterization and analysis of these up-regulated genes may reveal specific pathways associated with whitefly-species specific virus transmission.
Evaluation of perennial ryegrass cultivars for klendusity to ergot N. KAUR (1), J. Dung (2), S. Alderman (3), D. Walenta (4), K. Frost (1), P. Hamm (1)

(1) Hermiston Agricultural Research and Extension Center Oregon State University, Hermiston, OR, U.S.A.; (2) Central Oregon Agricultural Research Center Oregon State University, Madras, OR, U.S.A.; (3) USDA ARS Forage Seed and Cereal Research, Corvallis, OR, U.S.A.; (4) Union County Extension Service Oregon State University, LaGrande, OR, U.S.A.

Phytopathology 105(Suppl. 4):S4.70

Claviceps purpurea, causal agent of ergot, infects the unfertilized flowers of Lolium perenne (perennial ryegrass; PRG) resulting in the production of sclerotia instead of seed. PRG cultivars with short, uniform flowering periods or those that flower when ascospores are absent may escape infection. In 2014, twelve PRG cultivars were evaluated for klendusity to ergot. Plots $(9.1$ $\mathrm{m}$ by $1.2 \mathrm{~m}$ ) with 4 replications for each cultivar) were planted and artificially infested in the fall of 2013 by distributing $0.01 \mathrm{~kg}$ ergot uniformly over the plot area. Plants within each plot were examined weekly during anthesis from May 8, 2014 until harvest. When honeydew symptoms first appeared, 10 seed heads were arbitrarily collected from the plot and examined for honeydew (disease incidence) on June 11, 2014 and June 18, 2014. Ergot severity (average sclerotia per seed head out of 40 per plot) was determined at harvest. Significant correlations were observed between ergot severity and anthesis initiation date $(P=0.03 ; r=-0.31)$ and duration $(P<0.001 ; r=0.41)$, suggesting that ergot is reduced in cultivars that initiate anthesis later in the year or in cultivars with shorter anthesis periods. This information may have application in the development of new cultivars with greater klendusity to ergot.

Antimicrobial-independent disease suppression by lipopeptide iturin for a bacterial leaf disease on Oryza sativa

T. KAWASHIMA (1), K. Hashimoto (1), M. Ohbu (1), H. Shinohara (2), K. Yokota (1)

(1) Tokyo Univ of Agriculture, Tokyo, Japan; (2) Tokyo Univ of Agriculture, Kanagawa, Japan

Phytopathology 105(Suppl. 4):S4.70

Iturin is an antimicrobial cyclic lipopeptide produced by antagonistic Bacillus spp. It has been deduced that iturin plays a key role in disease suppression by depending on its antimicrobial activity against several kinds of plant pathogenic microbes. Our previous study suggested that the disease suppression by iturin is not only depending on its antimicrobial activity, we therefore examined the antimicrobial-independent disease suppression by iturin on a bacterial disease on rice, Oryza sativa. The causal agent of rice bacterial halo bright, Pseudomonas syringae pv. oryzae MAFF301529 was selected and used as an iturin-tolerant bacterial pathogen which showed a tolerance against iturin up to $50 \mu \mathrm{M}$ in liquid culture on its growth. The treatment of iturin on the roots of rice resulted significantly disease suppression against the bacterial leaf disease at the range from 1 to $2 \mu \mathrm{M}$ iturin in hydroponic culture. It is notable that $4 \mu \mathrm{M}$ of iturin treatment showed loss of disease suppression; whereas no growth inhibitions on rice by the same concentration of iturin treatment without pathogen infections. Our data revealed that the existing of antimicrobial-independent disease suppression by iturin on rice.

Sensitivities of fungal pathogens of pomegranate (Punica granatum L.) to seven different fungicides

A. N. KC (1), G. Vallad (1)

(1) University of Florida, Wimauma, FL, U.S.A

Phytopathology 105(Suppl. 4):S4.70

Recent surveys identified Neofusicoccum parvum, Lasiodiplodia theobromae, and two Colletotrichum sp. as major pathogens of pomegranate in Florida. The in vitro sensitivity of these pathogens to fungicides pyraclostrobin, tebuconazole, thiophanate methyl, pyrimethanil, mancozeb, boscalid, and copper sulfate was determined. Three isolates of each pathogen were tested against six different concentrations of each fungicide and replicated three times. The effective concentration to reduce radial growth by $50 \%$ (EC50) was calculated for each pathogen-fungicide combination. Effect of salicylhydroxamic acid (SHAM) on the sensitivity of each pathogen to pyraclostrobin was also assessed. Two Colletotrichum sp. and $N$. parvum were sensitive to pyraclostrobin with average EC50 value of $0.66 \mu \mathrm{g} / \mathrm{ml}$. Isolates of L. theobromae were not sensitive to pyraclostrobin $(\mathrm{EC} 50>100)$ and addition of SHAM significantly increased their sensitivity $(P<0.001)$ with average value of $0.19 \mu \mathrm{g} / \mathrm{ml}$. All species, except Colletotrichum sp. (EC50 for thiophanate methyl, pyrimethanil and mancozeb $>50 \mu \mathrm{g} / \mathrm{ml}$ ), were sensitive to tebuconazole, thiophanate methyl, pyrimethanil, and mancozeb. None of the tested species were sensitive to boscalid or copper compound (EC50 > 100). This study provides a preliminary result to the effectiveness of different 
fungicides to the major diseases of pomegranate in Florida. Additional disease assays and field trials to evaluate fungicide efficacy are in progress.

\section{Germination parameters and qPCR quantification of pycnidiospores of} Phyllosticta citricarpa, the causal agent of citrus black spot

M. KELLERMAN (1), G. Van Zyl (1), M. M. Dewdney (2), A. McLeod (3), P. H. Fourie (4)

(1) Citrus Research International/Stellenbosch University, Stellenbosch, South Africa; (2) Univ of Florida, Lake Alfred, FL, U.S.A.; (3) Stellenbosch University, Stellenbosch, South Africa; (4) Citrus Research International/ Stellenbosch University, Nelspruit, South Africa

Phytopathology 105(Suppl. 4):S4.71

The role of $P$. citricarpa pycnidiospores in disease establishment in new regions is often debated. In order to study specific infection parameters, viable pycnidiospores and a feasible inoculation method of leaves for infection studies is needed. Optimal pycnidiospore germination conditions have been determined by suspending spores in $0.3 \%$ citric acid (CA) or water and harvesting spores from 2-, 3- and 4-week-old cultures. Pycnidiospores oozing from pycnidia were also harvested at ooze-generations 1 to 4 . Spore suspensions were incubated on microscope slides for 24 and $48 \mathrm{~h}$ at $27^{\circ} \mathrm{C}$ in a moist chamber. Spores germinated more readily when suspended in CA, from 3 -week-old cultures, and first generation ( $\leq 90 \%$ germination). Spores suspended in water and harvested from older cultures or later generations had poor viability $(<2 \%)$. No difference in percent germination was observed at 24 and $48 \mathrm{~h}$. These germination parameters will enable the harvest of consistent viable pycnidiospores to be used in infection studies. Two published qPCR assays were adapted for pycnidiospore quantification of $P$. citricarpa and the morphologically similar endophyte, $P$. capitalensis, from filter paper spore traps. In spiked samples, the detection limit was 20,000 pycnidiospores. This method will be adapted to quantify ascospores, and allow for relative enumeration of the pathogen and the harmless endophyte ascospores on spore trap discs, which is impossible based on spore morphology alone.

\section{Phenotypic and genotypic characterization of QoI fungicide resistant} Cercospora sojina

H. M. KELLY (1), B. Vega (2)

(1) University of Tennessee, Jackson, TN, U.S.A.; (2) DuPont, Newark, DE, U.S.A.

Phytopathology 105(Suppl. 4):S4.71

QoI-resistance in Cercospora sojina, the causal agent of frogeye leaf spot (FLS) in soybean, has continued to be reported since it was first discovered in Tennessee in 2010. C. sojina isolates and infected field samples were analyzed using phenotypic and genotypic characterization. Phenotypic characterization included conidia germination assay using a discriminatory dose of azoxystrobin at $0.1 \mathrm{ppm}$, calculating $\mathrm{EC}_{50}$ values for multiple isolates to azoxystrobin, and analyzing variability in conidia germination. Genotypic characterization included using a qPCR protocol to quantify the point mutation at position 143 that replaces the amino acid glycine with alanine in the cytochrome b gene. A QoI-resistant and -sensitive isolate were compared in a greenhouse experiment using different inoculation ratios of the isolates. The same isolates were compared saprophytically on media. Preliminary data from 2014 FLS samples from Tennessee showed that phenotypic and genotypic detection of QoI resistance are significantly correlated and can be used to detect approximate proportions of QoI-resistance. Based on the 2 isolates used in the greenhouse and saprophytic studies the QoI-resistant isolate was more aggressive and virulent. Although, on media the sensitive isolate grew faster and had greater sporulation. These studies will be repeated with other QoI-resistant and -sensitive isolates; and analysis of FLS lesions with genotypic and phenotypic assays will be continued in 2015 .

\section{Molecular identification of intercepted Colletotrichum fungi at an APHIS- PPQ Plant Inspection Station}

A. H. KENNEDY (1), K. A. Beucke (2), S. L. Gallant (2), F. Zhang (2)

(1) USDA-APHIS-PPQ-National Identification Services, Beltsville, MD, U.S.A.; (2) USDA-APHIS-PPQ-San Francisco Plant Inspection Station, South San Francisco, CA, U.S.A.

Phytopathology 105(Suppl. 4):S4.71

The USDA-APHIS-PPQ identifies organisms intercepted at US ports on plants and plant products primarily at its Plant Inspection Stations and exclusively uses morphology as the character source. PPQ began pilot studies of molecular detection and identification technologies at PISs in 2014 to better understand their associated benefits and challenges. Among these was identification of intercepted Colletotrichum at the San Francisco PIS. This genus was ideal because it is frequently intercepted, contains q-rated fungi, and is well studied. Published laboratory methods were optimized for speed and extracting DNA directly from host tissue. A procedure was created and implemented as a plugin to the software Geneious Pro to automate bioinformatics tasks necessary for preparing multi-locus datasets and automating identifications. Identifications were based on a set of criteria utilizing both DNA sequence similarity and phylogenetics. Taxa were selected for pairwise comparisons and multiple sequence alignments from a reference database containing sequences from type and expertly identified specimens. One hundred nineteen interceptions from a wide range of hosts were studied. Sequence data useful for identification were generated from $75 \%$ of interceptions. Of these, $48 \%$ were identified to species-level and $52 \%$ to subgeneric clade. Additional details will be presented relating to methodology, identifications, undescribed species, host records, and potential quarantine impact.

Report of Candidatus Liberibacter caribbeanus, a new citrus- and psyllidassociated Liberibacter from Colombia, South America

M. L. KEREMANE (1), C. Ramadugu (2), A. Castaneda (3), J. E. Diaz (3), E. A. Peñaranda (3), J. Chen (4), Y. P. Duan (5), S. E. Halbert (6), R. F. Lee (7)

(1) USDA ARS - Citrus Germplasm Repository, Riverside, CA, U.S.A.; (2) University of California Riverside, Riverside, CA, U.S.A.; (3) Instituto Colombiano Agropecuario, Bogota, Colombia; (4) USDA ARS, Parlier, CA, U.S.A.; (5) United States Horticultural Research Laboratory, Fort Pierce, FL, U.S.A.; (6) Division of Plant Industry, Gainesville, FL, U.S.A.; (7) USDA ARS National Clonal Germplasm Repository for Citrus and Dates, Riverside, CA, U.S.A.

Phytopathology 105(Suppl. 4):S4.71

We report a new species, Candidatus Liberibacter caribbeanus (CLca) from both the Asian citrus psyllid and Citrus sinensis from Cordoba in the northeastern region Colombia, South America. Additional psyllids carrying CLca were found from Barranquilla in the northern part of Colombia close to the Caribbean Sea. Quantitative real time PCR assays using primers and probes routinely used for $\mathrm{Ca}$. L. asiaticus were capable of detecting CLca. About 5000 psyllids and 100 plant samples were tested for the presence of Liberibacter. We conducted conventional PCR and Sanger sequencing of the 16S rDNA region, followed by phylogenetic analysis, 16S metagenome analysis of the psyllid DNA, and PAC-BIO sequencing of the bacterium from psyllid. A new method, digital PCR, was utilized for sensitive detection of CLca. Confirmatory tests using multiple genomic regions of both CLca and CLas were conducted. CLca is about $92-96 \%$ similar to previously reported Liberibacters based on 16S rDNA sequence analysis. At this time, it is not clear if CLca can be cultured and if it causes HLB-like symptoms in citrus. The titer of the bacterium reaches very high levels in the psyllid. Further research to determine the pathogenicity of the organism and its role in citrus HLB is in progress.

Fitness of tetraconazole-resistant isolates of Cercospora beticola after exposure to different temperature regimes

M. F. R. KHAN (1), S. Arabiat (2)

(1) North Dakota State Univ \& Univ of MN, Fargo, ND, U.S.A.; (2) North Dakota State University, Fargo, ND, U.S.A.

Phytopathology 105(Suppl. 4):S4.71

Cercospora leaf spot (CLS) caused by Cercospora beticola is the major foliar disease of sugar beet in North Dakota and Minnesota. Growers use fungicides to manage CLS and tetraconazole-resistant isolates of C. beticola have been reported. Phenotypic stability of tetraconazole-resistant isolates of $C$. beticola after exposure to different temperature regimes, $-20^{\circ} \mathrm{C}$ (for 4 weeks), $4^{\circ} \mathrm{C}$ (for 4 weeks), $20^{\circ} \mathrm{C}$ (for 4 weeks), $-20^{\circ} \mathrm{C}$ (for 2 weeks) to $4^{\circ} \mathrm{C}$ (for 2 weeks), $20^{\circ} \mathrm{C}$ (for 1 week) to $4^{\circ} \mathrm{C}$ (for 1 week) to $-20^{\circ} \mathrm{C}$ (for 1 week) to $4^{\circ} \mathrm{C}$ (for 1 week), and $-20^{\circ} \mathrm{C}$ (for 1 week) to $20^{\circ} \mathrm{C}$ (for 1 week) to $-20^{\circ} \mathrm{C}$ (for 1 week) to $20^{\circ} \mathrm{C}$ (for 1 week) was evaluated. Resistant isolates of $C$. beticola had no fitness penalty as measured by spore production, spore germination, mycelium radial growth, and disease severity. However, isolate 09-347 resistant to tetraconazole reverted to a moderate resistance level after exposure to $-20^{\circ} \mathrm{C}$, and $-20^{\circ} \mathrm{C}$ to $4^{\circ} \mathrm{C}$ to $-20^{\circ} \mathrm{C}$ to $4^{\circ} \mathrm{C}$. It may be important to use effective nontriazole fungicides in the first application to control overwintering population of $C$. beticola triazole-resistant isolates, and use triazoles later in the season so as to prolong the usefulness of this class of fungicides.

Genomics and genetic approaches to understanding the interplay between Ralstonia solanacearum type III effectors and the resistance $\mathrm{R}$ genes

A. A. KHAN (1), G. S. Ali (2), D. J. Norman (2)

(1) Univ of Florida, Orlando, FL, U.S.A.; (2) University of Florida, Apopka, FL, U.S.A.

Phytopathology 105(Suppl. 4):S4.71

Ralstonia solanacearum, a soil borne gram negative bacterial plant pathogen causes bacterial wilt in more than 200 plant species. It is considered a species 
complex, classified into 4 different phylotypes with more than 100 identified type III effectors. These type III effectors are translocated to host plant cell cytosol and are the primary determinants of pathogenicity. Plants respond to and guard against these effectors via the Nucleotide Binding-Leucin Rich Repeats (NB-LRR) protein products of the $R$ genes that recognize these effectors. This response, usually hypersensitive cell death (HR) at the site of infection, is termed Effector Triggered Immunity (ETI) and results in disease resistance. Recognition of the effectors by $R$ genes can be direct or indirect. Host targets of a majority of the Ralstonia effectors are unknown. We have made a yeast-2-hybrid library of tomatoes infected with Ralstonia and have identified targets of the cloned conserved effectors. In tomatoes, we are using a reverse genetic approach based on available genomics information and RNAi to determine function of these effectors. In potatoes, we are using a forward genetics approach where we are using the HR response to individual type III effectors to identify plants with corresponding $R$ genes. We are currently screening cultivated potatoes and advance breeding lines that show resistance to Ralstonia. Environmental growth chamber work is being used to help quantify this resistance.

\section{Nitric oxide produced by Ralstonia solanacearum affects tomato defense responses}

L. M. KIIRIKA (1), B. Dalsing (2), C. Allen (1)

(1) University of Wisconsin, Madison, WI, U.S.A.; (2) University of Wisconsin, Madison, WI, U.S.A.

Phytopathology 105(Suppl. 4):S4.72

Nitric oxide (NO) is a key signaling molecule in plant defenses against microbial pathogens. Ralstonia solanacearum, the causal agent of bacterial wilt disease, can produce NO from nitrate via its denitrification pathway. The bacterial genes encoding this pathway are highly expressed during tomato infection, and $R$. solanacearum deletion mutants that cannot produce NO $(\triangle a n i A)$ or that accumulate $\mathrm{NO}(\Delta n o r B$ and $\Delta h m p X)$ were all reduced in fitness in tomato plants. Qualitative and quantitative assessments of NO production using the fluorescent probe DAF-FM showed that a burst of NO was released when wild-type $R$. solanacearum cells were incubated with tomato seedlings. It was not clear if this NO was produced by the bacterium, the host plant, or both. However, plants inoculated with the $\triangle a$ aniA mutant produced very little detectable $\mathrm{NO}$, while those inoculated with the $\triangle$ nor $B$ and $\Delta h m p X$ mutants had significantly higher NO accumulation. These results indicated that under our conditions, much of the NO was produced by the pathogen rather than by the plant. Interestingly, the NO-deficient $R$. solanacearum mutant $\triangle a$ aniA triggered lower expression of the tomato ethylene pathway defense gene PR1, while the NO-accumulating $\Delta h m p X$ mutant strain triggered significantly higher plant PR1 gene expression. Together these results suggest that pathogen-produced NO may play a significant role in induction of plant defenses.

\section{Quorum sensing controls Candidatus Liberibacter asiaticus interactions} with host plant and insect vector

N. KILLINY (1)

(1) University of Florida, Citrus Research and Education Center, Lake Alfred, FL, U.S.A.

Phytopathology 105(Suppl. 4):S4.72

The typical LuxR bacterial "quorum sensing" or cell-to-cell communication system consists of two components, LuxR protein and Acyl-Homoserine Lactone (AHL). Genome of Candidatus Liberibacter asiaticus (CLas) contains luxR gene that encodes LuxR protein and the absence of luxI to produce AHL. We have confirmed the functionality of the CLas-LuxR by constructing a luxR gene promoter fused with a GFP reporter to generate a functional CLas luxR::GFP monitor E. coli. Several AHLs as well as extractions from insect, Diaphorina citri- Asian citrus psyllid (ACP) vector of CLas, or from the citrus plant, have been shown to activate CLas-luxR. The plant derived extracts may functionally be related to AHL but likely to be structurally unrelated. As a response to infection by CLas, citrus plants may induce the production of AHL mimic(s), which would bind to LuxR and trigger cell aggregation, and consequently limit bacterial growth and movement in planta. Additionally, the psyllid extract AHL produced by the endosymbiontic bacteria may bind to CLas-LuxR. As a result of this binding, Clas form biofilm on the surface of ACP gut. We expressed CLas LuxR in citrus using the CTV-based vector system. These citrus plants showed evenly distributed severe symptoms when infected with HLB (by graft inoculation). However, infection of healthy LuxR-citrus plants by CLas positive ACP ('hot' psyllids) showed diminished CLas titer. Thus, study of how CLas cells communicate in citrus and psyllids would help understanding the pathogenicity and transmission of this bacterium and potentially will lead to identify compounds that interfere with its growth and biofilm formation.
Evaluation of treatments for eradication of Xanthomonas from seeds of Capsicum pepper

B. S. KIM (1)

(1) Kyungpook Natl Univ, Daegu, Korea

Phytopathology 105(Suppl. 4):S4.72

We have faced recurrent problem of bacterial spot caused by Xanthomonas campestris pv. vesicatoria on nursery seedlings of Capsicum pepper grown for breeding. We have had a small breeding program for resistance to bacterial spot and often inoculated young plants for screening for resistance. The disease occasionally spread to susceptible plants grown for breeding for some other objectives. We found that the disease is occurring on seedlings grown from the seeds obtained from the diseased plants. We tried dipping the seeds in $1 \%$ sodium hypochlorite solution for $30 \mathrm{~min}$ for disinfection of the seeds. However, the control effect was not satisfactory since the problem continued occurring. Therefore, we evaluated the disinfecting effects of hot water treatments at 52,54 , and $56^{\circ} \mathrm{C}$ for $30 \mathrm{~min}$, dipping $1 \%$ sodium hypochlorite solution, $3 \% \mathrm{H}_{2} \mathrm{O}_{2}, 1 \% \mathrm{HCl}$, and 1,000 -fold solution of Benoram for $30 \mathrm{~min}$ by direct plating the treated seeds, inoculated ahead with vacuum, on Tween medium B (TMB) and by washing the treated seeds, centrifugation and plating the concentrated washings on TMB. The results showed that dipping in $3 \%$ $\mathrm{H}_{2} \mathrm{O}_{2}$ or $1 \% \mathrm{HCl}$ solution for $30 \mathrm{~min}$ or hot treatment at $54^{\circ} \mathrm{C}$ or above for 30 in successfully eradicated the bacterial spot pathogen from seeds. Germination of pepper seeds was lightly and severely affected by $3 \% \mathrm{H}_{2} \mathrm{O}_{2}$ treatment and by hot water treatment at $56^{\circ} \mathrm{C}$, respectively, in the greenhouse sowings.

Biocontrol efficacies of Paenibacillus polymyxa against ginseng root rot caused by Cylindrocarpon destructans

Y. S. KIM (1), Y. Jeon (2)

(1) Andong Natl Univ, Andong, Korea; (2) Andong National University, Andong, South Korea

Phytopathology 105(Suppl. 4):S4.72

The use of a microorganism or its secretions to prevent plant disease offers an attractive alternative to synthetic fungicides without the negative effects of chemical control mechanisms. In this study, we evaluated the potential of Paenibacillus polymyxa strain YGB as a biological control agent against Cylindrocarpon destructans. The 92 bacteria isolated obtained from soil and ginseng root were subjected to in vitro screening tests for antagonism against $C$. destructans, out of which, $P$. polymyxa showed a strong antibiotic activity. Bacterial isolates displayed antagonistic activity against test-cultures and pathogens found in ginseng root discs. The conidial germination rates significantly reduced in the bacterial treated conidia compared to the untreated control. The metabolites of our isolates significantly affected the conidial germination of $C$. destructans. In in vivo experiments, pretreatment with the bacterial isolate significantly reduced disease severity with a higher control efficacy at an inoculum concentration of $10^{6} \mathrm{CFU} / \mathrm{ml}$ than at $10^{7} \mathrm{CFU} / \mathrm{ml}$. Wound periderm-like layers were formed beneath the inoculation sites. Examination through an optical microscope revealed that the wound periderm-like layer was initiated by the stimulated periclinal cell division of enlarged parenchymatous cells. These results suggest that the bacterial isolate has good potential as a microbial agent for the biocontrol of the ginseng root rot caused by $C$. destructans.

MoYAK1 protein kinase gene plays key roles in infection-related development and disease development in Magnaporthe oryzae K. S. KIM (1), J. Hur (1)

(1) Kangwon National University, Chuncheon, Korea

Phytopathology 105(Suppl. 4):S4.72

Magnaporthe oryzae is the pathogen responsible for the rice blast disease. Conidiation and appressorial differentiation are key processes for polycyclic dissemination and infection in many pathogens. Using DNA microarray analysis, a study of conidiation led to the discovery of the MoYAKl gene in $M$. oryzae from a subset of genes induced in a conidiation-specific mutant. MoYAKl was found to be orthologous to YAK1 in Saccharomyces cerevisiae. Although mechanistic roles of YAK1 were detailed in S. cerevisiae, YAK1 orthologues remained uncharacterized in filamentous fungi. Targeted disruption of MoYAK1 resulted in pleiotropic defects in M. oryzae development and pathogenicity. The AMoyakl mutant showed a severe reduction in aerial hyphal formation and conidiation. Conidia in the AMoyak1 were delayed in germination and decreased in glycogen content in a conidial age-dependent manner. The AMoyakl mutant exhibited changes in expression of hydrophobin-coding genes, leading to loss of surface hydrophobicity. Unlike the inability of the $\Delta$ Moyakl mutant to develop appressoria on conidial germ-tubes on an inductive surface, the mutant formed abnormal shapes of appressoria in response to exogenous cAMP and host-driven signals, which were defective in penetrating host tissues. These data indicate that MoYAKl is a protein kinase important for development and pathogenicity of $M$. oryzae. 
Screening of probiotics for livestock feeding rye silage

H. S. KIM (1), S. Kim (2), J. Kim (1), Y. S. Kwak (1)

(1) Gyeongsang National University, Jinju, Korea; (2) Gyeongsang National University, Jinu, Korea

Phytopathology 105(Suppl. 4):S4.73

Silage is high moisture content forage and feed of livestock. During silage fermentation, it may contaminate by toxin producer. The fungi can cause severe quality problem in silage production. After heading stage the biomass of rye increased up to $30 \%$. However, lignification accelerated and the macromolecule caused low fermentation efficiency and coefficient digestibility. This study was performed to develop probiotics which reduce growth of mycotoxigenic fungi and have the fibrinolysis ability to improve quality of silage. During the fermentation, dominant microorganisms were isolated and identified. In addition, antifungal activities were confirmed against mycotoxigenic fungi. The isolated probiotics also were tested rapid acidification ability, which is a critical characteristic of potential probiotic in high quality of silage produce. Finally, those selected strains were confirmed ability of fibrinolysis through the four enzyme plate assay. Total 180 isolate were isolated from rye silages from 0 day to 100 day fermentation period. Among the isolates, 8 isolates showed antimicrobial activities against $F$. moniliforme. Also 2 isolates among antimicrobial activities isolates had acidification abilities for various plant macromolecules. In enzyme plate assay test, 1 isolate was selected that has effective against the four enzymes. In case developed microorganism product was applied at other stock feed as well as rye, production of high quality forage will expand.

Transcriptomic analyses of the interaction between Rathayibacter toxicus and phage CS14Ф

J. G. KING (1), A. Sechler (2), C. M. Fennessey (1), B. Atha (1), W. L. Schneider (1)

(1) USDA ARS FDWSRU, Fort Detrick, MD, U.S.A.; (2) USDA ARS FDWSRU, Frederick, MD, U.S.A.

Phytopathology 105(Suppl. 4):S4.73

Rathayibacter toxicus is the bacterial plant pathogen responsible for causing annual ryegrass toxicity in forage grasses in Australia and is closely related to several other forage pathogens that inhabit other continents. The $R$. toxicus life cycle is complex and involves a nematode vector, host plant, the bacterium and commonly a phage (CS14 1 ). Little is known about toxin production or the basic biology of $R$. toxicus, although interaction with the phage has been implicated in the induction of toxin biosynthesis and our own laboratory results support this idea. Genome sequencing revealed the presence of a gene cluster in $R$. toxicus that has significant homology to toxin production systems in other closely related bacteria. However, the functionality of this gene cluster in R. toxicus remains to be demonstrated. Here, we used Illumina RNA-seq data and qPCR to analyze the transcriptome of $R$. toxicus before, during, and following infection with phage CS14Ф. Preliminary analyses suggest that toxin production is related to stress-response regulatory pathways. Fundamental attributes of the transcriptome will be discussed, along with candidate pathways for toxin induction as evidenced by transcriptome analyses and the recently sequenced phage CS14Ф genome.

\section{Quantifying the evolution of fungicide-resistance from seed and foliar} treatments: A modeling analysis

J. L. KITCHEN (1), F. van den Berg (1), N. D. Paveley (2), F. van den Bosch (1)

(1) Rothamsted Research, Harpenden, United Kingdom; (2) ADAS UK Ltd, Duggleby, Malton, United Kingdom

Phytopathology 105(Suppl. 4):S4.73

Systemic seed treatments can treat foliar diseases of cereals. These treatments can improve disease control; however, fungicidal efficacy can be reduced from selection for fungicide-resistance. Seed and foliar treatments may use the same fungicidal mode of action, yet there has been little research to assess the resistance risks. To explore resistance proliferation from seed and foliar treatments, we have developed a mathematical model to simulate an epidemic and resistance evolution of Zymoseptoria tritici on winter wheat. The model can compare different application strategies of seed and foliar treatments by calculating the fungicide effective life, before disease control is lost to resistance. The model simulates the leaf layers of a wheat canopy, simulates healthy, latent and infectious leaf tissue and models fungicidal activity. Fungicidal mixtures can also be input to the model. Simulations using current model parameterisations suggest that effective lives from two foliar treatments within a season are greater than or equal to those obtained from a seed and foliar treatment application. Adding a mixing partner to seed treatments can extend the effective life, when the mixing partner aids disease control and resistance does not evolve against it. Whether adding a mixing partner to the seed or foliar treatment delays resistance the most depends on the relative efficacies of both treatments. The model output could help inform resistance management decisions.

Using fluorescent labeling to gain insight into fire blight disease development

S. KLEE (1), G. Monshausen (1), T. McNellis (2)

(1) Penn State University, State College, PA, U.S.A.; (2) The Pennsylvania State University, State College, PA, U.S.A.

Phytopathology 105(Suppl. 4):S4.73

Fire blight is an economically damaging disease of apples and pears caused by the bacterium Erwinia amylovora. We have developed a versatile tool to help understand the roles of bacterial virulence genes in fire blight disease development: a strain of E. amylovora that carries an indispensible plasmid marking both the location of the bacteria and the expression of target virulence gene transcriptional promoters. This plasmid reporter system allows us to visualize the spatiotemporal expression of genes necessary for disease development within plant tissues. We are currently using this system to observe the in planta expression of $h r p N$, a gene necessary for fire blight disease development, with epifluorescent and confocal microscopy. The knowledge gained from these experiments will give us a better understanding of exactly when and where E. amylovora virulence genes are expressed in planta, and this may define novel targets for intervention in disease prevention.

Influence of native plasmids to fitness of Pantoea vagans strain C9-1

J. M. KLEIN (1), V. Stockwell (1), J. Loper (2)

(1) Department of Botany and Plant Pathology, Oregon State University, Corvallis, OR, U.S.A.; (2) USDA-ARS, Horticultural Crops Research Unit, Corvallis, OR, U.S.A.

Phytopathology 105(Suppl. 4):S4.73

Pantoea vagans strain C9-1 is a biological control agent for fire blight caused by Erwinia amylovora. We cured C9-1 of two of its three plasmids: pPag2, pPag3, and both pPag2 and pPag3, tested phenotypes of the derivatives, and evaluated blossom colonization in the field. pPag2 $(166 \mathrm{~kb})$ encodes for production of the antibiotic herbicolin I and tellurite resistance. We found that C9-1 lacking pPag2 were more sensitive to tellurite than C9-1. pPag3 (530 kb) carries genes for a carotenoid pigment, thiamine biosynthesis, iron acquisition, and UV tolerance. C9-1 lacking pPag3 formed white colonies, were thiamine auxotrophs, and produced smaller zones on CAS agar compared to the wild type C9-1. UV-tolerance did not differ between C9-1 and its derivatives lacking pPag3. Swarming patterns on nutrient broth-glucose solidified with $0.5 \%$ agar were influenced by pPag3. C9-1 formed wide dendritic swarms that coalesced, whereas C9-1 lacking pPag3 formed thin dendritic swarms. Biofilm formation was greater with derivatives lacking both pPag2 and pPag3 compared to the wild type. In an orchard trial, C9-1 and derivatives cured of pPag2 and/or pPag3 established similar population sizes on apple blossoms. Although some of the assayed phenotypes were negatively affected by the loss of one or two plasmids, the epiphytic fitness of C9-1 in orchards was not reduced by plasmid loss.

Identification of a new allele at the Rsv4 locus for resistance to Soybean mosaic virus in VIR 2980 soybean accession

M. KLEPADLO (1), P. Chen (1)

(1) University of Arkansas, Fayetteville, AR, U.S.A

Phytopathology 105(Suppl. 4):S4.73

Implementation of genetic resistance is considered to be the most effective method to control disease caused by Soybean mosaic virus (SMV). Already identified alleles of SMV resistance genes do not provide protection to all strains. The objective of the research was to identify source of SMV resistance in Korean soybean accession VIR2980. This accession was crossed to a susceptible parent Essex (rsv) for an inheritance study, and to differential parents PI96983 (Rsv1), L29 (Rsv3), and V94-5152 (Rsv4) for an allelism test. F2 and F2:3 plants coming from all four crossing combinations were screened with SMV-G7 strain in a greenhouse. The results revealed that the resistance in VIR2980 is controlled by a single dominant gene allelic to Rsv4 locus. This information was supported by two SSRs Satt634 and Satt296 genotyping which confirmed that the tested R-gene is located at linkage group D1b (Chr 2). VIR2980 plants exhibited a unique symptoms pattern when compared with already reported Rsv4 alleles in V94-5152, PI 88788, and Beeson. VIR2980 was fully resistant to SMV-G1 through G6, and resistant at early vegetative stages to G7, therefore, we propose that the new allele Rsv4-v should be assigned to the SMV resistance locus in VIR2980 accession. Soybean sources carrying the Rsv4 alleles are rare to find among the soybean germplasm and $R s v 4-v$ allele confers great resistance to most SMV strains, thus, this allele may be a good choice to implement it in breeding programs. 
Fine mapping of Rsv4 gene and identification of SNP markers for Soybean mosaic virus resistance

M. KLEPADLO (1), P. Chen (1)

(1) University of Arkansas, Fayetteville, AR, U.S.A.

Phytopathology 105(Suppl. 4):S4.74

Soybean mosaic virus (SMV) causes the most serious viral disease of soybeans. Chemical and cultural control of SMV is neither economical nor environment-friendly. Deployment of genetic resistance is considered to be the most effective alternative to control the disease. Three independent multiallelic loci for SMV resistance, $R s v 1, R s v 3$, and $R s v 4$, have been mapped previously, however, no exact location of these genes have been identified. The objective of this research was to map Rsv4 gene and develop SNP markers for direct detection of resistance conferred by this gene. Random SNP primers were designed to screen $2 \mathrm{cM}$ interval between two most closely linked SSR markers using iPLEX Genotyping Platform. Three SNPs were identified close to the Rsv4 locus displaying $100 \%$ matching with known genotypes having Rsv4. In addition, 293 lines from germplasm collection were genotyped using the Sequenom iPLEX Gold platform to confirm identified SNPs for Rsv4. Furthermore, a cross between V94-5152 (Rsv4) and Lee 68 (rsv) was made for SNPs validation, and 766 plants from F2 population were screened with three discovered SNPs closely linked to the Rsv4 locus using KASP array. For fine mapping, several SNPs on the two sides of the target SNP makers were added with $\mathrm{r}>0$ to validate the target SNPs. Rsv4 candidate gene has been proposed. The information from this research can be helpful in selecting crossing parents to accelerate efforts to develop multi-virus resistant crops.

Evaluation of cover crop susceptibility to Fusarium virguliforme, the causal agent of sudden death syndrome of soybean

R. KOBAYASHI-LEONEL (1), D. S. Mueller (1), L. F. S. Leandro (1)

(1) Iowa State University, Ames, IA, U.S.A.

Phytopathology 105(Suppl. 4):S4.74

A greenhouse study was conducted to determine if cover crops species can be infected by Fusarium virguliforme (Fv), a soilborne fungus that causes sudden death syndrome (SDS) of soybean. Sixteen cover crop species belonging to 14 different genera were planted in steam-pasteurized soil amended with sterile or Fv-infested sorghum. Plants were grown for 5 weeks at $25^{\circ} \mathrm{C}$ and assessed for fresh biomass (g), root necrosis and foliar symptoms (\% scale). Fv DNA in roots was quantified using qPCR. Inoculated alfalfa, crimson clover, red clover and pea had more root necrosis compared to their non-inoculated controls. Pathogen DNA quantities detected in inoculated roots of these 4 plant species were similar to those found in inoculated soybean roots $(P>$ 0.05 ). Inoculated alfalfa, corn, crimson clover, oat, red clover, sorghum and turnip plants had lower biomass compared to non-inoculated controls, but corn, oat and turnip had no root necrosis. Biomass reduction and root necrosis were not observed in inoculated hairy vetch, false flax, millet, mustard, rye, ryegrass, triticale and wheat. Fv DNA quantity in the roots of these species ranged from 0 to $43 \mathrm{pg}$ per $50 \mathrm{ng}$ total DNA and was lower $(P<0.05)$ than in soybean $(121 \mathrm{pg})$. Since grass and brassica crops did not develop root rot symptoms and had small amounts of Fv DNA in root tissue, they may be minor contributors to inoculum buildup in soil and preferred cover crop choices for fields with a history of SDS.

Fungicides and biocontrols for management of Sclerotium rolfsii on stevia A. KOEHLER (1), H. D. Shew (1)

(1) North Carolina State University, Raleigh, NC, U.S.A.

Phytopathology 105(Suppl. 4):S4.74

Stevia (Stevia rebaundiana) is an herbaceous perennial with leaves containing multiple glycosides that are extracted for use as a nonnutritive sweetener. Stevia was approved for use as a food additive by the USDA in 2008 and is now an emerging crop in the US. Stevia plantings began in North Carolina in 2011 and symptoms of southern blight caused by Sclerotium rolfsii were first observed in 2012. Disease develops within days after transplanting into infested soils and stem rot symptoms are observed simultaneously with the emergence of stevia from overwintering root systems. S. rolfsii produces abundant sclerotia that are able to overwinter in soil, making accumulation of sclerotia a concern for this perennial cropping system. Currently there are no fungicides labeled for use on stevia, which will limit expansion of commercial production. Fungicide and biocontrol field trials were initiated in 2014 to identify and optimize products with potential to be labeled for control of $S$. rolfsii on stevia. Trials were established in May and July. Two fungicides, tebuconazole and azoxystrobin, and two biocontrol products, clonotri + strepse and veramin, significantly reduced disease in comparison to nontreated control plots with $>50 \%$ mean disease. Treatments applied prior to planting or at the time of planting resulted in significantly lower disease than treatments that began three weeks after planting. Early season control is vital in the management of $S$. rolfsii on stevia.

RNA as fungicide: Towards an RNAi-based non-transgenic control of plant diseases

K. H. KOGEL (1), A. Koch (2)

(1) Justus Liebig University Giessen, Giessen, Germany; (2) Justus Liebig University, Giessen, Germany

Phytopathology 105(Suppl. 4):S4.74

To meet the increasing food and energy demands of a growing population, it will be necessary to roughly double crop yields worldwide over the next 40 50 years despite a changing climate. Meeting this challenge will require developing ground-breaking strategies that promote sustainable plant production. We have recently shown that host-induced gene silencing (HIGS) by transgenic expression of a $791 \mathrm{nt}$ long double-stranded (ds)RNA (CYP3-

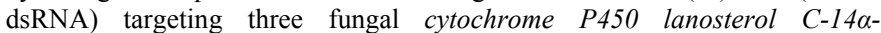
demethylase (CYP51) genes involved in ergosterol biosynthesis renders barley highly resistant against the pathogenic fungus Fusarium graminearum (Koch et al. 2013,m PNAS 110, 19324-19329). Here, we provide proof-of-concept that direct delivery of CYP3-dsRNA via spray application also effectively controls spreading of the fungus on barley leaves. Local application of CYP3dsRNA on leaves also resulted in systemic protection which is consistent with the finding that spray-delivered fluorescent-labelled dsRNA was detected in xylem and phloem of vascular leaf tissue. Our work provides evidence that RNA can be used as a fungicide against devastating microbial pathogens. Given their extreme specificity, applicability to diverse pathogens and ease of design, the use of target-specific inhibitory RNAs offers unprecedented potential as a new plant protection strategy.

CI5791 is a barley line that harbors high levels of resistance to Pyrenophora teres f. teres, causal agent of net form net blotch (NFNB)

V. M. KOLADIA (1), J. D. Faris (2), R. S. Brueggeman (1), T. L. Friesen (3)

(1) North Dakota State University, Department of Plant Pathology, Fargo, ND, U.S.A.; (2) Cereal Crops Research Unit, Northern Crops Science Laboratory, USDA-ARS, Fargo, ND, U.S.A.; (3) North Dakota State University, Department of Plant Pathology; Cereal Crops Research Unit, Northern Crops Science Laboratory, USDA-ARS, Fargo, ND, U.S.A.

Phytopathology 105(Suppl. 4):S4.74

The barley line CI5791 is resistant to most isolates of the net form net blotch (NFNB) pathogen Pyrenophora teres f. teres, with few isolates overcoming this resistance. The barley line Tifang is known to harbor a NFNB resistance gene on chromosome $3 \mathrm{H}$. We constructed a CI5791 $\times$ Tifang $\mathrm{F}_{6}$ recombinant inbred line (RIL) population using single seed descent. Genetic analysis of the RIL population was carried out using the Illumina Infinium Assay to produce a saturated SNP map. Inoculations were done using a global collection of ten $P$. teres f. teres isolates which included isolates collected from different barley growing regions of the world. Tifang was resistant to five isolates whereas CI5791 was resistant to all ten isolates. QTL analysis indicated that CI5791 and Tifang harbored resistance QTL on chromosomes $6 \mathrm{H}$ and $3 \mathrm{H}$, respectively. Interestingly, CI5791 also harbored resistance to two Japanese isolates that mapped to a $3 \mathrm{H}$ region similar to that of Tifang but in repulsion. $\mathrm{F}_{2}$ analysis of a CI5791 $\times$ Tifang cross indicated that resistance was dominant at both the $3 \mathrm{H}$ and $6 \mathrm{H}$ loci. A 3:1 R:S ratio was found when CI5791 $\times$ Tifang $\mathrm{F}_{2}$ individuals were inoculated with five of the isolates avirulent only on CI5791. RILs harboring both $3 \mathrm{H}$ and $6 \mathrm{H}$ resistance will be useful for combining sources of resistance to NFNB.

Three clonal lineages of the cocoa pathogen Phytophthora megakarya in Nigeria

O. O. Kolawole (1), E. M. GOSS (2)

(1) University of Ibadan and Cocoa Research Institute of Nigeria, Ibadan, Nigeria; (2) University of Florida, Gainesville, FL, U.S.A.

Phytopathology 105(Suppl. 4):S4.74

Black pod disease of cocoa caused by Phytophthora megakarya is the most destructive disease affecting cocoa production in Central and West Africa. $P$. megakarya is an aggressive and highly virulent pathogen that can cause 60 to $100 \%$ loss of cocoa pods. To reduce use of fungicides currently used to control the disease, breeding for resistant cultivars is a research priority. However, there is little knowledge of the population structure and diversity of the pathogen, which is critical for breeding resistance that will be effective and durable. We used genotyping-by-sequencing to obtain SNPs from 95 isolates of $P$. megakarya collected from cocoa pods in southern Nigeria. We found two genetically diverged groups of 80 and 15 isolates. The larger group was found in all states sampled, whereas the minor group was found only in the southeast cocoa-growing areas near the border with Cameroon. The dominant group comprised a single clonal group containing limited genetic varia- 
tion. The minor group included two closely related clonal lineages. We found no evidence of recent sexual reproduction. These data will be used to examine the evolution of the pathogen in Central Africa and to inform breeding efforts.

Nematicidal activity in extracts of chenopods

T. Kouser (1), D. I. Yates (2), K. D. GWINN (3)

(1) Webb School of Knoxville, Knoxville, TN, U.S.A.; (2) University of Tennessee and David Crockett High School, Knoxville, TN, U.S.A.; (3) Univ of Tennessee, Knoxville, TN, U.S.A.

Phytopathology 105(Suppl. 4):S4.75

Plants in the Family Chenopodiaceae (chenopods) have chemical properties that make them well suited for use in biologically-based management of nematodes. The objective of this research was to determine if the model nematode, Caenorhabditis elegans, is differentially inhibited by autoclaved aqueous extracts of chenopods. Chenopods, except a field-collected Chenopodium berlandieri, were grown in the greenhouse from commercial seed; all were air dried. Plant extracts $(4 \mathrm{~g}$ dry weight $/ 150 \mathrm{ml}$ water) were placed in 24-well-culture plates and diluted in culture medium (final concentration 0 to $50 \%$ ). Twenty adult nematodes or J4 larvae were added to each well. After incubation at room temperature for 1 day, nematodes were stimulated by prodding and were considered dead if they had minimal or no response. All nematodes in all extracts concentrations at or above $25 \%$ were dead. Extracts from Chenopodium album were the least active against the nematode. Extracts of C. quinoa and C. berlandieri (field-collected and 'Red Aztec Spinach') had strong activity against the nematode, but extracts from the phylogenetically-basal epazote (Dysphania ambroisioides) were most active. Matricidal hatching occurred in all treatments. After 1 week, there were more $\mathrm{J} 2$ and $\mathrm{J} 3$ larvae in no-extract controls than in the 20 or $30 \%$ extract treatments. Although these results are promising, further research is needed to fully characterize anti-nematodal activity of chenopods.

\section{Powdery mildew resistant cucurbit rootstocks confer tolerance to grafted} susceptible watermelon scions

C. S. KOUSIK (1), J. L. Ikerd (2), R. Hassell (3)

(1) USDA ARS, Charleston, SC, U.S.A.; (2) U.S. Vegetable Laboratory, USDA, ARS, Charleston, SC, U.S.A.; (3) Costal Research and Education Center, CREC, Clemson University, Charleston, SC, U.S.A.

Phytopathology 105(Suppl. 4):S4.75

Cucurbit powdery mildew (PM) caused by Podosphaera xanthii, can impact seedling growth and cause serious losses in greenhouse and open fields. We have developed watermelon and bottle gourd germplasm lines with high levels of resistance to PM. A PM susceptible watermelon cultivar Mickey Lee (ML) was grafted on various PM resistant rootstocks. USVL677-PMS (watermelon) and USVL848-PMS (bottle gourd) were used as susceptible rootstock checks. Grafted plants were inoculated by spraying with a PM melon race 1 conidial suspension $\left(2 \times 10^{4}\right.$ conidia/mL $)$ and rated for PM severity on a $0-10$ scale about two weeks after inoculation. Trials were repeated in a greenhouse three times (2012-2014). Severe PM was observed on the cotyledons of USVL677PMS and USVL848-PMS. Significantly less to no PM was observed on the cotyledons of PM resistant rootstocks. Severe PM was observed on plants of ML scion cotyledon that were grafted on susceptible rootstocks. Significantly $(P \leq 0.001)$ less powdery mildew was observed on the second true leaf of ML scions grafted on some of the PM resistant watermelon lines compared to those grafted on susceptible rootstocks. Not all PM resistant watermelon lines conferred resistance to true leaves of the susceptible scion. PM on the second true leaf and upper leaves was significantly less on ML scion grafted on two PM resistant bottle gourd rootstocks (USVL351-PMR and USVL482-PMR) and a hybrid rootstock. Resistant rootstocks may help manage PM of watermelon.

Influence of Fruit age on Phytophthora fruit rot development on susceptible and resistant watermelon germplasm

C. S. KOUSIK (1), J. L. Ikerd (2), W. W. Turechek (3)

(1) USDA ARS, Charleston, SC, U.S.A.; (2) U.S. Vegetable Laboratory, USDA, ARS, Charleston, SC, U.S.A.; (3) Horticulture Research Laboratory, USDA-ARS, Fort Pierce, FL, U.S.A.

Phytopathology 105(Suppl. 4):S4.75

Phytophthora fruit rot of watermelon caused by Phytophthora capsici is an emerging disease in the Southeastern United States. The disease has resulted in severe losses to watermelon growers in GA, SC and $\mathrm{NC}$ and is considered a top research priority by the National Watermelon Association. We released four germplasm lines (USVL203-PFR, USVL020-PFR, USVL782-PFR and USVL489-PFR) with high levels of resistance to Phytophthora fruit rot. To determine influence of fruit age on disease development, resistant and susceptible lines were planted at weekly intervals for five consecutive weeks in a field in 2011, 2012 and 2013. Flowers were routinely inspected and pollinated to produce fruit of differing ages. Fruit of varying ages were harvested on the same day in each year and inoculated with a 5-mm agar plug from an actively growing colony of $P$. capsici. Inoculated fruit were maintained in a humid chamber $\left(>95 \% \mathrm{RH}, 26 \pm 2^{\circ} \mathrm{C}\right)$ for five days after which data on disease development (lesion diameter \& sporulation intensity) was recorded for each fruit. Fruit of susceptible checks (Sugar Baby and PI 536464) had significantly larger lesions and greater sporulation than fruit from resistant germplasm lines at all fruit ages. Significantly lower amounts of $P$. capsici DNA was detected in fruit tissue of resistant germplasm than susceptible checks at all ages. These results suggest that resistance to Phytophthora fruit rot in watermelon is not correlated with fruit age.

\section{Antimicrobial activity of plant produced bacteriophage endolysin}

N. KOVALSKAYA (1), J. Foster-Frey (2), D. Donovan (2), G. Bauchan (3), R. W. Hammond (1)

(1) USDA-ARS-Molecular Plant Pathology Laboratory, Beltsville, MD, U.S.A.; (2) USDA-ARS-Animal Biosciences and Biotechnology Laboratory, Beltsville, MD, U.S.A.; (3) USDA-ARS-Electron and Confocal Microscopy Unit, Beltsville, MD, U.S.A.

Phytopathology 105(Suppl. 4):S4.75

The increasing spread of antibiotic-resistant microorganisms is a growing concern for modern animal production, agriculture and medicine. Phageencoded endolysins have acquired significant attention as antimicrobials due to their high efficiency and mechanism of action. To produce a functionally active Gram-negative bacterium bacteriophage CP933 endolysin in plants, a combination of transient expression and vacuole targeting strategies was employed. Cytoplasmic expression of the cp933 gene in Nicotiana benthamiana using Potato virus $\mathrm{X}$ - based transient expression vectors (pP2C2S and pGR107) resulted in death of the apical portion of experimental plants. To protect plants against the toxic effects of the CP933 protein, the cp933 coding region was fused at its $\mathrm{N}$-terminus to an $\mathrm{N}$-terminal signal peptide from the potato proteinase inhibitor I to direct CP933 to the delta type vacuoles. Plants producing the CP933 fusion protein did not exhibit the severe toxic effect and the level of expression was $0.16 \mathrm{mg} / \mathrm{g}$ of plant tissue. Antimicrobial assays revealed that the Gram-positive plant pathogenic bacterium Clavibacter michiganensis was the most susceptible to the plantproduced CP933 showing $18 \%$ growth inhibition, whereas the Gram-negative bacterium Escherichia coli (BL 21 (DE3)) was not affected significantly. Using vacuole protein targeting is a promising approach for the production of functionally active proteins that exhibit toxicity when expressed in plant cells.

\section{Discovery of a novel Grapevine vein clearing virus isolate in wild Vitis} rupestris vine

M. KOVENS (1), L. Hubbert (1), S. Honesty (1), Q. Guo (1), D. Pap (1), R. Dai (1), L. Kovacs (1), W. Qiu (1)

(1) Missouri State University, Springfield, MO, U.S.A.

Phytopathology 105(Suppl. 4):S4.75

Grapevines are infected by over 60 different viruses. Grapevine vein clearing virus $(\mathrm{GVCV})$ is associated with a severe vein clearing and vine decline disease that severely affects grape production and berry quality in Midwest vineyards. Symptoms include translucent vein-clearing, backward rolling, and chlorosis of the leaves as well as short, zig-zagged internodes and a decline of vine vigor. The genome sequence of GVCV-CHA that was isolated from grape cultivar 'Chardonel' in a commercial vineyard was published in 2011. It has a circular dsDNA genome of 7,753 bp, and encodes three open reading frames (ORFs) with four short ORFs within the intergenic region. A new isolate of GVCV, referred to as GVCV-VRU, was identified in a wild Vitis rupestris vine in its native site in the southwest region of Missouri. GVCVVRU associated symptoms are necrotic flecks along major veins in $V$. rupestris. Comparative analysis of GVCV-CHA and GVCV-VRU found that GVCV-VRU genome is two base pairs longer than GVCV-CHA genemoe and thyey shared $91.5 \%$ identical nucleotides. ORF II is the most variable region with a 9 bp insert only present in GVCV-VRU genome; GVCV-VRU and GVCV-CHA share only $83.3 \%$ nucleotide identity. The discovery of a new GVCV isolate in wild grapevine provides important clues on the evolution and epidemics of GVCV.

Evidence for independent evolution of resistance to AvrPto and AvrPtoB from the wild tomato species Solanum chmielewskii

C. M. KRAUS (1), K. R. Munkvold (2), G. B. Martin (3)

(1) Department of Plant Pathology and Plant-Microbe Biology, Cornell University, Ithaca, NY, U.S.A.; (2) Boyce Thompson Institute for Plant Research, Ithaca, NY, U.S.A.; (3) Boyce Thompson Institute for Plant Research, Department of Plant Pathology and Plant-Microbe Biology, Cornell University, Ithaca, NY, U.S.A.

Phytopathology 105(Suppl. 4):S4.75 
Resistance in tomato to Pseudomonas syringae pv. tomato (Pst) is activated upon recognition of either of two bacterial effectors, AvrPto and AvrPtoB, by a member of the Pto kinase family and the NB-LRR protein Prf. Pto recognizes both AvrPto and AvrPtoB, whereas another family member, Fen, recognizes only forms of AvrPtoB lacking the C-terminal E3 ubiquitin ligase. This ancient recognition is present in diverse tomato accessions and other Solanaceous plants. We screened wild relatives of tomato for resistance to Pst delivering either AvrPto or AvrPtoB and found a novel resistance phenotype in S. chmielewskii. These plants recognize AvrPtoB with E3 ligase activity, but not AvrPto and subsequent experiments demonstrated that AvrPtoB is being recognized by Pto and not Fen. Sequence comparisons between Pto proteins from resistant Rio Grande-PtoR and S. chmielewskii revealed that two residues at positions 49 and 51 required for AvrPto recognition are polymorphic. Site-directed mutagenesis of these two residues was not enough to recapitulate the recognition of AvrPto. A third residue at position 193, which has not been previously implicated in AvrPto recognition, was needed to activate signaling in response to AvrPto by Pto from S. chmielewskii. We hypothesize that this represents an intermediate evolutionary step in the arms race between Pst and tomato, in which a Solanum species has evolved the ability to recognize full-length AvrPtoB, but not yet AvrPto.

\section{Diversity of Alternaria brassicicola isolates collected in New York State}

R. A. KREIS (1), C. D. Smart (1), H. R. Dillard (2)

(1) Cornell University NYSAES, Geneva, NY, U.S.A.; (2) University of California, Davis, CA, U.S.A.

Phytopathology 105(Suppl. 4):S4.76

Alternaria brassicicola, causal agent of Alternaria Leaf Spot, is a common disease of brassica crops in New York State. Management of the disease can be difficult, and there have been unconfirmed reports of reduced fungicide efficacy. Alternaria brassicicola has no known sexual stage; however diversity has been observed in Australian populations. It has been unknown whether a diverse or clonal population of $A$. brassicicola can be found in New York, but this information is critical to understand the pathogen population and improve disease management. To study the diversity of the population, over 60 New York isolates of A. brassicicola were collected from 2010-2014 from a variety of brassica crops. Single conidial isolates were obtained, DNA was extracted and each isolate was assayed for allelic variation using 11 microsatellite loci. Preliminary results indicate diversity among New York isolates of A. brassicicola.

A reliable and highly sensitive, digital PCR-based assay for early detection of citrus Huanglongbing

K. R. Kremer (1), S. Shi (1), Y. Duan (2), Z. XIONG (1)

(1) University of Arizona, Tucson, AZ, U.S.A.; (2) USDA ARS USHRL, Fort Pierce, FL, U.S.A.

Phytopathology 105(Suppl. 4):S4.76

Huanglongbing (HLB) is caused by a phloem-limited bacterium, $\mathrm{Ca}$. Liberibacter asiaticus (Las) in the United States. The bacterium is often present at a low concentration and unevenly distributed in the early stage of infection, making reliable and early diagnosis a challenge. We have developed a promising and novel diagnostic assay based on digital PCR (dPCR) for early and reliable detection of HLB. dPCR partitions samples into 20,000 picoliter wells in a single reaction, with each well carrying out independent PCR reactions simultaneously. The large number of positive and negative wells can be fitted to a Poisson distribution to allow absolute and precise quantification of the target molecules and statistical assessment of the measurement. Using probes targeting the Las $16 \mathrm{~s}$ rDNA and the integrated prophage repeat sequences, we showed that as few as 1 to 2 copies of the targeted DNA molecules per microliter could be detected, with the prophage probe providing the best sensitivity. Early-stage HLB samples can be statistically differentiated from healthy individuals. Furthermore, this assay can quantitate the copy number of the $16 \mathrm{~S}$ rDNA and the phage repeat DNA simultaneously, permitting the tracking of lytic activities of the Las prophage/phage accurately. The dPCR-based assay will not only provide a reliable and early diagnostic tool but also an enabling technology to advance research on HLB therapies.

Spatial distribution of Phytophthora rubi and Pratylenchus penetrans in Northwest red raspberry fields

D. KROESE (1), I. Zasada (2), N. Grünwald (2), J. Weiland (2)

(1) Oregon State University, Corvallis, OR, U.S.A.; (2) USDA ARS HCRL, Corvallis, OR, U.S.A.

Phytopathology 105(Suppl. 4):S4.76

Phytophthora rubi, an oomycete that causes root rot, and Pratylenchus penetrans, a plant parasitic nematode, are two of the most economically important pathogens of red raspberry in the Pacific Northwest. Due to environmental and health concerns, control of these pathogens with fumigation, fungicides, or nematicides has become increasingly restricted. The objective of this study was to determine the spatial distribution of $P$. rubi and $P$. penetrans. To accomplish this, four raspberry 'Meeker' fields (4 ha, two each in Oregon and Washington) were sampled. Soil and root samples were collected using two sampling patterns: a uniform grid and a localized concentric, focal sampling scheme. A visual plant disease rating, GPS location, and soil textural data were also obtained for each sampling site. $P$. penetrans were extracted from both soil and roots and the presence of $P$. rubi was determined from roots with both a greenhouse bioassay and real-time PCR. P. rubi data were further analyzed to determine correlations with relative elevation and soil texture. While root rot symptoms tended to aggregate in discrete foci, $P$. rubi was found throughout each field, even in sites without symptoms. $P$. penetrans tended to be more concentrated in areas where there was less disease pressure from root rot. Knowing the spatial distribution of these pathogens within a field can provide useful information to direct and improve management decisions.

\section{Iris yellow spot virus epidemics in onion crops in Serbia}

B. B. KRSTIĆ (1), I. M. Stanković (1), A. B. Vučurović (1), K. N. Milojević (1), D. M. Nikolić (1), S. D. Teodorović (2), A. R. Bulajić (1)

(1) Institute of Phytomedicine, Department of Phytopathology, University of Belgrade-Faculty of Agriculture, Belgrade, Zemun, Serbia; (2) Forensics Department, Academy for Criminalistic and Police Studies, Belgrade, Serbia Phytopathology 105(Suppl. 4):S4.76

An extensive survey of Iris yellow spot virus (IYSV; Tospovirus, Bunyaviridae) presence was conducted in Serbia from 2005 to 2014,. A total of 139 crops from various locations were visually inspected and 730 samples of onion, garlic and leek were collected. Samples were analyzed by ELISA using commercial antisera and RT-PCR using nucleocapsid $(\mathrm{N})$ gene-specific primers. IYSV was detected for the first time in 2007, infecting one seed and one onion bulb in two distinct locations. In the years following the first outbreak (2008-2013), IYSV was not detected in any of the tested plants and was, thus, regarded as eradicated. During 2014, serious outbreaks of IYSV in onion crops planted from true seed were observed in eight locations. Infected plants showed symptoms suggestive of IYSV infection, with high incidence estimated at over $90 \%$, leading to nearly total crop failure. A substantial population of Thrips tabaci was also noted. Following the laboratory confirmation of IYSV presence, $\mathrm{N}$ gene sequence data from isolates collected in 2014 were used to characterize IYSV natural populations in Serbia. Phylogenetic analysis placed the selected Serbian IYSV isolates into two distant clades, together with isolates from 2007. Nucleotide sequence characteristics of Serbian IYSV isolates show lack of evidence for a recent introduction into the country and that two previously detected, distinct viral lineages are well established in Serbia and becoming epidemic.

\section{Real-time simultaneous detection of Loop-mediated AMPlification (LAMP) by Assimilating Probes}

R. KUBOTA (1), D. M. Jenkins (2)

(1) Diagenetix, Inc., Honolulu, HI, U.S.A.; (2) Univ of Hawaii At Manoa, Honolulu, HI, U.S.A

Phytopathology 105(Suppl. 4):S4.76

Isothermal nucleic acid amplification technologies, such as Loop-mediated isothermal AMPlification (LAMP), are simple, rapid, and specific diagnostic tools and appealing alternatives to PCR. Some common advantages are that reactions are extremely fast and do not require thermocycling, therefore the instrumentation can be simple, inexpensive, and portable. Assimilating Probes (AP) were developed to give more functionality to LAMP reaction such as real-time monitoring of reactions with higher sequence specificity. Here we report another application of AP, real-time monitoring of the duplexed LAMP assay for the detection of bacterial wilt pathogen Ralstonia solanacearum (Rs) and its quarantine subgroup of strains Race 3 Biovar 2 (R3B2), as well as Salmonella enterica and $\lambda$-phage DNA as an internal control. The detection limits of duplexed LAMP assays were one or two orders of magnitude higher due to competitive effects compared to individual reactions, which were less than 100 genomic copies. However, these results suggested that discrimination of quarantine agents such as R3B2 can be performed on-site by using rapid, specific, sensitive, and portable diagnostic tools.

Oxathiapiprolin, a new fungicide active ingredient for control of diseases caused by Oomycetes

P. KUHN (1), A. Tally (1), B. Druebbisch (1)

(1) Syngenta Crop Protection, LLC, Greensboro, NC, U.S.A.

Phytopathology 105(Suppl. 4):S4.76

Oxathiapiprolin is a new fungicide active ingredient (a.i.) that provides excellent control of economically important diseases caused by downy 
mildews, Phytophthora species, and Pythium ultimum. The mode of action is novel and mediated via interaction with an oxysterol binding protein, with an essential, but as yet uncharacterized, function. Oxathiapiprolin has been assigned FRAC Code U15. The high intrinsic activity of the molecule enables foliar a.i. use rates that are 10-100 times lower than commercially available Oomycete fungicides. Oxathiapiprolin has been shown to exhibit translaminar efficacy, and to provide protection to expanding leaves and the developing canopy. Similarly, it gives systemic disease control following soil drench application. With high potency and a single site mode of action, there is the potential for development of reduced sensitivity or resistance to oxathiapiprolin. In order to mitigate this possibility, label directions will incorporate strict stewardship guidelines on application régimes as well as products containing two modes of action. Federal registration of oxathiapiprolin is anticipated during the second half of 2015, with launch early in 2016. The first wave of crops to be registered will include potatoes, leafy vegetables, tobacco, cucurbits, and fruiting vegetables.

\section{Development of an assay for rapid detection of the lettuce downy mildew pathogen, Bremia lactucae}

S. G. KUNJETI (1), Y. J. Choi (2), A. Anchieta (3), M. Thines (4), R. Michelmore (5), S. T. Koike (6), C. Tsuchida (5), F. N. Martin (7), K. V. Subbarao (8), S. J. Klosterman (9)

(1) University of California, Salinas, CA, U.S.A.; (2) Biodiversity and Climate Research Center (BiK-F), Frankfurt, Germany, Frankfurt Main, Germany; (3) USDA, Salinas, Salinas, CA, U.S.A.; (4) Biodiversity and Climate Research Centre (BiK-F), Frankfurt, Germany; (5) University of California, Davis, Davis, CA, U.S.A.; (6) Cooperative Extension Monterey County, Salinas, CA, U.S.A.; (7) USDA-ARS, Salinas, CA, U.S.A.; (8) UC Davis, Davis, CA, U.S.A.; (9) USDA ARS, Salinas, CA, U.S.A.

Phytopathology 105(Suppl. 4):S4.77

Downy mildew of lettuce, caused by Bremia lactucae, causes chlorosis on leaves and adversely affects marketability. Though downy mildew on lettuce can be controlled by fungicide applications, it is costly to routinely apply fungicides to prevent the establishment of downy mildew. Repeated use of the chemicals also can lead to fungicide resistance in the pathogen. To specifically detect Bremia lactucae for the purpose of developing an early warning system, we designed a quantitative real-time PCR (qPCR) assay based on mitochondrial DNA sequence unique to Bremia lactucae. Specificity tests revealed that the qPCR assay is specific for detection of $B$. lactucae and not related Bremia species. This assay is sensitive, enabling detection of very low levels of inoculum that may be present in the field. Early detection of the pathogen, coupled with knowledge of other factors that favor downy mildew outbreaks, may enable disease forecasting for judicious timing of fungicide applications.

\section{Presence of viable oospores of Peronospora effusa in spinach seeds}

S. KUNJETI (1), A. Anchieta (2), R. Pena (3), K. V. Subbarao (4), S. T. Koike (5), S. J. Klosterman (2)

(1) University of California, Davis, Salinas, CA, U.S.A.; (2) USDA-ARS, Salinas, CA, U.S.A.; (3) USDA, ARS, Salinas, CA, U.S.A.; (4) University of California, Davis, Davis, CA, U.S.A.; (5) Cooperative Extension Monterey County, Salinas, CA, U.S.A.

Phytopathology 105(Suppl. 4):S4.77

Downy mildew on spinach (Spinacia oleracea) is caused by the oomycete pathogen, Peronospora effusa. This disease is widespread on spinach, and is destructive when environmental conditions are optimal because the crop becomes unmarketable. Though seedborne oospores were observed three decades ago in seed lots in Japan, follow up studies to document the levels of infestation in current seed lots have been unavailable. In this study, seed washes from 67 commercial seed lots were examined for the presence of oospores. Seven of the 67 seed lots were positive for the oospores. These seed lots were further tested for presence of Peronospora effusa using conventional PCR with primers specific for Peronospora sp. and ITS DNA sequencing of amplicons. Oospores from two seed lots were also tested for viability using two independent tests. One test involved a detection of plasmolysis in the oospores in a hypertonic $4 \mathrm{M}$ sodium chloride solution, and the other involved staining the oospores with trypan blue. In the former test, contraction of cytoplasm (plasmolysis) in the viable oospores was observed, which was absent in the non-viable oospores. In the latter test, viable oospores remained unstained. Both tests provided consistent results on each tested seed lot. The presence of viable oospores of $P$. effusa on spinach seed in some of the tested seed lots was confirmed and the potential of these oospores in initiating downy mildew infection in spinach is currently being evaluated.
Factors affecting cotyledon inoculation on lima bean stem blight caused by Botryodiplodia theobromae

C. H. KUO (1), W. H. Hsieh (2)

(1) National Chiayi University, Chiayi, Taiwan; (2) National Chung-Hsing University, Taichung, Taiwan

Phytopathology 105(Suppl. 4):S4.77

Seedling stem blight of lima bean (Phaseolus limensis Macf.) reported and pathogenicity tested by seed soaking method. Cotyledon inoculation method researched. Factors affecting cotyledon inoculation on lima bean stem blight caused by Botryodiplodia theobromae tested. The percentage of spore germination of $B$. theobromae was more than $87 \%$ both in distilled water and under $100 \%$ relative humidity at $25^{\circ} \mathrm{C}$; however, no spores germinated when the relative humidity fell below $89 \%$. Both unicellular and uniseptate conidia failed to germinate after being air dried for 48 and 72 hours, Spore germination was more than $98 \%$ for unicellular conidia and $80 \%$ for uniseptate conidia on water agar with water potential ranging from 0 to -10 bars at $25^{\circ} \mathrm{C}$. No germination at -40 bars or lower. The cotyledon-inoculation technique developed for disease assessment. The stem blight symptoms developed when lima bean (cv. Pai-Jen) was inoculated with unicellular and uniseptate conidia separately. The disease severity reached more than $60 \%$ when spore suspensions containing $10^{3}$ to $10^{5}$ conidia per $\mathrm{ml}$ used for inoculation on lima bean. When lima bean seedlings of different ages were inoculated with $B$. theobromae, the 5-day-old seedlings showed the most susceptible. The fungus was unable to cause infection on the seedlings whose cotyledons had dropped naturally or removed artificially. The result suggested that the cotyledons of lima bean provided an entry for successful infection of $B$. theobromae.

\section{The use of spectroscopy techniques in investigating the underlying} mechanism of resistance to Fusarium head blight in wheat

R. LAHLALI (1), L. Wang (2), S. Kumar (1), P. R. Fobert (2), G. Peng (3), E. Hallin (1), C. Karunakaran (1)

(1) Canadian Light Source Inc., Saskatoon, SK, Canada; (2) National Research Council Canada, Saskatoon, SK, Canada; (3) Agriculture and AgriFood Canada, Saskatoon, SK, Canada

Phytopathology 105(Suppl. 4):S4.77

Fusarium Head Blight (FHB) is the major disease of wheat in North America, causing severe losses in grain yield and quality. Breeding for cultivar resistance is considered the most practical way to manage this disease. In this study, different spectroscopy and microscopy techniques were applied to discriminate resistance in wheat genotypes against FHB. Synchrotron based spectroscopy and imaging techniques such as Fourier transform mid infrared (FTIR) microspectroscopy and X-ray fluorescence (XRF) spectroscopy were used to understand biochemical changes and trace elements in the rachis following FHB infection. Two cultivars, resistant (Sumai3) and susceptible (Muchmore) to FHB were investigated. Marked changes were observed in the biochemical composition of rachis of control and infected samples. The biochemical changes between the two cultivars were also different. The FTIR microspectroscopy data showed differences in the cell wall composition of the wheat cultivars before and after infection. XRF spectroscopy data revealed differences in trace elements between infected and control samples of the two cultivars. The chemical profiling using these spectroscopy techniques have a good potential as a tool for screening wheat genotypes for FHB resistance.

\section{Contribution of mid-season cover sprays to management of peach brown} rot at harvest

N. LALANCETTE (1), J. Gager (1), K. A. McFarland (1)

(1) Rutgers University, Bridgeton, NJ, U.S.A.

Phytopathology 105(Suppl. 4):S4.77

Protectant fungicides are routinely applied to peach trees during the period from shuck-split through early pre-harvest. These cover sprays are applied to control peach scab, rusty spot, and anthracnose. However, results from fungicide treatments in 2010 and 2012, which lacked the typical pre-harvest applications, indicated that these mid-season sprays may also provide extended residual activity for control of brown rot, caused by Monilinia fructicola. To confirm these observations and determine the mechanism of this control contribution, a field study was conducted during 2012 and 2013 in an experimental peach orchard. Four protectant fungicides (captan, sulfur, ziram, and thiram) were applied from shuck-split through sixth cover. Captan and thiram in 2012 and captan in 2013 significantly reduced brown rot at harvest. Estimation of fungicide levels on fruit, using an in vivo bioassay based on M. fructicola spore germination, showed that residual activity was the primary mechanism for control. Some anti-sporulant activity against blossom blight cankers was also detected, but this form of control was not sustained through the pre-harvest period. These findings indicate that residual activity from mid-season applications of protectant fungicides (i) can contribute significantly to the efficacy of pre-harvest programs and (ii) may 
play an important role in reducing selection for resistance among site-specific fungicides typically applied during the pre-harvest period.

Functional genomic analysis of soybean interactions with pathogenic and non-pathogenic isolates of Fusarium oxysporum

A. Lanubile (1), U. K. Muppirala (2), A. J. Severin (2), A. Marocco (1), G. P. MUNKVOLD (3)

(1) Univ Cattolica del Sacro Cuore, Piacenza, Italy; (2) Iowa State University, Ames, IA, U.S.A.; (3) Iowa State Univ, Ames, IA, U.S.A.

Phytopathology 105(Suppl. 4):S4.78

Fusarium oxysporum is one of the most common species causing soybean root rot and seedling blight in the U.S. In the present study, RNA-seq-based analysis was used for the first time to investigate the molecular aspect of the interaction of a partially resistant soybean genotype with pathogenic and nonpathogenic isolates of $F$. oxysporum at 72 and 96 hours post inoculation (hpi). Markedly different gene expression profiles were observed in response to the two isolates. A peak of differentially expressed genes (DEGs) was observed at $72 \mathrm{hpi}$ in soybean roots in response to both isolates, but the number of DEGs was about eight times higher for the pathogenic isolate compared to the nonpathogenic one. Furthermore, the magnitude of induction was much greater in response to the pathogenic isolate. This response included a stronger activation of defence-related genes, transcription factors, and genes involved in ethylene biosynthesis, secondary and sugar metabolism. In addition, 1130 fungal genes were differentially expressed between the $F$. oxysporum isolates in planta during the infection process. Interestingly, $10 \%$ of these genes encode plant cell-wall degrading enzymes, reactive oxygen species-related enzymes and fungal proteins involved in primary metabolic pathways. Such information may be useful in the development of new methods of broadening resistance of soybean to $F$. oxysporum, including the silencing of important fungal genes.

\section{Characterization of potato pathogenic Streptomyces species present in} Uruguay: Emerging pathogens?

M. I. Lapaz (1), E. Verdier (2), M. I. Siri (1), J. Huguet-Tapia (3), R. Loria (3), M. J. PIANZZOLA (1)

(1) Facultad de Química, Universidad de la República, Montevideo, Uruguay; (2) Ministerio de Agricultura y Pesca, Montevideo, Uruguay; (3) Plant Pathology Department, University of Florida, Gainesville, FL, U.S.A.

Phytopathology 105(Suppl. 4):S4.78

Common scab of potato is a disease that occurs worldwide and is caused by bacteria of the genus Streptomyces. This disease acquired global importance in recent years due to the emergence of new pathogens such as $S$. turgidiscabies and $S$. acidiscabies. The appearance of these has been associated with the ability of this genus to the horizontal transfer of pathogenicity genes located within pathogenicity islands, suggesting the possibility of the continuous emergence of new pathogenic species. We have a collection of 85 pathogenic strains. Phenotypic and genotypic characterization will be completed to know their pathogenic potential. The identification by primer species-specific PCR, sequence of $r p o \mathrm{~B}$ gene and Multilocus Sequence Analysis (MLSA) was performed. Some isolates have been identified as $S$. scabies, $S$. acidiscabies and S. europaeiscabiei. In our experience, MLSA is the best method to the identification of this genus, although that needs to be improved to identify all pathogenic species described. New housekeeping genes will be evaluated to incorporated in the characterization system MLSA. Some representative strains of the isolates will be sequenced. Characteristics of pathogenicity islands present, as potential virulence factors and genes associated with mobility, will be determined. The results of this work will contribute to understanding the mechanisms of pathogenicity, horizontal gene transfer and to improve the identification of Streptomyces pathogens.

Cumulative and residual effects of potato cropping system management strategies on soil physical, chemical, and biological properties R. P. LARKIN (1)

(1) USDA ARS, Orono, ME, U.S.A.

Phytopathology 105(Suppl. 4):S4.78

In field trials established in 2004, different 3-yr potato cropping systems focused on specific management goals of soil conservation (SC), soil improvement (SI), and disease-suppression (DS) were evaluated and compared to a 2-yr standard rotation (SQ) and a non-rotation control (PP) for their effects on a variety of soil properties under both rainfed and irrigated conditions. Systems were actively managed through 2010, with potato crops planted in subsequent years (2011-12) to examine residual effects. Cropping system significantly affected many parameters associated with soil health, with effects generally increasing over time as well as having lasting residual effects. All rotations increased aggregate stability, water availability, microbial biomass $\mathrm{C}$, and total $\mathrm{C}$ and $\mathrm{N}$ relative to no rotation (PP), and 3-yr rotations (SI, SC, DS) increased aggregate stability and water availability relative to the 2-yr rotation (SQ). The SI system, which included yearly compost amendments, resulted in greater increases in total and POM C and $\mathrm{N}$, Active C, microbial activity, water availability, CEC, and concentrations of $\mathrm{P}$, $\mathrm{K}, \mathrm{Ca}, \mathrm{Mg}$, and $\mathrm{S}$ than all other rotations. SI also reduced bulk density (BD) relative to all other systems, and $\mathrm{SC}$ reduced $\mathrm{BD}$ relative to remaining systems. Cropping systems that incorporate management practices such as increased rotation length and the use of cover crops, green manures, organic amendments, and reduced tillage can improve soil health.

Association of Grapevine rupestris stem pitting-associated virus in declining Cabernet Sauvignon grapevines grafted on Schwarzmann in California T. L. LAWLER (1), A. Rowhani (1), J. K. Uyemoto (2), M. R. Sudarshana (2) (1) UC Davis, Davis, CA, U.S.A.; (2) USDA-ARS, Davis, CA, U.S.A. Phytopathology 105(Suppl. 4):S4.78

In fall 2014 in Napa County, California, four year old grapevines of Cabernet Sauvignon (CS) (Vitis vinifera) on Schwarzmann (V. riparia X ( $V$. rupestris) rootstock, were found to exhibit red canopy and vine decline. Disease incidence was $15.2 \%(n=342)$. Several affected and healthy vines were uprooted and trunk sections, comprised of scion and rootstock, were excised and brought back to the laboratory. The trunk specimens were autoclaved briefly and bark removed to expose the woody cylinder. The rootstocks, but not scions, exhibited extensive stem pitting symptoms. Nucleic acid extracts were prepared from petioles and tested by RT-PCR or PCR. Affected grapevines tested negative for eight leaf roll-associated viruses, four vitiviruses and Grapevine red blotch-associated virus. Affected, but not healthy, vine samples tested positive for the Syrah strain of Grapevine rupestris stem pitting-associated virus (GRSPaV). Analysis of the nucleotide sequence of the RT-PCR products indicated that the GRSPaV isolate was $92 \%$ identical to GRSPaV Syrah strain in California and $98 \%$ identical to a GRSPaV isolate in Australia. The latter strain was detected in Syrah grapevines grafted on 1103 Paulsen ( $V$. berlandieri $\mathrm{X} V$. rupestris) that exhibited symptoms of red canopy, swollen graft union and stem pitting. This is the first reported incidence of GRSPaV associated with declining CS grapevines on Schwarzmann rootstock.

Interactive effects of water stress and Neofusicoccum parvum on Botryosphaeria dieback of grapevines

D. LAWRENCE (1), E. Galarneau (2), R. Travadon (3), K. Baumgartner (2)

(1) Univ of California, Davis, CA, U.S.A.; (2) USDA, Davis, CA, U.S.A.; (3) University of California, Davis, Davis, CA, U.S.A.

Phytopathology 105(Suppl. 4):S4.78

The relationship between vascular pathogens and water stress is an important topic in California agriculture, given successive years of drought and restrictions on water use. We examined the interactive effects of water stress and Neofusicoccum parvum on severity of Botryosphaeria dieback of grapevines. Cabernet Sauvignon plants were inoculated with $N$. parvum after wounding; control plants were wounded and inoculated with PDB. After 2 weeks, water stress was imposed on half of the inoculated and control plants. Water usage was estimated weekly for non-stressed plants to provide $20 \%$ of this volume for stressed plants. Leaf water potential was monitored weekly with a pressure chamber to maintain potentials of $>-8$ bars for non-stressed and $<-13$ bars for stressed plants. Leaves were collected for RNA extraction 2 weeks before imposing water stress ( 2 weeks post-inoculation, $2 \mathrm{WPI}$ ), and at 8 and 12 WPI. At 12 WPI, lengths of stem lesions were measured and used to determine the effect of the pathogen and/or water stress on the vascular tissue. Inoculated plants under water stress had the greatest lesion lengths, relative to other treatments. To examine the specificity of the host response to the pathogen, water-stressed plants were used to screen 13 grapevine genes that have been shown to be differentially regulated in leaves in response to $N$. parvum. Some markers were specific to infection, whereas others crossreacted with water stress in non-inoculated plants.

Evaluation of a strawberry powdery mildew risk index (Broome-modified Gubler-Thomas grape powdery mildew index) to time fungicide applications M. L. LEBLANC (1), O. Cuevas (1), K. Coons (2), J. C. Broome (3)

(1) Pacific Ag Research, San Luis Obispo, CA, U.S.A.; (2) Driscoll's, Santa Maria, CA, U.S.A.; (3) Driscoll's, Watsonville, CA, U.S.A.

Phytopathology 105(Suppl. 4):S4.78

The Gubler-Thomas powdery mildew risk index, developed for grapes based on temperature-driven fungal growth and reproduction modeling, was modified by Broome based on strawberry work suggesting days with temperatures between $15-25^{\circ} \mathrm{C}$ and when airborne inoculum is above a 250 conidia/m3 air/day threshold are favorable for strawberry powdery mildew (Podosphaera aphanis) growth. We evaluate the index for accurately predicting disease incidence for timing fungicide applications on proprietary 
variety $510 \mathrm{Q} 89$ planted in Santa Maria, CA. Hourly weather station temperature data was used to calculate the risk index and fungicide sprays were timed based on high disease risk (60 or above). A grower conventional fungicide regimen (quinoxyfen and myclobutanil) and organic standard (hydrogen dioxide and Bacillus subtilis QST 713 strain) were applied based on either the risk model, a weekly calendar program, or left untreated (control). The risk model-timed treatments received 11 and calendar-timed received 18 applications. There were no significant differences in marketable yield or powdery mildew AUDPC between the weekly or the risk model timed treatments for either farming system and average mildew percent control for the calendar and model sprayed plots was $35.27 \%$ of the control. The Broomemodified Gubler-Thomas powdery mildew index applied to strawberries has the potential for reducing treatment costs and environmental impacts while preserving disease control and yields.

\section{A new model to estimate leaf wetness duration in an apple orchard}

A. LECA (1), B. Boissonnier (2), V. Joubert (1), V. Philion (1)

(1) IRDA, Saint-Bruno de Montarville, QC, Canada; (2) Ecole Nationale Agronomique de Toulouse, Castanet-Tolosan, France

Phytopathology 105(Suppl. 4):S4.79

Leaf Wetness Duration (LWD), i.e. the time that free water remains on leaves after rain or dew events, is for many fungal species the significant parameter linking the epidemic risk to the plant microclimatic conditions. For disease control decision support systems such as RIMpro (ex: apple scab), it is often a crucial parameter. However, modeling LWD is not trivial and is generally empirical or very approximate, mainly because of the specific input variables needed such as wind, solar radiation, and water distribution in the canopy, requiring local measurements. Our recent works led to the development of a framework improving the physical modeling of LWD without on-site measurements by deriving input variables from high resolution short term weather forecasts (nowcasting) and a minimum of empirical values, in order to accurately adapt the model to different locations and pathosystems. The model takes into account the tree architecture, and physical and phenological properties of leaves. It has been validated for apple McIntosh cultivar using local weather and LWD (measured with impedance grid sensors) in 2014 in St-Bruno-de-Montarville, QC, Canada: hourly simulation compared to those data produced a LWD RMSE of 26 minutes. Since such sensors underestimate LWD, a correction factor was applied to compare simulation to most probable true LWD. The impact on risk assessment will be validated by using estimated LWD as an input value for RIMpro.

Characterization and identification of Pseudomonas syringae pv. syringae causing Bacterial shoot blight on apple

S. LEE (1), W. Cheon (1), Y. Jeon (1)

(1) Andong National University, Andong, South Korea

Phytopathology 105(Suppl. 4):S4.79

Pseudomonas syringae pv. syringae has a wide host range. Bacterial shoot blight occurred on the leaves of apple. To pathogens associated Apple leaf in Korea, Samples were collected in NorthenGyeongbuk province. Symptoms observed on cluster leaves included yellowing and fallen down. In some orchard, more than $60 \%$ of apple tree exhibited symptoms. Isolates were positive for tobacco hypersensitivity, levan formation, and negative for oxidase, potato rot, and arginine dehydrolase. Use of BiOLOG GN2 microplate and Release 4.20 system identified the isolate as Pseudomonas syringae pv. syringae with $87 \%$ similarity. Isolate were MIDI analysis as Pseudomonas syringae pv. syringae with $94.8 \%$ similarityThe $16 \mathrm{~S}$ rRNA of isolated bacterial was amplified by PCR using $27 \mathrm{~F}$ and $1492 \mathrm{R}$ primer set. A BLAST searches for sequence similarity of the 16S rRNA revealed $99 \%$ identity for $P$. s. pv. syringae. The resulting sequences were deposited (Accession Nos. KP713782, KP753380 and KP753381) in GenBank. Toxin test were using bioassay with Bacillus megaterium. Zone of inhibition indicate production of syringopeptin. The pathogenicity test was used apples leaves. Isolates were spraying $50 \mathrm{ml}$ of bacterial suspension $\left(10^{8} \mathrm{CFU} / \mathrm{ml}\right)$ the leaves. After 5days inoculation, leaves were showed blight symptoms. The results obtained on LOPAT test, BiOLOG analysis, MIDI, Toxin test, pathogenicity and molecular date corresponded with those P. s. pv. syringae causing bacterial shoot blight on apple.

Survey of walnut diseases in walnut plantations in South Korea and control efficacy of fungicides

S. H. LEE (1), J. H. Park (2), J. K. Lee (3)

(1) Warm-Temperate and Subtropical Forest Research Center, Korea Forest Research Institute, Jeju, South Korea; (2) Korea Forest Research Institute, Seoul, South Korea; (3) Kangwon National University, Chuncheon, South Korea

Phytopathology 105(Suppl. 4):S4.79
Walnut (Juglans sinensis) is an important non-timber forest product in South Korea, the domestic production of which annually amounts to approximately 900 ton, equivalent of the revenue of 9.7 million dollars. Walnut is mostly cultivated in warm-temperate areas in the country (around 1,200 ha). Considering its product value, a survey on walnut diseases was conducted throughout the walnut plantations in 2014. Types of diseases commonly found in the field sites were anthracnose, white mold, powdery mildew, Melanconis dieback, felt, and Phomopsis dieback. Among the sites, the plantations located in Buyeo-gun, Cheonan-si, Yeongdong-gun, and Gimcheon-si showed the highest anthracnose incidences and severity with the incidence of 5 to $30 \%$. The highest incidence was found in a plantation in Buyeo-gun mainly due to drought conditions during spring, resulting in decreased walnut production by $20 \%$. White mold, which was first reported to occur in Gimcheon-si and Muan-gun in 2011, was also observed in Buyeo-gun during the survey. The amount of damage by white mold increased especially in May, when the rainfall was heavy and intense. Eight fungicides were tested with various levels of concentration in field sites for control efficacy against anthracnose and white mold. Among the fungicides tested, tebuconazole $(25 \%, \times 2,000)$ and fluazinam $(50 \%, \times 1,000)$ turn out to be the most effective for anthracnose control, and propiconazole $(25 \%, \times 1,000)$ for white mold control.

Chestnut blight prevention effects of burlap sheet covering and cloth bandage for trees

S. H. LEE (1), J. H. Park (2), J. K. Lee (3)

(1) Warm-Temperate and Subtropical Forest Research Center, Korea Forest Research Institute, Jeju, South Korea; (2) Korea Forest Research Institute, Seoul, South Korea; (3) Kangwon National University, Chuncheon, South Korea

Phytopathology 105(Suppl. 4):S4.79

Chestnut is widely cultivated in South Korea and the main producing areas of chestnut used to be located in the Southern part of the country. However, the number of new plantations set in the central regions has increased. A recent survey on chestnut blight in the new plantation sites found that disease incidences range from 5 to $50 \%$, with the highest incidence in an orchard (5 ha) in Chunghwa-myun. The disease occurrences were found mainly associated with unremoved dead trees in adjacent areas and stem wounds made by mowers or winter injury. Based on the observations, the methods of burlap sheet wrapping of unremoved tree residue and cloth bandage for trees were examined for chestnut blight prevention effects in two orchards for 2013-2014. In the test, dead trees were cut, and twigs, branches, and logs left unremoved were collected. Then, they were piled together in one place and covered with a burlap sheet. The mean blight incidences during the test period were shown to decrease in the treated sites $(7.2 \%$ and $9.8 \%)$, compared to those in the untreated sites $(13.9 \%$ and $12.5 \%)$. It was also found that when tree stems were covered with the cloth bandage, the disease incidences decreased by half or more than those in the untreated sites. Keeping the tree residue under the sheet and protecting tree stems by covering them with the cloth bandage were shown to likely reduce the inoculum density and the degree of stem wounds, thus helping prevent the spread of the disease.

\section{Interplay between HrpS and HrpL in regulation of type III secretion} system in Erwinia amylovora

J. H. LEE (1), Y. Zhao (1)

(1) University of Illinois at Urbana-Champaign, Urbana, IL, U.S.A.

Phytopathology 105(Suppl. 4):S4.79

The type III secretion system (T3SS) is a key pathogenicity factor in Erwinia amylovora. Previous studies have demonstrated that T3SS in E. amylovora is transcriptionally regulated by an RpoN-HrpL sigma factor cascade, which is activated by the bacterial alarmone ppGpp. In this study, the DNA binding site of HrpS, a $\sigma^{54}$ enhancer binding protein, was identified for the first time in plant pathogenic bacteria. Complementation of the $h r p L$ mutant with promoter deletion constructs of the $h r p L$ gene and promoter activity analyses using various lengths of $h r p L$ promoter fused to a promoter-less $g f p$ delineated the upstream region for HrpS binding. Bioinformatic analysis revealed a dyad symmetry sequence between -138 to $-125 \mathrm{nt}$ (TGCAA-N4-TTGCA), which is conserved in the promoter of the $h r p L$ gene among plant enterobacterial pathogens. Results of qRT-PCR, electrophoresis mobility shift assay coupled with site-directed mutagenesis (SDM) showed that the intact dyad symmetry sequence was essential for HrpS binding and activation of hrpL gene expression. In addition, the role of the GAYTGA motif of HrpS in regulating $h r p L$ gene expression in E. amylovora was characterized by SDM and complementation of the hrpS mutant. Results showed that an Y100F substitution of HrpS complemented the hrpS mutant, whereas Y100A and Y101A substitutions did not. These results suggest that tyrosine $(\mathrm{Y})$ and phenylalanine (F) function interchangeably in the conserved GAYTGA motif of HrpS in E. amylovora. 
Identification of novel secreted virulence factors from Xylella fastidiosa using a TRV expression system

S. A. LEE (1), E. E. Rogers (1)

(1) USDA-ARS, SJVASC, Parlier, CA, U.S.A.

Phytopathology 105(Suppl. 4):S4.80

Xylella fastidiosa is a bacterium that causes leaf scorch diseases of agriculturally important crops including grapevines and almonds. Little is known about virulence factors that are necessary for $X$. fastidiosa to grow and cause disease in the xylem vessels of a plant host. Any protein secreted by the bacterium has the potential to interact with the plant host and affect disease. The objective of this study is to identify novel secreted proteins that play a role in the virulence of $X$. fastidiosa. The $X$. fastidiosa genome was screened to identify proteins with putative secretion signal sequences. Fifty-eight proteins were identified, cloned in the tobacco rattle virus (TRV) expression system and screened for pathogenesis in Nicotiana benthamiana. Eight of the fifty-eight proteins conferred increased virulence in $N$. benthamiana. Expression of these eight proteins in E. coli revealed that four proteins were secreted. Knock out mutants of the four secreted proteins have been constructed and inoculated to grapevines. In vitro assays such as cellular aggregation, surface attachment and sensitivity to chemical stresses are being performed to phenotypically characterize these proteins. Identification of the full repertoire of virulence factors is important to the understanding of leaf scorch diseases caused by $X$. fastidiosa.

The population of Sclerotinia sclerotiorum from common bean in Brazil is structured by mycelial compatibility groups

M. S. Lehner (1), T. J. Paula Júnior (2), J. E. S. Carneiro (1), E. S. G. MIZUBUTI (3)

(1) Univ Federal De Vicosa, Viçosa, Brazil; (2) EPAMIG, Viçosa, Brazil; (3) Univ Federal De Vicosa, Vicosa, Brazil

Phytopathology 105(Suppl. 4):S4.80

Microsatellite (SSR) markers and mycelial compatibility groups (MCGs) were used to characterize the population of Sclerotia sclerotiorum causing white mold on common bean in Brazil. A total of 300 isolates were studied and 154 SSR haplotypes and 32 MCGs were identified. Two MCGs were widely distributed and accounted for $70 \%$ of the isolates. Six SSR haplotypes were associated to more than one closely related MCGs. When the population was analyzed as a whole (all MCGs) or divided by geographic criteria there was no evidence of random association of alleles among the loci. Nevertheless, when it is analyzed by MCGs there was evidence of random mating, suggesting that outcrossing occurs within of the MCGs. There was strong differentiation between MCGs and $95.6 \%$ of total genetic variation was attributed to differences among MCGs. Thus, the common bean population of S. sclerotiorum in Brazil is structured by MCGs and breeders and pathologists should focus on the dynamics of MCGs in order to develop resistant cultivars and set white mold control strategies.

\section{Star jasmine (Jasminum multiflorum) plants in Hawaii are infected with} multiple tombusviruses

M. Leite de Oliviera (1), W. Borth (2), J. Carrillo (3), J. Hu (2), K. Neupane (3), S. Stubblefield (3), M. MELZER (2)

(1) Universidade Estadual Paulista, Botucatu, Brazil; (2) Univ of Hawaii At Manoa, Honolulu, HI, U.S.A.; (3) Leeward Community College, Pearl City, HI, U.S.A.

Phytopathology 105(Suppl. 4):S4.80

Star jasmine (Jasminum multiflorum) is a shrub with fragrant flowers commonly grown in Hawaii as a hedgerow or landscape accent plant. On the island of Oahu, Hawaii, star jasmine plants express a diverse array of viruslike foliar symptoms including ringspots, line patterns, mosaic, mottling, and leaf deformation, often on the same plant. To investigate a possible viral etiology, symptomatic tissue underwent double-stranded RNA (dsRNA) isolation, library construction, and sequencing. DsRNAs of approximately 4.2 and $1.6 \mathrm{kbp}$ were consistently isolated from symptomatic leaf tissues collected from three locations on Oahu. Conventional and high-throughput sequencing of a library constructed from these dsRNAs revealed the presence of several related viruses most similar to putative members of the family Tombusviridae: Rosa rugosa leaf distortion virus, Pelargonium ringspot virus, Pelargonium chlorotic ring pattern virus, and Elderberry latent virus. Reverse transcription PCR-based assays revealed these viruses are widely distributed on Oahu and are the presumptive causal agent(s) of these symptoms.

Enrichment of Clavibacter michiganensis subsp. michiganensis in tomato seeds extracts

F. M. V. LELIS (1), J. M. van der Wolf (1)

(1) Wageningen University and Research Centre, Wageningen, Netherlands

Phytopathology 105(Suppl. 4):S4.80
Clavibacter michiganensis subsp. michiganensis $(\mathrm{Cmm})$ is a Gram-positive bacterium which is considered to be the most serious seed-borne pathogen of tomato. Detection of the pathogen in seed can be difficult as infection levels are often low. An enrichment procedure in seed extract was developed to improve detection. Seeds were incubated overnight at $4^{\circ} \mathrm{C}$ in PBST and antibiotics Trimethoprim, Nalidixic acid and antifungal Nystatin commonly used in semi-selective media for $\mathrm{Cmm}$. Seeds were extracted with a stomacher and spiked with a $\mathrm{Cmm}$ suspension. After incubation for three days at $25^{\circ} \mathrm{C}$, a 10-100 fold increase of $\mathrm{Cmm}$ populations was found. No growth was found in PBST without added antibiotics. If seed lots were spiked with $1 \%$ internally infected seeds, even up to a 100.000 -fold increase in numbers was found. We used internally-infected seeds with a GFP-tagged strain of Cmm to allow localization of the bacteria in seeds. Indeed, the presence of the fluorescent bacteria in endosperm and/or embryo of seeds was confirmed with epifluorescence stereo- and confocal laser scanning microscopy. Enrichment of $\mathrm{Cmm}$ was more efficient if, after crushing seeds, unbroken seeds and large seed fragments were removed from the extracts. Resuscitation of bacteria, by incubation of seeds for $24 \mathrm{~h}$ in PBST prior to the supply of the selective antibiotics also supported the enrichment.

\section{Functional characterization of aphid candidate effectors and their} potential host targets

C. LENOIR (1), P. A. Rodriguez (2), M. Jaouannet (2), P. Birch (1), J. Bos (1) (1) The James Hutton Institute / The University of Dundee, Dundee, United Kingdom; (2) The James Hutton Institute, Dundee, United Kingdom Phytopathology 105(Suppl. 4):S4.80

Aphids are phloem-feeding insects form close associations with their host plants. Aphid stylets form an interface with the host plant where signals are exchanged. While feeding and probing, aphids secrete saliva, containing effector proteins, directly into the host-stylet interface. These effectors are predicted to interact with host targets to manipulate plant cell processes and promote infestation. This project aims to functionally characterize aphid candidate effectors and to identify and characterize their potential host targets. Five candidate effectors from the aphid species Myzus persicae were selected. To identify host targets, these candidate effectors were screened against a Nicotiania benthamiana Yeast-2-Hybrid library. Putative targets of interest were identified: a chitinase-like and a E3 SUMO ligase. These interactions were confirmed using co-immunoprecipitation and co-localization studies. Functional assays are performed with both candidate effectors and putative host targets to investigate their role in plant-aphid interactions. These include aphid performance assays on Nicotiana benthamiana transiently overexpressing putative plant targets and effectors as well as Arabidopsis thaliana transgenic overexpression and mutant lines. Understanding how aphid effectors manipulate host cell processes will provide novel insights into how we may develop durable and sustainable aphid control in economically important crops.

\section{Characterization of North American Grapevine Yellows}

P. LENZI (1), T. Stoepler (1), D. Melby (1), T. Wof (1)

(1) Virginia Tech, Winchester, VA, U.S.A.

Phytopathology 105(Suppl. 4):S4.80

North American Grapevine Yellows (NAGY) is an economically important disease that severely affects grapevines along the East Coast of the United States. It is caused by cell wall-less bacteria that colonize the plant's phloem and it is transmitted by insect vectors, like leafhoppers. Since many ecological and epidemiological aspects of NAGY are still poorly understood, this work aims to characterize the disease by identifying the vector species responsible for transmission, and to study the plant-pathogen interaction by determining the concentration of phytoplasmas in infected vines. We used transmission assays to identify 7 potential vector species of leafhoppers. Randomly collected leafhoppers were reared on artificial diets to determine their ability to transmit phytoplasmas while feeding. During the past season, we collected insects and allowed them to feed on NAGY-infected grapevine shoots to allow them to acquire phytoplasmas. After a latency period, insects were then separated by species and transferred onto healthy Chardonnay seedlings. We are currently monitoring these seedlings for NAGY symptoms that would confirm the ability of each species to vector phytoplasmas. Moreover, we are conducting quantitative PCR (qPCR) analysis to determine the titer of phytoplasmas in different plant organs and in different grapevine plants, since susceptibility to NAGY varies among different grapevine cultivars.

Quantification and determination of inoculum threshold levels of Fusarium commune in Douglas-fir nurseries

A. L. Leon (1), K. P. Coats (2), G. A. CHASTAGNER (2)

(1) Weyerhaeuser, Federal Way, WA, U.S.A.; (2) Washington State

University, Puyallup, WA, U.S.A.

Phytopathology 105(Suppl. 4):S4.80 
Damping-off of Douglas-fir seedlings by Fusarium commune causes crop losses in conifer nurseries in the Pacific Northwest. A more thorough understanding of the quantity and identity of soil-borne pathogens is needed in order to reduce use of increasingly restricted fumigants. A quantitative realtime PCR (qPCR) assay was developed to quantify $F$. commune and distinguish it from $F$. oxysporum, a less virulent and morphologically indistinguishable species. Standard curves designed with pure culture isolates had efficiencies of $\mathrm{r}^{2}=0.9910$ and $\mathrm{r}^{2}=0.9801$ for $F$. commune and $F$. oxysporum, respectively. Correlations between the average colony-formingunits per gram ( $\left.\mathrm{CFU} \mathrm{g}^{-1}\right)$, the current method of quantification, and the cycle threshold $\left(\mathrm{C}_{\mathrm{t}}\right)$ value of the $F$. commune $\mathrm{qPCR}$ assay ranged from $\mathrm{r}^{2}=0.5153$ to 0.9871 . Results of this study suggest that a qPCR assay for $F$. commune has the potential to determine the quantity of the pathogen in nursery soils. Disease thresholds for Fusarium spp. in Douglas-fir nurseries have been speculated upon but not published. Greenhouse inoculum threshold trials in which Douglas-fir seed were planted into soil infested at levels of 2000, 1000, 500,250 and $100 \mathrm{CFU} / \mathrm{g}$ were established to determine the mortality threshold for Douglas-fir seedlings. Results indicate that soil should be managed below

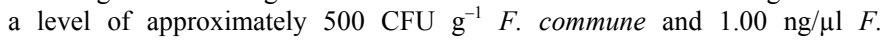
commune DNA to keep Douglas-fir damping off below 5\%.

\section{Screening the NAM parents for resistance to multiple Pythium species}

E. R. LERCH (1), A. E. Dorrance (2), A. E. Robertson (1)

(1) Iowa State University, Ames, IA, U.S.A.; (2) The Ohio State University, Wooster, OH, U.S.A.

Phytopathology 105(Suppl. 4):S4.81

Pythium is an oomycete that causes damping-off and root rot in soybeans. The most effective management tool to reduce stand losses due to damping-off is resistance, however relatively little is known regarding resistance in soybean to Pythium species. The goal of this project was to screen 47 lines from the Nested Associated Mapping (NAM) soybean parents for resistance to Pythium sylvaticum, $P$. lutarium, $P$. oopapillum, and $P$. torulosum. The cultivars, Archer and Sloan, were used as resistant and susceptible checks, respectively. Two assays were used to screen for resistance: a plate assay, which assesses susceptibility to seed rot, and a cup assay that assesses susceptibility to root rot. Seed rot severity among the parents ranged from 0.65 to 3.73 where $0=$ no seed rot and $4=$ seed rotted without germinating. Root rot severity among the parents ranged from 0.55 to $2.63,(0=$ no root rot; $3=$ severe rot $)$. Some parents showed resistance to seed rot but were susceptible to root rot and viceversa. A few parent lines showed resistance to multiple Pythium spp. Multiple Pythium spp. are often present in the same field and can cause stand and yield loss. Identification of sources of resistance to multiple Pythium spp. would be useful for soybean breeders to incorporate into soybean varieties.

\section{Two potato cultivars display different responses to the infection by} "Candidatus Liberibacter solanacearum"

J. LEVY (1), L. Cosme (1), A. Mendoza (1), J. C. Miller (1), C. Tamborindeguy (1), E. Pierson (1)

(1) Texas A\&M University, College Station, TX, U.S.A.

Phytopathology 105(Suppl. 4):S4.81

"Candidatus Liberibacter solanacearum" (Lso) is an important pathogen of potatoes and solanaceous plants in North, Central America and New Zealand, as well as in carrot in Europe. To better understand the molecular interaction of this pathogen and its plant host, we performed RNA-seq analysis using Illumina platform. Previous studies identified differences in symptom development between two potato cultivars NY138 and Atlantic after infection by Lso To understand the molecular mechanisms involved in each variety during this interaction we infested both varieties with psyllids harboring Lso or without Lso. RNA Samples were collected 3 weeks after infestation, before symptom development. We obtained $\sim 20$ million reads per sample which were mapped back to the reference genome. We used HTseq to count the number of unique reads mapped to each gene. On average $85 \%$ of the reads mapped to only one location in the genome. We used DEseq2 to perform differential expression analysis in R. Finally, we performed Gene Ontology, using gProfiler, on the genes that were differentially expressed. GO analyses reveals the enrichment for genes involved in nitrogen metabolism upon infection. In contrast, the genes down-regulated with infection in both varieties are related to photosynthesis and energy metabolism. The response of the varieties to the Lso infection is different. Expression level of selected plant genes, in defense mechanism and hormones regulation were confirmed by RT-qPCR.

cAMP-dependent protein kinase A globally regulates pathogenic differentiation in the rice blast fungus Magnaporthe oryzae

Y. LI (1)

(1) Purdue Univ, West Lafayette, IN, U.S.A.

Phytopathology 105(Suppl. 4):S4.81
The conserved cyclic AMP-dependent protein kinase A (cAMP/PKA) signaling pathway is one of the critical pathway contribute to a variety of cellular processes in eukaryotic cells responding to extracellular cues. In Magnaporthe oryzae, multiple genes involved in cAMP pathway are identified to be required for morphology of infection structure and pathogenicity. However, the role of PKA is not well characterized because of the functional redundance of the two PKA catalytic subunits CPKA and CPK2 in M. oryzae. In this study, we functionally characterized $c p k A c p k 2$ double mutant to get new insight into the critical roles of PKA in growth and pathogenicity. Unlike the $c p k A$ and the $c p k 2$ single mutant, the $c p k A c p k 2$ double mutant showed significant defect in growth, conidiation, appressorium formation and infection. The cross-talk between cAMP pathway and multiple MAPK pathways were detected in the $c p k a c p k 2$ double mutant. In response to absence of PKA, the activity of Osm1 was reduced; in contrast, PMK1, which is essential for appressorium formation, was surprisingly hyperactivated. Interestingly, the cpka cpk2 mutant is unstable, which frequently generate spontaneous suppressors. The spontaneous mutations partly rescued the growth rate, conidiation and appressorium formation defect. However, it failed to recover pathogenicity in the double mutant.

The gene HFII encoding a transcriptional coactivator in Fusarium oxysporum $\mathrm{f}$. sp. cubense is required for virulence on banana plants

M. LI (1), H. Nong (1), Z. Xu (1), X. Xie (1), P. Xi (1), L. Sun (1), Z. Jiang (1)

(1) Department of Plant Pathology, South China Agricultural University, Guangzhou, China

Phytopathology 105(Suppl. 4):S4.81

Fusarium oxysporum f. sp. cubense (FOC), the casual agent of banana fusarium wilt, is one of the most destructive pathogens threatening the banana production. However, the molecular mechanisms underlying the virulence and pathogenecity of this fungal pathogen are still poorly understood. In this study, we identified and characterized the disrupted gene in a T-DNA insertional mutant L715 of FOC with significantly reduced virulence on banana plants. The gene disrupted by T-DNA insertion in L715 harbors an open reading frame encoding a protein with high homology to transcriptional coactivator in Saccharomyces cerevisiae. The deletion mutant ( $\triangle H F I 1)$ of FOC couldn't produce any conidia when cultured on PDA plate, exhibited an impaired in fungal growth, and showed less cell wall-degrading enzymes (CWDEs) expression than the wild type. Furthermore, the deletion of HFII in FOC led to decline dramatically in virulence to the banana host. The mutant phenotypes were fully restored by complementation with the wild type HFII gene. Our data provide a first evidence for the critical role of $H F I 1$ in regulation of CWDEs expression and the virulence of $F$. oxysporum f. sp. cubense.

Reniform nematode (Rotylenchulus reniformis) manipulation of host root gene expression during syncytium formation in cotton (Gossypium hirsutum) W. LI (1), C. Wells (1), P. Agudelo (1)

(1) Clemson University, Clemson, SC, U.S.A.

Phytopathology 105(Suppl. 4):S4.81

Reniform nematode (Rotylenchulus reniformis) is a major yield-limiting pest of upland cotton (Gossypium hirsutum). To better understand the molecular mechanisms of the interaction between reniform nematode and cotton, we constructed comprehensive transcriptomic profiles of the nematode-induced feeding structures called syncytia. We sampled tissue from syncytial and nonsyncytial cotton roots at 3, 6, 9 and 12 days after inoculation (DAI). The extracted mRNAs were sequenced on the Illumina HiSeq 2000 platform and reference transcriptome was assembled de novo with Trinity pipeline. Annotations were assigned according to NR database in Blast2GO and differential expression was investigated using RSEM and DESeq2. Overall, $178,121,358$, and 89 genes were differentially expressed at 3, 6, 9 and 12 DAI, respectively $(\mathrm{FDR}=0.05)$. Among the common differentially expressed genes across more than three time points: thirteen genes were significantly upregulated, including sulfite reductase, expansin b1, an abc transporter, and a zinc finger transcription factor; two genes were significantly down-regulated: an extensin and a non-specific lipid transfer protein; only cytochrome p450 gene with reported functions in gibberellin deactivation and cell wall biosynthesis was depressed at 3DAI but up-regulated later. Comprehensive gene expression profiles of syncytial development significantly advance our current understanding of plant resistance to reniform nematode.

Induction of systemic acquired resistance against citrus Huanglongbing by exogenous application of functional analogs of salicylic acid

J. LI (1), P. Trivedi (1), N. Wang (1)

(1) University of Florida, Lake Alfred, FL, U.S.A.

Phytopathology 105(Suppl. 4):S4.81

Huanglongbing (HLB) is one of the most destructive diseases of citrus worldwide. Induction of systemic acquired resistance (SAR) has been widely 
deployed in agriculture to protect plants from pathogens. Naturally infected citrus trees in four different groves in Florida were sprayed with various SAR inducing compounds for two to four continuous growing seasons respectively. Each treatment was tested with 5 to 10 trees in each grove. After three or four applications for each season, the functional analogs of salicylic acid and plant defense inducers including $\beta$-aminobutyric acid (BABA), 2,1,3-benzothiadiazole (BTH), and 2,6-Dichloroisonicotinic acid (INA), individually or in combination, were able to reduce the HLB disease severity by 15 to $25 \%$, compared to the water-treated control; and slow the population growth of 'Candidatus Liberibacter asiaticus' (Las), the pathogen of HLB in planta. Effects of these treatments on fruit yield and quality were also significant as compared with the water-treated control. Overall, these findings indicate that SAR inducers could be a useful strategy for the management of citrus HLB.

Population dynamics and molecular mechanisms of DMI resistance in cucurbit gummy stem blight fungi

H. X. LI (1), K. L. Stevenson (2), H. Sanders (2), M. T. Brewer (1)

(1) University of Georgia, Athens, GA, U.S.A.; (2) University of Georgia, Tifton, GA, U.S.A.

Phytopathology 105(Suppl. 4):S4.82

Understanding pathogen population dynamics in response to fungicides is critical for improved disease management. Gummy stem blight (GSB) is a devastating disease of cucurbits caused by three morphologically similar, but genetically distinct Stagonosporopsis species: S. cucurbitacearum (syn. Didymella bryoniae), S. citrulli, and S. caricae. Management of GSB in the southeastern US relies heavily on DMI fungicides. Our objectives were to determine if DMI-resistant isolates are present in the southeastern US and, if so, to identify the molecular basis of resistance. Four of 246 isolates $(2 \%)$ collected in 2013 and 15 of 76 isolates $(20 \%)$ collected in 2014 tested for sensitivity to tebuconazole were resistant based on mycelial growth assays. All resistant isolates were $S$. caricae, which had not been previously found in the southeastern US. All sensitive isolates were $S$. citrulli, the only known cause of GSB in the southeastern US until now. An increased frequency of resistant S. caricae was also detected in 2014 field trials with decreased efficacy of tebuconazole. To investigate mechanisms of resistance, the Cyp51 gene and promoter of 8 resistant and 3 sensitive $S$. caricae isolates were sequenced. No sequence differences were associated with resistance. Increased expression of Cyp51 and/or AtrG (an ABC-transporter) in resistant isolates is currently being investigated. We aim to develop a molecular marker for detection of resistant isolates to improve GSB management.

\section{Host eIF4E functions in RNA replication during Bymovirus infection} H. Li (1), Y. SHIRAKO (1)

(1) ANESC, University of Tokyo, 1-1-1 Yayoi, Bunkyo-ku, Tokyo, Japan Phytopathology 105(Suppl. 4):S4.82

The eukaryotic translation initiation factor 4E (eIF4E) has been reported to be a host factor that is commonly involved in infections caused by monopartite plant RNA viruses in the genus Potyvirus in the family Potyviridae. The possible functions of eIF4E in potyvirus infection have been postulated to include viral genome translation, viral RNA replication and virus movement in host plants, but the mechanisms that underlie these functions remain unclear. We demonstrated previously that the expression of wheat eIF4E from RNA2 of the bipartite Wheat yellow mosaic virus (WYMV) in the genus Bymovirus in the family Potyviridae enabled WYMV replication in barley mesophyll protoplasts, which do not support the replication of wild-type WYMV. Here we examined an isolate of the bymovirus Barley yellow mosaic virus (BaYMV), which cannot replicate in a barley variety that exhibits allelic eIF4E-mediated resistance. The virus isolate could replicate in the resistant barley protoplasts when an eIF4E gene from a susceptible barley variety was expressed from a recombinant BaYMV RNA2 vector. These results indicated that host eIF4Es function in bymoviral RNA replication. BaYMV VPg was demonstrated to be a viral factor that overcomes eIF4E-mediated barley resistance. However, experimental evidence for the existence of physical interactions between eIF4E and bymoviral VPg, which could be obtained via a yeast two-hybrid assay and a co-immunoprecipitation analysis, is not yet available.

The PdeR-TriP interaction mediates a novel c-di-GMP signaling pathway to regulate virulence of Xanthomonas oryzae pv. oryzae

H. Li (1), F. Tian (1), D. Xue (1), H. Chen (1), X. Yuan (2), C. H. Yang (2), C. HE (1)

(1) Institute of Plant Protection, Chinese Academy of Agricultural Sciences, Beijing, China; (2) Department of Biological Sciences, University of Wisconsin-Milwaukee, Milwaukee, WI, U.S.A.

Phytopathology 105(Suppl. 4):S4.82

PdeK/PdeR, the two-component system (TCS), was previously shown to regulate the virulence of the rice bacterial blight pathogen Xanthomonas oryzae pv. oryzae (Xoo). The response regulator PdeR harbors phosphodiesterase (PDE) activity to degrade bacterial second messenger cyclic diGMP. To understand the downstream signaling pathway mediated by PdeR, we started with looking for its interacting partners in Xoo strain PXO99 . Here, we identified a response regulator PXO_04421, named as TriP, as an interactor of PdeR. Yeast two-hybrid $(\mathrm{Y} 2 \mathrm{H})$ and GST pull-down assays confirmed the PdeR-Trip interaction. Interestingly, the interaction was inhibited by high concentration of c-di-GMP. Virulence assays demonstrated that the triP mutant caused shorter lesion length on rice leaves than the wild type, and its ability to progress through the xylem tissue was also impaired. Moreover, both the $p d e R$ and the triP single mutant produced less exopolysaccharide (EPS), while the $p d e R /$ triP double mutant produced similar level of EPS with that of the triP mutant. These results indicated that TriP and PdeR are functionally related in regulation of bacterial virulence. Additionally, RNA-seq analysis revealed a considerable overlap in the upregulated genes in the two single mutants, which further implied that TriP might mediate a part of the PdeR-mediated signaling in Xoo.

Evaluating soybean cultivars for resistance to Phomopsis seed decay in Mississippi

S. LI (1), G. Sciumbato (2)

(1) USDA ARS CGRU, Stoneville, MS, U.S.A.; (2) Mississippi State University, Stoneville, MS, U.S.A.

Phytopathology 105(Suppl. 4):S4.82

Phomopsis seed decay (PSD) of soybean reduces seed quality, germination and seedling vigor. PSD has been problematic in most soybean production areas including Mississippi (MS). Planting resistant cultivars is one of the most effective means to control PSD. However, very few soybean cultivars resistant to PSD are currently available for planting in the US. In this study, 16 commercial soybean cultivars were evaluated for resistance to PSD with inoculated and non-inoculated treatments and two harvest times at R8 and R $8+2$ weeks growth stages (on-time vs. delayed harvest) in Stoneville, MS in 2012 and 2013. Those 16 cultivars were chosen based on the data from seed assays of 50 commonly planted cultivars in 2011. Significant differences in seed infection by Phomopsis longicolla, the causal agent of PSD, were observed among cultivars tested. Seed infection ranged from a low of $6 \%$ to a high of $76 \%$. These differences among cultivars were also reflected in visual seed quality and seed germination. Morsoy R2 491 had the lowest percentage of Phomopsis seed infection and highest germination rate in both harvest trials. However, some cultivars had low Phomopsis seed infection in the ontime harvest trial, but had high Phomopsis seed infection in the delayed harvest trial. Testing of cultivars at delayed harvest time or under the conditions which favor PSD disease development is important for identification of PSD-resistant cultivars.

Analysis of a MULE-cyanide hydratase gene fusion in Verticillium dahliae Z. LI (1), A. Anchieta (2), S. J. Klosterman (3)

(1) Cotton Research Institute, Anyang, China; (2) USDA-ARS, Salinas, CA, U.S.A.; (3) USDA ARS, Salinas, CA, U.S.A.

Phytopathology 105(Suppl. 4):S4.82

The genome of the phytopathogenic fungus Verticillium dahliae encodes numerous Class II "cut-and-paste" transposable elements, including those of a small group of MULE transposons. We have previously identified a fusion event between a MULE transposon sequence and sequence encoding a cyanide hydratase in one strain of $V$. dahliae. Phylogenetic analyses of cyanide hydratases from filamentous fungi showed that this particular cyanide hydratase had separate origins from others. That is, the fused gene is specific to $V$. dahliae. Additionally, reverse transcription PCR was used to verify the expression of this unique gene fusion and further enabled characterization of the gene structure. Currently the function of this cyanide hydratase-MULE fusion is unknown, and additional gene expression experiments are underway to gain insight on the function of this unique gene. This provides an example of a direct effect of repetitive DNA elements, such as transposons, on gene modification within the genome of $V$. dahliae.

Validation of novel microRNAs and prediction of their targets responding to the infection of Cucumber green mottle mosaic virus in cucumber C. LIANG (1), H. Liu (2), L. Luo (1), J. Li (1), C. N. Mortensen (3)

(1) Department of Plant Pathology/Beijing Engineering Research Center of Seed and Plant Health, China Agricultural University, Beijing, China, Beijing, China; (2) Molecular Plant Pathology, United States Department of Agriculture, Agricultural Research Service, Beltsville, Maryland, USA, Beltsville, MD, U.S.A.; (3) Department of Plant Biology and Biotechnology, Faculty of Life Sciences, University of Copenhagen, Frederiksberg C, Copenhagen, Denmark, Copenhagen, Denmark

Phytopathology 105(Suppl. 4):S4.82 
MicroRNAs (miRNAs) are a class of small, non-coding, single stranded RNA molecules. They are known to play a fundamental role in plant growth and development processes, stress responses and host-pathogen interaction. The previous research results showed that 8 novel miRNAs (csa-miRn1-3p, csamiRn2-3p, csa-miRn3-3p, csa-miRn4-5p, csa-miRn5-5p, csa-miRn6-3p, csamiRn7-5p and csa-miRn8-3p) were identified in cucumber plants in response to Cucumber green mottle mosaic virus (CGMMV) infection by highthroughput sequencing, bioinformatic analysis and prediction of secondary structure. In this study, these 8 novel miRNAs were confirmed to be real existence in cucumber leaves by quantitative real-time PCR (RT-qPCR), and their expression levels were changed at 1-42 days post inoculation (dpi) in CGMMV-infected cucumber leaves. It was revealed that csa-miRn1-3p, csamiRn2-3p, csa-miRn3-3p, csa-miRn4-5p, csa-miRn5-5p, csa-miRn6-3p and csa-miRn8-3p were predicted to have totally 211 target genes, which exert their regulatory functions mostly through target RNA cleavages, and no potential target gene for csa-miRn7-5p was predicted by psRNATarget software analysis. The analysis of regulation networks showed that 7 novel miRNAs carried their targets respectively and none of the targets was regulated by two or more miRNAs. The results are essential to clarify the role of novel miRNAs and their targets in cucumber developmental processes and interactions between CGMMV and cucumber.

\section{A newly developed GFP-expressing Barley stripe mosaic virus infectious} clone used to investigate seed transmission in barley in South Korea

H. J. LIM (1), E. Y. Seo (2), J. Kim (2), J. S. Gong (1), H. K. Ju (3), C. H. Park (1), D. Li (4), N. Kim (5), C. Jang (5), I. Hwang (5), J. Hammond (6), H. S. Lim (1)

(1) Chungnam National University, Deajeon, South Korea; (2) Chungnam National University, Daejeon, South Korea; (3) Chungnam National Universitiy, Deajeon, South Korea; (4) China Agricultural University, Beijing, China; (5) Central Research Institute of Kyung Nong Corporation, Gyeongju, South Korea; (6) United States Department of Agriculture-Agrocultural Research Service, Beltsville, MD, U.S.A.

Phytopathology 105(Suppl. 4):S4.83

Barley cultivation is expanding nationwide in South Korea due to the increasing popularity of health foods. We therefore investigated the virus status of barley crops around iksan and wanju. On one newly cultivated barley farm we observed several areas showing symptoms of Barley stripe mosaic virus (BSMV), and confirmed BSMV infection. The newly isolated Korean BSMV showed high sequence homology to previously reported US isolates. BSMV is known to be seed-transmitted, and Korean farmers save seed for replanting, potentially increasing levels of infection. In order to understand BSMV movement into seeds, we created an infectious BSMV clone expressing GFP. Instead of creating a TGB1- or gamma-b-GFP fusion protein, we created a GFP expression vector using a quadripartite virus with a partially duplicated beta RNA. In one copy of RNA beta, TGB1 was substituted by a multiple cloning site including GFP, maintaining TGB2 and TGB3. A functional copy of TGB1 was expressed from a second copy of RNA beta, in which the TGB2 and TGB3 start codons were deleted, and this copy was coinoculated with the newly designed GFP expression construct. GFP expression was stably detected in early stages of infection, but was not passaged to new plants. The quadripartite virus was then used to monitor BSMV movement into barley seed embryos, and delayed germination due to BSMV infection. Additionally we report retardation of BSMV replication by biological control agent 'FarmWorld'.

Development of TaqMan probe-based insulated isothermal PCR (iiPCR) for detection of Fusarium oxysporum f. sp. cubense race 4

Y. J. Lin (1), J. C. Hsu (1), Y. H. Chiu (1), L. L. Hong (1), T. D. Chang (1), P. F. L. Chang (2), Y. H. LIN (1)

(1) Dept. of Plant Medicine, National Pingtung University of Science and Technology, Pingtung County, Taiwan; (2) Dept. of Plant Pathology, National Chung Hsing University, Taichung City, Taiwan

Phytopathology 105(Suppl. 4):S4.83

Banana (Musa spp.) is cultivated worldwide and is one of the most popular fruits. Based on the statistics of FAO (Food and Agriculture Organization of the United Nations, Rome, Italy) and COA (Council of Agriculture, Executive Yuan, Taipei, Taiwan), banana was the third most important fruits in the world and Taiwan, respectively. Fusarium wilt of banana caused by $F$. oxysporum f. sp. cubense (Foc) has become one of the major limiting factors for banana production in the world. Disease monitoring is the basis of integrated pest management of diseases. The lack of rapid, accurate, and reliable device to detect and identify plant pathogens is one of the main limitations in integrated disease management. Insulated isothermal PCR (iiPCR), established on the basis of the Rayleigh-Bénard convective PCR method, is a rapid and low-cost platform for nucleic acid amplification within less than $60 \mathrm{~min}$. This study developed a novel, efficient detection method based on the TaqMan probe-based iiPCR method for rapid detection of Foc race 4. The iiPCR system is carried out in the R-tubeTM within the POCKIT Nucleic Acid Analyzer designed by GeneReach Biotechnology Corporation, Taichung, Taiwan. The approach will be applied to detect Foc-contaminated samples.

Interaction dynamics between the potato late blight resistance protein RB and pathogen effectors

Y. LIN (1), J. Jiang (1), D. Halterman (2)

(1) University of Wisconsin-Madison, Madison, WI, U.S.A.; (2) USDA/ARS Vegetable Crops Research Unit, Madison, WI, Madison, WI, U.S.A.

Phytopathology 105(Suppl. 4):S4.83

Potato Late blight, caused by the oomycete pathogen Phytophthora infestans, can effectively be controlled by $\mathrm{R}$ protein mediated resistance. The RB protein, from the diploid wild potato species Solanum bulbocastanum, can specifically recognize its corresponding pathogen effector IPI-O and contribute to resistance to most $P$. infestans isolates. IPI-O is a multigene family of effectors and most IPI-O proteins (e.g. IPI-O1, IPI-O2) can trigger a resistance response in plants with RB. Recognition has been shown to be mediated through direct protein-protein interaction. An IPI-O family member, IPI-O4, is not only able to elude RB detection, but can also block recognition of IPI-O1. To understand this, we are currently studying the interaction proteomics of RB and IPI-O proteins. RB with a double tag HA-Biotin along with IPI-O1/4 with a MYC tag were transiently expressed in Nicotiana benthamiana individually or cooperatively. After immunoprecipitation (IP) of RB protein using streptavidin labeled magnetic beads, the biotin tag has been cleaved from the recombined RB-HA-Biotin protein and isolated secondarily by HA tag to increase purity. However, positive results is hard to be identified by Co-IP between RB and IPI-O1/4 proteins. The interaction proteomics of $\mathrm{RB}$ and IPI-O1/4 individually or cooperatively will be helpful to identifying members of the RB protein complex.

Genome sequencing, genetic diversity and field detection of Cucumber green mottle mosaic virus using LAMP technology

K.-S. LING (1), R. Li (1)

(1) USDA-ARS, U.S. Vegetable Laboratory, Charleston, SC, U.S.A.

Phytopathology 105(Suppl. 4):S4.83

The recent outbreaks of Cucumber green mottle mosaic virus on cucumber, melon and watermelon in Australia, Canada, and the U.S. highlight the importance in implementing a cleaned seed program to manage this seedborne virus from introduction. Both Canadian and Australian isolates were closely related to the Asian genotype with $99 \%$ sequence identity. However, the California isolate was more divergent, which was more closely resembled those from Europe ( $95 \%$ sequence identity) than from Asia (90-92\% sequence identity). In addition to the genetic diversity, recent discovery of a strong serological specificity between the two genotypes causes concerns on the reliability of a serological test (i.e., ELISA), particularly for seed health test, where virus titer is typically lower. To improve reliability for a sensitive detection of CGMMV isolates in all genotypes, a loop-mediated isothermal amplification (LAMP) was developed with six oligonucleotides derived from a highly conserved region in a multi-sequence alignment of available 31 genome sequences. This LAMP system was confirmed to provide a sensitive detection to all genotypes of CGMMV. In combination with a simple crude tissue RNA preparation, this LAMP detection system was used to monitor CGMMV infection on greenhouse cucumber. The availability of this simple and sensitive detection would greatly enhance our ability to provide an early and reliable detection of CGMMV-infected plants as well as for seed health test.

Genomics study of limber pine genetic resistance to white pine blister rust J. J. LIU (1), A. W. Schoettle (2), R. A. Sniezko (3), N. Wang (4), A. Zamany (1), R. Sturrock (1), A. Kegley (3)

(1) Pacific Forestry Centre, Canadian Forest Service, Natural Resources Canada, Victoria, BC, Canada; (2) USDA Forest Service, Rocky Mountain Research Station, Fort Collins, CO, U.S.A.; (3) USDA Forest Service, Dorena Genetic Resource Center, Cottage Grove, OR, U.S.A.; (4) Academy of Agriculture and Forestry Science, Qinghai University, Xining, China Phytopathology 105(Suppl. 4):S4.83

Limber pine (Pinus flexilis) is a keystone species in high-elevation landscapes of western North America with ecological importance. Like other white pines species native to North America, limber pine trees are infected and killed by exotic white pine blister rust (Cronartium ribicola) throughout its geographical ranges, but few studies of genetic resistance mechanisms have been reported. Recently a single dominant resistance gene $(\mathrm{Cr} 4)$ was discovered for genetic control of an inherited stem disease-free trait by artificial rust inoculation 
(Schoettle et al. 2014). To provide genomic insight into molecular limber pine-blister rust interactions and find DNA markers for breeding selection of $\mathrm{Cr} 4$, we performed de novo sequencing of the transcriptome by RNA-seq analysis using stem and needle tissues. Using bioinformatic analyses, we identified a large number of gene members of the disease resistant families as well as candidate genes for conifer defense response upon disease infection. Genetic variations with focus on single nucleotide polymorphisms (SNPs) were determined by transcriptome comparison between resistant and susceptible families. SNPs found in disease resistance gene families and unique resistant seed families were selected and subjected to high-throughput SNP genotyping. We will present an update on our ongoing research on association and linkage analysis of P. flexilis SNP genotypes with phenotypic resistance to white pine blister rust.

\section{First report of Monilochaetes infuscans infecting Punica granatum}

B. LIU (1), R. Cadet (1), F. Zhang (2)

(1) USDA APHIS PPQ, Linden, NJ, U.S.A.; (2) USDA APHIS PPQ, San Mateo, CA, U.S.A

Phytopathology 105(Suppl. 4):S4.84

Monilochaetes infuscans causes scurf disease or soil stain of sweet potato (Ipomoea batatas), however, so far there is no report that this fungus can infect pomegranate (Punica granatum). An pomegranate interception from Colombia was sent to the plant pathology lab of the USDA APHIS PPQ, and Monilochaetes-like fungus was found from the lesion of the fruit skin of pomegranate, morphological character of this fungus match to the description of Monilochaetes infuscans. DNA sequence analysis was done based on ITS1 and ITS4 primers, and Blast search was used to search GenBank database, results showed that the sequences of this fungus are exactly match to the sequences of Monilochaetes infuscans deposited in GenBank. To our knowledge, this is the first report of Monilochaetes infuscans infecting Punica granatum.

Biological control of multiple plant diseases and plant growth promotion in the presence of pathogens by Plant Growth-Promoting Rhizobacteria (PGPR)

K. LIU (1), J. W. Kloepper (1), J. A. McInroy (1), C. H. Hu (1)

(1) Auburn University, Auburn, AL, U.S.A.

Phytopathology 105(Suppl. 4):S4.84

PGPR strains Bacillus aerophilus AP69 and B. amyloliquefaciens subsp. plantarum AP199, which previously demonstrated biological control of three different diseases in the growth chamber, were tested for both biological control of multiple plant diseases and plant growth promotion in the presence of pathogens in the greenhouse. Tests evaluated mixtures of AP69 and AP199, B. amyloliquefaciens subsp. plantarum strain GB03 was a positive control, a disease control, and a healthy control. The specific diseases tested in this study included bacterial spot of tomato (Xanthomonas axonopodis pv. vesicatoria (Xav)), bacterial speck of tomato (Pseudomonas syringae pv. tomato (Pst)), and damping-off of cucumber (Pythium ultimum). Results indicated that the two individual PGPR strains and their mixture significantly reduced severity of all three diseases compared to the disease control, and only the mixture significantly increased the root dry weight and four root morphology parameters in all three experiments while individual strains significantly increased some of these growth parameters. In the test of $P$. ultimum, AP199 and the mixture reduced disease severity and promoted plant growth better than GB03. Also in presence of Pst, plant growth of the mixture was equivalent or higher than the healthy control.

Soybean root rot, Rhizoctonia communities and their relation with other microbes and nematode communities

B. LIU (1), H. Wei (2), W. Shen (2), H. Smith (2), J. Correll (3)

(1) Univ of Arkansas, Fayetteville, AR, U.S.A.; (2) University of NebraskaLincoln, North Platte, NE, U.S.A.; (3) University of Arkansas, Fayetteville, AR, U.S.A.

Phytopathology 105(Suppl. 4):S4.84

Soybean root rot, caused by Rhizoctonia solani, has been one of the most serious soybean diseases in the North Central Region of the United States. There is no report on the relationship of Rhizoctonia root rot with soil physical and chemical properties, and microbial and nematode communities. A commercial soybean field with a long history of Rhizoctonia problem was examined using media culturing and molecular approaches to explore this relationship. Results demonstrated that the high disease incidence in sampled areas were positively correlated with high levels of $\mathrm{N}, \mathrm{P}, \mathrm{K}, \mathrm{Mn}$, populations of soil Rhizoctonia, thermophiles and fungi, root Rhizoctonia colonies, populations of lesion and stunt nematodes, and negatively correlated with high levels of $\mathrm{Ca}, \mathrm{Mg}, \mathrm{Na}, \mathrm{pH}$, and base saturation (BS), and populations of soil Pseudomonas based on correlation analysis and canonical correspondence analysis (CCA). Cluster analysis showed that the communities of both Rhizoctonia and bacteria in sampled areas were separated based on healthy and diseased plants using degenerate gradient gel electrophoresis (DGGE); however, the communities of Fusarium were very similar. There was no clear separation for Pythium and Trichoderma communities in sampled areas based on healthy and diseased plants. Moreover, the disease suppression seems to be more related to the quantity of soil microorganisms rather than specific species.

Virulence and secondary metabolite profiles of vascular competent and vascular incompetent pathotypes of Fusarium oxysporum f. $\quad$ sp. vasinfectum

J. LIU (1), A. A. Bell (1), R. L. Nichols (2)

(1) USDA ARS, College Station, TX, U.S.A.; (2) Cotton Incorporated, Cary, NC, U.S.A.

Phytopathology 105(Suppl. 4):S4.84

Fusarium wilt of cotton, caused by Fusarium oxysporum f. sp. vasinfectum (Fov), occurs in most cotton growing areas of the world. Pathotypes of Fov have been categorized into eight races based on virulence to different hosts. However, lack of reciprocal resistance reactions among cotton cultivars to putative races has made assignment problematic. We propose to subdivide Fov isolates into vascular competent (VC) and vascular incompetent (VI) pathotypes, based on their ability (VC) or inability (VI) to produce wilt in a standard stem-puncture assay. Isolates of the Australian biotypes, race 4 , and race 7 which had identical sequence type with race 4 based on several gene fragments, cause wilt when young cotton roots are dipped in conidial suspensions or when suspensions are injected into the soil around the plant. But these isolates fail to produce wilt when conidia are puncture-inoculated into stem and thus are classified as VI. Race 1, 2, 6 and 8 isolates produce wilt symptoms when the cotton is stem-puncture or root-dip inoculated, but not when conidia are inoculated into soil; thus they are classified as VC. These results correspond with field observations that VI isolates cause wilt without vectoring by nematodes, while $\mathrm{VC}$ isolates require co-infection by root knot nematodes to exhibit severe disease. VC isolates produce the heptaketides, nectriafurone, anhydrofusarubin lactol, and 5-O-methyljavanicin. VI isolates consistently produce fusaric acid.

Efficacy of SDHI fungicides to protect leaves and fruits from infections of apple scab (Venturia inaequalis) in apple orchards in Maule Region, Chile M. LOLAS (1), G. Diaz (1), M. Caceres (1), R. Mendez (1)

(1) Universidad de Talca, Talca, Chile

Phytopathology 105(Suppl. 4):S4.84

Apple scab in Chile is a main disease, thus growers use integrated control programs to avoid fruit damage, protecting or curing developing scab lesions. In the last years, representatives of the group SDHI (Succinate dehydrogenase inhibitors) have been tested. In these studies, the efficacy against primary and secondary infections of $V$. inaequalis was assessed for penthiopyrad, fluopyram and isopyrazam, in Royal Gala or Red Chief orchards located in Panguilemo or Colin, Maule Region, respectively, during seasons 2013-2014 and 2014-2015. The program used in each trial consisted of three-four applications made during the flowering period starting at the beginning $(5 \%)$; full bloom, petal fall and 7-10 days after petal fall. A completely randomized design with 4 replications was used at each location; the experimental unit consisted of 5 homogenous trees. Fungicide treatments, including a control which was spread only with water, were applied using a $150 \mathrm{~L}$ Impact sprayer. During 2013-2014, prevalence of apple scab in the control trees at the trials ranged from 9.4 to $74.4 \%$ for leaves and from 6.5 to $24 \%$ for fruits; during 2014-2015 season ranged from 47 to $85 \%$ for leaves and 13 to $35 \%$ for fruits. All SDHI treatments decreased significantly the prevalence of apple scab on leaves and fruits at the tested doses compared with the respective control. Therefore, penthiopyrad, fluopyram and isopyrazam showed high efficacy to protect apple leaves and fruits from scab infection.

Vectoring plant pathology: Outreach group brings scientific research from the laboratory to the classroom

E. Lookabaugh (1), A. KOEHLER (1), K. McCorkle (1)

(1) North Carolina State University, Raleigh, NC, U.S.A.

Phytopathology 105(Suppl. 4):S4.84

The North Carolina State University Plant Pathology Graduate Student Association developed an outreach committee to educate the public about plant pathology, disease diagnosis, and management. Outreach activities include informational public events held at the North Carolina Museum of Natural Science and high school education programs. The outreach group travels to high schools around North Carolina to raise awareness of plant pathology and allow students to gain hands on experience with biotechnology techniques used in plant disease diagnosis and management. Students are split 
into four rotating stations: diagnosis, culturing, DNA, and plant transformation. At the diagnosis station, students analyze symptomatic plants and practice using microscopes while they are introduced to major plant pathogen groups. In culturing, students are introduced to sterile technique and practice transferring their own cultures of bacteria and fungi. At the DNA station students are introduced to ways that scientists use and visualize DNA by extracting DNA from fruit. Plant transformation introduces techniques employed to develop pest-resistant plants and students simulate transforming a plant. The goal of this program is to allow students hands-on opportunities to learn new material and use technology not regularly available. The outreach committee recently received the Mathre Education Endowment Award to purchase portable gel electrophoresis systems to add as a new activity.

Evaluating poinsettia cultivars for partial resistance to Pythium root rot E. LOOKABAUGH (1), B. Shew (1)

(1) North Carolina State University, Raleigh, NC, U.S.A.

Phytopathology 105(Suppl. 4):S4.85

Thirty-four cultivars of poinsettia (Euphorbia pulcherrima) were screened for partial resistance to root rot caused by Pythium aphanidermatum. A variety of bract colors, response times, and vigors were represented. Commercial cuttings were potted in Fafard 4P potting media and six rice grains colonized with $P$. aphanidermatum were added to pots $3 \mathrm{wk}$ after transplanting. Plants were scored on a 1 to 5 scale for root rot $(1=$ none; $5=$ completely rotted $)$ and above-ground symptoms $(1=$ healthy; $5=$ dead plant $)$ ca. 9 wk after inoculation. Cultivars differed in root rot $(P<0.0001)$ and above-ground symptoms $(P<0.0001)$. The hybrid cultivars, 'Princettia Max White' and 'Luv U Pink' had the least root rot, with a mean rating of 2.25. The cultivar 'Sparkling Punch' had the most root rot, with a mean rating of 4.75 . Aboveground symptoms were nearly absent (mean rating 1.0) in 'Premier Red' whereas 'Sparkling punch' had the most severe symptoms with a mean aboveground rating of 4.75. Mean root rot ratings were correlated with aboveground symptom expression for most cultivars $(\mathrm{r}=0.6534, P<0.0001, \mathrm{n}=$ 34). The cultivars 'Princettia Dark Pink', 'Premier Red', and 'Jubilee Pink' were able to withstand high levels of root necrosis with minor above-ground symptom expression as compared to non-inoculated controls. The results suggest that certain cultivars exhibit partial resistance to Pythium root rot.

Distribution and abundance of Heterodera glycines and Macrophomina phaseolina in Ohio

H. D. LOPEZ-NICORA (1), A. C. M. Simon (1), B. C. Dossman (1), P. A. Paul (2), A. E. Dorrance (2), L. E. Lindsey (1), T. L. Niblack (1)

(1) The Ohio State University, Columbus, OH, U.S.A.; (2) The Ohio State University, Wooster, OH, U.S.A.

Phytopathology 105(Suppl. 4):S4.85

In the U.S. and worldwide, Heterodera glycines, the soybean cyst nematode $(\mathrm{SCN})$, is responsible for significant losses incurred by soybean growers every year. The fungus, Macrophomina phaseolina, is reported to affect more than 500 species of plants, causes significant yield loss in soybeans worldwide and can also affect corn. Both organisms are soil-borne pathogens and, once detected in a field, eradication is very unlikely. Our objective was to determine the presence, distribution, and abundance of both SCN and $M$. phaseolina in soybean and corn fields across Ohio. During 2013 and 2014, composite soil samples were collected from 370 fields in 60 counties (out of 88). Farmers volunteered to have their fields sampled and responded to a questionnaire about their awareness of SCN infestation in their fields. Samples were processed for SCN eggs $/ 100 \mathrm{~cm}^{3}$ and M. phaseolina colony forming units (CFU)/g soil with standard techniques. SCN was detected in more than $44 \%$ of the fields sampled; furthermore, SCN was detected in 51 counties. Additionally, more than $77 \%$ of the growers whose samples were from infested fields were unaware they had SCN. M. phaseolina was detected in $84 \%$ of the sampled fields. In fields where these pathogens were detected, SCN eggs $/ 100 \mathrm{~cm}^{3}$ soil ranged from 40 to 18,000 and M. phaseolina $\mathrm{CFU} / \mathrm{g}$ soil ranged from 1 to 77 . Information generated from this survey will be used to educate producers on the importance of these two pathogens in Ohio.

Optimization of the detection of viable 'Candidatus Liberibacter asiaticus' bacterium in citrus tissue

E. S. LOUZADA (1), O. Vazquez (1), S. J. Schneider (1), M. Kunta (1)

(1) Texas A\&M University Kingsville, Weslaco, TX, U.S.A.

Phytopathology 105(Suppl. 4):S4.85

Candidatus Liberibacter asiaticus (Clas), the supposed causal agent of huanglongbing (HLB) has been a major threat to the survival of the citrus industry worldwide because of its destructive nature and unavailability of any type of control. Clas is an uncultured bacterium that inhabits the phloem of infected citrus plant, and it is vectored by the Asian citrus Psyllid (Diaphorina citri Kuwayama). The annual viable Clas population dynamics in citrus trees is not known because the published methods available to identify viable Clas in the tissue are not adequate. Furthermore, to test the efficacy of any product or treatment to control the Clas, a reliable method to identify viable and dead bacteria is needed. Ethidium monoazide (EMA) and propidium monoazide (PMA) have been widely used to differentiate live from dead bacteria in varied research reports. PMA has proved to be more efficient than EMA, because EMA can also penetrate into viable bacteria cells. However, no suitable method has been reported in the case of bacterium inhabiting the interior of plant tissue. We will present the results of the optimization of PMA use to identify live and dead Clas, including a detailed protocol.

Untargeted metabolomic analysis reveals that the plant pathogenic bacterium Ralstonia solanacearum alters the composition of host plant xylem fluid

T. M. LOWE (1), A. L. Jancewicz (1), B. L. Dalsing (1), P. H. Masson (1), C. Allen (1)

(1) University of Wisconsin, Madison, WI, U.S.A.

Phytopathology 105(Suppl. 4):S4.85

The bacterial wilt pathogen Ralstonia solanacearum ("Rs") colonizes the vasculature of many plants. Although xylem sap is often considered to be nutrient-poor, $R s$ is highly adapted to this niche and reaches population sizes of $>1 \times 10^{10} \mathrm{CFU} / \mathrm{g}$ stem. Intriguingly, $R s$ grew better in xylem sap harvested from $R s$-infected tomato plants than in healthy sap. We hypothesize that $R s$ manipulates plant hosts to move nutrients into the xylem. Using metabolomics, we identified $>5$ sugars and $>10$ amino acids that were significantly enriched in infected sap. To see what $R s$ consumed in sap, we compared sap from infected plants after $3 \mathrm{~h}$ incubation $+/-R s$. Glucose was depleted suggesting, unsurprisingly, that glucose is a preferred carbon source for $R s$ in the plant. The infect sap contained more cell wall fragments (likely products of $R s$ 's cell-wall degrading enzymes) and tyramine and phenylalanine (metabolites involved in biosynthesis of antimicrobial phenylpropanoids). Overall, few compounds were enriched $>5$-fold, but putrescine was enriched by a striking 62-fold in $R s$-infected xylem sap. Putrescine production contributes to virulence of several animal pathogens and is associated with abiotic plant stress. To determine if $R s$ synthesizes putrescine in infected plants, we mutated adi and speB, which putatively encode putrescine biosynthesis enzymes in Rs. Additionally, we are testing whether exogenous putrescine influences the infection outcome on several host plants.

Computational pipeline to improve detection accuracy of plant pathogens from NGS datasets using signature oligonucleotides

C. LOWE (1), M. Zahariev (2), M. Liu (1), S. Hambleton (1), K. Seifert (1), C. A. Levesque (1), W. Chen (1)

(1) Agriculture and Agri-Food Canada, Ottawa, ON, Canada; (2) Skwez Technology Corp, Garibaldi Highlands, BC, Canada Phytopathology 105(Suppl. 4):S4.85

The spread of plant pathogens poses a significant risk to the agricultural sector. The use of amplicon-based metagenomics (ABM) for monitoring microbial communities, particularly for the presence of pathogenic or regulated species, has grown with the decreasing cost of next generation sequencing (NGS). Algorithms for sequence identification, such as BLAST and RDP classifier, implemented in the community accepted tools Qiime and Mothur rely on sequence similarity and therefore are suitable for biodiversity studies at higher taxonomic ranks but may lack the resolution to identify sequences at or below the species rank. The differentiation of species or subspecies is critical for the accurate detection of pathogens in food and crops. We developed an Automated Oligonucleotide Design Pipeline (AODP) that designs signature oligonucleotides (SO) with specificity and fidelity for members of a specified taxonomic group of any rank. SO for a taxon of interest can then be used to probe a NGS dataset in silico to improve the sequence identification at species or subspecies levels using the Oligo Fishing Pipeline (OFP). As such, AODP-OFP serves as a method for detecting and monitoring the distribution of targeted pathogens by improving the species/subspecies-level identification of NGS data. We demonstrate this proof of concept using agri-environmental surveys of fungal pathogens.

Identification of candidate effector proteins potentially involved in Fusarium graminearum-wheat interactions

S. LU (1), M. C. Edwards (1)

(1) USDA-ARS Cereal Crops Research Unit, Fargo, ND, U.S.A. Phytopathology 105(Suppl. 4):S4.85

Pathogen-derived small secreted cysteine-rich proteins (SSCPs) are known to be a common source of fungal effectors that trigger resistance or susceptibility in specific host plants. This group of proteins has not been well studied in Fusarium graminearum, the primary cause of Fusarium head blight (FHB), a 
devastating disease of wheat. Here we report the identification of candidate effectors from this fungus through a culture filtrate-based approach. Fungal mycelia were grown in liquid medium under nitrogen starvation mimicking pathogenesis conditions. Total secreted proteins were isolated from the culture filtrates and subjected to SDS-PAGE analysis which detected multiple species of proteins with molecular masses ranging from 10-150 kDa. Subsequent shotgun mass spectrometry and database searches identified a total of 153 secreted proteins, including 15 with characteristics of SSCPs $(<200$ amino acids, Cys content $\geq 2 \%$ ). Sequence analysis indicated that the 15 extracellular SSCPs ( $10 \%$ of the total SSCPs encoded in the $F$. graminearum genome) have no predicted functions and 10 of them appear to be unique to $F$. graminearum or species within the same genus. RT-PCR analysis confirmed that eight SSCPs are expressed in infected wheat heads, half of which have expression levels that correlate with the development of FHB symptoms. Gene knockout experiments are underway to determine the potential roles of these candidate effector proteins in F. graminearum-wheat interactions.

\section{A ToxA-like protein from Cochliobolus heterostrophus induces light-} dependent leaf necrosis with host selectivity on maize

S. LU (1), M. C. Edwards (1)

(1) USDA-ARS Cereal Crops Research Unit, Fargo, ND, U.S.A.

Phytopathology 105(Suppl. 4):S4.86

ToxA, the first discovered fungal proteinaceous host-selective toxin (HST), was originally identified from Pyrenophora tritici-repentis (Ptr), and its homologues have not been identified from any other ascomycetes except Parastagonospora nodorum. Here we report the identification of a ToxA-like protein from the maize pathogen Cochliobolus heterostrophus (Ch). ChToxA is encoded by a 535-bp open reading frame featuring a ToxA-specific intron with unusual splicing sites at conserved positions relative to PtrToxA. The protein shows $64 \%$ similarity to Ptr ToxA and is predicted to adopt a ToxAlike three-dimensional structure, although lacking the arginyl-glycyl-aspartic acid (RGD) motif. Reverse-transcriptase PCR revealed that the ChToxA gene is expressed at low levels in fungal cultures, but is up-regulated during fungal pathogenesis. Plant assays indicated that the recombinant ChToxA protein induces light-dependent leaf necrosis in a host-selective manner on maize inbred lines. Gene knockout experiments confirmed that chtoxa null mutants sustain reduced virulence on specific ToxA-sensitive maize lines. Database searches identified potential ChToxA homologues in other plant-pathogenic ascomycetes. These results suggest that $C$. heterostrophus is capable of producing proteinaceous HSTs in addition to virulence-related secondary metabolites. Further studies on ChToxA may provide new insights into effector evolution in host-pathogen interactions.

Evaluation of plant hormones and starter fertilizers on plant development in the presence of $M$. incognita or R. reniformis

J. A. LUANGKHOT (1), K. S. Lawrence (2), A. L. Smith (2)

(1) Auburn Univ, Auburn, AL, U.S.A.; (2) Auburn University, Auburn, AL, U.S.A.

Phytopathology 105(Suppl. 4):S4.86

The purpose of this project is to develop a system incorporating starter fertilizers and plant hormones to enhance cotton plant development in rootknot (Meloidogyne incognita) and reniform (Rotylenchulus reniformis) infested fields. Greenhouse trials were conducted to screen starter fertilizers and plant hormones to determine their effect on the nematode populations and plant development. The starter fertilizers with $M$. incognita found the phosphorus fertilizer (2-1-6) had $(P>0.05)$ higher numbers of $M$. incognita eggs than the control. No differences were detected among fertilizers on cotton with $R$. reniformis. The hormone blend (cytokinin, gibberellic acid, IBA) was tested by application methods. The hormone foliar spray supported higher $(P>0.05) R$. reniformis nematodes than all other hormone application methods. The methods, seed treatment + foliar spray, seed treatment + infurrow spray, and a seed treatment alone increased $(P>0.05)$ shoot fresh weight and the seed treatment + foliar spray increased root fresh weight. $M$. incognita responded to the in-furrow spray with increased $(P>0.05)$ egg populations, but all other methods were similar to the control. A combination of seed treatment + in-furrow + foliar spray had $(P>0.05)$ higher root fresh weights. Starter fertilizers and plant growth hormones are a way to increase plant mass and to promote an overall healthy plant stand. It should be considered that the larger plant may also support more nematodes.

\section{Evaluating peanut varieties for resistance to Sclerotinia sclerotiorum} P. LUJAN (1), S. Sanogo (2), N. Puppala (3)

(1) New Mexico State Univ, Las Cruces, NM, U.S.A.; (2) New Mexico State University, Las Cruces, NM, U.S.A.; (3) New Mexico State University, Clovis, NM, U.S.A.

Phytopathology 105(Suppl. 4):S4.86
Sclerotinia sclerotiorum is a fungal plant pathogen that affects over 400 plant species worldwide, including many economically important crops. $S$. sclerotiorum has been reported on Valencia peanut in New Mexico and Texas. Control of this pathogen with fungicides can be both expensive and not effective due to high disease pressure, thus the need for development of resistant varieties. This study was conducted to assess which varieties of peanut show resistance or partial resistance to isolates of $S$. sclerotiorum found in NM. Seeds from 30 peanut varieties belonging to 4 market groups were obtained from the NMSU Peanut Breeding Program and grown in a $25^{\circ} \mathrm{C}$ growth chamber. Once plants reached the 3 to 5 fully expanded leaf stage, they were inoculated with two $1-\mathrm{cm}$ mycelium plugs of $S$. sclerotiorum which were placed on the cotyledonary axils of each plant. Inoculated plants were then placed in a humidity box and maintained in a $20^{\circ} \mathrm{C}$ growth chamber. Plants were watered as needed and monitored daily for symptoms or signs of infection. Symptoms of infection occurred within one week postinoculation on several varieties. Two weeks following inoculation, all varieties were dead with the exception of one experimental line, which showed no symptoms or signs of infection. These results suggest that this experimental line can be used in peanut breeding program to develop varieties resistant to $S$. sclerotiorum.

\section{Azoxystrobin-resistant Rhizoctonia solani on rice in southwestern Louisiana \\ A. LUNOS (1), C. Hollier (1)}

(1) LSU Dept. of Plant Pathology \& Crop Physiology, Baton Rouge, LA, U.S.A.

Phytopathology 105(Suppl. 4):S4.86

Fungicide resistance is a growing problem within many pathosystems, including sheath blight (Rhizoctonia solani AG I-IA) of rice. In 2011, strobilurin fungicide resistance was confirmed in Louisiana's southwestern rice growing area. This study will determine fungicide sensitivity of $R$. solani isolates collected from 45 commercial rice fields across southwestern Louisiana. Rhizoctonia solani was isolated from symptomatic rice tissue then frozen on rye seed at $-20 \mathrm{C}$. Potato dextrose agar plates amended with serial dilutions of azoxystrobin from 0.005 to $10 \mathrm{ug} / \mathrm{ml}$ were used to determine the effective fungicide concentration that inhibits mycelial growth to $50 \%$ of the unamended control (EC50). The isolates were inoculated onto the amended plates and incubated for 28 hours at $25 \mathrm{C}$. Colony area was measured from digital images using Assess 2.0 software, adjusted to percent of the unamended control plate, and fitted to a dose response curve using GraphPad Prism 6 software. While EC50's tested so far were not significantly different from each other, they did serve to group the isolates into sensitivity categories. Two isolates were grouped with the resistant control (EC50's 0.503-0.540), three isolates with the sensitive control (0.006-0.037), and three intermediate (0.069-0.187 ug azoxystrobin $/ \mathrm{ml})$. This geographic survey will identify problem areas in southwestern Louisiana where alternative sheath blight management techniques will need to be applied.

Improved residential citrus host mapping and its potential influence on Asian Citrus Psyllid (ACP) population

W. LUO (1), T. Gottwald (2), G. McCollum (3)

(1) USDA ARS, Ft Pierce, FL, U.S.A.; (2) U.S. Horticultural Research Laboratory, USDA ARS, Fort Pierce, FL, U.S.A.; (3) USDA ARS, Fort Pierce, FL, U.S.A

Phytopathology 105(Suppl. 4):S4.86

Linking residential citrus cultivar/species preference to distribution is a challenging new area of host density mapping and modeling. Dooryard citrus preferences are heterogeneous and far from random. In addition to local climatic and environmental factors, we postulate that a range of demographic and socioeconomic factors can also affect the residential preferences for citrus types. Based on residential citrus huanglongbing (HLB) /ACP survey between 2013 and 2015, hundreds of thousands properties with dooryard citrus in seven counties of southern California was visited. Dooryard citrus tree distribution and associated ACP population counts were observed for each property. Principle component analysis was firstly used to characterize the variation of dooryard citrus distribution. Subsequently, we evaluated the relationship between demographic and socioeconomic factors and citrus tree preference using generalized linear model regression where statistically appropriate, and evaluated the fit of the relationship to determine the degree to which variable has significant association with preferences. Lastly, we summarized the spatio-temporal pattern of ACP finds in each citrus host type, and quantified the effect of citrus host on ACP tolerance. Improved residential citrus host mapping is a necessary step towards a comprehensive understanding of the ACP spread in residential areas and its influence on HLB epidemics. 
A mathematical model for the dynamics of Asian Citrus Psyllid (ACP) spread in different landscapes

W. LUO (1), T. Gottwald (2)

(1) USDA ARS, Ft Pierce, FL, U.S.A.; (2) U.S. Horticultural Research Laboratory, USDA ARS, Fort Pierce, FL, U.S.A.

Phytopathology 105(Suppl. 4):S4.87

The recent discovery of ACP populations in the California Central Valley increases the potential for HLB establishment. A spatially explicit ACP simulation model was developed in an attempt to understand the dynamics of ACP population changes, in particular, how ACP spreads in a mixture of residential and commercial citrus landscapes in the Central Valley. A 16 mile $^{2}$ area of Porterville, CA was used as the universe for ACP spread simulation. The mathematical model considers the following parameters for ACP progression: psyllid life span and mortality, net psyllid reproduction rate in natural environment, psyllid dispersal distance, citrus host type and density, new flush events, and the effect of different spray schemes. An initial ACP population was introduced in one/multiple locations before the simulation. We then modeled factors influencing ACP population variation and interaction with new flush availability in relation to temperature (e.g. seasonal effect) for a period of 3 years using a daily time step. ACP spread occured more frequently and spread was more rapid within commercial citrus clusters, but comparatively slower for low density or well separated residential areas. This study also evaluated the behavior and distribution of ACP populations subjected to different spray strategy scenarios for management/decisionmaking. A comparison between simulation outputs confirmed that coordinated synchronized sprays slowed ACP population development.

\section{Impact of postharvest practices on avocado contamination by Listeria} monocytogenes

D. MACARISIN (1), P. Evans (2), Y. Chen (2)

(1) U.S. Food and Drug Administration, College Park, MD, U.S.A.; (2) Center for Food Safety and Applied Nutrition, U.S. Food and Drug Administratio, College Park, MD, U.S.A.

Phytopathology 105(Suppl. 4):S4.87

Foodborne listeriosis has been commonly associated with the consumption of ready-to-eat meat and dairy products; however, tree fruits emerged as a new concern for Listeria monocytogenes contamination. In the 2014-2015 listeriosis outbreak associated with caramel apples, clinical isolates of $L$. monocytogenes were traced back to the fruit processing facility, indicating that fruits were contaminated during post-harvest processing. In 2014, FDA initiated a surveillance sampling of import and domestic whole avocados and identified L. monocytogenes contamination of skin and pulp in whole avocados. We investigated whether the L. monocytogenes on the fruit surface can infiltrate into the pulp during post-harvest handling. Surface of intact avocados was either spot-inoculated with or fruits were hydrocooled in a $L$. monocytogenes suspension. Incidence of L. monocytogenes internalization into intact avocados was $60 \%$ after spot-inoculation of stem scars and $100 \%$ after hydrocooling in contaminated water. Bacteria infiltration primarily took place through the stem or stem scar and then distributed within the mesocarp, in some instances reaching the bottom-end of the fruit. Populations of internalized L. monocytogenes colonized edible portions of the fruit reaching up to $7 \log \mathrm{CFU} \mathrm{\textrm {g } ^ { - 1 }}$ within 2 weeks after hydrocooling at a storage temperature of $3^{\circ} \mathrm{C}$. These studies will help to define contamination risks associated with current post-harvest practices in the production of avocados.

\section{Biological and genetic characterization of Botrytis cinerea isolates from a} commercial packinghouse in Pennsylvania

O. MACARISIN (1), V. L. Gaskins (1), J. Yu (1), W. M. Jurick II (1)

(1) USDA ARS - Food Quality Laboratory, Beltsville, MD, U.S.A.

Phytopathology 105(Suppl. 4):S4.87

The Unites States is the second largest producer of apples worldwide behind China, and is a multi-billion dollar industry. Gray mold, caused by the filamentous fungus, Botrytis cinerea, is one of the most devastating postharvest apple pathogens causing losses as high as $60 \%$ in storage. Fungicides are the primary method of control as host resistance to this fungus is lacking in commercial apple cultivars. Eight single spore $B$. cinerea isolates from decayed 'Fuji' and 'Gala' apples were obtained from a commercial packing facility with a history of postharvest fungicide use. Conventional Polymerase Chain Reaction (PCR) was used to amplify 4 different loci (Mrr1, G3PDH, HSP60, RPB2) from each isolate. The amplicons were sequenced directly and the $2 \mathrm{X}$ consensus nucleotide sequences were subjected to MegaBLAST analysis. Mycelial growth rate was conducted in vitro using different media (Potato Dextrose Agar, Malt Extract Agar and Tryptic Soy Agar) to optimize growth conditions and conidial production. Discriminatory doses of several technical grade postharvest fungicides will be analyzed to determine if shifts in baseline sensitivity has occurred. Data from this study will help identify if fungicide resistant $B$. cinerea strains have emerged and may enable scientists to develop strategies to prevent them from developing in the packinghouse and or during long-term storage.

Incidence of begomovirus and tospovirus diseases in processing tomato fields in three midwest states of Brazil in 2013 and 2014

M. MACEDO (1), R. Gilbertson (1), A. Bergamin Filho (2)

(1) Universidad of Davis, Davis, CA, U.S.A.; (2) Escola Superior de Agronomia Luiz de Queiroz, Piracicaba, Brazil

Phytopathology 105(Suppl. 4):S4.87

Begomoviruses and tospoviruses and are currently the main viruses that affect tomato production in Brazil. The objective of this study was to monitor the incidence of begomovirus and tospovirus diseases at different time-points at five locations in the states of Goiás (Luziania, Itaberaí, Morrinhos), Minas Gerais (Patos de Minas) and São Paulo (Guaira). In 2013, the evaluation was done at 60 and 80 days after transplanting (DAT) in three center pivotirrigated tomato fields for each location. In 2014, an additional time-point, 2030 DAT, was added. In each field, 100 plants at three points were randomly evaluated for begomovirus and tospovirus infection (visually). The incidence of begomovirus was higher in 2013 (4.6-89.3\%) than in 2014 (2.3-51.9\%), with the highest incidence in Goiás for both years. At 20-30 DAT (2014), begomovirus incidence varied from $8.0-20.0 \%$ in Goiás and $0.8-1.0 \%$ in the other two states. Interestingly, the incidence of begomovirus appeared to decrease in the middle of season and then increase at the end of the season. The incidence of tospovirus increased over time, but was low for both years: $0.03-1.6 \%$ in 2013 and $0.3-4.0 \%$ in 2014 . The highest incidence was in Guaíra. At 20-30 DAT (2014), the incidence of tospovirus ranged from 0.2 to $1.2 \%$. In conclusion, the begomovirus incidence varied depending on the production region, whereas the tospovirus incidence was low. The monitoring will be continued for one more year to confirm these results.

Populations of Fusarium oxysporum f. sp. cubense causing Panama disease of banana in Ecuador: Learning from the past for future perspectives

F. A. MAGDAMA (1), M. Jimenez-Gasco (1)

(1) The Pennsylvania State University, University Park, PA, U.S.A.

Phytopathology 105(Suppl. 4):S4.87

Despite Ecuador's leadership on banana production worldwide for export trade, there is still a lack of understanding about the status of Panama disease in the country. While current production relies on the resistant variety 'Cavendish', smallholders rely on susceptible bananas for local consumption and continue to be affected by Fusarium oxysporum f. sp. cubense (Foc) that caused the demise of 'Gros Michel'. We used a multi-locus sequence method based on the translation elongation factor 1-alpha gene (TEF) and the intergenic spacer region of the ribosomal DNA (IGS), to characterize 300 isolates of Foc obtained from symptomatic 'Gros Michel' plants from the main banana-producing areas of Ecuador, as well as some $F$. oxysporum root endophytes. Our phylogenetic analyses placed all Foc isolates in a single clade associated to VCG0120. The presence of only one mating type (MAT1-2) confirms the clonal dispersion of the fungus within the country and its introduction from a common place of origin. In addition, a positive PCR reaction using specific primers for detection of Tropical race 4 (TR4), corresponding pathogenicity and VCG analyses, revealed the presence of an endophitic non-pathogenic isolate of banana sharing genomic regions similar to those of TR4. This work provides new insights on the reliability and specificity of current quarantine-detection methods for TR4 and proposes the study of banana endophytes and their role in the emergence of pathogenicity.

An Argonaute sequence within the Iranian Beet black scorch virus satellite RNA

M. MAHILLON (1), C. Bragard (2), M. Mehrvar (3)

(1) Applied microbiology, Earth \& Life Institute, Université catholique de Louvain, Croix du Sud, 2 bte L7.05.03. 1348 Louvain-la-Neuve, Belgium, Louvain-la-Neuve, Belgium; (2) Applied microbiology, Earth \& Life Institute, Université catholique de Louvain, Croix du Sud, 2 bte L7.05.03. 1348 Louvain-la-Neuve, Belgium, Louvain-la-Neuve, Belgium; (3) Department of Plant Protection, School of Agriculture, Ferdowsi University of Mashhad, Mashhad, Iran, Mashad, Iran

Phytopathology 105(Suppl. 4):S4.87

Beet black scorch virus (BBSV) is a soil-borne Necrovirus found on sugar beet in China, Spain, USA, California and more recently in Iran. The virus is sometimes associated with a satellite RNA, found in China and Iran. Over 200 samples gathered in Iranian sugar beet fields have been analyzed for the presence of BBSV and its satellite, hence revealing a wide diversity. 61 different Iranian BBSB sources have been recorded, a large number of which were associated with the satellite. The whole genome of BBSV isolates and 
satellites from several Iranian sugar beet growing areas have been sequenced. Full length clones of BBSV were generated by simply amplifying the viral genome before cloning into the pDrive vector (Qiagen) before generating infectious RNA transcripts. Comparison of the virus sequences stress the numerous changes in the 3 ' untranslated terminal region at the nucleotide level, yet with limited impact on the structure. A sequence homologous to Argonaute was found lying within the satellite RNA. This finding raises the possibility that the satellite is acting as a suppressor of gene silencing, by targeting RNAi itself. As for other Tombusviridae, such finding stimulates interest in a better understanding of the intriguing interaction between the virus and its satellite RNA.

\section{Comparative genomics-based development of a LAMP detection assay for} boxwood blight

M. MALAPI-WIGHT (1), J. Demers (1), D. Veltri (2), J. A. Crouch (1)

(1) USDA ARS, Beltsville, MD, U.S.A.; (2) Rutgers University, New Brunswick, NJ, U.S.A.

Phytopathology 105(Suppl. 4):S4.88

Calonectria pseudonaviculata is an emergent pathogen in the U.S. and one of the causal agents of the boxwood blight disease. The fungus was first reported in the U.S. in 2011 and rapidly spread to 10 additional states and three Canadian provinces. A second causal fungus, $C$. henricotiae, has not yet been reported in North America. Affected plants show necrotic spots on leaves, followed by defoliation, severe dieback and eventual plant death. The main challenges for the diagnosis of these pathogens are 1) the ubiquitous nature of the Calonectria species in soil and plants; 2) the possibility of latent infections; and 3) the unavailability of Calonectria genome sequences for identification of specific DNA markers. In this study, we sequenced and assembled draft genomes of the two boxwood blight fungi and three species of the Nectriaceae to identify novel and specific regions for the diagnosis of these pathogens. Based on different comparative genomic-based approaches, three LAMP assays were developed. The assays identified all $C$. pseudonaviculata and $C$. henricotiae isolates tested from representative genotypes from as little as $100 \mathrm{pg}$ of DNA. No cross-reactions were observed with 11 additional Calonectria spp. and from other common fungal species and soil samples present in boxwood environments. The assays developed in this study will provide useful tools for monitoring and development of mitigation strategies towards the control of the boxwood blight fungi in the U.S.

\section{Conserved nematode signaling molecules elicit plant defenses and disease} resistance

M. MANOHAR (1), P. Manosalva (2), S. H. von Reuss (1), S. Chen (3), A. Koch (4), X. Wang (3), K. H. Kogel (4), P. W. Sternberg (5), V. M. Williamson (6), F. C. Schroeder (1), D. F. Klessig (1)

(1) Boyce Thompson Institute for Plant Research, Cornell University, Ithaca, NY, U.S.A.; (2) University of California, Riverside, Riverside, CA, U.S.A.; (3) Cornell University, Ithaca, NY, U.S.A.; (4) Justus Liebig University, Giessen, Germany; (5) California Institute of Technology, Pasadena, CA, U.S.A.; (6) University of California, Davis, CA, U.S.A.

Phytopathology 105(Suppl. 4):S4.88

Plant defense responses against pathogens are triggered by perception of conserved microbe-associated molecular patterns (MAMPs). However, it remained unknown whether plants are able to detect conserved molecular patterns derived from nematodes, which likely represent the most abundant animals on earth. Here we show that plant parasitic nematodes produce small molecules called ascarosides (ascr), an evolutionarily conserved family of nematode pheromones, and that plants respond to these molecules by activating systemic defenses against a broad spectrum of pathogens. Low concentrations of ascr\#18 $(1 \mathrm{nM}-1 \mu \mathrm{M})$, the most abundant ascaroside in plant parasitic nematodes, induce hallmark of defense responses in plants. Ascr\#18 induced both salicylic acid- and jasmonic acid-mediated defense signaling pathways and enhanced resistance to virus, bacteria and nematode in Arabidopsis. Furthermore, we show that ascr\#18 perception via roots or leaves increases resistance in tomato, potato, and barley to foliar bacterial, oomycete, or fungal pathogens. Our results indicate that monocots and dicots recognize ascarosides as a conserved molecular signature of nematodes that triggers conserved plant defense signaling pathways, similar to perception of MAMPs. Using potent small-molecule signals such as ascarosides to activate plant immune systems and protect plants against pathogens and pests has potential to improve the economic and environmental sustainability of agriculture.

Frogeye leaf spot management and the impact of fungicide phytotoxicity in Mississippi soybean

W. J. MANSOUR (1), T. H. Wilkerson (1), J. T. Irby (2), B. R. Golden (1), T. W. Allen (3)
(1) Mississippi State University, Stoneville, MS, U.S.A.; (2) Mississippi State University, Starkville, MS, U.S.A.; (3) Mississippi State Univ, Stoneville, MS, U.S.A.

Phytopathology 105(Suppl. 4):S4.88

Frogeye leaf spot (FLS), caused by Cercospora sojina Hara, has become a major foliar disease in the Mississippi soybean production system over the past three years. With the observation of quinone outside inhibitor (QoI) fungicide resistance in the $C$. sojina population throughout MS, alternative fungicide management practices need to be explored to effectively manage FLS in susceptible soybean varieties. Trials were conducted during 2013 and 2014 to determine fungicide efficacy on QoI-resistant FLS. Several different fungicide modes of action were considered alone and in combination at different application-timing strategies (either R2, R3, R5, or R5.5) in a FLSsusceptible variety. Observations were made pre- and several times postapplication on the presence and severity of FLS and more specifically phytotoxicity as a result of specific fungicide products. Fungicide products that contained demethylation inhibitors (DMI) significantly reduced FLS severity, by as much as $21 \%$, while significantly increasing yield by as much as $17.6 \%$ over the nontreated. However, DMI products that contained prothioconazole produced a significant amount of phytotoxicity. In some cases, the phytotoxicity as a result of prothioconazole was present in $70 \%$ of the leaf area within a plot compared to flutriafol and tetraconazole that produced minimal phytotoxicity, on the order of less than $2 \%$. However, yield was not determined to be reduced as a result of the fungicide phytotoxicity.

\section{Molecular and functional characterization of the movement protein of} Ourmia melon virus

P. MARGARIA (1), C. T. Anderson (1), M. Turina (2), C. Rosa (1)

(1) The Pennsylvania State University, University Park, PA, U.S.A.; (2) CNR, Torino, Italy

Phytopathology 105(Suppl. 4):S4.88

Ourmia melon virus (OuMV) has a genome composed of three +ssRNA molecules, each coding for a single protein. Our work focuses on the RNA2encoded movement protein (MP). We selected 11 amino acids for alanine scanning mutagenesis, either conserved in the MP of ourmia-, tombus- and aureoviruses (all phylogenetically distantly related), or charged and solventexposed. We infected Nicotiana benthamiana via agroinfiltration with OuMV infectious clones carrying the desired mutations. Viral movement was impaired for five of the mutants: the MP (and CP) were detected by Western blot analysis at the site of agroinfiltration for all the mutants, whereas no MP (or CP) was present in systemic tissues of the same plants or in new plants inoculated with tissues from the agroinfiltated area, suggesting that these substitutions impaired both systemic and local movement. EGFP-wtMP fusion was expressed through agroinfiltration in puntate spots at the cell periphery of infected N. benthamiana and Arabidopsis thaliana Col 0 and leaf staining with propidium iodide and aniline blue showed that the wt MP co-localizes at the cell wall, with plasmodesmata. So far, three eGFP-mutant MPs did not localize in foci. Isolated protoplasts infected with eGFP-mutant MPs showed absence of the tubule protrusions from the plasma membrane, typical of cells infected with eGFP-wtMP. We are currently using Arabidopsis mutants to test the requirements of specific cellular components for viral movement.

Is there local adaptation to temperature in Phytophthora infestans?

N. MARIETTE (1), A. Androdias (1), R. Mabon (1), R. Corbière (1), J. Montarry (1), D. Andrivon (1)

(1) INRA IGEPP, Le Rheu, France

Phytopathology 105(Suppl. 4):S4.88

As in several biotrophic parasites, the infectious cycle of the oomycete causing potato late blight, Phytophthora infestans, is highly dependent on climate and particularly temperature. In order to test the hypothesis of a local adaptation to temperature, we have compared the performances at four constant temperatures $\left(10^{\circ} \mathrm{C}, 14^{\circ} \mathrm{C}, 18^{\circ} \mathrm{C}\right.$ and $\left.24^{\circ} \mathrm{C}\right)$ of isolates collected in three geographical areas: Western Europe, Northern Europe and Mediterranean basin. Three traits linked to the parasite's infectious cycle were measured on the potato cultivar Bintje: latent period (LP), lesion growth rate (LGR) and sporulation capacity (SC). Isolates from Northern Europe had a significantly higher $\mathrm{SC}$ than others at $10^{\circ} \mathrm{C}$ while the opposite was observed at $18^{\circ} \mathrm{C}$, consistent with local adaptation to lower temperatures. Nevertheless, for the other traits, isolates from Northern Europe had lower performances at all temperatures tested. These results may reflect the need for sexual oospores to survive the harsh winters in Northern Europe. As P. infestans is a heterothallic species, a long LP and a slow LGR should favor the probability for multiple infections and the chance for mating. By contrast, as the formation of oospores is not needed for the inter-epidemic survival of $P$. infestans in Western Europe and in the Mediterranean basin, isolates colonizing their host rapidly (short LP and high LGR) would be selected there. 
Disease detection in hop rhizomes and plantlets to ensure clean yards in Wisconsin

M. E. MARKS (1), A. P. Geske (2), A. J. Gevens (2)

(1) Univ of Wisconsin, Madison, WI, U.S.A.; (2) University of Wisconsin, Madison, WI, U.S.A.

Phytopathology 105(Suppl. 4):S4.89

Hop (Humulus lupulus) production is expanding in Wisconsin. To ensure sustainability of production, it is critical that growers use pathogen-free plantlets or rhizomes to establish new hop yards. Diseases that can be harbored in plants include downy mildew (Pseudoperonospora humuli), powdery mildew (Podosphaera macularis), and several viruses. We partnered with three commercial hop producers to support their interests in identifying disease and excluding it from new yards. Our disease screen included microscopic analyses of plant tissues for downy and powdery mildew, in addition to PCRand ELISA-based tests for downy mildew, Arabis mosaic virus (ArMV), Apple mosaic virus (ApMV), Cucumber mosaic virus (CMV), and carlaviruses. From 10 Dec 2014 to 9 Mar 2015 we received 29 samples comprised of 8 cultigens. All plant tissues were asymptomatic upon receipt and were stored at $4^{\circ} \mathrm{C}$ until processed. Tests were completed with an average turnaround time of 8 days. All samples tested negative for powdery mildew, ArMV and CMV. One sample was positive for ApMV; 3 samples (10\%) comprised of 3 cultigens from a single producer were positive for downy mildew. Of predominance, 7 samples (24\%) comprised of 6 cultigens from all collaborators were positive for Carlavirus (non-specific). The detection of ApMV, Carlavirus, and downy mildew reinforces the need for continued and more extensive disease screening of hop propagative material prior to new yard establishment.

\section{Role of GATA transcription factors during infection of the rice blast fungus Magnaporthe oryzae}

M. MARROQUIN-GUZMAN (1), R. A. Wilson (2)

(1) Univ of Nebraska, Lincoln, NE, U.S.A.; (2) University of NebraskaLincoln, Lincoln, NE, U.S.A.

Phytopathology 105(Suppl. 4):S4.89

Magnaporthe oryzae is the most destructive rice pathogen worldwide and one of the most important hemibiotrophic microorganisms. During infection, sensing of Glucose 6-Phosphate (G6P) availability by Tps1 initiates fungal development in the plant. Subsequently, NADPH levels increase in response to G6P in a Tps1-dependent manner, which among other outcomes, results in the activation of Nut1 and Asd4, two members of the GATA family transcription factors. Here we attempted to identify the role of these GATA factors during rice blast infection. Using molecular genetics and biochemical approaches, we demonstrate that Asd4 controls the expression of a cryptic glutamine synthetase-encoding gene $(G L N 1)$ to modulate intracellular glutamine levels and inactivate TOR, in order to promote appressoria formation. Moreover, our results reveal that the nitrogen regulator Nut1 is needed in $M$. oryzae for expressing genes to assimilate nitrogen under starvation conditions and is needed for in planta growth but not appressoria formation. Taken together, the regulation of nitrogen metabolism by the GATA factors Asd4 and Nut1 play a critical role during different stages of rice blast infection. In addition, we describe the first report indicating that TOR is a negative-acting regulator of appressoria formation in M. oryzae, thus adding to our knowledge about how these specialized infection cells develop.

Population structure and host preference of Macrophomina phaseolina on strawberry

F. N. MARTIN (1), S. T. Koike (2), R. Arias (3), M. L. Ramon (4)

(1) USDA ARS, Salinas, CA, U.S.A.; (2) Cooperative Extension Monterey County, Salinas, CA, U.S.A.; (3) USDA-ARS, Dawson, GA, U.S.A.; (4) USDA ARS, Salinas, CA, U.S.A.

Phytopathology 105(Suppl. 4):S4.89

In recent years $M$. phaseolina has become an emerging disease problem in California strawberry production as preplant soil fumigation practices have shifted from traditional broadcast treatments to bed fumigation, causing serious losses in all production districts. A culture collection was established with isolates representing all strawberry production areas as well as other hosts in California. Pathogenicity tests have demonstrated a host preference, with isolates recovered from strawberry being highly aggressive on this host but having minimal impact on other hosts tested. Conversely, isolates from other hosts had limited impact on strawberry. Population analysis using SSR markers revealed a lineage of strawberry isolates on a separate clade from isolates recovered from other hosts in California. There were two exceptions to this grouping, one strawberry isolate grouped with isolates recovered from other hosts from the San Joaquin Valley (SJV) and a cantaloupe isolate from the SJV grouped with the strawberry isolates. More detailed pathogenicity and virulence assessments on strawberry are in progress with these isolates to clarify the relationship between genotype and virulence on this host.

\section{Subcellular localization and characterization of proteins of Maize mosaic} virus

K. M. MARTIN (1), M. M. Goodin (2), A. E. Whitfield (1)

(1) Kansas State University, Manhattan, KS, U.S.A.; (2) University of Kentucky, Lexington, KY, U.S.A.

Phytopathology 105(Suppl. 4):S4.89

Maize mosaic virus (MMV) infects corn and sorghum and is vectored by the corn planthopper, Peregrinus maidis. MMV is a Nucleorhabdovirus in the family Rhabdoviridae with the gene order: nucleoprotein $(\mathrm{N})$, phosphoprotein $(\mathrm{P})$, putative movement protein (3), matrix protein $(\mathrm{M})$, glycoprotein $(\mathrm{G})$ and polymerase (L). In silico analyses of all proteins determined that $\mathrm{N}$ contains a non-traditional nuclear localization signal (NLS); P, G and L have predicted NLSs but $M$ and 3 do not contain an NLS. In this study, we fused MMV proteins to the green fluorescent protein (GFP) and agroinfiltrated them into Nicotiana benthamiana. Confocal fluorescence microscopy revealed that when individually expressed, $\mathrm{N}$ localizes to the nucleus and also the cell periphery. $\mathrm{P}$ is strictly nuclear, whereas, 3 localizes to the cell periphery with little nuclear localization. M localizes outside of the nucleus and $\mathrm{G}$ localizes to the membranes surrounding the nucleus. When expressed in the presence of other viral proteins, $\mathrm{M}$ is able to enter the nucleus. Taken together, these localization data are consistent with the rhabdovirus model of replication, wherein; N, P, L and viral RNA form the viral RNP complex in the nucleus that associates with $M$ and then $G$ as the virion buds into the perinuclear space. Lastly, 3 then facilitates the movement of the virus out of the cell. These results suggest a complex interplay of rhabdoviral proteins to facilitate replication and systemic infection.

\section{Differential gene expression in Peregrinus maidis after infection with} Maize mosaic virus

K. M. MARTIN (1), K. Barandoc-Alviar (1), D. Rotenberg (1), A. E. Whitfield (1) (1) Kansas State University, Manhattan, KS, U.S.A.

Phytopathology 105(Suppl. 4):S4.89

Maize mosaic virus (MMV), a Nucleorhabdovirus, is a pathogen of corn and sorghum and is vectored by the corn planthopper, Peregrinus maidis. MMV is transmitted by $P$. maidis in a persistent, propagative manner, yet little is known about how virus infection occurs and how insect cells respond to infection. To address this, an RNAseq experiment with three biological replications was conducted to analyze global transcriptome changes in $P$. maidis adults exposed to MMV compared to non-exposed insects. Virus infection was confirmed in individual adults with endpoint RT-PCR, and realtime qRT-PCR was performed to quantify titer. RNAseq libraries were prepared from groups of pooled males and females with high titers and pools of non-exposed insects and multiplex-sequenced using Illumina Hi-Seq 2500 to generate single-end reads. Of the 68,003 de novo-assembled contigs, 144 were differentially-expressed; 77 were upregulated and 67 contigs were downregulated. Of the differentially-expressed contigs, 88 were provisionallyannotated using Blast2GO. Contigs associated with the nucleus were primarily upregulated and contigs categorized as integral components of membrane were predominantly down-regulated. Our findings support the hypothesis that MMV infection perturbs host genes associated with the nucleus and membrane composition, which is consistent with the current model for plant and insect infection by a Nucleorhabdovirus.

Role of lutein in Fusarium head blight resistance in the DH population Toronit x ACW211.12014

C. Martin (1), J. Dougoud (1), V. Susanne (2), B. Mauch-Mani (3), F. MASCHER (1)

(1) Agroscope IPV, Nyon, Switzerland; (2) Agroscope IDU, Zürich, Switzerland; (3) University of Neuchatel, Neuchatel, Switzerland

Phytopathology 105(Suppl. 4):S4.89

In wheat, Fusarium head blight (FHB) can cause severe yield losses, modifications in grain conformation and accumulation of mycotoxins. Several resistance mechanisms have been described to reduce the impact of the infection on different parts of the plant. Yet, resistance is always quantitative since no complete resistance has been described, to date. Plant secondary metabolites with antioxidant activity, such as phenolic acids, anthocyanins or carotenoids receive increasing attention for their role in resistance. The present work studies the role the role lutein, a carotenoid, on FHB resistance in grain and in the spike. The work based on the Toronit X ACW 211.12014 DH population $(\mathrm{n}=165)$, with Toronit as a lutein rich cultivar with moderate resistance and ACW 211.12014, a susceptible breeding line with a low lutein content. The population was screened in field and in greenhouse experiments with artificial infections, and disease symptoms and kernel resistance were 
assessed. Overall, the lutein content explained only very weakly the resistance of the spike in $\mathrm{cv}$. Toronit $(\mathrm{r}=0.13 ; \mathrm{p}<0.02)$. Yet, the content of lutein showed a negative significant correlation with the proportion of kernels damaged by the infection (FDK) $(\mathrm{r}=-0.23, \mathrm{p}<0.01)$. These results suggest that that lutein contributes to the resistance against kernel infection. Ongoing studies address the role of lutein in grains on accumulation of deoxynivalenol in kernels.

Identification of novel and diverse mycoviruses through metagenomic characterization of the viromes of five fungal pathogens of major crop plants

S. Y. L. MARZANO (1), B. D. Nelson (2), O. O. Ajayi (1), C. A. Bradley (1), T. J. Hughes (3), G. L. Hartman (4), K. N. Lambert (1), L. L. Domier (4)

(1) Department of Crop Sciences, University of Illinois, Urbana, IL, U.S.A.; (2) Department of Plant Pathology, North Dakota State University, Fargo, ND, U.S.A.; (3) Monsanto Co, Chesterfield, MO, U.S.A.; (4) USDAAgricultural Research Services, Department of Crop Sciences, University of Illinois, Urbana, IL, U.S.A.

Phytopathology 105(Suppl. 4):S4.90

Infection of plant pathogenic fungi by mycoviruses can attenuate their virulence on plants and vigor in culture. In this study, we described the viromes of 275 isolates of five widely dispersed plant pathogenic fungal species (Colletotrichum truncatum, Macrophomina phaseolina, Phomopsis longicolla, Rhizoctonia solani and Sclerotinia sclerotiorum) using a highthroughput sequencing-based metatranscriptomic approach. To characterize the viromes, sequence data derived from total RNA preparations were assembled de novo, and contigs with predicted amino acid sequence similarities to viruses in the non-redundant protein database were identified with BLASTX. The analysis identified partial or complete genome sequences of 63 mycoviruses, 48 of which had not been described previously. The novel mycoviruses showed affinity with 16 distinct lineages: Benyviridae, Bornaviridae, Bunyaviridae, Crysoviridae, Endornaviridae, Hypoviridae, Idaeovirus, Narnaviridae, Nyaviridae, Ophioviridae, Ourmiavirus, Partitiviridae, Rhabdoviridae, Tombusviridae, Totiviridae and Virgaviridae. Viruses were identified from families and genera that previously contained no fungalinfecting representatives, including Benyvirus, Bornaviridae, Idaeovirus, Ophiovirus, Ourmiavirus, Tombusviridae, and Tospovirus (Bunyaviridae). The metatranscriptomic screenings widened the range and diversity of mycoviruses and identified additional potential biological control agents.

Genotype-level interactions determine the degree of reduction of leaf rust on wheat by seed application of beneficial pseudomonads

F. MASCHER (1), A. Sharifi-Tehrani (2), S. Kellenberger (1), M. Farzaneh (2), M. Pechy-Tarr (3), C. Keel (3)

(1) Agroscope IPV, Nyon, Switzerland; (2) University of Tehran, Karaj, Iran; (3) University of Lausanne, Lausanne, Switzerland

Phytopathology 105(Suppl. 4):S4.90

Some bacteria have the capacity to reduce incidence and severity of plant diseases either by inhibiting the pathogen or by modulating the resistance response of the plant. Plants dispose of different resistance mechanisms that are influenced by the biotic and abiotic environment. The present experiments explored the effects of biocontrol strains of Pseudomonas fluorescens on the resistance of wheat varieties against brown rust disease caused by Puccinia triticina. Root inoculation with biocontrol pseudomonads reduced the disease severity on the leaves. The plant response depended on the genotype of both the microbes and the wheat varieties, suggesting a straight interaction at the molecular level.

\section{Genetic analysis of begomoviruses and betasatellites associated with} tomato leaf curl disease in Ghana

Y. J. MATER (1), L.-F. Chen (2), M. K. Osei (3), R. L. Gilbertson (4)

(1) University of California, Davis - Department of Plant Pathology, Davis, CA, U.S.A.; (2) University of California, Davis - Department of Plant Pathology, Davis, CA, U.S.A.; (3) CSIR-Crops Research Institute, Kumasi, Ghana; (4) University of California, Davis, Davis, CA, U.S.A.

Phytopathology 105(Suppl. 4):S4.90

Whitefly-transmitted geminiviruses (genus Begomovirus) are a constraint on tomato production in West Africa. In a survey of tomato fields in Ghana in 2012, samples with symptoms of begomovirus infection (leaf curl and yellow leaf curl) were collected. Twenty-three of 27 samples were positive for begomovirus infection. Results of PCR and sequencing revealed that 12 and 6 samples were infected with Tomato yellow leaf curl Mail virus (TYLCMLV) and Tomato leaf curl Ghana virus (ToLCGHV), respectively, which are known to occur in Ghana. The other five samples were infected with Tomato leaf curl Cameroon virus (ToLCCMV) or recombinant begomoviruses. Eight samples also were infected with betasatellites, and these were all variants of Tomato leaf curl Togo betasatellite (ToLCTGB). Selected begomoviruses and betasatellites were used to generate multimeric clones and infectivity was assessed. In Nicotiana benthamiana, ToLCGHV (AK2-5 and A3-5) and a Tomato leaf curl Kumasi virus recombinant (ToLCKuV-R, A4-2) induced upward leaf curling, vein enation and crumpling; ToLCCMV (T3-6) did not induce obvious symptoms. In tomato, ToLCGHV and ToLCKuV-R induced stunting, leaf curling and yellowing; ToLCCMV induced a symptomless infection. Co-inoculation of combinations of these begomoviruses and three ToLCTGB variants resulted in more severe symptoms, and revealed a differential interaction among the viruses and betasatellites.

Association of Diaporthe longicolla with zone lines on soybean stems and roots

F. M. MATHEW (1), A. Gebreil (1), K. A. Wise (2), C. A. Bradley (3), D. S. Mueller (4), M. I. Chilvers (5)

(1) South Dakota State University, Brookings, SD, U.S.A.; (2) Purdue University, West Lafayette, IN, U.S.A.; (3) University of Illinois, Urbana, IL, U.S.A.; (4) Iowa State University, Ames, IA, U.S.A.; (5) Michigan State University, East Lansing, MI, U.S.A.

Phytopathology 105(Suppl. 4):S4.90

In a late season survey of soybean diseases in Illinois, Indiana, Iowa, Michigan and South Dakota, black zone lines were observed on the lower, internal portions of the stems and roots. The surveys were performed by sampling three soybean fields per state. In each field, at least 10 plants were collected and examined for stem or root tissue containing dark, zone lines. Isolations were made from infected stems and roots by plating $5 \mathrm{~mm}$ pieces on acidified potato dextrose agar (PDA). Plates were incubated at $25^{\circ} \mathrm{C}$ for $14-\mathrm{d}$ under a 12-h photoperiod and hyphal tips of white colonies were transferred to acidified PDA. A total of 18 isolates producing black stromata in concentric patterns and alpha conidia were tentatively identified as a Diaporthe sp. (anamorph Phomopsis sp.). Alpha conidia exuded from the pycnidial ostiole in yellow drops and were ellipsoid with an average length of $6.6 \mu \mathrm{m}$ and width of $2.1 \mu \mathrm{m}$. Beta conidia and perithecia did not form on acidified PDA. DNA was extracted from the mycelia of 18 isolates, and the ribosomal DNA internal transcribed spacer region (ITS) were amplified using primers ITS5 and ITS4 and then sequenced. BLASTn analysis of the 600-bp fragment showed that the best match was Phomopsis longicolla strain STAM-35 (GenBank Accession No. AY745021) from Glycine max with identities 98 to $100 \%$. Pathogenicity work is in progress to confirm the association of Diaporthe longicolla with zone lines on soybeans.

Pathogenicity of Pythium species from the North Central region on soybean and corn screened at two temperatures

R. MATTHIESEN (1), M. Chilvers (2), A. Robertson (1)

(1) Iowa State University, Ames, IA, U.S.A.; (2) Michigan State University, East Lansing, MI, U.S.A.

Phytopathology 105(Suppl. 4):S4.90

Seedling disease caused by various Pythium spp. significantly reduces soybean and corn yield in the North Central region of the United States. Primarily managed using fungicide seed treatments, seedling disease is a risk when seed is planted when soil temperatures are below $\sim 13^{\circ} \mathrm{C}$ for corn and $\sim 18^{\circ} \mathrm{C}$ for soybean, and abundant rainfall occurs shortly after planting. The pathogenicity of four Pythium spp. recovered from diseased soybean seedlings collected in 10 states in the North Central region was evaluated using an in vitro assay on soybean and corn at $13^{\circ} \mathrm{C}$ and $23^{\circ} \mathrm{C}$. All species caused seed and root rot on both crops. Temperature had no effect on pathogenicity of $P$. lutarium and $P$. oopapillum, and no difference in the pathogenicity of strains collected from the various states was detected. P. sylvaticum was more pathogenic at $23^{\circ} \mathrm{C}$ compared to $13^{\circ} \mathrm{C}$ for strains from all states except Arkansas where the pathogenicity of one strain was not affected by temperature. Conversely, $P$. torulosum was more pathogenic at $13^{\circ} \mathrm{C}$ than at $23^{\circ} \mathrm{C}$ for all stains, except for one strain from Arkansas and one strain from Indiana, the pathogenicity of which was not affected by temperature. Understanding the diversity in pathogenicity of Pythium species from across the Midwest will improve our ability to develop and implement management strategies that reduce risk of seedling disease and improve farmer profitability.

Antagonistic activity of potential biocontrol agents screened against Pythium spp. that cause seedling disease

R. Matthiesen (1), M. MORGAN (2), A. Fakhoury (3), A. Robertson (2)

(1) Iowa State Univ, Ames, IA, U.S.A.; (2) Iowa State University, Ames, IA, U.S.A.; (3) Southern Illinois University, Carbondale, IL, U.S.A.

Phytopathology 105(Suppl. 4):S4.90

Pythium is an economically important pathogen that causes seedling disease on corn and soybean. Emerging resistance to fungicides has prompted research into additional management tools including biological control agents (BCA). The objective of this study was to assess activity of potential BCAs against Pythium spp. A dual culture assay was used to screen 14 strains 
representing Trichoderma, Mortierella, and Bionectria species against four Pythium species (12 strains). Pythium mycelial growth was measured on days $1,2,4$, and 7 to calculate percent of radial growth inhibition (PRGI). All species of Pythium were inhibited by all BCAs at day 4. M. elongata and B. ochroleuca inhibited growth of $P$. sylvaticum, $P$. lutarium, and $P$. oopapillum (PRGI $=40 \%, 23 \%$, and 24\%, respectively). Similarly, T. hamatum and $T$. harziarum inhibited growth of P. sylvaticum $(\mathrm{PRGI}=42 \%)$. P. torulosum was inhibited by $M$. elongata and B. ochroleuca on all days (PRGI $=12-58 \%)$. Neither an inhibition zone, nor parasitism was observed for any Pythium spp. or any BCA strain, respectively. Overgrowth by 8 to 10 BCAs occurred against each Pythium species. Identification of BCAs to reduce the risk of seedling disease caused by Pythium spp. and protect stand will be a useful management tool for farmers.

Eutypella spp. and Neoscytalidium hyalinum causing citrus branch canker and dieback in the Southern California desert regions

J. S. MAYORQUIN (1), D. H. Wang (1), M. Twizeyimana (1), A. Eskalen (1) (1) University of California, Riverside, CA, U.S.A.

Phytopathology 105(Suppl. 4):S4.91

In the Southern California desert regions citrus branch canker and dieback is commonly attributed to Neoscytalidium hyalinum, however preliminary evidence showed Eutypella spp. to be associated with disease in these areas as well. Numerous Eutypella spp. have been reported from citrus worldwide, but their potential role in citrus decline had not been fully investigated. To characterize the suite of fungi associated with citrus branch canker and dieback in this region, a total of 236 symptomatic branch samples were collected from seven commercial citrus groves between the summer of 2011 to the spring of 2013 in Imperial, Riverside and San Diego Counties. Surveys revealed $N$. hyalinum to be the most predominate canker fungus associated with disease in this area overall followed by Eutypella citricola, E. microtheca and an un-named Eutypella sp. Pathogenicity tests on three year old Lisbon lemon confirmed all three species of Eutypella as pathogens of citrus with the un-named Eutypella sp. being the most virulent among the four fungi tested. These results confirm the role of Eutypella spp. as pests of citrus and highlight the complexity of fungal pathogens that cause citrus branch canker and dieback in this area supporting a need for continued research into the epidemiology of this pathosystem for improved diseased management.

Mycotoxin management in maize (Zea mays (L.)) damaged by Lepidopteran insects pest

D. MAYS (1), G. Schuster (2), M. Sétamou (3), P. Porter (4), D. Wester (5), A. Tri (5)

(1) Department of Agriculture, Agribusiness, and Environmental Sciences, Texas A\&M University-Kingsville, Kingsville, TX, U.S.A.; (2) Texas A\&M University - Kingsville, Texas A\&M AgriLife Extension, Kingsville, TX, U.S.A.; (3) Texas A\&M University-Kingsville Citrus Center, Weslaco, TX, U.S.A.; (4) Texas A\&M AgriLife Extension Service, Lubbock, TX, U.S.A.; (5) Department of Animal and Wildlife Sciences; Texas A\&M University Kingsville, Kingsville, TX, U.S.A.

Phytopathology 105(Suppl. 4):S4.91

Mycotoxins pose animal health risks and reduce grain marketability. Lepidopteran damage on corn causes injury, which facilitates infection by fungal spore development. Atoxigenic strains of Aspergillus flavus can reduce contamination of aflatoxin and fumonisin. Producers need to know the optimal application period to maximize effectiveness of biocontrol products. This study was initiated to evaluate optimal timing of biocontrol products, AF-36 and Alfa-Guard ${ }^{\circledR}$ in relationship to insect activity. Treatments included no insect control (UTC), insect control, and application of both products at V8 and VT manually applied by hand at growth stages at a rate of $11.2 \mathrm{~kg} / \mathrm{ha}$ in a randomized complete block design. Fumonisin concentrations (ppm) for insect control applications were $65 \%$ lower $(\mathrm{P}<0.05)$ than untreated and biocontrol applications. We found no statistical difference in aflatoxin concentrations (ppb) among any treatment $(\mathrm{P}=0.375)$; however, aflatoxin concentrations of insect control and AF-36 VT were 21\% lower than control and other biocontrols. Insect damage $(\mathrm{cm}$ squared $)$ on the upper ear $(\mathrm{P}=0.032)$ and lower ear $(\mathrm{P}=0.157)$ were $52-91 \%$ lower for the insect control plots. AF-36 VT had $20-60 \%$ less insect damage than the control or other biocontrol treatments, suggesting timing may have implications for biocontrol success. Our results indicate that biocontrols had limited effects on mycotoxin concentrations and insect damage, with exception.

Root infection of corn by Clavibacter michiganensis subsp. nebraskensis G. Mbofung (1), J. Sernett (2), A. E. ROBERTSON (3)

(1) Iowa State University, Ames, IA, U.S.A.; (2) Monsanto, Huxley, IA, U.S.A.; (3) Iowa State Univ, Ames, IA, U.S.A.

Phytopathology 105(Suppl. 4):S4.91
Clavibacter michiganensis subsp. nebraskensis (Cmn) causes Goss's bacterial wilt and leaf blight (GWLB). Although the most common route of infection is via leaf wounding, GWLB via root wounding has also been reported. Furthermore, field observations suggest young plants are more susceptible to the wilt phase of GWLB. We hypothesized that resistance to systemic infection is root age dependent. Four corn hybrids, susceptible (S), moderately susceptible (MS), moderately resistant (MR) and resistant (R) to GWLB were grown to the V3 and V6 developmental stage. Either a single seminal root on V3 plants or a nodal root on V6 plants was wound inoculated with $10 \mu 1$ of $\mathrm{Cmn}$. Percent wilt incidence and leaf blight incidence were rated beginning at 10 days after inoculation (dai) through 26 dai. A three-way interaction (development stage*hybrid*root inoculation) was detected $(\mathrm{P}<0.0002)$. Incidence of GWLB differed depending on the development stage at which plants were inoculated $(\mathrm{P}<0.001)$. Wilt was observed on the $\mathrm{S}$, MS and MR hybrids that were inoculated at V3 $(37.5 \%, 25 \%$ and $6.25 \%$, respectively). No wilt was observed on any hybrid inoculated at V6. Similarly, leaf blight occurred on all hybrids inoculated with $\mathrm{Cmn}$ at $\mathrm{V} 3$, but only occurred, and at low incidence, on the S and MS hybrid that were inoculated at V6. These results suggest that root infection that occurs prior to V6 may result in GWLB that could lead to stand loss and consequently reduced yields.

New prospects, progress, and research at the Fungal Genetics Stock Center

K. MCCLUSKEY (1), J. F. Leslie (1)

(1) Kansas State University, Manhattan, KS, U.S.A.

Phytopathology 105(Suppl. 4):S4.91

Following changes in federal support for living collections, the Fungal Genetics Stock Center has relocated to the department of Plant Pathology at Kansas State University. The relocation included over thirteen thousand pounds of equipment and materials and was accomplished in one day. Despite this significant disruption, the collection continued to provide high quality resources with very few delays. Encompassing over 75,000 fungal strains, the FGSC is the largest collection of genetically characterized and modified fungal strains in the world and the vast majority of these strains are plant pathogen or plant associated fungi. As part of the leading approaches implemented at the FGSC, an increasing number of strains that have been subject to whole genome sequence analysis, including wild and classical mutant strains of over 30 species, are maintained and made available by the center. In addition to these strains, the FGSC holds 775 plasmids as well as gene and genome libraries for subset of the strains in the collection, further contributing to its global leadership in curating and distributing materials to researchers around the world. Existing collections at Kansas State complement the FGSC holdings and emphasize the synergy of bringing the FGSC to KSU.

Adaptation to host resistance genes by Phytophthora nicotianae

K. L. MCCORKLE (1), R. S. Lewis (1), H. D. Shew (1)

(1) North Carolina State University, Raleigh, NC, U.S.A.

Phytopathology 105(Suppl. 4):S4.91

Black shank, caused by Phytophthora nicotianae, is an important disease of tobacco worldwide. Host resistance is the easiest and most cost effective tool for disease control; however, the widespread deployment of complete resistance failed due to a rapid race shift in the pathogen from wild type race 0 to race 1. Partial resistance also is available, and while offering some protection to both races, severe losses may still occur. All partial resistance in commercial varieties is from variety Florida 301. To increase the level of partial resistance, variety Beinhart 1000 was investigated. Since all resistance genes place selection pressure on the pathogen, the objectives of our study were to determine how isolates adapt to varying sources and levels of partial resistance and if adaptations are specific to the source of resistance. Ten tobacco lines with resistance from Florida 301, Beinhart 1000, or single genes $W z$ or $P h p$ were grown in the greenhouse. Plants were inoculated with race 0 and race 1 isolates for six continuous generations. Adapted isolates were compared across generations for each variety and pathogen race combination. An increase in pathogen aggressiveness was observed over generations for both races on multiple varieties. More aggressive isolates had decreased incubation periods and increased root rot. Understanding how $P$. nicotianae adapts to resistance will improve recommendations for variety rotation to manage the pathogen and the disease.

Tobacco mosaic virus/Tomato mosaic virus contamination of tomato seed and transmission to transplants

M. McCullough (1), C. NISCHWITZ (2), B. Rhoads (3), A. Klomp (1)

(1) Utah State University, Logan, UT, U.S.A.; (2) Utah State Univ, Logan, UT, U.S.A.; (3) Utha State University, Logan, UT, U.S.A.

Phytopathology 105(Suppl. 4):S4.91 
In response to a Tobacco mosaic virus (TMV)/Tomato mosaic virus (ToMV) outbreak in Utah greenhouse tomatoes, seed was tested and verified positive with the virus. It is known that TMV/ToMV can be transmitted through seed, but this mode of transmission is frequently considered unimportant. The objective of this study was to determine how widespread TMV/ToMV seed contamination is and the rate of transmission from the seed to the seedlings. ELISA testing was used to confirm the presence of the virus on the seed. Reverse transcription-PCR was used to identify viral strains and confirm transmission from seed to seedling. Tomato seed from over 40 varieties were tested for TMV/ToMV. The contamination rate ranged from $0-100 \%$. Leaf samples taken from the seedlings at four weeks were negative. However, after three months up to $78 \%$ of plants grown from contaminated seed were infected. The results indicate that seed transmission of TMV/ToMV can be high. Introductions into greenhouse and field operations could be prevented by using disease-free seed that has been tested for TMV/ToMV. This is especially important for organic growers using untreated seed and could help prevent secondary spread of the virus to healthy tomato plants and other vegetables.

\section{A low temperature threshold is important for predicting clubroot development}

M. R. MCDONALD (1), T. Cranmer (2), T. Gludovacz (2), B. D. Gossen (3) (1) Univ of Guelph, Guelph, ON, Canada; (2) University of Guelph, Guelph, ON, Canada; (3) Agriculture and Agri-Food Canada, Saskatoon, SK, Canada Phytopathology 105(Suppl. 4):S4.92

Several studies have examined the relationships among temperature, infection by Plasmodiophora brassicae, and the development of clubroot on several Brassica spp. Under controlled conditions, the optimum temperature for each stage of the disease cycle was about $25^{\circ} \mathrm{C}$. In the field, seeding into cool soils reduced clubroot severity and cool temperatures prior to harvest also suppressed symptoms. To assess the impact of weather on clubroot development, canola (B. napus) cv. InVigor 5030 was seeded at 2-week intervals at a site naturally infested with $P$. brassicae in replicated studies at the Holland Marsh, Ontario, Canada, from 2011-2014. Plants were harvested at 4 and 6 weeks after seeding, and clubroot incidence and severity was assessed. Similar trials at the same site were conducted over 6 years with Brassica vegetable crops. Soil and air temperatures and rainfall, were recorded daily. Correlation analysis and stepwise regression were used to examine the relationships among clubroot severity and air and soil temperature, degree days (base of $12.5,14$ and $17^{\circ} \mathrm{C}$ ) and rainfall, on the separate crops and on the combined data. Mean daily air temperature in the first 3 weeks after seeding was positively correlated with clubroot incidence and severity, while none of the degree day data were correlated with clubroot symptoms. This indicates that temperatures above a critical threshold $\left(14^{\circ} \mathrm{C}\right)$ are not useful for predicting clubroot development under field conditions.

\section{Why is clubroot on canola more severe in Alberta than in Ontario,} Canada?

M. R. MCDONALD (1), A. Deora (2), F. Al-Daoud (3), B. D. Gossen (4)

(1) Univ of Guelph, Guelph, ON, Canada; (2) Syngenta, Guelph, ON, Canada; (3) University of Guelph, Guelph, ON, Canada; (4) Agriculture and AgriFood Canada, Saskatoon, SK, Canada

Phytopathology 105(Suppl. 4):S4.92

Clubroot, caused by Plasmodiophora brassicae, has spread rapidly and become an important disease of canola (Brassica napus) in the Canadian province of Alberta since it was first identified there in 2003. In contrast, clubroot has caused losses on vegetable crops in Ontario since the 1930's but is not a major disease on canola and is not spreading quickly. Field trials were conducted to examine the reaction of spring canola cultivars to pathotype 6 , the predominant pathotype in Ontario in 2011, 2012, and 2014. Cultivars were designated as resistant if they had disease severity index (DSI) values $<30$. Severe clubroot developed on the clubroot-susceptible line ACSN39 each year (66, 100, and $71 \mathrm{DSI}$, respectively). Resistant lines were identified each year: 22 of 33 lines in 2011, 3 of 13 lines in 2013, and 7 of 22 lines in 2014. All of the cultivars known to be resistant to pathotype 3 , which is the dominant pathotype in Alberta, were also resistant to pathotype 6, but lines resistant to pathotype 6 were generally susceptible to pathotype 3 . The low level of clubroot on canola in Ontario can be attributed, in part, to the resistance of many canola cultivars to pathotype 6 . Long cropping rotations, alkaline soil $\mathrm{pH}$, and seedling in fall or early spring, when low temperatures reduce infection and subsequent clubroot severity, may further reduce the risk of establishment of $P$. brassicae in Ontario fields and its impact once established.
A food grade oil product induces resistance to diseases and insect pests in vegetable crops

M. R. MCDONALD (1), I. Teshler (2), M. Fefer (2)

(1) Univ of Guelph, Guelph, ON, Canada; (2) Petro-Canada Lubricants Inc, Mississaugua, ON, Canada

Phytopathology 105(Suppl. 4):S4.92

A food grade oil product, in conjunction with various adjuvants $(\mathrm{FGO}+\mathrm{A})$, was evaluated in replicated field trials for the suppression of diseases and insect pests of onion and carrot, in the Holland Marsh region of Ontario, Canada. The products were applied 5 times a season and compared to standard fungicides or insecticides. Stemphylium leaf blight of onion, caused by Stemphylium vesicarium, was reduced from $10 \%$ in the untreated check to $2 \%$, when disease pressure was low. The FGO+A was as effective as the fungicide axozystrobin plus difenoconazole. No differences were found when disease pressure was much higher (severity $44-76 \%$ ), in a previous trial. Spraying with $\mathrm{FGO}+\mathrm{A}$ also reduced the number of onion thrips (Thrips tabaci) from 29 per plant in the untreated check to 10 per plant in treated plots. Carrot leaf blights caused by Alternaria dauci and Cercospora carotae were reduced from $48 \%$ disease severity index in the untreated check to $35 \%$ in 2013 and from $52 \%$ to $36 \%$ in trials in 2014 . The number of dead leaves was reduced from 6.3 per plant to 4.3. Disease suppresseion was equivalent to that of fungicide boscalid. The incidence of aster yellows (caused by Candidatus phytoplasma asteris) on carrot was high in 2013 and applications of $\mathrm{FGO}+\mathrm{A}$ reduced the incidence of aster yellows from $19 \%$ in the check to $<2 \%$. This novel food grade oil supressed both diseases and insect pests on two very different vegetable crops.

Engaging the public to report occurrence of a new disease, basil downy mildew

M. T. MCGRATH (1)

(1) Cornell Univ, Riverhead, NY, U.S.A.

Phytopathology 105(Suppl. 4):S4.92

A web page was set up in Google Docs in 2009 for logging reports on occurrence of basil downy mildew (BDM). It was anticipated that extension pathologists would be the primary users, so they were contacted about the monitoring program. However, gardeners have logged most reports. Growers have also reported. These reporters while searching the internet for what happened to their basil found an article about BDM that included requests for occurrence information with links to each year's report page. Attempt was made to confirm all reports by requesting photographs from the reporter; symptom descriptions were also used. After 6 years of reporting, not only is knowledge about BDM occurrence in the USA substantially better than it could have been without public help, reporters in the spreadsheet comments section provided valuable additional information including environmental conditions when BDM appeared, impact, and management practices tried. This new disease, first observed in Florida in fall 2007, was reported in eight states in 2008. Since then through the monitoring program it has been reported in 42 states plus District of Columbia. Total of 49, 63, 63, 75, 64, and 284 reports were logged in 2009, 2010, 2011, 2012, 2013, and 2014 from 20, 26, $22,26,20$, and 35 states, respectively. Reports also came from Argentina, Australia, Mexico, Baja California, Grand Cayman, Costa Rico, Puerto Rico, Jamaica, Quebec, Ontario, British Columbia, South Africa, and South Korea.

Virulence assessment of representative isolates of the Phytophthora cinnamomi population in California on different avocado rootstock selections

B. McKee (1), M. Crowley (1), J. Mayorquin (1), G. Douhan (1), M. L. Arpaia (1), A. Eskalen (1), P. MANOSALVA (1)

(1) University of California Riverside, Riverside, CA, U.S.A.

Phytopathology 105(Suppl. 4):S4.92

Phytophthora cinnamomi $(P c)$ is the causal agent of Phytophthora root rot of avocado and is the most serious disease of avocado worldwide. In a recent study, two distinct clades of A2 mating type isolates from avocado were found in the avocado-growing regions of California based on the analysis of molecular markers. One clade (A2 Clade I) contained isolates from both Northern and Southern California and consisted of isolates collected from 1989 to 2010. The second clade (A2 Clade II) consisted of only unique isolates from Southern California collected from 2009 to 2010 and it was hypothesized that these isolates may have been recently introduced. Therefore, the virulence of representative isolates from each clade on six avocado rootstocks was investigated using a mixture of three isolates from each clade in various combinations. Preliminary results demonstrated that two of the three isolates from the A2 Clade II group as well as their mixtures were more aggressive than the isolates from the A2 Clade I group. In addition, it was found that the resistance phenotype of some rootstocks has an effect on the behavior of some of these isolates. The increasing population diversity of 
$P c$ in California and the presence of more aggressive isolates argue that screening for resistant rootstocks of avocados needs to be performed with a mixture of isolates representing the genetic diversity of this oomycete in California.

A survey of Phytophthora species causing root rot on Abies in U.S. Christmas tree farms

K. MCKEEVER (1), G. Chastagner (1)

(1) Washington State University, Puyallup, WA, U.S.A.

Phytopathology 105(Suppl. 4):S4.93

Multiple Phytophthora species are known to cause root rot (PRR) on Abies. Information about the community structures and habits of these regionallyvariable pathogens has received little study in the past 30 years. A contemporary survey could update literature with current pathogen and host ranges; reveal previously unreported Phytophthora-fir combinations; and inform about varying host susceptibilities leading to improved management advice. 32 Christmas tree farms were visited in 7 U.S. states representing 4 major production regions to isolate and identify Phytophthora species causing disease on Abies. Symptomatic tissues from lesion margins on roots and crowns were plated on PARPH-cV8 selective media to recover Phytophthora. Cultures were identified by DNA sequencing of the nuclear ITS and mitochondrial cox I regions. It was determined that $P$. cambivora is the prominent PRR species in western WA and OR, while P. cinnamomi was prevalent in $\mathrm{CA}$ and $\mathrm{NC}$. In the eastern US, the undescribed $P$. taxon 'kelmania' was isolated in great frequency and was found to be capable of killing fir species considered to be tolerant of PRR. Completion of Koch's Postulates confirmed pathogenicity of 12 unpublished Phytophthora-fir combinations. Supplementing established knowledge regarding Phytophthora species prevalence and Abies sensitivities will enhance crop productivity by improving recommendations for site modifications including water abatement and proper host species deployment.

Novel PCR-mediated assay for detection, identification and quantification of Clavibacter michiganensis subsp. nebraskensis

R. R. MCNALLY (1), C. A. Ishimaru (2), D. K. Malvick (2)

(1) University of Minnesota, St. Paul, MN, U.S.A.; (2) University of Minnesota, St. Paul, MN, U.S.A.

Phytopathology 105(Suppl. 4):S4.93

Clavibacter michiganensis is a Gram-positive bacterium responsible for a wide range of important plant diseases. The corn disease Goss's wilt is caused by C. michiganensis subsp. nebraskensis (CMN). As of 2014, 15 states and three Canadian provinces have reported Goss's wilt, an emerging plant disease. Despite the significance of Goss's wilt, diagnosis relies on symptoms, signs, and isolation of CMN. Antibody-based assays are expensive, nonspecific, and incapable of distinguishing C. michiganensis subspecies. We have developed an assay for the specific, sensitive, and inexpensive identification and quantification of CMN via PCR-mediated detection of a predicted gene in CMN. The assay with two primers was tested for specificity using DNA isolated from diverse bacterial and fungal samples as well as from healthy and $\mathrm{CMN}$-infected corn tissues. In all cases, the assay proved an effective tool for 1) distinguishing CMN from other microorganisms, including other C. michiganensis subspecies, and 2) for direct detection of CMN in infected host tissues. To determine sensitivity using standard PCR, our assay was tested with decreasing quantities of DNA template from CMN. CMN was detected in samples containing fewer than $10 \mathrm{CMN}$ genomes $(\sim 1$ $\mathrm{fg} / \mathrm{ul})$. A real-time, quantitative assay based on the same gene target is under development. This assay should prove an economical, accurate and sensitive means for the identification, detection, and quantification of CMN.

Expression of a single-chain antibody recognizing an extracellular loop of the Candidatus Liberibacter asiaticus NodT protein in Citrus $\mathbf{x}$ paradisi

T. MCNELLIS (1), J. Sinn (2), V. Orobovic (3), T. Gottwald (4)

(1) Penn State Univ, University Park, PA, U.S.A.; (2) The Pennsylvania State University, University Park, PA, U.S.A.; (3) University of Florida, Lake Alfred, FL, U.S.A.; (4) U.S. Horticultural Research Laboratory, USDA ARS, Fort Pierce, FL, U.S.A.

Phytopathology 105(Suppl. 4):S4.93

The gram-negative, phloem-limited bacterium Candidatus Liberibacter asiaticus (CLas) is believed to be the cause of citrus greening disease, or 'huanglongbing' (HLB), and is now endemic through the state of Florida. Most commercial citrus varieties are highly susceptible to HLB, and few options exist for development of resistant cultivars by conventional breeding. The CLas genome sequence reveals the presence of a copy of the nodT gene, which encodes a predicted outer-membrane efflux protein with amino acid sequence homology to the Escherichia coli TolC protein. The available crystal structure of E. coli TolC, coupled with the amino acid sequence homology between TolC and NodT, allowed us to predict the position of a 21-aminoacid extracellular loop of NodT. We hypothesized that antibodies recognizing this extracellular loop of NodT could be expressed in citrus phloem to counteract citrus greening disease development and CLas multiplication. Single-chain antibodies (scFvs) binding to a peptide corresponding to the predicted extracellular loop of CLas NodT were identified by screening a phage display antibody combinatorial library. The $\mathrm{scFv}$ with strongest affinity to the peptide was fused to the C-terminus of the Flowering Locus T (FLT) protein by gene fusion and expressed in 'Duncan' grapefruit (Citrus $\mathrm{x}$ paradisi) by stable Agrobacterium-mediated transformation. Expression of the FLT-scFv protein and effects on grapefruit HLB susceptibility will be discussed.

Phomopsis associated with spruce decline: Virulence studies on six spruce species

C. K. MCTAVISH (1), D. W. Fulbright (1), A. M. Jarosz (1)

(1) Michigan State University, East Lansing, MI, U.S.A.

Phytopathology 105(Suppl. 4):S4.93

Spruce decline is found throughout Michigan and is characterized by needle loss and branch dieback. Disease is most pronounced on Colorado blue spruce (Picea pungens). Previous work confirmed that Phomopsis is commonly isolated from cankers on spruce exhibiting spruce decline. Phomopsis isolates collected from infected trees fell into five groups based on ITS sequence. Management of spruce decline requires information on the susceptibility of multiple spruce species to the different groups of Phomopsis. Six species were chosen for testing based on popularity or possible replacements for blue spruce. Norway $(P$. abies), white $(P$. glauca), Black Hills $(P$. glauca var. densata), Serbian ( $P$. omorika), Meyer ( $P$. meyeri), and Colorado blue spruce ( $P$. pungens) were inoculated with isolates representing three of the five Phomopsis groups isolated from trees exhibiting spruce decline. Trees were inoculated by inserting an agar plug containing Phomopsis into a small wound and canker area was measured 78 days post-inoculation. Phomopsis isolates from groups 4 and 5 were highly virulent, and similar statistically $(\mathrm{p}=0.31)$. Group 3 was less virulent, but still caused small cankers. Colorado blue spruce was the most susceptible species while Black Hills and Meyer spruce were resistant but not immune. The remaining three spruce species displayed intermediate levels of susceptibility. Our results suggest that alternative spruce species may be useful as landscape plantings.

Characterization of Phomopsis isolated from spruce exhibiting spruce decline symptoms

C. K. MCTAVISH (1), D. W. Fulbright (1), A. M. Jarosz (1)

(1) Michigan State University, East Lansing, MI, U.S.A.

Phytopathology 105(Suppl. 4):S4.93

Spruce decline is characterized by needle loss and branch dieback, and is found throughout Michigan. Previous work determined that Phomopsis is commonly isolated from cankers associated with this decline and isolates were variable based on morphology and virulence. Further characterization of these Phomopsis isolates was carried out with DNA sequencing using ITS1 and ITS4 primers. Five groups were found, which differed by one to 11 substitutions and/or insertions along the approximately 600bp sequence. Group 3 displayed 95\% identity to Phomopsis occulta based on BLAST searches, which agrees with earlier published reports. However, this group also displayed $99 \%$ identity to Diaporthe eres. Phylogenetic analyses yielded congruent topologies using neighbor-joining, maximum parsimony, maximum likelihood, and Bayesian methods, and all placed group 3 as a member of $D$. eres. Group 3 was also the least virulent group. Phylogenetic analyses using ITS sequences placed the more virulent groups 4 and 5 in an unresolved portion of the phylogenetic tree. It is important to resolve these two important groups to determine if this is a new species. Therefore, future work will involve using the $\beta$-tubulin gene to resolve the species identity of each of the 5 groups.

Using comparative genomics to identify new resistance sources against the tomato pathogens Pseudomonas syringae pv. tomato and Ralstonia solanacearum

M. E. MECHAN LlONTOP (1), C. R. Clarke (1), C. L. Monteil (1), D. C. Haak (1), B. A. Vinatzer (1)

(1) Virginia Tech, Blacksburg, VA, U.S.A.

Phytopathology 105(Suppl. 4):S4.93

Comparative genomics is an excellent tool for the identification of virulence genes. Pseudomonas syringae pv. tomato (Pto) and Ralstonia solanacearum race 3 biovar2 (R3bv2) are both economically important pathogens and genetic resistance against these pathogens is extremely limited. Therefore, we compared type III-secreted effector repertoires among isolates of these pathogens and isolates of related crop pathogens and, in the case of Pto, 
isolates collected in non-agricultural environments. This allowed for the identification of effectors that are highly conserved among pathogen isolates and that can be expected to be important for pathogen fitness. Disease assays of Pto and Agrobacterium-mediated transient assays of Pto and R3bv2 effectors were then used to screen wild tomato relatives and other plant species for putative sources of resistance. Our results show that some tomato genotypes are resistant to Pto regardless of the expression of AvrPto1, which suggests that they may recognize effectors other than AvrPto1, which is so far the only effector recognized by any cloned tomato resistance gene. Additionally, one R3bv2 effector triggered a plant cell death response indicative of disease resistance in a resistant eggplant genotype but not in a susceptible genotype. We will show progress in characterizing the interactions of Pto with resistant tomato genotypes and of Rs R3bv2 effectors with plant genotypes carrying putative cognate resistance genes.

Improving crop rotation efficiency for Soybean cyst nematode management

R. MEDINA (1), C. G. Taylor (1)

(1) Ohio State University, OARDC, Wooster, OH, U.S.A.

Phytopathology 105(Suppl. 4):S4.94

Soybean cyst nematode (Heterodera glycines; $\mathrm{SCN}$ ) has significant impact on the soybean industry, costing $\$ 90$ million dollars annually. SCN has been managed mostly through the use of resistant cultivars and non-host crop rotations. Recently, resistance-breaking populations of SCN have made the use of SCN-resistant cultivars far less effective in controlling this nematode. Reliance on crop rotation has now become significantly more important for SCN control. However, little is known about how widely used non-host crops can impact SCN populations. Previous reports suggest that SCN numbers can be reduced during rotations with maize but the mode of action in this interaction is unknown. This study aimed to identify maize lines from the Nested Associated Mapping series of maize breeding population that are most suitable for decreasing SCN populations through the production of allelopathic chemicals in root exudates. Collected root exudates were screened for their ability to affect SCN hatching. Of the 27 maize lines tested, we observed lines that repeatedly altered SCN hatching rates. This exudateinduced hatching response suggests that genetic factors that regulate these differences might be identifiable through breeding and/or gene expression analysis. More immediate benefits of this research are to identify maize lines that should be used in rotation with soybean to help reduce SCN numbers in the field.

Draft genome sequence of a Pantoea ananatis strain associated with cotton bud/boll blight

E. G. MEDRANO (1), W. Mantooth (2), A. A. Bell (3)

(1) USDA ARS CPRU, College Station, TX, U.S.A.; (2) USDA ARS, College Station, TX, U.S.A.; (3) USDA-ARS, College Station, TX, U.S.A.

Phytopathology 105(Suppl. 4):S4.94

Known to have a cosmopolitan distribution, Pantoea ananatis strains have been isolated from hosts including plants, animals, and humans. Pantoea ananatis, strain CFH 7-1, was isolated from a diseased, greenhouse-grown cotton boll previously caged with a field-collected cotton fleahopper. A high quality draft, whole genome sequence of strain CFH 7-1 was generated using Roche 454 technology based on both shotgun and paired-end libraries. Over $30 \mathrm{Mb}$ of sequence data was produced. The sequence data was manually annotated using the NCBI blast program. Over 4000 putative genes were identified. Amongst the potential open reading frames identified, pathogenicity matches were revealed including type IV and VI secretion systems. Collectively, these data advance knowledge of the $P$. ananatis infection process of cotton bolls based on a genomics approach.

Development and optimization of a weather-based decision aid for soybean foliar fungicide applications in Virginia

H. L. MEHL (1), T. Zhou (1), D. Holshouser (1)

(1) Virginia Tech Tidewater AREC, Suffolk, VA, U.S.A.

Phytopathology 105(Suppl. 4):S4.94

Over the past decade, soybean producers have increased inputs, including foliar fungicides, to maximize yields. Typically, fungicide applications are made at beginning pod (R3), but yield responses are inconsistent. One contributing factor may be application of fungicides when disease pressure is low and conditions are not conducive to disease development. The objective of this work is to develop a weather-based decision aid for soybean foliar fungicide applications in Virginia. Environmental parameters favorable for disease development were determined previously. R3 fungicide applications were compared to weather-based applications at 7 on-farm locations and 2 research stations in 2014. Weather stations collected environmental data including temperature and relative humidity. Dominant foliar diseases were frogeye leaf spot (Cercospora sojina) and Cercospora blight (C. kikuchii). Yield response to fungicide applications was significant in 5 of 9 trials. When a yield response occurred, R3 applications were equal to or better than weather-based applications suggesting R3 is the optimal time to apply fungicide. However, yield response was greatest when weather conditions conducive to disease development occurred within 7 days of R3. Additional years of data including a range of environmental conditions and detailed assessments of crop and disease development are needed to optimize a weather-based decision aid for foliar fungicide applications in soybean.

Pre-planting risk assessment models for Stagonospora nodorum blotch in winter wheat

L. MEHRA (1), C. Cowger (1), P. S. Ojiambo (1)

(1) North Carolina State University, Raleigh, NC, U.S.A.

Phytopathology 105(Suppl. 4):S4.94

Stagonospora nodorum blotch (SNB) caused by Parastagonospora nodorum, is a major disease of wheat. Pre-planting factors such as previous crop, tillage, host genotype, disease history, and location of a field affect disease intensity. However, the risk of SNB due to these factors has not been quantified. Experiments were conducted at several locations in North Carolina from 2012 to 2014. For modeling purposes, unique cases were randomly divided into training, validation, and test dataset and this process was repeated 15 times. Multiple regression (MR), classification and regression tree (CART), and logistic regression approaches were used to relate area under disease progress curve (AUDPC) and maximum disease severity (DS) to pre-planting factors. Generally, MR models explained $73 \%$ and CART models explained $68 \%$ of the variation in AUDPC. When DS was dichotomized into low and high disease level, logistic regression correctly classified 61 to $85 \%$ cases in test datasets. Similarly, when MR and CART were used to predict DS and then dichotomized into low and high disease level, they correctly classified 73 to $94 \%$ and 76 to $91 \%$ cases in test datasets, respectively. Previous crop, tillage type, location, and cultivar resistance were the most important predictors of AUDPC and DS. Once optimized, these models can provide important information on the risk of disease occurrence during the season and thereby guide growers in making pre-planting management decisions for SNB.

Characterization of Bacillus amyloliquefaciens strain BAC03 for plant growth promotion

Q. Meng (1), H. JIANG (2), J. Hao (3)

(1) Univ of Maine, Spencer, IA, U.S.A.; (2) Univ of Maine, Orono, ME, U.S.A.; (3) Univ of Maine, orono, ME, U.S.A.

Phytopathology 105(Suppl. 4):S4.94

Previous studies has demonstrated Bacillus amyloliquefaciens strain BAC03 is an antagonist against Streptomyces scabies, as well as promotes plant growth. However its mechanisms on plant growth promotion needs to be investigated. In this study, BAC03 was tested for potential growth promotion on nine selected plants types, including beet, carrot, cucumber, pepper, potato, radish, squash, tomato, and turnip, at the concentration of $10^{5}$ colony forming unit $\mathrm{cm}^{-3}$ potting mix under greenhouse conditions. Results showed that BAC03 increased the growth at various levels of some tested plants in different plant parts. Application of BAC03 at 10 days after planting was associated in radish with the highest biomass gain compared to applications at other stages. Multiple applications of BAC03 giving the same total amount of inoculum resulted in higher weights of radish roots and leaves compared to the controls. BAC03 produced indole-3-acetic acid and ammonia, as well as showing a 1aminocyclopropane-1-carboxylate deaminase activity, some of which could be potentially related to plant growth promotion. Volatiles released from BAC03 might be responsible for suppressing seed germination and seedling growth for a short period, and impacting on the growth of S. scabies. Acetoin and 2,3butanediol were detected as major components of the volatile by using gas chromatography-mass spectrometry analysis.

Potassium and phosphorus effects on disease severity of charcoal rot of soybean

A. MENGiSTU (1), X. Yin (2), N. Bellaloui (3), A. M. McClure (2), D. D. Tyler (2), K. N. Reddy (4)

(1) USDA ARS, Jackson, TN, U.S.A.; (2) University of Tennessee, Jackson, TN, U.S.A.; (3) USDA-ARS, Jackson, TN, U.S.A.; (4) USDA-ARS, Stoneville, MS, U.S.A.

Phytopathology 105(Suppl. 4):S4.94

The effects of potassium (K) and phosphorus (P) fertilizers on charcoal rot of soybean [Glycine $\max$ (L.) Merr.] are unknown. Therefore, the severity of charcoal rot was studied at five levels of $\mathrm{K}(0,37,75,111$ and $149 \mathrm{~kg}$ ha-1) and a level that was equal to the recommended fertilizer application rate based on annual soil $\mathrm{K}$ testing; and five levels of $\mathrm{P}(0,10,20,30$ and $40 \mathrm{~kg}$ ha-1) and a level that was equal to the recommended $\mathrm{P}$ fertilizer application rate 
based on annual soil testing under field conditions in 2008, 2009 and 2010 at Jackson and Milan, TN, USA. Analysis of variance showed no significant effect from treatments or treatment-by-year interactions. However, analysis of colony forming unit (CFU) of Macrophomina phaseolina level from soil showed a significant effect from $\mathrm{P}$ but not from $\mathrm{K}$ application. Analysis using treatment as a linear trend effect indicated that tissue CFU as a function of $\mathrm{K}$ rates showed a positive but moderate significant slope at both Jackson and Milan in 2009 suggesting a modest increase in disease severity in that particular year. Further increase in $\mathrm{K}$ rate however, resulted in a negative slope at Milan in 2010. The CFU from soil also showed a similar positive significant slope for P in Milan in 2009 and 2010 but not for 2008. Our results showed that increasing the $\mathrm{K}$ and $\mathrm{P}$ application rates over both the recommended rate and control did not result in any consistent increase or decrease in disease severity of charcoal rot.

Variability in virulence as determined by honeydew production by strains of Claviceps purpurea (Fr.) Tul. on eight genotypes of wheat

J. MENZIES (1), A. Gordon (2), D. O'Sullivan (3)

(1) Agriculture and Agri-Food Canada, Morden, MB, Canada; (2) National Institute of Agricultural Botany, Cambridge, United Kingdom; (3) University of Reading, Reading, United Kingdom

Phytopathology 105(Suppl. 4):S4.95

Ergot, caused by Claviceps purpurea has steadily increased in incidence and severity on the Canadian prairies since 1999. The development of resistant wheat lines is being explored. The variability in pathogenicity among strains of $C$. purpurea in nature is not known. The objective of this work was to study variability in pathogenicity among different strains of $C$. purpurea for honeydew production on wheat lines differing in levels of resistance. Forty seven pathogen strains from the Canadian prairies and the United Kingdom were inoculated onto 3 durum wheat (Triticum aestivum $\mathrm{L}$. var. durum) lines ('Melita', 'Kyle', and 9260B-173A) and 5 hexaploid wheat (T. aestivum) lines ('Cadillac', 'Vista', 'Kenya Farmer', 'Lee', and HY630). Nine spikes per wheat line were needle inoculated prior to anthesis for each pathogen strain using a conidial suspension. Each spike was assessed for honeydew production 14 days after inoculation using a scale of 1 to 4 ; $1=$ no honeydew visible, $2=$ honeydew confined within glumes, $3=$ honeydew exuding from florets in small drops, and 4=large drops of honeydew running down the spike. There were significant differences among the 8 wheat genotypes for the mean honeydew production. Pathogen strains were considered to be virulent when they produced a honeydew rating of 2.5 or higher. Different virulence phenotypes were identified, and there was a wide range of genetic variability among strains of $C$. purpurea.

Pestalotiopsis spp., a newly discovered root pathogen of strawberry transplants

J. MERTELY (1), M. Chamorro (2), N. A. Peres (2)

(1) Univ of Florida-GCREC, Wimauma, FL, U.S.A.; (2) University of Florida-GCREC, Wimauma, FL, U.S.A.

Phytopathology 105(Suppl. 4):S4.95

Florida strawberries are planted in the fall for fruit production during the winter and spring. Rapid establishment of transplants is necessary for early production when market prices are high. We have repeatedly isolated Pestalotiopsis spp. from weak and dying strawberry transplants in commercial fields and suspect it may contribute to establishment difficulties. Four strawberry root isolates were tested for pathogenicity in replicated greenhouse experiments. In Aug. 2013, the roots of cut-top transplants (cultivar Strawberry Festival) were briefly dipped in inoculum and planted in pots filled with fumigated field soil. The inoculum consisted of 10-day-old PDA cultures bended with sterile water. After 2 weeks, stunting and/or premature dying of older leaves was observed on 16 of 20 inoculated plants, and 2 of 10 control plants. Pestalotiopsis was subsequently reisolated from necrotic crowns of several plants. In Aug. 2014, the same isolates were retested using inoculum calibrated to $2 \times 10^{5}$ conidia/ml. After 2 weeks, plant mortality ranged from 17 to $83 \%$, depending on the isolate, whereas none of the controls had died. Several collapsed partially green plants developed a basal crown rot from which Pestalotiopsis was reisolated. Based on spore morphology and host preference, our isolates appear to be P. longisetula, a known pathogen of strawberry fruit and leaves. However, sequencing of the ITS region suggest a close relationship to $P$. clavispora.

Identification of Stewart's wilt of maize (Zea mays L.) caused by Pantoea stewartii subs. stewartii in the State of Mexico, Mexico

M. MEZZALAMA (1), B. Martinez-Cisneros (2), G. Juarez-Lopez (2), N. Valencia-Torres (2)

(1) CIMMYT, Texcoco, Mexico; (2) CIMMYT, Texcoco, Mexico

Phytopathology 105(Suppl. 4):S4.95
Two hundred yellow colonies were isolated from leaf fragments detached from the edges of symptomatic leaves of 19 maize plants showing typical Stewart's wilt, collected in the State of Mexico, in August 2011. The isolates tested positive to ELISA and were characterized with conventional microbiological metabolic and pathogenicity test as Pantoea stewartii subsp. stewartii. The reference strain ATCC 29228 of P. stewartii subsp. stewartii was used as a control. All isolates tested negative to hydrolysis of esculin, indole production and motility. All isolates were positive in real time PCR with the specific primers $340,354,353 F A M, 356$ and 357 . Sequencing of 30 of the 200 isolates was performed with the 16S rRNA gene universal primers $27 f$ and $1495 r$. The 30 sequences were tested on BLAST and they were $99 \%$ identical to $P$. stewartii subsp. stewartii (AF373198.1). Sequences of 17 isolates were published (GenBank Accessions No. from KM508073 to KM508089). The pathogen is regulated in many countries of the world mainly because it is seed borne, although at the very low level. The main vector of the pathogen in the field is the corn flee beetle Chaetocnema pulicaria. In Mexico it has a very restricted distribution and both the pathogen and the vector have been reported before. To our knowledge this is the first time that the presence of the pathogen has been confirmed and identified to a subspecies level by $16 \mathrm{~S}$ rRNA gene sequencing.

Molecular analysis of host resistance and pathogenicity of rice blast in East Africa

E. MGONJA (1), G. L. Wang (1), M. Bellizzi (1)

(1) The Ohio State University, Columbus, OH, U.S.A.

Phytopathology 105(Suppl. 4):S4.95

Despite a number of efforts to improve rice production in East Africa (EA), the average yield is still very low, ranging between 1 to $1.5 \mathrm{t} / \mathrm{ha}$. Rice blast disease caused by a fungus Magnaporthe oryzae is one of the major rice production constraints in most rice growing areas of EA. Genome Wide Association Study (GWAS) uses natural population of lines or germplasm collections and the linkage disequilibrium (LD)-based association to rapidly map target genes in a large collection of diverse genotypes. In our study, we used GWAS to map rice blast resistance genes in 190 African rice cultivars. We conducted virulence and variability tests for $60 \mathrm{M}$. oryzae isolates from EA and identified 6 most virulent isolates, which were used to inoculate a total of 190 rice cultivars. We conducted Genome Wide Association analysis using GAPIT bioinformatics software and identified two quantitative trait loci (QTL), which are strongly associated with the phenotype and may contain resistance genes against $6 \mathrm{M}$. oryzae isolates. This study aimed at generating the information on the level of blast resistance among the East African rice germplasm and determines the variability and pathotypes of $M$. oryzae across the East African region. The results and methodologies used in this study may apply to the disease control for important crops like wheat and corn indirectly because rice blast is the model pathogen system for plant and fungal interactions.

Development of molecular markers for downy mildew (race $F$ of Phytophthora phaseoli) resistance in lima bean

T. T. MHORA (1), R. J. Wisser (2), T. A. Evans (3), E. G. Ernest (4), M. E. Patzoldt (2), N. F. Gregory (3), N. M. Donofrio (3)

(1) Univ of Delaware, Newark, DE, U.S.A.; (2) University of Delaware, NEWARK, DE, U.S.A.; (3) University of Delaware, Newark, DE, U.S.A.; (4) University of Delaware Carvel Research and Education Center, Georgetown, DE, U.S.A.

Phytopathology 105(Suppl. 4):S4.95

Lima bean is the cornerstone of the Delaware vegetable processing industry. This crop is affected by severe downy mildew epidemics caused by the oomycete Phytophthora phaseoli. There are six documented races of this pathogen $(\mathrm{A}-\mathrm{F})$, with $\mathrm{F}$ currently prevalent in fields, creating a need to breed for resistance. $\mathrm{An}_{2}$ population from a cross between a race $\mathrm{F}$ susceptible and resistant parent was used to identify markers linked to race $\mathrm{F}$ resistance. Segregation ratios in the $F_{2}$ indicate a single dominant resistance locus. Genotyping-by-sequencing (GBS) in bulk segregant analysis (BSA) was utilised on this population to identify markers for this locus. A custom reference free bioinformatics pipeline was used to analyse the sequence data and determine candidate single nucleotide polymorphisms (SNP). Filters applied to these candidate SNP loci included statistical validation of allele frequency expectations. Of the 146 loci identified, 12 were statistically significant, exhibiting a signature of phenotypic association. Seven of these loci were successfully converted into Kompetitive Allele Specific PCR (KASP) assays, which were used to genotype all 216 of the aforementioned $F_{2}$ population. Six markers were linked to each other and were significantly associated with race $\mathrm{F}$ resistance. The race $\mathrm{F}$ resistance locus mapped between two markers located $\sim 4.5 \mathrm{cM}$ apart. These markers may be used in breeding programs and will be used to genotype a lima bean diversity panel. 
Investigation of host gene expression modulation by Triticum Mosaic Virus N. MIHELICH (1), R. Roberts (1), K. S. Browning (2), A. M. Rakotondrafara (1) (1) University of Wisconsin-Madison, Madison, WI, U.S.A.; (2) University of Texas - Austin, Austin, TX, U.S.A.

Phytopathology 105(Suppl. 4):S4.96

Viruses have evolved unique strategies to take over the cellular protein synthesis machinery of their host for efficient infection. Although viruses cause serious economic damage to agricultural crops, little is known about their mechanism of translation. The Potyviridae family is the largest and most agronomically influential group of plant viruses. Here we propose to investigate the ability of the recently-emerged Triticum Mosaic Virus (TriMV) to modulate host gene expression to favor its own translation. TriMV stands out within the Potyviridae family, having a long translational enhancer in its 5' untranslated region that has been shown to direct translation stronger than other Potyvirus translational enhancers. TriMV translation is dependent upon the cap-binding eIF4F complex and its isoform eIFiso4F. Our goal is to measure changes in the accumulation of the host translation factors upon TriMV infection in wheat. Better knowledge of the mechanism of viral infection can be a basis for the advancement of crop protection.

A systematic approach to develop species-specific markers for Phytophthora spp. using a mitochondrial locus

T. D. MILES (1), G. Robideau (2), F. N. Martin (3), G. Bilodeau (2), M. Coffey (4)

(1) California State University Monterey Bay, Seaside, CA, U.S.A.; (2) Canadian Food Inspection Agency, Ottawa, ON, Canada; (3) USDA-ARS, Salinas, CA, U.S.A.; (4) UC Riverside, Riverside, CA, U.S.A.

Phytopathology 105(Suppl. 4):S4.96

The genus Phytophthora contains many species that can cause significant damage to agriculture and native ecosystems. A genus and species-specific diagnostic assay has been developed based on mitochondrial gene order differences that allows for the systematic development of species-specific TaqMan probes. Previous research validated this marker system for 14 Phytophthora spp. This research has been developed further by adding over 100 in silico species-specific probes and refined the system by normalizing annealing temperature and probe concentration across all assays. Furthermore, we have fully validated 30 additional species-specific probes against over 136 different taxa in two laboratories and conducted technology transfer experiments on several thermocyclers. All probes were found to be speciesspecific and could be multiplexed with a genus-specific probe and a plant internal control (IC). The lower limit of linear detection using purified DNA was $50-100 \mathrm{fg}$ in all assays. Additionally, a RFLP system for species identification was developed using the restriction enzymes AseI and DraI; this was adapted for terminal RFLP fragment analysis using fluorescently labeled primers to facilitate conducting community analysis. This system represents a comprehensive, hierarchal approach to increase detection capability and provide important tools for community analysis of Phytophthora species.

Systematic development of species-specific assays for important Phytophthora spp. using recombinase polymerase amplification

T. D. MILES (1), F. N. Martin (2)

(1) California State University Monterey Bay, Seaside, CA, U.S.A.; (2) USDA-ARS, Salinas, CA, U.S.A.

Phytopathology 105(Suppl. 4):S4.96

A platform for systematically developing rapid field-deployable isothermal assays for identification of Phytophthora at a genus and species specific level has been developed which focuses on the mitochondrial atp9-nad9 locus utilizing a technique known as recombinase polymerase amplification (RPA). This approach was used to develop species-specific RPA assays for $P$. cinnamomi, $P$. kernoviae, P. ramorum, $P$. rubi, and P. tentaculata. These assays have been validated against 21 Pythium species, 1 Phytopythium sp. and over 100 Phytophthora species. Additionally, these assays were validated for sensitivity by diluting purified DNA from $10 \mathrm{ng}$ down to $1 \mathrm{fg}$. In general most RPA assays were capable of detecting the respective Phytophthora species between a range of $1 \mathrm{pg}-100 \mathrm{fg}$, which is similar to sensitivities with qPCR. When feasible, each assay was also validated with infected plant tissue collected in California. These RPA assays have many benefits over traditional diagnostic techniques because they don't require DNA extraction and can be run directly in the field in less than 25 min. Techniques for sequencing of amplified products have also been developed to confirm species identification.

Development of microsatellite markers in the watermelon pathogen Fusarium oxysporum f. sp. niveum using comparative genomics

N. F. MILLER (1), L. M. Cano (1), L. M. Quesada-Ocampo (1)

(1) North Carolina State University, Raleigh, NC, U.S.A.

Phytopathology 105(Suppl. 4):S4.96
Fusarium wilt of watermelon, caused by Fusarium oxysporum f. sp. niveum (Fon), is one of the most important diseases affecting watermelon; nonetheless, little is known about the population structure of this pathogen. Simple Sequence Repeats (SSRs), or microsatellites, are preferred markers in population studies that can be easily developed using publicly available genomic data. Twelve complete genomes of Fusarium oxysporum isolates have been released, although none of these isolates belong to the forma specialis that infects watermelon. In order to find SSRs likely to be polymorphic in $F$. oxysporum f. sp. niveum for future population studies, all twelve predicted transcriptomes were utilized to find conserved microsatellites. The Fusarium oxysporum f. sp. lycopersici $(\mathrm{Fol})$ transcriptome was used as a reference, and blastn alignments were used to identify orthologous transcripts across the twelve isolates. The MIcroSAtellite identification tool (MISA) was used to extract repeats of 1-6 bp from orthologous transcripts for each isolate. Identified SSRs in the individual MISA outputs were mapped to the reference Fol transcriptome to identify conserved and polymorphic SSRs. 96 SSR-containing transcripts were conserved among all 12 isolates, and 68 of these were polymorphic among isolates. After validation, these 68 conserved polymorphic transcripts will provide a valuable resource for Fon population studies.

Preliminary assessment of differential susceptibility of soft red winter wheat cultivars to Lolium and Triticum pathotypes of Magnaporthe oryzae

K. MILLS (1), P. Paul (2), L. Madden (2), G. Peterson (3)

(1) Ohio State Univ, Orrville, OH, U.S.A.; (2) The Ohio State University, Wooster, OH, U.S.A.; (3) USDA ARS Foreign Disease-Weed Science Research Unit, Fort Detrick, MD, U.S.A.

Phytopathology 105(Suppl. 4):S4.96

In South America, wheat blast epidemics caused by the Triticum pathotype of Magnaporthe oryzae (MoT) have resulted in yield loss as high as $40-100 \%$. In 2011, wheat blast, incited by the Lolium pathotype of M. oryzae (MoL) was identified on a spike in Kentucky, suggesting that MoL could pose a threat to wheat production in the US. Subsequent pathogenicity studies have shown that some MoL isolates were indeed just as virulent as MoT on US hard red winter wheat. Two studies were conducted to compare the reactions of 86 northern soft red winter wheat (SRWW) cultivars to a Brazilian MoT isolate (T-25) and a US MoL isolate (P2-1). The MoT study was conducted in a BSL3 facility at Fort Detrick, MD, and the MoL study in growth chambers in Wooster, OH. In both studies, 5-25 spikes of each cultivar were sprayinoculated, and then incubated for $48 \mathrm{hrs}$ at high relative humidity and temperatures between 24 and $26^{\circ} \mathrm{C}$. Blast severity was quantified as percent diseased spikelets. SRWW cultivars exhibited a wider range of reactions to MoL than to MoT. For MoL, average severity ranged from 2 to $78 \%$, with a mean of $27 \%$, compared to 70 to $100 \%$ for MoT, with a mean of $92 \%$. These results suggest that some SRWW cultivars may be partially resistant to MoL but less so to MoT. MoL may pose a threat to SRWW production in the US if conditions are conducive for disease development and a susceptible cultivar is planted.

Complete genome of the select agent Rathayibacter toxicus isolate SA0304 from South Australia

A. MOHAMMAD (1), G. Y. Busot (1), R. Mann (2), B. Rodoni (2), S. Liu (1), J. P. Stack (1)

(1) Department of Plant Pathology, Kansas State University, Manhattan, KS, U.S.A.; (2) Department of Primary Industries, La Trobe University, Bundoora, Australia

Phytopathology 105(Suppl. 4):S4.96

The Select Agent Rathayibacter toxicus is a gram-positive, toxin-producing bacterium that causes morbidity and mortality in livestock that consume contaminated annual ryegrass (Annual Ryegrass Toxicity, ARGT). Livestock deaths occur annually in Australia due to ARGT raising concerns about the potential global spread of $R$. toxicus in ryegrass hay and seed. A genetic analysis identified three distinct populations of $R$. toxicus in Australia, including one not previously reported. Whole-genome sequencing was undertaken to develop a better understanding of this new population collected in South Australia in March 2014. A complete genome of $R$. toxicus isolate SA03-04 (population-A) was obtained through de novo assembly (HGAP3 assembly pipeline: Hierarchical Genome Assembly Process) using 101X depth of PacBio sequences. The $R$. toxicus genome is a single chromosome $2.369 \mathrm{Mb}$ in size with $61.4 \% \mathrm{GC}$ content. The genome annotation identified 2290 coding sequences (CDS) including, 45 tRNA CDS and 6 rRNA CDS (LSU rRNA, SSU rRNA and 5S rRNA were 3117-, 1486- and 121-bp in length, respectively). Genome analyses will provide a better understanding of the genetic constitution and evolutionary history of this high consequence bacterium. Signature genomic regions are being identified to develop effective diagnostic tools for plant biosecurity applications. 
Early detection of Huanglongbing using mass spectrometry-based proteomics and machine learning

J. MOHR (1), J. Chavez (2), J. Ramsey (3), J. Mahoney (4), T. Thannhauser (5), K. Howe (5), M. Alexander (1), K. Godfrey (6), E. Chin (6), C. Slupsky (6), J. Bruce (2), M. Cilia (5)

(1) Cornell University, Ithaca, NY, U.S.A.; (2) University of Washington, Seattle, WA, U.S.A.; (3) Boyce Thompson Institute, Ithaca, NY, U.S.A.; (4) Boyce Thompson Institute, Ihtaca, NY, U.S.A.; (5) USDA-ARS, Ithaca, NY, U.S.A.; (6) UC Davis, Davis, CA, U.S.A.

Phytopathology 105(Suppl. 4):S4.97

Huanglongbing (HLB) is the most serious disease of citrus and is associated with infection by Candidatus Liberibacter asiaticus (CLas). Typically, infected trees have no visible symptoms for the first few months, making it difficult to correctly diagnose infected trees. Current detection strategies rely on real-time, quantitative PCR for detection of CLas 16s rDNA, but the success of this approach depends on the presence of a sufficiently high titer of CLas in the host tissue sampled. Measuring systemic changes in plant proteins in response to HLB offers an opportunity for early detection of HLB infection that does not rely on direct detection of the pathogen. Using tandem mass tag labeling and high resolution mass spectrometry, we constructed proteomic profiles of HLB-positive Lisbon Lemon at two-week intervals over the first several months of infection. Samples from the same set of trees were analyzed by RNAseq to generate an annotated database of expressed genes and predicted proteins to facilitate analysis of proteomic data. Proteins differentially expressed between control and infected samples were identified at each time point, and evaluated for their use as potential biomarkers. A drastic change in protein response between control and infected lemon trees was measured at 10 -weeks post-grafting. A support vector machine model was generated and trained using selected differentially expressed proteins, which may improve early detection in field samples.

Discovery and profiling of small RNAs responsive to stress conditions in the plant pathogen Pectobacterium atrosepticum

L. N. MOLELEKI (1), S. Kwenda (1), V. Gorshkov (2), E. Rubagotti (1), P. Birch (3)

(1) University of Pretoria, Pretoria, South Africa; (2) Kazan Institute of Biochemistry and Biophysics, Kazan Scientific Center, Russian Academy of Sciences, Kazan, Russia, Kazan, Russia; (3) The James Hutton Institute, Dundee, Scotland

Phytopathology 105(Suppl. 4):S4.97

Small RNAs (sRNAs) have emerged as important regulatory molecules and have been studied in several bacteria. However, to date, there have been no whole-transcriptome studies on sRNAs in any of the Soft Rot Enterobacteriaceae (SRE) group of pathogens. Although the main ecological niches for these pathogens are plants, a significant part of their life cycle passes in adverse soil environments where mechanisms of survival and adaptation are still poorly understood. In this study strand-specific RNA-seq analysis and in silico sRNA predictions revealed numerous sRNA candidates in Pectobacterium atrosepticum including those that are induced during starvationactivated stress response. This approach enabled detection of 137 known sRNAs and sRNA candidates under starvation conditions; 25 of these sRNAs were predicted for this bacterium in silico. Functional annotations were computationally assigned to $68 \mathrm{sRNAs}$. The expression of sRNAs in $P$. atrosepticum was compared under growth-promoting and starvation conditions: 63 sRNAs were differentially expressed with 57 sRNAs up-regulated under nutrientdeficient conditions. Conservation analysis indicated that most of the identified sRNAs are conserved within the SRE. Subsequently, we identified 9 novel sRNAs within the $P$. atrosepticum genome. A large number of these sRNAs is starvation-induced, suggesting their role in bacterial adaptive response.

Forensic application of SSR typing to identify probable sources of Fusarium proliferatum strains associated with salmon blotch of onions I. R. Moncrief (1), C. D. Garzon (2), S. M. Marek (2), A. Gamliel (3), J. P. Stack (4), Y. Isack (3), J. FLETCHER (2)

(1) Harry S. Truman College, Chicago, IL, U.S.A.; (2) Oklahoma State University, Stillwater, OK, U.S.A.; (3) ARO Volcani Institute, Bet Dagen, Israel; (4) Kansas State University, Manhattan, KS, U.S.A.

Phytopathology 105(Suppl. 4):S4.97

Microbial forensics tools can help to identify potential pathogen sources. Since 2008, unprecedented outbreaks of salmon blotch of onion, caused by the mycotoxigenic fungus Fusarium proliferatum $(F p)$, have occurred in southern Israel. Though presumed to be of natural causes, the disease allowed us to validate plant pathogen forensic tools in a field application. To test whether onion sets grown in northern Israel and shipped to southern farms for planting could be a source of the fungus, the diversity and structure of $F p$ populations from different locations and sample matrices were assessed by simple sequence repeat (SSR) typing. $F p$ isolates $(\mathrm{n}=216)$ from onion sets, field soil, nearby date palm orchards, weeds, local vegetation, and onion bulbs were characterized. Of 17 SSR loci screened with genomic DNA from all $F p$ isolates, six amplified a range of isolates from different locations and hosts. Resulting PCR amplicons were analyzed by capillary electrophoresis. Data analysis (UPGMA, AMOVA, and STRUCTURE) revealed two distinct $F p$ populations: 1) northern Israel isolates from onion sets, and 2) southern Israel isolates from onion bulbs, soil, weeds, or palm trees. That the two populations were genetically distinct eliminates the northern-grown onion sets as the source of the $F p$ causing salmon blotch in southern Israel. The work demonstrated that SSR typing is useful for fungal strain discrimination and can be useful for forensic investigation of plant diseases.

Frequency of pathogenic fungi isolated from symptomatic grapevines in California

J. Monis (1), L. A. MILIES (2), M. Vernon (1)

(1) Eurofins/STA Laboratories Inc, Gilroy, CA, U.S.A.; (2) Eurofins STA Laboratories, Gilroy, CA, U.S.A.

Phytopathology 105(Suppl. 4):S4.97

Grapevine vascular diseases caused by fungal pathogens are detrimental to vineyard establishment and production. A diversity of fungal species were recovered from symptomatic grapevine samples processed in our laboratory during the 2013 and 2014 calendar year. The grapevine samples included sections of root, rootstock, graft union, trunk and cordon. Symptomatic samples were dissected, surface-sterilized, and plated onto media developed for the isolation of fungi. Fungal growth was observed, sub-cultured as necessary, and incubated for a period of 2-4 weeks to allow for sporulation. Recovered fungi were identified based on colony morphology, microscopic examination, and further molecular sequencing. The most frequently isolated fungi belonged to the Botryosphaeriaceae and Diatrypaceae families, Cylindrocarpon, Phaeoacremonium, Phaeomoniella, and Phomopsis genera. Additionally, significantly more fungal species of the genera Cylindrocarpon, Phaeoacremonium, and Phaeomoniella were isolated from the lower sections of the vines. Botryosphaeriaceae and Diatrypaceae and Phomopsis spp. were isolated from sections above the graft union. The observed trends and frequency of detection of specific pathogenic fungi in the vine sections will be further discussed.

Effects of application parameters on spray deposition and uniformity on wheat stem

W. B. MORAES (1), H. E. Ozkan (2), D. C. Richard (3), H. Zhu (3), L. V. Madden (1), P. A. Paul (1)

(1) Department of Plant Pathology, The Ohio State University, Wooster, OH, U.S.A.; (2) Department of Food, The Ohio State University, Columbus, OH, U.S.A.; (3) USDA-ARS Application Technology Research Unit, Wooster, OH, U.S.A.

Phytopathology 105(Suppl. 4):S4.97

Recent concerns about stem rust, caused by Puccinia graminis f. sp. tritici, have led to questions being asked about fungicide application technology to adequately protect the wheat stem. Two field experiments were conducted in 2010 and 2011 to evaluate the effects of application parameters on spray deposition and uniformity on wheat stem. Dye was applied to separate plots at head emergence using a Hagie Model 254 self-propelled agricultural sprayer with a 15 -m-long boom and different combinations of nozzles, droplet sizes, nozzle orientation, boom pressures and heights, drive speeds, and spray volumes, with and without the aid of an air assisted spray system. Stems were harvested and dye quantified. Bayesian and spatial analyses were used to determine the uniformity of spray distribution on stems. The lowest amplitude of the highest posterior density interval of the mean and variance parameters were observed in the marginal posterior distribution of the treatment in which medium droplets were applied without air, with the boom at $45 \mathrm{~cm}$ above the canopy at $165.5 \mathrm{kPa}, 11.3 \mathrm{Km} \cdot \mathrm{h}^{-1}$, and $93.5 \mathrm{~L} \cdot \mathrm{ha}^{-1}$. The spatial distribution of dye was most uniform with this treatment, covering 80 to $92 \%$ of the plot with between 4 and $6 \mu \mathrm{L} \cdot \mathrm{g}^{-1}$ of dye in 1 or 3 continuous areas. Additionally, this was the only treatment for which there was no spatial dependence, suggesting the lack of spray patches with significant structures in the lower canopy, and hence uniform coverage of the stems.

First report of the teleomorph of Neofusicoccum mediterraneum, a pathogen of olive

J. Moral (1), M. Pérez Rodríguez (2), T. MICHAILIDES (3), A. TraperoCasas (2)

(1) Univ De Cordoba, Cordoba, Spain; (2) University of Cordoba, Cordoba, Spain; (3) University of California-Davis Kearney Agricultural Research and Extension Center, Parlier, CA, U.S.A.

Phytopathology 105(Suppl. 4):S4.97 
Neofusicoccum mediterraneum causes very devastating diseases such as shoot blight of olive and panicle and shoot blight of pistachio. The life cycles of these diseases are based on the asexual stage (conidial inoculum) of the pathogen since no sexual spore-producing structures have been observed. In 2010 and 2011, we monitored blighted olive branches in an orchard located at southern Spain. The fruiting structures from 50 branches were removed and $25 \%$ were identified morphologically as $N$. mediterraneum, $12 \%$ as Cytospora sp., and $1.3 \%$ as pseudothecia that belonged to genus Botryosphaeria. The asci measured $87.7-158 \times 18.6-21.3 \mu \mathrm{m}$ and were clavate and bitunicate; the ascospores measured (15.8-)20.0-21.7(-26.5) $\times(5.9-) 8.4-9.4(-11.1) \mu \mathrm{m}$ and were rhomboid, smooth, hyaline and aseptate. The monoascosporic isolates showed a complete homology with $N$. mediterraneum according to ITS and EF regions. Moreover, crosses of eight brother ascospores were performed on water agar (WA) and PDA media and olive leaves and shoots on WA plates, which were incubated at several temperatures $\left(10-25^{\circ} \mathrm{C}\right)$ and humidities. Likewise, inoculated branches were hung on olive trees far from other inoculum sources for 2.5 years. Pseudothecia of $N$. mediterraneum were developed only on inoculated branches hung on the trees after 2 years. This is the first time that the teleomorph of $N$. mediterraneum is observed worldwide, a fact that justifies reappraising the current life cycle of this pathogen.

Investigation of the potential disease complex concerning Pythium aphanidermatum and Meloidogyne incognita on cucumber K. A. MORRIS (1)

(1) University of Georgia, Tifton, GA, U.S.A.; (2) Virginia Tech, Suffolk, VA, U.S.A.

Phytopathology 105(Suppl. 4):S4.98

A growth chamber trial in 2011 demonstrated a possible disease complex between Pythium aphanidermatum (PA) and Meloidogyne incognita (RKN) on cucumber. Two growth chamber trials were conducted in 2014 to investigate this potential disease complex. Treatments included cucumber seedlings inoculated with: PA alone, RKN alone, PA + RKN, and an untreated control. Each pathogen was inoculated to 14 day old cucumber seedlings that were planted into $5 \mathrm{~cm} \times 25 \mathrm{~cm}$ cone-tainers containing a soil mixture of 3 parts sand, 3 parts sterilized field soil, and 1 part germinating mix. Inoculated pots received $1500 \mathrm{~J} 2$ stage RKN juveniles or $100 \mathrm{~g}$ of sand-corn meal PA inoculum. Once inoculated, cucumbers were placed in a growth chamber at 28 $\mathrm{C}, 12 \mathrm{~h}$ photoperiod, and $75 \%$ humidity. Plants were monitored daily for 3 weeks for symptoms of PA infection. The presence of PA was determined on symptomatic plants isolating hypocotyl sections onto PDA and observing the colony morphology and microscopic characteristics of PA. In each trial, plants inoculated with both pathogens demonstrated significantly greater plant death than pots inoculated with either pathogen alone. A significant plant vigor reduction was noted in trial 2 when the two pathogens occur together in the soil. These results indicate that a disease complex between RKN and PA exists in cucumber.

\section{Assessment of an antimicrobial peptide source to control Pseudomonas plant pathogens}

E. A. MOYA-ELIZONDO (1), T. P. Quezada (2), M. Reyes Salinas (3), M. A. Reyes Navarro (3)

(1) Universidad de Concepcion, Chillán, Chile; (2) Universidad de Concepción, Chillán, Chile; (3) Avance Biotechnologies Chile S.A., Santiago, Chile

Phytopathology 105(Suppl. 4):S4.98

Pseudomonas syringae pv. syringae (Pss) is responsible of the stone canker disease on cherry fruit plants. Antimicrobial peptides (AMPs) are small and variety of proteins with activity on lipid bilayer of bacterial membrane produced by multicellular organisms. The biopesticide $3 \mathrm{Tac}-\mathrm{I} / \mathrm{Beta}$ is based in the action of three Trichoderma strains with bactericidal action on stone cherry canker. AMP activity of this product on bacterial membrane of Pss was assessed by using Transmission Electronic Microscopy (TEM). Effect on Pss population was assessed by using in vitro tests and pot and field experiments. TEM study showed a marked damaged on the membrane of Pss, affecting the shape of the bacterium by changing the continuity of the membrane. In vitro tests showed formation of inhibition halos around disc treated with the filtered product on a bacterial lawn. Bacterial growth, expressed as optical density of cell culture, was reduced in around a $12 \%$ when was used 3 Tac-I/Beta, while a concentrated AMP solution of the filtered product showed reduction around $34.8 \%$ at 18 hours of co-culture. Preventive spraying of 3 Tac-I/Beta on cherry plants wounded and inoculated with Pss $24 \mathrm{~h}$ after the spraying showed differences in canker longitude with the untreated control and was not different of copper based treatments $(\mathrm{P}<0.10)$. Bud damage reduction was observed in a field trial $(\mathrm{P}<0.05)$. Results suggest that $3 \mathrm{Tac}-\mathrm{I} / \mathrm{Beta}$ is a new alternative to control cherry canker.
Relationships between fungal communities of the crown and genotypes of 2,4-DAPG and phenazine-producing Pseudomonas bacteria in Chilean wheat crops

E. A. MOYA-ELIZONDO (1), H. A. Doussoulin (2), N. L. Arismendi (1)

(1) Universidad de Concepción, Chillán, Chile; (2) Universidad Austral de Chile, Valdivia, Chile

Phytopathology 105(Suppl. 4):S4.98

2,4-diacetylphloroglucinol (2,4-DAPG) and phenazine (PCA)-producing Pseudomonas have been associated with control of wheat crop pathogens, but the relationship between communities of pathogens and genotypes of these bacteria has been little studied. Frequency of fungi associated with the crown of wheat, incidence and severity that they cause, and their relationships with the presence of 2,4-DAPG and PCA-producing Pseudomonads were evaluated in four commercial wheat crops located in La Araucania and Los Lagos Regions, during two crops seasons. Portions of the first culm internode were cultured in an artificial medium for fungi isolation. Roots were used for the detection of 2,4 DAPG and PCA-producing Pseudomonas spp. by PCR with specific primers of phlD and phzCD genes. Phaeosphaeria, Fusarium, Rhizoctonia, and Microdochium were repeatedly isolated $(52.6 \%, 22.1 \%$, $7.8 \%$, and $4.9 \%$, respectively). Genetic composition of 2,4 DAPG and PCAproducing Pseudomonas spp. varied between fields and sampling periods. Genetic groups $\mathrm{A}, \mathrm{B}, \mathrm{D}, \mathrm{K}, \mathrm{L}$, and $\mathrm{P}$ associated to the phlD gene were detected. Presence of 2,4-DAPG-producing bacteria benefited health of the crop, relating their existence with increasing yield and plant height, and reduction of incidence and severity of microorganisms on the first internode. This phenomenon showed a synergistic effect in those fields that had a greater diversity of crown fungi under environmental conditions of southern Chile.

\section{Small RNA from the wheat stripe rust fungus Puccinia striiformis f.sp.} tritici

N. A. MUETH (1), S. H. Hulbert (1)

(1) Washington State University, Pullman, WA, U.S.A.

Phytopathology 105(Suppl. 4):S4.98

Wheat stripe rust (Puccinia striiformis $f$. $s p$. tritici) is a costly global disease that burdens farmers with yield loss and high fungicide expenses. This sophisticated parasite develops haustoria inside living host cells, meanwhile suppressing the plant immune system. Understanding the genetic factors behind pathogen development may be the key to more effective control strategies. Developmental processes in most eukaryotes are regulated by small noncoding RNA molecules (sRNA), yet some fungi lack RNA interference (RNAi) capabilities. We extracted RNA from infected wheat leaves and confirmed that genes required for RNAi are expressed in stripe rust. Then, we size-selected for small RNA and performed deep sequencing. Reads likely to be of fungal origin were identified by mapping to the stripe rust genome and excluding reads present in uninfected controls. Sequencing and bioinformatics results were verified by RT-PCR. The $P$. striiformis sRNA library has all the features of functional small RNAs from known RNAi-equipped species, including microRNA-like sequences that originate from hairpin-shaped precursors. Finally, sRNA-target prediction software was used to identify possible target genes in both stripe rust and wheat. To our knowledge, this is the first report of small RNAs from any Puccinia species. These findings lay the groundwork for functional analysis of gene regulation on both sides of the wheat/stripe rust interaction.

Molecular epidemiology of cassava mosaic geminiviruses in Zambia

R. M. MULENGA (1), J. Ndunguru (2), P. C. Chikoti (1), D. W. Miano (3), J. P. Legg (4), O. J. Alabi (5)

(1) Zambia Agriculture Research Institute, Mount Makulu Central Research Station, Chilanga, Lusaka, Zambia; (2) Mikocheni Agricultural Research Institute, Dar-Es-Salaam, Tanzania; (3) University of Nairobi, College of Agriculture and Veterinary Sciences, Department of Plant Sciences and Crop Protection, Kangemi, Nairobi, Kenya; (4) International Institute of Tropical Agriculture, Dar es Salaam, Tanzania; (5) Department of Plant Pathology \& Microbiology, Texas A\&M AgriLife Research and Extension Center, Weslaco, TX, U.S.A.

Phytopathology 105(Suppl. 4):S4.98

A diagnostic survey was conducted between April and May 2014 to determine the status of cassava mosaic geminiviruses in Zambia. Cassava mosaic disease (CMD) symptoms were mostly mild to moderately severe across farmers' fields in Western, Northwestern, Northern, Luapula, Lusaka and Eastern provinces of Zambia although plants with very severe symptoms were also observed in Eastern, Lusaka and Luapula provinces. Sixty percent of the 226 survey samples were positive in PCR using primers amplifying a $550 \mathrm{bp}$ product specific to the core coat protein $(\mathrm{CCP})$ region of most geminiviruses. Sequence analysis of the cloned $550 \mathrm{bp}$ products revealed the presence of 
African cassava mosaic virus (ACMV), East African cassava mosaic virus (EACMV) and East African cassava mosaic Malawi virus (EACMMV) as single or mixed infections of different proportions in the CCP-positive samples. Further analysis showed that $59.6 \%$ of these samples had single infection of ACMV $7.4 \%$ had EACMMV alone, $1.5 \%$ had EACMV alone, $27.5 \%$ had ACMV+EACMMV and 3.8\% had ACMV+EACMV. Rolling circle amplification and sequence analysis of the complete DNA A genome of three EACMMV isolates showed that they shared $98.2-99.9 \%$ identity among themselves, 97.9-98.4\% with DNA A of EACMMV isolate MK (AJ006460), and 98.2-98.7\% with EACMMV isolate MH (AJ006459). The results provide the first molecular evidence for the presence of ACMV, EACMV and EACMMV in Zambia and represent the first report of EACMMV in Zambia.

Extent and severity of Caliciopsis canker in New England, U.S.A: An emerging disease of eastern white pine

I. Munck (1), K. BRODERS (2), W. Livingston (3), K. Lombard (4), T. Luther (5), W. Ostrofsky (6)

(1) USDA Forest Service, Durham, NH, U.S.A.; (2) Department of Biological Sciences, University of New Hampshire, Durham, NH, U.S.A.; (3) Scool of Forest Resources, University of Maine, Orono, ME, U.S.A.; (4) New Hampshire Department of Resources and Economic Development, New Hampshire Division of Forests and Lands, Concord, NH, U.S.A.; (5) NA State and Private Forestry, USDA Forest Service, Durham, NH, U.S.A.; (6) Maine Forest Service, Maine Department of Agriculture, Conservation and Forestry, Augusta, ME, U.S.A.

Phytopathology 105(Suppl. 4):S4.99

Caliciopsis canker is an emerging problem in pine growing regions of eastern North America where Caliciopsis pinea infects eastern white pine (Pinus strobus), $P$. echinata, and $P$. virginiana. In Europe, the pathogen infects $P$. pinaster and $P$. radiata. Although reports of damage caused by $C$. pinea were common in the 1930s, since then there has been little progress towards understanding its epidemiology. The objective of this study was to identify areas at greatest risk of $C$. pinea damage. During 2014, 58 sites across New England with $>75 \%$ white pine basal area were surveyed. Most sites $(72 \%)$ had Caliciopsis canker signs or symptoms. In sites with Caliciopsis canker, $36 \%$ of the overstory pines were symptomatic. Poletimber was more likely to be symptomatic ( $50 \%$ trees) than sawtimber ( $30 \%$ trees) $(\alpha=0.05)$. White pine density for sites with Caliciopsis canker was 311 trees/ha compared to 220 trees/ha for sites without Caliciopsis canker $(\mathrm{p}=0.1)$. Caliciopsis canker symptoms occurred more frequently $(\mathrm{p}=0.1)$ in sites with excessively drained, coarse textured soils derived from glacial outwash $(86 \%)$ or sites with poorly drained soils and low fertility (78\%) than in sites with well drained, more fertile soils $(59 \%)$. This is a problem for forest managers because coarse textured soils derived from glacial outwash are common in New England and white pine is the only significant commercial species to grow well on these sites where soil fertility is too poor for hardwoods.

\section{Study of new grapefruit cybrids for potential citrus canker resistance in} Florida

M. M. MURATA (1), C. D. Chase (1), A. A. Omar (2), J. Grosser (2), J. H. Graham (2)

(1) University of Florida, Gainesville, FL, U.S.A.; (2) Citrus Research and Educational Center (CREC) - University of Florida, Lake Alfred, FL, U.S.A. Phytopathology 105(Suppl. 4):S4.99

Host resistance is the most desirable strategy for control of citrus canker caused by Xanthomonas citri subsp. citri. A process called cybridization, the combination of cytoplasm and nucleus from different species, is a useful somatic hybridization approach for Citrus improvement. Several putative cybrids were created through the fusion between nucellar callus cells of Meiwa kumquat (Fortunella crassifolia), a citrus canker resistant species, and mesophyll cells of three selections of grapefruit (Citrus paradisi), a citrus canker susceptible. Four mitochondrial (mt) introns, a mt ribosomal RNA spacer region, and four chloroplast (cp) DNA regions were selected to genotype the generated clones for cytoplasmic DNA polymorphisms between kumquat and grapefruit. For disease resistance screening, a pick-point inoculation assay was processed to evaluate the response of 11 cybrids clones to Xcc. Mt intron nad7i2, mt rRNA spacer and two cp DNA regions confirmed cybrids with $\mathrm{mt}$ genome and $\mathrm{cp}$ genome originating from the nucellar callus parent kumquat. The inoculation assay was performed twice and showed variation for citrus canker resistance among the cybrid clones. Two clones presented significantly smaller lesions after pick-point inoculation. More clones are under evaluation. These cybrids have the potential to provide citrus canker resistance in commercial grapefruit varieties and contribute to the study of cytoplasmic organelles in plant disease resistance.
Evaluation of the effects of soybean seed treatments on yield and soybean cyst nematode (Hetrodera glycines) populations

K. M. MUSIL (1), N. J. Arneson (1), L. J. Giesler (1)

(1) University of Nebraska-Lincoln, Lincoln, NE, U.S.A.

Phytopathology 105(Suppl. 4):S4.99

There have recently been commercial seed treatments released that are registered as being able to reduce soybean cyst nematode populations (SCN). During the 2014 growing season these seed treatments were evaluated for their effects on yield and SCN population density (eggs/100 cc's of soil). Four fields were located across Nebraska, where three were infested with SCN and the fourth field was non-infested. The three seed treatments were Cruiser Maxx Advanced (Syngenta), Clariva Complete Beans (Syngenta), and Poncho VOTiVO (Bayer Crop Science). All three seed treatment combinations contained the same fungicides and an insecticide with the same mode of action. Clarivia Complete Beans and Poncho VOTiVO also contained an additional component that is a microbial agent. Clarivia pn (Pasteuria nishizawae) is the active ingredient in Clarivia Complete Beans, while Poncho VOTiVO contains Bacillus firmus as its active ingredient for the microbial component. Average yields in the SCN infested fields ranged from 45 to 55 $\mathrm{bu} / \mathrm{A}$ and initial SCN population densities ranged from 1,100 to 4,300 eggs/100 cc's of soil. No statistical differences were found among the three treatments in either yield or SCN reproduction at any individual location or when the SCN infested locations were combined. Based on the results of this study, more field evaluations are needed to determine if the addition of microbial agents to chemical seed treatment are effective and profitable for SCN management.

Phylogenetic analysis of the genus Xanthomonas by comparison of partial 23S ribosomal RNA gene sequences

I. S. MYUNG (1), M. J. Yoon (1), J. Y. Lee (2), Y. K. Lee (2), H. Shim (2)

(1) National Academy of Agricultrual Science, Wanju, South Korea; (2)

NAAS, Wanju, South Korea

Phytopathology 105(Suppl. 4):S4.99

Phylogenetic relationship of 28 reference species of the genus Xanthomonas was assessed based on analysis of the 23S rDNA sequences. The levels of sequence similarity among all of the Xanthomonas species were $98.85 \%$ $\pm 1.0 \%$. The phylogeny indicates a classification with two main groups. Group I was separated into further clades (IA and IB), supported by a high bootstrap value of $100 \%$. Group IA comprised $X$. citri $^{\mathrm{T}}, X$. fuscans $^{\mathrm{T}}, X$. alfalfae ${ }^{\mathrm{T}}, X$. euvesicatoria $^{\mathrm{T}}, X$. perforans $^{\mathrm{T}}, X$. melonis $^{\mathrm{T}}$ and $X$. codiaei $^{\mathrm{T}}$, whilst group IB comprised $X$. axonopodis ${ }^{\mathrm{T}}, X$. oryzae $^{\mathrm{T}}, X$. vasicola $^{\mathrm{T}}, X$. bromi $^{\mathrm{T}}, X$. campestris $^{\mathrm{T}}$, $X$. dyei $^{\mathrm{T}}, X$. fragariae $^{\mathrm{T}}, X$. arboricola $^{\mathrm{T}}, X$. cassavae $^{\mathrm{T}}, X$. cucurbita $^{\mathrm{T}}, X$. pisi $^{\mathrm{T}}$, $X$. cynarae $^{\mathrm{T}}, X$. hortorum $^{\mathrm{T}}, X$. gardneri $^{\mathrm{T}}$, and $X$. vesicatoria ${ }^{\mathrm{T}}$. Group II contained $X$. translucens $^{\mathrm{T}}, X$. hyacinth $^{\mathrm{T}}, X$. theicola $^{\mathrm{T}}, X$. sacchari $^{\mathrm{T}}$ and $X$. albilineans ${ }^{\mathrm{T}}$. The sequence similarity between the species in the cluster IA was $99.84 \pm 0.11 \%$ corresponding to $4.10 \pm 3.05$ nucleotide differences (ND). Cluster IB exhibited $99.69 \pm 0.15 \%$ corresponding to $9.31 \pm 4.36$ ND. The cluster II exhibited $98.95 \pm 0.41 \%$ corresponding to $28.40 \pm 11.20 \mathrm{ND}$. A phylogenetic tree discriminated all the type strains of Xanthomonas, except for $X$. alfalfa and $X$. euvesicatoria, which had the same sequence. The $23 \mathrm{~S}$ rRNA gene can be used for identifying strains of the genus Xanthomonas in species level. In addition the primer sets used in this study can be used for phylogenetic relationships of the genera Stenophomonas and Xylella.

Spatiotemporal population structure of Pseudoperonospora cubensis isolates in Michigan and Ontario, Canada

R. NAEGELE (1), L. M. Quesada-Ocampo (2), J. Kurjan (3), C. Saude (4), M. K. Hausbeck (3)

(1) Michigan State Univ, East Lansing, MI, U.S.A.; (2) North Carolina State University, Raleigh, NC, U.S.A.; (3) Michigan State University, East Lansing, MI, U.S.A.; (4) Canadian Tobacco Research Foundation, Tillonsburg, ON, Canada

Phytopathology 105(Suppl. 4):S4.99

Cucurbit downy mildew (CDM), caused by the oomycete pathogen Pseudoperonospora cubensis, is a devastating disease that affects cucurbit species worldwide. Many cucurbits can be infected by this pathogen, but cucumbers are the most susceptible. Michigan is the number one producer of cucumbers for pickling in the U.S., and Ontario, Canada produces approximately $75 \%$ of the nation's greenhouse-grown cucumbers. This pathogen is dispersed by wind currents into Michigan or other northern regions from overwintering sites and introduced annually. Similar population structure among $P$. cubensis populations was identified within the northern U.S. and Canada, but not between the northern and southern U.S. in previous studies. To further evaluate the regional and temporal population structure of P. cubensis in Michigan and Ontario, Canada, sporangia from CDM lesions were collected from field-grown cucurbit foliage in 2011. Population structure 
and genetic diversity were assessed in 262 isolates using nine simple sequence repeat (SSR) markers. Five genetic clusters were detected and change in population structure varied by site and sampling date within a growing season.

Protein signatures from wild type and reduced genomic compliments of Rhizoctonia solani isolates

B. Narayanaswamy (1), J. BIRCH (1), S. Bharathan (1), M. Chey (1), R. Connacher (1), B. Michalides (1), A. Long (1), M. Cubeta (2)

(1) Indiana Univ of PA, Indiana, PA, U.S.A.; (2) North Carolina State Univ, Raleigh, NC, U.S.A.

Phytopathology 105(Suppl. 4):S4.100

Amongst genetically isolated anastomosis groups (AG's), Rhizoctonia solani, a soil-borne, plant pathogenic fungus, shows an increased genetic variability between these groups. Sub-groups of these AG's groups can be further differentiated via colony morphology, virulence, genetic sequences, biochemical characteristics and host ranges. Data from specific protocols and standard operating procedures developed for protein purifications of filamentous fungi using ToPI-DIGE kit will be presented. Proteins were purified from select $R$. solani isolates that included wild-type, heterokaryons, and compared to those that had reduced genomic complements, homokaryons. The concentration and quality of the protein were assayed using BeckamCoulter DU 800 Spectrophotometer. All such high-quality proteins were subjected to Agilent Protein Chip Technology protein separation and 2Dprotein profiling. Preliminary results suggests considerable variation in the protein from the homokaryon and the wild-type isolates. Proteins Spot statistics data from 2D-gel preparations indicated nearly $81 \%$ of the proteins were similar while there was an under-expression of $10.4 \%$ in the wild-type and $8.7 \%$ over-expression in the homokaryon. Additional comparisons are being made between viral-infected versus non-infected $R$. solani isolates.

The potential global climate suitability of Huanglongbing (HLB) and its vector (Asian citrus psyllid) using two correlative models

H. A. NAROUEI KHANDAN (1), S. Halbert (2), A. van Bruggen (1)

(1) University of Florida, Gainesville, FL, U.S.A.; (2) Florida Department of Agriculture and Consumer Services, Division of Plant Industry, Gainesville, FL, U.S.A.

Phytopathology 105(Suppl. 4):S4.100

Two species distribution models (SDMs), MaxEnt and Multi-Model, were used to predict the global and local potential distribution of HLB caused by Candidatus Liberibacter asiaticus and its vector Asian citrus psyllid (ACP). The current global distribution of HLB and ACP was gathered from online databases, literature review and personal communications with specialists. The long-term climate data (19 bio-climatic variables) were sourced from the Worldclim website. The USA occurrence data of HLB and ACP were not used in model calibration to evaluate the model predictions at independent locations. Both models successfully predicted Florida as highly suitable for HLB and ACP establishment, which is in agreement with the rapid spread and current distribution of HLB and ACP in Florida. The models also predicted that a limited area in California is climatically favorable for HLB establishment, but the probability of establishment was predicted to be much lower compared to Florida. Principal Component Analysis was also used to investigate the climate similarity of regions with proven HLB presence with the California and Florida climate. PCA analysis showed that the climate in areas around Los Angeles overlapped with the climate of regions where HLB is currently present. On a global scale, HLB predictions from SDMs combined with expert knowledge could be informative for countries such as Australia, New Zealand, and European countries, where HLB has not been reported thus far.

In vitro assessment of foliar fungicides against major pathogens of soybean

S. S. NAVI (1), X. Yang (2), A. Rajasab (3)

(1) Iowa State Univ, Ames, IA, U.S.A.; (2) Iowa State University, Ames, IA, U.S.A.; (3) Tumkur University, Vishwavidyanilaya Karyalaya, Tumkur, India Phytopathology 105(Suppl. 4):S4.100

Effects of four Strobilurin (picoxystrobin, fluoxastrobin, pyraclostrobin and azoxystrobin), two premixes of Strobilurin and Triazole (pyraclostrobin + fluxapyroxad and trifloxystrobin + prothioconazole) and one pyrazolecaroxamide (fluxapyroxad) fungicides were tested against the growth and reproduction of seven soybean pathogens. In a modified dual culture disc procedure, a single 6-mm culture disc (CD) from 7-d old cultures of fungi that cause anthracnose, charcoal rot, pythium and rhizoctonia root rots, brown spot and white mold and 20-d old culture of fungus that causes sudden death syndrome was placed on one end inside periphery of 9-cm PDA plates (except for brown spot) and on the opposite end 6-mm sterilized blotter disc with 50$\mu l$ fungicide (FD) at a labeled rate. Fungicides against brown spot fungus were tested by spreading $50-\mu 1$ spore suspension on to PDA plates and placing FD in the center. During 24-d incubation in $12 \mathrm{~h}$ photoperiod, growth rates, and percent reduction in the growth of a pathogen and inhibition zone $(\mathrm{mm})$ formation in presence of a fungicide and water were assessed daily. Some of the fungicides showed significant effects $(P<0.05)$ on reduction in the growth of fungi and on the formation of inhibition zone either between CDs and FDs or around FDs. SEM from the fungistatic zones showed either deformed hyphae and spores or no reproduction of spores.

Effect of Pyraclostrobin spray either solo or in combination with other chemicals on soybean diseases and yield response in Iowa from 2003 to 2013

S. NAVI (1), X. Yang (2), X. Li (2), L. Jing (3)

(1) Iowa State Univ, Ames, IA, U.S.A.; (2) Iowa State University, Ames, IA, U.S.A.; (3) Inner Mongolia Agricultural University, Hohhot, China

Phytopathology 105(Suppl. 4):S4.100

Twenty eight fungicide trials were set up in a RCBD with four replications each with $3-\mathrm{m}$ wide (four $76-\mathrm{cm}$ rows) $\times 13.8-\mathrm{m}$ long plots in multiple locations of Iowa State University Research farms, from 2003 to 2013. Test products Pyraclostrobin $\left(\right.$ Headline $^{\circledR}$ ) alone or combination with insecticides and other fungicides were sprayed using $\mathrm{CO}_{2}$ backpack 3-m hand boom/ XR8003 tips. Pre- and post-spray disease ratings were recorded weekly from one week before spray through one week before the harvest. Plots were harvested using a John Deere 4420 combine and yields were measured in $\mathrm{bu} / \mathrm{ac}$ converted to $13 \%$ grain moisture. Major diseases assessed were septoria brown spot (SBS), frogeye leaf spot (FELS), sudden death syndrome (SDS) and sclerotinia stem rot or white mold (WM). Headline, either solo or in combination with insecticides ( 1 out of 5 at a time) and other fungicides ( 1 or $>1$ of 12) showed significant $(P<0.05)$ yield advantages over unsprayed controls across 18 roundup ready soybean varieties. Also, results showed wide-ranging yield advantages of sprays among varieties. Headline (solo or combination) significantly $(P<0.05)$ suppressed SBS, and FELS. Across 11 seasons, average yield advantage was $5 \mathrm{bu} / \mathrm{ac}$ (range 2 to $8 \mathrm{bu} / \mathrm{ac}$ ), even under low diseases pressure. Although no significant advantage of Headline (solo or combination) in plots with SDS and WM, but significant $(P<0.05)$ yield increase was observed over unsprayed controls indicating plant health benefits of spray(s).

\section{Complete genome sequence of the Alfalfa latent virus}

L. G. NEMCHINOV (1)

(1) USDA ARS MPPL, Beltsville, MD, U.S.A.

Phytopathology 105(Suppl. 4):S4.100

Alfalfa latent virus (ALV) is a member of the carlavirus group and occurs symptomlessly in alfalfa (Medicago sativa). It is recognized as a strain of Pea streak virus (PeSV). No complete genomic sequence of PeSV or ALV is available. ALV-infected tissue was obtained from ATCC (PV-264 isolate from Lancaster County, NE). Total RNA was extracted with the TRIzol (Life Technologies) and used in primer walking. The 5' end of the genome was amplified with the RACE system (Life Technologies). Sequenced PCR fragments were assembled into contigs and the complete genome consensus sequence was generated. Independently, total RNA was used as a template for Illumina RNA sequencing. Paired-end libraries were cleaned by removing host plant DNA. Using Bowtie2, the reads were mapped to the Medicago truncatula genome, mitochondrial DNA and plastid DNA. Reads that did not map were assembled with Trinity and queried against known plant viruses. A single contig was selected that was $99.8 \%$ identical to the PCR-derived ALV sequence. The 8,041 nt-long consensus sequence was produced and used for further analysis. The ALV genome contains five tentative ORFs encoding a viral replicase, three movement proteins and a capsid protein. The sequenced ALV isolate from Nebraska differs substantially from the Czech ALV and Wisconsin PeSV isolates. Absence of clear 3' proximal nucleotide acidbinding protein typical for carlaviruses indicates ALV divergence from other members of the genus Carlavirus.

Predicting in-season infection risk of cucurbit host types by Pseudoperonospora cubensis using weather variables

K. N. NEUFELD (1), A. P. Keinath (2), P. S. Ojiambo (1)

(1) North Carolina State University, Raleigh, NC, U.S.A.; (2) Clemson University, Charleston, SC, U.S.A.

Phytopathology 105(Suppl. 4):S4.100

Pseudoperonospora cubensis, the causal agent of cucurbit downy mildew (CDM), is considered the most damaging disease of cucurbitaceous crops worldwide. Following initial infection, disease development is primarily determined by weather variables such as temperature, relative humidity and moisture. Three cucurbit host types, cucumber, cantaloupe and squash, were exposed to prevailing weather conditions over a 24 and $48 \mathrm{~h}$ period in an 
infected cucurbit field from 2012 to 2014. In each experiment, disease severity was assessed at 5 and 7 days after exposure. Mean temperature, relative humidity and cumulative solar radiation, leaf wetness hours and rainfall were collected over the exposed periods. Data from $24 \mathrm{~h}$ on disease occurrence were fitted to a logistic regression model to identify weather variables associated with an increased disease severity of 1 to $3 \%$. Temperature, relative humidity, and leaf wetness significantly $(P<0.05)$ affected disease development for cucumber and squash, while temperature was the main factor for cantaloupe. The model with superior predictive ability in cucumber and squash included variables for morning and afternoon mean temperature and relative humidity, while the model with superior predictive ability in cantaloupe included variables for mean temperature. Cutpoints on receiver operating characteristic curve that minimize the overall error rate and associated costs of disease control will be presented and discussed.

Diverse Pseudomonas syringae associated with foliar diseases of watermelon, cantaloupe, and squash

E. A. NEWBERRY (1), M. L. Paret (2), C. T. Bull (3), J. B. Jones (4), B. Babu (5), E. M. Goss (4), I. Rubio (6), T. Jardini (7), P. Roberts (8)

(1) University of Florida, Gainesville, FL, U.S.A.; (2) Department of Plant Pathology, North Florida Research and Education Center, University of Florida, Quincy, FL, U.S.A.; (3) USDA ARS, Salinas, CA, U.S.A.; (4) Department of Plant Pathology, University of Florida, Gainesville, FL, U.S.A.; (5) Department of Pant Pathology, North Florida Research and Education Center, University of Florida, Quincy, FL, U.S.A.; (6) California State University, Monterey Bay, CA, U.S.A.; (7) UC Davis, Davis, CA, U.S.A.; (8) Department of Plant Pathology, Southwest Florida Research and Education Center, University of Florida, Immokalee, FL, U.S.A. Phytopathology 105(Suppl. 4):S4.101

Pseudomonas syringae pv. lachrymans is widely reported as the causal agent of angular leaf spot of cucurbits. Reports of $P$. syringae pathovars syringae and aptata causing foliar diseases on melon indicates that a broader diversity of organisms within $P$. syringae may be responsible for cucurbit diseases. In this study we have collected 54 isolates recovered in various years from watermelon, cantaloupe, and squash hosts in Florida, Georgia, and California. Isolates were fluorescent on King's medium B agar and identified as Pseudomonas syringae through biochemical and 16S rRNA analysis. Variability was observed in level of fluorescence, levan production, and ice nucleation ability. Multilocus sequence analysis of four housekeeping genes (gyrB, rpoD, glt1, gapA) revealed the presence of multiple groups all belonging to genomospecies 1 . All isolates were genetically diverged from $P$. s. pv. lachrymans. Phylogenetic analysis including pathotype sequences within $P$. syringae sensu lato identified two groups forming sister clades with $P$. syringae pathovars papulans and dysoxyli, another grouped with aptata and atrofacians. All other isolates formed distinct clades. The identification of clonal strains recovered from Florida, Georgia, and California over multiple years indicates potential dispersion of the pathogen through seed. Pathogenicity and host range tests show that watermelon, squash, and cantaloupe are susceptible to wide range of genetic diversity among $P$. syringae.

Development of a sanitizing agent for use on field equipment to reduce the spread of olive knot in mechanized olive production in California

K. NGUYEN (1), H. Forster (1), J. Adaskaveg (1)

(1) Department of Plant Pathology and Microbiology, University of California Riverside, Riverside, CA, U.S.A.

Phytopathology 105(Suppl. 4):S4.101

New methods in olive production such as high-density planting, mechanical harvesting, and automated hedging have increased the risk of spreading Pseudomonas savastanoi pv. savastanoi (Psv), the causal agent of olive knot. Routine disinfection of field equipment with sanitizers could reduce spread of Psv to healthy trees by preventing field equipment from becoming inoculum sources. Oxidizers such as chlorine or peroxyacetic acid are not used due to their corrosive properties and ineffectiveness in the presence of organic material. We conducted in vitro direct contact (DC) toxicity and hard surface disinfection assays (HSD) to evaluate the efficacy of sanitizers. A quaternary ammonium compound (i. e., Deccosan 321 - D321) was tested due to its potential for registration since it is currently used in Florida for equipment disinfestation for diseases of other crops. In DC assays, D321 at $5 \mathrm{mg} / \mathrm{L}$ or 25 $\mathrm{mg} / \mathrm{L}$ reduced Psv growth by $97.3 \%$ or $100 \%$, respectively, when a $1 \times 10^{8}$ $\mathrm{CFU} / \mathrm{ml}$ suspension of Psv was exposed for $60 \mathrm{sec}$. In HSD assays where PVC pipes were contaminated with macerated olive tissue containing $1 \times 10^{6}$ $\mathrm{CFU} / \mathrm{ml}$ Psv, D321 at the suggested rate of $2000 \mathrm{mg} / \mathrm{L}$ reduced bacterial growth by $99.8 \%$ in $90 \mathrm{sec}$, proving it to be highly effective under an organic load. In comparison, chlorine was less effective. Based on these findings,
D321 was submitted for registration for use on olives in California and is expecting approval by 2015 .

Efficacy of kasugamycin against olive knot caused by Pseudomonas savastanoi pv. savastanoi and its potential use in California olive production

K. NGUYEN (1), H. Forster (2), J. Adaskaveg (2)

(1) University of California Riverside, Colton, CA, U.S.A.; (2) University of California Riverside, Riverside, CA, U.S.A.

Phytopathology 105(Suppl. 4):S4.101

Pseudomonas savastanoi pv. savastanoi (Psv) is an economically important pathogen of olives worldwide. Infections occur through wounds formed naturally (e.g., hail, frost) or by human activity (e.g., mechanical pruning and harvesting). An important control practice is the protection of wounds with chemical treatments such as copper. Moderate and high copper tolerance was detected in strains from California surveys. In field trials, copper was less effective against these strains. With limited alternatives to copper available, we evaluated the antibiotic kasugamycin as an additional management option. Baseline sensitivities were determined for kasugamycin using over 100 strains with the spiral gradient endpoint method and compared with streptomycin and oxytetracycline. Minimum inhibitory concentrations ( $\geq 95 \%$ growth inhibition) ranged from 1.89 to $6.29 \mathrm{mg} / \mathrm{L}$ for kasugamycin, 0.20 to $0.54 \mathrm{mg} / \mathrm{L}$ for oxytetracycline, and 0.14 to $1.77 \mathrm{mg} / \mathrm{L}$ for streptomycin. In field trials where lateral twig wounds or leaf scars were treated and then inoculated with a copper tolerant strain, kasugamycin reduced the incidence of knot formation by $>63 \%$ from that of the control, similar to that of the other antibiotics. Kasugamycin-copper mixtures provided up to $80 \%$ reduction in disease incidence while copper hydroxide alone reduced disease incidence by $<18 \%$. Kasugamycin was recently registered for use against fire blight on pome fruit and is currently in the IR-4 residue program for olive.

Distribution of azoxystrobin resistance in nonpathogenic Alternaria alternata isolates of citrus

K. R. NICOLETTA (1), B. Vega (2), M. M. Dewdney (3)

(1) University of Florida, Lake Alfred, FL, U.S.A.; (2) DuPont Crop Protection-Discovery, Newark, DE, U.S.A.; (3) Univ of Florida, Lake Alfred, FL, U.S.A.

Phytopathology 105(Suppl. 4):S4.101

Currently, the greatest proportion of tangerine-infecting A. alternata isolates in Florida are QoI-resistant populations; however the selection pressure of QoI fungicides has not been measured for non-pathogenic A. alternata isolates. Pathogenic and nonpathogenic isolates of $A$. alternata are found on tangerine cultivars, and 126 isolates of the 1,258 total collected were confirmed nonpathogenic to cultivars Dancy, Minneola, Murcott, and Sunburst through phenotypic approaches. Also, the absence of the ACT-toxin production was molecularly confirmed in 37 isolates by amplifying the ACTTR locus. Isolates were tested for azoxystrobin (AZ) sensitivity using the resazurin-based microtiter assay. Isolates with an effective concentration to reduce growth by $50 \%\left(\mathrm{EC}_{50}\right)$ values greater than $5.0 \mu \mathrm{g} / \mathrm{ml}$ were categorized resistant. Of the isolates, $75 \%$ were resistant to $\mathrm{AZ}$. The mean $\mathrm{EC}_{50}$ value for the 32 sensitive isolates was $0.044 \mu \mathrm{g} / \mathrm{ml}$. Twenty of the sensitive isolates came from Murcott and 12 from Sunburst cultivars. A $\chi^{2}$ comparison of the populations of pathogenic and nonpathogenic resistant isolates was not significantly different $\left(\chi^{2}=0.699, P=0.403\right)$ among cultivars. The presence of the G143A mutation in the cytochrome $b$ gene was determined in a subset of 37 isolates using PCR-restriction fragment length polymorphism with the developed primers CytF1/CytR1 and restriction enzyme Fnu4HI. All non-pathogenic QoIresistant isolates carried the G143A mutation.

Identification and characterization of an Alfalfa mosaic virus isolate associated with tuber necrosis in a potato variety

X. NIE (1), D. De Koeyer (2), Z. Liang (2), V. Dickison (2), M. Singh (3), G. Hawkins (4)

(1) Agric \& Agri-Food Canada, Fredericton, NB, Canada; (2) Potato Research Centre, AAFC, Fredericton, NB, Canada; (3) Agri Certification Services, Fredericton, NB, Canada; (4) McCain Produce, Florenceville, NB, Canada Phytopathology 105(Suppl. 4):S4.101

RT-PCR tests for 10 common potato viruses detected Potato virus $S$ (PVS), Potato virus $M(\mathrm{PVM})$ and Potato virus $Y$ tuber necrosis strain $\left(\mathrm{PVY}^{\mathrm{NTN}}\right)$ in potato tubers exhibiting extensive internal necrosis in a potato variety. Progeny plants developed mosaic symptoms and tested positive for the above viruses/virus strain by ELISA, RT-PCR and bioassay. As expected, tubers from these plants developed severe internal necrosis. However, single or mixed infections with PVS, PVM and PVY ${ }^{\mathrm{NTN}}$ on plants of the same variety did not cause any visible internal or external necrosis, suggesting that an unusual virus/virus strain might be the causal agent of internal tuber necrosis. 
Using next generation sequencing for detection of virus-derived small RNAs, Alfalfa mosaic virus (AMV), in addition to PVS/PVM/PVY, was detected in the original samples. After passing through tobacco and a PVY-resistant potato breeding line, AMV was purified. Inoculation of various potato varieties with the purified AMV induced calico-symptoms, typical for the virus. Preliminary scanning of the tubers from the inoculated plants indicated the presence of internal necrosis in some of the varieties including the one from which the original samples were obtained. Phylogenetic analysis of the complete genome of the AMV isolate with those available in the databases indicated that at least two clustering groups can be found for each of the three genomic RNAs in AMV.

\section{Effect of soil potassium levels on Iris yellow spot virus in onion}

C. NISCHWITZ (1), H. Schwartz (2), E. Petrizzo (3), M. McCullough (3), B. Rhoads (4)

(1) Utah State Univ, Logan, UT, U.S.A.; (2) Colorado State University, Fort Collins, CO, U.S.A.; (3) Utah State University, Logan, UT, U.S.A.; (4) Utha State University, Logan, UT, U.S.A.

Phytopathology 105(Suppl. 4):S4.102

For onion growers in Utah and Colorado Iris yellow spot virus (IYSV) is the most important and difficult to manage disease problem. Mineral nutrients in soil and/or tissue have been shown to affect diseases on other crops. To determine if nutrition could be an option to manage IYSV incidence and symptom expression, soil and tissue samples were collected from onion fields in Utah and Colorado and analyzed for nutrient composition. Onion leaves were collected and tested by ELISA to determine IYSV incidence. Soil K by itself and in ratios with $\mathrm{P}$ and $\mathrm{Zn}$ affected IYSV incidence in fields based on regression analysis. The higher the soil $\mathrm{K}$ levels the more likely IYSV occurred. A field trial was set up in Utah with four levels of available soil K. Leaves from all onion plants were analyzed by ELISA for IYSV and visual ratings of plants was started when first symptoms appeared. The results showed a clear trend between increased levels of $\mathrm{K}$ and percent IYSV symptomatic plants and a higher incidence of infected plants. Increasing soil $\mathrm{K}$ levels not only increased levels of $\mathrm{K}$ in plant tissue, it also led to a dramatic increase in available soil Mn and Mn levels in plant tissue and decrease in the soil and tissue Mg:Mn ratio. The mechanism behind the increased IYSV symptomatic plants and IYSV incidence with increasing soil $\mathrm{K}$ levels is currently unknown. Monitoring soil $\mathrm{K}$ levels could be an effective addition to existing IPM programs to manage IYSV in onion.

\section{Analysis of FST1 mutant of Fusarium verticillioides reveals global impact on transcriptome}

C. NIU (1), C. P. Woloshuk (2), G. A. Payne (3)

(1) Purdue Univ, West Lafayette, IN, U.S.A.; (2) Purdue University, West Lafayette, IN, U.S.A.; (3) North Carolina State University, Raleigh, NC, U.S.A.

Phytopathology 105(Suppl. 4):S4.102

Fusarium verticillioides is a fungal pathogen of maize, and produces fumonisins in infected kernels. Fumonisins are toxic secondary metabolites that are detrimental to the health of humans and livestock. Our research has focused on the molecular regulation of fumonisin biosynthesis. Deletion of the gene FST1 was previously shown to reduce fumonisin production and virulence. Further studies have revealed that the mutation causes increased sensitivity to hydrogen peroxide, decreased production of macroconidia, and reduced mycelial hydrophobicity. To better understand the function of FST1, we compared the transcriptomes of the wild type and FSTI mutant strain $(\Delta \mathrm{fst} 1)$ when grown on autoclaved maize kernels. Our analysis indicated that $93 \%$ of the genes in $F$. verticillioides genome were transcribed in both strains. Among these transcripts, 2677 were differentially expressed ( $\mathrm{P}$ value $<0.01$ and an absolute fold difference $>2$ ). Based on gene ontology analysis, we assigned 961 genes to one of 13 molecular function categories. The results provide evidence that disruption of FST1 affects several metabolic and developmental pathways in F. verticillioides. FST1 appears to connect the expression of several gene networks, including those involved in secondary metabolism, cell wall structure, conidiogenesis, virulence, and resistance to reactive oxygen species. Furthermore, the results support our hypothesis that FST1 functions within the framework of environmental sensing.

\section{Management of blueberry, Vaccinium spp. replant disease with pine bark soil amendment and pre-plant fumigation}

J. P. Noe (1), G. B. JAGDALE (2), W. T. Holladay (1), P. M. Brannen (1)

(1) University of Georgia, Athens, GA, U.S.A.; (2) Univ of Georgia, Athens, GA, U.S.A.

Phytopathology 105(Suppl. 4):S4.102

Blueberry (Vaccinium spp.) replant disease (BRD) is characterized by poor growth, yellowing and reduced yields. Since BRD incidence is associated with high population densities of Mesocriconema ornatum, we hypothesized that a combination of tactics, including pre-plant fumigation and pine bark soil amendments, would suppress $M$. ornatum populations, which in turn would control BRD. We tested the efficacy of pine bark soil amendment with and without pre-plant application of soil fumigants on two varieties each of rabbiteye (RBE; Prince and Vernon) and southern highbush (SHB; Farthing and Legacy) at two different sites in Georgia in 2013 and 2014, respectively. Control plots received no pine bark or fumigants. Overall $M$. ornatum population densities were lower in the fumigated plots than in the control plots for RBE and SHB species. Nematode population densities in RBE plots remained lower in bark-treated than non-bark plots, but differences due to bark were not observed in the SHB plots during the first growing season. RBE plant volumes at the end of the second growing season were higher in barkamended than in non-bark plots. SHB plant volumes were higher in fumigated than control plots at the end of the first growing season. Our results showed that the addition of pine bark soil amendment to a robust protocol of pre-plant soil fumigation may provide a more sustainable level of management for blueberry replant disease.

The evaluation of a high throughput microtiter plate assay to examine the sensitivity of a soybean oomycete community to mefenoxam and ethaboxam

Z. A. NOEL (1), A. Rojas (2), J. Jacobs (2), D. McDuffee (3), M. I. Chilvers (2)

(1) Michigan State University, East Lansing, MI, U.S.A.; (2) Michigan State University, East Lansing, MI, U.S.A.; (3) Valent USA Corp, Indianapolis, IN, U.S.A.

Phytopathology 105(Suppl. 4):S4.102

Seed treatments may be an effective tool to manage soybean seedling stand establishment issues due to oomycete root and stem rot pathogens. It is important to examine fungicide sensitivity of Pythium spp. and Phytophthora spp. on a community scale, because multiple species may infect soybean roots. However, fungicide sensitivity studies rarely focus on pathogen communities due to time, labor and funding constraints. The objectives of this study were to develop a high throughput microtiter plate fungicide sensitivity assay and evaluate the sensitivity of an oomycete pathogen community to mefenoxam and ethaboxam. Isolates were collected from 12 major soybeanproducing states across the Midwest in 2011 and 2012. Preliminary results indicate that $\mathrm{EC}_{50}$ obtained from the microtiter plate assay was significantly correlated with the $\mathrm{EC}_{50}$ of a poison plate assay and reduces the time required for evaluation. Of the 98 Pythium spp. and 32 Phytophthora spp. isolates tested, all have been sensitive to mefenoxam $\left(\mathrm{EC}_{50} \leq 1 \mu \mathrm{g}\right.$ a.i. $\left./ \mathrm{mL}\right)$. The mean $\mathrm{EC}_{50}$ of 29 Phytopththora sojae isolates to ethaboxam was below $0.1 \mu \mathrm{g} / \mathrm{mL}$. This research aims to be the foundation for future fungicide sensitivity monitoring efforts of oomycete root and stem rot pathogens and provide important data that may influence the use of soybean seed treatments.

Antagonistic and symbiotic activity of fluorescent Pseudomonas and rhizobia associated with root nodules of Vigna radiata and Leucaena leucocephala

R. Noreen (1), J. ARA (2), V. Sultana (3), S. Ehteshamul-Haque (1)

(1) Department of Botany, University of Karachi, Karachi, Pakistan; (2) Department of Food Science \& Technology, University of Karachi, Karachi, Pakistan; (3) Department of Biochemistry, University of Karachi, Karachi, Karachi, Pakistan

Phytopathology 105(Suppl. 4):S4.102

Fifteen isolates each of fluorescent Pseudomonas and rhizobia isolated from the root nodules of mungbean (Vigna radiata) and Leucaena leucocephala were evaluated for antifungal, nematicidal activity along with the ability to produce siderophore, cyanide, phosphate solubilizing capacity, indole acetic acid production as well as production of volatile antifungal compound. All the strains of fluorescent Pseudomonas and rhizobia showed inhibitory effect on the growth of Macrophomina phaseolina, Fusarium solani and F. oxysporum by producing volatile compounds, while against Rhizoctonia solani all fifteen isolates of Pseudomonas and ten isolates of rhizobia caused growth inhibition. All isolates also showed significant nematacidal activity against the second stage juveniles of Meloidogyne javanica. While 11 strains of fluorescent Pseudomonas and 13 stains of rhizobia showed positive result in siderophore production, all strains of fluorescent Pseudomonas and 8 isolates of rhizobia found to produce cyanide. Wheras 12 isolates of fluorescent Pseudomonas and 7 isoaltes of rhizobia were found positive for phosphate solubilization. Fluorescent Pseudomonas showed significant increase in root and shoot mass and enhanced number of nodule initials, mature nodules and concentration of fixed nitrogen in mungbean plant. Role of root nodule associated fluorescent Pseudomonas seems important in rhizobia-plant symbiosis. 
Next generation sequencing for identifying known and novel viruses in the Asian citrus psyllid, the vector of $C$. Liberibacter asiaticus

S. NOURI (1), N. Salem (2), B. Falk (1)

(1) University of California, Davis, CA, U.S.A.; (2) University of Jordan, Amman, Jordan

Phytopathology 105(Suppl. 4):S4.103

The Asian citrus psyllid, Diaphorina citri Kuwayama, is the vector of Candidatus Liberibacter asiaticus which causes Huanglongbing, a devastating disease that threatens the entire U.S. citrus industry. To discover D. citri virus candidates which might be useful for virus-based biological control, we used deep sequencing, RT-PCR and Sanger sequencing. D. citri transcriptome and small RNAs were sequenced using next generation Illumina-based sequencing for selected, worldwide D. citri populations. de novo assemblies followed by BLAST searches using Blastx and tBlastx suggested possible known and novel viruses. Here, we are reporting viruses tentatively named Diaphorina citri reovirus (DCRV), Diaphorina citri densovirus (DCDV), Diaphorina citri bunyavirus (DCBV) and Diaphorina citri picorna-like virus (DCPLV). DCPLV is a novel Picorna-like virus discovered in D. citri from Brazil, China and Taiwan. DCPLV showed 31-35\% identities to viruses of the genus Iflavirus at the protein level. However, genetic analysis revealed a genome organization distinct for DCPLV. Bioinformatics analysis predicted one possible Open Reading Frame (ORF) of 9402 bp. In contrast to iflaviruses, the DCPLV structural proteins were located C-terminally to the non-structural proteins in the polyprotein. Bioinformatics analysis followed by RT-PCR, Sanger sequencing and RACE strategy completed the genome of DCPLV. The positive-sense, single-stranded RNA genome of DCPLV is approximately $10 \mathrm{~kb}$.

Curtovirus capsid protein interaction with a GroEL- like protein produced by the bacterial endosymbiont of the vector beet leafhopper T. NUSAYR (1), R. Creamer (1)

(1) New Mexico State University, Las Cruces, NM, U.S.A.

Phytopathology 105(Suppl. 4):S4.103

The economically important Curly top diseases (CTDs) are caused by curtoviruses (Geminiviridae). The vector of CTDs, beet leafhopper (Circulifer tenellus), can feed on the phloem of more than 300 plant species. Plant viruses must avoid degradation while present in their insect vectors' hemolymph. GroEL-like proteins (GroLp), produced by insect-vector endosymbionts, have been identified in other plant virus vectors, such as the luteoviruses and begomoviruses, as proteins interacting with the virus. It was found that only one type of GroLp produced from one type of endosymbiont can interact with and protect the virus. Curtoviruses must also avoid degradation while present in their insect vectors' hemolymph. The beet leafhopper (LH) harbors endosymbiotic bacteria able to produce a GroLp which can interact with the capsid protein $(\mathrm{CP})$ of curtoviruses protecting them. The virus $\mathrm{CP}$ is important for transmission by an insect vector. Mutation of the CP led to loss of transmission but not infectivity. Our research investigated the presence of GroLp in the LH and a possible interaction with curtovirus CP using wild type and mutated CP. We used ELISA, PCR capture method, and other protein interaction assays. We also investigated the interaction between some plant viruses and GroEL (Escherichia coli). The location of GroLp and CP in different body organs of the leaf hopper was also examined. Our results have been in agreement with previous findings.

\section{Diplodia shoot blight on ponderosa pine following hail in northeast} Oregon

\section{B. W. OBLINGER (1)}

(1) USDA Forest Service, Bend, OR, U.S.A.

Phytopathology 105(Suppl. 4):S4.103

Ponderosa pine is an important timber species and plays various ecological roles in drier forest types of the Pacific Northwest. Diplodia shoot blight epidemics in ponderosa pine have been reported previously in parts of western North America but not in northeast Oregon (NE OR). Shoot blight caused by Diplodia sp. was recently observed at multiple locations in NE OR. Symptoms were noted on ponderosa pine growing near portions of the Grande Rhonde, Wenaha, Wallowa and Minam Rivers. Severe shoot blight was detected following a hailstorm surrounding Troy, OR. To monitor health of pines with shoot blight and severity of shoot blight in Troy, pole- to sawtimber-sized trees with varying severities were evaluated after the 2012 growing season and revisited after two growing seasons. Each third of tree crowns was visually rated for shoot blight severity (0-2). After two yr, 31\% of trees had died and those dead had higher initial severity $(\mathrm{p}<0.0001$ based on a Mann-Whitney test) than those still living. Severity on trees still living did not change $(\mathrm{p}=0.21)$ after two yr (increased on $38 \%$ and did not change on all others). Based on PCR assays, D. pinea was detected on cones and shoots (unknown whether other Diplodia spp. also are present). Results document
Diplodia shoot blight damage in NE OR and the potential for this disease to predispose trees to mortality. Future climatic conditions influencing the frequency of hailstorms may affect incidence and severity of this disease.

New and re-emerging fungal pathogens affecting Brassicaceae plants in western Oregon: Black leg, light leaf spot, and white leaf spot

C. M. OCAMB (1), C. Mallory-Smith (1), W. J. Thomas (1), M. Serdani (2),

M. L. Putnam (2)

(1) Oregon State University, Corvallis, OR, U.S.A.; (2) Oregon State University - Plant Clinic, Corvallis, OR, U.S.A.

Phytopathology 105(Suppl. 4):S4.103

Disease surveys in Brassica and radish (Raphanus sativus) seed production fields in the Willamette Valley of western Oregon during spring of 2014 detected new and re-emerging diseases of economic importance to Brassicaceae crops. Prior years' surveys of specialty Brassica seed crops in this region revealed other diseases but not the following: black leg (Phoma lingam, teleomorph: Leptosphaeria maculans), light leaf spot (Cylindrosporium concentricum, teleomorph: Pyrenopeziza brassicae), and white leaf spot and gray stem (Pseudocercosporella capsellae, teleomorph: Mycosphaerella capsellae). Black leg was observed in 43 of 61 sites examined in seven counties, including oilseed, cover, forage, and vegetable Brassica seed crops as well as on field mustard (B. rapa var. rapa) and western yellowcress (Rorippa curvisiliqua). This is a first report for light leaf spot in the US; it was detected on fall-planted Brassica crops including oilseed, vegetable seed crops, cover crops, and field mustard in six counties on 25 of the 61 sites examined. This disease was also observed in radish seed crops. White leaf spot and gray stem occurred on canola, specialty Brassica seed crops, forage Brassica crops, and field mustard in 13 of 61 locations, representing five counties. Disease incidence and symptomology varied among the host species examined. The potential impact of black leg, light leaf spot, and white leaf spot on Brassicaceae crops in the region is being assessed.

Rpi-blb2-mediated hypersensitive cell death caused by Phytophthora infestans AVRblb2 requires SGT1, but not EDS1, NDR1-mediated signaling

S. K. OH (1)

(1) Chungnam National University, Deajeon, South Korea

Phytopathology 105(Suppl. 4):S4.103

Potato Rpi-blb2 encodes a protein with a coiled-coil-nucleotide binding site and leucine-rich repeat (CC-NBS-LRR) motif that recognizes the Phytophthora infestans AVRblb2 effector and triggers hypersensitive cell death (HCD). To better understand the components required for Rpi-blb2mediated HCD in plants, we used virus-induced gene silencing to repress candidate genes in Rpi-blb2-transgenic Nicotiana benthamiana plants and assayed the plants for AVRblb2 effector. Rpi-blb2 triggers HCD through NbSGT1-mediated pathways, but not NbEDS1- or NbNDR1-mediated pathways. In addition, the role of salicylic acid (SA), jasmonic acid (JA), and ethylene (ET) in Rpi-blb2-mediated HCD were analyzed by monitoring of the responses of NbICS1-, NbCOI1-, or NbEIN2-silenced or Rpi-blb2::NahGtransgenic plants. Rpi-blb2-mediated HCD in response to AVRblb2 was not associated with SA accumulation. Thus, SA affects Rpi-blb2-mediated resistance against $P$. infestans, but not Rpi-blb2-mediated HCD in response to AVRblb2. Additionally, JA and ET signaling were not required for Rpi-blb2mediated HCD in N. benthamiana. Taken together, these findings suggest that $\mathrm{NbSGT1}$ is a unique positive regulator of Rpi-blb2- mediated HCD in response to AVRblb2, but EDS1, NDR1, SA, JA, and ET are not required.

Pathogenicity of Diaporthe species infecting soybeans (Glycine max L.) in South Dakota

P. Okello (1), A. Gebreil (1), T. Olson (1), B. Kontz (1), A. Micijevic (1), F. MATHEW (2)

(1) South Dakota State University, Brookings, SD, U.S.A.; (2) South Dakota State Univ, Brookings, SD, U.S.A.

Phytopathology 105(Suppl. 4):S4.103

Diaporthe/Phomopsis complex cause diseases of economic importance to soybean production in the U.S. In 2005, stem canker compromised soybean yield ( $>1.3$ million tons) in South Dakota more than any other U.S. states. Recently, stem canker has become an emerging problem in the North Central U.S. The goal of this study was to characterize and determine the pathogenicity of the Diaporthe species associated with stem canker in South Dakota. To determine the identity of Diaporthe sp., over 200 isolates were cultured from infected samples collected from soybean fields in South Dakota between 1999 and 2014. The internal transcribed spacer (ITS) regions and the translation elongation factor $1-\alpha(E F 1-\alpha)$ of representative 18 isolates were sequenced, analyzed, and compared with their morphological characteristics. The molecular analysis of ITS and EF1- $\alpha$ sequences in comparison with those 
of type specimens allowed the identification of Diaporthe longicolla and Diaporthe caulivora, to be associated with stem canker in South Dakota. Pathogenicity tests conducted by inoculating two-week-old soybean plant of cv. 'AG1835' confirmed that the Diaporthe isolates belonging to the two species were pathogenic. Future work will include interaction of the two Diaporthe species with soybean cyst nematode ( $\mathrm{SCN}$ ) as well as screening early maturity soybean varieties for resistance to the Diaporthe species identified in South Dakota.

Velista $^{\circledR}$ (penthiopyrad) baseline sensitivity of Sclerotinia homoeocarpa isolates

G. OLAYA (1), R. Linley (1), K. Edlebeck (1), M. Agnew (1)

(1) Syngenta Crop Protection, Vero Beach, FL, U.S.A.

Phytopathology 105(Suppl. 4):S4.104

The baseline sensitivity of Slerotinia homoeocarpa isolates was established for penthiopyrad $\left(\right.$ Velista $\left.^{\circledR}\right)$, a succinate dehydrogenase inhibitor (SDHI) fungicide (FRAC group 7). The sensitivity to penthiopyrad of 31 isolates never exposed to SDHI fungicides collected before 2004 and 50 isolates exposed to SDHI fungicides collected after 2004 was determined in vitro. The first SDHI fungicide introduced in the turf market to control the dollar spot pathogen $S$. homoeocarpa was boscalid in 2003. Sensitivity of each isolate was determined by comparing the colony radial growth on $1 / 2$ strength potato dextrose agar plates either amended or un-amended with penthiopyrad. The mean sensitivity distributions (ED50 values) of $S$. homoeocarpa isolates, never exposed and exposed to SDHI fungicides were different and two baselines were established for both populations. Sensitivities of isolates never exposed to SDHI fungicides ranged from 0.114 to 7.350 (geometric mean: 1.229) $\mathrm{mg} / \mathrm{L}$, whereas sensitivities of isolates exposed to SDHI fungicides ranged from 0.369 to 11.372 (geometric mean: 3.019) $\mathrm{mg} / \mathrm{L}$. Baseline sensitivity information will be used to track changes in sensitivity in $S$. homoeocarpa populations after the commercial introduction of Velista ${ }^{\circledR}$. The use of the SDHI fungicides in the turf market is increasing and new fungicides are being introduced (flutolanil, boscalid, fluxapyroxad, penthiopyrad) and the selection pressure of less sensitive or resistant isolates could increase.

Sensitivity of Phytophthora infestans isolates to the fungicide oxathiapiprolin

G. OLAYA (1), R. Linley (1), K. Myers (2), W. Fry (2), K. Paul (3), K. Edlebeck (1)

(1) Syngenta Crop Protection, Vero Beach, FL, U.S.A.; (2) Cornell University, Ithaca, NY, U.S.A.; (3) Syngenta Crop Protection, Greensboro, NC, U.S.A.

Phytopathology 105(Suppl. 4):S4.104

The sensitivity to the fungicide oxathiapiprolin of 74 individual isolates of Phytophthora infestans was determined under in-vitro conditions. Isolates were recovered from potato, tomato or nightshade from different states in the United States and from other countries including Canada, Mexico, Ecuador, Colombia, Uruguay, and South Africa. Sensitivity of each isolate was determined by comparing the colony radial growth on diluted rye agar plates amended or not with oxathiapiprolin. The baseline sensitivity distributions (ED50 values) of the $P$. infestans isolates ranged from 0.00000684 to 0.00021176 with a geometric mean of $0.00004148 \mathrm{mg}$ oxathiapiprolin/L. Oxathiapiprolin baseline information is essential for the implementation of future sensitivity studies aiming in the early detection of changes in sensitivity of $P$. infestans to oxathiapiprolin. Oxathiapiprolin is a new class of fungicides (piperidinyl thiazole isoxazoline) developed by DuPont. Oxathiapiprolin is active against downy mildews and Phytophthora species, and it is generally weak against Pythium species. Syngenta has an exclusive license from DuPont to develop and sell products containing oxathiapiprolin for foliar and soil use in North America and for lawn and garden uses globally.

Complete genome sequences of xanthomonads causing common bacterial blight of common bean reveal conserved and divergent features

M. L. O'LEARY (1), G. Coaker (1), R. L. Gilbertson (1)

(1) University of California, Davis, Davis, CA, U.S.A.

Phytopathology 105(Suppl. 4):S4.104

Common bacterial blight of bean, caused by Xanthomonas axonopodis pv. phaseoli (Xap) and X. fuscans subsp. fuscans (Xff), is a major foliar disease of common bean. Although symptoms caused by Xap and Xff are indistinguishable, they represent distinct species based on genetic and phenotypic (the production of brown pigment by Xff strains in culture) characteristics. Pathogenic variability among Xap and Xff strains has been observed, but there is no apparent race structure. To gain further insight into the nature of the variability among genetically distinct xanthomonads that cause the same disease, the genomes of three globally predominant Xap strains, two East African Xap strains, and three Xff strains, all of which have distinct genotypes, were determined utilizing the Pacific Biosciences RS II platform. The single chromosome of each of these xanthomonads was circular, $\sim 5 \mathrm{Mb}$ and had $\sim 65 \% \mathrm{GC}$ content. Depending on the species/strain, there were one to three plasmids, one of which is conserved among all these bacteria and harbors putative virulence factors. The Xap and Xff strains have Type III secretion systems and significant overlap in the Type III secreted effector repertoire, including TAL effectors. Comparisons among globally predominant Xap and East African Xap strains revealed large genomic rearrangements, reinforcing the considerable genetic variability among Xap strains.

Optimization of a resazurin-based assay testing sensitivity of Botrytis cinerea to respiration-inhibitor fungicides

M. S. OLIVEIRA (1), L. G. Cordova (1), N. A. Peres (1)

(1) University of Florida, Wimauma, FL, U.S.A.

Phytopathology 105(Suppl. 4):S4.104

Resistance of Botrytis cinerea to fungicides is rapidly developed due to its abundant sporulation and high genetic variability. Conidial germination test is the common assay to determine resistance to respiration inhibitor fungicides; however, this test requires time, space, and its measurements are subjective. Our objective was to adapt a resazurin-based assay to evaluate $B$. cinerea sensitivity to boscalid and pyraclostrobin and determine the effective dose to provide $50 \%$ control of the fungus $\left(\mathrm{EC}_{50}\right)$ in both fungicides. Eleven isolates were tested in three liquid media (Complete Medium, Minimal Medium and HA Medium with $\mathrm{pH}$ 6.5), four conidial concentrations (from $10^{2}$ to $10^{5}$ conidia $/ \mathrm{ml})$, eight fungicide concentrations $(0,0.001,0.01,0.1,0.5,1,5$, and $10 \mu \mathrm{g} / \mathrm{ml}$ for boscalid and $0,0.001,0.01,0.1,0.5,1,10$, and $50 \mu \mathrm{g} / \mathrm{ml}$ for pyraclostrobin), and evaluated from 16 to $32 \mathrm{~h}$ after plating. HA at $24 \mathrm{~h}$ and $10^{4}$ conidia/ml were the optimal conditions for evaluation of resazurin reduction. The $\mathrm{EC}_{50}$ calculated for the sensitive isolates $(\mathrm{n}=5)$ varied between 0.39 and $3.25 \mathrm{ppm}$ in the conidial germination test, and between 0.13 and $0.20 \mathrm{ppm}$ in the resazurin-based assay. For the resistant isolates $(\mathrm{n}=6)$, the $\mathrm{EC}_{50}$ was higher than $10 \mathrm{ppm}$ on boscalid and higher than $50 \mathrm{ppm}$ on pyraclostrobin. Our findings suggest that this rapid and reliable technique can be used on a large scale to evaluate the sensitivity of $B$. cinerea to respiration inhibitor fungicides.

Effect of cultivar and cluster maturity on ripe rot of grape caused by Colletotrichum acutatum and Colletotrichum gloeosporioides

C. OLIVER (1), M. Nita (2)

(1) Virginia Polytechnic Institure and State University, Winchester, VA, U.S.A.; (2) Virginia Tech, Winchester, VA, U.S.A.

Phytopathology 105(Suppl. 4):S4.104

Ripe rot of grape is caused by two species complexes: Colletotrichum acutatum and Colletotrichum gloeosporioides. To investigate effect of cultivar selection and cluster maturity on the development of ripe rot, field and potted vine cluster inoculations were conducted during 2012-2014 growing seasons. Grape clusters were inoculated at five different cluster developmental stages with $C$. acutatum or $C$. gloeosporioides spores $\left(5 \times 10^{5}\right.$ spores $\left./ \mathrm{ml}\right)$, clusters were then bagged for 24 hours for incubation, and visual assessment of berries were made at the end of the season. In 2013, Cabernet sauvignon resulted in the highest disease incidence ranging $29-34 \%$ among timings of inoculation, followed by Cabernet Franc (15-17\%), Chardonnay (6-14\%) and Merlot (4$7 \%$ ). For 2014, overall mean disease incidence was lower than the previous year, ranging $3-8 \%$ disease incidence. With the potted vine studies, Chardonnay resulted with $15-32 \%$ disease incidence followed by Cabernet sauvignon (1-6\%), Merlot (1-2\%) and Petit Verdot (0-2\%). We have identified that a certain level of infection could take place on most cultivars tested from bloom to the near harvest, and there were significant differences in disease incidence among cultivars $(P<0.05)$, but little or no differences between the fungal species. Results from this study help growers to determine the critical period for ripe rot management.

Evaluation of Allium sativum and Trichoderma asperellum for the control of cowpea anthracnose disease caused by Colletotrichum lindemuthianum D. B. OLUFOLAJI (1), M. A. Ajayi (1)

(1) Federal Univ of Technology, Akure, Nigeria

Phytopathology 105(Suppl. 4):S4.104

Cowpea is an important source of protein for millions of poor Africans who cannot afford other sources like meat, milk and fish. In Nigeria, beans anthracnose caused by Colletotrichum lindemuthianum (Sacc. and Magnus), has caused yield loss of up to $75 \%$. Thus, screen house studies was carried out between August and September 2014, using two concentrations of Allium sativum I. (garlic) crude extract, $30 \%$ and $45 \%$, (w/v) and Trichoderma asperellum (Samuel,Lieckf) at $10^{3}$ and $10^{6}$ spore $/ \mathrm{ml}$ to manage the disease. They were applied as foliar spray prophylacticaly to control C. lindemuthi- 
anum on cowpea. The experiment was replicated thrice and laid out in a Completely Randomized Design. It was observed that, the least values of $4.17 \%$ and 1.33 , disease incidence and severity respectively, and the highest number of pods, 10.33, were recorded for cowpea plants treated with $T$. asperellum at $10^{6}$ spores $/ \mathrm{ml}$ and $72 \mathrm{hrs}$ prophylactic application. These values were not significantly different $(\mathrm{P}=0.05)$ from those of crude garlic extract at $45 \%$ concentration and $48 \mathrm{hrs}$ prophylactic application, but were significantly different from the control and other treatments. Trichoderma asperellum at $10^{6}$ spores $/ \mathrm{ml}$ and garlic extract at $45 \%$ concentration can therefore be used to control C. lindemuthianum the anthracnose pathogen of cowpea.

Monitoring virus and phytoplasma incidences in Texas peach orchards: 2014 and 2015

K. ONG (1), S. C. Rhodes (2), M. Giesbrecht (2)

(1) Texas A\&M AgriLife Extension Service, College Station, TX, U.S.A.; (2) Texas A\&M AgriLife Extension Service, College Station, TX, U.S.A. Phytopathology 105(Suppl. 4):S4.105

As a continuation effort to monitor pathogens of concern to the stone fruit industry in Texas, eight (8) commercial peach orchards in 7 counties were surveyed for the presence of Plum Pox Virus (PPV) and European Stone Fruit Yellows (ESFY) phytoplasma in 2014. Additionally, some samples were also evaluated to monitor incidences of Prune Dwarf Virus (PDV) and Prunus Necrotic Ringspot Virus (PNRSV). A total of 1224 foliar peach and plum samples were tested for PPV and 390 samples were tested for PDV and PNRSV. For virus detection, each sample was tested by ELISA using Agdia ELISA kits and following USDA APHIS PPQ and National Plant Diagnostic Network protocols. The 1224 samples tested negative for all five strains of PPV. Of the 390 samples tested for the two other viruses of interest, PDV was detected in 13 samples from 3 different counties and PNRSV were detected in 49 samples from 2 different counties. ESFY was not observed or detected in the surveyed peach orchards. This survey effort will continue in 2015 to ensure early detection if these pathogens emerges in Texas.

Identification of genes associated with biofilm formation in the phytopathogen Pectobacterium subsp. brasiliense

E. M. ONKENDI (1), I. Toth (2), L. N. Moleleki (1)

(1) University of Pretoria, Pretoria, South Africa; (2) The James Hutton Institute, Dundee, Scotland

Phytopathology 105(Suppl. 4):S4.105

Biofilms play a key role in enhancing the infection process of many phytopathogens. The importance of biofilm formation in virulence has been well investigated in vascular phytopathogens such as Xylella and Xanthomonas spp. However, within Pectobacterium genus, members of the Enterobacteriaceae family, there are few such studies. Significantly, the highly virulent and aggressive soft rot phytopathogen Pectobacterium carotovorum subsp. brasiliense $(P c b)$ forms biofilms in planta to colonise a susceptible potato plant stems. However genes involved in $P c b$ biofilm formation have not been identified. In the present study, a library of 2,000 Tn5-tagged mutants of $P c b 1692$ strain was screened to isolate biofilm defective mutants. A total of 19 mutants were found to be reduced in their ability to form biofilms. All biofilm defective mutants were shown to have a significant reduction in virulence when inoculated into potato tubers. Furthermore, all biofilm defective mutants were attenuated in their ability to elicit hypersensitive response in tobacco leaves after $48 \mathrm{~h}$ of infiltration. Some of the disrupted genes in the biofilm defective mutants have been associated with bacterial aggregation and virulence (such as CstA and cytolysin activator) while others may be novel. Therefore, the role of each gene identified in this study will broaden our understanding of biofilm, colonization and virulence in $P c b$, a potato phytopathogen of global importance.

Host specificity of Corynespora cassiicola causing target spot on blueberry and tomato in Florida

R. B. ONOFRE (1), G. Vallad (2), N. A. Peres (2)

(1) University of Florida - Gulf Cost REC, Wimauma, FL, U.S.A.; (2) University of Florida, Wimauma, FL, U.S.A.

Phytopathology 105(Suppl. 4):S4.105

Corynespora cassiicola causes target spot of tomato (Solanum lycopersicum L.) and has been found recently causing a similar leaf spot disease of blueberry (Vaccinium corymbosum L.) in Florida. Leaf symptoms on both hosts consist of circular to irregular reddish-brown necrotic spots. The host specificity of $C$. cassiicola isolates from tomato and blueberry, two widely grown crops in West Central Florida, was studied. Three isolates recovered from blueberry plants (14-671, 14-673 and 14-679) and two isolates from tomatoes (1P and 7P) were used. All isolates were inoculated on ten 2-yr-old healthy blueberry plants $\mathrm{cv}$. Jewel and fifteen 60-day-old healthy tomato plants cv. Taste Lee. Two blueberry plants and three tomato plants were used per isolate. A suspension of 1 to $2 \times 10^{4}$ conidia per $\mathrm{mL}$ was sprayed to runoff. Plants were kept in humid chamber for $36 \mathrm{~h}$ and incubated in the greenhouse. Experiments were conducted twice. After 5 days, identical symptoms to those described above were observed on the leaves of blueberry plants inoculated with all blueberry and tomato isolates. However, only tomato isolates caused target spot symptoms on tomato plants. C. cassiicola re-isolated from the lesions was identical to the original cultures. All the controls remained symptomless. The genetic relatedness of blueberry and tomato isolates will be further investigated to determine the origin of the $C$. cassiicola isolates that are host specific to blueberry.

Searching for synchrony: Seeking the early events in SW-5 resistance N. A. ORDAZ (1), C. A. Stafford-Banks (1), S. P. Dinesh-Kumar (2), D. E. Ullman (3)

(1) University of California, Davis, Oakley, CA, U.S.A.; (2) University of California, Department of Plant Biology and The Genome Center, College of Biological Sciences, Davis, CA, U.S.A.; (3) University of California, Davis, Davis, CA, U.S.A.

Phytopathology 105(Suppl. 4):S4.105

Tomato spotted wilt virus (TSWV) is the type member of the genus Tospovirus, the only plant infecting member of the family Bunyaviridae. Economically, TSWV results in millions of dollars of damage in tomatoes alone. TSWV is transmitted by several thrips species, the most important of which is Frankliniella occidentalis, the Western flower thrips (WFT). The most significant gene for TSWV resistance in tomato is Sw5. The long term goal of our study is aimed at revealing early and discernable transcriptional and proteomic changes triggered by Sw5-mediated recognition of TSWV. A prerequisite for conducting these experiments is a robust system for synchronizing and optimizing TSWV infection in tomato with mechanical and thrips inoculation. We hypothesized that plant age would be a significant component in synchronizing TSWV infections across a large number of plants. With mechanical inoculations we demonstrated that tomato $c v$. Celebrity plants with three expanded leaves resulted in $100 \%$ infection. The rate of infection decreased with age of the plant: 2 leaves (95\%), 3 leaves $(100 \%), 4$ leaves $(95 \%), 5$ leaves $(84 \%), 6$ leaves $(61 \%), 7$ leaves $(68 \%)$. Enzyme-linked immunosorbent assay (ELISA) showed that virus could be detected in thrips larvae 24 hours post-acquisition. ELISA readings indicated that TSWV titers increased incrementally up to 96 hours post-acquisition.

Developing a molecular diagnostic for Fusarium oxysporum f.sp. cubense tropical race 4 through Diversity Array Technology genotyping

N. I. ORDONEZ ROMAN (1), M. Salacinas (1), C. Schoen (1), O. Mendes (1), A. Kilian (2), G. Kema (1)

(1) Wageningen University and Research, Wageningen, Netherlands; (2) Diversity Arrays Technology Pty Ltd, Canberra, Australia

Phytopathology 105(Suppl. 4):S4.105

Early and reliable detection of Fusarium oxysporum f.sp. cubense (Foc) tropical race 4 (TR4) is required for effective monitoring of this banana (Musa) pathogen. To date, TR4 - originally identified in Taiwan - has already spread throughout Southeast Asia and was recently discovered outside this region. Traditional morphological Foc characterization is not feasible, as microscopic structures are similar within strains belonging to the $F$. oxysporum complex. Vegetative Compatibility Group (VCG) analyses, yet proven to be largely consistent, are time consuming and not always possible. Rapid molecular based methods are preferential due to their accuracy. The detection of unique genomic regions is a vital step for development of strainspecific probes. In this study, the Diversity Array Technology Sequencing (DArTseq) approach was used for the discovery of unique genome regions for primer design. DNA was extracted from 29 strains representing the overall VCG diversity of Foc. Using a stringency of call rate $>0.66$, reproducibility $=$ 100 and $\mathrm{Q}$ value $>2.7$ we selected 15,900 markers that included unique DArTseq markers for three TR4 strains-VCG01213. Alignments of the DArTseq sequences with the reference genome of Foc TR4 II-5 allowed upand downstream extension of the $\sim 69$ base pair sequences that enabled the design of Foc TR4 specific primers for the rapid detection of TR4 strains in different sample matrices from a range of geographically different locations.

Detached leaf culture technique for the study of switchgrass rust

G. ORQUERA (1), C. Garzón (1), S. Marek (1)

(1) Oklahoma State University, Stillwater, OK, U.S.A.

Phytopathology 105(Suppl. 4):S4.105

Switchgrass rust caused by Puccinia emaculata can significantly reduce biomass yield and feedstock quality. Lack of pure single-spore cultures of this obligate biotroph complicates molecular characterization of individual genotypes within heterogeneous populations. Thus, a detached leaf technique was developed to culture single pustules and single spores of $P$. emaculata. 
Prior to inoculation, $5 \mathrm{~cm}$ switchgrass leaf segments were surface-sterilized. Urediniospores of an Oklahoma population of $P$. emaculata, maintained on several varieties of switchgrass in a growth chamber, were used as inoculum. Spores from single pustules were isolated directly from infected tissue using a sterile pipet tip. Single spores were isolated by spreading urediniospores on water agar to induce germination and using agar plugs containing single germinated spores to inoculate detached leaves. Inoculated leaves $(n=120)$ were placed in a pipet tip box in a dew chamber for 24 hours and then maintained on water agar. Chlorotic flecks were observed after 8 or 12 days and uredinia formation after 12 or 18 days on leaves inoculated with single pustules or single spores, respectively. Benomyl was used to suppress mycoparasitic fungi without affecting $P$. emaculata. This method was used to isolate four single-spore and 19 single-pustule cultures of $P$. emaculata. These isolates will be used to characterize interactions of $P$. emaculata with genotypes of switchgrass.

Use of propidium monoazide and qPCR to quantify viable and nonviable conidia of Aspergillus flavus in almond soils

A. ORTEGA-BELTRAN (1), Y. Luo (1), T. J. Michailides (1)

(1) University of California, Davis, Kearney Agricultural Research and Extension Center, Parlier, CA, U.S.A.

Phytopathology 105(Suppl. 4):S4.106

Aflatoxins, produced mainly by Aspergillus flavus and A. parasiticus, are carcinogenic compounds that pose serious health threats. In California, almonds are occasionally contaminated with aflatoxins. However, climate change is increasing the frequencies of aflatoxin contamination events. The atoxigenic isolate Aspergillus flavus AF36 is a biocontrol agent registered with the EPA for use in California, Arizona, and Texas to limit aflatoxin accumulation of cottonseed, maize, and pistachio. Registration of AF36 for use in almond is being sought. However, use of multiple atoxigenics may allow for additive and long-term reduction in aflatoxin-producing potential of fungal communities associated with cultivated and non-cultivated areas. Use of atoxigenics (i) adapted to target crops, (ii) highly competitive, and (iii) able to thrive in distinct soil types would enhance long-term residence of the applied atoxigenics throughout a target area. Using propidium monoazide (PMA), a DNA-binding dye, along with real-time polymerase chain reaction (qPCR) allows for quantification of viable and nonviable conidia. Survival of atoxigenics in distinct almond soil types was monitored with a PMA-qPCR protocol. Results revealed differential soil adaptation among the examined atoxigenics. These results will aid in the selection of atoxigenics to integrate a biocontrol mixture for future registration with the EPA to limit aflatoxin accumulation of almond and other susceptible crops.

Glycoside Hydrolase family 3 genes may play a role in Phytophthora sojae pathogenicity

M. D. OSPINA-GIRALDO (1), A. Parish (1)

(1) Lafayette College, Easton, PA, U.S.A.

Phytopathology 105(Suppl. 4):S4.106

Carbohydrate-Active Enzymes are involved in the metabolism of complex carbohydrates. Glycoside hydrolase (GH) family 3, in particular, includes enzymes with $\beta$-glycosidase, xylan 1,4- $\beta$-xylosidase, and glucan 1,3- $\beta-$ glycosidase activities, whose substrates are glucose derivatives commonly found in the plant cell wall. Therefore, it is hypothesized that GH family 3 may play a role in the Phytophthora sojae infection process by targeting the cell wall components. Genome examination using bioinformatic approaches has revealed the presence of 27 gene sequences belonging to GH family 3 in P. sojae. The majority of sequences were located on scaffolds 1,2 and 8; on average, these sequences were $2200 \mathrm{bp}$ in length and many contained 1 or 2 introns, some of which largely exceeded the average intron length in Phytophthora spp. Phylogenetic analysis indicated that most sequences can be classified in three major clades, and bootstrap values supported the occurrence of multiple paralogous genes. Four GH family 3 genes were chosen for expression studies in mycelium grown in vitro and during plant infection, using real-time reverse-transcription PCR (qPCR). Analysis of the results obtained by qPCR showed differences in the level of expression of two of these genes at different time-points during the infection process when compared to mycelial expression. These results suggest a potential role for GH family 3 enzymes in the infection of soybean plants by $P$. sojae.

Effect of cultivar resistance, soil management and soil solarization on spatiotemporal development of Verticillium wilt of olive: A long-term study

E. Ostos (1), R. Porras (2), F. J. Lopez-Escudero (1), A. Trapero-Casas (1), J. MORAL (3)

(1) University of Cordoba, Cordoba, Spain; (2) Biogeos S.L., Cordoba, Spain;

(3) University of Cordoba, Cordoba, Spain

Phytopathology 105(Suppl. 4):S4.106
One of the major constraints for olive cultivation is Verticillium wilt, caused by Verticillium dahliae. Because no control measure applied singly is effective, an integrated disease management (IPM) strategy is recommended for its control. Here we study the effect of an IPM strategy applied during 12 years in an orchard ("Grañón") of susceptible cv. Picual and consisting in: chemical control of weed, soil solarization and replantation of the dead trees with the resistant cv. Frantoio. For that, we compare the disease progress on "Grañón" orchard with its progress on a neighbor orchard "Ancla" with analogous conditions, except that the soil is plowed and the susceptible cv. Picual is used for replanting. Verticillium wilt epidemics progressed faster in "Ancla" orchard than in the "Grañón". The annual mortalities were $6.10 \%$ for the olives cv. Picual grown on "Ancla" orchard, and $3.25 \%$ and $0.51 \%$ for the olives cvs. Picual and Frantoio, respectively, grown on "Grañón". The annual mortality of susceptible cultivar was positively correlated with the spring rainfall (March-May). The disease incidence and severity of treatments were Picual-"Ancla" > Picual-"Grañón" > Frantoio-"Grañoon". The Aggregation Index and Beta-Binomial distribution suggested aggregation on diseased trees. The main effect of replanting died olives was a decreasing of aggregation of Verticillium wilt. In conclusion, this IPM strategy has helped maintain a low level of disease and orchard profitability.

The role of science in regulatory decision making: Pesticides and honeybee health

D. OUIMETTE (1), J. Fajardo (2), P. Lewis (3), K. Eversole (4)

(1) Dow AgroSciences LLC, Indianapolis, IN, U.S.A.; (2) USDA, Washington, DC, U.S.A.; (3) EPA, Washington, DC, U.S.A.; (4) Eversole Associates, Bethesda, MD, U.S.A.

Phytopathology 105(Suppl. 4):S4.106

Science plays a critical role in the development and implementation of regulations and policy at the Federal level for plant health. Occasionally, regulatory issues capture the attention of the public decisively enough to result in Congressional action or modifications in policy. Recently, considerable attention has been focused on the decline in honeybee colonies which are critical for more than $\$ 15$ billion in crop value each year. Questions have been raised as to whether these declines are the result of the potential adverse effects of pesticides. Here, we use honeybee health as a model to portray the role of science in helping policymakers understand complex issues that may require changes in laws, regulations, or policies. Honeybee health has been shown to be affected by a number of factors, including entomopathogens, parasitic mites, poor nutrition, genetics, breeding, hive management, and exposure to environmental toxicants, including pesticides. Each of these factors must be addressed with scientific research that can properly balance the demands of regulatory decision-making, agricultural production, and stakeholder advocacy. Scientific input from a variety of sources, including the use of independent scientific advisory panels, peer-reviewed research, as well as stakeholder input provides a foundation for making sound and informed regulatory decisions. A broader array of cases is proposed for a future APS session.

Small RNA deep sequencing revealed that mixed infection of known and unknown viruses were common in field collected vegetable samples

C. Padmanabhan (1), Y. Zheng (2), R. Li (3), Z. Fei (4), K.-S. LING (5)

(1) USDA-ARS, U.S. Vegetable Laboratory, Charleston, SC, U.S.A.; (2) Boyce Thompson Institute for Plant Research, Ithaca, NY, U.S.A.; (3) USDAARS, U.S, Vegetable Laboratory, Charleston, SC, U.S.A.; (4) Boyce Thompson Insitute for Plant Research, Ithaca, NY, U.S.A.; (5) USDA ARS, Charleston, SC, U.S.A.

Phytopathology 105(Suppl. 4):S4.106

In an effort to characterize the causal agents for plant diseases in field collected samples using the small RNA deep sequencing technology, numerous known or novel viruses and viroids were identified. In many cases, a mixed infection with multiple pathogen species was common. Such situation complicated the accurate disease diagnosis and determination of the actual causal agent for the disease. With mixed infection of multiple viruses, the traditional concept of disease diagnosis based on specific virus detection may need to be fundamentally changed. Therefore, proper strategies could be deployed for effective disease management based on the actual involvement of multiple viruses on the crop. Using nearly 200 sRNA libraries prepared from various vegetable samples collected from around the world, and a bioinformatics pipeline developed specifically for virus identification with deep sRNA sequencing datasets, many viruses and viroids were tentatively identified. Their characterization for validation and complete genome sequences were carried out through gap filling and 5' RACE analysis. The 
preliminary analysis and the advantage of small RNA sequencing for virus identification will be presented.

\section{Understanding and managing a Diplodia blight outbreak on slash pine in} Florida

C. A. PAEZ (1), J. A. Smith (2)

(1) University of Florida, Alachua, FL, U.S.A.; (2) University of Florida, Gainesville, FL, U.S.A

Phytopathology 105(Suppl. 4):S4.107

Massive dieback and mortality of slash pines (Pinus elliottii) was reported in 2012 in central Florida. To understand and clarify the causal agent and disease biology, field plots were established to assess disease progression over time, isolations and inoculations were conducted to confirm the causal agent and relationships between abiotic factors and disease were examined. Identified based on sequencing of the ITS-rDNA, the following fungi were isolated from resinous cankers: Diplodia sapinea, Diplodia scrobiculata, Lasiodiplodia theobromae, Lasiodiplodia pseudotheobromae. At sites that were severely affected, Diplodia pinea and Lasiodiplodia theobromae were frequently recovered and at sites that were slightly affected, Diplodia scrobiculata was more frequent. To understand how abiotic factors could be affecting the disease outbreak, a foliar nutrient analysis was performed in trees exhibiting different stages of the disease. Mean nitrogen levels were significantly higher ( $p$-value $<0.05$ ) in trees exhibiting moderate to severe disease severity versus healthy trees. Virulence of each pathogen will be evaluated on pines with differing levels of nitrogen in a greenhouse experiment. The results from this work are being used to manage the disease and improve silviculture of slash pine buffer plantings in urban areas of Florida.

\section{Antibody-based diagnosis of Citrus Huanglongbing and stubborn disease using pathogen secreted proteins as detection markers}

D. PAGLIACCIA (1), E. Hawara (1), K. Clark (1), J. Shi (1), A. Mulchandani (2), T. T. Tran (2), G. Vidalakis (1), M. Wenbo (1)

(1) University of California, Riverside, Department of Plant Pathology \& Microbiology, Riverside, CA, U.S.A.; (2) University of California, Riverside, Department of Chemical and Environmental Engineering, Riverside, CA, U.S.A.

Phytopathology 105(Suppl. 4):S4.107

Citrus industry is facing threats worldwide from insect-transmitted and phloem-colonizing bacterial pathogens associated with diseases such as Huanglongbing (HLB) and Stubborn Disease (CSD). HLB and CSD diagnosis is challenging due to low titer and erratic distribution of the associated/causative bacteria in the infected trees. We seek to develop serological detection methods using pathogen secreted proteins as markers. Methods based on pathogen-specific secreted proteins, which are readily distributed in the infected trees through vascular flows, could better cope with the erratic distribution of the pathogen; therefore, improving disease diagnosis. We have identified secreted proteins from the HLB associated bacteria Candidatus Liberibacter asiaticus and the CSD causative agent Spiroplasma citri and generated antibodies that can specifically bind to selected secreted proteins. Using these antibodies, specific signals were picked up from HLB and CSD infected plants using direct tissue imprint immunoblots. For HLB, signals were also observed from plant tissue imprints of asymptomatic field samples, providing promise of early detection before the development of physical symptoms. A quantitative enzyme-linked immunosorbent assay (ELISA) is also under development using these antibodies for HLB and CSD. On-going efforts and progress on the development, evaluation and validation of these novel secreted proteins-based detection methods will be reported.

Distribution of endophytic bacteria-Bacillus amyloliquefaciens SPX1 in tomato tissues and its potential on control of tomato bacterial wilt

H. R. PAN (1), Y. J. Chen (2), W. H. Chung (1)

(1) National Chung Hsing University, Taichung, Taiwan; (2) Kaohsiung District Agricultural Research and Extension Station, Pingtung, Taiwan Phytopathology 105(Suppl. 4):S4.107

Previous studies indicated that endophytic bacteria (EB) could protect host plants from pathogenic infection. The EB of Bacillus amyloliquefacines SPX1 was able to inhibit the Ralstonia solanacearum and control the bacterial wilt (BW) of sweet potato. The objectives of this study are to analyze the distribution of SPX1 in tomato tissue and evaluate its efficacy on control of tomato BW. The inoculation tests indicated that SPX1 could spread in tissues of various hosts, including sweet potato, cucumber, tomato, etc. Further experiments revealed that SPX1 remained detectable in the whole stem of tomato 28 DAI. Thus, the movement of SPX1 strain proved to be systemically distributed in tomato. SPX1 maintained at a higher population $\left(6.0 \times 10^{4} \mathrm{cfu} / \mathrm{g}\right.$ tissue) in hypocotyls than in stems based on dipping method with hypocotylcutting seedlings. In addition, results from using the transformed strain, SPX1/DH5 $\alpha$ (pAD43-25), with green fluorescent protein marker as inoculum demonstrated that the SPX1 could enter intercellular spaces and colonize xylems of tomato. Seven days after inoculation, SPX1 was detected from vascular bundles, cortex, epidermis and secondary roots. As for disease control, SPX1 was shown to reduce the disease severity of BW by $95.0 \%$ to $62.5 \%$ in tomato that had grown in the SPX1-infested soil $\left(10^{6} \mathrm{cfu} / \mathrm{g}\right.$ soil) for 28 days. Thus, the $B$. amyloliquefaciens SPX1 strain could potentially be used as a bio-agent on controlling $\mathrm{BW}$ of tomato.

Exogenously derived RNAi signals targeting essential genes of Puccinia triticina shows enhanced resistance to leaf rust disease in wheat

V. PANWAR (1), M. Jordan (2), B. McCallum (2), P. Fobert (3), G. Bakkeren (4)

(1) National Research Council of Canada, Morden, MB, Canada; (2) Agriculture and Agri-Food Canada, Morden, MB, Canada; (3) National Research Council of Canada, Saskatoon, SK, Canada; (4) Agriculture and Agri-Food Canada, Summerland, BC, Canada

Phytopathology 105(Suppl. 4):S4.107

Wheat rusts caused by fungal pathogens are devastating diseases causing huge yield losses in global wheat production regions. Available natural resistance against rust fungi has often proved to be ephemeral owing to changes in the pathogen population. Further, the obligate biotrophic nature of rust pathogens obviates the use of many established genetic methods for genome manipulation studies. A novel control strategy is the prevention of infection by feeding exogenously generated lethal RNAi signals to these fungi targeting their essential genes. We show that stable in planta expression of RNAi sequences derived from two leaf rust fungus Puccinia triticina $(P t)$ essential pathogenicity genes, namely MAP-kinase (PtMAPK1) and cyclophilin $(P t C Y C)$, effectively suppress leaf rust disease in wheat. Transgenic wheat plants expressing these $P t$ gene segments as hairpin RNAi constructs conferred effective disease suppression in $\mathrm{R}_{1}$ and $\mathrm{R}_{2}$ generations. In some generated wheat lines, fungal biomass was reduced to $80 \%$ of control plant infections. RNA analysis confirmed the presence of $P t$ gene-specific short interfering RNAs in transgenic plants which effectively down regulated the expression of the target endogenous genes in $P t$ colonizing wheat leaves. Our results indicate that host derived silencing of essential fungal genes can be used as an effective strategy for developing durable rust disease resistance in wheat plants.

\section{Global transcriptional response to heat shock and grapevine wood in} Lasiodiplodia theobromae

M. PAOLINELLI-ALFONSO (1), J. M. Villalobos-Escobedo (2), J. F. LopezHernandez (2), M. Rendon-Anaya (2), A. E. Herrera-Estrella (3), R. Hernandez-Matinez (4)

(1) Centro de Investigacion CIentifica y Educacion Superior de Ensenada (CICESE), Ensenada, Mexico; (2) Laboratorio Nacional de Genomica para la Biodiversidad (LANGEBIO), Irapuato, Mexico; (3) Laboratorio Nacional de Genómica para la Biodiversidad (LANGEBIO), Irapuato, Mexico; (4) Centro de Investigacion Cientifica y Educacion Superior de Ensenada (CICESE), Ensenada, Mexico

Phytopathology 105(Suppl. 4):S4.107

Lasiodiplodia theobromae is Botryosphaeriaceae fungus that causes grapevine trunk disease, especially in regions with hot climate. To test for the hypothesis that heat shock (HS) triggers its pathogenicity, a transcriptomic study in the presence or absence of grapevine wood (GW) was performed. A total of 19860 "de novo" assembled transcripts were obtained with an average length of $658 \mathrm{bp}$ and an N50=2472 bp. Fourty-nine per cent showed homology with other Botryosphaeriaceae fungi, such as Neofusicoccum parvum and Macrophomina phaseolina; functional annotation was obtained for $38 \%$ of them. In total, 1225 genes were up-regulated and 1114 down-regulated in all evaluated conditions (11\%). Genes up-regulated in the presence of GW but down-regulated with HS, were found mainly involved in O-glycosyl hydrolysis, while genes induced by HS were enriched in oxido-reductase activity. One of the transcripts heavily induced in response to HS encodes a putative intradiol ring cleavage dioxygenase, a key enzyme in the benzoate (and phenolics) degradation metabolic pathway. L. theobromae grows well using only benzoate as carbon source, suggesting that its presence promotes mycelial growth during HS. The marked change in carbon-source utilization, from glucose-based to amino acid-based and oxido-reductive metabolism together with the induction of putatively secreted pathogenicity factors suggest that a change to a pathogenicity behavior occurs in response to HS. 
Prevalence of Pepper mild mottle virus in a nationwide survey in South Korea, and production of an infectious clone of the major isolate of PMMoV

J. S. PARK (1), J. S. Gong (2), J. K. Kim (2), E. Y. Seo (2), N. g. Kim (3), C. Jang (3), I. Hwang (3), J. Hammond (4), H. G. Kim (2), H. S. Lim (2)

(1) Chungnam National University, DaeJeon, South Korea; (2) Chungnam National University, Daejeon, South Korea; (3) Central Research Institute of Kyung Nong Corporation, Gyeongju, South Korea; (4) United States Department of Agriculture-Agrocultural Research Service, Beltsville, MD, U.S.A.

Phytopathology 105(Suppl. 4):S4.108

Pepper mild mottle virus (PMMoV) affects yields of peppers in South Korea. The distribution and incidence of viral diseases in Korean pepper fields and greenhouses were examined in a nationwide survey. We detected PMMoV in 15 of 181 domestic pepper samples collected. In this study, sequence analysis of the coat protein of isolates collected revealed seven strains correlating with the collection regions. The isolates share greater than $95 \%$ coat protein amino acid identity with each other. In addition we determined whether PMMoV was seed transmitted from the pepper samples collected. Seed transmission rates varied from $12.5 \%$ to $86 \%$, depending on the regions and the isolates. In order to determine which viral proteins affect seed transmission, we constructed infectious clones from five isolates of PMMoV, which were then evaluated for seed transmission frequency. In addition we also investigated the ability of the commercial biological control agent 'FarmWorld' to reduce seed transmission.

\section{Multiple disease resistance in dry edible pinto bean breeding lines} obtained by marker-assisted selection

J. S. PASCHE (1), R. S. Lamppa (2), J. M. Osorno (2), P. Miklas (3)

(1) North Dakota State Univ, Fargo, ND, U.S.A.; (2) North Dakota State University, Fargo, ND, U.S.A.; (3) USDA-ARS, Prosser, WA, U.S.A.

Phytopathology 105(Suppl. 4):S4.108

Numerous diseases limit yield potential and affect the quality of dry beans in North Dakota (ND) including rust (Uromyces appendiculatus), common bacterial blight (CBB; Xanthomonas axonopodis pv. phaseoli; Xap) and anthracnose (Colletotrichum lindemuthianum). The objective of this research was to verify resistance in pinto bean breeding lines developed based on marker-assisted selection (MAS) for rust $(U r-5)$, CBB (SU91 and SAP6 QTL), and anthracnose $\left(\mathrm{Co}-4^{2}\right)$ resistance genes. No pinto bean cultivars resistant to new races of $U$. appendiculatus and C. lindemuthianum in ND are available, and limited resistance to Xap is available. A total of 63 breeding lines from the MAS program, selected for agronomic performance in ND and Washington State in 2014, were challenged by the pathogens in the greenhouse. Plants were placed in a misting chamber for 24 hours following inoculation of the first trifoliate leaves with Xap and primary leaves with race 20-3 U. appendiculatus, and for 5 days following inoculation of primary leaves with race $73 \mathrm{C}$. lindemuthianum. Two leaves from each of four plants (replicates) were rated 10,14 and 21 days after inoculation for anthracnose, rust and $\mathrm{CBB}$, respectively. Four lines were rated as highly resistant and 11 more had high to moderate resistance to all three diseases. Additional lines displayed high resistance to two diseases. These lines possess good agronomic characteristics and have excellent potential as future cultivars.

Monitoring the spread of Phytophthora ramorum at a quarantine site reveals new and rare pathogens of woody plants in Northern California T. Pastalka (1), S. Rooney Latham (2), K. Suslow (1), W. SCHWEIGKOFLER (1)

(1) Dominican Univ of California, San Rafael, CA, U.S.A.; (2) California Department of Food \& Agriculture, Sacramento, CA, U.S.A.

Phytopathology 105(Suppl. 4):S4.108

The National Ornamental Research Site at Dominican University of California (NORS-DUC) is a quarantine research nursery established in 2009 to study invasive plant pathogens like Phytophthora ramorum, causal agent of Sudden Oak Death and Ramorum blight. In order to fulfill federal and state regulations, the possible movement of pathogens from the research site must be monitored using a sentinel plant system with host plants of $P$. ramorum. Symptoms on the sentinel plants are studied using culturing, immuno tests and sequence analysis. Symptom development follows a clear seasonal pattern with a peak in the rainy season (Dec. to Mar.). To date, P. ramorum has not been detected on sentinel plants, indicating limited aerial spread of the pathogen under unfavorable climatic and environmental conditions. $P$. ramorum-like symptoms on sentinel plants were associated with a number of Oomycetes including $P$. syringae, $P$. multivora and $P$. cf. fallax and a fungus closely related to Neofusicoccum cryptoaustrale. P. fallax has previously only been isolated from crown dieback symptoms of Eucalyptus in New Zealand and Australia and N. cryptoaustrale from Eucalyptus in South Africa. Host ranges of the P. cf. fallax and Neofusicoccum sp. isolates detected in CA are unknown and they might represent new invasive species in the U.S.A. In addition, monitoring of other host plants near the quarantine site revealed the presence of several other new fungi, including Cryptosporiopsis $s p$.

Epistatic interaction among rust resistance genes in common bean M. PASTOR-CORRALES (1), G. Valentini (2), Q. Song (3), P. Cregan (3) (1) USDA ARS, Beltsville, MD, U.S.A.; (2) Universidade Estadual de Maringá, Maringa, Brazil; (3) USDA/ARS, Beltsville, MD, U.S.A.

Phytopathology 105(Suppl. 4):S4.108

Resistance in common bean to the rust pathogen is conferred by single and dominant genes. The resistant phenotype of these genes is expressed either as a hypersensitive reaction (HR), visualized as necrotic spots without sporulation or as tiny uredinia (R). Epistatic interaction, in which one gene masks the phenotype of another gene, has been mentioned often in the literature for some rust resistance genes, however little is known about these interactions. We conducted a study using phenotypic and genetic markers to elucidate the interaction between the $U r-4$ and $U r-5$ rust resistance genes. A total of six $F_{1}$ plants and $182 F_{2}$ plants from a Mexico $309(U r-5)$ x Early Gallatin $(U r-4)$ cross plus the two parents, and 10 check cultivars, were inoculated with races 40, 44, 53 and 108 of $U$. appendiculatus. Races 53 and 108 were used to detect the presence of $U r-5$ and $U r-4$, respectively, and races 40 and 44 to determine the plausible epistatic interaction between these genes. We also used simple sequence repeat genetic markers that were previously identified as closely linked to the $U r-4$ and $U r-5$ genes. Inoculation of the 182 $\mathrm{F}_{2}$ plants produced a segregation ratio of $12 \mathrm{R}$ : $3 \mathrm{HR}$ : $1 \mathrm{~S}$. The molecular analysis on the $182 \mathrm{~F}_{2}$ plants using the SSR markers gave the same results as the phenotypic evaluation, except for one plant. These results indicate a dominant epistatic interaction, where the $U r-5$ gene is epistatic to $U r-4$ and the dominant allele of $U r-5$ hides the effects of $U r-4$.

Factors affecting sporangial germination of Peronospora belbahrii, a causal organism of basil downy mildew

J. S. PATEL (1), S. Zhang (2), M. I. Costa de Novaes (3)

(1) Univ of Florida IFAS, Homestead, FL, U.S.A.; (2) TREC - Univ of Florida, Homestead, FL, U.S.A.; (3) Sponsored by CNPq, University of Florida, Homestead, FL, U.S.A.

Phytopathology 105(Suppl. 4):S4.108

Peronospora belbahrii is an obligate oomycete causing downy mildew on basil. The pathogen was first detected on basil from Homestead of Florida in 2007 and since then the disease has spread to more than 30 U.S. states. Due to recent emergence of the disease, only few management strategies are available to control the disease. Therefore, it is imperative to understand biology of the pathogen for developing effective management strategies. The main objective of this study was to determine the effect of temperature and age of sporangia on germination of the $P$. belbahrii sporangia. The freshly produced sporangia were plated on $1.5 \%$ water agar, and placed at $5,10,15$ and $20^{\circ} \mathrm{C}$. The greatest germination rate was found for the sporangia placed at $10^{\circ} \mathrm{C}$. Further, the sporangia of various ages, i. e. $0,1,2,3,4$, and 5 days after sporulation (DAS) were evaluated for germination on $1.5 \%$ water agar at $10{ }^{\circ} \mathrm{C}$. Germination of the sporangia occurred at $3 \mathrm{~h}$ after plating and exponentially increased until $6 \mathrm{~h}$. Sporangia collected 0,1 , and 2 DAS germinated at a significantly greater level compared to the sporangia collected 3,4 , and 5 DAS. Results from this study indicate that freshly produced sporangia of $P$. belbahrii could cause severe downy mildew on basil at cool temperatures.

Factors influencing the survival of Fusarium oxysporum f. sp. lactucae on crop residue

K. R. PAUGH (1), T. R. Gordon (1)

(1) UC Davis, Davis, CA, U.S.A.

Phytopathology 105(Suppl. 4):S4.108

The causal agent of Fusarium wilt in lettuce, Fusarium oxysporum f. sp. lactucae, is a soilborne fungal pathogen that can survive and reproduce on crop residue in absence of the host. In a field experiment either dried or fresh crop residue naturally infested with $F$. oxysporum f. sp. lactucae was incorporated into the upper $20 \mathrm{~cm}$ of fallow soil in $0.3 \mathrm{~m}^{2}$ micro-plots. Preliminary results indicated that drying infested crop residue prior to its incorporation in soil reduced soil populations of the pathogen by 52 and 37 percent following incorporation in late August 2013 and 43 and 27 percent following incorporation in early October 2013 at 6 and 9 months, respectively. Fragmenting and oven-drying infested crop residue at $30^{\circ} \mathrm{C}$ has similarly demonstrated a reduction in recovery of the pathogen from crop residue. In a greenhouse experiment either fragmented or whole pieces of infested crop residue were incorporated in field soil in $10 \mathrm{~cm}$ diameter pots to assess the impact of fragmentation on the survival of pathogen populations in soil. Additional greenhouse experiments are currently in progress to assess the 
effect of a 2 -fold increase in soil nitrogen and/or an approximately 15 -fold increase in the soil density of a competitive soil fungus, Fusarium equiseti, on pathogen populations in soil. Samples in each experiment were collected at 13 month intervals to evaluate the impact of each treatment on pathogen populations in soil over time.

Pathogenicity and phylogenetics of the celery anthracnose pathogen, Colletotrichum fioriniae ( $=$ C. acutatum sensu lato)

J. PAVEL (1), B. Liu (1), C. Feng (1), J. C. Correll (1), S. R. May (2), B. K. Gugino (2), M. del Mar Jimenez-Gasco (2), M. K. Hausbeck (3), R. N. Raid (4), S. T. Koike (5)

(1) Dept. Plant Pathology, University of Arkansas, Fayetteville, AR, U.S.A.; (2) Dept. of Plant Pathology and Environmental Microbiology, Pennsylvania State University, State College, PA, U.S.A.; (3) Dept. of Plant, Soil, and Microbial Sciences, Michigan State University, East Lansing, MI, U.S.A.; (4) Dept. of Plant Pathology, University of Florida, Belle Glade, FL, U.S.A.; (5) University of California Cooperative Extension, Salinas, CA, U.S.A. Phytopathology 105(Suppl. 4):S4.109

Celery anthracnose was first described in Australia in 1966 and was reported in the U.S. in 2010. Symptoms of the disease include leaf, petiole, and crown lesions and necrosis along with an unusual and severe leaf curling. The pathogen causing celery anthracnose was described as a member of the species complex Colletotrichum acutatum sensu lato. Recent phylogenetic analysis of C. a . sensu lato has led to 29 species being differentiated and described. Sequence analysis of the glutamine synthetase gene intron of celery anthracnose isolates in this study indicated that all isolates were closely related to one another and belong to the recently described species $C$. fioriniae. In addition, vegetative compatibility testing is being evaluated to determine the genetic diversity among the celery anthracnose pathogen to determine if celery isolates are clonal. A diverse collection of over 50 isolates within $C$. a. sensu lato, including celery and non-celery isolates of $C$. fiorniae, has been examined for the ability to cause typical celery anthracnose symptoms. Both celery isolates and many non-celery isolates in the species complex were able to infect celery, cause typical leaf, petiole, and crown lesions and necrosis, as well as cause the unusual leaf curl type symptoms.

A population analysis of Puccinia emaculata using single spores in Oklahoma and Virginia

J. C. PAVLU (1), G. Orquera (2), O. Arias (3), A. Moya (4), S. M. Marek (5), C. Garzón (5)

(1) Oklahoma State University, Perkins, OK, U.S.A.; (2) Oklahoma State University, Stillwater, OK, U.S.A.; (3) Escuela Politécnica del Ejército, Sangolquí, Ecuador; (4) PUCE - Pontificia Universidad Católica del Ecuador, Quito, Ecuador; (5) Oklahoma State University, Stillwater, OK, U.S.A. Phytopathology 105(Suppl. 4):S4.109

Native to the North American prairies, Panicum virgatum, most commonly known as Switchgrass, has been chosen for biofuel production due to its low input cost of production and high feed stock quality. However, a rust pathogen (Puccinia emaculata) has become a major threat to Switchgrass causing significant widespread biomass reductions. The objective of this study was to spatially and temporally analyze the population dynamics of switchgrass rust over a period of two years (2011 and 2012) between two different states, Virginia and Oklahoma. Individual $P$. emaculata urediniospores were collected and whole genome amplified. Following confirmation of the rust genus and species, 20 previously designed microsatellite loci were screened. Four microsatellites consistently produced polymorphic amplicons. Our results indicate that the 2011 population from Virginia was the most unique, while the 2011 and 2012 Oklahoma populations and the 2012 Virginia population were not significantly different. These findings suggest a possible movement of alleles through migration along the Puccinia pathway. Population analysis of representative population samples over space and time, will allow a better understanding of the epidemiology and movement of the rust pathogen, which will lead to the development of best management practices.

Evaluation of native and non-native phytoalexins in suppressing in vitro, in vivo, and in planta growth of Sclerotinia sclerotiorum

M. L. PAWLOWSKI (1), C. B. Hill (1), A. V. Lygin (1), O. V. Zernova (2), J. M. Widholm (1), V. V. Lozovaya (1), G. L. Hartman (1)

(1) University of Illinois, Urbana, IL, U.S.A.; (2) University of Illinois, Champaign, IL, U.S.A.

Phytopathology 105(Suppl. 4):S4.109

Sclerotinia stem rot has been a major threat to soybean production worldwide. Partial resistance found in crop germplasm has been only marginally effective in managing diseases caused by Sclerotinia sclerotiorum. Genetic manipulation of the innate defenses in plants may offer additional sources of resistance. Phytoalexins are important components of plant innate defenses and genetic manipulation resulting in increased or decreased levels of phytoalexin accumulation in plant tissues has been shown to significantly impact plant resistance. Resveratrol and pterostilbene are phytoalexins native to Vitis vinifera and other plant species that are known for their antimicrobial activity. Transgenic expression of these phytoalexins in other hosts has increased disease resistance. The objectives of our research were to determine if resveratrol and pterostilbene could suppress growth of S. sclerotiorum. Mycelial growth on pterostilbene-amended agar was reduced by $53 \%$ and $50 \%$, at $25 \mu \mathrm{g} / \mathrm{ml}$ and at $18 \mu \mathrm{g} / \mathrm{ml}$, respectively. A dual concentration of 25 $\mu \mathrm{g} / \mathrm{ml}$ pterostilbene with $100 \mu \mathrm{g} / \mathrm{ml}$ resveratrol reduced mycelial growth by $69 \%$. Evaluation of in planta and in vivo responses of soybean plants transformed to synthesize resveratrol and pterostilbene to $S$. sclerotiorum is in progress.

The interesting case of soybean seedborne Fusarium spp.: From identity to pathogenicity

R. PEDROZO (1), J. J. Fenoglio (1), C. R. Little (1)

(1) Kansas State University, Manhattan, KS, U.S.A.

Phytopathology 105(Suppl. 4):S4.109

Although Fusarium spp. are among the most important pathogen groups on soybeans, their identity and importance as seedborne pathogens remain unclear. Thus, the objective of this work was to determine the identity of soybean seedborne Fusarium spp. and to study the potential of some species to impact seedling vigor and seed quality in pathogenicity assays. Seed was collected from 408 soybean samples produced in ten counties in the state of Kansas, USA during three growing seasons, 2010-2012. A semi-selective medium (PCNB) was used for fungal isolations and identification was based upon morphological characters and PCR. Isolate pathogenicity was assessed in the laboratory and greenhouse. The three-year screening effort showed that nine Fusarium species were seedborne. F. semitectum (FSE) was the most frequently encountered species $(56.42 \%)$, followed by $F$. proliferatum (FPR; $19.25 \%$ ), $F$. verticillioides (FVE; 13.20\%), F. acuminatum (FAC; $2.85 \%$ ), $F$. equiseti (FEQ; 2.80\%), F. thapsinum (FTH; 2.22\%), F. fujikuroi (FFU; $1.95 \%), F$. oxysporum (FOX; $0.98 \%$ ) and $F$. graminearum (FGR; $0.33 \%$ ). Regarding their pathogenicity, only soybean seeds artificially infested with FPR, FGR, FTH, FFU and FOX reduced seed germination when compared with non-inoculated seeds (control) in both environments. Additional experiments are underway to further explore soybean seedborne Fusarium pathogenicity behavior and its potential implication on seed health testing.

Understanding the emergent fruit rot disease of Winterberry holly F. Peduto Hand (1), S. LIN (1), N. J. Taylor (2), R. H. Zondag (3)

(1) The Ohio State University, Columbus, OH, U.S.A.; (2) C. Wayne Ellett Plant \& Pest Diagnostic Clinic, Raynoldsburg, OH, U.S.A.; (3) The Ohio State University Extension, Painesville, OH, U.S.A.

Phytopathology 105(Suppl. 4):S4.109

Winterberry holly (Ilex sp.) is a deciduous ornamental shrub harboring colorful berries, whose cut stems are used in holiday decorations. Since 2012, winterberry producers in Ohio and several other states in the Midwestern and Eastern United States are confronted with a fruit rot problem of unspecified cause that is reducing yield of saleable plant material, income, and opportunities for market expansion. Spore trapping trials were set up in Spring 2014 at two orchards in northern Ohio. Microscope slides coated with petroleum jelly were hung on the plants and allowed to trap spores weekly. Spores were washed off the slides and plated onto PDA amended with antibiotics. The most represented colonies were counted and subcultured and subjected to identification. Colony counts were also correlated to meteorological data, including rainfall and average temperature. Fruit infection was monitored biweekly on 100 randomly collected berries. Any observed fungal colonies were subcultured onto PDA plates and subjected to identification. A few isolates were selected to conduct preliminary pathogenicity tests. The most represented fungal species recovered from the spore traps included Colletotrichum sp., Alternaria sp. and Fusarium sp. Species of Alternaria were also consistently isolated from the fruit samples. Preliminary pathogenicity tests of Alternaria sp. and Colletotrichum sp. showed that different cultivars might affect the ability of the pathogens to infect.

Comparative transcriptomic analysis of Burkholderia glumae reveals the important role of tep $R$ gene in regulating a multitude of cellular processes J. PENG (1), J. H. Ham (1), S. Osti (1), I. K. Barphagha (1)

(1) Louisiana State University, Baton Rouge, LA, U.S.A.

Phytopathology 105(Suppl. 4):S4.109

Burkholderia glumae causes bacterial panicle blight in rice by producing the phytotoxin, toxoflavin, as a major virulence factor. Production of toxoflavin is 
negatively regulated by tep $R$, which encodes a sigma 54-dependent response regulator. In order to gain insights into the comprehensive biological functions of tepR, the genome-wide transcriptional profile dependent on tepR was analyzed through comparative transcriptome analysis between a tepR defective mutant and the wild-type strain, resulting in the identification of 238 differentially expressed genes (DEGs). Gene Ontology (GO) enrichment analysis indicated that genes involved in flagella assembly and stress response were highly enriched in tepR defective strain. Kyoto Encyclopaedia of Genes and Genomes (KEGG) pathway enrichment further revealed that tepR positively regulates the gene cluster encoding a type six secretion system (T6SS) and the genes for a branched-chain amino acid ABC Transporter system, but negatively regulates the genes for thiamine, cysteine and methionine metabolism. As homologues of tepR are found in a number of pathogenic Burkholderia species, the modulation of bacterial behavior through the regulatory action of tepR is likely to be a widely occurring phenomenon in this group of bacterial pathogen.

\section{LSU Summer Technical Sharing Sessions: Graduate students teaching graduate students}

J. PENG (1), R. R. Sweany (1), A. Lunos (1)

(1) Louisiana State University Agricultural Center, Baton Rouge, LA, U.S.A. Phytopathology 105(Suppl. 4):S4.110

Plant pathology researchers study pathogens, plants, and their interactions using vastly different techniques; lack of knowledge of all techniques can result in communication barriers between scientists. The objective of our Summer Technical Sharing Sessions (STSS) was to promote graduate student sharing and learning of techniques from different labs in LSU's Department of Plant Pathology and Crop Physiology. The Graduate Student Association (https://lsuppcpgsa.wordpress.com/) implemented STSS in the summer of 2014. Seven students from different labs shared techniques ranging from field, lab and computer based research; typically 10-15 students attended each session. Students learned about several forms of chromatography, yield estimation, evaluation of fungicide resistance, quantification of chlorophyll and xanthophylls, phylogeny construction and about the lac-operon. The STSS provided a great opportunity for graduate students to understand peers' research and comprehend the diversity of techniques used within the department. This learning, sharing and teaching exposure in turn increased student comradery, communication and teaching skills. With the success of last year, our department will host STSS annually and increase the diversity of techniques covered. We firmly believe STSS could benefit all the agriculturerelated departments especially as research techniques are becoming more diverse and complex.

\section{Tomato gene has homology to a defense related gene in Arabidopsis thaliana \\ J. PEREIRA (1), J. B. Jones (2), Z. Mou (2)}

(1) Univ of Florida, Gainesville, FL, U.S.A.; (2) University of Florida, Gainesville, FL, U.S.A.

Phytopathology 105(Suppl. 4):S4.110

Genetic transformation of plants has offered new opportunities compared to traditional breeding practices, which reduces the occasional transference of genes. This technique allows for the transference of a specific DNA sequence. Genetic modified crops have had difficult acceptance due to many external factors. Complementation assay has been used for several research possibilities, among them, to verify if the genotype of one organism can rescue the function absent in the other organism. The predicted protein sequence of a tomato gene has homology to a defense related gene in Arabidopsis thaliana. The gene in Arabidopsis confers resistance to fungal diseases. This same gene confers resistance to bacterial disease when transformed in tomato. In each instance, homologous genes may have the same function. The aim of this work was to verify if the tomato homologous gene has the same function as the Arabidopsis defense related gene. Arabidopsis mutant plants were transformed with the tomato homologous gene. After the seed screening, the transformants showed wild type phenotype. More studies must be done for further confirming evidence. If both genes have the same function, the homologue gene in tomato could be overexpressed to increase resistance to diseases. This tomato plant, supported by such methods, would not be transgenic, making its acceptance in the market more affluent.

Incubation in soil reduces sporulation and risk of epidemic development from leaf disks infested by Phytophthora ramorum

E. K. PETERSON (1), J. L. Parke (1), N. J. Grünwald (2)
(1) Oregon State University, Corvallis, OR, U.S.A.; (2) USDA-ARS Horticultural Crops Research Laboratory, Corvallis, OR, U.S.A. Phytopathology 105(Suppl. 4):S4.110

Soilborne inoculum has been proposed as an important contributor to the local persistence of the sudden oak death pathogen Phytophthora ramorum, however current research has only assessed recovery of this pathogen from infested leaf debris. Two additional aspects of the disease cycle are being investigated at the National Ornamental Research Site at Dominican University of California: the infection of leaf baits at the soil surface, and sporangia production from inoculum incubated in soil. Rhododendron leaf disk inoculum was buried in soil at 5 and $15 \mathrm{~cm}$ in June 2014. Subplots were baited with non-infested leaf disks, and buried inoculum was recovered and placed in water to induce sporulation. Isolation of $P$. ramorum from soilincubated disks remained stable after an initial decline observed within the first two weeks. Sporangia production was greatest from inoculum at $15 \mathrm{~cm}$, but was reduced for inoculum at both depths. With the onset of cooler temperatures in autumn we observed a moderate increase in sporangia production, but have not yet isolated $P$. ramorum from baits placed above inoculum introduced in June. Our results indicate recovery of $P$. ramorum from infested leaf debris may overestimate the risk soilborne inoculum poses to new infections due to decreases in sporulation capacity. While cooler and more constant temperatures and moisture levels at lower depths may contribute to greater survival and sporulation, dispersal from these depths may be limited.

The Leaf Doctor: A new application for quantifying disease severity S. J. PETHYBRIDGE (1), S. C. Nelson (2)

(1) Cornell University, Geneva, NY, U.S.A.; (2) University of Hawaii at Manoa, Honolulu, HI, U.S.A.

Phytopathology 105(Suppl. 4):S4.110

The Leaf Doctor (LD) is a new, semiautomatic iPhone app to quantify plant disease severity. The user collects or imports a photograph of a symptomatic plant organ superimposed individually on a solid black background or one modified by photo editing software. The iterative assessment algorithm allows users to select up to eight different hues representing healthy tissues. A threshold slider sets the value for the proximity of healthy to diseased pixels. The end point occurs when only the symptomatic tissues are colored blue. The algorithm then calculates the percentage of diseased pixels. The accuracy, precision, and robustness of LD were evaluated for six different diseases. Disease severity estimates calculated by LD were highly accurate $\left(R^{2} \geq 0.79\right)$ compared to those obtained by the PC program, Assess version 2.0. The accuracy of LD was slightly impaired for diseases having less discrete color contrasts between diseased and healthy tissues, such as powdery mildew and rust. Advantages of LD include zero cost, high portability, reduced times for image processing, ease of use, and the ability to adapt analyzed images to create realistic standard area diagrams. LD can be downloaded from iTunes for use on a mobile Apple platform.

A functional gene cluster for toxoflavin biosynthesis in the genome of the soil bacterium Pseudomonas protegens Pf-5

B. J. Philmus (1), B. T. Shaffer (2), Q. Yan (1), J. E. LOPER (3)

(1) Oregon State University, Corvallis, OR, U.S.A.; (2) USDA-ARS, Corvallis, OR, U.S.A.; (3) USDA ARS, Corvallis, OR, U.S.A.

Phytopathology 105(Suppl. 4):S4.110

Toxoflavin is a broad-spectrum toxin best known for its role in virulence of Burkholderia glumae, which causes panicle blight of rice. A gene cluster containing homologs of toxoflavin biosynthesis genes (toxA-E) of B. glumae is present in the genome of Pseudomonas protegens Pf-5, a biological control strain that produces many antimicrobial compounds. Repeated efforts to detect toxoflavin production by Pf-5 were unsuccessful so we attempted to associate antimicrobial activity with the gene cluster. Recognizing that this antimicrobial activity may be difficult to discern from the activities of other metabolites, we derived a mutant of Pf-5 (LK185) that lacked production of all of Pf-5's known antibiotics. LK185 suppressed P. syringae DC3000 on an iron-amended medium (KMB-Fe), whereas toxC or toxD derivatives of LK185 did not suppress DC3000. We then observed that LK185 grown in KMB-Fe broth without shaking produced toxoflavin whereas tox $C$ and tox $D$ mutants did not produce toxoflavin. We propose that the biosynthetic pathways for toxoflavin and riboflavin share the first two steps because deletion of toxE diminished but did not eliminate toxoflavin production, suggesting that the paralog RibD compensates for the loss of ToxE. We also report that $\operatorname{tox} M$, which is present in the toxoflavin cluster of Pf-5 but not $B$. glumae, encodes a monooxygenase that detoxifies toxoflavin; introduction of toxM into $P$. syringae DC3000 confered toxoflavin resistance. 
Development of a PCR-based diagnostic assay for the detection of Magnaporthe oryzae (Triticum pathotype) from infected wheat

M. PIECK (1), L. Chen (2), A. Ruck (1), M. L. Farman (2), B. Valent (3), G. Peterson (4), K. F. Pedley (4)

(1) USDA-ARS Foreign Disease- Weed Science Research Unit, Ft. Detrick, MD, U.S.A.; (2) Department of Plant Pathology, University of Kentucky, Lexington, KY, U.S.A.; (3) Kansas State University, Department of Plant Pathology, Manhattan, KS, U.S.A.; (4) USDA-ARS Foreign Disease Weed Science Research Unit, Ft. Detrick, MD, U.S.A.

Phytopathology 105(Suppl. 4):S4.111

Wheat blast disease, caused by the Triticum pathotype of the fungus Magnaporthe oryzae, is responsible for sporadic widespread losses of wheat production in Brazil, Bolivia, Paraguay, and northern Argentina. To date, wheat blast has only been reported in South America. The potential for introduction of the pathogen to the U.S. is a significant concern due to the lack of resistance in elite wheat cultivars and the ineffectiveness of fungicide treatments during outbreaks. An important first step in preventing the spread of the South American M. oryzae (Triticum pathotype) isolates into the U.S. is the development of a rapid, accurate and sensitive diagnostic assay that can distinguish between the $M$. oryzae (Triticum pathotype) population from South America and the closely related M. oryzae (Lolium and Oryzae pathotype) populations that are endemic to the U.S. We used whole genome sequencing and a custom bioinformatics pipeline to identify 20 loci unique to the South American M. oryzae (Triticum pathotype) genome. Conventional polymerase chain reaction (PCR) assays were optimized for one of these markers, MoT3. The MoT3 marker was detected in 109 of 110 South American M. oryzae (Triticum pathotype) isolates collected from infected wheat heads, while 169 of the $170 \mathrm{M}$. oryzae isolates collected from 14 different host species from seven different countries tested negative for the MoT3 marker. Pathogenicity testing of the two outlying isolates is currently underway.

\section{Screening avocado germplasm for resistance to laurel wilt}

C. PISANI (1), M. A. Ritenour (2), E. Stover (3), R. C. Ploetz (4), D. N. Kuhn (5), O. A. Gutierrez (6)

(1) Univ of Florida-Indian River Research Education Center, Fort Pierce, FL, U.S.A.; (2) University of Florida-Indian River Research Education Center, Fort Pierce, FL, U.S.A.; (3) USDA Horticultural Research Laboratory, Fort Pierce, FL, U.S.A.; (4) University of Florida-Tropical Research Education Center, Homestead, FL, U.S.A.; (5) USDA- Subtropical Horticulture Research Station, Miami, FL, U.S.A.; (6) USDA-Subtropical Horticulture Research Station, Miami, FL, U.S.A.

Phytopathology 105(Suppl. 4):S4.111

In the US, avocado (Persea americana Mill.) is grown commercially in California and Florida. It is now threatened by laurel wilt (LW), caused by Raffaelea lauricola, a nutritional symbiont of an introduced Asian ambrosia beetle, Xyleborus glabratus. LW-resistant germplasm is needed for currently threatened production areas, as well as areas where avocado has potential as an alternative crop (e.g. where citrus is impacted by huanglongbing). Half-sib families were generated from diverse open pollinated source trees in the USDA Miami avocado germplasm collection and planted at the U.S. Horticultural Research Laboratory in Fort Pierce, FL. Trees were inoculated with an isolate of $R$. lauricola during 2 consecutive years. Repeated inoculations were conducted on surviving trees, and trees were monitored for external symptom development. Trees of 'Argui-1' and 'Booth-8' showed the lowest disease severity with $>37 \%$ of trees displaying LW symptoms in less than $20 \%$ of their canopies, over both years. The most susceptible groups were seedlings of 'No.21', with trees showing LW symptoms in $69 \%$ of their canopies in 2013 and 61\% in 2014. 'Ettinger' and 'Winter Mexican' seedlings had the greatest mortality from LW disease after the first year's inoculations. DNA from all trees has been extracted by the USDA Miami laboratory where data will be analyzed to identify alleles associated with resistance and susceptibility.

Candidatus Liberibacter asiaticus effector proteins localize in chloroplasts or mitochondria when expressed in plant cells

M. PITINO (1)

(1) USDA ARS, Fort Pierce, FL, U.S.A.

Phytopathology 105(Suppl. 4):S4.111

Candidatus Liberibacter asiaticus' (Las) is the globally prevalent species of Liberibacter pathogens that cause huanglongbing (HLB), a worldwide destructive disease of citrus. Like other bacterial pathogens, Las encodes different effectors to regulate host responses. In this study, we characterize several putative Las effector genes via agrobacterium-mediated transient expression and/or stable transformation since Las is not culturable. Using Nterminal and/or C-terminal GFP tagging, we were able to visualize two putative effectors targeting the mitochondria and two putative effectors targeting the chloroplasts of inoculated plants or transgenic plants. In addition, the expression of these effector genes was confirmed with polyclonal antibodies against their respective antigens. The association of the effectors with pathogenesis was determined in transgenic plants and through transient expression at cellular and molecular levels. Transgenic citrus plants expressing one of the chloroplast-targeting effector genes displayed HLB-like symptoms with yellow shoots and retarded growth. A dramatic proliferation of root hairs was observed in transgenic Arabidopsis expressing one of the mitochondrion-targeting effector genes. To identify molecules that interfere with the function of these effectors and disrupt Las infection, we have developed a novel in vitro screening system that can evaluate small molecules effective against Las effectors in less than two weeks.

Comparative analysis of Cucumber green mottle mosaic virus in California: Evidence to two introductions

T. PITMAN (1), T. Tian (2), B. Aegerter (3), B. Falk (1)

(1) Univ of California, Davis, CA, U.S.A.; (2) California Dept of Food \& Agric, Sacramento, CA, U.S.A.; (3) Univ of California Coop Ext, Stockton, CA, U.S.A.

Phytopathology 105(Suppl. 4):S4.111

Cucumber green mottle mosaic virus (CGMMV) is a +ssRNA virus in the genus Tobamovirus, family Virgaviridae. CGMMV infects cucurbit crops, causing green mottling and mosaic symptoms on leaves, and uneven ripening and flesh deterioration in the fruit. CGMMV is both seed-borne and mechanically transmissible. CGMMV occurs in Europe, the Middle East, and Asia, and is exotic to North America. There have been two outbreaks of CGMMV in California in consecutive years, which occurred in 2013 in Yolo County and in 2014 in San Joaquin County. In this study we examined the genetic variation, basic biology, and diagnostic approaches for detecting CGMMV in plants and cucurbit seeds. We sequenced the genomes of both isolates and compared them to world-wide isolates available in GenBank, with results indicating two separate introductions for 2013 and 2014. We have also examined the rate of seedling infection from contaminated seed via seedling grow-out experiments, with testing for individual seeds and seedlings by ELISA, RT-PCR, and qRT-PCR. Seeds tested positive for CGMMV by ELISA $100 \%$, but only $1-5 \%$ of seedlings developed infections in grow-out assays. We also used contaminated seeds homogenized in inoculation buffer for rub inoculations to Nicotiana benthamiana plants, resulting in $40-60 \%$ infection rate. Taken together, this suggests that seed transmission of CGMMV has a much higher potential infection rate than actually occurs.

MiR858 mediated post-transcriptional gene silencing of MYB83 regulate Arabidopsis susceptibility to the beet cyst nematode, Heterodera schachtii S. PIYA (1), T. J. Baum (2), T. Hewezi (1)

(1) University of Tennessee, Knoxville, TN, U.S.A.; (2) Department of Plant Pathology, Iowa State University, Ames, IA, U.S.A.

Phytopathology 105(Suppl. 4):S4.111

MicroRNAs are small non-coding endogenous RNA molecules that play important regulatory roles in plants by controlling the transcriptional activity of their targets. In Arabidopsis, miR858 is encoded by a single gene and regulates the expression of two MYB transcription factors (MYB12 and 83), which contain the miR858 binding site. Promoter:GUS activity analysis of miR858 and its MYB12 and MYB83 targets in response to Heterodera schachtii infection pointed to a role of miR858 in the post-transcriptionally regulation of MYB83 in the nematode feeding sites. Overexpression of miR858 in Arabidopsis resulted in a statistically significant reduction in plant susceptibility to $H$. schachtii. The negative impact of increased miR858 expression on nematode susceptibility was further supported by our data showing that overexpression of target mimicry for miR858 resulted in significant increase in plant susceptibility to cyst nematode relative to wildtype plants. Interestingly, overexpression of a miR858-resistant version of MYB83 resulted in statistically significant increases in nematode susceptibility compared to wild-type control. Taken together, these findings suggest a novel functional role of miR858/MYB83 regulatory system in plantnematode interactions.

Laurel wilt of avocado: Epidemiology and management of a recalcitrant disease of an important crop

R. PLOETZ (1), D. Carrillo (2)

(1) Univ of Florida, Homestead, FL, U.S.A.; (2) Univ Florida, Homestead, FL, U.S.A.

Phytopathology 105(Suppl. 4):S4.111

Laurel wilt is caused by Raffaelea lauricola, which is a nutritional symbiont of an Asian ambrosia beetle, Xyleborus glabratus. Recently, commercial production of an important crop in the family, avocado (Persea americana), 
has been affected in southern Florida. There are significant gaps in what is known about this emerging problem. Xyleborus glabratus introduced $R$. lauricola into the US and has played a primary role in the laurel wilt epidemic on native members of the Lauraceae, but it may not be important in the avocado system. Since its introduction to the US, an unprecedented lateral transfer of $R$. lauricola has occurred to least nine other species of ambrosia beetle, some of which may be important in the epidemic on avocado. Sanitation is a key control measure, but low titers and inconsistent distributions of $R$. lauricola in affected trees hinder its detection, especially during early stages of an outbreak when sanitation would be most effective. Soon after orchards are affected, the pathogen spreads via root-grafts and control becomes exceedingly difficult. Improved management of this disease will rely on: better understandings of the host $\times$ pathogen interaction and epidemiology of the disease in avocado orchards; better information on the identity of vectors, their dynamics and the roles they play in the disease cycle on avocado; and better application measures, increased duration, and greater efficacy of pesticides against this disease.

\section{Influence of silicon on the development of anthracnose disease on grain sorghum}

C. Hollier (1), S. POKHREL (1)

(1) Louisiana State Univ Agricultural Center, Baton Rouge, LA, U.S.A. Phytopathology 105(Suppl. 4):S4.112

Sorghum anthracnose, caused by Colletotrichum sublineolum P. Henn, has been found to reduce sorghum yields by up to $28 \%$ in Louisiana over the past 10 years. Although fungicides are available for management, there was a desire to investigate soil amendments as a tool for disease management. Silicon ( $\mathrm{Si}$ ) has been found to reduce bean and cabbage anthracnose in previous studies, therefore, the aim of this study was to assess anthracnose severity on a moderately susceptible grain sorghum hybrid when supplemented with increasing rates of Si. In the greenhouse, the sorghum hybrid Pioneer 84G62 was grown for five weeks in silt loam soil supplemented with lime (to maintain a constant level) and five rates of $\mathrm{Si}(0$, $0.2,0.4,0.6$ or $0.8 \mathrm{t} / \mathrm{ha}$ ) as Wollastonite. Plants were inoculated at the $4^{\text {th }}$ leaf stage and maintained with 24 hours of dew. Anthracnose severity, assessed as a percentage, was found to be $8,5,7,6$ and $4 \%$ respectively for the five rates of Si resulting in a reduction of disease by 50 percent in the $0.8 \mathrm{t} \mathrm{Si} / \mathrm{ha}$ treatment. The area under disease progress curve was 127.85 (no-lime control), 125.6 (lime control), 92.2, 110.65, 105.35 and 75.575 sq. units, respectively, for the five rates of Si. Plant $\mathrm{Si}$ content was greatest $(12.3 \mathrm{mg} / \mathrm{g})$ in the $0.4 \mathrm{t} \mathrm{Si} / \mathrm{ha}$ treatment and lowest $(6.3 \mathrm{mg} / \mathrm{g})$ in the $0 \mathrm{t} \mathrm{Si} / \mathrm{ha}$ treatment (with lime). No significant differences were found with the addition of lime or in plant $\mathrm{Si}$ among the $0.4,0.6$ and $0.8 \mathrm{t} \mathrm{Si} /$ ha treatments.

Control of the wheat curl mite, vector to Wheat streak mosaic virus, with pesticide applications on spring wheat

C. POL (1), M. Burrows (1), Z. Miller (2)

(1) Montana State University, Bozeman, MT, U.S.A.; (2) Western Agricultural Research Center, Corvallis, MT, U.S.A.

Phytopathology 105(Suppl. 4):S4.112

Wheat streak mosaic virus (WSMV) is the most prevalent virus of wheat in the Great Plains region of the United States, and occurs worldwide where wheat is grown. No known chemical control options exist for the vector, the wheat curl mite (WCM, Aceria tosichella Keifer). To identify pesticides for WCM management, spring wheat (Triticum aestivum cv. Choteau) was infested with viruliferous WCM in the growth chamber and treated. Treatments included Organic Materials Review Institute (OMRI) approved products and common wheat insecticides: oils, soaps, biocontrols, thiamethoxams, organophosphates, carbamates, chitosan, sulfur, pyrethroids, etoxazols and hexythiazoxes. The population growth rate was measured 5 days after treatment. Sixteen of the 19 treatments had no effect on mite population growth rate $(p>0.05)$. Carbamate (insecticide group 1A) and organophosphate (insecticide group 1B) treatments reduced the population growth rate of WCM after treatment compared to the control $(p=0.01$ and $p<0.0001$, respectively). The most promising treatments were Chlorpyrifos organophosphate treatments, commercial products "Lorsban 4E" and "Lorsban Advanced". Chlorpyrifos decreased WCM populations on plants from $50-100$ mites to less than 10 mites $(p<0.05)$. Further testing is required to see if these results are repeatable under field conditions and best practices for wheat curl mite management. Economic feasibility will also be assessed.

Discovery of blueberry genes for mummy berry resistance

J. POLASHOCK (1), K. Shim (2), T. Smolinski (2)

(1) USDA ARS, Chatsworth, NJ, U.S.A.; (2) Delaware State University,

Dover, DE, U.S.A.

Phytopathology 105(Suppl. 4):S4.112
One approach to discovering disease resistance genes is to challenge a plant thought to be resistant with the pathogen followed by analysis of the transcriptome. We have used this approach in an attempt to identify genes expressed in blueberry that are associated with resistance to the flower infection phase of mummy berry disease (causal agent Monilinia vacciniicorymbosi). The flower infection phase requires that the conidia germinate on the stigma, grow down the stylar canal, and infect the ovary. We demonstrated that the highbush blueberry cultivar Bluejay is fairly resistant to this disease while 'Berkeley' is very susceptible. The growth of the fungus down the style of 'Bluejay' flowers is inhibited to the point where the fungus fails to reach the ovary. Thus 'resistance' may be expressed in the stylar canal. Open flowers of both cultivars were pollinated and then inoculated with mummy berry conidia. Total RNA was isolated from the styles two days postpollination/inoculation. RNASeq libraries were prepared and sequenced using the Illumina HiSeqII platform. The resulting sequences were trimmed and assembled producing 37,354 contigs for 'Bluejay' and 36,202 for 'Berkeley' of at least $200 \mathrm{nt}$. Blastn was used to determine putative gene functions. Analysis of genes differentially expressed between the resistant and susceptible cultivars should identify genes potentially associated with resistance and/or susceptibility.

\section{Phylogeny of Tobacco streak virus in cranberry}

J. POLASHOCK (1), L. Wells (2), P. McManus (2), E. Saalau Rojas (3)

(1) USDA ARS, Chatsworth, NJ, U.S.A.; (2) University of WisconsinMadison, Madison, WI, U.S.A.; (3) University of Massachusetts Amherst, Amherst, MA, U.S.A.

Phytopathology 105(Suppl. 4):S4.112

Tobacco streak virus (TSV) was first reported in cranberry in 2001 during routine virus testing of germplasm sent to Scotland. The prevalence of this virus in the United States was unknown, and no symptoms were associated with infection of this virus in cranberry. In 2012, cranberry fruit with scarring were identified in Wisconsin. Subsequent ELISA testing showed the plants bearing scarred fruit were infected with TSV. More extensive testing suggested the virus was present in several commercial cranberry fields in Wisconsin, Massachusetts, and New Jersey. Phylogenetic analysis was performed using the coat protein gene sequence to help ascertain the genetic variability in TSV within and between all three (WI, MA, NJ) growing regions. Preliminary data indicate wide variation in the coat protein sequence. These data argue against recent introduction of a single virus clone. It is more likely that the virus has been present in cranberry for some time and is naturally diverging or that there were multiple introductions into commercial cranberry. The vector is currently unknown, but thrips have been suggested. Further research should help determine the source(s) and mode(s) of spread of the virus in cranberry.

Survey for incidence and distribution of grapevine viruses in British Columbia

S. Poojari (1), J. Boule (1), N. DeLury (1), T. Lowery (1), M. Rott (2), A. M. Schmidt (2), J. R. URBEZ-TORRES (1)

(1) Agriculture and Afri-Food Canada / Pacific Agri-Food Research Centre, Summerland, BC, Canada; (2) Canadian Food Inspection Agency (CFIA), North Saanich, BC, Canada

Phytopathology 105(Suppl. 4):S4.112

British Columbia (BC) is the second largest grape-growing region in Canada. Viruses are considered significant constraints for grapevine production worldwide. Among them, Grapevine leafroll associated viruses (GLRaVs) are considered to be the most wide-spread reducing vine growth and fruit quality. To understand the current status of grapevine viruses in $\mathrm{BC}$, surveys were conducted during the 2013 and 2014 growing seasons to record the incidence of GLRaVs, Grapevine fanleaf virus (GFLV), Grapevine fleck virus (GFkV), Arabis mosaic virus (ArMV), and the recently discovered Grapevine red blotch-associated virus (GRBaV). A total of 1,957 random-composite and 63 target-individual grapevine samples from 22 different cultivars were collected from 113 vineyard blocks in different grape-growing regions of $\mathrm{BC}$ and tested for the presence of GLRaVs by DAS-ELISA. Among GLRaVs, the most common was GLRaV-3 (17.2\%), followed by GLRaV-2 (5.5\%), GLRaV 4-9 (4.2\%) and GLRaV-1 (1.4\%). GFLV incidence was detected at $0.5 \%$ from 998 composite samples, whereas GFkV was detected at $29.2 \%$ from 788 composite samples. No positives were detected for GRBaV from 539 composite and 195 targeted samples tested using PCR. Similarly, no positives were detected for ArMV from 998 composite samples. Insect vector identification and transmission studies are in progress to develop strategic management programs for major grapevine viral diseases in $\mathrm{BC}$. 
Resistance in pea to the root rot pathogen Fusarium avenaceum L. D. PORTER (1)

(1) USDA ARS, Prosser, WA, U.S.A.

Phytopathology 105(Suppl. 4):S4.113

Fusarium avenaceum $(\mathrm{Fa})$ is a major root rot pathogen impacting dry pea production in Montana, North Dakota, and Canada. Currently there are no fungicides identified that can manage this pathogen. Host resistance is the most economical and environmentally friendly means to manage this disease. Pea accessions (444 lines) from the Pisum Collection located in Pullman, WA and commercial cultivars (28 lines) commonly grown in the USA were evaluated for resistance to $F a$ using a greenhouse screening method that has been successful in identifying germplasm with field resistance to Fusarium solani. Of the pea accessions evaluated, 34 lines demonstrated high levels of partial resistance to $F a$ in repeated greenhouse tests, with disease severity scores of 1 or less on a scale from 0 to 5 , with 5 being the most severe. The accessions originated from 15 countries, with India (6), Ethiopia (5) and Greece (4) representing the greatest number of accessions. The resistant accessions, in general, had pigmented flowers and pigmented seed coats with yellow cotyledons. The commercial cultivars were highly susceptible (scores of 4 or greater) to $F a$, except for Austrian winter peas, which had excellent partial resistance (score of 2 or less). Outstanding partial resistance to $F a$ is found in the Pisum germplasm and efforts to incorporate genes associated with this resistance into white-flowered commercial cultivars is desired by the industry.

\section{Transcriptome analysis of resistant and susceptible alfalfa cultivars} infected with root-knot nematode Meloidogyne incognita

O. A. Postnikova (1), M. Hult (2), J. Shao (1), A. Skantar (2), L. G. NEMCHINOV (1)

(1) USDA ARS MPPL, Beltsville, MD, U.S.A.; (2) USDA ARS Nematology Lab, Beltsville, MD, U.S.A.

Phytopathology 105(Suppl. 4):S4.113

Root-knot nematodes (RKN, Meloidogyne spp.) are widely distributed and economically important sedentary endoparasites of agricultural crops and they may inflict significant damage to alfalfa fields. No studies have been published on global gene expression profiling in alfalfa infected with RKN or any other plant parasitic nematode. In this work, we performed root transcriptome analysis of resistant (cv. Moapa 69) and susceptible (cv. Lahontan) alfalfa cultivars infected with RKN Meloidogyne incognita, a major pest worldwide. A total of 1,701,622,580 pair-end reads were generated on an Illumina Hi-Seq 2000 platform from the roots of both cultivars and assembled into 45,595 and 47,590 transcripts in cvs Moapa 69 and Lahontan, respectively. Bioinformatic analysis revealed a number of common and unique genes that were differentially expressed in susceptible and resistant lines as a result of nematode infection. Although the susceptible cultivar showed a more pronounced defense response to the infection, feeding sites were successfully established in its roots. Basal gene expression levels under normal conditions differed between the two cultivars as well, which may confer advantage to one of the genotypes toward resistance to nematodes. Functional roles of the differentially expressed genes were predicted. Candidate genes that contribute to protection against $M$. incognita in alfalfa were proposed.

\section{Rhizobiome responses to new tomato rootstock systems}

R. POUDEL (1), A. Jumpponen (2), M. Kennelly (1), C. Rivard (3), K. Garrett (4)

(1) Kansas State University/ Plant Pathology Department, Manhattan, KS, U.S.A.; (2) Kansas State University/ Department of Biology, Manhattan, KS, U.S.A.; (3) Kansas State University / Horticulture Forestry \& Rec, Manhattan, KS, U.S.A.; (4) Kansas State University/ Plant Pathology Department \& University of Florida / Institute for Global Food Systems and Plant Pathology Department, Manhattan, KS, U.S.A.

Phytopathology 105(Suppl. 4):S4.113

Rhizosphere microbiomes are critical for nutrient exchange between plants and soil, determining plant performance and health. Microbial communities are under biotic and abiotic pressure and shift in response to interventions such as agricultural practices. We analyzed the impacts of grafting and rootstock genotypes on bacterial communities in the roots and rhizosphere of tomatoes using high-throughput sequencing. Our study included tomato plants representing non-graft, self-graft, and hybrid graft treatments. 16S rRNA sequences revealed significantly $(\mathrm{p}<0.001)$ more diverse bacterial communities in the rhizosphere than in the roots across all the treatments, although a large proportion (85\%) of classified OTUs were shared. Proteobacteria, Actinobacteria, Firmicutes, Bacteroidetes, and Planctomycetes were the most abundant phyla in all rootstock-scion combinations, where the proportion of the last three phyla was lower in the roots. The number of unique OTUs in the rhizosphere was twenty times higher than in roots. OTUs specific to rootstock genotypes, and to grafted versus non-grafted plants, were identified for both the root and the rhizosphere, and substantially more unique OTUs were found in grafted plants. The results of this study provide novel insights to the roles of grafting and rootstock genotype in the selection of microbial taxa. Ultimately, a better understanding of microbial responses to rootstock management will support improved vegetable production.

Xylem vessels diameter affects resistance to the vascular fungal pathogen Phaeomoniella chlamydospora in grapevine

J. POUZOULET (1), E. Scudiero (1), P. E. Rolshausen (1)

(1) University of California Riverside, Riverside, CA, U.S.A.

Phytopathology 105(Suppl. 4):S4.113

The fungus Phaeomoniella chlamydospora is a vascular pathogen of grapevine, Vitis vinifera. In vineyards, characteric symptoms include tigerstripe leaves, necrotic spots on berries, apoplexy and vine decline. To date, no grapevine cultivar is known to be completely resistant to $P$. chlamydospora. However, previous field observations and scientific studies indicate that grapevine cultivars vary in susceptibility towards this pathogen. The goal of our study was to understand the underlying mechanisms responsible for these differences. Grape cuttings of four commercial cultivars ('Merlot', Cabernet Sauvignon', 'Chardonnay' and 'Thompson') representing a continuum between low and high susceptibility were experimentally inoculated with $P$. chlamydospora and grown in glasshouse. Ten weeks post-inoculation, colonization of host tissue was assessed by quantitative PCR. Our results showed that $P$. chlamydospora was able to colonize susceptible cultivars at a faster rate than resistant cultivars. We analyzed several morphological features of grapevine xylem and found that increase in large vessels diameter correlated negatively with the degree of resistance. In addition, we showed that vessels diameter affects inter-tylosis boundary network and the time course of vessels occlusion. We discuss how this concept could be use to mitigate the impact of $P$. chlamydospora infection in field.

Characterization of Sec-translocon dependent extracytoplasmic proteins and essential signal peptidase I of Candidatus Liberibacter asiaticus

S. PRASAD (1), N. Wang (1)

(1) University of Florida, Lake Alfred, FL, U.S.A.

Phytopathology 105(Suppl. 4):S4.113

Citrus Greening is a destructive disease in citrus plants globally. A comprehensive view of the molecular interactions of the causative agent, Candidatus Liberibacter asiaticus (Las) with its plant and vector hosts (citrus and Diaphorina citri respectively) has not yet been established, owing to constraints in maintenance of a pure culture of the bacterium. Interestingly, Las contains a complete Sec-translocon, a potent system for the transportation of Las proteins including the virulence factors into the extracytoplasmic milieu. We characterize the Sec-translocon dependent, signal peptide (SP) containing extracytoplasmic proteins of Las. A total of 150 Las proteins were predicted to contain SP targeting them out of the cytoplasm using four dedicated online algorithms. Experimental validation of the predictions was conducted with $E$. coli based phoA gene fusion assays. The study revealed 89 proteins to be extracytoplasmic in the annotated Las proteome. Further comparison analyses of these 89 proteins of Las were carried out with Liberibacter crescens BT-1. Additionally, we assessed the function of Las signal peptidase I (lep) responsible for cleaving the SP of most exported proteins, the Las lep restored the growth of otherwise conditionally lethal amber mutants of lep gene in E. coli. This is the first study describing the Sectranslocon dependent extracytoplasmic proteome of Las and will contribute in the identification of Sec-translocon dependent effector proteins.

Pyrimidine salvage by Uracil Phosphoribosyltransferase (UPRT) of Phytophthora infestans in late blight disease in Solanum tuberosum K. PRIETO (1), M. Garavito (2), J. J. Vásquez (2), B. H. Zimmermann (2), S. Restrepo (2)

(1) Universidad de los Andes, Bogota, Colombia; (2) Universidad de los Andes, Bogotá, Colombia

Phytopathology 105(Suppl. 4):S4.113

Phytophthora infestans expresses UPRT as an enzyme of the pyrimidine salvage pathway to synthesize uridine monophosphate (UMP). Although little is known about its nutritional requirements in the host, our group observed up and down-regulation in different stages of infection using qRT-PCR and microarrays. The objective of this study was to characterize UPRT and its role in infection of potato plants. Kinetic parameters for substrates PRPP and uracil, as well as for uracil analogs were determined for recombinant UPRT enzymes from Solanum tuberosum and $P$. infestans. The enzymes showed low substrate affinities compared to organisms such as E. coli, Leishmania and 
Toxoplasma gondii and suggested that 5-fluorouracil can act as a good alternative substrate for both UPRTs. We also assayed silenced PiUPRT for sporulation and germination in detached potato leaves in the presence and absence of uracil and analogs as substrates for the enzyme. The silenced strains and the chemical treatments showed slightly different phenotypes compared to wild type strain. The effect was in all cases stronger in later stages such as sporulation. Our results were in agreement with the previous genomic results and combined with kinetic data suggest that uracil recycling seems more important in late events, when availability of pyrimidine pools from the host might be increased. Additionally, 5-fluorouracil reduced sporulation, being a candidate to prevent the dispersion of the pathogen.

Control of Pythium ultimum on cucumber by different composts and biochars in potted plants

M. Pugliese (1), M. L. GULLINO (2), G. Castella (3), A. Garibaldi (3)

(1) DISAFA, University of Torino, Italy, Grugliasco, Italy; (2) University of Torino, Grugliasco Torino, Italy; (3) University of Torino, Agroinnova, Grugliasco, Italy

Phytopathology 105(Suppl. 4):S4.114

Pythium ultimum is a widespread and important pathogen affecting several crops, vegetables in particular. The use of compost to suppress diseases caused by P. ultimum can provide different results, according to the type of wastes and the composting process. The aim of this research was to evaluate the suppressiveness of composts produced from different origins for controlling P. ultimum in potted plants. Composts originated from green wastes and/or municipal biowastes, using a traditional composting system or an integrated biogas/composting system were used. They were blended with a peat substrate at different dosages $(10,20$ and $50 \%$ vol./vol.) 14 days before seeding or transplanting. Pathogens were mixed into the substrate at 0.5 or $1 \mathrm{~g}$ of wheat kernels $\mathrm{L}^{-1} 7$ days before seeding. Seeds of cucumber were sown into $2 \mathrm{~L}$ pots in greenhouse. The number of alive plants and above ground biomass were measured 24-25 days after seeding or transplanting. The number of diseased cucumbers was significantly reduced by increasing dosages of municipal compost. However in some cases phytotoxicity effects were observed.

Effect of Rhapsody (Bacillus subtilis) on post-harvest pathogen development on fresh market tomatoes

Z. K. PUNJA (1), G. Rodriguez (1)

(1) Simon Fraser University, Burnaby, BC, Canada

Phytopathology 105(Suppl. 4):S4.114

Fungi commonly recovered from fruit lesions on fresh market tomatoes in British Columbia are species of Penicillium, Botrytis, Rhizopus and Alternaria. The effect of Rhapsody (Bacillus subtilis) applications made once monthly on disease incidence and severity by these fungi was determined in 2 commercial greenhouses in 2012-2013. Populations of the bacteria and disease incidence were monitored weekly from harvested fruit during the study. Weekly variation in the level of disease was observed, due to variation in fungal populations within the greenhouse, as well as unidentified cultural and environmental factors. A significant reduction in disease incidence and severity was observed on fruit treated with Rhapsody in each week of the study. Post-harvest storage temperature $\left(13{ }^{\circ} \mathrm{C}\right.$ vs. $\left.21^{\circ} \mathrm{C}\right)$ and incubation time (12 vs. 16 days) also had a significant effect on final disease levels. Rhapsody-treated fruit incubated at $13^{\circ} \mathrm{C}$ had $0-5 \%$ fruit infection, compared to $>30 \%$ disease on untreated fruit at $21^{\circ} \mathrm{C}$. A major source of post-harvest fungi on fruit, particularly Penicillium, was from stem and calyx tissues. Rhapsody applications prevented spread of these fungi onto the fruit. Rhapsody applied once every 4 weeks was sufficient to maintain high populations of Bacillus on the fruit surface, providing significant post-harvest disease control on fruit during storage. When combined with storage at $13^{\circ} \mathrm{C}$ for no more than 12 days, fruit infection was reduced to negligible levels.

Evaluation of biological control products for management of powdery mildew on greenhouse cucumbers

Z. K. PUNJA (1), D. Collyer (2), A. Tirajoh (2)

(1) Simon Fraser Univ, Burnaby, BC, Canada; (2) Simon Fraser University, Burnaby, BC, Canada

Phytopathology 105(Suppl. 4):S4.114

Three biological control products - Rhapsody (Bacillus subtilis strain QST $713,1 \%$ rate), Prestop (Gliocladium catenulatum strain J1446, 1\% rate) and Mycoflora PRO (Bacillus subtilis, B. amyloliquefaciens, B. pumilus, $0.2-0.5 \%$ rate) were evaluated for management of powdery mildew (Podosphaeria xanthii) on cucumber cultivar Picowell RZ. Aqueous suspensions of the 3 products were applied to cucumber plants prior to the onset of disease (preventative) and after disease had reached approximately 10 colonies per leaf (eradicative). Four applications were made at weekly intervals to foliage and compared to plants receiving water sprays. Disease development was assessed in week 5 as the total number of colonies developing on treated leaves on 12 replicate plants per treatment. The experiment was conducted twice during 2013-2014. Rhapsody provided a significant reduction of mildew colony development, from an average of 13.75 colonies per leaf in the control plants to 2.5 colonies for the preventative sprays, and from an average of 38.6 colonies to 8.2 colonies in the eradicative sprays. Mycoflora PRO was the next best treatment, with an average of 6.6 colonies per leaf in the preventative spays and 15.2 colonies in the eradicative sprays. By comparison, Prestop was the least effective, and treated plants showed an average of 9.6 colonies per leaf in the preventative sprays and 19.2 colonies in the eradicative sprays.

Newly observed diseases of blueberries in the Pacific Northwest

M. L. PUTNAM (1), M. Serdani (2), M. S. Wiseman (2)

(1) Oregon State Univ, Corvallis, OR, U.S.A.; (2) Oregon State UniversityPlant Clinic, Corvallis, OR, U.S.A.

Phytopathology 105(Suppl. 4):S4.114

The Oregon State University Plant Clinic has received numerous northern highbush blueberry plants (Vaccinium corymbosum) with diseases not previously diagnosed in the Pacific Northwest. Fungi were isolated from surface-disinfected symptomatic tissue using PDA. All fungal identifications were based on morphological features and were confirmed with ITS sequencing of cultures derived from a single spore or hyphal tip. In 2011 and 2013, Verticillium dahliae was recovered from 'Bluetta' bushes from Yakima, WA. The plants showed leaf reddening, defoliation, and branch dieback in the absence of vascular streaking. During 2013, Lasiodiplodia theobromae was recovered from 'Draper' and 'Duke' plants from different farms in the Yakima area. Affected plants had browning of internal stem tissues, defoliation, and dieback in the absence of cankers. During 2014 we confirmed the presence of the silver leaf fungus, Chondrostereum purpureum, from the cultivars Draper and Liberty showing non-specific dieback in the Willamette Valley in western Oregon. Symptomatic plants have been observed for more than 10 years, and the disease appears to be widely distributed in the Valley; one grower removed 300 bushes from a field due to silver leaf. These diseases represent a threat to the nearly 20,000 acres of blueberry production in the region.

Using next-generation sequencing to develop species-specific molecular diagnostics for cucurbit downy mildew

L. QUESADA-OCAMPO (1), S. Withers (1), E. Gongora-Castillo (2), D. Gent (3), P. Ojiambo (1)

(1) North Carolina State University, Raleigh, NC, U.S.A.; (2) Michigan State University, East Lansing, MI, U.S.A.; (3) USDA-ARS, Corvallis, OR, U.S.A. Phytopathology 105(Suppl. 4):S4.114

Cucurbit downy mildew, caused by Pseudoperonospora cubensis, is the most economically important disease limiting cucurbit production in the United States. Growers experience significant losses yearly due to severe defoliation of plants that reduce fruit yield and quality. Currently, the disease can only be controlled through expensive fungicide programs that are initiated when an outbreak has been confirmed in the state by visual inspection of advanced symptoms and pathogen sporulation. Early detection of $P$. cubensis prior to infection by using spore traps or in the initial stages of infection by using molecular tools could help reduce fungicide applications and production costs. To address this need, Illumina next generation sequencing and comparative genomics between P. cubensis and its sister species P. humuli (hop downy mildew) was used to identify candidate genomic regions for species-specific molecular detection of $P$. cubensis. We compared the transcriptomes of diverse $P$. cubensis and $P$. humuli isolates, selected candidate genomic regions unique to $P$. cubensis, and tested these candidates against a diverse $P$. cubensis and $P$. humuli panel to confirm specificity. In addition to developing a molecular diagnostic tool for $P$. cubensis, this project has generated genomic data for two economically important downy mildew pathogens that can increase our understanding of evolutionary relationships and host specificity within and between $P$. cubensis and P. humuli.

\section{Piriformospora indica a possible biological control agent}

M. RABIEY (1), M. W. Shaw (1)

(1) University of Reading, Reading, United Kingdom Phytopathology 105(Suppl. 4):S4.114

Piriformospora indica (Sebacinacea) forms mutualistic symbioses with a broad range of host plants, assisting in nutrient acquisition, increasing biomass production and resistance/tolerance to fungal pathogens/abiotic stresses. Fusarium crown rot (FCR) and head blight (FHB) are two of the most important diseases of wheat globally. There is no significant control strategy available. Inoculation of wheat seedlings with $\mathrm{UK}$ isolates of $F$. culmorum, $F$. 
graminearum and/or $P$. indica under glasshouse and simulated UK autumn conditions showed that the amount of Fusarium DNA in roots grew rapidly in Fusarium-inoculated alone. In pots co-inoculated with $P$. indica, Fusarium DNA was reduced with no visible symptoms; suggesting $P$. indica can protect wheat seedlings from FCR damage. Winter and spring wheat samples were inoculated with $P$. indica (at sowing) and/or $F$. graminearum (at flowering) under natural conditions. $P$. indica reduced FHB disease severity and mycotoxin deoxynivalenol in the grains. The effect of $P$. indica on soil/plant nutrients and on the most important weeds is being tested in the 2014-15 growing season. $P$. indica viability under UK weather conditions was tested in four different soil types after winter/summer by PCR/RT-PCR. $P$. indica was still alive in the soil after 4 months and in $6 / 20$ pots after 8 months, will be tested again this year. The effect of $P$. indica on other soil microorganisms was tested by PCR-Denaturing Gradient Gel Electrophoresis.

Pythium species from Minnesota soybean fields, their relative pathogenicity to soybeans and corn, and their sensitivity to seed treatment fungicides

L. RADMER (1), G. Anderson (1), D. M. Malvick (1), J. E. Kurle (1)

(1) University of Minnesota, St. Paul, MN, U.S.A.

Phytopathology 105(Suppl. 4):S4.115

Pythium species are a major cause of seedling diseases in soybean and corn, two crops often planted in rotation. Although they are known to cause seed decay, damping-off and root rot, their importance as pathogens in Minnesota is unknown. Our objectives were to determine the pathogenicity on corn and soybean and the sensitivity to seed treatment fungicides for Pythium spp. isolated from soybean fields. Pathogenicity was determined in an in vitro seed assay and a seedling assay. The in vitro assay was performed at $15^{\circ} \mathrm{C}, 20^{\circ} \mathrm{C}$, and $25^{\circ} \mathrm{C}$, representative of a Minnesota spring, to examine if temperature affects pathogenicity. Of 21 species evaluated, seven of the Pythium spp. were pathogenic on both soybean and corn, reducing root growth by $20 \%$ or more. Two Pythium and one Phytopythium spp. were pathogenic only on soybeans. Contrary to observations that Pythium spp. are cool weather pathogens, the aggressiveness of most isolates increased as the temperature increased. Sensitivity to the fungicides azoxystrobin, ethaboxam, mefenoxam, pyraclostrobin, or trifloxystrobin was tested for the ten pathogenic species. Fungicide sensitivity varied up to more than $80 \%$ between species within the same fungicide treatment. The fungicides had differing efficacies against Pythium and Phytopythium spp. The results confirm that Pythium spp. are a significant cause of seedling disease on soybean and corn in Minnesota but that fungicides are still effective control measures.

Improved management of tomato foliar diseases by conventional and organically acceptable products

M. RAHMAN (1)

(1) West Virginia Univ, Morgantown, WV, U.S.A

Phytopathology 105(Suppl. 4):S4.115

Tomato foliar diseases (Early blight, Late blight and Septoria leaf spot) cause significant yield losses in West Virginia. This study was conducted to determine the efficacy of Organic Materials Review Institute (OMRI) approved products and resistant varieties in managing foliar diseases for two consecutive years (2012 and 2013). In addition, efficacy of newer fungicides in controlling foliar diseases was determined in 2014. Trials were conducted at WVU research farms in a randomized complete block design (RCBD) with four replications of each treatment. Treatments were applied on a 10-14 day interval starting from the early bloom and continued for eight to ten weeks. All treatments with OMRI approved products provided significant $(P<0.001)$ reduction of Early blight (EB) compared with non-treated control except Champ application initiated at the first symptom appearance and Actinovate. Regalia alternated with Champ provided statistically similar EB and Late blight (LB) suppression compared to the application of Champ only. Although Mountain Fresh Plus F1 (MF+) and Juliet F1 showed excellent resistance to EB but were seriously affected by LB and Septoria leaf spot (SLS) in 2012 and 2013, respectively. Among the conventional fungicides tested in 2014 as stand alone or in schedule, Revus Top was the most effective against both LB and SLS followed by Quadris Top and Catamaran. Fontelis, Kocide and Tanos schedule significantly reduced SLS severity but not LB.

A global analysis of the emergence and evolutionary lineages of Potato virus Y strains

G. Raikhy (1), S. Gray (2), H. PAPPU (1)

(1) Washington State University, Pullman, WA, U.S.A.; (2) USDA-ARS, Ithaca, NY, U.S.A.

Phytopathology 105(Suppl. 4):S4.115

Potato virus $Y$ (PVY), an important viral pathogen of potato, was reported from more than 25 countries on six continents. Due its biological complexity and economic importance, the origin, evolution and spread of PVY have lately been of great interest. Using the complete genomic sequences of 149 PVY isolates reported from around the world, we investigated the migration and evolutionary patterns of virus and its various naturally occurring strains. PVY is a biologically active and dynamic virus and exists as several distinct strains that cause a wide range of phenotypes in potatoes. Several criteria that could provide insights into PVY evolution were used. These included the substitution rate, divergence times, recombination events, and phylogeographic patterns. We found that recombination has been a hallmark of PVY evolution, and that the $\mathrm{N}$ strain has a different evolutionary history from that of the $\mathrm{O}$ strain. The $\mathrm{N}$ strain had a significantly more recombination events compared to the $\mathrm{O}$ strain. In $\mathrm{N}$ and NTN strains, clear crossover points for recombination were detected while they lacked in the $\mathrm{O}$ strain. It was estimated that the PVY genes had evolved at rates between 1.44 and $2.39 \times$ $10^{-4}$ substitutions/site/year, in line with viruses having an RNA genome. Our results showed that PVY probably spread from a single population from Europe to other parts of the world. Evidence suggests frequent gene flow between populations in Europe and USA.

Identification of suppressors of hypersentive response from Puccinia spp. infecting wheat

S. RAMACHANDRAN (1), S. Hulbert (1)

(1) Washington State University, Pullman, WA, U.S.A.

Phytopathology 105(Suppl. 4):S4.115

Fungi that cause rust disease (genus Puccinia) are economically important pathogens of wheat. Upon infection the fungus secretes several effectors that help it to colonize the plant. Some of these effectors may function to suppress plant defense responses allowing the pathogen to survive in living tissues. In this study we mined the available transcriptome of Puccinia spp. infecting wheat to look for potential suppressors of hypersensitive response (HR). Predicted secreted proteins smaller than 400 amino acids, enriched in cysteine residues and expressed two fold or higher in the haustorium as compared to infected tissue were selected for screening in Nicotiana benthamiana. These proteins were screened against five cytotoxic effector-R gene combinations (mutated Pto gene, Cp/Rx, ATR13/RPP13, Rpt2/RPS-2 and GPA/RBP-1) when co-inoculated or inoculated 24 hours before the HR-causing construct. A sub-set of the proteins screened were found to suppress HR when inoculated 24 hours prior to the HR causing constructs. The effectors also show a differential response to the different cell death constructs, indicating that Puccinia can interfere with a variety of host defense pathways. The function of some of these proteins was further confirmed by making nested deletions. This study provides the first evidence for suppressors in Puccinia. In the long run, a thorough understanding of the infection process will facilitate better management practices for this disease.

Citrus Huanglongbing tolerance in Australian Citrus Relatives, Microcitrus and Eremocitrus

C. RAMADUGU (1), M. L. Keremane (2), S. E. Halbert (3), E. Stover (4), D. G. Hall (5), G. T. McCollum (5), M. L. Roose (6), R. F. Lee (2)

(1) Univ of California, Riverside, CA, U.S.A.; (2) USDA ARS National Clonal Germplasm Repository for Citrus and Dates, Riverside, CA, U.S.A.; (3) Division of Plant Industry, Gainesville, FL, U.S.A.; (4) United States Hortucultural Research Laboratory, Fort Pierce, FL, U.S.A.; (5) United States Horticultural Research Laboratory, Fort Pierce, FL, U.S.A.; (6) University of California Riverside, Riverside, CA, U.S.A.

Phytopathology 105(Suppl. 4):S4.115

Tolerance, or resistance to citrus huanglongbing will be important as a long term solution for this disease. In a field trial conducted with over 1000 plants belonging to different genera in the sub-family Aurantioideae, we observed field tolerance in many Australian citrus relatives. To confirm these results in a controlled greenhouse setting, we have exposed 140 plants to Candidatus Liberibacter asiaticus via psyllid feeding under no-choice conditions for a period of two weeks. We have maintained these seedlings under greenhouse conditions and conducted detailed analysis on the presence of the pathogen and level of tolerance observed in various plants after exposure to the pathogen. We have analyzed the samples using qPCR and droplet digital PCR (ddPCR) to estimate the bacterial titer in accessions that were either susceptible, tolerant, or resistant in field studies. To confirm the presence or absence of the pathogen in plants with questionable qPCR results, ddPCR analysis was conducted targeting multiple genomic regions of Candidatus Liberibacter asiaticus. Since these experiments are conducted using open pollinated seeds where the pollen parent is not known, we have also conducted about 2000 pollinations using Microcitrus, Eremocitrus, pummelo, and mandarin as both seed and pollen parents. The targeted crosses were also conducted with known HLB tolerant cultivars of mandarins like Temple tangor and Fallglo mandarin. 
Sensitivity of various genotypes of Xanthomonas campestris pv. vitians to bacteriophages isolated from soil and water in lettuce production fields G. J. RAMOS (1), I. Rubio (2), A. Wright (3), R. J. Hayes (4), C. T. Bull (5) (1) Science and Math Institute, Hartnell College, Salinas, CA, U.S.A.; (2) Department of Plant Pathology, University of Wisconsin, Madison, WI, U.S.A.; (3) Hartnell College, Salinas, CA, U.S.A.; (4) USDA, ARS, Salinas, CA, U.S.A.; (5) USDA ARS, Salinas, CA, U.S.A.

Phytopathology 105(Suppl. 4):S4.116

Xanthomonas campestris pv. vitians $(\mathrm{Xcv})$, the causal agent of bacterial leaf spot of lettuce, causes lettuce yield reduction worldwide. Bacteriophages are potential biological control agents and tools for characterizing the diversity of $X c v$. Bacteriophages were isolated from soil (XcvP11) and water (XcvP12, $\mathrm{XcvP14}$ ) from lettuce fields using strain $\mathrm{BS} 0340$ as a host. $X c v$ strains were previously categorized into at least six multilocus sequence types (A, B1, B2, $\mathrm{C}, \mathrm{D}$ and $\mathrm{E}$ ) using concatenated sequences of $r p o D$, dnaK, fyuA, gyrB, and gap1. Plaques were purified using either strains BS0340 or BS0347 representing sequence types $\mathrm{B} 2$ and $\mathrm{B} 1$, respectively. Bacteriophage sensitivity was evaluated for $\mathrm{Xcv}$ strains from a broad geographic distribution. Lysis was recorded for bacteriophage samples $(5 \mathrm{ml})$ pipetted onto solidified soft agar containing test bacteria and poured over nutrient agar. All four $\mathrm{Xcv}$ type E strains were lysed by XcvP11, 12 and 14. Xcv strains from types A and $\mathrm{C}$ were negative for lysis by each bacteriophage. Approximately 81 and 45 percent of strains of types B1 and B2, respectively, were sensitive to the bacteriophages. For individual $X c v$ strains, reactions were consistent for all three bacteriophages. In order to use bacteriophage for disease management, bacteriophage mixtures will be needed to cover $X c v$ diversity. $X c v$ strains not lysed by $\mathrm{XcvP} 11,12$ and 14 will be used as hosts to isolate additional bacteriophages to use in mixtures.

\section{A thief in the blight: What can Erwinia amylovora bacteria steal from} their host?

L. RAMOS (1), J. Sinn (1), B. Lehman (2), E. Pfeufer (1), K. Peter (3), T. McNellis (1)

(1) The Pennsylvania State University, State College, PA, U.S.A.; (2) Penn State University - Fruit Research Extension Center, Biglerville, PA, U.S.A.; (3) The Pennsylvania State University / Penn State University - Fruit Research Extension Center, State College, PA, U.S.A.

Phytopathology 105(Suppl. 4):S4.116

Fire blight is the most destructive bacterial disease of apples, which are the second most consumed fresh fruit in the United States. The causal agent of fire blight is Erwinia amylovora. In this study, we tested whether E. amylovora is able to extract sufficient pyrimidine and arginine from the host to allow for bacterial growth and fire blight disease development. An E. amylovora $\arg D$ mutant, which had a disrupted arginine biosynthesis pathway, was an arginine auxotroph and did not cause disease or grow in host tissue. An E. amylovora pyrC mutant, which had a disrupted pyrimidine biosynthesis pathway, was a uracil auxotroph, yet still caused fire blight disease in apple tree shoots and grew in apple and pear immature fruits. Our study suggests that E. amylovora is able to obtain sufficient pyrimidine, but not arginine, from the host tissue to support bacterial growth and fire blight disease development. This study provides new information about $E$. amylovora food acquisition potential while growing in the host and could help develop novel possible fire blight controls. Furthermore, we found that the plasmid used to complement the $\arg D$ mutant was stably maintained in $E$. amylovora cells growing in planta. This plasmid could be a useful tool for functional analysis of E. amylovora genes in planta.

Impact of cover crop termination methods on diseases of wheat and lentil N. B. RANABHAT (1), M. E. Burrows (1), Z. J. Miller (1), E. A. Lehnhoff (1), F. D. Menalled (1)

(1) Montana State University, Bozeman, MT, U.S.A.

Phytopathology 105(Suppl. 4):S4.116

Crop rotation, cover cropping, and livestock integration may improve the sustainability of farming systems, but there is very little data on their effect on plant disease intensity. We investigated the impact of cover crop termination method on disease development in Bozeman, Mt. The experiment was began in 2013 with three systems of weed/cover crop management including sheep grazed organic (OG), organic tilled (OT) and conventional no-tillage (CNT). Each system (main plot) was split into five crop rotations (safflower/clover; clover; winter wheat; lentil; winter wheat). For two years, data were collected in the wheat and lentil phases of the rotations at three growth stages: tillering, flowering and matured. Incidence and severity of plant disease were recorded on 25 plants/subplot and subsets were chosen for identification of causal species. Management system did not impact disease incidence in wheat $(P=0.38)$ in both years and in lentil in $2013(P=0.9)$. CNT had lower disease incidence $(P<0.001)$ but OG and OT had similar disease incidence in lentil in
2014. The impact of management system on disease incidence depended on the crop phase and stage of wheat plants in $2014(P<0.001)$ and only with stages of wheat in $2013(P=0.2)$. This result suggests that disease incidence depends on plant stages irrespective of management system thus cropping system diversification of including targeted sheep grazing will not contribute to increased disease intensity.

Plant microbiota-driven facilitation of the persistence, growth and internalization of human pathogens on leafy greens

L. I. RANGEL (1), J. H. J. Leveau (1)

(1) University of California Davis, Davis, CA, U.S.A.

Phytopathology 105(Suppl. 4):S4.116

A common property among culturable bacteria that inhabit the phyllosphere is the production of the plant hormone indole-3-acetic acid (IAA). We are interested to know how this property contributes to the ability of human enteropathogenic bacteria to persist and survive on leafy greens. For the first part this study, we identified the sources and abundances of bacterial IAA producers from field-grown lettuce. Using quantitative measurements of in vitro IAA production in combination with $16 \mathrm{~S}$ rDNA gene amplicon sequencing, we discovered that among the culturable bacteria on these lettuce leaves, the genera Erwinia, Serratia and Arthrobacter had both IAAproducing and non-producing representatives. Of the IAA-producing isolates, Serratia was found to generate IAA at the highest relative rate. In the second part of this study, we demonstrated the effect of in vitro IAA exposure on the survival of attenuated Escherichia coli O157:H7. Pre-exposure of attenuated E. coli $\mathrm{O} 157: \mathrm{H} 7$ to IAA resulted in significantly prolonged survival of the bacteria in a nonnutritive environment. Next, we will assess whether IAA producers are active in vivo and whether the IAA that is produced has an impact on the foliar survival of attenuated E. coli $\mathrm{O} 157: \mathrm{H} 7$. By looking into the environmental factors that modify the production and response to bacterially produced IAA we hope to advance our understanding of how to predict or mitigate the establishment of human pathogens on leafy greens.

Soybean Respiratory burst oxidase homologs are required for white mold disease development

A. RANJAN (1), D. L. Smith (2), M. Kabbage (2)

(1) Univ of Wisconsin, Department of Plant Pathology, Madison, WI, U.S.A.;

(2) Department of Plant Pathology, University of Wisconsin, Madison, WI, U.S.A.

Phytopathology 105(Suppl. 4):S4.116

The plant membrane localized NADPH oxidases, also known as respiratory burst oxidase homologs (Rbohs), play crucial roles in various cellular activities including plant disease responses and cell death. Sclerotinia sclerotiorum is a cosmopolitan fungal pathogen that causes Sclerotinia stem rot (SSR) in soybean. It induces apoptotic-like programmed cell death (PCD) in its host plant, a process that requires generation of reactive oxygen species (ROS). Under pathogen attack, Rbohs act as major source of ROS, catalyzing the conversion of oxygen to superoxide anion $\left(\mathrm{O}_{2}{ }^{-}\right)$, a reactive free radical. In this study, we investigate the role of soybean Rbohs (GmRbohs) in Sclerotinia stem rot disease development. Using sequence similarity searches, we have identified 19 putative GmRboh genes in the soybean genome. Phylogenetic analysis of these genes indicates six distinct phylogenic groups. Expression analysis of GmRbohs in soybean at different time points following $S$. scleoriorum challenge indicates that a subset of these genes is highly upregulated during disease development. Conversely, other GmRbohs were down-regulated in the same time course. Virus induced gene silencing (VIGS) of specific GmRbohs resulted in the arrest of disease progression and abolished typical symptom development. Our results indicate the requirement of GmRbohs in Sclerotinia stem rot disease development and provide a potential target for the generation of white mold resistant soybean lines.

Lipopolysaccharide modulates the vector-pathogen interface of the bacterial phytopathogen, Xylella fastidiosa

J. RAPICAVOLI (1), N. Kinsinger (1), T. M. Perring (1), C. M. Johnston (1), S. Walker (1), M. C. Roper (1)

(1) University of California, Riverside, CA, U.S.A.

Phytopathology 105(Suppl. 4):S4.116

Xylella fastidiosa $(\mathrm{Xf})$ is a gram-negative, xylem-limited bacterium that causes serious diseases in economically important crops, such as Pierce's disease (PD) of grapevine. Lipopolysaccharide (LPS) is the dominant macromolecule displayed on the cell surface of Gram-negative bacteria. Due to its abundance, it mediates bacterial interactions with the environment and potential hosts. LPS is comprised of a conserved lipid A-core oligosaccharide component and a variable O-antigen. Through a mutation made in wzy (XP0836), which encodes an $\mathrm{O}$-antigen polymerase, we demonstrate that a structural alteration to the O-antigen causes a change in the physiochemical properties of the cell 
surface. Moreover, this alteration impairs attachment of $X$. fastidiosa to a cuticular substrate and to insect foreguts. Consequently, mutation of the $\mathrm{O}$-antigen significantly affects acquisition of $X f$ by an efficient insect vector, the blue-green sharpshooter, Graphocephala atropunctata (Signoret). Details of the kinetics of biofilm formation within insect foreguts is relatively unknown and, thus far, little attention has been given to the predominant surface polysaccharide, LPS, and its role in this complex biological process.

\section{Transgenic management of aflatoxin contamination in corn through host induced gene silencing}

Y. RARUANG (1), Q. Wei (2), R. Brown (2), D. Bhatnagar (2), Z. Chen (1)

(1) Louisiana State Univ Agric Center, Baton Rouge, LA, U.S.A.; (2) Southern Regional Research Center, USDA-ARS, New Orleans, LA, U.S.A. Phytopathology 105(Suppl. 4):S4.117

Maize (Zea mays L.) is one of the major crops susceptible to Aspergillus flavus infection and subsequent contamination with aflatoxins, the most potent naturally produced carcinogenic secondary metabolites. This pathogen has the potential to cause severe economic losses due to aflatoxin contamination. The gene encoding $A$. flavus $O$-methyltrasferase (omtA, aflP), a key enzyme involved in the aflatoxin biosynthetic pathway, was selected as a possible target for suppression through host induced gene silencing (HIGS). In this study, an RNAi vector containing the omtA gene was constructed and introduced into the immature B104 maize immature zygotic embryos through Agrobacterium transformation. Twelve transgenic plants from twelve independent transformation events were produced. PCR analysis of the genomic DNA from leaf tissue confirmed the presence of transgene in six out of the twelve plants. Real time PCR analysis of RNA isolated from transgenic leaf tissues showed a high variation of fungal target gene expression among the transgenic leaf tissues. Kernel screen assays were performed to determine aflatoxin level in the mature transgenic seeds seven days after inoculation with A. flavus. It was found that maize kernels containing the omtA gene had less aflatoxin than those that did not contain the gene. The results from these preliminary studies suggest that the HIGS using omtA gene can reduce aflatoxin contamination in maize.

Characterization of stuA mutants in the mycotoxigenic maize pathogen Fusarium verticillioides

M. RATH (1), N. J. Crenshaw (2), S. E. Gold (2)

(1) Dept. of Plant Pathology, University of Georgia, Athens, GA, U.S.A.; (2) Toxicology and Mycotoxin Research Unit, USDA-ARS, Athens, GA, U.S.A. Phytopathology 105(Suppl. 4):S4.117

Fusarium verticillioides is a major pathogen of maize, causing root, stalk and ear rots and seedling blight. It also produces fumonisin mycotoxins. Ingestion of fumonisin-contaminated corn causes acute toxicity in livestock and is a potential carcinogen to humans. StuA, an APSES protein class transcription factor, is a global regulator with stuA homologs regulating sporulation, mycelial and several other developmental processes in different fungal species. In $F$. graminearum, StuA is associated with regulation of pathogenicity, spore development and secondary metabolism. We hypothesize that the stuA homolog in $F$. verticillioides directly or indirectly regulates the production of fumonisin. Characterizing stuA mutants will help determine genes regulated by $s t u A$ and consequently should provide insight into fumonisin production and pathogenicity of $F$. verticillioides. stuA deletion mutants are being characterized phenotypically and preliminary data indicates mutants have slightly slower vegetative growth and reduced sporulation. More importantly, maize kernels inoculated with stuA mutants had reduced fumonisin accumulation compared to the wild-type $F$. verticillioides, as determined by a standard cracked corn assay. Further phenotypic and biochemical characterization of the mutants is underway. We plan to report construction, phenotypic characterization and initial global gene expression analysis of stuA mutants.

Characterization of apple replant disease-associated microbial communities over multiple growth periods using next-generation sequencing

A. J. REED (1), M. Mazzola (1)

(1) USDA ARS, Wenatchee, WA, U.S.A.

Phytopathology 105(Suppl. 4):S4.117

Replant disease in apple occurs as a result of incompletely understood and variable complexes of soil-borne pathogens that can build up over time in orchard soil. This disease limits economic viability of newly established orchards on replant sites and results in reduced productivity for the life of the orchard. Replant disease susceptible rootstock M.26 and disease-resistant rootstock G210 were planted in soil from two orchards. DNA was extracted from roots and root-associated rhizosphere soil at the end of three growing periods. The bacterial 16S rRNA gene and fungal ITS region were sequenced on the Illumina platform to characterize the microbial community, identify known and unknown potential pathogens involved and to track community changes over successive plantings. Sequencing results from the first growing period showed that potentially pathogenic Fusarium spp. were relatively rare ( $0.25 \%$ to $1.5 \%$ of total fungi) and contained few sequences of other known fungal pathogens. After the third growing period, the percentage of Fusarium spp. sequences was generally higher (up to $>16 \%$ ), and additional pathogenic species were detected including the Cylindrocarpon-like phytopathogen Ilyonectria radicicola. Agrobacterium tumefaciens was detected in root material (up to $3.5 \%$ of total bacteria) and rarely in soil. Results indicate that both rootstock genotype and rhizosphere microbiota are important factors in tolerance to replant disease.

Strategies for improving the Kinnow scab/malanose management plan: A major export quality issue in Pakistani citrus

A. REHMAN (1), A. U. Malik (2), M. Yasin (3), M. Ahsan (4), H. Bashir (4), M. W. Alam (1), B. Saleem (5), K. Riaz (1)

(1) Department of Plant Pathology University of Agriculture Faisalabad 38040- Pakistan, Faisalabad, Pakistan; (2) Institute of Horticultural Sciences, University of Agriculture Faisalabad 38040- Pakistan, Faisalabad, Pakistan; (3) Institute of Soil and Environmental Sciences, University of Agriculture Faisalabad 38040- Pakistan, Faisalabad, Pakistan; (4) Department of Entomology, University of Agriculture Faisalabad 38040- Pakistan, Faisalabad, Pakistan; (5) Department of Agriculture Extension, Government of Punjab -Pakistan, Sargodha, Pakistan

Phytopathology 105(Suppl. 4):S4.117

Recently, in Punjab-Pakistan, citrus scab/melanose in Kinnow fruit causing rind blemishes has become worst resulting in increased fruit rejection $(20-$ $50 \%$ ) and decreased export price in the international market. A 2013 survey of the target areas depicted higher level of scab/ melanose and citrus canker issues (15\% of the visited orchards) followed by citrus wither tip, gummosis and decline. Promising collected samples indicated the presence of fungal pathogens viz; Elsinoe fawcettii, Colletotrichum gloeosporioides, Alternaria citri and Fusarium oxysporum with variable frequencies. Organoleptic studies showed that total soluble sugars (TSS) were less in scab affected Kinnow as compared to the healthy fruits. Fungicides like Nativo ${ }^{\circledR}$ (Tebuconazole+ Trifloxystrobin), Cabriotop $^{\circledR} \quad$ (Pyraclostrobin+ Metiram), Score $^{\circledR}$ (Difenoconazol), Topsin M (thiophenate methyl), Copper oxychloride and Champion $^{\circledR}$ (Copper hydroxide) at $5,10,25,35$ and $50 \mu \mathrm{g} / \mathrm{mL}$ concentrations inhibited the in vitro mycelial growth of $E$. fawcettii and others invariably (Score, 45 and $77 \%$ against $E$. fawcettii and A. citri respectively and Topsin M $100 \%$ against $C$. gloeosporioides). Fruit assays depicted that Nativo, Score and Topsin $\mathrm{M}(50 \mu \mathrm{g} / \mathrm{mL})$ were statistically significant in reducing scab/melanose symptoms when tried in different combinations and could be recommended as preventive and curative treatment for the management of Kinnow Scab.

Interspecific competition for colonization of maize host between Fusarium proliferatum and Fusarium verticillioides

A. REYES GAIGE (1), J. P. Stack (1)

(1) Department of Plant Pathology, Kansas State University, Manhattan, KS, U.S.A.

Phytopathology 105(Suppl. 4):S4.117

Fusarium proliferatum (Fp) and Fusarium verticillioides (Fv) are seed-borne pathogens of maize. They are often asymptomatic in seed eluding symptombased detection. Although co-colonization of individual seed occurs, some seed lots are predominantly colonized by a single species. Experiments were conducted in non-sterile soil to determine if interspecific competition influenced establishment of an introduced isolate of $\mathrm{Fp}$ or Fv. Green fluorescent (GFP), hygromycin resistant Fp (Fp-G) and monomeric red fluorescent (mRFP) hygromycin resistant $\mathrm{Fv}$ (Fv-R) were developed to provide markers to track establishment in new environments. Heat-killed $\left(75^{\circ} \mathrm{C}\right.$ water bath for 20 minutes) maize seeds were colonized with Fp-G or Fv-R by immersion in a spore suspension for 16 hours. The ability of Fp-G and Fv-R to colonize viable seed already colonized by the other species was determined. Controls included competition with non-colonized cured seeds, and naturally colonized viable seeds. Plants were retrieved from soil after 14 days and observed for fluorescence. PCR amplification of partial GFP and mRFP gene sequences confirmed that Fp-G and Fv-R effectively competed against each other to colonize roots and stems of the maize plant. Fv-R colonized roots of $50 \%$ of the plants, and stems of $30 \%$ of the plants originally colonized with Fp-G; Fp-G colonized roots of 30\% of the plants, and stems of $10 \%$ of the plants originally colonized with Fv-R. 
Using Genotyping-by-sequencing (GBS) to study the population genetics of the fungus Fusarium proliferatum

A. REYES GAIGE (1), W. Yue (1), C. Toomajian (1), J. P. Stack (1)

(1) Department of Plant Pathology, Kansas State University, Manhattan, KS, U.S.A.

Phytopathology 105(Suppl. 4):S4.118

Fusarium proliferatum (Fp) is a toxigenic plant pathogen with a wide host range including symptomatic and asymptomatic hosts. In maize, Fp is seedborne yet most often asymptomatic which facilitates its long-distance dispersal. Genotyping-by-sequencing (GBS) was used to characterize $64 \mathrm{Fp}$ isolates from 6 commercial maize hybrids grown in the U.S. Green fluorescent (GFP), hygromycin-resistant $\mathrm{Fp}$ transformation of one of the isolates was used as an out-group. Euclidean distance and kinship analyses identified greater genetic similarity among isolates from the same hybrid than among isolates from different hybrids; with the exception of a hybrid in which isolates showed high variation (distance ranging from 0.11 to 0.81 ). Phylogenetic analyses grouped isolates from similar geographical location in three major clades. One clade grouped 48 isolates coming from 4 maize hybrids in Nebraska, another grouped 10 isolates coming from 2 maize hybrids in Illinois, and the other grouped 6 GFP isolates. Principal Component Analysis (PCA) grouped the isolates by geographical origin too. SNP analysis is being used to identify polymorphic genomic regions to develop specific primers for the detection of individual isolates in field experiments. The polymorphisms were detected by aligning similar reads from all isolates. The specificity of the primers will be confirmed by obtaining unique amplicons in $\mathrm{PCR}$, and by sequencing and aligning those amplicons to the original polymorphic read.

\section{The role of Avr4 in Cercospora kikuchii virulence and cercosporin biosynthesis}

J. S. REZENDE (1), Z. Y. Chen (1)

(1) Louisiana State University Agricultural Center, Baton Rouge, LA, U.S.A. Phytopathology 105(Suppl. 4):S4.118

The Avr4 effector, secreted by Cladosporium fulvum, has been demonstrated to be involved in pathogen virulence. Recent study further demonstrated that Avr4 is highly conserved among several Cercospora species, indicating a potential important role of this gene in the fungus virulence. Therefore, further investigation to determine whether this fungal effector gene is present in Cercospora kikuchii, the causal agent of soybean cercospora leaf blight (CLB) disease, and whether it plays any role in CLB development, is of great interest. These studies can provide important information in understanding host-pathogen interaction and in developing new approaches to enhance resistance to $C$. kikuchii infection in soybean. In the present study, we have cloned the avr4 gene from C. kikuchii, and mutants lacking the expression of avr4 have been created by homologous recombination using fusion PCR to generate a construct containing the hygromycin cassette fused with avr4 $5^{\prime}$ and $3^{\prime}$ fragments. Virulence assays using soybean leaves revealed that the avr 4 mutants have reduced fungal growth. Furthermore, disruption of the avr 4 gene resulted in mutants with significant reduced cercosporin toxin biosynthesis (CTB) and CTB genes expression. The results indicate that in addition to the previous reported chitin binding activity to protect pathogen during infection, Avr4 also contributes to the virulence of Cercospora kikuchii on soybean through regulation of cercosporin biosynthesis.

Use of bacterial strains degrading 3-hydroxy palmitate methyl ester, the Ralstonia quorum sensing signal, as biocontrol agents

K. RIAZ (1), S. Genin (2), D. Faure (3), Y. Dessaux (4)

(1) Univ of Agriculture Faisalabad, Faisalabad, Pakistan; (2) Laboratoire des Interactions Plantes Micro-organismes, UMR CNRS-INRA 2594/441, F31320 Castanet Tolosan, France, Toulouse, France; (3) Département de Microbiologie Institut de biologie intégrative de la cellule (Institute of integrative cell biology) CNRS - Bât 23 Avenue de la terrasse 91198 Gif sur Yvette CEDEX France, Gif sur Yvette, France; (4) Département de Microbiologie Institut de biologie intégrative de la cellule (Institute of integrative cell biology) CNRS - Bât 23 Avenue de la terrasse 91198 Gif sur Yvette CEDEX France, Gif sur Yvette, Pakistan

Phytopathology 105(Suppl. 4):S4.118

In Ralstonia solanaceraum, causing tomato wilts, most of the pathogencity and virulence related determinants (e.g. Plant cell wall degrading enzymes and extrapolysaccharides) are regulated in a cell density dependent manner through quorum sensing (Qs) utilizing 3-hydroxy-palmitate, methyl ester (3OH PAME) as the major Qs signaling molecule. In this study, several bacteria belonging to Acinetobacter sp., Chryseobacterium sp, Pseudomonas sp., and Delftia $s p$ degrading 3-OH PAME could be isolated from various soil-root horizons through selective enrichment procedures based on growth on QS signal analogues assisted with biosensor assays. In addition to 3-OH PAME these bacteria could also degrade several classes of NAHL molecules. Twenty isolates were identified and assayed as potential quenchers of the virulence of Ralstonia solanacearum in-planta. Some of these strains indeed delayed the appearance of the symptoms of the disease and reduced the aggressiveness of the pathogen confirming the value of QS interference strategies in the development of biocontrol strategies aimed at plant pathogens.

\section{Screening for ISR-eliciting bacteria in citrus}

N. RIERA (1), U. Handique (1), N. Wang (2)

(1) University of Florida, Lake Alfred, FL, U.S.A.; (2) University of Florida CREC, Lake Alfred, FL, U.S.A.

Phytopathology 105(Suppl. 4):S4.118

Citrus greening (or HLB) presents an unprecedented challenge to Florida citrus industry. The causative agent in Florida is Candidatus Liberibacter asiaticus, a gram negative alphaproteobacteria, which is transmitted by the psyllid vector Diaphorina citri. Despite many efforts taken, no effective control has been found for the disease. Some rhizospheric beneficial bacteria are able to trigger Induced Systemic Resistance (ISR), priming the plant with enhanced capacity against a broad spectrum of pathogens and herbivores. In previous studies, beneficial bacteria were isolated from healthy citrus trees in severely HLB-infected groves. Nine bacterial strains isolated from healthy citrus rhizosphere from the genus Burkholderia, Pseudomonas, Stenotrophomonas, Bacillus and Rhodoccocus were screened for ISR-eliciting ability. Plant defense responses were monitored using qRT-PCR by studying expression of selected defense genes of the leaf tissue. Genes from the Ethylene/Jasmonate and Salicylic Acid pathways as well as Pathogenesis Related (PR)-proteins were used as markers. To further characterize the ISR against other pathogens, Xanthomonas citri subsp. citri, the causal agent of citrus canker, was used to challenge plants pretreated with beneficial bacteria and non-treated control plants. Disease severity of treated and non-treated plants was compared. Exploring and exploiting beneficial bacteria-plant interactions can contribute to sustainably control phytopathogens.

The integration of fungicide application and host resistance on blast progress on wheat under field conditions

J. A. RIOS (1), F. A. Rodrigues (1), V. S. Rios (1), P. A. Paul (2), M. A. Souza (3), L. Araújo (1)

(1) Department of Plant Pathology, Universidade Federal de Viçosa, Viçosa, Brazil; (2) Department of Plant Pathology, The Ohio State University, Wooster, OH, U.S.A.; (3) Department of Crop Science, Universidade Federal de Viçosa, Viçosa, Brazil

Phytopathology 105(Suppl. 4):S4.118

Wheat blast, caused by Pyricularia oryzae, continues to cause substantial losses in all major wheat-producing regions of Brazil. Fungicide and cultivar resistance are major components of management programs to minimize such losses. Two field experiments (EXP1 and EXP2) were conducted to evaluate the effect of fungicide and host resistance on blast development, as well as selected physiological parameters of the wheat plants. Plots of wheat cultivars BR-18 (partially resistant) and Guamirim (susceptible) were either treated $(+\mathrm{F})$ with epoxiconazol + piraclostrobina or left nontreated (-F), and inoculated with at spore suspension of $P$. oryzae at anthesis. Blast severity rated on spikes and leaves was used to estimate area under blast progress curve (AUBPC) for each treatment combination. In EXP1, BR-18+F and Guamirim $+\mathrm{F}$ resulted in significantly lower AUDPC, 58 and $83 \%$ reduction for spikes and 70 and $62 \%$ for leaves, respectively, than the untreated checks. The corresponding values for EXP2 were 90 and $92 \%$ and 68 and $64 \%$ for spike and leaves, respectively. BR-18 had significantly lower AUBPC than Guamirim in both experiments. Maximal photosystem II quantum yield $\left(F_{\mathrm{v}} / F_{\mathrm{m}}\right)$, maximal fluorescence $\left(F_{\mathrm{m}}\right)$, effective PS II quantum yield (Y(II)), quantum yield of regulated energy dissipation (Y(NPQ)) were superior in treated than nontreated plants. Reduction of blast symptoms in association with better photosynthetic performance occurred in the fungicide-treated resistant cultivar.

Evolutionary origins of US and famine-era lineages of Phytophthora infestans

J. RISTAINO (1), A. Saville (2), M. Martin (3), M. T. P. Gilbert (4)

(1) North Carolina State University, Raleigh, NC, U.S.A.; (2) Department of Plant Pathology, NC State University, Raleigh, NC, U.S.A.; (3) Center for Theoretical and Evolutionary Genomics, University of California, Berkeley, CA, U.S.A.; (4) Center for Geogenetics, Natural History Museum, University of Copenhage, Copenhageno, Denmark

Phytopathology 105(Suppl. 4):S4.118

Phytophthora infestans, causal agent of the Irish potato famine, is a threat to food security globally and an important pathogen in the southeastern US on both tomato and potato. USABlight (www.usablight.org) was launched in 
2011, and lineages in the US have been genotyped. The mefenoxam sensitive US-22, more common on tomato in 2009, has been displaced by US-23, which infects both hosts. The genetic relationships of 18 of the 24 clonal lineages found in the US since the 1970s were examined using 12 simple sequence repeat (SSR) markers. The US-1 lineage formed a cluster distinct from most modern US lineages in DPCA and STRUCTURE analyses of SSR loci. Five clusters of modern US lineages were identified, and some recent US lineages showed similarity to Mexican lineages with the exception of US-1 and US-23. Mitochondrial haplotype data, SSR and NGS data from herbarium specimens were used to track the emergence of lineages. The US-1 (Ib) lineage increased in frequency in the mid-20th century in the US and globally and then declined. Populations from European and US 19th century outbreaks shared allelic diversity and were distinct from US-1 and modern aggressive US lineages. US and EU famine era populations shared allelic diversity with lineages found in South America. The evolutionary relationship of historic and recent US lineages will be discussed in the context of possible pathogen migration routes.

Harnessing the power of endemic fungal communities in the boxwood rhizosphere

Y. RIVERA (1), P. Ndukwe (2), L. Cornelius (2), J. A. Crouch (2)

(1) USDA ARS, Beltsville, MD, U.S.A.; (2) USDA-ARS, Beltsville, MD, U.S.A. Phytopathology 105(Suppl. 4):S4.119

Calonectria pseudonaviculata (Cps) is a newly emergent, non-native pathogen that causes boxwood blight, a disease that can rapidly lead to plant death. In this study, we evaluated native fungal rhizosphere communities to identify natural enemies of Cps. Rhizosphere soil from 40 mature boxwood plants from two arboreta collections was sampled (20 species/cultivars) and metagenomic analyses performed from 454 pyrosequencing of the ITS. Communities were structured according to arboretum site, not boxwood species. However, only minor differences in overall community diversity were observed, with $4 \%$ of the 15,699 OTUs differentially represented. Among boxwood species, differences were greater in community diversity and less pronounced in composition. Just four genera made up 30\% or more of the total fungal community, and only four OTUs were found at all 40 sites. Fusarium and Mortierella were the most widespread and dominant fungal genera. Thirty species of Trichoderma - a genus known for its fungal antagonistic properties - were identified, but were minority constituents in all but one sample. Trichoderma cultured from the soil samples reduced Cps growth by as much as $99.4 \%$ in dual-culture experiments, outperforming commercial strain T22. Growth inhibition by Trichoderma varied between Cps isolates. Ecological context can significantly impact plant physiology, and our data support the theory that indigenous fungal communities could influence boxwood blight development.

Comparative genome analysis of three oomycete species causing downy mildew disease in specialty crop production

Y. RIVERA (1), C. Salgado-Salazar (2), D. Veltri (3), J. A. Crouch (4)

(1) USDA-ARS; Rutgers University, Beltsville, MD, U.S.A.; (2) USDA-ARS, Rutgers University, Beltsville, MD, U.S.A.; (3) Rutgers University, New Brunswick, NJ, U.S.A.; (4) USDA-ARS, Beltsville, MD, U.S.A.

Phytopathology 105(Suppl. 4):S4.119

Downy mildews are economically important diseases affecting a wide variety of crops worldwide. In recent years, downy mildew epidemics have become increasingly prevalent, resulting in significant losses to specialty crops worldwide. Because of the biotrophic nature of these organisms, genomic resources are often scarce, especially relative to oomycetes capable of growth in pure culture such as Phytophthora and Pythium. To understand the diversity of three organisms responsible for these recent epidemics, we sequenced the genomes of three species within the Peronosporaceae: Plasmopara halstedii (isolated from Helianthus annuus and Rudbeckia fulgida), Plasmopara obducens (isolated from Impatiens walleriana) and Peronospora sp. (isolated from Agastache mexicana). Nextera XT and TruSeq Nano libraries were prepared from sporangial masses collected from leaves and sequenced using Illumina sequencing-by-synthesis technology. De novo read assemblies estimated the genome sizes at $70 \mathrm{Mb}$ for $P$. halstedii and Peronospora sp. while the genome of $P$. obducens was estimated at over $200 \mathrm{Mb}$. Analyses of gene predictions, carbohydrate-active enzymes and protein secretion were used to compare the draft genomes generated in this study and publicly available genomes in the Peronosporales.

Eicosapolyenoic fatty acids induce resistance to Phytophthora capsici and alter oxylipin metabolism in tomato

S. M. ROBINSON (1), R. M. Bostock (1)

(1) UC Davis, Davis, CA, U.S.A.

Phytopathology 105(Suppl. 4):S4.119
The eicosapolyenoic fatty acids, arachidonic (AA) and eicosapentaenoic (EPA) acids, are absent from higher plants and most plant pathogens, but present in plant pathogenic oomycetes. During infection, AA and EPA released from Phytophthora species serve as microbe associated molecular patterns (MAMPs) that elicit plant defense responses. Tomato plants whose roots are pre-treated with AA or EPA and subsequently inoculated with Phytophthora capsici display increased root and crown lignification and decreased susceptibility to crown rot and collapse. Recognition and action of AA and EPA in plants may occur via different mechanisms than other studied MAMPs. These include disruption of host membrane integrity and multifaceted alteration of oxylipin metabolism. RT qPCR analyses reveal increased expression of 9-lipoxygenase (9-LOX), 9-divinyl ether synthase (9DES), 13-lipoxygenase (13-LOX) and 13-allene oxide synthase (13-AOS) genes in AA-treated tomato roots compared to controls, with 9-DES showing the greatest increase. Similar changes in gene expression occur in P. capsiciinfected tomato roots. The oxylipin profile of AA-treated tomato roots differs greatly from controls. Further characterization of oxylipin metabolism and induced resistance responses in AA-treated plants is ongoing. This research will increase understanding of oxylipins in plant defense and may identify novel host targets for controlling oomycete pathogens.

Fungal diseases of Cannabis sativa in British Columbia, Canada G. RODRIGUEZ (1), A. Kibler (1), P. Campbell (1), Z. K. Punja (2) (1) Agrima Botanicals, Maple Ridge, BC, Canada; (2) Simon Fraser Univ, Burnaby, BC, Canada Phytopathology 105(Suppl. 4):S4.119

A number of previously unreported fungal diseases were observed in a commercial growing facility of Cannabis sativa being produced hydroponically for medicinal use in British Columbia, Canada (under approved Health Canada regulatory requirements) during 2013-2014. These included root rot caused by Pythium dissotocum and P. myriotylum and bud rot caused by Botrytis cinerea. As well, Penicillium olsonii was found colonizing dried packaged product and was recovered from air samples in the growing facility. All fungi were recovered on agar media and identified by PCR using ITS1 - ITS4 primers and nucleotide sequence comparison using BLASTn. Powdery mildew infection on leaves was identified to be caused by Golovinomyces (Erysiphe) cichoracearum by PCR. Pythium dissotocum isolates were confirmed to cause root rot by inoculation of rooted vegetatively propagated plants grown in a hydroponic system. Ten days following inoculation at $25^{\circ} \mathrm{C}$, plants appeared stunted and roots were rotting and discolored. The pathogen was recovered from diseased tissues. Some isolates of $F$. oxysporum were found to be pathogenic and caused symptoms of root browning and rot. Freshly harvested flower buds inoculated with $B$. cinerea and incubated at high humidity at $25^{\circ} \mathrm{C}$ developed symptoms of rot and decay within 9 days and the pathogen was reisolated from inoculated tissues. Isolates of $P$. olsonii were frequently isolated from dried product and could negatively affect product quality.

Genomes come from organisms--the continued importance of voucher specimens in fungal identification

M. ROMBERG (1), C. Blomquist (2)

(1) USDA APHIS PPQ NIS, Beltsville, MD, U.S.A.; (2) California Department of Food and Agriculture, Sacramento, CA, U.S.A.

Phytopathology 105(Suppl. 4):S4.119

Identification of fungi increasingly relies upon DNA sequence comparison. Only a fraction of described fungal species have been sequenced. These sequences often do not represent type or vouchered material. There is an ongoing need to preserve plant tissue with sporulating fungi and cultures of fungi that can be grown on artificial media. This is especially important for confirmation of a first report of a fungus in a given area or on a new host. For example, a historical powdery mildew specimen collected in California in the $19^{\text {th }}$ century, never formally described, matched a fungus found recently in California showing that the mildew was endemic, avoiding destruction of a greenhouse of a new ornamental hybrid. In another example, an ascomycete on Commelina was intercepted in Florida. A fungus with the same morphology was described in 1943 as Physalospora commelina. The type of this species, and the only known specimen, is held at the USDA National Fungus Collections. Examination of the type showed morphological discrepancies with the original description, but it was consistent with the intercepted fungus. A pure culture of the intercepted fungus was obtained and DNA sequence comparison utilized to determine its proper systematic placement and provide support for epitypification. The case studies presented illustrate the importance of voucher specimens for current and future systematic research as well as their use in trade and quarantine situations. 
A bioassay method for detection and quantification of latent infections of Colletotrichum spp. in olive fruits

J. Romero (1), F. Cherifi (2), A. E. Santa-Barbara (1), J. MORAL (3), L. F. Roca-Castillo (1), A. Trapero-Casas (1)

(1) Univ. de Cordoba, Cordoba, Spain; (2) Univ. de Cordoba, Córdoba, Spain; (3) Univ De Cordoba, Cordoba, Spain

Phytopathology 105(Suppl. 4):S4.120

Anthracnose of olive (Olea europaea), caused by Colletotrichum spp., is the most important disease adversely affecting the olive oil quality. Early detection of latent infections on olives helps to determine the control strategy in early autumn. However, there are no techniques for latent infections in olives that are easy to use, fast and economical. This study compared six treatments on fruit of two olive cultivars, 'Hojiblanca' (highly susceptible to disease) and 'Arbequina' (moderately susceptible). Tests were made with naturally infected and artificially inoculated olives. Treatments evaluated were paraquat (with and without fruit wound), etephon, freezing, ethanol and sodium hydroxide, all at different concentrations and exposure times. There were two control treatments which included washed and disinfested olives, or unwashed and no disinfested olives. Incidence of symptomatic olives (\%) and $\mathrm{T}_{50}$ (days until the appearance of $50 \%$ symptomatic olives) were the parameters estimated to compare treatments. Sodium hydroxide at $0.05 \%$ for more than 1 day of exposure showed equal or better results than Paraquat, the technique being used to date. Sodium hydroxide $0.05 \%$ for 96 hours detected $4.4 \%$ more of diseased olives in 8.9 days less than the paraquat treatment used as reference. This treatment was economical and safe. Because of this, sodium hydroxide technique could be a great tool in making decisions about fungicide applications on commercial farms.

\section{Plant pathology career and internship opportunities at Monsanto}

S. ROSENBERGER (1)

(1) Monsanto Vegetable Seeds, Woodland, CA, U.S.A.

Phytopathology 105(Suppl. 4):S4.120

Twenty years from now, the earth's population will need 55\% more food than it can produce now. Today, Monsanto is working with farmers around the world to do something about it. We employ nearly 22,000 dedicated employees who are focused on helping farmers produce healthy, nutritious food in a sustainable way. Monsanto has professional development programs and a history of building careers in more than 60 countries. We have established industry-leading products because we give professionals like you the freedom to make real decisions.

Integration of crop rotations and fertility to enhance soil health, plant health, and disease management in Michigan potato production N. ROSENZWEIG (1), K. Steinke (1), L. Steere (1), W. W. Kirk (1) (1) Michigan State University, East Lansing, MI, U.S.A.

Phytopathology 105(Suppl. 4):S4.120

An experimental research trial was established evaluating fertility and rotational potato crop management. A randomized complete split-block design with four replications was used. The split-block included organic and inorganic fertilizer treatments. Tubers were harvested, weighed, graded, and assessed for common scab. Potato petioles were sampled twice over the growing season for nutritional status. Bulk and rhizosphere soil was sampled from treatments. The bacterial 16S rRNA was targeted using next-generation sequencing. Total number of DNA sequences identified to phyla, class, order, family and genus was $28,81,140,300$ and 814 respectively. There was no difference in plant respiration by crop rotation. Tuber yield in back-to-back potato was $2.91 \mathrm{~kg} / \mathrm{ha}$, compared to $3.05 \mathrm{~kg} / \mathrm{ha}$ potato following corn. There was no significant interaction in rotation by fertility. Tuber yield in organic back-to-back potato fertility programs was $3.23 \mathrm{~kg} / \mathrm{ha}$ and inorganic back-toback was $2.60 \mathrm{~kg} / \mathrm{ha}$. Moreover tuber yield in organic fertility programs of potato following corn was $3.27 \mathrm{~kg} / \mathrm{ha}$ and inorganic was $2.82 \mathrm{~kg} / \mathrm{ha}$. Scab severity was significantly lower $(10-15 \%)$ in the organic potato following corn fertility treatment compared to the inorganic back-to-back potato fertility treatment $(P=0.044)$.

Application of anaerobic soil disinfestation in Florida; a brief review E. N. ROSSKOPF (1), C. Shennan (2), N. Kokalis-Burelle (3), D. M. Butler (4), P. Serrano-Perez (5), M. Rodríguez-Molina (5), J. C. Hong (6)

(1) USDA ARS, Ft Pierce, FL, U.S.A.; (2) University of California-Santa Cruz, Santa Cruz, CA, U.S.A.; (3) USDA, ARS, Fort Pierce, FL, U.S.A.; (4) University of Tennessee, Knoxville, TN, U.S.A.; (5) Centro de Investigaciones Científicas y Tecnológicas de Extremadura (CICYTEX), Badajoz, Spain; (6) USDA ARS, Fort Pierce, FL, U.S.A.

Phytopathology 105(Suppl. 4):S4.120
Anaerobic soil disinfestation (ASD) is a pre-plant, biologically-based alternative to chemical fumigation for controlling multiple soil-borne pathogens and weeds. Application of ASD typically consists of incorporating a labile carbon source, covering the soil with a gas-impermeable plastic, and irrigating the soil to saturation. During this process, the environment under the plastic becomes anaerobic, and many of the byproducts produced by anaerobic organisms inhibit the survival of plant pathogens. Appling ASD in Florida includes the use of feed grade molasses as the carbon source, and incorporating in composted broiler litter (CBL). Clear or opaque film can be used. ASD in Florida has been used to control plant pathogens in diverse crops including tomato, bell pepper, eggplant, cucumber, strawberry, and fresh cut flowers. Comparing ASD to methyl bromide or other soil fumigants, disease ratings and fruit yields for ASD-treated plots were similar to chemical fumigation. Various types of plastic mulch and different nitrogen sources have been evaluated to determine their effectiveness for use in ASD. Current experiments are focused on minimizing disruption to the typical field production practices used by Florida growers, and adapting the approach for spring crop production cycles.

A rapid and reliable isothermal diagnostic assay for detecting Soybean sudden death syndrome (SDS) pathogen Fusarium virguliforme

M. G. ROTH (1), A. Rojas (2), J. Wang (1), M. I. Chilvers (1)

(1) Michigan State University, East Lansing, MI, U.S.A.; (2) Michigan State University, East Lansing, MI, U.S.A.

Phytopathology 105(Suppl. 4):S4.120

Some plant diseases are difficult to detect until the disease has progressed extensively, like sudden death syndrome (SDS) in soybean caused by Fusarium virguliforme. Quantitative polymerase chain reaction (qPCR) and enzyme-linked immunosorbent assays (ELISA) are the most common molecular diagnostic techniques currently used, but they use lengthy procedures, expensive equipment, and are limited to lab conditions. In comparison, isothermal amplification techniques like recombinase polymerase amplification (RPA) provide similar diagnostic data with multiple advantages. An RPA assay can provide real-time detection or end point detection much faster than qPCR or ELISA, uses less expensive lab equipment, and only requires crude DNA extractions for pathogen detection. In addition, RPA assays can be performed on a completely portable platform and used directly in the field. The objective of this project was to develop an RPA assay to rapidly and reliably detect $F$. virguliforme from soybean roots. This RPA design uses primers and a probe to amplify the $F$. virguliforme intergenic spacer (IGS) region, and assay specificity was confirmed against six closely related Fusarium species. With similar specificity and sensitivity to current techniques, the portability of this assay may allow faster detection at the point of contact and facilitate quicker management decisions. Additional RPA assays could provide similar advantages for other diseases that are difficult to diagnose.

Utilization of differential line and vegetative compatibility group methods for the characterization of isolates of Magnaporthe oryzae from Africa F. ROTICH (1), S. Mutiga (1), C. Feng (1), J. Harvey (2), D. Silué (3), D. Tharreau (4), T. Mitchell (5), G. L. Wang (5), N. Talbot (6), J. Correll (1) (1) University of Arkansas, Fayetteville, AR, U.S.A.; (2) Biosciences eastern and central Africa (BecA)-ILRI Hub, Nairobi, Kenya; (3) Africa Rice center (AfricaRice), Cotonou, Benin; (4) French Agricultural Research Center for International Development (CIRAD), Montpellier, France; (5) The Ohio State University, Columbus, OH, U.S.A.; (6) University of Exeter, Exeter, United Kingdom

Phytopathology 105(Suppl. 4):S4.120

Rice blast, caused by Magnaporthe oryzae, is a disease that can cause severe losses in rice yields. Recent studies showed that demand for rice is increasing faster than the increase in production in Africa, and there is a need to mitigate production challenges such as rice blast. Breeding for resistance to rice blast in Africa requires knowledge of $M$. oryzae diversity and the history of resistance in the pathosystem. A core collection isolates of $M$. oryzae $(n=100)$ from nine African countries (Benin, Burkina Faso, Ghana, Kenya, Mali, Nigeria, Tanzania, Togo, and Uganda) were assessed for vegetative compatibility groups (VCG) and for disease reactions based on an International Rice Research Institute set of differential lines and African interspecific cultivars. Preliminary analysis showed that VCGs were unique for each country, but the virulence phenotypes of isolates were not associated with the country of origin. Avirulence was observed in a range $25 \%-75 \%$ of the subset $(n=49)$ of the isolates that were pathotyped using the 31 differential lines. The $P i 9$ resistance gene was most effective against the core set of isolates, conferring resistance against $75 \%$ of the isolates, followed by unknown resistances in two African interspecific cultivars, which conferred 
resistance against $56 \%$ of the isolates. Findings of this study will facilitate resistance breeding efforts to enhance effective control of rice blast in Africa.

Nonpathogenic Geosmithia species associated with the walnut twig beetle, Pityophthorus juglandis, in California walnut orchards

T. V. ROUBTSOVA (1), T. L. Nguyen (1), M. A. Yaghmour (1), S. J. Seybold (1), R. M. Bostock (1)

(1) UC Davis, Davis, CA, U.S.A.

Phytopathology 105(Suppl. 4):S4.121

The walnut twig beetle (WTB), Pityophthorus juglandis, is the vector of Geosmithia morbida, the causal agent of thousand cankers disease (TCD) responsible for dieback and death of Juglans nigra throughout the USA, and now a threat to English walnut, Juglans regia, in California orchards. Flighttrapped WTB from a California walnut orchard carried fungi that are morphologically similar to Geosmithia species. Isolates were purified and identified by using ITS sequence analysis. Two of these isolates were confirmed to be Geosmithia lavendula and one was confirmed to be a closely related Geosmithia species. Inoculation of excised J. regia branch sections indicated that these species are not pathogenic on this host. Isolates of another Geosmithia species obtained from the Phaff collection at UC Davis that were originally isolated from oak trees in California were determined to be Geosmithia fassatiae by sequence analysis. In pathogenicity tests, these $G$. fassatiae isolates caused cankers on J. regia branch sections. G. fassatiae was previously confirmed to be pathogenic on oak trees, and is carried by western oak bark beetle, Pseudopityophthorus pubipennis, and related species. The ecological importance of additional Geosmithia species in association with the WTB is unclear and needs further study.

Single race screening nurseries elucidate race-specificity involved in adult plant resistance to stem rust in wheat cultivar Thatcher

M. ROUSE (1), J. Briggs (2)

(1) USDA ARS Cereal Disease Lab, St. Paul, MN, U.S.A.; (2) University of Minnesota, St. Paul, MN, U.S.A.

Phytopathology 105(Suppl. 4):S4.121

Adult plant resistance (APR) in wheat to stem rust caused by Puccinia graminis f. sp. tritici $(P g t)$ is resistance that is effective only at the adult plant stage of development. Wheat varieties that exhibit APR to stem rust have been shown to possess multiple quantitative trait loci (QTL). We tested the racespecificity and epistatic interactions of wheat stem rust APR QTL. Wheat cultivar 'Thatcher' is susceptible as a seedling and resistant as an adult plant to $P g t$ races TPMKC, RCRSC, and TTKST (TTKST is a member of the Ug99 race group). We previously assessed 160 RILs derived from the cross 'Thatcher'/'McNeal' in Kenya against Pgt race TTKST, and identified four QTL that displayed epistatic interactions. We also assessed the same population for two seasons in Rosemount, Minnesota, in two separate stem rust screening nurseries each inoculated with a single Pgt race (races TPMKC and RCRSC). The QTL with the largest effect, coincident with the Sr12 locus, remained effective to all races in all environments. However, other QTL including previously detected $Q S r . c d l-1 A L$ displayed consistent race-specific effectiveness. These results suggest that wheat stem rust APR QTL can be race-specific, similar to what has been demonstrated in other pathosystems. This finding suggests that assessing field reaction to multiple Pgt races is necessary to select for non-race-specific resistance.

Identification of Brevipalpus yothersi Baker, as vector and possible primary host of cytoplasmic citrus leprosis viruses

A. ROY (1), J. S. Hartung (2), J. Shao (2), G. Leon (3), M. J. Melzer (4), J. J. Beard (5), G. Otero-Colina (6), G. R. Bauchan (2), R. Ochoa (2), R. H. Brlansky (7), W. L. Schneider (1)

(1) USDA-ARS, Frederick, MD, U.S.A.; (2) USDA-ARS, Beltsville, MD, U.S.A.; (3) Centro de Investigación La Libertad, CORPOICA, Villavicencio, Colombia; (4) University of Hawaii, Honolulu, HI, U.S.A.; (5) Queensland Museum, South Brisbane, Australia; (6) Colegio de Postgraduados, Campus Montecillo, Texcoco, Mexico; (7) Univ of Florida, Lake Alfred, FL, U.S.A. Phytopathology 105(Suppl. 4):S4.121

The citrus leprosis complex is one of the most important emerging viral threats to US citrus production. Several viruses are associated with leprosis, including Citrus leprosis virus $C(\mathrm{CiLV}-\mathrm{C})$ and Citrus leprosis virus $\mathrm{C2}$ (CiLV-C2) (genus Cilevirus), Hibiscus green spot virus 2 (HGSV-2), (Higrevirus), Citrus leprosis virus $N$ (CiLV-N) and Citrus necrotic spot virus (proposed genus Dichorhavirus). All leprosis viruses are transmitted by or associated with flat mites (Brevipalpus spp.). Suspected non-viruliferous and viruliferous Brevipalpus mites were collected from Colombian and Mexican leprosis-free and -infected citrus groves, respectively. B. yothersi from Colombia and Mexico and B. californicus from Mexico were identified as vectors of citrus leprosis, utilizing low temperature scanning electron microscopy. Conventional and reverse strand specific RT-PCR detection assays were developed to identify the viruses and assess replication in mites. All three major leprosis viruses (CiLV-C, $-\mathrm{C} 2$ and $-\mathrm{N}$ ) as well as replication intermediates were detected from viruliferous $B$. yothersi. A circulativepersistent-propagative virus-vector relationship was established, corroborating phylogenetic analyses of the RNA-dependent RNA polymerase 2 domains and p24 gene amino acid sequences. Further analysis suggested that the Cilevirus and Higrevirus genera may have originated in mites, with Citrus spp. being a secondary host.

Using supervised machine learning to identify host-microbe interaction factors in the soft rot pathogen, Pectobacterium carotovorum

I. RUBIO (1), B. Ma (1), A. Charkowski (1), N. Perna (1)

(1) University of Wisconsin-Madison, Madison, WI, U.S.A

Phytopathology 105(Suppl. 4):S4.121

Genome sequencing provides many insights into the genes involved in hostmicrobe interactions. However, traditional bioinformatics approaches struggle with unknown genes and have high false positive rates, discouraging experimental verification. To try to overcome these limitations, we used a supervised machine learning strategy to generate a list of novel candidate genes involved with host-microbe interactions. To test this new approach, we selected the soft rot pathogen, Pectobacterium carotovorum, due to its worldwide importance, large amount of genomic data available, and tractability in laboratory environments. 30 different genes were chosen which consisted of 10 genes of unknown function that were predicted to be important for host-microbe interactions, 10 genes that were not, and 10 random genes. Site directed mutagenesis was conducted for the genes predicted to be important for host-microbe interactions. Mutants were tested with multiple plant assays to determine any impairment in survival, growth, persistence or disease development in potato. Preliminary data showed that 8 mutants predicted to be important for plant-microbe interaction macerated potato tubers. Petiole assays with the same mutants demonstrated that 4 mutants were impaired in virulence. In stem assays, only two mutants showed reduced virulence. Our long-term goal is to develop a generalized machine learning tool to aid in identification of microbial genes involved in complex traits.

RNAi Mediated Silencing of Triticum mosaic virus coat protein gene induces resistance to virus in transgenic wheat

J. L. RUPP (1), L. Cruz (1), J. P. Fellers (2), H. N. Trick (1)

(1) Kansas State University, Manhattan, KS, U.S.A.; (2) USDA-ARS, Manhattan, KS, U.S.A.

Phytopathology 105(Suppl. 4):S4.121

Triticum mosaic virus (TriMV) is one of three viruses of the wheat mosaic complex affecting wheat in the Great Plains of the United States. Currently, there are no resistant commercial varieties. We have evaluated the use of RNAi to generate resistance to TriMV. A RNAi expression vector was created from the sequence of the coat protein of TriMV and immature embryos of the wheat cultivar 'Bobwhite' were co-transformed by biolistic particle delivery system with the RNAi expression vector and pAHC20, which contains the bar gene for glufosinate selection. After tissue culture and plant recovery, putative transformed plants were analyzed with PCR and RT-PCR for the presence and expression of the appropriate RNAi TriMV CP gene. To determine viral resistance, $T_{1}$ progeny were mechanically inoculated with TriMV. In the $T_{1}$ generation, resistance was seen in up to $80 \%$ of the plants evaluated for the TriMV CP construct. These plants have undergone single plant selection up to the $\mathrm{T}_{6}$ generation and continue to show high level of resistance when challenged with the virus. Crosses have been made with the virus susceptible winter wheat, 'Overley.' Real-time PCR results show a decrease in viral titer up to 20 -fold in the $\mathrm{T}_{6}$ transgenic lines, the $\mathrm{F} 1$ crosses, and the $\mathrm{BC} 1 \mathrm{~F} 1$ compared to control plants. This research provides evidence that this RNAi silencing construct can provide stable resistance to TriMV and has great potential benefits to both breeders and producers.

Baseline sensitivity to fluopyram and fungicide resistance phenotypes of Botrytis cinerea populations from table grapes in California

S. SAITO (1), M. J. Themis (2), C. L. Xiao (3)

(1) USDA ARS, Parlier, CA, U.S.A.; (2) University of California-Davis, Kearney Agricultural Center, Parlier, CA, U.S.A.; (3) USDA Agricultural Research Service, Parlier, CA, U.S.A. Phytopathology 105(Suppl. 4):S4.121

Gray mold caused by Botrytis cinerea is a major postharvest disease of table grapes grown in the Central Valley of California. Understanding fungicide resistant phenotypes of $B$. cinerea is important to the development of preharvest fungicide programs for control of postharvest gray mold. Baseline sensitivity to fluopyram, and sensitivities to boscalid, cyprodinil, fenhexamid, fludioxonil, and pyraclostrobin were determined on agar media for 80 and 212 
B. cinerea isolates collected from vineyards at various locations in the region, respectively. All isolates were sensitive to fluopyram with $\mathrm{EC}_{50}$ values ranging from 0.001 to $0.054 \mu \mathrm{g} / \mathrm{mL}$. Seven fungicide-resistant phenotypes were detected; $85.0,23.1,13.7$, and $94.8 \%$ of the isolates were resistant to boscalid, cyprodinil, fenhexamid, and pyraclostrobin, respectively. All isolates were sensitive to fludioxonil. Frequencies of resistance to two, three or four fungicides were 56.1, 23.6, and 6.1\%, respectively. Most fungicides failed to control gray mold on detached table grapes inoculated with respective fungicide-resistant phenotypes. Our results suggest that alternation of different classes of fungicides will be needed to control postharvest gray mold and that fluopyram could be an effective fungicide integrated into a pre-harvest fungicide program for control of gray mold in table grapes.

Use of scanning electron microscopy to visualize how oxathiapiprolin affects the initial infection process of potato leaves by Phytophthora infestans S. E. SALAS (1), C. P. Shepherd (2), N. M. Donofrio (3), D. H. Powell (4) (1) DuPont Crop Protection / University of Delaware, Newark, DE, U.S.A.; (2) DuPont Crop Protection, Newark, DE, U.S.A.; (3) College of Agriculture and Natural Resources, Plant and Soil Sciences, University of Delaware, Newark, DE, U.S.A.; (4) DBI BioImaging Center, University of Delaware, Newark, DE, U.S.A.

Phytopathology 105(Suppl. 4):S4.122

Oxathiapiprolin is a novel fungicide recently discovered by DuPont and the first member of a new class of fungicides (thiazolyl- piperidine- isoxazolines) with a unique target site in Oomycetes. In-vitro and whole plant studies have shown oxathiapiprolin to affect multiple stages in the life cycle of Phytophthora infestans, the causal agent of late blight. Scanning electron microscopy (SEM) was used to visualize the impact of oxathiapiprolin on initial infection using treated and untreated potato leaves inoculated with a $P$. infestans sporangial suspension. Two inoculation methods were tested. In Method 1, potato plants were inoculated using a hand held applicator, placed in a dew chamber at $15^{\circ} \mathrm{C}$ for $3 \mathrm{~h}$ and dried at $20^{\circ} \mathrm{C}$ for $2 \mathrm{~h}$. In new Method 2, developed for the study, potato leaf discs in a Petri dish containing water-agar were inoculated with an air-assisted mist applicator and incubated at $15^{\circ} \mathrm{C}$ for $5 \mathrm{~h}$. The first method failed, resulting in collapsed spores on the untreated leaves. The new inoculation method proved successful resulting in welldefined, hydrated sporangia and zoospores on the untreated leaves. SEM observations using the new method confirmed inhibition of sporangial germination, zoospore release, and zoospore germination on oxathiapiprolin treated leaves. Oxathiapiprolin (DuPont global trademark name Zorvec ${ }^{\mathrm{TM}}$ ) is highly effective against Oomycete pathogens while its new mode of action makes it a valuable tool for resistance management.

Spike colonization, fungal spread and deoxynivalenol accumulation in Fusarium head blight susceptible and resistant wheat cultivars

J. D. SALGADO (1), L. V. Madden (2), P. A. Paul (2)

(1) The Ohio State University, Wooster, OH, U.S.A.; (2) The Ohio State University, Wooster, OH, U.S.A.

Phytopathology 105(Suppl. 4):S4.122

Resistance to Fusarium head blight (FHB) in wheat does not always parallel resistance to deoxynivalenol (DON) accumulation. Under highly favorable weather conditions, FHB resistant cultivars may accumulate DON at levels comparable to that of susceptible cultivars. The goal of this study was to better understand and quantify spike colonization, fungal growth and DON spread in spikes of resistant (Truman) and susceptible (Cooper) cultivars grown under controlled conditions. Spikes were point-inoculated and then separate groups were incubated in growth chambers at one of three temperatures $(15,20$ and $25^{\circ} \mathrm{C}$ ) and high relative humidity ( $\mathrm{RH}>90 \%$ ) for 3 to 21 days after inoculation. Visual symptoms, fungal growth, and DON were quantified on spikelets at regular intervals above and below the point of inoculation. Mean FHB severity (0 to $95 \%$ ) increased with increasing temperature and moisture duration. Beyond the point of inoculation, DON was detected earlier (6 days) at higher temperatures $\left(20\right.$ and $\left.25^{\circ} \mathrm{C}\right)$ than at $15^{\circ} \mathrm{C}(9$ to 12 days, for Cooper and Truman, respectively). At all temperatures, DON content and fungal spread from the point of inoculation tended to be greater for the susceptible than the resistant cultivar during the first 15 days of high $\mathrm{RH}$. However, comparable spread was observed in the two cultivars at 20 and $25^{\circ} \mathrm{C}$ and more than 15 days of exposure to high RH. After 21 days at high RH, DON had spread throughout the spike in both cultivars.

Emergence of the impatiens downy mildew epidemics in the U.S. corresponded with a shift in pathogen population structure

C. SALGADO-SALAZAR (1), Y. Rivera (1), J. A. Crouch (2)

(1) USDA-ARS, Rutgers University, Beltsville, MD, U.S.A.; (2) USDA-ARS, Beltsville, MD, U.S.A.

Phytopathology 105(Suppl. 4):S4.122
Plasmopara obducens is the newly emergent oomycete responsible for impatiens downy mildew disease (IDM). Since outbreaks began in 2004, IDM has almost eliminated U.S. impatiens production. In this study, we evaluated if there was a change in $P$. obducens population structure associated with the epidemic. We generated a $199-\mathrm{Mb}$ genome assembly for $P$. obducens $(94 \%$ of the non-repetitive genome), and identified 4,531 microsatellite motifs. We genotyped 148 pre- and post-epidemic $P$. obducens samples using 37 microsatellite markers. Population structure analysis identified six populations (P1-P6). Populations P1-P4 shared an identical rDNA profile, but populations P5 and P6 were divided between four rDNA groups. Prior to IDM epidemics, $\mathrm{P} 1$ and P2 dominated, existing as pure P1 or P2 genotypes $(\sim 65 \%)$, or moderately admixed P1/P2. The first epidemic IDM specimen collected in 2004 from TN showed an admixed microsatellite genotype: P1/P2/P5 (30:50:20). All post-epidemic samples clustered in populations P3-P6, with $50 \%$ showing admixed genotypes. In post-epidemic P3-P6 populations, admixture with P1 and P2 allelic profiles was uncommon. These data show that the recent emergence of IDM in the U.S. corresponded with a major shift in $P$. obducens populations, with endemic P1/P2 genotypes supplanted by novel genotypes. The presence of admixed individuals indicates gene flow between $P$. obducens populations, which may present difficulties for durable disease control implementation.

Transmission of root-knot nematodes, Meloidogyne hapla and Meloidogyne incognita, to tomato by Helix aspersa

K. R. SANCHEZ (1), E. P. Caswell-Chen (2)

(1) Univ of California, Davis, CA, U.S.A.; (2) University of California, Davis, CA, U.S.A.

Phytopathology 105(Suppl. 4):S4.122

Helix aspersa is a pest in California agriculture and nursery settings. Here we report that second-stage juveniles (J2s) of the root-knot nematode species Meloidogyne hapla and M. incognita were consumed by $H$. aspersa and emerged from snail fecal pellets to successfully infect and reproduce on tomato host plants. Individual carrot discs $(1.75 \mathrm{~g})$ were infested with an aqueous suspension of $1000 \mathrm{~J} 2 \mathrm{~s}$ of the two nematode species. The fecal pellets from snails fed a single infested carrot disc were collected on each of 5 days subsequent to snail feeding, and placed at the base of individual tomato seedlings held in growth chambers. After 2 months the tomato plants showed infection with galled roots, egg masses, and substantial nematode reproduction with the ratio of final J2s from roots to initial J2s fed to snails (i.e., $\mathrm{P}_{\mathrm{f}} / \mathrm{P}_{\mathrm{i}}$ ) of 3.5 and 9.1 for $M$. hapla and M. incognita respectively. Tomato shoot weights were significantly reduced by infection with both $M$. hapla and M. incognita. Helix aspersa ingested root-knot nematode J2s and those J2s survived ingestion and passage through the alimentary canal, deposition in fecal pellets, emergence from pellets, and were capable of moving into the soil and successfully locating roots, infecting, and reproducing. The passive, phoretic dispersal of plant-parasitic nematodes through endozoochory in $H$. aspersa may represent a significant, unrecognized means of nematode invasion and colonization within agricultural fields.

Integration of a spring-planted mustard cover crop and mustard seed meal for control of Verticillium wilt in chile pepper

S. SANOGO (1), P. Lujan (1), R. Rudolph (1), M. Uchanski (1), S. Walker (1), E. Tahtamouni (1)

(1) New Mexico State Univ, Las Cruces, NM, U.S.A.

Phytopathology 105(Suppl. 4):S4.122

Mustard (Brassica spp.) cover crops are beneficial in improving soil properties. However, using mustard cover crops as green manure may be challenging because excellent stand establishment is essential to obtain sufficient biomass at the optimum growth stage for incorporation into the soil. Mustard seed meals may be used to complement or remedy failure of mustard cover crops, and offer flexibility to producers in terms of amount and timing of application. Combining the use of mustard cover crops (MCC) and mustard seed meals (MSM) has potential to reduce soilborne diseases. This study was conducted in a field with history of Verticillium wilt. Seed of 'Caliente 199' (Brassica juncea), a high-performing mustard cover crop was planted in early spring on February 17, 2014. Aboveground biomass of the mustard cover crop was $2.55 \mathrm{~kg} / \mathrm{m}^{2}$. Eight weeks after planting, the cover crop was disked and incorporated into soil. Mustard seed meal was then applied with a manure spreader at two rates $(0,1265$, and $1680 \mathrm{~kg} / \mathrm{ha})$ before seed beds were shaped. The green chile cultivar, AZ-1904 (Capsicum annuum) was direct seeded three weeks later. Disease assessment was conducted on 21 August 2014. Average disease incidence was between 20 and $30 \%$ across the three treatments. MCC and MSM may serve as viable components of soil and disease management in a chile rotation system. 
Reduction in the population of Phytophthora capsici and disease severity in chile pepper by extracts from pecan shell and husk tissues

S. SANOGO (1), P. Lujan (1), J. Idowu (2)

(1) New Mexico State Univ, Las Cruces, NM, U.S.A.; (2) New Mexico State University, Las Cruces, NM, U.S.A.

Phytopathology 105(Suppl. 4):S4.123

Tissues of pecan (Carya illinoensis) have been shown to contain fungitoxic substances. This study assessed the effects of extracts from pecan shell and husk on the production of sporangia and chile pepper infection by Phytophthora capsici, which attacks several vegetable crops worldwide. Aqueous extracts of 5, 10, 15 and $20 \%$ were prepared from ground tissues. Mycelium plugs from a 5-7 day-old V8 culture of $P$. capsici were placed in filter-sterilized extracts, and incubated at $26^{\circ} \mathrm{C}$ in the dark for $72 \mathrm{~h}$. Control plugs were placed in sterile distilled water. Zoospores of $P$. capsici were added to extracts, and placed on a rotary shaker. At 24,48 and $72 \mathrm{~h}$ after incubation, an aliquot of each extract was plated on a medium selective for $P$. capsici to assess the population of the pathogen. Sporangia production was less in pecan husk than in pecan shell extract. Population of $P$. capsici was significantly reduced in pecan tissue extracts at $72 \mathrm{~h}$ after incubation. In growth chamber studies, seedlings of a chile pepper cultivar susceptible to $P$. capsici were subirrigated with infested tissue extracts. Disease severity was significantly reduced when chile plants were subirrigated with infested extracts compared to plants subirrigated with infested water. Results indicate that pecan shell and husk tissues may be used in the treatment of irrigation water to reduce the activity of $P$. capsici in chile pepper.

Parametrization of evolutionary mechanisms of Ralstonia solanacearum in Brazil using genealogies and the coalescent approach

T. R. SANTIAGO (1), G. Caetano-Anolles (2), C. A. Lopes (3), E. Mizubuti (4)

(1) Universidade Federal de Viçosa, Viçosa, Brazil; (2) University of Illinois, Urbana, IL, U.S.A.; (3) Embrapa Hortaliças, Gama, U.S.A.; (4) Univ Federal De Vicosa, Vicosa, Brazil

Phytopathology 105(Suppl. 4):S4.123

Parametrization of evolutionary processes that affect populations of plant pathogenic bacteria can be useful to understand adaptation, mainly host adaptation, and ultimately to seek effective management strategies. Ralstonia solanacearum (Rs) causes bacterial wilt, a disease that affects several plant species and that is particularly severe to many crops in the tropics. Four phylotypes of Rs are characterized and their origins are geographically related. Phylotype II is an "ancestral lineage" originated in Brazil, but its evolutionary history has not been thoroughly investigated. The genealogies of 7 genes (5 in the chromosome and 2 in the megaplasmid) of 189 Brazilian isolates of Rs were reconstructed to study its evolutionary history. Four lineages were identified using a nonparametric multivariate method. The estimated mutation rate varied from $10^{-5}$ to $10^{-6}$. Mutation was more important than recombination in the Brazilian population of Rs. Population expansion was detected for phylotype IIA. Recent introduction of phylotype I in Brazil was also identified. We anticipate that the development of resistant cultivars to bacterial wilt will be a major challenge in all host crops.

\section{What is flag smut of wheat?}

K. G. SAVCHENKO (1), L. M. Carris (1), J. Demers (2), L. A. Castlebury (3) (1) Department of Plant Pathology, Washington State University, Pullman, WA, U.S.A.; (2) Systematic Mycology \& Microbiology Laboratory, USDAARS, Beltsville, MD, U.S.A.; (3) Systematic Mycology and Microbiology Laboratory, USDA-ARS, Beltsville, MD, U.S.A.

Phytopathology 105(Suppl. 4):S4.123

Urocystis agropyri, causal agent of flag smut of wheat, is a quarantineregulated pathogen of wild and cultivated grasses with a wide host range and worldwide distribution. Currently U. agropyri is subject to strict quarantine regulations in countries that prohibit the entry of wheat from any region where the pathogen is known to occur. The fungus was first described from Elymus repens; however, using a morphological concept G.W. Fischer in 1953 placed a large number of species in synonymy with $U$. agropyri, including $U$. tritici from wheat. The present study is a first attempt to clarify the taxonomy and phylogeny of flag smut of grasses using molecular analyses. Internal transcribed spacer rDNA and translation elongation factor 1 alpha loci were amplified from 22 fresh and herbarium specimens of infected Alopecurus, Beckmannia, Bromus, Calamagrostis, Elymus, Lolium, Phleum, Secale and Triticum. Results indicate that there are several distinct lineages of flag smut, with $U$. hispanica from Aegilops being the closest relative of flag smut of wheat. The specimens from Elymus repens grouped in a clade distinct from the specimens from wheat and rye. Recognition that $U$. tritici is genetically distinct from $U$. agropyri s. str. will impact regulatory policy and facilitate the development of diagnostic tests.
Use of Trichoderma harzianum Th2 to control seedborne pathogens on barley and wheat

M. M. SCANDIANI (1), J. Zapiola (2), A. Faura (3), L. Gasoni (2), A. Luque (4), G. Gonzalez Anta (3)

(1) Rizobacter Argentina S.A., Pergamino, Argentina; (2) Instituto de Microbiología y Zoología Agrícola, Hurlingham, Argentina; (3) Rizobacter, Pergamino, Argentina; (4) Centro de Referencia de Micología, Rosario, Argentina

Phytopathology 105(Suppl. 4):S4.123

Barley and wheat represent the most important alternatives for agriculture in winter in Argentina. Bipolaris sorokiniana (Bs), Drechslera spp. and Fusarium graminearum $(\mathrm{Fg})$ survive in seeds and cause foliar or root diseases. Trichoderma spp. has been widely used to control diseases caused by soilborne pathogens. In the present study, the previously selected strain of Trichoderma harzianum, Th2, was applied as seed treatment on barley and wheat, to control fungi in seeds. Naturaly infested barley and wheat seed samples were treated with Th2. An untreated check and a chemical check, treated with difenoconazole+mefenoxam (Compinche) were included. A total of 6 samples of each crop had been placed on blotters and incubated using the deep freezing method. The volatile and non-volatile metabolites were determined for the interaction Th2 with $\mathrm{Bs}, \mathrm{Fg}$ and Drechslera tritici-repentis $(D t r)$ in vitro tests. Th2 significantly reduced the incidence of Bipolaris sorokiniana, Drechslera spp., Alternaria spp., and Fusarium spp., compared to the untreated check. Strong inhibition of Dtr and Bs was observed with non-volatile metabolites. The seed treatment with $T$. harzianum $\mathrm{Th} 2 \mathrm{can}$ be an effective tool to control seedborne pathogens on barley and wheat.

\section{In vitro antagonism of Trichoderma species against Fusarium tucumaniae} and Fusarium virguliforme

M. M. SCANDIANI (1), A. I. Faura (2), W. Vargas (3), A. Luque (4), G. Gonzalez Anta (2)

(1) Rizobacter Argentina S.A., Pergamino, Argentina; (2) Rizobacter, Pergamino, Argentina; (3) Centro de Estudios Fotosintéticos y Bioquimicos, Rosario, Argentina; (4) Centro de Referencia de Micología, Rosario, Argentina

Phytopathology 105(Suppl. 4):S4.123

Soybean is the most important crop in Argentina, and it can be severely affected by different sudden death syndrome Fusarium causing species. The dominant specie is $F$. tucumaniae $(\mathrm{Ft})$, followed by $F$. virguliforme ( $\mathrm{Fv})$. These pathogens infect soybean roots early after planting and seed treatments with fungicides or antagonistic organisms would be a good tool for an integrated disease management. To determine the potential antagonistic variation of isolates of Trichoderma, primarily $T$. koningiopsis and $T$. harzianum, were compared for the control of $\mathrm{Ft}$ and $\mathrm{Fv}$. In vitro paired cultures of 27 isolates of Trichoderma were started simultaneously with $\mathrm{Ft}$ and $\mathrm{Fv}$ on PDA. The cultures were incubated at $22^{\circ} \mathrm{C}, 12 \mathrm{~h}$ fluorescent light$12 \mathrm{~h}$ darkness, during 15 days, and were scored for antagonism at 7 and 15 days, on a 1-5 scale, where 1: Trichoderma completely overgrew the pathogen and covered the entire medium surface, and 5: the pathogen completly overgrew the Trichoderma and ocuppied the plate surface. With Ft, 9 isolates of Trichoderma rated class 1 . Of those 9, only 3 were highly antagonistic to the isolate of Fv. Both SDS Fusarium causing species developed inhibition zones against some strains of Trichoderma. The results show the potential SDS biocontrol by native soil Trichoderma spp.

Management of powdery mildew on wild mint within protected lands G. T. SCHIERMAN (1), C. Y. Kadooka (2), J. Uchida (2), C. Smith (2)

(1) PEPS, Honolulu, HI, U.S.A.; (2) University of Hawaii at Manoa, Honolulu, HI, U.S.A.

Phytopathology 105(Suppl. 4):S4.123

The Hawaiian Islands is an isolated oceanic archipelago with numerous climate zones, and abundant microclimates. Habitats within these isolated environments contain species that have evolved with unparalleled diversity. Because of Hawaii's remarkable adaptable radiation, approximately $90 \%$ plants are endemic. Hawaii has a rich ecology; however this pristine framework has been disrupted due to the introduction of invasive species and many native species now face extinction. A native Hawaiian mint (Phyllostegia) which once grew naturally at high elevations above the gulches of Hawaii's mountain ranges, is now only remains within protected forestry plots, and re-establish possibly due to infection by powdery mildew and attack from other pests. Disease control is difficult as there are few fungicides which are approved within natural forested areas. Furthermore, we do not want to introduce such compounds into relatively intact natural areas. Powdery mildew is highly prevalent in wild populations and has the ability to overwhelm its host at early stages of plant growth. Additional fungal plant pathogens such as Fusarium have been isolated from dying mint, and these 
may pose an even greater threat to wild populations. Beneficial fungi, such as Trichoderma have also been isolated and cultured. In laboratory tests, Trichoderma has provided enhanced vigor to propagated plants, and has the potential to hasten plant development during highly susceptible stages.

\section{Cacao black pod and wilt control research}

G. SCHIERMAN (1), J. Uchida (2), C. Y. Kadooka (2)

(1) PEPS, Honolulu, HI, U.S.A.; (2) University of Hawaii at Manoa, Honolulu, HI, U.S.A.

Phytopathology 105(Suppl. 4):S4.124

Cacao (Theobroma cacao) grows within a limited geographical climate and Hawai' $i$ is the only state currently producing cacao commercially. In Hawai' $i$ cacao production is growing, with farms located on Oahu, Maui, Kauai and the Big Island with both large and small operations. Cacao black pod and tree wilt are severe fungal pathogens and have recently been found on mature trees and are infecting over $70 \%$ of some fields. At a large farm in Waialua on the island of Oahu, several hundred acres of cacao are under production, and at each plot tree diseases have become major problems. Farm managers have requested help to identify the cause of the dieback, as the problem has increased within the recent years. Trees with wilt symptoms exhibited blackening of immature pods that shrivel and prematurely drop. Pods that remain on the tree are often malformed and contain small misshaped seeds. On infected trees branches that are affected lose leaves, with complete defoliation as the disease progresses. In this experiment a survey will be conducted to collect possible fungal pathogen throughout the State. Samples of diseased pods and branches will be collected for pathogen isolation and pathogenicity tests will be conducted on young cacao seedlings at the University of Hawaii at Manoa. In early collections, Lasiodiplodia, Phoma, Phomopsis, colletotrichum, and Fusarium have been isolated. Pathogenicity tests will be done and disease management options will be developed.

Osmoprotectants and carriers for formulating co-cultures of Gramnegative biocontrol agents active against potato dry rot in storage

D. SCHISLER (1), P. Slininger (1), N. Olsen (2), L. Woodell (2)

(1) USDA ARS MWA NCAUR, Peoria, IL, U.S.A.; (2) University of Idaho, Kimberly, ID, U.S.A.

Phytopathology 105(Suppl. 4):S4.124

Pseudomonas fluorescens strains S11:P:12, P22:Y:05, and S22:T:04 suppress four important storage potato maladies; dry rot, late blight, pink rot, and sprouting. When grown as a three-strain co-culture, the efficacy and consistency of the strains are enhanced over blends of individually cultured strains. A dry formulation of these co-cultured strains is needed to develop a commercial biocontrol product. After $24 \mathrm{~h}$ of cell growth in a liquid medium and resuspension in a buffered solution, the survival of each component strain at 1 and 7 days after drying was enhanced by the presence of trehalose and fructose $(\mathrm{P}<0.05$, FPLSD) and S11:P:12 cell survival was reduced compared to the other strains of the co-culture. Trehalose and fructose formulated cocultures reduced dry rot by $74 \%$ and $25 \%$, respectively. Cells survived drying better combined with the diatomaceous earth Kenite 700 compared to perlite $(\mathrm{P}<0.05)$ though populations of S11:P:12 and P22:Y:05 were less than S22:T:04. All treatment combinations of carrier and osmolyte reduced dry rot by 65 $83 \%$ when applied to tubers seven days after co-cultured cells were dried. Results of a small pilot-scale trial testing dried co-cultured biocontrol products for efficacy in reducing dry rot on potatoes in storage will also be reported.

A smartphone app for plant disease and fungicide resistance management G. SCHNABEL (1), M. J. Hu (1), G. Edison (1), R. Pargas (1)

(1) Clemson University, Clemson, SC, U.S.A.

Phytopathology 105(Suppl. 4):S4.124

We developed a new smartphone application, MyIPM, to promote Integrated Disease Management for sustained peach and strawberry production in the southern United States. The app is available in the Google Play Store and Apple Store. It features about a dozen of the most important diseases of the two fruit crops. For each disease there are pictures of signs and symptoms, descriptions of the causal agent, and a 2-min audio from the regional specialist. The app features chemical and biological control options, including a list of registered active ingredients for each disease that are sortable by FRAC codes and southeastern spray guide-published efficacy. The app also features field EIQ values as published by the Cornell IPM Program. The active ingredients are linked to registered trade names. MyIPM also features some audio recordings from regional specialists on peach and strawberry IPM issues. Our vision is that this app provides a valuable tool for growers and specialists alike that supplements current spray guides. The unique display of active ingredients, color-coded by chemical classes, provides a useful tool to promote resistance management. MyIPM is fed by an external database that can be updated through an authoring tool and is free of charge. It is expandable to more crops and could, with minor programming modifications, also be useful for entomologists.

Insect E-probe Diagnostic Nucleic acid Analysis (EDNA): A novel bioinformatic tool for detection of vectors and pathogens in insect trap metagenomes

W. SCHNEIDER (1), A. Stone (2), D. Sherman (2), M. Malapi-Wight (3), J. Crouch (3), S. Andreason (4), J. Daniels (4), A. Wayadande (4), F. OchoaCorona (4)

(1) USDA ARS FDWSRU, Ft. Detrick, MD, U.S.A.; (2) USDA ARS FDWSRU, Ft Detrick, MD, U.S.A.; (3) USDA ARS SMML, Beltsville, MD, U.S.A.; (4) Oklahoma State University, Stillwater, OK, U.S.A.

Phytopathology 105(Suppl. 4):S4.124

Plant pathogen detection takes many forms. In simple cases, researchers are attempting to detect a known pathogen from a known host utilizing targeted nucleic acid or antigenic assays. However, in more complex scenarios researchers may not know the identity of a pathogen, or they may need to screen for a wide array of pathogens from a single sample. Metagenomics represents a possible broad range diagnostic approach, with next generation sequencing generating a comprehensive profile of all organisms in a given nucleic acid sample. However, the vast amount of data gathered makes metagenomic analysis computationally problematic. EDNA is a bioinformatic tool that greatly reduces the amount of computational work in metagenomic data diagnoses. A prime target for EDNA may be insect traps, which represent diverse samples that may include vectors of a wide array of viral and bacterial pathogens. Currently insects from traps are analyzed morphologically and tested for related pathogens individually. To test if this process could be simplified, EDNA was applied to the detection of vectors and plant pathogens in individual insect and simulated trap samples. In proof of concept experiments EDNA identified insect vectors from 454 data, while vectors plus bacterial pathogens and viral pathogens were identified using the Illumina platform. These data indicate that EDNA analysis of metagenomes may provide a viable means for screening insect trap samples for vectors and pathogens.

Potential quail consumption of aflatoxin through contaminated deer corn G. SCHUSTER (1), B. Newman (2), S. Henke (2), A. Fedynich (2)

(1) Texas A\&M University - Kingsville, Texas A\&M AgriLife Extension, Kingsville, TX, U.S.A.; (2) Caesar Kleberg Wildlife Research Institute, Texas A\&M University-Kingsville, Kingsville, TX, U.S.A.

Phytopathology 105(Suppl. 4):S4.124

Mycotoxins are secondary metabolites produced by certain molds that are capable of causing diseases called mycotoxicosis, leading to death in avian wildlife. Deer corn is commonly fed to supplement avian wildlife and usually has 5-100 ppm fumonsin and $<50 \mathrm{ppb}$ of aflatoxin. Short-term, acute doses of aflatoxin of $100 \mathrm{ppb}$ can be detrimental Northern Bobwhites (Colinus virginianus) health. This project was initiated to determine how storage conditions impact mycotoxin concentrations in supplemental feed in South Texas. Treatments included deer corn in open air, trailer, pavilion, steel container, aluminum container, and plastic container mimicking normal storage and feeder type structures. Aflatoxin concentrations increased significantly after 8 weeks of exposure. Aflatoxin levels were twice the lethal concentration $(>100 \mathrm{ppb})$ when stored in enclosed trailer. The steel containers reached aflatoxin levels of $150 \mathrm{ppb}$, almost 3 times the original concentration. This is of concern since most wildlife feeders are made of steel or aluminum. Aflatoxin concentration was higher within the perimeter of the containers than the central core, due to condensation. Our data suggest this storage method can influence exposure risk to bobwhites.

Molecular differentiation of Claviceps isolates from Kentucky bluegrass and perennial ryegrass in Oregon and Washington

J. C. Scott (1), N. Kaur (2), S. C. Alderman (3), D. L. Walenta (4), P. B. Hamm (2), K. E. Frost (2), J. K. S. DUNG (1)

(1) Oregon State University, Madras, OR, U.S.A.; (2) Oregon State University, Hermiston, OR, U.S.A.; (3) USDA-ARS NFSPRC, Corvallis, OR, U.S.A.; (4) Oregon State University, La Grande, OR, U.S.A.

Phytopathology 105(Suppl. 4):S4.124

Ergot, caused by the fungus Claviceps purpurea, is an economically important seed replacement disease of Kentucky bluegrass (Poa pratensis) and perennial ryegrass (Lolium perenne) seed crops. Recently, C. purpurea ecotypes G1, G2, and G3 were defined as three separate species: C. purpurea sensu stricto, C. humidiphila, and C. spartinae, respectively. A collection of 25 Claviceps isolates collected from $P$. pratensis and L. perenne in Oregon (OR) and Washington (WA) between 2010 and 2014 were characterized using RAPD patterns, ITS sequences, and mating type. Three isolates from $P$. pratensis and nine isolates from $L$. perenne collected in OR exhibited RAPD patterns corresponding to $C$. purpurea and ITS sequences grouped the isolates with ITS 
sequences from $C$. purpurea previously deposited in GenBank. Nine isolates from $P$. pratensis collected in WA produced RAPD patterns and ITS sequences corresponding to $C$. humidiphila; however, one isolate each from OR and WA exhibited RAPD patterns associated with $C$. humidiphila but ITS sequences grouped them with $C$. purpurea. One isolate each from $P$. pratensis and $L$. perenne produced RAPD patterns matching C. purpurea but formed their own subclade using ITS sequences. Mating type PCR assays detected the MAT1-11 gene in 18 isolates and the MAT1-2-1 gene in two isolates; five isolates possessed both genes. This is the first study to identify different Claviceps species infecting $P$. pratensis and L. perenne seed crops in OR and WA.

Genetics governing the differential response to boscalid of Microsphaeropsis tanaceti and Stagonosporopsis tanaceti

J. SCOTT (1), T. Pearce (1), S. Pilkington (1), F. Hay (1), C. Wilson (2)

(1) Tasmania Institute of Agriculture, Burnie, Australia; (2) Tasmania Institute of Agriculture, Hobart, Australia

Phytopathology 105(Suppl. 4):S4.125

Microsphaeropsis tanaceti, the cause of tan spot disease of pyrethrum (Tanacetum cineariifolium), is an emerging pathogen in Australian crops. Through its emergence, it has displaced Stagonosporopsis tanaceti, the cause of ray blight disease, as the dominant pathogen during the spring growing period. Screening of the fungicide response of these pathogen populations indicated that $M$. tanaceti has developed levels of resistance to the fungicide boscalid, while $S$. tanaceti has maintained susceptibility. This is despite both species being exposed to boscalid over an equal time period. Characterisation of the succinate dehydrogenase $(S d h)$ gene complex was conducted to examine the basis for the response profiles of the two species. Point mutations at codon 277 of the $S h d B$ gene were evident in the majority of boscalid-resistant $M$. tanaceti individuals. These mutations change the amino acid from histidine (susceptible) to arginine or tyrosine (resistant) in the resultant protein. Additionally, a smaller proportion of boscalid-resistant individuals had mutations resulting in amino acid translation changes at one of three codons in the $S h d C$, or at one codon in the $S h d D$ gene. Mutations in the $S h d$ gene complex corresponding with fungicide resistance were absent in the $S$. tanaceti individuals sequenced.

\section{Epidemiological conditions promoting Rhizopus soft rot and Fusarium} root rot of sweetpotato

A. C. SCRUGGS (1), L. M. Quesada-Ocampo (1)

(1) North Carolina State University, Raleigh, NC, U.S.A.

Phytopathology 105(Suppl. 4):S4.125

Sweetpotato production is limited by many postharvest diseases including Rhizopus soft rot (RSR) and Fusarium root rot (FRR). However, little is known about the epidemiological conditions that promote RSR and FRR. The effects of temperature $\left(13,23\right.$, and $\left.29^{\circ} \mathrm{C}\right)$, relative humidity $(75,85,95 \%)$, and inoculum level (3-, 5-, and 7-mm diameter mycelia plugs) on disease progression of RSR and FRR were investigated in controlled growth chamber studies. Six wounded, 'Covington' sweetpotatoes were inoculated with an actively growing mycelia plug of Rhizopus stolonifer, Fusarium solani, or F. proliferatum, and incubated in the dark at the desired temperature and humidity. Control roots were included and the experiment was repeated twice. Disease progression was recorded for RSR and FRR as percent decay and lesion diameter, respectively, for two weeks. Sporulation on infected roots was also recorded. Area under the disease progress curve (AUDPC) values were calculated and subjected to an ANOVA. Significant differences were observed for RSR and FRR across the temperature, humidity, and inoculum level treatments. Disease progression of RSR and FRR was consistently higher at $29^{\circ} \mathrm{C}, 95 \%$ humidity, and $7 \mathrm{~mm}$ inoculum level as compared to lower temperatures, humidities, and inoculum levels. These findings will contribute in providing accurate recommendations to growers so that environmental conditions in storage and curing facilities may be optimized to reduce postharvest diseases.

Etiology and management of Fusarium spp. causing cryptic cankers in cold-stored, bare-root propagated almond seedlings

A. J. SEIDLE (1), T. R. Gordon (2), R. M. Bostock (2)

(1) University of California, Davis, Sacramento, CA, U.S.A.; (2) University of California, Davis, Davis, CA, U.S.A.

Phytopathology 105(Suppl. 4):S4.125

From 1997-2001, a canker disease that developed during cold-storage of dormant bare-root trees or that emerged in the field soon after planting resulted in the loss of hundreds of thousands of stone fruit seedlings in California fruit and nut tree nurseries. Fusarium acuminatum and Fusarium avenaceum were identified as the primary causal agents. Mild desiccation stress leading to reduced bark turgidity correlated with significantly increased susceptibility of almond branches to F. acuminatum. In 2010-11, the disease reemerged in at least one nursery, resulting in substantial losses of cold-stored almond seedlings. The incidence of Fusarium spp. in the almond propagation system, DNA sequencing, and vegetative compatibility tests are being utilized to better understand the disease etiology and to identify potential sources of inoculum. Pathogenic Fusarium spp. have been isolated at every stage in the production system including non-symptomatic almond seedlings, wheat rotation cover crops and residues in nursery fields, cold storage facility air and surfaces, and nursery equipment. The effect of bark turgidity and seasonal changes in susceptibility of almond seedlings along with fungicide protection at critical stages of production are also being investigated. Understanding pathogen diversity, predisposing factors, and contributing cultural practices will help guide disease management.

First report of resistance to azoxystrobin in Phytophthora cactorum on strawberry

T. SEIJO (1), E. Zuchelli (2), N. A. Peres (3)

(1) Univ of Florida - GCREC IFAS, Wimauma, FL, U.S.A.; (2) Universidade de Passo Fundo, Passo Fundo, Brazil; (3) University of Florida, Wimauma, FL, U.S.A.

Phytopathology 105(Suppl. 4):S4.125

Phytophthora cactorum causes leather rot of fruit or Phytophthora crown rot (PhCR) in strawberry. Leather rot occurs sporadically, but can be severe during conducive weather conditions. Epidemics with up to $50 \%$ fruit loss have been reported. In Florida's annual strawberry winter production system, $\mathrm{PhCR}$ can be severe when transplants of a susceptible cultivar infected with $P$. cactorum are overhead irrigated for 10 days to promote establishment during warm weather in October. Mortality and stunting of $>50 \%$ of transplants can lead to entire fields being replanted. Both diseases are controlled with chemicals. Mefenoxam alternated with phosphites are used to control PhCR. Currently azoxystrobin is labelled for control of leather rot but not PhCR. $P$. cactorum isolates from both leather rot and PhCR were collected from multiple strawberry fields in Florida between 1997 and 2015. Isolates were tested for sensitivity to azoxystrobin at concentrations of $0,0.01,0.1,1.0,10$, and $50 \mathrm{mg} / \mathrm{L}$ amended with SHAM $(100 \mathrm{mg} / \mathrm{L})$. The isolates were separated into two groups, sensitive isolates, with $\mathrm{EC}_{50}$ values of less than $1.0 \mathrm{mg} / \mathrm{L}$, and resistant isolates all having $\mathrm{EC}_{50}$ values greater than $50 \mathrm{mg} / \mathrm{L}$. Resistance was found among both leather rot and PhCR isolates collected after 2010. This is the first report of resistance to azoxystrobin in P. cactorum from strawberries.

Identification of visual phenotypes by Tobacco rattle virus VIGS expression of a Nicotiana benthamiana cDNA library

E.-Y. SEO (1), J.-K. Kim (1), S. Lim (2), S.-H. Yu (1), J. Hammond (3), H.-S. $\operatorname{Lim}(1)$

(1) Chungnam National University, Daejeon, South Korea; (2) Korea Research Institute of Bioscience and Biotechnology and University of Science and Technology, Daejeon, South Korea; (3) United States Department of Agriculture - Agricultural Research Service, Beltsville, MD, U.S.A.

Phytopathology 105(Suppl. 4):S4.125

Virus-induced gene silencing (VIGS) is an efficient and rapid method to identify plant gene functions. One of the most widely used VIGS vectors is Tobacco rattle virus (TRV) which has been used successfully for RNA interference (RNAi) in N. benthamiana and tomato. We have modified a TRV VIGS vector to contain the Gateway system for high throughput cloning, and utilized this system to express a library of $N$. benthamiana cDNA. Random c.300 bp N. benthamiana cDNA fragments were generated by ultrasonication and inserted into the TRV VIGS vector by Gateway cloning. About 1000 plants of $N$. benthamiana were agroinfiltrated with randomly selected TRV cDNA constructs in Agrobacterium tumefaciens GV 2260. Distinct visible phenotypes were identified in eight of the inoculated $N$. benthamiana plants. Sequence analysis and BLAST searches of the eight inserted $N$. benthamiana gene fragments were carried out to identify the corresponding genes. Six fragments were matched with $N$. benthamiana EST data, and the remaining two fragments identified from $N$. tabacum sequences. A leaf yellowing phenotype was observed in plants infected with VIGS constructs Nos. 30 (CK296346.1), 39 (GO603828.1), 46 (GO608093.1), 75 (AM804954.1), and 85 (GO606553.1). Constructs 29 (CK290013.1), 33 (ES886892.1) and 60 (AM811216.1) induced leaf malformation and necrosis. Identification of the selected genes by VIGS will aid further analysis to determine the relationship between VIGS phenotype and gene function.

Chilling injury during imbibition of soybean seed increases incidence of damping-off caused by Pythium torulosum

M. SERRANO (1), A. Robertson (1)

(1) Iowa State University, Ames, IA, U.S.A.

Phytopathology 105(Suppl. 4):S4.125

Pythium sylvaticum and P. torulosum are Oomycetes which cause dampingoff of soybeans in Iowa. Periods of cool weather soon after planting are 
frequently associated with high incidence of damping-off. The relationship between chilling injury during seed imbibition and reduction in germination caused by Pythium spp. was evaluated using an in vitro assay. Seeds were imbibed in a solution of PEG 6000 or sterile water for 12 hours at $0^{\circ} \mathrm{C}$ or at room temperature $\left(20-22^{\circ} \mathrm{C}\right)$. After imbibition, seeds were placed in Petri dishes on 3 day-old cultures of either P. sylvaticum or P. torulosum, or in a non-inoculated control of dilute V8 agar. The plates were incubated for 7 days at room temperature. No seeds germinated in $P$. sylvaticum plates, indicating a higher virulence of this species, and no differences between imbibition treatments were detected $(\mathrm{P}>0.05)$. Seed germination on plates inoculated with $P$. torulosum was low when the seed was imbibed in water at $0{ }^{\circ} \mathrm{C}(30 \%$ germination) in comparison to seed imbibed in PEG at $0^{\circ} \mathrm{C}(86 \%$ germination) $(\mathrm{P}<0.05)$, suggesting chilling injury may predispose soybeans to damping-off caused by $P$. torulosum. These results indicates that chilling injury predisposes soybean to infection by some Pythium spp., and may explain why cool weather at planting increases the risk of damping off.

Fungicide use in maize: Results from the 2009 Midwestern crop management survey

D. A. Shah (1), P. D. Esker (2), C. A. Bradley (3), A. E. Robertson (4), S. P. Conley (5), P. A. PAUL (6)

(1) Kansas State University, Manhattan, KS, U.S.A.; (2) Universidad de Costa Rica, San José, Costa rica; (3) University of Illinois, Urbana-Champaign, IL, U.S.A.; (4) Iowa State University, Ames, IA, U.S.A.; (5) University of Wisconsin, Madison, WI, U.S.A.; (6) The Ohio State University, Wooster, OH, U.S.A.

Phytopathology 105(Suppl. 4):S4.126

Corn growers (CGs) and certified crop advisors (CCAs) in IA, IL, OH and WI were surveyed on their perceptions and practices regarding foliar fungicide use in maize from 2005 to 2009. Among CCAs $73 \%$ recommended spraying maize with foliar fungicides during 2005 to 2009 , whereas $35 \%$ of CGs sprayed maize with foliar fungicides during that period. The odds of foliar fungicide use by those aged 34 or younger were four times the odds of foliar fungicide use by those aged 55 or older. Foliar fungicide use was a novelty: $84 \%$ of CGs used them in maize production for the first time during 2005 to 2009. Among CCAs who recommended spraying maize, $98 \%$ did so for grain maize (as opposed to seed and silage maize), and of CGs who used foliar fungicides $94 \%$ sprayed maize for grain. Headline was the most popular product. Overall, $94 \%$ of CCAs reported seeing a yield gain in response to foliar fungicide application, substantially higher than the $65 \%$ of CGs who did. The most common yield gain reported by both CCAs and CGs was between 0.31 and 0.57 metric tons per hectare (5 to $9 \mathrm{bu} / \mathrm{acre}$ ). Overall, the survey results revealed that foliar fungicide use in maize grown in these four states was adopted quite rapidly during the 2005 to 2009 seasons, and that the practice was mainly seen as yield-enhancing.

Exploring the dark Side: Identification of candidate Ug99 effectors that interact with the barley $r$ pg $4 /$ Rpg 5 locus

R. SHARMA POUDEL (1), S. Solanki (1), R. Brueggeman (1)

(1) North Dakota State University, Fargo, ND, U.S.A.

Phytopathology 105(Suppl. 4):S4.126

Stem rust caused by the pathogen Puccinia graminis $f$. sp. tritici (Pgt) is an important disease of wheat and barley. The rye stem rust resistance gene Rpg5 was shown to confer resistance against all three formae specialis of $P$. graminis (wheat, rye and oat) and is effective against many prevalent wheat stem rust races, including the highly virulent race TTKSK (Ug99). Rpg5 has been cloned and partially characterized and the data suggest that rpg4/Rpg5mediated resistance is an early PAMP triggered immunity response that primes a later effector triggered immunity response. The protein structure of Rpg5 suggests that resistance is dependent on Rpg5 as the integrated decoy Rprotein, which is manipulated by a Pgt effector. This research focuses on identifying and understanding the various effectors produced by $P g t$ during the rpg4/Rpg 5 mediated defense response in barley. Genotype-by-sequencing is being utilized on 40 Pgt isolates, collected in ND since 1970, selected by phenotyping of barley differentials to identify isolates that are both virulent/avirulent to Rpgl and rpg4/Rpg5. Association mapping will be performed utilizing the phenotypic and genotypic data, and complimented with the Pgt genome sequence in order to identify candidate effectors that possibly target the integrated protein kinase decoy domain of Rpg5. The AM will be complemented with RNAseq data of rust isolates, QCCJ (Avrrpg4/Rpg5+) and HKHJ (AvrRpg1+) to help identify candidate effectors.

Latent virus survey in pome and stone fruit nurseries in Oregon D. Sharma Poudyal (1), S. Lane (1), J. Grant (1), N. OSTERBAUER (1)

(1) Oregon Department of Agriculture, Salem, OR, U.S.A.

Phytopathology 105(Suppl. 4):S4.126
Oregon is a major producer of certified virus-tested pome and stone fruit nursery plants. All plants in the program must be certified virus-free by a clean plant center before entering Oregon's program. The recent report of the latent virus Cherry capillovirus $A$ (CVA) in certified plants grown by the Clean Plant Center Northwest (CPCNW) prompted a survey of Oregon's certified plant material for this and other latent viruses (Apple chlorotic leafspot virus (ACLSV), Apple stem grooving virus (ASGV), and Apple stem pitting virus (ASPV). A total of 350 leaf samples of Malus, Prunus, Pyrus, and Cydonia were collected from 23 nurseries in Clackamas, Marion, Multnomah, Washington, and Yamhill counties. The samples were tested for ACLSV, ASGV, and ASPV using commercially available ELISA test kits with virus-specific antisera. All of the samples tested were free of ACLSV, ASGV, and ASPV. For CVA detection, 312 Prunus leaf samples were collected from 14 nurseries located in Clackamas, Multnomah, Washington, and Yamhill counties. The samples were tested with a RT-PCR assay that used CVA-specific primers. CVA was detected in 56 of the 312 samples, indicating this virus was present in $17.9 \%$ of the certified plants tested. Based on this survey, Oregon's certified Malus, Prunus, Pyrus, and Cydonia nursery plants are free of ACLSV, ASGV, and ASPV; however, CVA was detected in some Prunus nursery plants.

\section{High-throughput single seed PCR detection of Cephalosporium gramineum in wheat}

H. SHENG (1), K. L. Klos (2), Z. Sexton (1), T. D. Murray (1)

(1) Washington State University, Pullman, WA, U.S.A.; (2) USDA Small Grains and Potato Germplasm Research, Aberdeen, ID, U.S.A.

Phytopathology 105(Suppl. 4):S4.126

Cephalosporium stripe of wheat is caused by Cephalosporium gramineum. This fungus survives primarily in colonized residue, but is also transmitted by seed in low frequency. A high-throughput PCR method of detecting $C$. gramineum in single wheat seed was developed based on amplification of the ITS region of the rDNA. Field-grown seed of four winter wheat cultivars were collected from 16 inoculated plots over 3 years (48 total seed lots). Isolation of $C$. gramineum on a semi-selective medium was attempted from 2016 seeds from each lot. DNA of 384 seeds from the same lots was extracted individually in 96-deep well plates and PCR conducted on each seed. $C$. gramineum was isolated from 0 to $0.74 \%$ of the seed. Based on PCR, $C$. gramineum was detected in 1.6 to $7.6 \%$ of the seed. PCR products of 25 positive samples were sequenced and had 98 to $100 \%$ similarity with $C$. gramineum sequences in GenBank. The single-seed PCR assay improved the accuracy and speed of detecting C. gramineum in wheat seed compared with cultural methods and was about 10 times more sensitive. DNA of positive seed samples was subjected to real-time PCR to quantify C. gramineum. Based on 27 positive seeds, 24.9 to $52125.0 \mathrm{fg} / \mathrm{seed}$ of $C$. gramineum DNA was present in infected seed. A rapid and accurate real-time quantitative PCR will facilitate detection of $C$. gramineum in winter wheat seed lots and help to improve our understanding of seed transmission of Cephalosporium stripe.

Safeguarding agriculture through nomenclature support and incorporating standard scientific names in national regulatory and extension databases

B. B. SHEW (1), M. A. Cubeta (1), W. C. Allen (2), L. A. Castlebury (3), E. M. Luke (4), M. A. Hill (4), J. F. Hegarty (4), J. M. McKemy (5), M. K. Romberg (5), B. Randall-Schadel (6), N. Gregory (7)

(1) North Carolina State University, Raleigh, NC, U.S.A.; (2) North Carolina State University, Beltsville, MD, U.S.A.; (3) USDA ARS Systematic Mycology \& Microbiology Lab, Beltsville, MD, U.S.A.; (4) Purdue University, West Lafayette, IN, U.S.A.; (5) USDA ARS SMML, Beltsville, MD, U.S.A.; (6) USDA APHIS CPHST, Raleigh, NC, U.S.A.; (7) University of Delaware, Newark, DE, U.S.A.

Phytopathology 105(Suppl. 4):S4.126

The National Plant Diagnostic Network (NPDN), Federal identifiers and quarantine inspectors must be able to accurately identify and reference scientific names of microorganisms. The International Code of Nomenclature (ICN) now requires use of a single name for all species. However, two names, representing sexual and asexual stages, often are used for plant pathogenic fungi, causing confusion. Nomenclature is being investigated and conflicts resolved using references maintained by the USDA ARS Systematic Mycology and Microbiology Lab (SMML), the National Fungus Collection, Mycobank, and USDA APHIS PPQ National Identification Services and CPHST. The Center for Environmental and Regulatory Information Systems (CERIS), Purdue University, manages databases for NPDN and USDA APHIS. More than 3,000 names, including 200 plant pathogens, were reviewed for accuracy and updated in the NPDN and SMML databases. The SMML database was updated by more than 7,300 names and more than 1,500 entries were added or verified for newly described type specimens of fungal 
pathogens, voucher specimens from published first reports, and specimens intercepted at ports of entry. Updates are available through the National Fungus Collections website. Database design at CERIS ensures usability and commonality for all NPDN users. NPDN lab management systems can adapt their software to use the new naming conventions, and modifications allow for pest groupings and a large number of synonyms per pest.

Two amino acids of cassava novel cap-binding proteins critical for their interaction with Cassava brown streak virus VPg

S. SHI (1), M. A. Mandel (1), Z. Xiong (1)

(1) University of Arizona, Tucson, AZ, U.S.A.

Phytopathology 105(Suppl. 4):S4.127

Cassava brown streak disease is caused by two distinct species of RNA viruses: Cassava brown streak virus (CBSV) and Ugandan Cassava brown streak virus (UCBSV). Covalently linked to the 5'-terminus of both viral RNA genomes is a genome-linked viral protein ( $\mathrm{VPg}$ ), which is functionally analogous to the cap structure of mRNA and serves as the binding site for eukaryotic translation initiation factor 4E (eIF4E and eIF(iso)4E) during viral RNA translation. We have previously found two novel cap-binding proteins (nCBPs), cassava $013732 \mathrm{~m}$ and $015501 \mathrm{~m}$, to interact with CBSV and UCBSV VPgs positively in the yeast two-hybrid assay. Furthermore we found that the full-length nCBPs are essential for the eIF4E-VPg interaction. Homology-based protein structure modeling identified a number of candidate amino acids of nCBPs potentially involved in their interaction with the viral VPgs. Site-directed mutagenesis of selected amino acids showed that two amino acids, P99/S161 respectively in $013732 \mathrm{~m}$ and $\mathrm{P} 104 / \mathrm{S} 166$ in $015501 \mathrm{~m}$, are important for the nCBP-VPg interaction in the yeast two hybrid system. Bimolecular fluorescence complementation assay using split $\mathrm{YFP}^{\mathrm{N} 155}$ and $\mathrm{YFP}^{\mathrm{C} 86}$ fusion proteins confirmed the interaction between two cassava nCBPs and VPgs of CBSV and UCBSV, and the inability to interact with the viral VPgs in living plant cells by nCBP mutants with the single or double amino acid substitutions.

Local and regional distribution of banana Xanthomonas wilt in space and over time in Kagera, Tanzania

M. M. SHIMWELA (1), J. B. Jones (1), R. C. Ploetz (2), F. D. Beed (3), W. S. Lee (1), A. H. C. van Bruggen (4)

(1) University of Florida, Gainesville, FL, U.S.A.; (2) University of Florida, Homestead, FL, U.S.A.; (3) AVRDC, Bangkok, Thailand; (4) Univ of Florida, Gainesville, FL, U.S.A.

Phytopathology 105(Suppl. 4):S4.127

Banana is an important food and cash crop in the Kagera region of Tanzania. Banana Xanthomonas wilt (BXW) is an important factor in the production of this crop, as all cultivars are susceptible, and yield can be reduced to zero. BXW, first reported in Kagera in 2006, is caused by Xanthomonas campestris pv musacearum $(\mathrm{Xcm})$, which is spread by farm tools, infected planting materials and nectar feeding insect vectors. Cultural control practices have not prevented further spread. Disease survey data collected between 2007 and 2011 were used for spatiotemporal cluster analyses of BXW in ArcGIS and SAS. The relationship between BXW clusters and environmental measures was examined using bivariate correlations and Mann-Whitney U tests. Rapid disease progress was observed and statistically described over the 5-year period. Significant BXW clusters $(\mathrm{Z}>1.96)$ expanded consistently in Muleba and Bukoba districts. Weak negative correlations were obtained between the normalized difference vegetation index (NDVI), temperature, elevation and BXW clusters. NDVI, precipitation and temperatures were significantly different between high and low incidence clusters. BXW clusters were positively affected by temperature and precipitation but not by elevation (M$\mathrm{W}$ test). These findings suggest that BXW spreads rapidly over short distances and occurred at high incidences in high rainfall zones.

Survival of microsclerotia of two species of boxwood blight pathogen when exposed to sanitizers

N. SHISHKOFF (1)

(1) USDA ARS FDWSRU, Frederick, MD, U.S.A.

Phytopathology 105(Suppl. 4):S4.127

Boxwood blight is caused by two species of Calonectria in Europe, $C$. pseudonaviculata (Cps) and C. henricotiae (Che). Only Cps is known in the US, but preparations need to be made for introduction of this second species. Three sterilants previously tested on Cps were used to compare efficacy against microsclerotia of both species. Two isolates of each species were exposed to $10 \%$ bleach, $70 \%$ ethanol, and Zerotol at 1:50 v/v. Microsclerotia (ms) were produced on cellulose sheets covering the surface of glucose-yeast extract-tyrosine agar plates, detached, added to water and run through a series of nested sieves to capture $\mathrm{ms}$ in the 120-350 um range. These were added to mesh bags and dipped in treatment solutions or water for $0,5,10$, or $15 \mathrm{~min}$, rinsed, and plated on media. All treated ms had reduced survival compared to water controls. Ethanol killed microsclerotia of both species within $5 \mathrm{~min}$, but in Zerotol and bleach treatments, ms of Che were showed significantly lower survival than those of Cps after $15 \mathrm{~min}$ (11-24\% and $81-90 \%$, respectively). Further tests separating different size categories of ms and exposing them to Zerotol suggested the difference was not completely related to size of ms.

Anaerobic soil disinfestation amendment carbon rate affects germination and parasitism of sclerotia of Sclerotium rolfsii

U. SHRESTHA (1), M. M. Dee (1), B. H. Ownley (1), D. M. Butler (1)

(1) University of Tennessee, Knoxville, TN, U.S.A.

Phytopathology 105(Suppl. 4):S4.127

The effect of anaerobic soil disinfestation (ASD) amendment rates of 2, 4, 6 and $8 \mathrm{mg} \mathrm{C} / \mathrm{g}$ soil (C:N ratio 30:1) on sclerotia of Sclerotium rolfsii was investigated in a replicated pot study. Two polyethylene mesh bags with 10 sclerotia were buried at 5 to $10 \mathrm{~cm}$ depth in amended pots containing field soil and sand. Pots were irrigated to saturation, covered with black plastic to generate anaerobic conditions, and incubated at 15 to $25^{\circ} \mathrm{C}$. Two unamended, untreated controls, one with plastic and one without, were included. Soil pH and cumulative anaerobic activity (i.e., redox potential) were assessed. After 3 weeks, sclerotia bags were retrieved and grown on antibiotic-amended PDA at room temperature to assess sclerotial germination and Trichoderma parasitism. Sclerotial germination, Trichoderma parasitism and anaerobic activity were strongly correlated $(P<0.001)$. Across different $\mathrm{C}$ rates $(2$ to 8 $\mathrm{mg} \mathrm{C} / \mathrm{g}$ ), there was no difference in anaerobic activity; however, it was higher than both controls. All C rates above $2 \mathrm{mg} \mathrm{C} / \mathrm{g}$ significantly suppressed $S$. rolfsii; sclerotial germination was lowest at 4 and $6 \mathrm{mg} \mathrm{C} / \mathrm{g}$ soil (4 to $7 \%)$ and highest in controls (62 to 99\%). C rate influenced Trichoderma parasitism of sclerotia and was greatest at 2 and $4 \mathrm{mg} \mathrm{C} / \mathrm{g}$ soil (90 to $99 \%$ ) and lowest for controls (4 to $10 \%)(P<0.001)$. Results suggest that ASD using $\mathrm{C}$ amendment application rates of at least $4 \mathrm{mg} \mathrm{C} / \mathrm{g}$ soil reduces sclerotia germination and enhances beneficial Trichoderma parasitism.

Identification of genomic variants of rice associated with disease resistance to sheath blight and bacterial panicle blight through NGS sequencing

B. K. Shrestha (1), D. H. Oh (2), M. Dassanayake (3), J. H. HAM (1)

(1) Louisiana State Univ Agric Ctr, Baton Rouge, LA, U.S.A.; (2) Louisiana State University, Baton Rouge, LA, U.S.A.; (3) Louisiana State University, Baton Rouge, LA, U.S.A.

Phytopathology 105(Suppl. 4):S4.127

Sheath blight and bacterial panicle blight cause severe yield reductions of rice in many rice-growing regions around the world. Vertical resistance has not been found for these rice diseases, unlike blast or bacterial leaf blight, and only a few rice cultivars and lines show certain levels of quantitative resistance to these diseases. Jupiter, a medium-grain cultivar, and LM-1, a mutant line derived from the disease-susceptible cultivar Lemont, have been shown high levels of quantitative resistance to sheath blight and bacterial panicle blight and used as genetic resources for breeding of disease-resistant rice in LSU AgCenter. For better understanding of the genetic backgrounds related to the quantitative disease resistance to these major rice diseases, the whole genomes of Jupiter and LM-1 were resequenced along with those of Trenasse (disease-susceptible long-grain cultivar), Bengal (disease-susceptible medium-grain cultivar) and Lemont (the disease-susceptible parental line of LM-1), using an Illumina platform. Analyses of the draft sequences revealed DNA polymorphisms including synonymous and non-synonymous single nucleotide polymorphisms and small insertions and deletions among the five rice genotypes as well as the known or putative biological functions of polymorphic rice coding sequences. This information greatly contributes to our ongoing genetic/genomic study of rice disease resistance and breeding program to develop new disease-resistant rice cultivars.

Managing quarantine-significant post harvest diseases in Pacific Northwest apple orchards

P. Sikdar (1), M. MAZZOLA (2)

(1) Washington State University, Wenatchee, WA, U.S.A.; (2) USDA-ARS, Wenatchee, WA, U.S.A.

Phytopathology 105(Suppl. 4):S4.127

Phacidiopycnis washingtonensis and Sphaeropsis pyriputrescens are two recently reported quarantined pathogens that cause speck rot and sphaeropsis rot, respectively, in apple. Due to quarantine regulation, export of apple from Washington State to China was banned from 2012 through 2014. Previous studies demonstrated that pycnidia of $P$. washingtonensis and $S$. pyriputrescens survive on twig cankers of the pollinator 'Manchurian' crabapple, and serve as a primary source of inoculum to incite fruit infection in the orchard. The goal of this study was to evaluate orchard pruning as part 
of a systems approach to control speck rot and sphaeropsis rot in post-harvest storage. The research was conducted at 4 different locations in Washington State, and two treatments were compared: pruning cankered crabapple twigs at flower bloom followed by fungicide treatment at harvest and a no treatment control treatment. Fruits were harvested at commercial harvest dates, stored at $0^{\circ} \mathrm{C}$ in RA and disease development recorded up to 8 months post-harvest. It was observed that the pruning treatment followed by fungicide application was highly effective in controlling postharvest speck rot and sphaeropsis rot in storage. These findings are economically important to ensure uninterrupted export of disease free apple to international markets.

\section{Genetic diversity of Acidovorax citrulli in Brazil}

G. M. SILVA (1), R. R. Walcott (2), R. M. Souza (1), F. H. Medeiros (1)

(1) Federal University of Lavras, Lavras, Brazil; (2) The University of Georgia, Athens, GA, U.S.A.

Phytopathology 105(Suppl. 4):S4.128

Bacterial fruit blotch (BFB), caused by A. citrulli, is a serious disease of cucurbitaceous plants. The first major outbreak of BFB in Brazil occurred in 1991 on watermelon plants in Sao Paulo state. Since then, BFB has emerged as a major threat to the Brazilian watermelon and melon industry. In Brazil BFB field outbreaks on watermelon have been sporadic. On the other hand, BFB outbreaks occur regularly on melon during the raining season in the northeast region, which includes more than $80 \%$ of the country's melon production. To assess the genetic diversity of $A$. citrulli in Brazil, a collection of 67 strains recovered from melon and watermelon outbreaks were compared using pulsed-field gel eletctrophoresis (PFGE) of SpeI-digested whole cell DNA. Of the 67 Brazilian strains analyzed 7 haplotypes were observed, which included 4 that were unique and previously unreported. Ninety eight percent of the Brazilian strains clustered with group I, which includes strains that are mainly recovered from melon and non-watermelon cucurbits. Only one strain clustered with group II, which includes strains recovered mainly from watermelon. These results suggest that the Brazilian A. citrulli population is mainly comprised of group I strains, and provide a possible explanation for the increased incidence and severity of BFB on melon relative to watermelon in Brazil.

\section{Cercosporin concentration and fungal biomass suggest two modes of} pathogenesis for Cercospora kikuchii in soybean

E. C. SILVA (1), T. G. Garcia (2), A. V. Lygin (3), A. K. Chanda (4), C. L. Robertson (2), B. M. Ward (2), R. W. Scneider (2)

(1) Louisiana State Univ, Agricultural Center, Baton Rouge, LA, U.S.A.; (2) Agcenter, Louisiana State University, Baton Rouge, LA, U.S.A.; (3) University of Illinois, Urbana, IL, U.S.A.; (4) University of Minnesota, Northwest Research and Outreach Center, Crookston, MN, U.S.A.

Phytopathology 105(Suppl. 4):S4.128

Cercospora leaf blight, caused in soybean by $C$. kikuchii, is characterized by two symptoms, purple leaves (thought to be caused by an accumulation of cercosporin) and blight. However, these symptoms are not correlated with each other. We hypothesized that the pathogen has two modes of pathogenesis, one inducing a plant reaction (purple leaf symptoms) and the other causing blight. To test this hypothesis we designed an experiment with two objectives: 1) to determine the relationships among fungal biomass and cercosporin concentration in leaves as related to the two symptoms, and 2) to determine the concentrations of anthocyanins in symptomatic leaves. Five soybean cultivars were planted at three locations in Louisiana. Leaves showing different severities of the two symptoms were collected for quantification of anthocyanins, cercosporin and fungal colonization using qPCR. The pathogen was detected in all leaves, including those showing no symptoms; however, fungal biomass was three-fold higher in blighted leaves as compared to purple leaves. Cercosporin was not detected in symptomless leaves, but it was present at low concentrations ( $5 \mu \mathrm{g} / \mathrm{g}$ average) in purple leaves and much higher concentrations $(15 \mu \mathrm{g} / \mathrm{g}$ average) in blighted leaves. No anthocyanins were detected in any leaves. These results showed that the purple color is not caused by accumulations of cercosporin or anthocyanins. We also showed that there are two modes of pathogenesis for C. kikuchii.

\section{Biofilm forming Stenotrophomonas maltophilia isolated from chlorotic} streak in maize

H. V. SILVA-ROJAS (1), P. Aguirre-Rayo (2), T. B. Uribe-Cortes (3), R. Oropeza-Navarro (4), J. M. Aguirre-Rayo (3)

(1) Colegio de Postgraduados, Edo de Mexico, Mexico; (2) Instituto Tecnologico, Ciudad Altamirano, Mexico; (3) Colegio de Postgraduados, Texcoco, Mexico; (4) Universidad Nacional Autonoma de Mexico, Cuernavaca, Mexico

Phytopathology 105(Suppl. 4):S4.128

Maize is one of the most important crops for food and bioenergy production. Since 2007 chlorotic streaks on leaves have been observed in the Highland
Valley region of Mexico. Isolations done on different growth media as well as physiological and molecular approaches revealed the presence of Pantoea agglomerans, $P$. ananatis and Stenotrophomonas maltophilia. With the aim to know whether S. maltophilia was is the causative of chlorotic streaks and if they have the capacity for biofilm-forming this research was conducted. During 2014, a total of 60 strains were streaked on King B (KB) medium, after $48 \mathrm{~h}$ pure colonies were used for DNA extraction. MLST sequencing and phylogenetic reconstruction with Bayesian inference confirmed that isolated belonged to $S$. maltophilia. Pathogenicity tests were carried out on three week old seedlings. Chlorotic streak symptoms appeared two weeks after inoculation, no symptoms in control plants were observed. The bacterium was reisolated to fulfill Koch's postulates. To quantify biofilm formation, cells were grown overnight in KB broth and put into 96-well polystyrene microtiter plates. Total cell growth was measured at OD620, using a Bio-Tek Elx808 microplate reader, with the Kc4 software for 24, 48 and $72 \mathrm{~h}$. Biofilm-forming varied between 0.3 and 2.5 relative units at $72 \mathrm{~h}$, controls showed no biofilmformation. These results will be useful for the management of bacterial diseases in maize seed production.

Influence of cropping practices and soil characteristics on the presence and abundance of plant-parasitic nematodes in corn fields in Ohio

A. C. Simon (1), T. L. Niblack (1), P. A. PAUL (1)

(1) The Ohio State University, Columbus, OH, U.S.A

Phytopathology 105(Suppl. 4):S4.128

Based on results from a survey of 425 corn fields in Ohio in 2013 and 2014, at least one of nine plant-parasitic nematode genera, Helicotylenchus, Pratylenchus, Hoplolaimus, Xiphinema, Tylenchorhynchus, Paratylenchus, Criconemella, Paratrichodorus, and Heterodera, along with several members of the subfamily Tylenchinae counted together, was detected in each field, with maximum individual-field population densities ranging from 26 to 1,164 nematodes per $\mathrm{cm}^{3}$ of soil, depending on the genus. Binary and ordinal logistic regression models were fitted to the data to estimate the odds of each genus being present, and the lesion, lance, spiral, and pin nematodes exceeding critical damage thresholds based soil region, cropping sequence, tillage, and soil $\mathrm{pH}$, silt content, and electrical conductivity. Based on odds ratios, all covariates affected nematode populations, but soil region had the greatest and most consistent effect. Dagger and ring nematodes were more likely to be present in region 6 than any of the other regions, but, lance, stunt, pin, stubby-root, and spiral nematodes were more likely to be present in regions 1-5 than 6 . The odds of spiral, lance, and pin nematode populations being at moderate-high risk levels in region 3 and 4 were greater than the odds in region 6 , but region 1 was more likely than 6 to have lance nematode populations at high risk levels. Associations between other covariates and nematode presence and density varied among genera.

Frequency and heterogeneity of plant-parasitic nematodes in corn fields in Ohio

A. C. Simon (1), T. L. Niblack (1), P. A. PAUL (1)

(1) The Ohio State University, Columbus, OH, U.S.A

Phytopathology 105(Suppl. 4):S4.128

Corn fields in Ohio were surveyed in 2013 and 2014 to determine the frequency and heterogeneity of plant-parasitic nematodes. Soil samples were collected from 425 fields (15-16 from each of 28 counties) between growth stages V3 and V6. Nine genera of plant-parasitic nematodes, namely Helicotylenchus, Pratylenchus, Hoplolaimus, Xiphinema, Tylenchorhynchus), Paratylenchus, Criconemella, Paratrichodorus, and Heterodera, along with several genera in the subfamily Tylenchinae counted together, were identified. The spiral and tylenchus nematodes were detected in 94 and $96 \%$ of the fields, whereas the lesion, pin, lance, stunt, and dagger nematodes were recovered from $80,57,48,48$, and $37 \%$ of the fields, respectively. The stubby-root, cyst, and ring nematodes were the least frequent, each being present in less than $13 \%$ of the fields. Based on results from generalized linear mixed model analyses of the frequency data, county-level heterogeneity varied among genera, with variance estimates ranging from 0.56 to 3.23 . However, except for comparisons between the stunt and pin, stunt and dagger, and lance and dagger nematodes, the $95 \%$ confidence intervals around the estimates overlapped considerably, suggesting comparable county-level heterogeneity across genera. The stunt nematode was more variable in terms of frequency at the county level than the dagger and pin nematodes, and the lance nematode was more variable than the dagger nematode at this same spatial scale.

Red chili pepper: Flavorant or health risk

P. SINGH (1), M. S. Islam (2), P. J. Cotty (1)

(1) University of Arizona, Tucson, AZ, U.S.A.; (2) ARS, USDA, Tucson, AZ,

U.S.A.

Phytopathology 105(Suppl. 4):S4.128 
Aflatoxins are hepatotoxic carcinogens that frequently contaminate crops resulting in economic losses and health hazards. The filamentous fungus Aspergillus flavus is the most frequently implicated causal agent of aflatoxin contamination. Red chili pepper (Capsicum spp.) is one of the most consumed spices worldwide. Chilies are frequently contaminated with unacceptable levels of aflatoxins because many chilies are produced in warm regions that favor A. flavus growth. The current study examined prevalence of aflatoxin contamination in dried red chili, and identified causal agents of chili contamination with aflatoxin. Chili (whole, ground and crushed) and paprika were purchased from different cities across USA and Bangladesh and tested for total aflatoxin using a lateral flow assay. Overall, $93 \%$ of US samples and all samples from Bangladesh contained detectable levels of aflatoxins. The highest concentrations of aflatoxins detected in the US and Bangladesh were $68.8 \mu \mathrm{g} \mathrm{kg}^{-1}$ and $105.7 \mu \mathrm{g} \mathrm{kg}^{-1}$, respectively. The $\mathrm{L}$ morphotype of A. flavus was the most frequently isolated member of Aspergillus section Flavi. No fungal colonies were detected from $60 \%$ of US samples contaminated with aflatoxins, probably reflecting fumigation or treatment with heat or irradiation prior to supply to markets. Relationships among aflatoxin producers and atoxigenic $A$. flavus associated with chilies were determined by comparison of both DNA sequence and simple sequence repeats.

\section{Barriers to movement and spread of Radopholus similis in Anthurium}

B. SIPES (1), R. Myers (2), J. Lichty (3), K. Sewake (3)

(1) Univ of Hawaii At Manoa, Honolulu, HI, U.S.A.; (2) USDA ARS PBARC, Hilo, HI, U.S.A.; (3) Univ of Hawaii At Manoa, Hilo, HI, U.S.A. Phytopathology 105(Suppl. 4):S4.129

Radopholus similis, the causal agent of anthurium decline, is challenging to manage in anthurium cut flower production. Management can include preplant soil fumigation and the planting of nematode-free anthurium into the field. However, nematodes reinfect the newly planted anthurium quickly through movement from nearby infections or from fumigation escapes. Plant bags and weed mat may serve as barriers to nematode movement and assist in controlling the spread of $R$. similis in anthurium. A factorially arranged experiment with treatments of $\pm R$. similis, $\pm \mathrm{bag}$, and \pm weed mat was established in a shade house. Plots consisting of 9 anthurium plants arranged in 3 rows were replicated three times. The two center plants were inoculated with $1000 R$. similis and the four corner sentinel plants were sampled 6 months later. Roots were collected and placed in a mist chamber to extract nematodes. Samples from plots positive for nematodes were notated as having nematode spread. Within 6 months of inoculation, sentinel plants growing in the ground in cinders (commercial standard practice) were infected with nematodes whether the inoculated plants were also in the ground, in bags or in bags on weed mat. In one plot, sentinel plants in bags placed on weed mat had also become infected with nematodes. In general, weed mat was a more effective barrier preventing movement of $R$. similis to uninfected plants more than growing the anthurium in bags.

\section{Assessment of endophyte recovery in rubber trees: Culture vs. metagenomics approaches}

D. SKALTSAS (1), L. A. Castlebury (2), P. Chaverri (1)

(1) University of Maryland, College Park, MD, U.S.A.; (2) USDA ARS

Systematic Mycology \& Microbiology Lab, Beltsville, MD, U.S.A.

Phytopathology 105(Suppl. 4):S4.129

Hevea brasiliensis is the primary species for producing tapped natural rubber. Plantation grown trees suffer from a variety of diseases that reduce production or can destroy entire plantation areas. Endophytic fungi that have a highly specialized relationship with their wild hosts are considered ideal candidates for developing biological controls for diseases. The objectives of this project were to (1) compare the diversity of cultured and unculturable endophytes, and (2) explore host associations or preferences of endophytes obtained from wild Hevea and its sister genus Micrandra. Plant tissue samples were collected from adult and seedling trees located in the Amazon basin. Cultured fungal endophytes were screened with ITS nrDNA. DNA was extracted directly from adult sapwood tissue and ITS2 was sequenced using an Illumina MiSeq. In adult trees, Trichoderma spp. were most abundant using culture techniques while direct sequencing found Acremonium spp. to be most abundant. In seedlings, 800 of the 1,500 endophytes cultures recovered from seedlings were Diaporthe spp. A MOTHUR analysis with a $98 \%$ similarity criterion resulted in 31 putative Diaporthe species. Interestingly, no Trichoderma species were isolated from the seedlings. Understanding the diversity, host associations, and spatial distribution of endophytes in Hevea and related hosts will provide tools for a more integrated and sustainable approach to disease management of plantation grown trees.
Optimizing late blight forecasts for improved late blight management

I. M. SMALL (1), L. Joseph (1), Y. Wu (1), W. E. Fry (1)

(1) Cornell University, Ithaca, NY, U.S.A.

Phytopathology 105(Suppl. 4):S4.129

Late blight is a perennial concern for potato and tomato producers in rain-fed production systems. Calendar-based fungicide application strategies are often employed for the management of late blight regardless of existing disease levels, cultivar resistance, or prevailing weather. Such strategies may result in economically and environmentally inefficient disease management. Forecastguided fungicide schedules are influenced by prevailing weather and host resistance. The objective of this study was to evaluate and optimize late blight forecasts for fungicide scheduling using computer simulation. Simulation experiments utilized 10 years of observed weather data from 59 locations in potato producing states representing the equivalent of 770 field experiments. For each season at each location, forecast-guided application schedules for susceptible, moderately susceptible, and moderately resistant potato cultivars were compared to calendar-based schedules regarding their expected number of fungicide applications and simulated disease progress. Forecast-guided schedules resulted in fungicide schedules that improved late blight suppression, relative to a calendar-based schedule. Based on simulation results, opportunities for improvement of forecasts were identified and optimization conducted.

\section{Bioactivity of an endophytic bacterium in Betula occidentalis}

T. SMART (1), M. Sullivan (2), M. Roberts (2)

(1) Brigham Young Univ, Provo, UT, U.S.A.; (2) Brigham Young University, Provo, UT, U.S.A.

Phytopathology 105(Suppl. 4):S4.129

Essentially every plant maintains populations of endophytes which commonly produce secondary metabolites possessing antibacterial, antifungal, and anticancer properties. In Betula occidentalis, more commonly known as water birch, endophytic associates with plants of the same genus have been discovered to have of substantial bioactivity. Despite this, B. occidentalis has yet to be studied. In this study, endophytic fungi and bacteria of $B$. occidentalis were extracted, isolated, and assessed for bioactivity. The most prominent of these was an unknown bacterium exhibiting an array of bioactive traits. This bacterium was first isolated on LB agar plates and then inoculated in M1D and lysogeny broths. After two weeks of growth the broth was filtered, assayed for bioactivity, lyophilized, and then extracted via liquidliquid methods. Assays using the extract confirmed inhibition and acted as an assay-guided fractionation technique. Controls included pure $\mathrm{MeOH}$, vancomycin, and ampicillin. Our results indicate that several endophytes of $B$. occidentalis have antimicrobial activity. The most prominent of these is found in a particular bacterium of $B$. occidentalis. With significant inhibition occurring in sclerotinia, botrytis, and fusarium these endophytes may have application in agriculture and turf.

Disease diagnosis, habitat decline and fungal diversity associated with endangered Gondwanan conifers in New Caledonia

J. SMITH (1), A. Black (2), F. Tron (3), N. Anger (4), T. Dreaden (2)

(1) Univ of Florida, Gainesville, FL, U.S.A.; (2) University of Florida, Gainesville, FL, U.S.A.; (3) Conservation International, Noumea, New Caledonia; (4) Institut Agronomique Neo-Caledonien, Noumea, New Caledonia

Phytopathology 105(Suppl. 4):S4.129

New Caledonia's endemic conifers represent some of the most unique and diverse Gymnosperm forests on the planet. Due to small population sizes, microhabitat niches, invasive species, climate change and mining, many of these endangered trees are at risk. This study focuses on field observations of symptoms and the disease diagnosis of four critically endangered endemic conifers: Agathis montana, Araucaria humboldtensis, A. schmidii and Callitris neocaledonica. Due to its significance to indigenous cultures, special attention was given to A. montana, which is experiencing a rapid decline in its population. Field observations and laboratory studies suggest that numerous opportunistic pathogens (including novel fungal taxa) are causing foliage/shoot dieback, cankers on main stems and in some cases, root rot on this species. Habitat degradation due to invasive pigs is a primary inciting factor. Resinous cankers and dieback was observed affecting the two Araucaria species, and cankers and root disease was observed affecting $C$. neocaledonica. Studies are underway to assess pathogenicity of recovered fungi, resolve identities and describe novel taxa and examine fungal communities using next generation community sequencing. The results from this study are being used to develop better management strategies for these endangered relict species. 
Creation and implementation of STAR-D; a Laboratory Accreditation Program for the National Plant Diagnostic Network (NPDN) laboratories K. SNOVER-CLIFT (1), D. Dailey O’Brien (1), P. Shiel (2), K. Burch (2), G. Dennis (2)

(1) Cornell University, Ithaca, NY, U.S.A.; (2) USDA-APHIS-PPQ-CPHST, Raleigh, NC, U.S.A.

Phytopathology 105(Suppl. 4):S4.130

The System for True, Accurate and Reliable Diagnostics (STAR-D), Laboratory Accreditation Program is the result of an effort put forth by the National Plant Diagnostic Network (NPDN) and their collaboration with the USDA-APHIS-PPQ-CPHST to establish and implement a laboratory accreditation system for the NPDN's Land Grant University and State Department of Agriculture plant diagnostic facilities. STAR-D was created using the ISO-17025 national laboratory standard and the American Association of Veterinarian Laboratory Diagnosticians (AAVLD) accreditation system as models. This program is improving all aspects of early pathogen and pest detection for the network members by establishing requirements and standards. The goal is to have STAR-D accredited labs recognized by their clients as suppliers of superior lab services and as fully prepared to utilize their diagnostic capacity in response to urgent sample surges. In April 2014, the NPDN STAR-D Board granted the first two NPDN laboratories accreditation status after a three year implementation period and successful execution of a complete system audit. A third laboratory, the Nevada Department of Agriculture, gained accreditation status in May 2015. Implementation of STAR-D among NPDN laboratories is well underway with many labs incorporating the program's requirements and standards, with a trained auditor pool and with other labs ready for the final step of an external audit.

Expanding Phytophthora ramorum sample processing in New York: Searching for $P$. kernoviae, identifying species, and evaluating preliminary test methods

K. SNOVER-CLIFT (1), M. Daughtrey (2), T. Allen (1), S. Jensen (1)

(1) Cornell University, Ithaca, NY, U.S.A.; (2) Cornell University, Riverhead, NY, U.S.A.

Phytopathology 105(Suppl. 4):S4.130

Cornell University's Plant Disease Diagnostic Clinic (PDDC) has provided Phytophthora ramorum suspect sample processing for numerous state and national agencies since 2004. Processing trace forward/back events, national and observational surveys, and Farm Bill project samples triggered questions about our goals and procedures. Since we began, 3,695 suspect $P$. ramorum samples have been processed through Cornell's PDDC with very few containing the target pathogen. Many contained a Phytophthora species but were not identified to the species level due to time and funding limitationsyet identifying species might provide valuable information about other important Phytophthora species that impact nursery crops in New York State. $P$. kernoviae, in particular, should be looked for, as this pathogen was found in Europe during their P. ramorum surveys. Currently only the ELISA procedure is accepted for preliminary testing to the Phytophthora genus level by regulatory agencies, even though the ImmunoStrip ${ }^{\circledR}$ appears to offer an ideal diagnostic tool for small sample sets. The 2014 objectives for $P$. ramorum sampling added three new components: 1) sequencing Phytophthora positive samples that were not identified as $P$. ramorum, 2) testing $P$. ramorum suspect samples using real time PCR ITS1 and ITS2 protocols for $P$. kernoviae, and 3) a comparison of the ELISA Phytophthora species procedure with the ImmunoStrip test.

Proteomic and metabolic analysis of clubroot resistance mediated by the resistance gene Rcrl from Brassica rapa ssp. chinensis

T. Song (1), R. LAHLALI (2), L. McGregor (1), K. A. Aliferis (3), S. Jabaji (3), F. Yu (1), G. Peng (1)

(1) Agriculture and Agri-Food Canada, Saskatoon, SK, Canada; (2) Canadian Light Source Inc., Saskatoon, SK, Canada; (3) Department of Plant Science, Macdonald Campus of McGill University, Sainte-Anne-de-Bellevue, QC, Canada

Phytopathology 105(Suppl. 4):S4.130

Clubroot disease, caused by the soil-borne phytopathogen Plasmodiophora brassicae is one of the most devastating diseases on Brassica crop species worldwide. For decades, host resistance has been the major tool in management of clubroot. The CR gene Rcrl was previously identified from Brassica rapa ssp. chinensis. In this work, proteomic and metabolic approaches were employed to investigate the molecular mechanisms of the disease resistance conferred by Rcrl. Functional analysis of differentially accumulated proteins identified a potential signaling pathway associated with the resistance by $R c r l$ that was distinct from other commonly reported modes of recognition receptors for fungal and bacterial pathogens. This novel signaling pathway appeared to act in a calcium-independent way through an unknown cascade of mitogen-activated protein kinases (MAPK) and would require the ubiquitin-26S proteasome, which was previously demonstrated to function in abiotic stresses, especially the cold stress. Furthermore, our study also identified a range of biological processes that were differentially regulated in resistant plants, as well as metabolites that were differentially accumulated in the samples carrying Rcrl. The work presented showed that clubroot resistance mediated by Rcrl would consist of induced pathogenrecognition and signaling pathways in defense responses, as well as suppression of pathogen-induced re-programming of host metabolism favoring clubroot development.

\section{Infection process of Villosiclava virens on rice}

J. H. Song (1), C. LUO (1)

(1) Huazhong Agricultural University, Wuhan, China

Phytopathology 105(Suppl. 4):S4.130

Rice false smut disease caused by the fungus Villosiclava virens is an increasing threat to rice production in the world. The infection process of $V$. virens is not yet completely understood despite best efforts. In the present study, the infection process of GFP-labeled strain G2 in rice spikelets was monitored. At 1 to 3 day post inoculation (dpi), conidia germinated at the outer surface of spikelets and fungal hyphae extended to the interior of spikelets mostly from the apices of the spikelets, some hyphe also entered spikelets through cracks at the middle or basal part of the lemma and palea, finally reached the floral organs, e.g., filaments, styles and lodicules. At 4 to 7 dpi, filaments were firstly observed to be infected, then stigmas and styles were covered and gradually infected by fungal mycelia. Over the next days, fungal mycelia developed quickly and covered all of the floral organs. Under scanning electron microscopy, infection sites were observed on the surface of stigmas and styles, and the inner space of style tissue was found to be filled with hyphae under transmission electron microscopy. After invading the styles, fungal hyphae extended to the intercellular space but did not penetrate into host cells. At the later stages, many host cells in styles appeared to be digested. Although a few ovaries were infected by the fungal mycelia via infected styles, in most of the infection, the mycelia did not reach ovaries.

Chemical control as strategy for reducing the impact of leaf anthracnose and host resistance management in sorghum

A. G. C. SOUZA (1), L. V. Cota (2), D. D. Silva (2), F. E. Lanza (2), R. V. Costa (2), E. A. Guimarães (3)

(1) Univ Federal de Vicosa, Belo Horizonte, Brazil; (2) EMBRAPA, SETE LAGOAS, Brazil; (3) Universidade Federal de Lavras, Lavras, Brazil Phytopathology 105(Suppl. 4):S4.130

Anthracnose, caused by Colletotrichum sublineolum, can cause losses up to $50 \%$ of the sorghum production in Brazil. The most efficient method for disease control is host resistance, however, the use of fungicides can reduce more than $70 \%$ the losses. Therefore, our aim was to evaluate the interaction between chemical control and genotype resistance methods to leaf anthracnose control. The experiments were carried out in the EMBRAPA experimental area, Sete Lagoas-MG, Brazil, during the 2008 to 2011 years. In all experiments, the disease was assessed weekly by using a severity scale range from 1 $(0 \%)$ to $9(>75 \%)$. To estimate the time and dose of fungicides and sorghum resistance were used as follows genotypes: BRS304, BRS310, BRS308, MR43, DKB599, and AG1060. It was used four commercial mixtures of triazoles and strobilurins fungicides with doses range from 0 to $0.8 \mathrm{~L} / \mathrm{ha}$. We evaluated the time of fungicide application as follows: i- 45 days after emergence (DAE); ii- 45 and 60 DAE; iii- 45, 60 and 75 DAE. The application of Epoxiconazole + Pyraclostrobin $(\mathrm{E}+\mathrm{P})$ was the most effective to reduce the disease severity. The application of " $\mathrm{E}+\mathrm{P}$ " led to an increase of grain production in 58,87 and $100 \%$ with one, two or three times of fungicide applications, respectively. The higher resistance levels were observed with BRS308 and AG1060 (severity was lower than 14\%). The use of genotype rotation integrated with chemical control can be useful to host resistance management.

Host plant resistance against Tomato spotted wilt virus in peanut genotypes and its impact on virus and vector fitness

R. SRINIVASAN (1), S. Sunadaraj (2), A. Culbreath (2), D. Riley (2), H. Pappu (3)

(1) Univ of Georgia, Tifton, GA, U.S.A.; (2) University of Georgia, Tifton, GA, U.S.A.; (3) Washington State University, Pullman, WA, U.S.A.

Phytopathology 105(Suppl. 4):S4.130

Tomato spotted wilt virus (TSWV) is a constraint to peanut production in the Southeast. Peanut genotypes with field resistance to TSWV are often planted. Nevertheless, the mechanism of resistance against TSWV and/or thrips is unknown. Subsets of TSWV-resistant and susceptible genotypes were inoculated with TSWV. The incidence of TSWV infection was up to $90 \%$ and 
did not vary between resistant and susceptible genotypes. Resistant and susceptible genotypes exhibited typical TSWV symptoms. However, qPCR indicated that some resistant genotypes accumulated fewer viral copies than susceptible genotypes. To assess if resistant genotypes impacted the virus, parsimony and principal component analyses were performed using nucleocapsid protein $(\mathrm{N})$ amino acid sequences from susceptible and resistant genotypes, and from old (collected 12 yrs. ago) and new TSWV isolates. Amino acid sequence analyses indicated consistent differences between old and new isolates. Also, there was evidence for non-synonymous substitutions but not for positive selection. Purifying selection, population expansion, and differentiation seem to have influenced the TSWV populations temporally rather than positive selection due to host resistance. Choice and no-choice tests indicated that some resistant genotypes suppressed thrips feeding and fitness than susceptible genotypes, thereby suggesting that field resistance could be imparted due to differential effects on the virus and/or the vector.

\section{Unraveling the microbial profile of the rhizosphere of SDS-suppressive} soils in Soybean fields

A. Y. SROUR (1), L. Leonardo (2), D. Malvick (3), A. Fakhoury (1)

(1) Southern Illinois University, Carbondale, IL, U.S.A.; (2) Iowa State University, Ames, IA, U.S.A.; (3) University of Minnesota, St. Paul, MN, U.S.A.

Phytopathology 105(Suppl. 4):S4.131

Sudden Death Syndrome (SDS) incidence and severity in soybean fields is often found unevenly distributed across the same field. While certain spots in the field are highly conducive to SDS, other regions appear to be naturally suppressive to the disease. The role of microbes and mechanisms involved in SDS control was investigated in different soybean fields in IL, IA and MN in 2010, 2011 and 2012. By using highly informative barcodes specific to Bacteria, Fungi, Archaea and Nematodes, and coupling with Illumina Mi-Seq sequencing, we identified key microbial taxa likely to be involved in suppression of SDS. A total of $18,000,000$ reads were mapped against the NCBI-NT database and analyzed with MEGAN5 for comparative taxonomic analysis. Our data revealed significant differences in bacterial and fungal community structure and composition between suppressive and conducive soils. At least 20 taxa were found to be attributed to SDS-suppressive soils, however only three genera consisting of Fusarium oxysporum sp. complex, Metacordyceps and Myceliophtora were found to be consistently correlated with SDS suppression. Others such as Proteobacteria, Mycobacteria and Trichoderma sp. were found to dominate in some suppressive soils. This suggests that SDS suppression is dependent on multiple contributors and that their relative abundance plays a key role in determining the incidence of the disease.

Emergence of a new population of the select agent Rathayibacter toxicus J. P. STACK (1), M. Arif (1), G. Y. Busot (1), R. Mann (2), B. Rodoni (2), S. Liu (1)

(1) Kansas State Univ, Manhattan, KS, U.S.A.; (2) Victoria Department of Environment and Primary Industries, Bundoora, Australia

Phytopathology 105(Suppl. 4):S4.131

The U.S. Select Agent Rathayibacter toxicus is a toxigenic gram-positive bacterium responsible for a lethal disease of livestock and wildlife in Australia. The genetic structure of $R$. toxicus populations was determined to evaluate the efficacy of detection protocols and to understand the potential for emergence of new strains. Two genetic analyses were applied to 54 isolates of R. toxicus collected in Australia from 1973 to 2014; multi-locus sequence typing (MLST) and inter-simple sequence repeat (ISSR) analysis. Commonly used MLST gene targets detected no genetic variation among these $54 R$. toxicus isolates. An MLST based on seven strategically selected genes representing an array of cellular functions from metabolism and replication to cell defense detected substantial genetic variation. The concatenated MLST sequences comprised $5533 \mathrm{bp}(0.2 \%$ of the $2.369 \mathrm{Mb} R$. toxicus genome). Analysis of the MLST data partitioned the 54 isolates into three distinct populations. To analyze the genome more generally, a total of 94 ISSR loci (69\% polymorphic) were generated using ten ISSR primers. Both the genebased MLST and the neutral-locus ISSR analyses resolved the same three distinct populations of $R$. toxicus, designated population-A, -B and -C. Population-A isolates/genotypes were not represented in archived culture collections nor were they previously reported; population-A may be comprised of emerging isolates of $R$. toxicus.

Identification and characterization of fungi causing leaf spot and crown rot of bermudagrass in Florida

P. STAVORNVISIT (1), P. F. Harmon (2), J. A. Rollins (1)

(1) University of Florida, Gainesville, FL, U.S.A.; (2) Univ of Florida,

Gainesville, FL, U.S.A.

Phytopathology 105(Suppl. 4):S4.131
Leaf spot and crown rot diseases are common on bermudagrass putting greens in Florida. Turfgrass managers rely on fungicide applications to maintain turfgrass quality during extended periods of disease-favorable environmental conditions. From January 2013 through June 2013, Florida golf course superintendents reported difficulty managing Bipolaris leaf spot and crown rot diseases despite fungicide use. Our objective was to collect and identify bermudagrass leaf spot pathogens from across Florida to be used in future fungicide sensitivity research. A total of 81 fungal isolates were obtained from leaf spot and crown rot samples submitted by golf course superintendents to the University of Florida Plant Diagnostic Center. Taxonomic identities of the isolates were inferred from a phylogeny of their ITS sequences and reference sequences in the NCBI GenBank database. Of the 55 isolates that were placed in the Bipolaris genus, 45 of these were identified as $B$. cynodontis. Eight isolates were identified as $B$. hawaiiensis, and a single isolate of $B$. micropus and B. australiensis also were identified. Species of Curvularia and Exserohilum made up the 26 additional isolates. Draft genomes of two of the $B$. cynodontis isolates were assembled and will be used for future research into fungicide sensitivity.

Genetic approaches to identifying pathogens causing root rot problems of common bean

J. R. STEADMAN (1), G. Godoy-Lutz (2), C. Mukuma (2), S. V. Fernandes (2)

(1) Univ of Nebraska, Lincoln, NE, U.S.A.; (2) University of Nebraska, Lincoln, NE, U.S.A.

Phytopathology 105(Suppl. 4):S4.131

Climate change leading to drought and water-saturated soils contribute to widespread development of root/crown rot causing fungi in dry beans. To aid breeding for disease resistance, different approaches were used to identify predominant root/crown rot pathogens. Traditional isolations from diseased tissue on culture media and identification by morphological features was compared to identification directly from DNA extracted from tissue blotted on FTA cards with specific ITS rDNA primers and further Sanger sequencing or direct 454 pyro-sequencing. Samples collected in bean fields of breeding lines and land races and analyzed by both methods identified mostly different species of Fusarium compared with other fungi associated with root/crown rot symptoms. 454 pyrosequencing revealed more fungal species/strains per sample than direct culturing from tissue. Overall Fusarium oxysporum and Fusarium solani were the most prevalent isolates in samples of diseased beans in Africa and Nebraska. Pathogenicity of identified fungal isolates was tested on a dry bean line by four inoculation methods, using either the axil of the primary leaves, cut stem, detached leaf or the root. Analysis of variance of the disease score $(\mathrm{p}<0.001)$ at the 0.05 significance level showed the cut stem (straw test) to be the best to separate pathogenic from nonpathogenic isolates of F. oxysporum, F. solani, Macrophomina phaseolina, Pythium ultimum and Rhizoctonia solani.

Endophyte community composition is associated with dieback occurrence in an invasive tree

T. V. STEINRUCKEN (1), A. Bissett (2), J. R. Powell (1), A. K. H. Raghavendra (3), R. D. van Klinken (3)

(1) Hawkesbury Institute for the Environment, University of Western Sydney, Penrith NSW, Australia; (2) CSIRO Agriculture Flagship, Canberra, Australia; (3) CSIRO Biosecurity Flagship, Brisbane, Australia

Phytopathology 105(Suppl. 4):S4.131

Dieback is pervasive in many populations of invasive woody weeds globally. Previous studies on dieback have focused on specific potential causative biotic agents, but most cases remain unexplained. The potential role of endophytic microbial communities in dieback, including the relative importance of endophytes with pathogenic or protective capabilities, remains poorly studied. We tested whether changes in archaeal, bacterial and fungal endophyte community structure is associated with dieback occurrence in the invasive, leguminous tree, Parkinsonia aculeata L. (parkinsonia). We sampled roots, stems and stem tips from healthy and dieback-affected parkinsonia and conducted terminal restriction fragment length polymorphism (T-RFLP) analysis on DNA extracted from these samples using domain-specific primers for archaea, bacteria and higher fungi. Microbial community composition strongly differed with parkinsonia disease status (archaea, bacteria and fungi) and plant part (archaea and fungi). Plant part and disease status effects were strongest in archaea. We also found evidence implicating both pathogenic and potentially protective endophytes in the onset of dieback. This is the first study that has shown significant associations between changes in endophyte community composition and dieback presence. Our results highlight the complexity of those changes and provide support for the hypothesis that diverse pathogenic and protective endophytes may be implicated in dieback. 
Extreme genetic diversity in populations of Exobasidium maculosum, an emerging blueberry pathogen

J. STEWART (1), T. Glenn (2), M. T. Brewer (3)

(1) Dept of Plant Pathology, University of Georgia, Athens, GA, U.S.A.; (2) Environmental Health Science, University of Georgia, Athens, GA, U.S.A.; (3) Dept. of Plant Pathology, University of Georgia, Athens, GA, U.S.A.

Phytopathology 105(Suppl. 4):S4.132

Exobasidium leaf and fruit spot of blueberry, caused by Exobasidium maculosum, is an emerging disease that has rapidly increased in prevalence throughout the southeastern USA. It has severely reduced fruit quality in some plantings. Our objectives were to understand the genetic basis of disease emergence and if populations are structured based on geography, host species, or tissue type. We used multilocus sequencing and restriction site associated DNA markers (ddRAD) to estimate the genetic diversity of 88 isolates of $E$. maculosum from different blueberry host species in the southeastern USA and northeastern North America. Results show that E. maculosum from the Southeast and Northeast are not different species, but are distinct populations due to either geographic isolation or host specialization. Population differentiation was detected within the Southeast based on geography but no structure was found based on original host species or tissue type. Although genetically structured, populations were extremely diverse. Each of the 88 isolates had a unique multilocus haplotype. These results suggest that the emergence of this disease is not due to a recent introduction, host jump, or the evolution of more aggressive genotypes of E. maculosum, but could be due to an increasing host population or an environmental change. Future work will examine the underlying basis of the extreme genetic diversity in $E$. maculosum.

Population genomic analyses of the brown root-rot pathogen, Phellinus noxius, examine potential introductions to the Pacific islands

J. E. STEWART (1), M. S. Kim (2), L. Shuey (3), N. Sahashi (4), Y. Ota (4), S. Ashiglar (5), R. L. Schlub (6), P. G. Cannon (7), N. B. Klopfenstein (5)

(1) Department of Plant Pathology, University of Georgia, Athens, GA, U.S.A.; (2) Department of Forestry, Environment and Systems, Kookmin University, Seoul, Korea; (3) School of Biological Sciences, The University of Queensland, Brisbane, Australia; (4) Forestry and Forest Products Research Institute, Ibaraki, Japan; (5) USDA Forest Service, Rocky Mountain Research Service, Moscow, ID, U.S.A.; (6) University of Guam, ANR/CES/CNAS, University Station, Mangilao, Guam, U.S.A.; (7) USDA Forest Service FHP, Region 5, Vallejo, CA, U.S.A.

Phytopathology 105(Suppl. 4):S4.132

Phellinus noxius is a vastly destructive, fast-growing pathogen that affects a wide range of woody hosts in pan-tropical areas, including Asia, Australia, and Oceania. This pathogen causes brown root-rot disease on cacao, coffee, and rubber, as well as diverse fruit, nut, ornamental, and other native/exotic trees. This study is aimed toward performing a wide-scale, population-genetic study of P. noxius isolates from eastern Asia, Pacific islands, and Australia to determine the regional population structure, estimate diversity in recently observed populations, and assess the potential suitable climate space for specific genotypes. Isolates were collected from Japan, Australia, and the Pacific islands of Palau, Yap, Guam, Saipan, Pohnpei, and Kosrae. Sequencing data were generated by Illumina sequencing of double-digest reduced representation libraries (ddRAD). Preliminary results of the ddRAD single nucleotide polymorphism data show multiple genotypes of $P$. noxius that are structured geographically. Isolates from Pacific islands showed reduced levels of genetic diversity, which supports the hypothesis of potential introductions to some Pacific islands. Continued analyses will examine levels gene flow among populations, if population structure by host is present, and use bioclimatic modeling to predict the potential spread of specific genotypes.

Characterization of Pseudomonas syringae from blueberry fields in Oregon and Washington

V. STOCKWELL (1), B. T. Shaffer (2), R. Bennett (1), J. Lee (1), J. Loper (3)

(1) Oregon State University, Corvallis, OR, U.S.A.; (2) USDA-ARS, Corvallis, OR, U.S.A.; (3) USDA-ARS, Horticultural Crops Research Unit, Corvallis, OR, U.S.A.

Phytopathology 105(Suppl. 4):S4.132

Bacterial canker, caused by Pseudomonas syringae, is a common disease that kills buds and stems in blueberry fields in Oregon and western Washington. Management is primarily through application of copper; antibiotics are not registered for blueberry. Little is known about the diversity of $P$. syringae from blueberry or the prevalence of copper or antibiotic resistance. We collected blue-fluorescent, oxidase-negative isolates of $P$. syringae from blueberry fields in Oregon in 2013 and Washington in 2014. Through MLSA of housekeeping genes (cts, $r p o D$ and $g y r B$ ), we found that 50 isolates from
Oregon were associated with 5 of the 13 known phylogroups of $P$. syringae. Representatives of four phylogroups caused disease symptoms on cantaloupe seedlings and three also caused HR on tobacco. Only $1 \%$ of 139 isolates from Oregon and Washington were resistant to streptomycin or oxytetracycline. In contrast, $57 \%$ of isolates of $P$. syringae from blueberry in Washington were tolerant of $50 \mathrm{ppm}$ Kasumin, a newly registered antibiotic for crops. Copper resistance in P. syringae from blueberry was common; $55 \%$ and $87 \%$ of isolates in Oregon and Washington, respectively, grew on CYE medium with $0.32 \mathrm{mM} \mathrm{CuSO}_{4}$. With the emergence of copper-resistance in $P$. syringae from blueberry, alternative control measures for bacterial canker are needed.

Pathogenicity, vegetative compatibility, and genetic diversity in Verticillium dahliae from sugar beet and historical strains

C. A. STRAUSBAUGH (1), I. A. Eujayl (2), F. N. Martin (3)

(1) USDA ARS NWISRL, Kimberly, ID, U.S.A.; (2) USDA-ARS NWISRL, Kimberly, ID, U.S.A.; (3) USDA-ARS, Salinas, CA, U.S.A.

Phytopathology 105(Suppl. 4):S4.132

Verticillium wilt of sugar beet is a disease problem that has received very little attention in the literature, but has been reported to reduce sucrose production and purity. To improve our understanding of Verticillium wilt, a survey of sugar beet plants with wilt symptoms (leaves with yellow or necrotic vein delimited sectors) in Idaho was conducted in 2007 (5 roots from 40 fields; 200 roots total) and 2008 (5 roots from 45 fields; 225 roots total). Verticillium dahliae was isolated from all plants, while Fusarium oxysporum was also isolated from 19 and $21 \%$ of the plants in 2007 and 2008, respectively. From a collection of $109 \mathrm{~V}$. dahliae sugar beet isolates, all isolates had the MAT1-2 mating type and $95 \%$ of isolates evaluated for vegetative compatibility group (VCG) were $4 \mathrm{~A}(1,1$, and $3 \%$ were noncompatible, VCG 4B, and VCG 2B, respectively). All the VCG 4A isolates had the same mitochondrial haplotype based on sequencing from regions 3 ( $\operatorname{cox} 3$ to $n a d 6$ ) and 5 ( $\operatorname{cox} 1$ to $r n l$ ). In greenhouse pathogenicity tests on sugar beet cultivar Monohikari, the VCG 4A isolates produced more foliar symptoms $(P<0.0001)$ than VCG 1, 1A, 2A, 2B, 3, and 4B isolates, but none of the VCGs could consistently reduce root or top weight.

Superior performance of a silver based nanocomposite in relation to copper for management of bacterial spot of tomato in the greenhouse

A. L. STRAYER (1), I. Ocsoy (2), W. Tan (2), J. B. Jones (3), M. L. Paret (4) (1) Univ of Florida, Gainesville, FL, U.S.A.; (2) Center for Research at the Bio/Nano Interface, Department of Chemistry and Shands Cancer Center, University of Florida, Gainesville, FL, U.S.A.; (3) Department of Plant Pathology, University of Florida, Gainesville, FL, U.S.A.; (4) Department of Plant Pathology, North Florida Research and Education Center, University of Florida, Quincy, FL, U.S.A.

Phytopathology 105(Suppl. 4):S4.132

Bacterial spot of tomato, caused by four, Xanthomonas spp., is one of the most damaging diseases of tomato that occurs worldwide. Growers rely heavily on the use of copper-based bactericides, although most strains are copper-tolerant, rendering copper ineffective. This study was undertaken to characterize bactericidal activity of a silver-based nanocomposite, AgdsDNA-GO, and its potential as an alternative to copper. In vitro assays using copper-tolerant and copper-sensitive $X$. perforans strains revealed that $\mathrm{Ag}$ dsDNA-GO at rates as low as $10 \mu \mathrm{g} / \mathrm{ml}$, which contains the equivalent of less than $2 \mu \mathrm{g} / \mathrm{ml}$ of silver, killed all bacterial cells within $15 \mathrm{~min}$ of exposure. Thus, even at low concentrations, the silver in the nanocomposite had high antibacterial activity. In contrast, the equivalent rates $(10,25$, and $50 \mu \mathrm{g} / \mathrm{ml})$ of copper were unable to significantly reduce the population of the coppertolerant strain after $24 \mathrm{~h}$ of exposure. All copper concentrations killed the copper-sensitive strain, but required exposure for more than $1 \mathrm{~h}$. Greenhouse studies on artificially infected transplants treated with either 75 or $100 \mu \mathrm{g} / \mathrm{ml}$ Ag-dsDNA-GO significantly reduced disease severity when compared with copper-mancozeb and untreated controls ( $\mathrm{p}$-value of 0.05). This study highlights the use of a silver-based nanocomposite as an alternative to copper in tomato transplant production.

A TaqMan-based real-time multiplex PCR assay to detect select agent strains of Ralstonia solanacearum

M. J. STULBERG (1), Q. Huang (2)

(1) FNPRU, USDA/ARS, Beltsville, MD, U.S.A.; (2) USDA ARS, Beltsville, MD, U.S.A.

Phytopathology 105(Suppl. 4):S4.132

Ralstonia solanacearum race 3 biovar 2 strains have the ability to cause brown rot of potato in temperate climates. Due to the absence of these strains in the U.S. and because of the potential risk they pose to the potato industry, the U.S. government has listed them as select agents. Vegetatively produced geraniums are a host and have the potential to carry and spread the pathogen 
through trade. We designed two primer and probe sets to recognize select agent strains, a primer and probe set that recognizes phylotype II strains of $R$. solanacearum, and an internal plant primer pair and two probes that can recognize a variety of plant species including both potato and geranium. We multiplexed one of the select agent-specific primers and probe with the phylotype II and plant internal control sets to provide two layers of identification of a positive select agent sample and to prevent false-negatives. The TaqMan-based uniplex and multiplex real-time PCR assays were tested against 90 R. solanacearum species complex strains including 34 select agents and 52 phylotype II strains, and nine out-group bacterial species. They were also tested against symptomatic and asymptomatic tomato and geranium plants. We also designed an extraction buffer for a simple, quick and effective DNA extraction from geranium plants for use in real-time PCR assays.

Management of Rhizoctonia diseases of sugar beet under a replant scenario with various fungicide application methods in the High Plains W. L. STUMP (1), W. Cecil (1), A. Burkhardt (1)

(1) University of Wyoming, Laramie, WY, U.S.A.

Phytopathology 105(Suppl. 4):S4.133

Rhizoctonia solani, a soil-borne fungus that causes seedling disease and root and crown rot disease (RRCR) is a major problem in sugar beets. When growers have to replant, soils are typically warmer and hence an increased Rhizoctonia infection risk. A study was done to determine which single fungicide application method would provide the best season-long management of Rhizoctonia. In a split-split plot design, two sugar beet cultivars were planted at three dates into soil inoculated with infested barley grain. Using normal field rates and appropriate checks, a Kabina seed treatment was compared to four in-furrow treatments and a foliar band application. Measured were plant stands as an indirect measure of seedling disease, canopy decline due to RRCR, and root yield. Collectively, in-furrow treatments and the seed treatment improved final stands $22 \%$ and $12.4 \%$ compared to the inoculated check. With time the seed treatment failed having late season canopy decline (25\%) equivalent to the inoculated check (29.5\%). All infurrow and foliar band fungicide treatments resulted in canopy declines similar to the non-treated check (3.5-8\%). The Priaxor in-furrow treatment and the Quadris foliar band treatment resulted in root yields equivalent or greater than the non-inoculated check. In a replant situation, a single fungicide applied in-furrow or as a single foliar band can provide season-long management of diseases caused by Rhizoctonia under moderate disease.

Whole genome sequencing and analyses of Xylella fastidiosa subsp. fastidiosa Strain GV156 causing Pierce's disease of grapevine in Taiwan C. C. Su (1), W. L. Deng (2), H. T. Shih (1), F. J. Jan (2), C. J. Chang (3), J. CHEN (4)

(1) Taiwan Agricultural Chemicals and Toxic Substances Research Institute, Taichung, Taiwan; (2) Department of Plant Pathology, National Chung Hsing University, Taichung, Taiwan; (3) Department of Plant Pathology, University of Georgia, Griffin, GA, U.S.A.; (4) USDA ARS PWA, Parlier, CA, U.S.A. Phytopathology 105(Suppl. 4):S4.133

Xylella fastidiosa is a nutritionally fastidious Gram-negative bacterium causing Pierce's disease (PD) of grapevines. PD was first reported in Anaheim, California in 1892 and is currently endemic in California and the southeastern U.S. PD also was found outside U.S. but limited to the Americas with the exception of Kosovo in Europe and Taiwan in Asia. In Taiwan, grapevines with PD-like symptoms were first observed in Nantou County in 2002. Later research indicated that the causal agent was a strain of $X$. fastidiosa. This study further characterized the Taiwan PD bacterium by whole genome sequencing and analyses. Strain GV156 was first triple-cloned in PD2 medium. Total DNA was extracted and sequenced using the Illumina MidSeq platform. Using $X$. fastidiosa subsp. fastidiosa Strain M23 as reference, the GV156 genome was determined to be 2,521,639 bp. Comparison of sequences of five housekeeping genes, ssr (16S rRNA), gyrB (DNA gyrase subunit B), dnaK (chaperone protein), rpo D (RNA polymerase sigma factor), and ton $\mathrm{B}$ (outer membrane receptor), showed that GV156 was $100 \%$ identical to the genome sequences of five $X$. fastidiosa subsp. fastidiosa strains available in GenBank. However, comparison at the highly variable genomic loci such as pspB detected strain variations. Further characterization of the Taiwan PD strain is underway.

\section{Managing impatiens downy mildew in Florida}

S. N. SUAREZ (1), T. J. Shekels (1), A. J. Palmateer (2)

(1) University of Florida, Homestead, FL, U.S.A.; (2) Univ of Florida, Homestead, FL, U.S.A.

Phytopathology 105(Suppl. 4):S4.133

The focus for managing impatiens downy mildew has been evaluating residual control. Mefenoxam, fluopicolide and phosphonate-containing products show evidence of residual control. With the risk of fungicide resistance development it's imperative to tank mix and rotate for controlling downy mildew. Separate efficacy trials were conducted to evaluate five phosphonatecontaining products and two tank-mix combinations with fluopicolide. All chemicals were applied to visibly disease-free, 7-wk old, seed-raised impatiens as foliar sprays to runoff using a hand-pressurized sprayer. Phosphonate treated plants received an application and were transplanted into landscape beds 3 weeks later followed by two additional applications at 1 and 4 weeks post-transplanting. Plants treated with fluopicolide received two $7-$ day applications before transplanting, and two additional applications at 4 and 8 weeks post-transplanting. Disease severity was statistically lower for plants treated with $2 \mathrm{fl} \mathrm{oz}$ of Adorn tank mixed with either Pageant Intrinsic or Daconil Ultrex when compared to plants treated with $2 \mathrm{fl} \mathrm{oz}$ rate of Adorn alone. All phosphonate treated plants had statistically lower disease severity and AUDPC values than the untreated control plants. Plants treated with AgriFos remained disease free 7 days longer and disease severity and AUDPC values were statistically lower than all other treatments.

\section{Detection of spinach downy mildew during latent infection}

C. S. SUBBARAO (1), A. Anchieta (2), K. V. Subbarao (3), S. J. Klosterman (2)

(1) Salinas High School, Salinas, CA, U.S.A.; (2) USDA-ARS, Salinas, CA, U.S.A.; (3) University of California, Davis, Davis, CA, U.S.A.

Phytopathology 105(Suppl. 4):S4.133

Spinach downy mildew (DM) is caused by Peronospora effusa. In California, where nearly $75 \%$ of the US fresh spinach is grown, DM causes millions of dollars in losses annually. The disease is controlled by fungicide applications in conventional production, and some of these fungicides are applied whether or not infection has occurred on spinach because of the explosive nature of these epidemics. It was therefore hypothesized that if DM infection could be detected during the latent period, fungicide applications could be targeted only when the pathogen is present. In turn, this may prevent epidemics from developing. Polymerase chain reactions (PCR) with $P$. effus $a$-specific primers were used to detect the pathogen DNA from potential infections on 50 samples weekly for six weeks. Spinach plants were randomly sampled every 3 $\mathrm{m}$ within the beds, from a $36 \mathrm{~m}$ plot, consisting of four beds. Only a few of the 50 samples (usually in clusters throughout the plot) provided Peronosporaspecific amplicons in the initial four weeks. However, a few weeks later, $\sim 90 \%$ of the samples were positive. Initial samples were asymptomatic and yet the PCR enabled detection of DM, confirming detection in a latent period. In conclusion, using PCR, DM pathogen DNA was detected on symptomless spinach plants, and application of fungicides on plants within the field and nearby is expected to prevent further DM development. The approach requires additional validation in larger commercial fields.

Molecular characterisation of reniform nematodes of the genus Rotylenchulus Linford \& Oliveira, 1940 (Rotylenchulidae, Tylenchida) S. A. SUBBOTIN (1), E. Van den Berg (2), J. E. Palomares-Rius (3), N. Vovlas (4), L. R. Tiedt (5), P. Castillo (3)

(1) Plant Pest Diagnostic Center, California Department of Food and Agriculture, Sacramento, CA, U.S.A.; (2) Biosystematics Programme, ARCPlant Protection Research Institute, Queenswood, South Africa; (3) Institute for Sustainable Agriculture (IAS), Spanish National Research Council, Córdoba, Spain; (4) Istituto per la Protezione Sostenibile delle Piante, Bari, Italy; (5) North West University, Potchefstroom, South Africa Phytopathology 105(Suppl. 4):S4.133

Reniform nematodes of the genus Rotylenchulus comprise a few species, which kidney-shaped mature females are semi-endoparasitic on roots. These nematodes are widely distributed across tropical and subtropical regions of the world and associated with numerous agricultural and horticultural crops. Nematode surveys of plant-parasitic nematodes undertaken during the last years in several crops including sugarcane, sorghum, maize, grapevine and wild and cultivated olives in Italy, South Africa, Spain and USA revealed heavy soil infestations by several reniform nematodes, including Rotylenchulus clavicaudatus, $R$. leptus, $R$. macrosoma, $R$. macrodoratus, $R$. reniformis, $R$. sacchari and an unknown species parasitizing sugarcane in South Africa. These species were characterized and differentiated from each other using the D2-D3 of 28S rRNA, ITS rRNA, hsp 90 and coxI mtDNA gene sequences. In this study we also provided phylogenetic relationships within Rotylenchulus as inferred from the Bayesian analysis of these genes. PCR with species-specific primers was developed for rapid and accurate diagnostics of the pathogenic species $R$. reniformis. Sequence and phylogenetic analysis revealed two distinct and divergent paralogs of rRNA genes for $R$. reniformis and an unknown Rotylenchulus species. 
PiAF, a predicted protein-protein interactome of Aspergillus flavus

A. SUBEDI (1), B. M. Musungu (2), M. Geisler (2), R. L. Brown (3), D. Bhatnagar (3), A. M. Fakhoury (1)

(1) Department of Plant, Soil and Agriculture Systems, Southern Illinois University, Carbondale, IL, U.S.A.; (2) Department of Plant Biology, Southern Illinois University, Carbondale, IL, U.S.A.; (3) Southern Regional Research Center, USDA-ARS, New Orleans, LA, U.S.A.

Phytopathology 105(Suppl. 4):S4.134

Aspergillus flavus is a fungal plant pathogen known for the production of aflatoxin in many crops including corn, cotton, and peanut. Development of PiAF, a predicted protein-protein interaction (PPI) map of A. flavus allows the modeling of interactions for many of the primary metabolic processes, biological processes, and developmental processes in the fungus, helping thus in acquiring a better understanding of pathogenesis. PPI interactome maps have already been generated through the use of high throughput experimental methods in model organisms such as human, fruit fly, and arabidopsis. In this study, a PPI map for A. flavus was developed using the Inparanoid to determine one to one orthologies and many to many orthologies. Reference proteomes from baker's yeast, fission yeast, and human were used in the analysis process. Proteins of interest, such as putative pathogenicity genes and developmental proteins hypothesized to be involved in aflatoxin production were used as focal hubs in the analysis of the network. Putative pathogenicity related proteins were predicted based on their interactions with already known proteins. PiAF was also used to mine for novel pathogenicity proteins. The information from a PPI map of one of the primary aflatoxin producing fungi, A. flavus, will aid in understanding the uncharacterized functional genomics of the fungus as well as in improving the systematic analysis of protein-protein interactions in fungi.

Evidence for the horizontal spread of Grapevine red-blotch associated virus in California vineyards

M. R. SUDARSHANA (1), T. L. Lawler (2), B. W. Bahder (2), S. Bag (3), A. Li (2), M. M. Anderson (2), J. K. Uyemoto (4)

(1) USDA ARS, Davis, CA, U.S.A.; (2) UC Davis, Davis, CA, U.S.A.; (3) University of California-Davis, Davis, CA, U.S.A.; (4) USDA-ARS, Davis, CA, U.S.A.

Phytopathology 105(Suppl. 4):S4.134

Grapevine red-blotch associated virus (GRBaV) is a proposed member of the family Geminiviridae. It was first reported in 2012, in grapevines exhibiting red blotch disease in the states of California and New York. Since then it has been recognized in major wine grape production areas in the USA and also in Canada. To investigate if the virus was spreading within the vineyards, the disease incidence was monitored from 2011 to 2014 in an experimental vineyard of Cabernet Sauvignon (CS) established in 2001. The disease incidence was $46.5 \%(\mathrm{n}=480)$ in 2011 which gradually increased to $61.9 \%$ $(n=465)$ in 2014. Symptomatic and asymptomatic vines were tested in 2014 by qPCR for GRBaV infection and all symptomatic vines were positive for the virus. High resolution melting analysis and sequencing of the PCR product indicated that majority of the isolates detected in 2011 and 2012 belonged to clade 1, while the new infections in 2013 and 2014 were largely by isolates belonging to clade 2 . Taken together, our results provide evidence for the spread of GRBaV in the vineyards.

Examination and classification of secondary metabolites from endophytes of Cornus sericea

M. SULLIVAN (1), B. Geary (1), T. Smart (1), M. Roberts (1)

(1) Brigham Young University, Provo, UT, U.S.A

Phytopathology 105(Suppl. 4):S4.134

Cornus Sericea, best known as Red Dogwood, is traditionally used by Native Americans for relief from colds, fevers and rashes. Most of these properties are due to unknown metabolites. Because endophytes often produce metabolites similar to their hosts, it is likely that endophytes of $C$. sericea also produce these compounds. The objective of this study was to determine to what extent endophytes are responsible for the medicinal and bioactive properties of C. Sericea. Plant samples were collected from Utah's Wasatch Front. Roots and stems were washed in $50 \%$ bleach, flame sterilized, and placed on water agar. Over 3 weeks, all endophytes were isolated and cultured on $1 / 10$ PDA. The extracts were assayed to determine the presence of bioactive withanolides by growing them in the presence of known parasites and measuring inhibition. Bioactive isolates were grown in PDB and M1D broths and allowed to culture for 2 weeks, then filtered, lyophilized, and then extracted via liquid-liquid chromatography. The extract was evaporated to dryness then purified via column chromatography with DCM and $\mathrm{MeOH}$ as the main solvent systems. Isolation was accomplished using analytical and preparative TLC. Each endophyte producing bioactive secondary metabolites was characterized genetically by sequencing the $16 \mathrm{~S}$ RNA region and morphologically by SEM and light microscopy. Characterization of secondary metabolites was determined using a combination MS, NMR, and other imaging techniques.

Host range and phylogenetic diversity of Corynespora cassiicola, cause of target spot of cotton in the southeastern USA

L. SUMABAT (1), R. Kemerait (2), M. T. Brewer (1)

(1) University of Georgia, Athens, GA, U.S.A.; (2) University of Georgia, Tifton, GA, U.S.A.

Phytopathology 105(Suppl. 4):S4.134

Corynespora cassiicola is a ubiquitous pathogen causing emerging plant diseases worldwide including target spot of cotton, which has significantly increased in incidence and severity throughout the southeastern USA. Target spot has also increased on tomato and soybean within the same region. Our aim is to understand the emergence of target spot by comparing phylogenetic relationships of isolates from cotton with other hosts and determining their host range. Thirty-nine isolates were collected from different hosts in the southeastern USA and a total of 1709 nucleotides were sequenced. Across the four gene regions, C. cassiicola from the southeastern USA clustered based on host of origin, regardless of geographic location. Results revealed no genetic diversity among isolates from cotton in the southeastern USA and showed them to be genetically distinct from isolates from different hosts of origin. Thirty-two isolates from different hosts were tested for pathogenicity on cotton, soybean, tomato, and cucumber cultivars revealing significant differences. Isolates originally from cotton were more aggressive on cotton than isolates from other hosts. Soybean, tomato, and cucumber were only susceptible to isolates that originated from the same host, indicating host specialization among these isolates. These results suggest that emerging epidemics in the USA are caused by introduction of host specialized isolates or the evolution of more aggressive strains on each host.

Variable aflatoxin production in 48-well plates: Evidence for aflatoxin stimulatory and inhibitory volatile-chemicals produced by Aspergillus flavus

R. R. SWEANY (1), K. E. Damann (1)

(1) Louisiana State University Agricultural Center, Baton Rouge, LA, U.S.A. Phytopathology 105(Suppl. 4):S4.134

Aspergillus flavus produces toxic and carcinogenic aflatoxins in corn, peanuts, cotton and treenuts. Aflatoxin production is highly variable between strains, growing conditions, replicates and experiments. We demonstrated that increasing the number of inoculated wells in 48-well plates increased aflatoxin production. Here we reinvestigated the relationship. Either 12, 24, 36 or 48 wells containing glucose-salts medium were inoculated. Medium was either solid or liquid and $\mathrm{pH} 4$ or unbuffered. Parafilm was placed between the lid and wells or charcoal between wells filled with $\mathrm{pH} 4$ liquid-medium to control gas exchange. Aflatoxin per well was measured after 4 days growth at $25^{\circ} \mathrm{C}$. Mean aflatoxin production increased as more wells were inoculated on both $\mathrm{pH} 4$ and unbuffered solid-medium, and pH4 liquid-medium. More aflatoxin was produced on solid than liquid medium. Parafilm limited gas exchange and lowered aflatoxin concentration in a plate with 48 inoculated wells equivalent to only 12 inoculated wells. Volatile organic compound (VOC) adsorbent charcoal increased aflatoxin indicating the removal of an inhibitory VOC. Aflatoxin production is modulated by cultures growing in close proximity; the mechanism is undetermined but is likely due to sensing metabolism products, e.g. VOCs, $\mathrm{CO}_{2}, \mathrm{H}_{2} \mathrm{O}$. Culture arrangement should be considered in experimental design and interpretation of aflatoxin production in vitro.

Genomic signatures of host jumping onto raspberry and strawberry in two Phytophthora sister taxa

J. F. TABIMA (1), D. Shen (2), B. Kronmiller (1), B. J. Knaus (3), C. M. Press (3), I. A. Zasada (1), B. M. Tyler (1), N. J. Grünwald (3)

(1) Oregon State University, Corvallis, OR, U.S.A.; (2) Nanjing Agricultural University, Nanjing, Chile; (3) USDA ARS, Corvallis, OR, U.S.A.

Phytopathology 105(Suppl. 4):S4.134

Contrasting the genomes of sister taxa with narrow but different host ranges provides an ideal system for studying host adaptation. Selection pressures imposed by different hosts are expected to lead to adaptation by gradual divergence of populations and eventual speciation due to reproductive isolation. This process of host-mediated speciation is expected to leave signatures (such as mutations, recombination, indels, or duplications) in the corresponding pathogen genomes. We sequenced and annotated the genomes of two sister species in the plant pathogen genus Phytophthora, P. rubi and $P$. fragariae, to study which genomic signatures are involved in host adaptation (P. rubi and P. fragariae exclusively infects the genus Rubus or Fragariae, respectively). Genomes were assembled using SOAPdenovo version 1.05. Gene calling was performed using MAKER. The genomes of $P$. rubi and $P$. 
fragariae yielded 9,434 scaffolds and an estimated 18,268 genes for $P$. rubi and 8,511 scaffolds and 17,832 genes for $P$. fragariae. Functional annotation showed a similar number of genes involved in different biochemical pathways, such as sugar processing, metabolism, and amino acid synthesis between the two species. Positive selection (dN/dS) was detected for three pairs of orthologous effector proteins in the RxLR class. Work is ongoing to identify other candidate genes under selection and genomic signatures that may have been involved in the process of speciation via host jumping.

Genetic diversity and population structure of begomoviruses infecting sweet potato

M. N. TAHIR (1), F. Li (2), P. Lan (2), D. Mollov (1), G. R. Kinard (1), R. Li (3)

(1) USDA-ARS, Beltsville, MD, U.S.A.; (2) Yunnan Agricultural University, Kunming, China; (3) USDA ARS, Beltsville, MD, U.S.A.

Phytopathology 105(Suppl. 4):S4.135

Begomoviruses infecting sweet potatoes (Ipomoea batatas) exhibit high genetic diversity, and approximately eight species including Sweet potato leaf curl virus (SPLCV) have been described from different regions around the world. In this study, the complete genomic sequences of 17 geographically distinct SPLCV isolates were obtained. Comparative analyses with 141 isolates of other sweet potato begomoviruses were conducted to determine population structure, genetic diversity and underlying evolutionary forces. A mean diversity value of $11.6 \%$ was obtained in the population, with highest diversity found in the European subpopulation $(13.0 \%)$ and lowest in the South American subpopulation (9.3\%). With the exception of $\mathrm{C} 3$ and $\mathrm{C} 4$, four other genes (V1, V2, C1 and C2) were under negative selection pressure. A global analysis using Species Demarcation Tool (SDT) revealed the presence of 10 species, with most isolates (110) as SPLCV. Phylogenetic analysis using the genomic sequences separated the isolates into 16 clusters, which were different from the SDT grouping. Different topologies were obtained when individual genes were analyzed, indicating the occurrence of recombination. RDP4 analysis revealed that 141 of the 158 isolates were naturally occurring recombinants among different known and unknown viruses/isolates, suggesting genetic recombination is major driving force in evolution of these viruses/strains.

Oxathiapiprolin, a new tool for control of soil-borne Phytophthora diseases

A. TALLY (1), P. Kuhn (1), J. Haskell (1), B. Druebbisch (1)

(1) Syngenta Crop Protection, Greensboro, NC, U.S.A.

Phytopathology 105(Suppl. 4):S4.135

Oxathiapiprolin is a new fungicide active ingredient (a.i.) that provides excellent control of downy mildews and late blight. It is also active on soil diseases caused by Phytophthora. Although Pythium species appear to be less sensitive to the a.i. (P. ultimum is the exception), research is underway to determine what diseases caused by Pythium spp. can be controlled with oxathiapiprolin. The mode of action is novel with no cross resistance to any other commercially available Oomycete fungicide, making it an excellent partner for mefenoxam which has been used for over 30 years for disease management of these difficult pathogens. Trials on management of Phytophthora capsici and P. nicotianae have been conducted for the last several years at various universities. These studies have evaluated effect of rates, placement, timing, intervals, and application methods. While foliar rates range from 12-35 g ai/ha, the soil rates are higher ranging from 50-140 g ai/ha. Activity on P. capsici is excellent, following delivery of the fungicide in transplant water or via drip application. Likewise, for black shank of tobacco, control is very good, especially with early, preventive applications. Trials are underway to develop a program approach to integrate oxathiapiprolin with other products having different modes of action. Federal registration of oxathiapiprolin is anticipated during the second half of 2015, with launch early in 2016.

High throughput semi-automated robotic nucleic acid extraction and purification protocol optimized for citrus tissues

S. H. TAN (1), T. Dang (1), B. Ramirez (1), M. Voeltz (1), N. Siddiqui (1), E. Varady (1), B. Nguyen (1), G. Greer (1), S. Bodaghi (1), F. Osman (1), D. Pagliaccia (1), G. Vidalakis (1)

(1) University of California, Riverside, CA, U.S.A.

Phytopathology 105(Suppl. 4):S4.135

A semi-automated, high throughput, total nucleic acid extraction and purification procedure optimized for citrus tissues was developed. The procedure utilizes the Cryo-station and Geno Grinder 2010 (SPEX SamplePrep) for sample processing and the MagMAX ${ }^{\mathrm{TM}}$ Express-96 using the 5x MME-96 Viral RNA Isolation Kit (Applied Biosystems). The downstream detection of viral RNA with reverse transcription quantitative real time polymerase chain reaction (RT-qPCR) requires high quality RNA as defined by concentration, purity, and integrity. RNA concentration and purity were assessed by spectrophotometry at $260 \mathrm{~nm}$ and $260 / 280$ ratio, respectively. The RNA concentration of 968 citrus samples ranged from 9.040 to $886.000 \mathrm{ng} / \mu \mathrm{l}$ with an average of $118.895( \pm 95.195, \mathrm{n}=968)$. The majority of the samples $(84.9 \%)$ had concentration $\geq 50 \mathrm{ng} / \mu \mathrm{l}$ while $82.6 \%$ of the samples had concentrations $50-400 \mathrm{ng} / \mu \mathrm{l}$. The RNA purity ratio $260 / 280$ was higher than the desirable 1.8 (low protein contaminants) for the majority of the tested samples $(98.7 \%)$ with a mean value of $2.303( \pm 0.244, n=968)$. The RNA integrity of 439 samples was evaluated by RT-qPCR reactions targeting the mRNA of the NADH dehydrogenase citrus gene. The mean Cq value of the RT-qPCR determining RNA integrity was $21.427( \pm 2.811, \mathrm{n}=439)$ with maximum and minimum values 29.471 and 16.224 , respectively. The cost of supplies for the presented total nucleic acid extraction and purification procedure was estimated at US $\$ 4.03$ per sample.

Systemic movement by Clavibacter michiganensis subsp. michiganensis in the tomato vasculature

M. A. TANCOS (1), C. D. Smart (2)

(1) Cornell Univ, Geneva, NY, U.S.A.; (2) Cornell University NYSAES, Geneva, NY, U.S.A.

Phytopathology 105(Suppl. 4):S4.135

The Gram-positive bacterium Clavibacter michiganensis subsp. michiganensis is an economically devastating seed-disseminated pathogen causing bacterial canker of tomato. Historically, C. michiganensis subsp. michiganensis has been characterized as being non-motile; however, preliminary in planta experiments have demonstrated basipetal movement. In order to complement the in vivo studies, microfluidic chambers were utilized to mimic xylem vessels by providing a flow rate similar to that in planta. When observed in microfluidic chambers, non-flagellated C. michiganensis subsp. michiganensis actively moved against the media flow at speeds averaging $6 \mu \mathrm{m} / \mathrm{min}$, which were similar to speeds observed in planta. In addition to possible motility, systemic movement of $C$. michiganensis subsp. michiganensis is facilitated by the exploitation of nutrients. Interestingly, $C$. michiganensis subsp. michiganensis and a small number of vascularcolonizing bacteria possess putative expansin proteins that share high structural similarity to expansins found in plants. These proteins are found in the plant cell wall and are involved in cell wall loosening during cell growth. The function of the bacterial expansin remains unknown, but is hypothesized to play a role in colonization and nutrient acquisition. Therefore, a $C$. michiganensis subsp. michiganensis putative expansin gene was explored at both a microscopic and genetic level in order to better understand the role in pathogenesis.

Identification of novel avirulence genes through screening gain-ofvirulence mutants of Magnaporthe oryzae

D. TATE (1), J. Hu (1), T. Mitchell (1)

(1) The Ohio State University, Columbus, OH, U.S.A.

Phytopathology 105(Suppl. 4):S4.135

More than 700 million tons of rice is produced on an annual basis constituting roughly $20 \%$ of all calories consumed by humans. Infections such as rice blast, caused by the fungi Magnaporthe oryzae, have led to major losses with rice blast being the main limiting factor in many regions. The devastation caused by this disease has inspired the intense study of its infection dependent pathways. By using the gene-for-gene model, many resistance (R)-genes have been identified; allowing breeders to craft rice cultivars that are resistant to many strains of the fungus. However, much is still unknown concerning the cognate avirulence (Avr)-genes because of their obscure sequence patterns. The goal of this study is to find corresponding Avr genes to the Pi 2 R-gene in hopes of better understanding the strong relationship between this semibiotrophic pathogen and its host. Magnaporthe oyzae strain KJ201 was utilized after demonstrating that rice plants containing Pi2 R-genes are resistant to its infection. By performing Agrobacterium tumefaciens-mediated transformation, infections, and TAIL-PCR, we have identified five candidate genes. These genes have been cloned into the strain CHINOS, which does not naturally contain the Avr gene associated with the Pi-2 R-gene. By infecting $\mathrm{Pi}-2 \mathrm{R}$ rice plants with these genes cloned into CHINOS, and assessing their susceptibility to the fungus we will be able to identify and characterize the Avr gene leading to resistance in rice.

Role of Wheat streak mosaic virus-encoded proteins in disease development S. TATINENI (1)

(1) USDA ARS, Univ of Nebraska, Lincoln, NE, U.S.A.

Phytopathology 105(Suppl. 4):S4.135

Eriophyid mite-transmitted Wheat streak mosaic virus (WSMV) and Triticum mosaic virus (TriMV) are the type species of Tritimovirus and Poacevirus 
genera, respectively, in the family Potyviridae. TriMV and WSMV exhibit differential symptom phenotypes on wheat: TriMV elicits mild mosaic and mottling while WSMV induces severe chlorotic streaks, leaf yellowing and stunting of plants. Co-infection of wheat by WSMV and TriMV causes synergistic interaction with an increased symptom phenotype. The role of WSMV- and TriMV-encoded proteins in disease development is unknown. In this study, the role of WSMV proteins in disease development was examined by engineering individual WSMV cistrons in pTriMV between P1 and HCPro cistrons. Wheat plants infected with TriMV vectors expressing WSMV cistrons were examined for symptom phenotype. TriMV expressing WSMV 6K1, NIa-VPg, NIa-Pro or CP induced more severe symptoms than wild-type TriMV. Expression of WSMV 6K1 in TriMV induced severe mosaic and leaf yellowing, NIa-VPg and CP induced severe chlorotic streaks, mosaic and leaf deformation with moderate stunting of plants, and NIa-Pro induced severe stunting with shortened internodes with dark-green leaves. TriMV with WSMV P1, HC-Pro, P3, CI, 6K2, or NIb induced symptoms similar to those of the wild-type TriMV. These data suggest that WSMV 6K1, NIa-VPg, NIaPro and CP enhanced the symptom phenotype of TriMV, and these proteins might be involved in disease development of WSMV.

\section{Trivapro $^{\mathrm{TM}}$ : A new fungicide combining three different modes of action} for disease control on corn, soybeans, and wheat

E. C. TEDFORD (1), A. Tally (2), A. Fisher (2)

(1) Syngenta Crop Protection, Greensboro, NC, U.S.A.; (2) Syngenta Crop Protection, LLC, Greensboro, NC, U.S.A.

Phytopathology 105(Suppl. 4):S4.136

Trivapro $^{\mathrm{TM}}$ is a new fungicide that has been specifically developed for use on corn, soybeans, and wheat. This product combines three active ingredients; a succinate dehydrogenase inhibitor (SDHI), a strobilurin, and a triazole. All three active ingredients have different modes of action (FRAC Groups 3, 7, and 11) and are not cross-resistant with each other. This is a powerful combination that provides not only broad spectrum preventive control, but also curative activity. Solatenol ${ }^{\mathrm{TM}}$ fungicide (the SDHI component) has higher intrinsic activity than other SDHI fungicides, providing long lasting residual activity against key pathogens. Solatenol fungicide binds strongly to the wax layer on leaf surfaces and slowly penetrates into the plant. The strobilurin and triazole components are both systemic, moving within the plants xylem tissues to areas of new growth. This combination of high intrinsic activity and binding with Solatenol fungicide along with systemic mobility of the strobilurin and triazole components provides for long lasting disease control and protection to the developing canopy. Federal registration of Trivapro fungicide is anticipated in 2015.

Identifying Xanthomonas oryzae pv. oryzae strains and adapted sources of resistance among rice breeding lines in Mali

C. Tekete (1), H. Doucoure (2), M. Dembele (3), M. Doumbia (1), S. Dao (1), K. Dagno (4), S. Sarra (5), S. Cunnac (2), O. Koita (1), V. VERDIER (6)

(1) Laboratoire de Biologie Moléculaire Appliquée, Université des Sciences Techniques et Technologiques de Bamako, Bamako, Mali; (2) IRD, UMR Interactions Plantes Microorganismes Environnement, IRD-Cirad-Université de Montpellier, Montpellier, France; (3) Institut d'Economie Rurale de Niono, Niono, Mali; (4) Institut d'Economie Rurale, Programme Sorgho-CRRA de Sotuba, Bamako, Mali; (5) Institut d'Economie Rurale de Niono, Niono, Mali; (6) IRD, UMR Interactions Plantes Microorganismes Environnement, IRDCirad-Université de Montpellier, 34394 Montpellier Cedex 5, France

Phytopathology 105(Suppl. 4):S4.136

A major constraint to rice yield in Mali is a disease caused by Xanthomonas oryzae pv. oryzae (Xoo), causal agent of bacterial blight (BB). The use of rice cultivars with introgressed resistance $(\mathrm{R})$ genes is the most economically way to control BB disease. The choice of BB gene(s) for deployment depends on the effectiveness of that gene against prevalent races of Xoo. Until 2007 one Xoo race was reported in Mali. No breeding strategy was developed for controlling BB nor has R gene effective against Xoo strains been identified in currently used rice germplasm in Mali. Since 2009 and in order to have a more complete overview of $\mathrm{BB}$ distribution and Xoo race prevalence we surveyed different rice cultivated regions in Mali. A collection of Xoo strains originated from two main rice cultivated regions was obtained. Virulence typing of Xoo strains was conducted on different sets of rice breeding lines. Near-isogenic lines (NILs also known as IRBB lines) available for various BB genes (known as $X a$ ) was used for race characterization. We will report on the i) distribution of $\mathrm{BB}$ disease, ii) virulence of Xoo strains on rice accessions, iii) distribution and prevalence of Xoo races, iiii) identification of resistance sources among rice germplasm in Mali. The emergence of new races without deployment of $X a$ resistance genes and the potential use of identified resistance sources among rice germplasm cultivated in Mali will be discussed.
Applications of mobile microscopy in plant pathology

A. L. TESTEN (1), S. A. Miller (1)

(1) The Ohio State University OARDC, Wooster, OH, U.S.A.

Phytopathology 105(Suppl. 4):S4.136

Microscopes are an essential tool for plant pathologists, necessary for diagnostics, education, and research. Due to their size and expense, microscopes are usually limited to laboratory settings. However, the advent of smartphones with high-quality cameras has led to the development of lowcost, portable, mobile microscopes. These microscopes range from simple (a ball lens taped to a smartphone camera) to sophisticated (a 3D printed microscope with a smartphone dock). The portability, ease of use, and cost of these microscopes allow for applications in a wide range of settings by individuals with differing experiences with microscopes. We evaluated several low-cost, mobile microscopes, including the FoldScope and the PlantScope (CellScope), for applicability to plant pathology. Microscopes were evaluated under a range of conditions, typical of those found in plant pathology, including field and classroom settings. Microscopes were assessed for: i) suitability for mobile field diagnostics of tomato diseases in domestic and international settings, ii) usefulness as an educational tool in a plant disease diagnostics course, and iii) usefulness at extension events. As lowcost, mobile microscopes become more available, their acceptance and applications in plant pathology are likely to grow.

Resistant wild watermelon rootstocks for managing root-knot nematodes in grafted watermelon

J. A. THIES (1), A. Levi (1)

(1) USDA, ARS, Charleston, SC, U.S.A.

Phytopathology 105(Suppl. 4):S4.136

The southern root-knot nematode (RKN), Meloidogyne incognita is an important re-emerging pest of watermelon worldwide. We have developed RKN-resistant wild watermelon (WW) Citrullus lanatus var. citroides rootstocks for managing RKN in grafted watermelon. We evaluated 11 rootstock /scion ('Tri-X 313' seedless watermelon) combinations plus selfgrafted (SG) and non-grafted (NG) Tri-X 313 in RKN infested fields. Rootstocks were 4 resistant WW parental lines (RKVL 301, RKVL 316, RKVL 317, and RKVL 318) and 4 of their F1 hybrid crosses, 'Emphasis' bottle gourd (BG), 'Strong Tosa' squash hybrid (SQ), and 'Ojakkyo' WW. Three WW parental lines, three WW F1 hybrids, and 'Ojakkyo' had fewer $(P$ $<0.05$ ) RKN eggs than 'Tri-X 313' (SG and NG), 'Emphasis', and 'Strong Tosa'. Three RKVL parents and all four F1 hybrids had heavier fruit weights $(P<0.05)$ than SG 'Tri-X 313', 'Emphasis' BG, and 'Strong Tosa' SQ rootstocks. SQ and BG rootstocks were highly susceptible to RKN with high RKN reproduction and low yields. Overall, the wild watermelon rootstocks (parents and F1 hybrids) were resistant to RKN and performed well as rootstocks for grafted watermelon. These wild watermelon lines should be useful sources of RKN-resistance for development of watermelon rootstocks and germplasm.

Optimizing fungicide application intervals based on airborne Erysiphe necator (grape powdery mildew) inoculum concentration

L. THIESSEN (1), W. Mahaffee (2)

(1) Oregon State University, Corvallis, OR, U.S.A.; (2) USDA ARS, Corvallis, OR, U.S.A.

Phytopathology 105(Suppl. 4):S4.136

Using inoculum detection to initiate fungicide programs has been shown to reduce fungicide applications used to manage grape powdery mildew in the Oregon and California; however, quantification of airborne inoculum may be used to further optimize subsequent fungicide application intervals. Quantitative polymerase chain reaction (qPCR) allows for spore samples collected via impaction spore traps to be easily enumerated and a minimum spore threshold to be set for adjusting the length between fungicide applications. Impaction spore traps were placed at 6 commercial vineyards in the Willamette Valley of Oregon in both 2013 and 2014 growing seasons. Samples were collected from spore traps bi-weekly from budbreak until veraison. Fungicides were applied at the longest interval recommended by fungicide chemistry label until 10 spores were detected. When 10 spores or greater were detected, fungicides were applied at the shortest interval recommended by the fungicide chemistry label. Disease incidence was assessed visually on a weekly basis at each vineyard. In both years, disease in both plots was lower than $3 \%$ incidence, and adjusted interval plot disease incidence was not significantly different from grower standard control treatment plots $(P=0.44$ in 2013 and $P=0.29)$. In both 2013 and 2014, growers saved an average of 2 fungicide applications during the growing season. 
Population biology of Pseudoperonospora cubensis, the causal agent of Cucurbit downy mildew

A. THOMAS (1), I. Carbone (1), P. Ojiambo (1)

(1) North Carolina State University, Raleigh, NC, U.S.A.

Phytopathology 105(Suppl. 4):S4.137

Cucurbit downy mildew caused by Pseudoperonospora cubensis is considered the most important disease of cucurbits worldwide. The disease resurged in the US in 2004, when the pathogen overcame host resistance that had been effective for more than 40 years. Introduction of a new lineage, a new pathotype or new genetic recombinants of the pathogen, have been hypothesized as mediators of the disease resurgence. Comparative genome analyses has previously revealed the existence of two distinct evolutionary lineages of $P$. cubensis in the US with one lineage predominantly of the A1 mating type and associated mainly with cucumber, while the other was A2 and associated with squash, cantaloupe and watermelon. Signatures of sexual recombination suggested that host-specific lineages are not genetically isolated. Inoculation experiments using a total of 21 isolates and 15 host differentials revealed the presence of new pathotypes in the US, namely III and VI that previously existed only in Asia. Further studies are being conducted to examine the population genetic structure of $P$. cubensis in the US using a larger sampling of isolates from both host lineages and genome wide markers generated using double digest Restriction Associated DNA Sequencing (ddRADSeq). Variation from ddRADSeq will be examined to better understand the impact of contemporary sexual recombination on hostlineage evolution and to track the seasonal movement of the pathogen using island metapopulation models.

\section{Preliminary insights into the epidemiology of Blueberry shock virus in} cranberry

S. THOMAS-SHARMA (1), L. Wells (1), V. Kartanos (1), E. Saalau-Rojas (2), P. McManus (1)

(1) University of Wisconsin, Madison, WI, U.S.A.; (2) UMass Cranberry Station, E. Wareham, MA, U.S.A.

Phytopathology 105(Suppl. 4):S4.137

Blueberry shock virus (B1ShV) is emerging as one of two Ilarviruses associated with berry scarring in cranberry in WI and MA (the other, Tobacco streak virus (TSV)). Infected cranberry shoots (uprights) produce scarred, disfigured berries that turn red prematurely. Fields in WI suspected to have BlShV were sampled in 2013 and 2014, and BlShV was detected by DASELISA from cultivars LeMunyon, Mullica Queen, Pilgrim and Stevens. Before bloom, detection of B1ShV was $54 \%$ and $49 \%(n=35)$ when current season leaves and previous season leaves, respectively, were tested. Uprights with symptomatic berries had a lower detection in current season leaves $(11 \%$ positive; $n=79)$ in contrast to previous season leaves $(63 \%$ positive; $n=72)$. At fruit set, $98 \%$ of asymptomatic berries $(n=40)$ and $96 \%$ of symptomatic berries $(n=80)$ were positive for BlShV. BlShV was not detected in pollen washes but detected $(100 \%$ positive; $n=16)$ in pollen extracts, suggesting internalization within pollen. In 2014, 5 to $10 \%$ incidence of fruit scarring was observed in two different beds in MA. Symptomatic fruit from cultivars Howes and Stevens tested positive for BlShV and TSV. In WI and MA, individual uprights did not test positive for both BlShV and TSV, although the viruses were sometimes found in the same bed. Studies on the extent and impact of BlShV on cranberry production are currently underway.

\section{'Shiny' interfaces for interactive data collection and reporting in Plant Pathology}

S. THOMAS-SHARMA (1), Y. Xing (2), G. Forbes (3), K. Garrett (2)

(1) University of Wisconsin, Madison, WI, U.S.A.; (2) University of Florida, Gainesville, FL, U.S.A.; (3) International Potato Center, Beijing, China Phytopathology 105(Suppl. 4):S4.137

In an interdisciplinary and multi-stakeholder field such as Plant Pathology, innovative data collection and reporting platforms that encourage stakeholder interactions can be particularly useful. Shiny is a new package in the $\mathrm{R}$ programming environment developed by RStudio. Shiny allows R users to build interactive web applications that can be used to explore and analyze datasets, drawing on the other popular statistical analysis and graphics packages in $\mathrm{R}$. These applications can be readily shared among individuals whether they have $\mathrm{R}$ expertise or not, increasing their utility to multiple stakeholders. Shiny interfaces can be particularly useful to make large datasets accessible to the public, without restricting data summaries to a predetermined subset of variables. In addition to interactive reporting, Shiny can be used to develop interactive questionnaires and surveys for data collection. We developed an interactive questionnaire for collecting parameter estimates in expert elicitation from researchers, for a seed degeneration risk assessment model of vegetatively-propagated crops. The application allows experts to visualize the implications of their parameter estimates and to modify their response through real-time updating of graphs and figures. The results are then saved into a format that can be directly used for data analysis. Such an interactive questionnaire could improve the accuracy, efficiency, intuitiveness and reproducibility of data collection.

The isolation and characterization of a strain of Cucumber mosaic virus isolated from an epidemic affecting Phaseolus vulgaris in Upstate New York

J. Thompson (1), J. Langenhan (2), K. Watters (2), J. Lucks (2), M. FUCHS (1), K. Perry (2)

(1) Cornell University, Geneva, NY, U.S.A.; (2) Cornell University, Ithaca, NY, U.S.A.

Phytopathology 105(Suppl. 4):S4.137

In some years Cucumber mosaic virus (CMV) reaches epidemic proportions in bean in Upstate New York. During the years 2004 to 2014, CMV was isolated from over 50 infected plant samples from bean fields in the Finger Lakes region. Three fragments of the CMV genome spanning central regions of each of the three genomic RNAs were amplified using reverse transcription-PCR and sequenced. Phylogenetic analyses showed all virus isolates clustered within subgroup IA with low levels of diversity. The isolates of this study along with global sequences were analyzed bioinformatically for recombination, reassortment, selection pressures and temporal structure. Clones of one of the isolates (Bn57) were engineered for the production of infectious in vitro RNA transcripts initiated from a $\mathrm{T} 7$ promoter, and infections resulted in symptoms consistent with those of the original field isolate. These infectious clones were used to assess symptom determinants and the effects of virus infection on plant growth. In addition, and with the aim of further exploring the biology of legume-infecting and other CMVs, the RNA structures of regions of the genome of Bn57-CMV were determined using Selective 2-Hydroxyl Acylation analyzed by Primer Extension sequencing or SHAPE-Seq. These latter results provide the starting point for in-depth studies into the structure and function of CMV RNA.

Dead but alive -crop and weed residues aid survival of new pathogenic Diaporthe/Phomopsis species on soybean, sunflower, other hosts in Australia

S. M. THOMPSON (1), Y. P. Tan (2), R. G. Shivas (2), S. M. Neate (3), E. A. Aitken (4)

(1) University of Southern Queensland, Australia, Toowoomba Qld, Australia; (2) Department of Agriculture, Fisheries and Forestry, Brisbane, Australia; (3) University of Southern Queensland, Toowoomba Qld, Australia; (4) University of Queensland, Brisbane, Australia

Phytopathology 105(Suppl. 4):S4.137

Diaporthe/Phomopsis species cause damaging cankers on sunflower and soybeans. This study reveals that many Diaporthe species found in Australian broadacre cropping systems survive on both live and dead crop and weed hosts Unincorporated crop and weed stubble is commonly found in zero and low till cropping systems where herbicides are often used for weed control. Although living plants are well recognised as a 'green bridge' facilitating transmission of pathogens to crop hosts between seasons, the role of weed residues as a 'brown bridge' in aiding the survival of some groups of pathogens has not been previously studied. Of 27 novel Diaporthe species identified, 11 species have been newly described to date, including numerous from sunflower, soybean and other crop hosts. Early pathogenicity testing results indicate that some species are virulent on multiple hosts. Molecular analyses show that cankers on a single host may result from a complex of species. These findings have significant implications as even with effective rotational strategies, the overlooked weed residues on the surface are an effective 'brown bridge' and inoculum reservoir between pathogenic Diaporthe species and crop hosts. A number of species have also been isolated from asymptomatic live hosts such as maize. The role of endophytic infection of crops and weeds by Diaporthe species as a survival aid needs further investigation and suggests a case for the redefinition of the word 'host'.

Fluorescent Pseudomonas spp. associated with cranberry (Vaccinium macrocarpon Ait.)

J. THOMSON (1), A. Harrison (1), S. Gadagkar (1), C. Bull (2), S. Soby (1) (1) Midwestern University, Glendale, AZ, U.S.A.; (2) United States Department of Agriculture - Agricultural Research Service, Salinas, CA, U.S.A.

Phytopathology 105(Suppl. 4):S4.137

Cranberry is an ideal model system for comparing microbes associated with wild and cultivated plants because they are genetically similar and grow in the same geographic region of southeastern Massachusetts. Several hundred fluorescent Pseudomonas from wild and cultivated cranberry plants and soils 
were isolated as a first step for comparing wild and cultivated cranberry phytobiomes. Studying fluorescent pseudomonads as indicators of bacterial populations could contribute to an overall understanding of changes in the plant-associated microbiota that can be attributed to fertilizer and pesticide use, irrigation, or other agricultural practices. In an attempt to identify differences between the bacterial populations associated with cultivated and wild cranberry plants, soil and plant tissue Pseudomonas isolates were characterized and identified using genotypic and phenotypic analyses, including 16S rRNA, MLSA, and Biolog GENIII phenotyping. Cultivable Pseudomonas isolates clustered into five or six distinct groups. Less than $2 \%$ of isolates were identifiable as known Pseudomonas 'species' ( $P$. putida and $P$. entomophila), indicating that a large majority of cranberry bog isolates represent new members of this genus. At least one clade of these organisms was found only in wild cranberry bogs, leaving open the questions of distribution, rates of Pseudomonas evolution under environmental conditions, and selection due to cultivation practices.

Interaction of Ralstonia solanacearum and root-knot nematode on potato A. M. TOHAMY (1), M. A. Bekhiet (2), A. M. Kella (2)

(1) Plant Pathology Research Insitute, Giza, Egypt; (2) Plant Pathology Research Institute, Giza, Egypt

Phytopathology 105(Suppl. 4):S4.138

The interaction of $R$. solanacearum Race 3 biovar 2, the cause of brown rot disease, and root-knot nematode (M. incognita race 2) tested individually or in combination was studied on potato plants (cvs. Diamant and Spunta). Results showed that potato cv. Spunta was highly susceptible to brown rot disease than cv. Diamant. The possible combinations of $M$. incognita and $R$. solanacearum revealed that potato plants inoculated with $M$. incognita 10 days prior to bacterial inoculation showed higher bacterial wilt disease rating than those inoculated with both pathogens simultaneously. However, inoculation potato plants with $R$. solanacearum 10 days prior inoculation with $M$. incognita showed less disease rating than those of the other treatments. Typical bacterial wilt symptoms were observed on potato plants, 24 days old. The rate of stem colonization by $R$. solanacearum in potato cv. Spunta was higher than its corresponding in cv. Diamant. Also, the interaction between root-knot nematode and bacterial wilt pathogen exhibited more bacterial colonization to both stems and tubers than those infected with bacterial wilt pathogen alone. In addition, all plants infected with $M$. incognita showed gall development on roots. The higher galling index was noticed on potato plants cv. Diamant either inoculated with M. incognita alone or in combination with $R$. solanacearum at the same time or 10 days after nematode inoculation.

Finding "the right stuff": The search for regulatory patterns underlying defense response gene expression to improve quantitative resistance in rice

B. W. TONNESSEN (1), R. Mauleon (2), N. Alexandrov (2), J. E. Leach (1) (1) Colorado State University, Fort Collins, CO, U.S.A.; (2) International Rice Research Institute, Los Banos, Philippines

Phytopathology 105(Suppl. 4):S4.138

Diseases inflicting rice diminish the potential yield by about one third worldwide. Mitigating this issue through breeding and engineering of rice varieties that contain single resistance genes has produced plants that are resilient. The method is effective, but pathogens are able to overcome this selective pressure sometimes after only a few generations. A more sustainable resistance could be attained by selecting for enhanced expression of plant defense response (DR) genes that elicit basal defense against a broad spectrum of pathogens. DR gene promoters can affect timing and intensity of expression, and rice varieties with good basal resistance have been shown to harbor polymorphisms that are beneficial in the genome-wide defense response. Recently, the genomes of a diverse panel of 3000 rice varieties were sequenced, providing a valuable resource for understanding the subtle, pleiotropic mechanisms of basal resistance. In this project, in silico analysis has elucidated patterns in the regulatory regions of DR genes that are enriched in resistant phenotypes. These short sequences may contribute to beneficial regulation of the defense response. Discoveries from this work could be used to provide markers for breeding rice varieties with stable, broad spectrum resistance.

Control of Penicillium expansum with yeasts recovered from various plant materials

V. H. TOURNAS (1), E. J. Katsoudas (2)

(1) Center for Food Safety and Applied Nutrition/FDA, College Park, MD, U.S.A.; (2) Office of Regulatory Affairs/FDA, Jamaica, NY, U.S.A.

Phytopathology 105(Suppl. 4):S4.138

Over 400 yeast strains were isolated from various plant materials including fruits and leaves from ornamental pear (Pyrus calleryana), red and green seedless grapes, Red Delicious apples, and fruit salads and screened for their potential to act as biocontrol agents (BCAs) against the patulin-producing mould, Penicillium expansum, in vitro. Candida oleophila was used as positive BCA control. Two isolates from Red Delicious apples, one from $P$. calleryana fruit, one from red seedless grapes and one from pineapple fruit salad caused inhibition of the pathogen when incubated at $25^{\circ} \mathrm{C}$ for one week. The inhibition was limited to the area covered by the yeast growth. When incubation was extended for a second week, mould growth completely covered the yeast isolates originated from $P$. calleryana and grapes, while sporadic Penicillium structures appeared on the apple and fruit salad isolates and $C$. oleophila colonies. The activity of these strains, although substantial, was lower than the one exhibited by $C$. oleophila. These BCA yeast strains were also tested for viability retention when stored in aqueous suspensions at $5^{\circ} \mathrm{C}$ for up to 4 weeks. The highest viability $(89 \%)$ was observed in $C$. oleophila followed by the apple strains $(85-86 \%)$ and the lowest $(61 \%)$ was noted in the strain isolated from fruit salad.

\section{Potentially-toxigenic and spoilage postharvest fungi from pome and stone} fruits

V. H. TOURNAS (1), J. S. Kohn (2), E. J. Katsoudas (2)

(1) Center for Food Safety and Applied Nutrition/FDA, College Park, MD, U.S.A.; (2) Northeast Regional Lab/ORA/FDA, Jamaica, NY, U.S.A. Phytopathology 105(Suppl. 4):S4.138

A total of 41 pome fruit samples (Gala, Golden Delicious and Red Delicious apples, and D'Anjou, Bartlett and Bosc pears) and 57 stone fruit samples (including black, red and white cherries, black and red plums, nectarines, and peaches) were tested for fungal contamination using the direct plating method. The results of the study showed that the vast majority $(90-100 \%)$ of the pome fruit, nectarine and peach samples carried live fungi. The most common moulds encountered in apples and pears were Penicillium spp., Penicillium expansum and Alternaria spp. found in 39, 49 and $39 \%$ of the overall samples, respectively. Fusarium and Monilinia species were less frequent. The most common moulds in stone fruits were Alternaria, Monilinia and Penicillium spp. found in 23,29 an $18 \%$ of the samples, respectively, while Cladosporium spp. and $P$. expansum were recovered at a lower rate and frequency.

Ralstonia solanacearum extracellular nucleases aid bacterial virulence by degrading root extracellular traps and modulating biofilm formation

T. M. TRAN (1), A. MacIntyre (1), M. Hawes (2), C. Allen (1)

(1) University of Wisconsin-Madison, Madison, WI, U.S.A.; (2) University of Arizona-Tucson, Tucson, AZ, U.S.A.

Phytopathology 105(Suppl. 4):S4.138

Ralstonia solanacearum is a soil-borne bacterium that causes bacterial wilt on a wide range of hosts. To defend against the pathogen, plant roots cast a NETlike matrix of extracellular DNA studded with antimicrobial proteins such as histones that trap and kill invading bacteria. Possession of an inventory of nucleases, therefore, could assist the pathogen during early infection by degrading the root tip extracellular DNA matrix. We found that $R$. solanacearum genomes encode two secreted functional endonucleases that are unique to pathogenic Ralstonia species. Mutants lacking these genes had reduced virulence on plant hosts, reduced competitive fitness in tomato plants and impaired root attachment. These results suggest that $R$. solanacearum extracellular nucleases help the pathogen succeed during plant root infection. However, when we bypassed the roots and injected the nuclease-deficient mutants directly into tomato stems, they were still less virulent and less able to spread in plant stems than the wild-type strain. This indicates that these enzymes have an additional function beyond degradation of DNA traps at the root. Because nuclease mutants form abnormally thick biofilms in vitro and biofilms can be degraded by purified nucleases, we hypothesize that $R$. solanacearum also uses its extracellular nucleases to modulate biofilms in planta to facilitate bacterial spread in host xylem.

Influence of pruning systems on trunk pathogens and other fungi colonizing grapevine wood

R. TRAVADON (1), P. Lecomte (2), B. Diarra (2), D. P. Lawrence (1), J. Vallance (2), H. Ojeda (3), P. Rey (2), K. Baumgartner (4)

(1) Univ of California, Davis, CA, U.S.A.; (2) INRA, UMR 1065 SAVE, Université de Bordeaux, ISVV, Villenave d'Ornon, France; (3) INRA, Unité Expérimentale de Pech Rouge, Gruissan, France; (4) USDA-Agricultural Research Service, Davis, CA, U.S.A.

Phytopathology 105(Suppl. 4):S4.138

The main infection courts for fungal grapevine trunk pathogens are pruning wounds. Disease control practices typically involve modifications to pruning. Pruning practices requiring fewer/smaller wounds may thus be associated with fewer trunk pathogens and less wood necroses. We examined woodcolonizing fungi in the trunks of 14-year-old vines (Vitis vinifera 'Mourvèdre' 
at site A, 'Syrah' at site B), either spur-pruned or minimally (min-) pruned. At each site, the trunks of eight min-pruned and eight spur-pruned vines were characterized in terms of proportions of necrotic wood and the community structure of the 88 cultivable fungal taxa identified through ITS sequencing. Spur-pruned vines had more wood necroses $(35 \%$ vs. $20 \%$ in min-pruned vines) and a greater diversity of taxa, but not a greater abundance of trunk pathogens. Instead, Canonical Correspondence Analysis revealed different trunk pathogens: Diaporthe ampelina and Togninia minima were associated with spur pruning, and D. foeniculina, Neofusicoccum parvum, and Phaeomoniella chlamydospora with min-pruning. Among the 15 most abundant taxa, eight were more common in spur-pruned vines (e.g., wood endophytes Aureobasidium pullulans, Bionectria ochroleuca), compared to only five in min-pruned vines (e.g., Paraconiothyrium brasiliense, Pestalotiopsis microspora). Spur-pruned vines were associated with more plant pathogenic taxa, which may contribute to greater levels of wood necrosis.

Elevated $\mathrm{CO}_{2}$ increases wheat susceptibility to Barley yellow dwarf virus P. TREBICKI (1), N. Nancarrow (2), A. Freeman (3), N. Bosque-Pérez (4), A. Yen (2), G. Fitzgerald (5)

(1) Department of Economic Development, Horsham, Australia; (2) Department of Economic Development, Bundoora, Australia; (3) Department of Economic Development, Horsham, Australia; (4) University of Idaho, Moscow, ID, U.S.A.; (5) Department of Economic Development, Ballarat, Australia

Phytopathology 105(Suppl. 4):S4.139

Understanding how future climate conditions and increasing atmospheric carbon dioxide will shape agricultural production is essential in order to sustain or improve current crop production levels. Pests and diseases are major contributors to the reduction of crop yield and are responsible for raising input costs. Barley yellow dwarf virus (BYDV) can cause serious crop disease transmitted by many aphid species. This virus causes yellowing and stunting of plants and adversely affects the yield and quality of wheat, barley and oats as well as other economically important crops in Australia and worldwide. In this study, we used Australian Grains Free $\mathrm{Air} \mathrm{CO}_{2}$ Enrichment (AGFACE) facility and plant growth chambers to understand the insect, plant and pathogen interactions. We exposed infected and uninfected wheat and the bird cherry-aphid (Rhopalosiphum padi), the main vector of BYDV, to ambient and $\mathrm{eCO}_{2}$ (550ppm FACE, $650 \mathrm{ppm}$ chambers) levels. We examined the severity of BYDV in plants, aphid biology in both virus free and BYDV infected plants, as well as plants and virus response under these two $\mathrm{CO}_{2}$ scenarios. Despite the positive effect of $\mathrm{eCO}_{2}$ on yield and wheat growth, our study showed detrimental $\mathrm{CO}_{2}$ effects on grain quality and BYDV severity. We found that BYDV titre significantly increased in the leaves of wheat plants grown under $\mathrm{eCO}_{2}$ conditions, leading to a decrease in yield. We also found a decrease in aphid fecundity on uninfected plants; however, no changes in aphid reproduction were detected on BYDV infected plants. In addition, changes to wheat physiology mediated by $\mathrm{eCO}_{2}$, resulted in significantly extended aphid feeding activity on uninfected plants. Other experimental data from both the FACE facility and environmental growth chambers will be described and the results from these experiments presented.

Inorganic nitrogen metabolism in Ralstonia solanacearum: Functions and regulation

A. TRUCHON (1), B. Dalsing (1), C. Allen (1)

(1) University of Wisconsin, Madison, WI, U.S.A.

Phytopathology 105(Suppl. 4):S4.139

Tomato xylem is a low-oxygen and generally low nutrient habitat, but it does contain inorganic nitrogen species, including $\sim 30 \mathrm{mM}$ nitrate $\left(\mathrm{NO}_{3}{ }^{-}\right)$. The bacterial wilt pathogen Ralstonia solanacearum exploits these attributes of the xylem environment by using inorganic nitrogen species as terminal electron acceptors to generate ATP via denitrification. We used a series of $R$. solanacearum mutants to show that the NarG - AniA - NorB - NosZ denitrification pathway contributes not only to respiration on $\mathrm{NO}_{3}^{-}$, but also to detoxification of reactive nitrogen species like nitrite $\left(\mathrm{NO}_{2}{ }^{-}\right)$and nitric oxide (NO). The pathogen needed the full denitrification pathway for wild-type virulence on tomato plants, demonstrating its importance in a relevant biological context. To explore this key pathway's regulation, we mutated Rsp0958, a gene highly expressed in planta that encodes a possible homolog of the NO-binding protein NorA, which indirectly controls expression of the NO reductase NorB in $R$. eutropha. The $R$. solanacearum $\Delta$ norA mutant grew poorly in aerobic culture but grew normally under anaerobic conditions in culture and in planta, consistent with direct measurements showing that tomato xylem is a hypoxic environment. Preliminary results indicate that the $\Delta$ norA mutant is reduced in virulence on tomato plants, possibly because without NorA to sequester NO, the denitrification pathway was misregulated.
Alternatively, directly toxic levels of $\mathrm{NO}$ may accumulate in the $\Delta$ norA mutant.

Molecular identification of eggplant-infecting begomoviruses in Southeast Asia

W. S. TSAI (1), L. Kenyon (2)

(1) National Chiayi University, Chiayi, Taiwan; (2) AVRDC - The World Vegetable Center, Shanhua, Tainan, Taiwan

Phytopathology 105(Suppl. 4):S4.139

Since the eggplant-infecting begomoviruses were firstly recognized as causing yellow mosaic disease on eggplant (Solanum melongena) crops in West Thailand in 2001, the viruses have been detected in China, India, Iran, Laos, Pakistan and Vietnam. However, more recently, greater incidence of yellow mosaic symptoms have been observed in eggplant crops in Southeast Asia. Seventeen symptomatic samples were collected including four from Java, Indonesia in 2009, eight from Northern Thailand in 2012, two from Laos in 2010 and three from Vietnam in 2013. All samples were confirmed for begomovirus infection and the $1.5 \mathrm{~kb}$ begomoviral partial DNA-A products from PCR with primer pair- PAL1v1978B/PAR1c715H were sequenced. All the sequences from the samples from Indonesia, Laos and Vietnam, and four of the eight samples from Northern Thailand had nucleotide sequence identity $>94 \%$ to previous identified Tomato yellow leaf curl Kanchanaburi virus isolates. The sequences from the other four samples from Northern Thailand shared $>92 \%$ nucleotide sequence identity with each other and had highest nucleotide sequence identity $(<87 \%)$ with Pepper leaf curl virus. However, the full-length DNA-A sequences are required to determine if these represent a new begomovirus species, or the same species with eggplant as a new host. The results provide further evidence for the increasing virus diversity in Southeast Asia and for the spread of an eggplant-infecting begomovirus to the Java, Indonesia.

\section{Simple devices for determining grain moisture}

T. TUBBS (1), C. Woloshuk (1)

(1) Purdue University, West Lafayette, IN, U.S.A.

Phytopathology 105(Suppl. 4):S4.139

Maize producers in developing countries such as those in Sub-Saharan Africa, have major challenges in drying and storage. Methods used by farmers to determine grain dryness are subjective, relying on grain appearance, hardness, and the sound it makes when shaken. Maize is often not dried to levels safe for storage, resulting in spoilage and the accumulation of aflatoxin. The objective of this research was to establish a device for using low-cost hygrometers to measure grain moisture. We assessed the efficacy of two hygrometer types (digital and color strips). Maize was equilibrated to various moisture contents $(10,12,14,16,18$ and 20 percent), and placed in a closed containers with a digital and strip hygrometer, which were incubated at various temperatures $\left(18^{\circ} \mathrm{C}, 22^{\circ} \mathrm{C}, 25^{\circ} \mathrm{C}, 27^{\circ} \mathrm{C}, 30^{\circ} \mathrm{C}\right.$ and $\left.37^{\circ} \mathrm{C}\right)$. For each moisture and temperature, the time required to reach the equilibrium relative humidity (ERH) was determined and the equilibrium moisture content (EMC) was calculated. Grain from each moisture/temperature combination was also stored for one month and fungal growth and aflatoxin were measured. Our results indicated that with the digital hygrometer, EMC of the maize could be determined within as little as 30 minutes. The color strip also provided a consistent ERH measurement in the same amount of time. Based on the mold growth/aflatoxin analysis, an ERH at or below $70 \%$ on the color strip would indicate safe for storage over the tested temperature range.

Evaluation of Sclerotium cepivorum distribution in naturally infested commercial fields in Central California

T. TURINI (1), A. Biscaro (2), R. M. Davis (3)

(1) Univ of California Coop Ext Svc, Fresno, CA, U.S.A.; (2) University of California Cooperative Extension, Ventura, CA, U.S.A.; (3) University of California, Davis, CA, U.S.A.

Phytopathology 105(Suppl. 4):S4.139

Many fields in California's Central Valley are infested with Sclerotium cepivorum, which causes white rot of garlic and onions. Due white rot's devastating effects and presence of extremely long-lived sclerotia, susceptible crops are not grown fields known to be infested. This becomes more difficult as the of infested fields increases. Current treatment options are very costly, so economic returns may be achieved by treating specific areas of a field. To evaluate the potential for minimizing treated area, onion plants expressing symptoms in 12 commercial fields were mapped with a GPS unit from 2010 to 2014. Post-crop soil samples were collected from specific areas of 4 onion fields in which disease was limited to one portion of the field and not larger than 0.25 acres. Sclerotia counts of those soil samples were quantified in laboratory. The sclerotia levels where disease had been observed were relatively low or even absent. In all fields levels were greater than 5 sclerotia 
per $500 \mathrm{cc}$ soil, which can cause substantial economic damage, from 300 to $750 \mathrm{ft}$ from the site where disease was observed. This suggests that tillage practices have resulted in a dilution of inoculum and substantial distribution within the field. It may be possible to avoid treating an entire field, particularly in large fields. However, considering cultural practices in the fields evaluated, large areas should be treated.

Effects of Clariva seed treatment on soybean cyst nematode (Heterodera glycines) population densities and soybean yields in Iowa in 2014

G. L. TYLKA (1), C. C. Marett (1), A. E. Robertson (1), M. Serrano (1), T. A. Mueller (2)

(1) Iowa State University, Ames, IA, U.S.A.; (2) Iowa Soybean Association, Ankeny, IA, U.S.A.

Phytopathology 105(Suppl. 4):S4.140

Twenty-four field experiments were conducted in Iowa in 2014 to assess the effects of Clariva seed treatment (active ingredient Pasteuria nishizawae) on Heterodera glycines reproduction and soybean yields. Nine small-plot experiments with 4-row-wide by 5.2-meter-long plots were planted with seed of the moderately resistant soybean variety Asgrow AG2433 treated with either Clariva Complete Beans or CruiserMaxx Advanced plus Vibrance. The seed-treatment packages varied only in the presence or absence of Clariva. Treatments were replicated 12 times in each experiment. Fifteen strip-trial experiments consisted of 12- to 24-row-wide by 305 - to 457-meter-long strips of the same two seed-treatment packages. Variety AG2433 was used in eight strip trials; other resistant soybean varieties were used in seven trials. All but two trials had five or more replications. Soil samples were collected at planting and harvest to assess $H$. glycines population densities in each small plot and from sampling points in many of the strip trials. Reductions $(\mathrm{P}=0.03$ to 0.15 ) in season-long $H$. glycines reproduction were associated with Clariva in three of the nine small-plot experiments. Yields were determined from each plot and strip in every experiment. Mean yields ranged from 3.14 to 4.87 metric tons per hectare in small-plot experiments and from 2.47 to 6.16 metric tons per hectare in strip-trial experiments. Yields of treatments were different $(\mathrm{P}=0.11$ to 0.20$)$ in eight of 24 experiments.

\section{Host range testing and molecular analysis of Puccinia psidii in Hawaii} J. UCHIDA (1), C. Kadooka (1)

(1) Univ of Hawaii At Manoa, Honolulu, HI, U.S.A.

Phytopathology 105(Suppl. 4):S4.140

Since it was first detected on 'ōhi'a Metrosideros polymorpha in 2005, Puccinia psidii has been found on thirty eight Myrtaceae hosts in Hawaii. To determine if the rust in Hawaii is a single strain, or if it consists of multiple strains, host range testing on various Myrtaceae plants and analysis of DNA with SSR markers were conducted with rust collected from various hosts. When the rust was found on new a new host, spores were taken from pustules and inoculated onto healthy rose apple Syzygium jambos to increase the total amount of urediniospores. After ten days, the rose apple leaves were covered with pustules, and the spores were then used in host range testing. Inoculated were: rose apple Syzygium jambos, 'ōhi'a Metrosideros polymorpha, guava Psidium guajava, allspice Pimenta dioica, mountain apple Syzygium malacense, Surinam cherry Eugenia uniflora, wax apple Syzygium samarangense, and Rhodomyrtus Rhodomyrtus tomentosa. Results show that all of the rust isolates tested show the same infection profile on the eight Myrtaceae hosts. Molecular analysis of DNA extracted from spores and used for PCR reaction with nine SSR primers further characterize the Hawaii isolates as a single strain

The best timing of fungicide applications for control of narrow brown leaf spot of rice in main and ratoon crops

S. S. UPPALA (1), X. G. Zhou (1)

(1) Texas A\&M AgriLife Research, Beaumont, TX, U.S.A.

Phytopathology 105(Suppl. 4):S4.140

Narrow brown leaf spot (NBLS) is a foliar disease of rice caused by Cercospora janseana that causes significant yield losses. Timely application of fungicides is the key to minimizing production costs and maximizing yields. The present study was conducted to evaluate different application timings of the fungicide propiconazole (PropiMax @ $0.73 \mathrm{~L} / \mathrm{ha}$ ) on main and ratoon crops of rice (cv. Presidio or Cocodrie) in fields naturally infected with NBLS. Field experiments were established at Eagle Lake, Texas in 2010, 2011, 2012 and at Beaumont, Texas in 2014. Various main crop fungicide application timings (at panicle differentiation, booting, heading or 7 days after heading) in combination with ratoon crop with or without the fungicide application at the booting stage were evaluated. NBLS severity was visually rated prior to harvest in both main and ratoon crops. All fungicide timing treatments resulted in a reduction in NBLS compared to the untreated control. Most efficient control on the main and ratoon crops was achieved when propiconazole was applied at booting, heading or 7 days after heading in main crop. An additional fungicide spray in main or ratoon crop resulted in further reduction in NBLS but did not significantly increase yield. Results of this study suggest that single application of propiconazole between booting and up to 7 days after heading in main crop is sufficient for control of NBLS of rice in main and ratoon crops under the conditions evaluated.

\section{Grapevine trunk diseases in British Columbia}

J. R. URBEZ TORRES (1), P. Haag (1), J. Boule (1), D. O'Gorman (1)

(1) Pacific Agri-Food Research Centre / Agriculture and Agri-Food Canada, Summerland, BC, Canada

Phytopathology 105(Suppl. 4):S4.140

Grapevine trunk diseases (GTD) are caused by many different fungi and are recognized as one of the major threats to the industry's future economic sustainability. British Columbia (BC) is the second largest grape-growing region in Canada and its grape-industry contributes over \$2 billion CAD to the total country's economic impact. The objectives of this study were to identify the fungi associated with GTD in BC and determine their significance and spread. Field surveys were conducted in 191 vineyards that included assesment of foliar symptomatology from over 60,000 vines and isolations from 463 symptomatic vines. Results revealed the presence of all GTD in BC, including Botryosphaeria dieback, esca, Eutypa dieback, young vine decline (black foot and Petri disease), and Phomopsis dieback. Morphological studies along with DNA analyses of four genes (ACTIN, $\beta$-tubulin, TEF-1 $\alpha$, ITS15.8S-ITS2) allowed the identification of over 40 fungal species belonging to 20 different genera, including novel species. Pathogenicity tests for all identified fungi were completed and the characteristic esca and Eutypa dieback foliar symptoms were reproduced when vines were artificially inoculated with Phaeomoniella chlamydospora and different Togninia/Phaeoacremonium species and Eutypa lata, respectively under greenhouse conditions. This study represents the first attempt to demystify the status of GTD in BC, a young and emerging grape-growing region with unique climatic conditions.

Macroarray detection and identification of young vine decline fungal pathogens

J. R. URBEZ TORRES (1), P. Haag (1), D. O'Gorman (1)

(1) Pacific Agri-Food Research Centre / Agriculture and Agri-Food Canada, Summerland, BC, Canada

Phytopathology 105(Suppl. 4):S4.140

Young vine decline (YVD) is responsible for important economic losses to the grapevine industry worldwide. To date, several fungi in the genera Cadophora, Campylocarpon, Cylindrocadiella, Ilyonectria, Cylindrocarpon, Phaeomoniella, Phaeoacremonium, and Togninia have been identified to be associated with this complex disease. YVD fungi are also known to occur in planting material. Therefore, early detection is critical to assure longevity of newly established vineyards. A DNA-macroarray containing 102 oligonucleotides complementary to portions of the $\beta$-tubulin gene was developed for detection of YVD fungi. Its specificity was tested against 72 different species from eight genera from different geographical regions, including 37 YVD species. A total of 61 species including 34 YVD pathogens were detected and discriminated by the DNA-macroarray. Detection limit of the array was below $0.1 \mathrm{pg}$ of genomic DNA. The array was validated against artificially inoculated cuttings and soil as well as commercial planting material, showing the latter a high incidence of YVD fungi otherwise not detected by traditional plating and culturing. The high level of specificity demonstrated by this DNA-macroarray showed it to be a promising detection tool for rapid and accurate identification of YVD pathogens in a single test, which has the potential to be used in commercial diagnostics and/or by the grapevine nursery industry to determine the health status of the planting material.

Implementation of a DNA-macroarray diagnostic tool to assess the health status of grapevine nursery propagation material

J. R. URBEZ TORRES (1), D. T. O'Gorman (1)

(1) Pacific Agri-Food Research Centre / Agriculture and Agri-Food Canada, Summerland, BC, Canada

Phytopathology 105(Suppl. 4):S4.140

Young vine decline (YVD) is a complex disease caused by a wide range of taxonomical unrelated fungi and responsible for important economic losses to the grapevine industry worldwide. YVD fungi are known to occur in nursery propagated material and thus, detection prior to planting is critical to assure longevity of newly established vineyards. Accordingly, the goals of this study were i) to assess the potential of a recently developed YVD-DNA-macroarray (capable of simultaneous detection and identification of up to 34 YVD pathogens) to evaluate the health status of grapevine propagated material and 
ii) to compare this technique against currently used fungal pathogen detection tools in the nursery industry. Ready to plant material was provided by several nurseries and included different rootstock-scion combinations and self-rooted plants. For each plant, total DNA was obtained from roots, rootstock basal end, graft-union, and scion and processed with the DNA-macroarray. The DNA-macroarray successfully detected and identified several YVD fungi and showed Phaeomoniella chlamydospora to be the most prevalent YVD pathogen followed by Cadophora luteo-olivacea, Togninia-Phaeoacremonium spp., and Ilyonectria spp. Among all different plant parts, YVD pathogens were detected primarily in the rootstock basal end. The DNA-macroarray was shown to be an accurate, sensitive and a much faster, detection tool than other techniques used in the nursery such as traditional plating.

Field evaluation of Quintec and Actigard for managing bacterial spot and speck on nine fresh market tomato varieties in Florida

G. E. VALLAD (1), H. Adkison (1), R. Willis (1), S. Newman (1), J. Seibert (1) (1) University of Florida, Wimauma, FL, U.S.A.

Phytopathology 105(Suppl. 4):S4.141

Bacterial spot and speck caused by Xanthomonas perforans and Pseudomonas syringae pv. tomato, respectively, are common diseases of tomato production in Florida. The prevalence of copper-tolerant strains has compromised the efficacy of copper-based bactericides. Field trials were established to assess the efficacy and crop safety of programs using Quintec (quinoxyfen) and Actigard (acibenzolar-S-methyl) for managing bacterial spot and speck across nine tomato varieties: BHN602, Charger, FL47, FL91, HM8849, Rally, Ridgerunner, SevenTYIII, and TastiLee. Based on AUDPC values and the number of diseased fruit, weekly applications of Quintec, Quintec alternated with copper sulfate + mancozeb, and copper sulfate + mancozeb were ineffective for managing bacterial spot and speck compared to the non-treated control; while weekly applications of Actigard, or the FarMoreB seed treatment + Actigard exhibited lower severity and fewer diseased fruit. All treatments improved marketable yields compared to the non-treated control. Across all treatments, the tomato varieties Charger, Rally, Ridgerunner, FL91, and TastiLee exhibited the lowest AUDPC values. No treatment by variety interactions were detected for any evaluation of disease or fruit yields. Although symptoms of physiological leaf curl were associated with Quintec treatments, there was no apparent effect on yield or plant vigor. Overall, Actigard provided the best level of disease control with improved yields.

A new mechanistic model to simulate effects of diurnal temperature oscillations on potato late blight development

A. H. C. VAN BRUGGEN (1), S. K. Shakya (2), H. A. Narouei Khandan (3), J. L. Andrade-Piedra (4), E. M. Goss (3), N. S. Dufault (3)

(1) Univ of Florida, Gainesville, FL, U.S.A.; (2) Oregon State University, Corvallis, OR, U.S.A.; (3) University of Florida, Gainesville, FL, U.S.A.; (4) CIP, Lima, Peru

Phytopathology 105(Suppl. 4):S4.141

Global climate change is associated with increased average temperatures and changes in diurnal temperature ranges. Previously, it was shown that the optimum temperature curve for the latency development rate of Phytophthora infestans (US-23) on potato leaflets (Red Lasoda) was steeper under constant $\left(10-27^{\circ} \mathrm{C}\right)$ than under oscillating temperatures (with amplitudes of $5^{\circ} \mathrm{C}$ and $10^{\circ} \mathrm{C}$ ) in a growth chamber. The optimum curves for lesion growth rate, infection efficiency and sporulation were increased under oscillating temperatures with amplitude of $5^{\circ} \mathrm{C}$ but decreased with amplitude of $10^{\circ} \mathrm{C}$ compared to constant temperatures. Using these results, a mechanistic model (BLIGHTSIM) was developed to simulate late blight under oscillating versus constant temperatures, and predict potential late blight outbreaks under climate change. BLIGHTSIM is a modified Susceptible (S), Latent (L), Infectious (I) and Removed (R) compartmental model with hourly temperature and relative humidity as input variables. Parallel box car trains were introduced in the model to simulate and limit lesion growth. The model was calibrated with data obtained from growth chamber experiments and validated with field data from Ecuador. The model provided a good fit $\left(\mathrm{R}^{2}\right.$ of regression of simulated on observed data) to both growth chamber and field data. BLIGHTSIM will be incorporated in a potato growth model and used to study effects of changes in average temperature and diurnal oscillations on potato late blight.

Characterization and comparative analysis of disease resistance genes from the genomes of rosaceous species using the RosaR80 framework L. VAN ECK (1), J. M. Bradeen (1)

(1) University of Minnesota, St. Paul, MN, U.S.A.

Phytopathology 105(Suppl. 4):S4.141

The rapid increase in the number of publicly available, high quality genomes for species in the Rosaceae promises to advance gene discovery and targeted allele mining for plant improvement. We extracted and annotated NB-LRR genes from the genome sequences of apple, peach and strawberry using the RosaR80 framework, which defines homology groups at a threshold of $80 \%$ nucleotide identity for the NB domain. A total of 1,560 NB DNA sequences were assigned to 273 distinct RosaR80 lineages. Allele diversification patterns and $R$ locus attributes across species were visualized from phylogenetic analysis of this dataset. $R$ genes have diversified significantly between the species analyzed, with widely varying representation across the three genomes. Apple accounts for $42 \%$ of species-specific RosaR 80 lineages, and also shares 53 lineages with peach, consistent with current phylogeny. Only five lineages are shared between all three species. Patterns of $R$ gene evolution suggest that, in response to pathogens, strawberry evolved broad representation of distinct $R$ gene lineages with low levels of allelic diversification, while peach and especially apple exhibit high levels of allelic diversification within several lineages. In particular, members of a large apple-specific lineage seem to be linked to mapped genomic positions of apple scab resistance. We speculate that these diversified species-specific lineages are actively evolving in response to selection by pathogens.

Microsatellite characterization of Penicillium digitatum, causal agent of green mold of citrus

E. Varady (1), G. Vidalakis (1), G. W. DOUHAN (1)

(1) University of California, Riverside, CA, U.S.A.

Phytopathology 105(Suppl. 4):S4.141

Penicillium digitatum $(P d)$ is one of the most important post harvest pathogens of citrus on a global scale causing significant annual losses due to fruit rot. However, little is known about the diversity of $P d$ populations both within the field and subsequently after processing where significant post harvest treatments for control could influence the population dynamics. The genome of $P d$ was recently sequenced, providing an opportunity to determine the microsatellite distribution within $P d$ to develop markers that could be valuable tools for studying the population biology of this pathogen. In the analyses, mono, di, tri, tetra, penta, and hexanucleotide microsatellites within the genome of $P d$ were restricted to 12 repeats for mononucleotides and above while the rest were restricted to 5 repeats and above. A total of 3,134 microsatellite loci were detected; $66.73,23.23,8.23,1.24,0.16$, and $0.77 \%$ were detected as mono, di, tri, tetra, penta, and hexanucleotide repeats, respectively. As consistent with other ascomycte fungi, the genome size of $P d$ does not seem to correlate with the density of microsatellite loci. However, significantly longer motifs of mono, di, and tetranucleotide repeats were identified in $P d$ compared to 10 other published ascomycete species with repeats of over 800, 300, and 900 motifs found, respectively. Specific loci are currently being tested to determine their utility at studying the population genetics of this important pathogen of citrus.

Selecting an inoculation method to assess the efficacy of biological treatments against verticillium wilt of olive trees

A. Varo-Suarez (1), J. MORAL (2), A. Trapero-Casas (1)

(1) Univ. de Cordoba, Cordoba, Spain; (2) Univ De Cordoba, Cordoba, Spain Phytopathology 105(Suppl. 4):S4.141

Verticillium wilt of olive (Olea europaea), caused by the soil-borne fungus Verticillium dahliae, is one of the most serious diseases of this crop because it can kill trees and is difficult or impossible to control. One of the major constraints to develop control measures against this disease is the lack of inoculation methods to evaluate the effectiveness of treatments. In this study, we compared five inoculation methods for screening biological control agents (BCAs): i) planting in naturally infested soil; ii) planting in soil infested with microsclerotia (iii); planting in soils infested with different doses of cornmeal sand medium (CMS) colonized by the fungus; iv) root dipping on a conidial suspension; v) root impregnation with a paste of PDA medium colonized by the fungus. Soil infested with the CMS medium at $10 \% \mathrm{w} / \mathrm{v}$ performed the best, and its effectiveness was further tested in olive plants treated with 6 different BCAs. This assay allowed separate treatments for efficacy, since highly effective treatments ( $>90 \%$ reduction in disease severity) until little or no effective treatments $(<10 \%$ reduction in disease severity). The selected inoculation method is being used to screen more than 200 biological treatments for their efficacy against verticillium wilt in potted olive plants before their final evaluation under field conditions.

Mycotoxins detection on malting barley grains in the high lands of México L. M. VASQUEZ-SILLER (1), M. R. Zamora-Díaz (2), R. Gómez-Mercado (2), E. M. Rodríguez-Campos (1), K. C. Ordóñez-Morales (1)

(1) Universidad Autónoma Agraria Antonio Narro (UAAAN), Saltillo, Mexico; (2) Instituto Nacional de Investigaciones Forestales Agrícolas y Pecuarias (INIFAP), Texcoco, Mexico

Phytopathology 105(Suppl. 4):S4.141 
Several specie of the Fusarium genus infect barley grains used for brewing industry. Such fungi could modify the organoleptic, physical and chemical traits of beer, affecting its quality for industry and its safety for human consumers, because they can produce mycotoxins (secondary metabolites generated by such fungi) associated to human and livestock diseases. Mycotoxins content on stored grain of two malting barley varieties, Adabella and Esmeralda, from five barns in Hidalgo State of México were measured. Grains mixed samples were made from six points taken at 1 and $1.5 \mathrm{~m}$ depths in each variety per barn. Working samples of 200 seeds were microbiologically analyzed and toxigenic fungi counted to know which mycotoxin could be present for further analysis. Fusarium graminearum and Fusarium verticillioides were detected at an average of 1.8 and $1.4 \%$ respectively. Thus, deoxynivalenol and total fumonisins were determined using enzyme-linked immunosorbent assay (ELISA) kits (Romer Labs ${ }^{\circledR}$ ), on extracts of $20 \mathrm{~g}$ from $1.5 \mathrm{~kg}$ of milled grains mixed samples. Significant differences were observed among levels of deoxynivalenol and fumonisins detected in the barns with a general average at $0.12 \mathrm{ppm}$ and $0.07 \mathrm{ppm}$ respectively. The deoxynivalenol detected level could be considered safety if comparing with $0.5 \mathrm{ppm}$ of this mycotoxin, which is the standard detection level adopted by buyers of malting barley in the United States of America.

Distribution of ' $\mathrm{Ca}$. Liberibacter asiaticus' in roots of sour orange rootstock grafted with sweet orange and in leaves of grapefruit trees O. Vazquez (1), E. S. Louzada (1), G. Yanev (2), M. Devanaboina (2), M. KUNTA (1)

(1) Texas A\&M University Kingsville, Weslaco, TX, U.S.A.; (2) The University of Texas - Pan American, Edinburg, TX, U.S.A.

Phytopathology 105(Suppl. 4):S4.142

In August 2013, five naturally infected huanglongbing (HLB)-asymptomatic Valencia sweet orange trees grafted onto sour orange rootstock were uprooted and a total of 160 root samples were collected from horizontally growing roots starting at the trunk and extending to 3.6 meters, and vertically up to 1.5 meters. Similarly, a total of 133 symptomatic leaf samples were collected from four Rio Red grapefruit trees on sour orange rootstock including four quadrants of North, East, South, and West directions. The distances for these symptomatic leaves ranged from $15 \mathrm{~cm}-245 \mathrm{~cm}$ from the trunk. To detect the presence of Candidatus Liberibacter asiaticus (Clas) in these tissues, quantitative PCR and conventional PCR assays were performed. For leaf samples, the ANOVA test indicates overall statistically significant (p-value 0.0026) differences in mean number of bacteria cells with respect to distance and direction. In direction North, where the number of Clas cells detected was significantly higher than in directions West and South, titers was observed in distance ranging from 90 to $180 \mathrm{~cm}$ with highest frequency in the intervals 90 $-115 \mathrm{~cm}$ and $130-180 \mathrm{~cm}$ from the trunk. The bacterial concentrations for horizontal roots were higher compared to the vertical roots. The nucleotide sequences obtained for root and leaf samples shared $98.4-100 \%$ identity among themselves. BLASTn analysis for the sequences showed $100 \%$ identities with several Clas nucleotide sequences.

\section{Mapping the mitochondrial genomes of seven anastomosis groups of Rhizoctonia solani}

R. Verma (1), D. LAKSHMAN (2), D. Roberts (3), I. Misner (4), N. Alkharouf (5), A. Pain (6)

(1) USDA-ARS, FNPRU, Beltsville, MD, U.S.A.; (2) USDA ARS, FNPRU, Beltsville, MD, U.S.A.; (3) USDA-ARS, SASL, Beltsville, MD, U.S.A.; (4) University of Maryland, College Park, College Park, MD, U.S.A.; (5) Towson University, Towson, MD, U.S.A.; (6) King Abdullah University of Science and Technology, Thuwal, Saudi Arabia

Phytopathology 105(Suppl. 4):S4.142

The soilborne, basidiomycetous pathogen Rhizoctonia solani (sensu lato) constitutes at least 13 anastomosis groups (AGs) and several subgroups. Isolates of some $R$. solani AGs are important plant pathogens of economically important crops like vegetables, ornamentals, cereals, turfgrasses, fiber crops, and forest trees. AGs are genetically isolated populations showing degrees of host-specificity, ecological adaptability, sensitivity to fungicides and pathogenicity. The draft genome sequences of $R$. solani AG1-1A, AG1-1B, and AG8 have been published. However, the mitochondrial draft genomes of only the AG3 and AG1-B are publicly available and were used as reference genomes in this analysis. The mitochondrial genomes of AG1-1C, AG2, AG3T5, AG4-R118-11, AG5, AG6, and AG8 were assembled from Illumina paired-end sequences and compared for their gene contents and synteny. The mitochondrial genome sequences of the $R$. solani AGs were estimated to vary from $2 \%$ to $5 \%$ of the respective total genome sequences. This investigation will elucidate mitochondrial genome structure, organization, and the molecular evolution of those selected AGs of the broad group of plant pathogens.
Effects of wheat varietal resistance level and rainfall characteristics on splash dispersal of septoria tritici blotch

T. Vidal (1), P. Lusley (2), C. GIGOT (3), M. Leconte (4), F. Suffert (4), C. de Vallavieille-Pope (4), L. Huber (5), S. Saint-Jean (5)

(1) AgroParisTech / INRA EcoSys, 78850 Thiverval-Grignon, France; (2) INRA / AgroParisTech EcoSys, Thiverval Grignon, France; (3) Quantitative Biology and Epidemiology Lab, Plant Pathology Department, University of California, Davis, CA, U.S.A.; (4) INRA Bioger, Thiverval-Grignon, France; (5) AgroParisTech / INRA EcoSys, Thiverval-Grignon, France

Phytopathology 105(Suppl. 4):S4.142

Septoria tritici blotch is an important splash-dispersed disease, causing high yield losses in Europe. Plant disease propagation results from spore dispersal and susceptibility of plant tissues. An experiment was performed in order to study differents aspects of the disease dispersal cycle. Three wheat varieties with contrasted resistance levels were grown in greenhouse conditions until flowering. Plant canopies of each variety received rains of two different raindrop diameter distributions generated by a rain simulator. A linear inoculum source consisting of an aqueous suspension of spores was placed in the middle of each canopy. Horizontal and vertical spore fluxes were measured using traps composed of microscope slides. Varietal resistance was assessed in parallel. After incubation, leaves sampled in canopies were collected and scanned. Spore traps slides were photographed using a microscope combined with a digital camera. Disease area measurement and automatic spore counting were achieved using an image analysis software. Both disease and spore fluxes decreased with the distance from the inoculum source and lower mean raindrop diameter. Disease levels depended on variety and leaf level. Vertical and horizontal gradients of spore fluxes and disease varied in function of rain type and variety. Combining all these results made it possible to disentangle components of splash-dispersed disease propagation for a single dispersal event.

Characterization of the transcriptional profiling of the migratoy root lesion nematode infection on important crop and floral species

P. VIEIRA (1), S. Wantoch (2), J. Eisenback (3), K. Kamo (2)

(1) Dept. of Plant Pathology, Physiology, and Weed Science, Virginia Tech and Floral and Nursery Plants Research Unit, USDA, BELTSVILLE, MD, U.S.A.; (2) USDA-ARS/US National Arboretum, Floral and Nursery Plants Research Unit, USDA, Beltsville, MD, U.S.A.; (3) Dept. of Plant Pathology, Physiology, and Weed Science, Virginia Tech, Blacksburg, VA, U.S.A.

Phytopathology 105(Suppl. 4):S4.142

Worldwide crop losses due to plant-parasitic nematodes have been estimated at $\$ 118$ billion annually, with Pratylenchus spp. (commonly known as root lesion nematodes) ranking third in terms of economic losses. Global parasitehost transcriptomes constitute an excellent tool to provide a general overview of the molecular dialogue established between the pathogen and the host, and ultimately for the identification of the main host molecular pathways and individual genes involved during the host-pathogen interaction. Local transcriptional changes upon Pratylenchus penetrans infection were evaluated at two time points after nematode infection ( 3 and 7 days), using mRNA-seq analyses in an economically important crop (soybean) and a floral species (Lilium longiflorum). Preliminary data on the soybean data sets allowed the identification of a dynamic expression of genes in infected roots, suggesting a strong involvement of several metabolic pathways such as the phenylalanine and phenylpropanoid biosynthesis. The regulation of plant defense genes, programed cell death, and reduction oxidation genes appear to play an important role during root lesion disease induction. Comparative transcriptome analyses between the obtained results for soybean and lilies will be presented, providing important insights into the molecular mechanisms involved in such nematode-plant interactions.

Development of a quantitative loop-mediated isothermal amplification assay to detect Magnaporthe oryzae airborne inoculum in turf ecosystems C. VILLARI (1), W. F. Mahaffee (2), T. K. Mitchell (1), K. F. Pedley (3), M. Pieck (3), F. Peduto Hand (1)

(1) Department of Plant Pathology, The Ohio State University, Columbus, OH, U.S.A.; (2) USDA ARS, Corvallis, OR, U.S.A.; (3) USDA ARS, Fort Detrick, MD, U.S.A.

Phytopathology 105(Suppl. 4):S4.142

Grey Leaf Spot (GLS) is a detrimental disease of perennial ryegrass caused by a host-specialized form of Magnaporthe oryzae (Lolium pathotype, $M o L$ ). In order to improve turf management, a quantitative loop-mediated isothermal amplification (LAMP) assay coupled with a simple spore trap is being developed to monitor GLS airborne inoculum. LAMP has been shown to be suitable for implementation by practitioners to initiate and time fungicide applications in vineyards. Since the most common conserved regions such as ITS, LSU and Calmodulin lack the needed heterogeneity among the different 
host-specialized forms of M. oryzae, LAMP primers were designed to a nonannotated genomic DNA region unique to $M o L$. Reactions were performed on a Real-Time PCR Detection System and optimized to provide a response in approximately 30 minutes. Quantification of the inoculum was obtained by comparison with a standard curve developed using 6 independent serial spore dilutions. The LAMP assay was specific to the host-specialized forms of $M$. oryzae and was able to detect greater than 500 spores, indicating that it is capable of detecting the pathogen at the very beginning of the disease epidemic. The implementation of this assay could be a useful decision support tool to guide initiation and timing of fungicide applications for GLS management.

A new exclusively genome-based species-independent taxonomic framework for all life forms applied to Pseudomonas syringae

B. A. VINATZER (1), H. A. Elmarakeby (1), A. J. Weisberg (1), C. L. Monteil (1), L. S. Heath (1)

(1) Virginia Tech, Blacksburg, VA, U.S.A.

Phytopathology 105(Suppl. 4):S4.143

Next generation sequencing is revealing an immense genetic diversity of bacteria. In order to classify, describe, and name this diversity, we urgently need a new taxonomic framework that is applicable to all different levels of diversity, i.e., above and below the currently defined species level. Additionally, description and naming of taxonomic groups need to become much faster than the current practice of publishing peer-reviewed descriptions of named bacterial species in order to keep up with the ever-increasing speed of microbial discoveries. We previously proposed to assign to every individual genome-sequenced organism a code, called Life Identification Number (LIN), which can precisely reflect its similarity to all related organisms. Here we go one step further and propose the concept of genome similarity-based taxonomic groups, which we call LIN groups. LIN groups are independent of any species concept and organisms auto-assemble into LIN groups based solely on genome similarity. Genetic, phenotypic, and epidemiological descriptions can be linked to LIN groups whereby type strains provide the connection between LIN groups and named species. We show the potential of the proposed system for the taxonomy of plant pathogenic bacteria and regulatory purposes using publicly available genomes of the plant pathogen Pseudomonas syringae.

\section{Real time imaging of cucurbit infection by Erwinia tracheiphila}

C. M. VRISMAN (1), G. Rajashekara (1), S. A. Miller (1)

(1) Ohio State Univ, Wooster, OH, U.S.A.

Phytopathology 105(Suppl. 4):S4.143

Bacterial wilt, caused by E. tracheiphila, is a major problem of cucurbit crops in the Midwestern US. Strains of E. tracheiphila exhibit preference for the host genus, Cucumis or Cucurbita, from which they were isolated. We used bioluminescent $E$. tracheiphila TedCu10-BL\#9 to visualize bacterial colonization in these plants. The original strain TedCu-10 was isolated from Cucumis sativus. Transformation was performed using electroporation. Second true leaves of Cucumis and Cucurbita plants were inoculated with 100 $\mu 1$ of a TedCu10-BL\#9 suspension containing $10^{8} \mathrm{CFU} / \mathrm{ml}$. Plants were imaged daily using IVIS Lumina System III. Colonization of bioluminescent TedCu10-BL\#9 inside Cucumis plants (melon and cucumber) was observed 1 day post inoculation (DPI). At 2 DPI bacterial movement down the main stem was observed. Colonization of roots was also detected at 2 DPI. Colonization of the entire plant was visualized 4 DPI and plants were wilted at 6 DPI. No productive infection of Cucurbita plants (squash and pumpkin) was observed. In the future, nectaries, stems and roots will be inoculated with bioluminescent TedCu10-BL\#9 to determine the efficiency of these routes of infection. Host preference of a Cucurbita-derived strain transformed with the lux genes will also be assessed.

Evidence that Tomato mottle leaf curl virus from Northeastern Brazil is an indigenous New World monopartite begomovirus

S. VU (1), T. Melgarejo (2), L.-F. Chen (1), J. de Souza (1), M. Macedo (1), A. Nagata (3), R. L. Gilbertson (1)

(1) University of California, Davis - Department of Plant Pathology, Davis, CA, U.S.A.; (2) Departamento de Fitopatologia, Universidad Nacional Agraria La Molina, Lima, Peru; (3) Embraba Vegetables, Brasilia, DF, Brazil Phytopathology 105(Suppl. 4):S4.143

A diversity of whitefly-transmitted begomoviruses (family Geminiviridae, genus Begomovirus) infect many crops in Brazil. In 2014, tomatoes with begomovirus-like symptoms (stunting and upcurled leaves with swollen veins) and high populations of whiteflies were observed in the State of Piaui, Brazil. Leaf tissues from twenty symptomatic plants were applied onto FTA cards, total genomic DNA extracted and PCR analysis performed with degenerate DNA-A and DNA-B primers. The expected $\sim 1.2 \mathrm{~kb}$ DNA-A fragment was amplified from all samples, whereas no DNA-B fragment was amplified. Sequence analysis revealed the DNA-A fragment had highest identity (>90\%) with the DNA-A component of Tomato mottle leaf curl virus (ToMoLCV). The full-length genomic DNA of one isolate (C2) was generated by RCA, digested with NdeI and cloned. The C2 genomic DNA is 2630 nucleotides, shares $94 \%$ sequence identity to ToMoLCV-PE:BR and has a genome organization typical of the DNA-A components of New World (NW) bipartite begomoviruses. Phylogenetic analyses placed it in a distinct clade with other ToMoLCV isolates from Brazil. To confirm the monopartite nature of the virus and fulfill Koch's postulates, a multimeric $\mathrm{C} 2$ clone was generated and transformed into Agrobacterium tumefaciens. Tomatoes agroinoculated with the $\mathrm{C} 2$ multimeric clone developed symptoms similar to those observed in the field. Together, these results establish that ToMoLCV is the first NW monopartite begomovirus from Brazil.

The occurrence of Tobacco ringspot virus in a wine grape (Vitis vinifera L.) cultivar in Washington State

L. Walker (1), B. BAGEWADI (1), A. Schultz (2), R. A. Naidu (3)

(1) Department of Plant pathology, Washington State University, Prosser, Prosser, WA, U.S.A.; (2) Harttrup Frams Inc., Wapato, WA, Wapato, WA, U.S.A.; (3) Department of Plant Pathology, Washington State University, Prosser, Prosser, WA, U.S.A.

Phytopathology 105(Suppl. 4):S4.143

During a survey of vineyards in Washington State, a vineyard block planted with a red-berried wine grape (Vitis vinifera $\mathrm{L}$.) cultivar was observed with vines showing symptoms of grapevine fanleaf disease. The symptoms consisted of severe leaf distortion, short canes with reduced internodes and overall stunting of affected vines. Symptomatic vines produced smaller clusters with berries of uneven size and ripeness compared to clusters from non-symptomatic vines. Leaf samples from symptomatic and nonsymptomatic vines were tested by enzyme-linked immunosorbent assay using antibodies specific to nematode-transmitted viruses known to infect grapevines. Symptomatic samples tested positive only for Tobacco ringspot virus (TRSV; genus Nepovirus, family Secoviridae). Total nucleic acids from symptomatic and non-symptomatic leaves were subjected to reverse transcription-polymerase chain reaction using primers specific to RNAdependent RNA polymerase and coat protein genes encoded by the RNA 1 and 2 segments of TRSV, respectively. The virus-specific DNA fragments of expected size were amplified only from symptomatic samples. The amplicons were cloned and nucleotide sequence determined. A comparison of these sequences with corresponding sequences from GenBank confirmed the presence of TRSV. Cucumber baiting assays indicated the potential spread of TRSV by nematodes. A preliminary analysis of grapes indicated significant negative impact of TRSV on fruit quality attributes.

Validation of transcript SSR markers in Pseudoperonospora cubensis from commercial and non-commercial cucurbits

E. C. WALLACE (1), L. M. Quesada-Ocampo (1)

(1) North Carolina State University, Raleigh, NC, U.S.A.

Phytopathology 105(Suppl. 4):S4.143

The proccess of Simple Sequence Repeat (SSR) or microsatellite identification can be streamlined in non-model organisms due to increased availability of genomes and transcriptomes. SSRs derived from transcripts in particular show promise as useful markers due to their high polymorphism and transferability across species. Informative molecular markers are needed for understanding more about the population structure of Pseudoperonospora cubensis, the foliar pathogen causing cucurbit downy mildew. This study aimed to identify SSRs found in expressed regions of the P. cubensis genome. The MIcroSAtellite identification tool (MISA) was used to extract locations and frequencies of 2-6 bp repeats and reported that $10 \%$ of the 23,522 sequences examined contained SSRs. Primer3 identified 2,088 primers to amplify detected SSRs. A subset representative of the SSR motifs found was screened against a diverse panel of $P$. cubensis isolates from commercial and non-commercial cucurbit hosts as well as two $P$. humuli (hop downy mildew) isolates. Of the 100 SSRs tested, sixteen SSR loci were polymorphic across downy mildew isolates and nine differentiated $P$. cubensis isolates from $P$. humuli isolates. Markers displaying polymorphism were further investigated via fragment analysis. These results demonstrate that MISA and publically available transcriptomes provide a vast source of genetic markers that can be used to characterize populations of $P$. cubensis and possibly other downy midlews.

Fatty acid profiling to characterize California strains of Xylella fastidiosa C. WALLIS (1), J. Chen (1)

(1) USDA ARS PWA, Parlier, CA, U.S.A.

Phytopathology 105(Suppl. 4):S4.143 
Different strains of Xylella fastidiosa cause diseases such as Pierce's disease of grapevine, citrus variegated chlorosis, and bacterial leaf scorch of hardwoods. However, more research is needed to better define subspecies and strains of $X$. fastidiosa in order to improve both regulations concerning this bacteria and management of diseases it causes. Traditional, cell membrane fatty acid profiling has proven to be useful as a phenotypical characteristic to define bacterial species and subspecies. This study was conducted to develop fatty acid profiling techniques for $X$. fastidiosa to complement ongoing genome-based approaches to better distinguish subspecies and strains. Results have so far verified differences in fatty acid composition between $X$. fastidios $a$ ssp. fastidiosa and ssp. multiplex. There also was some evidence suggesting greater diversity than already established for $X$. fastidiosa based upon additional, smaller-scale differences in fatty acid composition which were dependent on location and host of isolation. Completion of this research will improve understanding about $X$. fastidiosa evolution, with potential impacts on regulatory efforts and management of Xylella-caused diseases.

Soybean cyst nematodes of Ohio: Deciphering mechanisms of virulence E. WALSH (1), B. Cassone (2), A. Grenell (3), T. Miller (2), C. G. Taylor (2) (1) Ohio State Univ, Wooster, OH, U.S.A.; (2) Ohio State University, OARDC, Wooster, OH, U.S.A.; (3) The College of Wooster, Wooster, OH, U.S.A.

Phytopathology 105(Suppl. 4):S4.144

Soybean cyst nematodes (SCN; Heterodera glycines) are the most yieldreducing pest of soybean in North America. While the practice of rotating with non-host crops remains the most effective control option, the demand for soybean production limits this practice. Resistant soybean cultivars are widely utilized for management purposes, however SCN populations are quickly adapting to overused sources of resistance. Counties across Ohio were surveyed for SCN populations and their virulence against the available sources of resistance was tested with the HG Type assay. Of the populations tested to date, $96 \%$ are infective on PI 88788 , the most prevalent source of resistance in commercial varieties. The objective of this study is to determine how SCN populations have overcome this source of resistance. Nine SCN populations from across Ohio were chosen that vary in their HG Type and/or level of virulence. These populations were grown on both PI 88788 and a susceptible cultivar, Lee 74 . Female SCN were harvested 15 days after inoculation, total RNA was extracted, and submitted for sequencing on the Illumina Hi-Seq 2500. We hypothesize that populations virulent on PI 88788 will share expression patterns not present in avirulent populations, and that expression differences between virulent populations on PI 88788 versus the susceptible check will provide insight into what mechanisms may be important to overcoming this source of resistance.

Stripe rust epidemics of wheat and barley and races of Puccinia striiformis identified in the United States in $\mathbf{2 0 1 4}$

A. WAN (1), X. Chen (2)

(1) Washington State University, Pullman, WA, U.S.A.; (2) USDA ARS, Pullman, WA, U.S.A.

Phytopathology 105(Suppl. 4):S4.144

In 2014, stripe rust of wheat, caused by Puccinia striiformis f. sp. tritici (Pst), occurred in the lowest level and distributions in the last decade due to the low levels of inoculum in the spring and the drought and hot weather conditions in the spring and summer. Stripe rust of barley, caused by P. striiformis f. sp. hordei $(P s h)$, was reported only in California, Oregon and Washington continually at low levels. Stripe rust samples collected from 14 states were tested on 18 wheat and 12 barley differentials for identifying Pst and Psh races, respectively. From recovered $18 P s h$ samples, five previously existing $P$ sh races were detected, of which PSH-33 (virulent only on Topper and Abed Binder 12 of the differentials) and PSH-48 (virulent only on Topper) were predominant with frequencies of $44 \%$ and $33 \%$, receptively. From 261 viable Pst samples, 33 Pst races were detected including 5 new races (PSTv-119, PSTv-120, PSTv-121, PSTv-122, and PSTv-123). PSTv-52 (34\%), PSTv-37 (18\%), PSTv-4 (9\%), PSTv-79 (7\%), and PSTv-48 (6\%) were the top five frequent races. PSTv-52 and PSTv-37 were detected in every epidemiological regions, while PSTv-4, PSTv-79 and PSTv-48 were detected only in the western U.S. High virulence frequencies were detected for $Y r 6, Y r 7, Y r 8, Y r 9$, Yr17, Yr27, Yr43, Yr44, and YrExp2; moderate for Yr1, YrTr1, and YrTye; low for $Y r 10, Y r 24, Y r 32$, and $Y r S P$; and none for $Y r 5$ and $Y r 15$.

Studying aeciospores and survival of teliospores revealed no sexual reproduction of Puccinia striiformis f. sp. tritici in the Pacific Northwest M. WANG (1), A. Wan (1), X. Chen (2)

(1) Washington State University, Pullman, WA, U.S.A.; (2) USDA ARS, Pullman, WA, U.S.A.

Phytopathology 105(Suppl. 4):S4.144
Puccinia striiformis f. sp. tritici (Pst), causing wheat stripe rust, can infect barberry under controlled conditions, but it is not clear if it can infect barberry under natural conditions like P. graminis f. sp. tritici (Pgt), the stem rust pathogen. In 2010-2013, aeciospores from individual aecia collected from barberry were inoculated on susceptible wheat 'Nugaines', and identified using internal transcribed spacer (ITS) markers and sequences. Of 920 aecia tested, $93 \%$ were $P g t ; 7 \%$ other $P$. graminis formae speciales; and none was Pst. In these years, teliospore survival was monitored from telial formation in the summer until the following summer by germination testing of periodically sampled telia. Pst teliospores readily germinated in the summer when separated from the wheat plant tissue and incubated under optimal moist and temperature conditions. The germination rate of Pst teliospores decreased from $50-90 \%$ in July-August, when susceptible young barberry leaves are not and moist conditions are hardly available in the region, to less than $1 \%$ in the following March; and no any viable teliospores were observed after May when susceptible young barberry leaves were produced. In contrast, Pgt teliospores that formed in late July to August remained in dormancy until the following spring, and the germination rate gradually increased from $1 \%$ in February to $90 \%$ in May to infect susceptible young barberry leaves. Thus, Pst cannot infect barberry in the Pacific Northwest.

Phylogenetic and morphological identification of the novel pathogen of Rheum palmatum leaf spot in Northwest China

Y. WANG (1), A. O. Charkowski (2), C. X. Rong (3), C. Y. Zeng (1), C. H. Gang (1)

(1) Gansu University of Traditional Chinese Medicine, Lanzhou, China; (2) University of Wisconsin-Madison, Madison, WI, U.S.A.; (3) Gansu Agricultural University, Lanzhou, China

Phytopathology 105(Suppl. 4):S4.144

A new spot disease was observed on leaves of Rheum palmatum (Chinese rhubarb) in Northwest China (Gansu Province) starting in 2005. A Septorialike fungus was isolated and completion of Koch's postulates confirmed that the fungus was the casual agent of the leaf spot disease. Morphology and molecular methods were combined to identify the pathogen. The fungus produced conidiomata pycnidia and the conidia were 2-5 septate, 61.2-134.1 $\mu \mathrm{m}$ in length and 3.53-5.3 $\mu \mathrm{m}$ in width, which is much larger than the known Spetoria species that infects Polygonaceae species. Phylogenic analysis of the internal transcribed spacer region (ITS) confirmed that this Septoria-like fungus is within the Septoria genus but distinct from known Septoria species. Together, these morphological and phylogenetic data support that the $R$. palmatum infecting Septoria strain is a newly-described plant pathogenic species.

Pseudoperonospora saposhnikovia nov. sp on Saposhnikovia divaricata

Y. WANG (1), A. O. Charkowski (2), L. Jin (1), Y. Y. Zhang (3), T. Zhu (1)

(1) Gansu University of Traditional Chinese Medicine, Lanzhou, China; (2) University of Wisconsin-Madison, Madison, WI, U.S.A.; (3) Longxi Agricultural Science and Technology Center, Lanzhou, China

Phytopathology 105(Suppl. 4):S4.144

The new oomycete species Pseudoperonospora saposhnikovia, which causes powdery mildew on Saposhnikovia divaricata (Apiaceae), a medicinal plant native to China, is described, illustrated, and differentiated from other Pseudoperonospora spp.

Evaluation of isothermal amplification methods for rapid HLB detection J. Wang (1), Z. LIU (1), G. Wei (1), W. Li (1), M. Nakhla (1)

(1) USDA APHIS PPQ CPHST, Beltsville, MD, U.S.A.

Phytopathology 105(Suppl. 4):S4.144

HLB (Huanglongbing) is one of the most devastating citrus diseases threatening the citrus industry. Various methods based on the real-time qPCR and conventional PCR have been successfully developed and are currently in use for HLB detection. Recently isothermal amplification techniques such as the loop-mediated isothermal amplification (LAMP) and recombinase polymerase amplification (RPA) have been used as simple, fast and on-site detection tools for plant pathogens with portable equipment. We are evaluating three isothermal amplification detection techniques (LAMP SmartDART, AmplifyRP Acceler8 kit and DNAble kit) focusing on the rapid detection of Candidatus Liberibacter asiaticus (CLas), especially in field settings. DNAs extracted from HLB-infected citrus plants grown in the greenhouse were used to determine analytic sensitivity and specificity. The evaluation using environmental samples will also be presented to assess the simplicity and reliability of those techniques. This evaluation represents a valuable contribution to the usage of rapid and reliable HLB detection techniques. 
Quick survey of avirulence genes in field isolates of Magnaporthe oryzae in the past 60 years

X. WANG (1), T. Bianco (2), M. Lin (2), Y. Wamishe (3), Y. Jia (2)

(1) Rice Research and Extension Center, Division of Agriculture, University of Arkansas, Stuttgart, AR, U.S.A.; (2) USDA, ARS, Dale Bumpers National Rice Research Center, Stuttgart, AR, U.S.A.; (3) Cooperative Extension Service, Division of Agriculture, University of Arkansas, Stuttgart, AR, U.S.A.

Phytopathology 105(Suppl. 4):S4.145

Avirulence $(A V R)$ genes in Magnaporthe oryzae determine deployment of effective corresponding resistance $(R)$ genes. Instability of $A V R$ genes is the major cause for resistance breakdown. Information on the presence or absence (P/A) of $A V R$ genes can be used as a predictor of the stability of deployed $\mathrm{R}$ genes in different rice growing regions. The $\mathrm{P} / \mathrm{A}$ of $A V R$ genes, $A V R-P i 9$, AVR-Pital, AVR-Pik, AVR -Pizt, and ACE1 (AVR-Pi33), in 1000 field isolates collected from the southern USA were examined using $A V R$ gene specific PCR primers. M. oryzae isolates stored on desiccated filter papers were used directly for PCR amplification. This survey revealed $74 \%, 65 \%, 82 \%, 20 \%$, and $76 \%$ of the tested isolates carrying AVR-Pi9, AVR-Pital, AVR-Pizt, AVR$P i k$, and $A V R-P i 33$, respectively. The results indicated that $R$ genes, $P i-9, P i-$ $t a, P i-k, P i-z t$, and $P i 33$, are still effective in preventing rice blast disease in the USA. However, the effectiveness of the resistance can vary by variety grown, location and season. The underlying mechanisms of $A V R$ gene instability are being investigated and results will be reported.

\section{Integration of apple rootstock genotype with reduced Brassica seed meal} application rates for replant disease control

L. WANG (1), M. Mazzola (2)

(1) Washington State University, Wenatchee, WA, U.S.A.; (2) USDA-ARS, Wenatchee, WA, U.S.A.

Phytopathology 105(Suppl. 4):S4.145

Pre-plant soil application of Brassica seed meal (SM) formulations can provide fumigant level control of apple replant disease. However, due to high cost of the SM treatment relative to non-tarped soil fumigation, reduced application rates would likely accelerate commercial adoption of this technology. A Brassica juncea/Sinapis alba (1:1) SM formulation was applied to replant orchard soil at 1, 2 or 3 (standard) ton per acre. Regardless of rate, all SM treatments effectively suppressed Pratylenchus penetrans root densities and increased tree biomass relative to the no-treatment control for all rootstock genotypes. The replant susceptible rootstocks M9 and MM106 showed a general increase in tree growth at increasing SM rates; however, there was no additional increase in growth when application rate was elevated from 2 ton to 3 ton when used in conjunction with the replant tolerant rootstocks G41 and G210. After five months growth, rhizosphere microbial communities were similar for the control and 1 ton per acre SM treatment, but the 2 and 3 ton per acre rate possessed communities that were similar to each other but distinct from the control. These findings suggest that a reduced SM application rate when employed with the appropriate apple rootstock genotype may provide sufficient replant disease control.

Detection of viable Xanthomonas fragariae cells in strawberry using propidium monoazide and long-amplicon quantitative PCR

H. WANG (1), W. W. Turechek (2)

(1) Univ of Florida/USDA-ARS, Ft Pierce, FL, U.S.A.; (2) USDA-ARS, Ft. Pierce, FL, U.S.A.

Phytopathology 105(Suppl. 4):S4.145

Xanthomonas fragariae causes angular leaf spot disease in strawberry. Pathogen survival is dependent upon its association with host tissue. Consequently, transmission to field production sites occurs almost exclusively through infected or contaminated planting stock. Thus, it is important to estimate the proportion of plants containing viable bacterial cells to aid the decision process for disease management. Current diagnostic tools for $X$. fragariae lack the capability to differentiate living from dead cells, so we developed a real-time PMA-qPCR protocol to facilitate this need. Propidium monoazide (PMA) is a DNA-binding dye that can enter the broken membrane of dead cells and bind to its remnant DNA, thus blocking the DNA from amplification in downstream PCR. To increase the likelihood of PMA binding to amplification sites, primers were designed to facilitate long-amplicon qPCR. The procedure was tested with DNA from PMA-treated samples with mixtures of living and heat-killed cells in defined proportions, and with amplicons of different sizes. Results showed that PMA-qPCR conducted with the largest amplicon $(\sim 1 \mathrm{~kb})$ provided the best efficiency for differentiating viable from dead $X$. fragariae cells. This assay will allow a more accurate characterization of the proportion of plants with living $X$. fragariae, and may contribute to improved disease control.
Identification and confirmation of root-knot nematode and Fusarium wilt disease resistance traits in cotton substitution lines

C. WANG (1), M. Ulloa (2), S. Saha (3), D. M. Stelly (4), J. N. Jenkins (3), P. A. Roberts (5)

(1) Northeast Institute of Geography and Agroecology, Chinese Academy of Sciences, Harbin, China; (2) USDA-ARS, Plant Stress and Germplasm Development Research, Lubbock, TX, U.S.A.; (3) USDA-ARS, Genetics and Precision Agriculture Research, Mississippi State, MS, U.S.A.; (4) Texas A\&M University, College Station, TX, U.S.A.; (5) University of California, Riverside, Riverside, CA, U.S.A.

Phytopathology 105(Suppl. 4):S4.145

A recombinant inbred line population between Upland Gossypium hirsutum TM-1 and Pima G. barbadense 3-79 was used previously to identify QTL determining response to both root-not nematode and Fusarium wilt races 1 (FOV1) and 4 (FOV4), economically important diseases in cotton. To confirm QTLs and to identify new QTLs, 32 cotton chromosome substitution (CS) lines developed in the TM-1 background including 25 CS-B lines from the 379 donor were used to examine phenotypic response to both diseases in inoculation assays. Three CS-B lines $(04,22 \mathrm{sh}, 18)$ had lower nematodeinduced root-galling indices than TM-1, 3-79. The previously identified QTLs $\mathrm{Mi}-\mathrm{GI}_{b}-\mathrm{CO} 4_{1}$ and $\mathrm{Mi}-\mathrm{Gl}_{b}-\mathrm{C22}{ }_{1}$ associated with root-galling response in 3-79 were located in the substitution regions of CS-B04 and 22sh, indicating that these 3-79 substitution chromosomes caused higher resistance. CS-B14sh supported greater nematode egg numbers due to 3-79 chromosome introgression, which agreed with QTL $M i-E G R_{h}-C 14 s h$ association and egg production in TM-1. In the FOV1 test, CS-B16 had less severity of wilt disease symptoms than the TM-1 parent, which confirmed the major FOV1 resistance QTL FOV1-C16 in 3-79. CS-BNTN17-11 and CS-B17 showed greater FOV4 infection symptoms, which confirmed the localization on chromosome 17 of an additional FOV4 resistance gene from Upland TM-1. This work demonstrated that CS lines are a valuable genetic resource for localizing disease resistance trait determinants in cotton.

Global population genetic diversity and phylogenetic analysis of Phytophthora palmivora

J. WANG (1), M. D. Coffey (2), E. M. Goss (1)

(1) University of Florida, Gainesville, FL, U.S.A.; (2) University of California

Riverside, Riverside, CA, U.S.A.

Phytopathology 105(Suppl. 4):S4.145

Phytophthora palmivora (Butler) is an important heterothallic oomycete plant pathogen found throughout the tropics and subtropics. It has a wide host range and causes substantial losses to fruit and vegetable crops. High genetic diversity was previously found in isozymes and RAPDs genotyped in a collection of isolates from Southeast Asia, supporting the hypothesis of Southeast Asia as the center of origin for P. palmivora. To date, the diversity and phylogeny of the global population of $P$. palmivora have not been investigated by gene sequencing methods. We applied multilocus sequence typing to $P$. palmivora isolates collected around the world. We sequenced one mitochondrial locus (cox2 and spacer) and three nuclear genes ( $\beta$-tubulin, pelota and trp 1 ). These data produced considerable genetic variation and heterozygosity. Given the high observed heterozygosity, we inferred haplotypes for each of the three nuclear genes for further analysis. The inferred alleles of isolates generally segregated into different phylogenetic groups, which indicates that these alleles have experienced significantly different evolutionary histories. We found higher genetic diversity, on average, in the Asia-Pacific than in the Americas. These data will contribute to the clarification of contemporary global population genetic diversity and historical migration routes of this major tropical pathogen.

The effect of relative humidity on colonization of citrus twigs by Phyllosticta citricarpa, the citrus black spot pathogen

N. Y. WANG (1), M. M. Dewdney (1)

(1) University of Florida, Lake Alfred, FL, U.S.A.

Phytopathology 105(Suppl. 4):S4.145

Citrus black spot, caused by $P$. citricarpa, in Florida has led to substantial economic loss to local growers since March 2010. In the subtropical conditions of Brazil, conidia produced on dead twigs were demonstrated to be an important inoculum source of $P$. citricarpa. While alternative wetting and drying cycles are critical for pseudothecia and ascospore formation of $P$. citricarpa on leaf litter, conditions needed for pycnidia and conidia production on twigs remain unclear. To determine the conditions for pycnidia and conidia formation of $P$. citricarpa, inoculated twigs were incubated at various relative humidities (RH; $43,72,82$, and $100 \%$ ) at $24^{\circ} \mathrm{C}$ under a 12 -h light cycle. The formation of pycnidia and conidia was observed at 3,5 , and 7 weeks postinoculation at 82 and $100 \% \mathrm{RH}$ but not at 43 and $72 \% \mathrm{RH}$, indicating that $\mathrm{RH}$ from 82 to $100 \%$ would be suitable for pycnidia and conidia production of $P$. 
citricarpa. While no pycnidia or conidia were observed on twigs at 43 and $72 \% \mathrm{RH}$, approximately $10 \mathrm{pg}$ of $P$. citricarpa DNA per twig was detected using qPCR, implying that $P$. citricarpa may survive under such conditions. Given that frequent summer rains and high \% RH also occur in Florida, the importance of conidia produced on dead twigs should not be underestimated. The information obtained could improve our understanding of conditions required for inoculum production of $P$. citricarpa on twigs, ultimately leading to the development of better control techniques.

Population genetic analyses of Fusarium virguliforme reveal the population structure of $F$. virguliforme isolates from North and South America

J. WANG (1), J. L. Jacobs (1), M. I. Chilvers (1)

(1) Michigan State University, East Lansing, MI, U.S.A.

Phytopathology 105(Suppl. 4):S4.146

Fusarium virguliforme is a destructive plant pathogen responsible for soybean sudden death syndrome (SDS), which results in severe yield loss of soybean. SDS was first reported in Arkansas in the 1970s, since then it has been reported in surrounding states. In South America, SDS was first discovered in Argentina and Brazil in the early 1990s and subsequently in other South American countries. In recent years, F. virguliforme has been detected in South Africa and Malaysia; however, little is known about the migration history of this pathogen. In this study, we conducted a population genetic analysis to demonstrate the genetic diversity and population structure of $F$. virguliforme isolates collected from multiple sites in the US and Argentina using microsatellite markers. Principal component analysis demonstrated that $F$. virguliforme isolates from Argentina clustered distinctly from the U.S. F. virguliforme isolates, with the exception of the Arkansas population, which overlapped with both Argentina and U.S. isolates. In a structure analysis, there was a clear distinction of population structure composition between Argentina and U.S. F. virguliforme populations, aside from the Arkansas population, which comprised both genotypes present in Argentina and US populations. In addition, the genetic diversity indices of the Arkansas populations were higher than the rest of the populations. These results suggest migration of $F$. virguliforme between continents.

\section{Greenhouse hemp in Kentucky exhibits many common diseases}

N. WARD-GAUTHIER (1), J. Beale (2), B. Amsden (2), E. Dixon (2)

(1) Univ of Kentucky, Lexington, KY, U.S.A.; (2) University of Kentucky, Lexington, KY, U.S.A.

Phytopathology 105(Suppl. 4):S4.146

Section 7606, Legitimacy of Industrial Hemp Research, of the 2013 Farm Bill authorizes university and state departments of fourteen states to grow industrial hemp (Cannabis sativa $\mathrm{L}$. varieties with less than $3 \%$ THC). In 2014, licensed farmers in Kentucky grew industrial hemp in fields for fiber and in greenhouses for medicinal extracts. These medicinal cultivars contain low THC and high cannabidiol, a cannabinoid that is reported to reduce epileptic seizures. Industrial hemp has not been grown in the US since 1957, and disease surveys have not been reported in greenhouse environments. On multiple occasions during 2014, samples of suspect diseases and unusual symptoms were collected from two greenhouses in eastern and central Kentucky. The following diseases were confirmed by morphological characteristics or PCR amplification: powdery mildew, botrytis gray mold in buds, botrytis canker, Fusarium canker, and Pythium root rot. Analysis of disease occurrence in these systems will make risk assessments more effective and serve as the first step for disease management protocols and educational programs.

Identifying and characterizing fungal pathogens causing seedling diseases on soybean through a multi-state survey

A. J. WARNER (1), E. Graves (1), T. Pojoski (1), J. Bond (1), A. Fakhoury (1)

(1) Southern Illinois Univ, Carbondale, IL, U.S.A.

Phytopathology 105(Suppl. 4):S4.146

Seedling diseases are both common and very destructive on soybean, Glycine max. They are caused by a variety of plant pathogens including fungi, bacteria, and oomycetes. The goal of this project is to identify fungal pathogens that cause seedling diseases of soybean through a multi-state effort. We used two different approaches for genus and species determination. The first approach relied on morphological features using microscopy and the second through the sequencing of specific genetic loci. In the sequencing approach, up to three different barcodes were targeted. The internal transcribed spacer (ITS), the elongation factor EF-1 $\alpha$, and the intergenic spacer (IGS) regions were sequenced for the isolates to confirm speciation. The data produced from this project includes identification of fungal isolates from eight different states, six fields per state, and at least 25 isolates per field.
Data also involves correlating species and genera distribution to factors including geographical distribution, cultural practices, soil type, and climatic conditions. Over 3,000 fungal isolates were processed for identification to help determine the predominant causal agents of seedling diseases on soybean in the production fields where the isolates were collected.

Identification of unknown sterile fungi as Rhizoctonia zeae and potential for biological control for fungal root diseases of sugar beet K. M. WEBB (1), R. M. Harveson (2)

(1) USDA ARS, SBRU, Fort Collins, CO, U.S.A.; (2) University of Nebraska, Panhandle Research and Extension Center, Scottsbluff, NE, U.S.A.

Phytopathology 105(Suppl. 4):S4.146

Several soilborne diseases routinely damage sugar beet in production areas of the Central High Plains, and it is becoming more common to find fields infested simultaneously with multiple pathogens. Due to a shortage of available fungicides for effective management of multiple diseases, alternative techniques such as biological control, are being sought for disease control. In past years, unidentified, sterile fungi have been isolated in conjunction with pathogens from infected sugar beet seedlings. In previous studies, two promising isolates were compared with Laetisaria arvalis and tested as potential biocontrol treatments in a field naturally infested with multiple sugar beet root diseases. Data indicated that these fungi provided some level of protection against a complex of soilborne diseases. In this work these isolates, 'Hall' and 'R47' were putatively identified as Rhizoctonia zeae based on morphological and molecular characterization. Plating assays determined that both isolates were able to inhibit the radial growth of multiple sugar beet root pathogens in vitro, including Rhizoctonia solani, Fusarium oxysporum f. sp. betae, Phoma betae, and Pythium aphanidermatum. Pathogenicity testing on sugar beet also indicated that neither isolate were pathogens of sugar beet. Based on these results and combined with earlier field studies, these isolates could have a potential role in integrative management strategies for several sugar beet diseases.

Spatio-temporal characterization of plant pathogenic Streptomyces species in Wisconsin

B. J. WEBSTER (1), L. A. Wanner (2), A. J. Gevens (1)

(1) University of Wisconsin, Madison, WI, U.S.A.; (2) USDA ARS, Beltsville, MD, U.S.A.

Phytopathology 105(Suppl. 4):S4.146

Potato common scab (CS) is a persistent disease that affects tuber quality and marketability. CS-causing Streptomcyes species inhabit most potatoproducing soils and vary based on location. To date, species incidence has not been associated with specific locations within the state. With the increase in incidence and severity of CS in potato, we were interested in exploring the species diversity of plant pathogenic Streptomyces in Wisconsin. Strains were isolated from tuber lesions during 2003-2006 and 2012-2013 from potato production regions in Wisconsin. Approximately 335 strains were characterized based on PCR analysis, sequencing of variable regions in the 16S rRNA region, and PCR-RFLP of the ITS region with Hpy99I. Most strains were isolated from potatoes grown in Langlade, Waushara, Portage, and Oneida counties. Of these, Streptomyces scabies predominated in Langlade, Waushara, and Portage counties across all sampling years. $S$. stelliscabiei was most prevalent in Oneida County based on 16S rRNA sequence analyses. Almost 50\% of isolates from 2003-2006 were identified as S. scabies, while nearly $40 \%$ of the strains isolated from similar regions were S. scabies during 2012-2013. The second most prevalent, S. stelliscabiei, comprised $13 \%$ of isolates during 2003-2006, but increased in prevalence to 17\% during 2012-2013. Diversity of Streptomyces species across Wisconsin potato producing regions may influence the varied CS response to varieties and treatment programs.

Development of a CANARY (Cellular Analysis and Notification of Antigen Risk and Yield) assay for detection of Citrus leprosis virus

G. Wei (1), F. Nargi (2), Z. LIU (1), J. Wang (1), M. K. Nakhla (1)

(1) USDA-APHIS-PPQ-S\&T-CPHST, Beltsville Lab, Beltsville, MD, U.S.A.;

(2) Bioengineering Systems \& Technologies, MIT Lincoln Laboratory (MITLL), Lexington, MA, U.S.A.

Phytopathology 105(Suppl. 4):S4.146

Citrus leprosis (caused by CiLV) is a devastating disease in South and Central America and is threatening US citrus industry. We are developing a CANARY assay for detection of CiLV-C. CANARY is a rapid, simple and sensitive antibody-based assay. The system consists of two components: 1) Virus capture using magnetic beads coated with single-chain fragment variable antibody derived from a monoclonal antibody (MAb) and 2) The CANARY assay in which the CANARY biosensor, engineered with a virusspecific MAb, interacts with the captured target virus inducing bio- 
luminescent light emission. Seventeen CiLV-C-specific CANARY clones were created at MIT-LL and tested in CPHST Beltsville Lab for assay feasibility, capture efficacy, specificity and sensitivity of the reaction between the biosensor and the target virus. CANARY assays were conducted using a luminometer and data were analyzed by MIT-LL developed CAGE software. In initial screenings, two CANARY cell lines were selected from the seventeen CANARY clones based on their specificity and sensitivity. The preliminary results suggest that the CiLV CANARY assay is specific for the CiLV-C strain and has the potential to become a screening technique for detection of CiLV in infected plants. The two selected CANARY cell lines have been engineered with a longevity gene to extend shelf-life and the longevity lines will be used in the final stage of method development for detection of CiLV.

Identification and biological characteristics of the pathogenic sf-19 strain of grape sour rot from Beijing

Y. WEI (1), C. Wang (1), X. Zhao (1), Q. Shang (1), Z. Liu (1)

(1) Beijing University of Agriculture, Beijing, China

Phytopathology 105(Suppl. 4):S4.147

Sour rot is one of the most important diseases causing great production loss and lower quality of grape and occurs in main grape producing regions in China. However, etiology of the disease is ambiguous at worldwide. In August of 2010 and 2011, a survey was conducted in Fangshan, Tongzhou, Changping and Yanqing District, Beijing, China. It was shown that the incidence of grape sour rot was around $22 \%-70 \%$. Berries showing typical symptoms of grape sour rot were collected and taken to laboratory. The strain sf-19 was isolated from the diseased samples by the tissue culture technique. Pathogenicity test of the strain was conducted in Lab. It found that the strain had strong pathogenicity to the Centennial Seedless variety of grape. Using molecular and Biolog identification method for classification of the strain sf19, the results showed that it was Hanseniaspora uvarum. For the strain sf-19 biological characteristics research, it found that the optimum culture time of the strain was $36 \mathrm{~h}-60 \mathrm{~h}$, the optimum $\mathrm{pH}$ was $3-7$, the optimum growth temperature was $28^{\circ} \mathrm{C}-30^{\circ} \mathrm{C}$. In addition, the strain produced the cell-wall degrading enzymes (CWDEs) in an induced culture media. And they were PG, PMG and Cx. Using a scanning electron microscope, it showed that grape peel tissues were ruptured by the strain suspension and the enzymes it produces. Financial support for this research was provided by Beijing Municipal Natural Science Foundation (6132008).

Interaction between seedborne Aspergillus section Nigri and soilborne Pythium species on maize seedling growth

L. K. WEIENETH (1), G. P. Munkvold (2), A. E. Robertson (1)

(1) Iowa State University, Ames, IA, U.S.A.; (2) Iowa State Univ, Ames, IA, U.S.A.

Phytopathology 105(Suppl. 4):S4.147

While many pathogens of maize seedlings have been studied extensively in isolation, little is known about their interactions with each other. In this study we investigated the relationship between Aspergillus section Nigri, seedborne fungi that cause ear rot and seedling disease, and Pythium spp., which are soilborne and cause seedling disease. Maize seeds inoculated with one of four strains of Aspergillus or not inoculated were planted in cups filled with noninfested sterile field soil, or soil infested with P. sylvaticum or P. torulosum. The cups were placed in a growth chamber at $25^{\circ} \mathrm{C}$ in a $\mathrm{RCBD}$ and assessments done at 7 and 14 days after planting (DAP). An interaction was detected between Pythium and Aspergillus on seedling height at 7 DAP and percent emergence. Percentage healthy mesocotyl, height at 14 DAP, and shoot weight were reduced by Pythium only. Root weight was affected by both Pythium and Aspergillus, but with no interaction. For the variables with an interaction, $P$. torulosum caused more severe symptoms when associated with seed-borne Aspergillus, while $P$. sylvaticum caused severe symptoms regardless of the presence of Aspergillus spp. The results suggest that seedborne Aspergillus can exacerbate seedling disease caused by Pythium spp. under some conditions. This highlights the need for further study of seedling pathogens with reference to the entire soil ecosystem, rather than simply in isolation.

Root removal to improve disease management in replanted Washington red raspberry fields

J. E. WEILAND (1), I. A. Zasada (2), L. W. DeVetter (3), T. Walters (4), R. Rudolph (3), S. Watkinson (3)

(1) USDA ARS, Corvallis, OR, U.S.A.; (2) USDA-ARS, Corvallis, OR, U.S.A.; (3) Washington State University, Mount Vernon, WA, U.S.A.; (4) Walters Ag Research, Anacortes, WA, U.S.A.

Phytopathology 105(Suppl. 4):S4.147
Washington leads the nation in the production of red raspberries for processing. Soilborne pathogens are a production constraint in this $\$ 61$ million industry with growers relying on preplant soil fumigation for their management. However, current fumigation methods can be ineffective, leading to replant issues and decreased crop lifespan. It is suspected that residual root debris remaining in the field after destroying the previous crop serves as a refuge protecting pathogens from lethal doses of fumigant. Residual roots would therefore act as a bridge for reintroducing pathogens to young raspberry plants in replanted fields. In 2014, three root removal devices (a Lundeby plant lifter, a potato harvester, and a beach cleaner) were tested for their ability to remove roots from fields slated for fumigation and replanting. All three devices were equally effective and lifted about $95 \%$ of residual roots, but the potato harvester was fastest. A field trial was then implemented with fumigation as the main plot and root removal as the subplot to determine the effect of root removal on reducing pathogen populations. Ten weeks after root removal and fumigation, no treatment was effective at reducing Pratylenchus penetrans populations. However, both root removal alone and fumigation alone reduced Fusarium and Pythium populations, with the combined root removal + fumigation treatment providing the best control and reducing soil populations by 64 and $69 \%$, respectively.

Disease-suppressive soils induce systemic resistance in Arabidopsis thaliana against Pseudomonas syringae pv. tomato

D. M. WELLER (1), J. A. van Pelt (2), C. M. J. Pieterse (2), P. A. H. Bakker (2)

(1) USDA-ARS, Washington State University, Pullman, WA, U.S.A.; (2) Utrecht University, Utrecht, Netherlands

Phytopathology 105(Suppl. 4):S4.147

Two-week-old Arabidopsis thaliana ecotype Col-0 seedlings were transferred into an autoclaved sand-soil mixture amended with 10 or $20 \%$ (weight/weight) soil that is suppressive to either take-all or Rhizoctonia root rot of wheat from fields in Washington State. These soils contain population sizes greater than $10^{5}$ colony forming units per gram of wheat root of 2,4-diacetylphloroglucinol or phenazine-1-carboxylic acid-producing pseudomonads, respectively. Plants were challenge-inoculated with Pseudomonas syringae pv. tomato three weeks later. Both suppressive soils induced resistance in Arabidopsis against P. syringae similar to the well-studied Pseudomonas strains WCS417r, Q2-87 (produces 2,4-diacetylphloroglucinol) and 2-79 (produces phenazine-1carboxylic acid). Pasteurization of the suppressive soils before adding them into the sand-soil mixture eliminated 2,4-diacetylphloroglucinol- and phenazine-1-carboxylic acid-producing pseudomonads from the Arabidopsis rhizosphere and significantly reduced induced systemic resistance activity. However, population sizes of total aerobic culturable bacteria were similar in the rhizosphere of plants grown in the mixes with pasteurized and raw suppressive soils. This is the first report of induced systemic resistance activity by take-all and Rhizoctonia suppressive soils and the ability of phenazine-1-carboxylic acid to induce resistance.

Evaluation of the common bean (Phaseolus vulgaris) core collections for resistance to soybean cyst nematode (Heterodera glycines)

L. WEN (1)

(1) Univ of Illinois, Urbana, IL, U.S.A.

Phytopathology 105(Suppl. 4):S4.147

Soybean cyst nematode (SCN), Heterodera glycines, is an obligate, sedentary endoparasite pathogen that infects soybean and a few other hosts including common bean (Phaseolus vulgaris). In soybean, but not common bean, there are several genes known to control resistance to SCN. The long-term goal of this research is to identify resistance genes in common bean and compare them to the known resistance genes in soybean. To complete the first step of this goal, over 360 accessions in the Mexico (MX) and Central/South American (CA/SA) Phaseolus Core Collections were inoculated with 2,000 eggs/juveniles of SCN HG type 2.5.7. In the first evaluation, $22(6.9 \%)$ were resistant, $52(16.5 \%)$ moderately resistant, 113 (35.3\%) moderately susceptible, and $132(41.3 \%)$ susceptible. Of the 22 resistant accessions, only one was from Andean gene pool while 21 were from Middle American gene pool. The result of this study will be used in association mapping for SCN resistance, and the resistant accessions found will be used as parents for developing mapping population to study the inheritance of $\mathrm{SCN}$ resistance in P. vulgaris.

Interaction of nematicdes and biostimulants for nematode management on cucumbers and carrots

B. WESTERDAHL (1)

(1) University of California, Davis, CA, U.S.A.

Phytopathology 105(Suppl. 4):S4.147 
Two randomized complete block field trials with five replicates were conducted to evaluate the effectiveness compared to an untreated control $(\mathrm{p}=0.05)$ of timing of pre-plant applications $(0,7,14$, or 21 days) of Nimitz (fluensulfone; MCW-2; ADAMA USA, Raleigh, NC); with and without multiple irrigations; and with and without the addition of a plant growth regulator (PGR; More Power; StollerUSA, Houston, TX), and soil penetrant (SP; Forte; J. R. Simplot Company, Boise, ID) for management of root-knot nematode, Meloidogyne javanica. The trials, one on cucumber, and one on carrots, had 16 identical treatments, and were adjacent to each other in the same field. Both crops are commonly used as indicators of nematicide effectiveness. For carrots, marketability of roots without nematode damage is most important. In contrast, for cucumbers, fruit yield is the most important factor. Overall, for cucumbers, there appears to be an advantage to using the PGR + SP additives. For example, the 7-day pre-plant treatments with PGR + SP with and without multiple irrigations, increased yields, while the same treatments without additives did not. Overall, both with and without PGR + SP, there appears to be an advantage to longer pre-plant intervals (14 and 21 days), both with and without additional irrigations. In contrast, in carrots, we did not see an advantage of the PGR + SP treatments.

Rapid detection of the zebra chip pathogen in infected plants and psyllids using loop mediated isothermal amplification

P. S. WHARTON (1), J. Woodhall (2), K. Fairchild (3)

(1) Univ of Idaho, Aberdeen, ID, U.S.A.; (2) Food and Environment Research Agency, York, United Kingdom; (3) University of Idaho, Aberdeen, ID, U.S.A.

Phytopathology 105(Suppl. 4):S4.148

Zebra chip (ZC) is an important disease of potato, caused by psyllid-borne bacterium Candidatus Liberibacter solanacearum (Lso). Symptoms are relatively easy to identify in tubers due to the characteristic banding patterns, but it is difficult to identify symptoms in potato foliage. The presence of psyllids on potatoes doesn't necessarily indicate that the crop will be infected with ZC as not all psyllids carry the bacterium. Although Lso bacteria can be routinely detected in infected psyllids, stems and tubers using real-time PCR it still takes up to 4 days for results from psyllid testing and up to 3 days for tuber testing as samples have to be shipped to appropriate labs for analysis. Not all psyllids found in monitoring programs are infected, so time is of the essence when it comes to analysis. A robust rapid diagnostic tests for the detection of Lso was developed using loop mediated isothermal amplification (LAMP). In this study, a species specific LAMP assay for Lso was used with the Genie II instrument to detect Lso in samples originating from infected psyllids, potato stems and tubers. Using a PEG/sodium hydroxide DNA extraction buffer, in field detection was possible of naturally infected psyllid, stem and tuber samples within 5 to 30 minutes depending on the levels of bacteria. With a rapid diagnostic LAMP test, growers would be able to make better management decisions as to if they actually need to spray to control potentially infected psyllids or not. Oxathiapiprolin, a new fungicide active ingredient for control of foliar
diseases caused by Oomycetes on cucurbits and fruiting vegetables

K. WHITTEN BUXTON (1), P. Kuhn (2), A. Tally (2), B. Druebbisch (2)

(1) Syngenta Crop Protection, Vero Beach, FL, U.S.A.; (2) Syngenta Crop Protection, LLC, Greensboro, NC, U.S.A.

Phytopathology 105(Suppl. 4):S4.148

Oxathiapiprolin is a new fungicide active ingredient (a.i.) that provides excellent control of downy mildew (Pseudoperonospora cubensis) on cucurbits and late blight (Phytophthora infestans) on fruiting vegetables. The mode of action is novel with no cross resistance to any other commercially available Oomycete fungicide. The high intrinsic activity of the molecule enables foliar a.i. use rates that are 10-100 times lower than current standards. Oxathiapiprolin has been shown to exhibit translaminar efficacy, and to provide protection to expanding leaves and the developing canopy. The fungicide is rainfast within 30 minutes and has excellent residual activity and crop safety. Field trials have shown that fungicide programs which include oxathiapiprolin provide increased disease control and yield protection when compared with spray programs using many currently available Oomycete fungicides. Since oxathiapiprolin has high potency and a single site mode of action, there is the potential for the selection of strains with reduced sensitivity. In order to mitigate this possibility, label directions will incorporate strict stewardship guidelines on application régimes as well as use of mixtures containing products with different modes of action. Federal registration of oxathiapiprolin is anticipated during the second half of 2015, with launch early in 2016. Cucurbits and fruiting vegetables will be included in the first wave of crops to be registered.
Fungal community structure associated with Eastern Hemlock and Hemlock Woolly Adelgid infestation

K. WICKERT (1), M. T. Kasson (1)

(1) West Virginia University, Morgantown, WV, U.S.A.

Phytopathology 105(Suppl. 4):S4.148

Hemlock woolly adelgid (HWA) is an exotic pest of native hemlock throughout the eastern United States. A potential management tool against HWA is the use of Lecanicillium muscarium, an entomopathogenic fungus recovered from HWA and other insects. In order to understand the efficacy of L. muscarium as a biocontrol, its biology and ecology on hemlock and HWA as well as its interactions among the present fungal communities, must be investigated. Fungi from diseased and healthy eastern hemlock tissues, as well as live and dead HWA from four sampling locations in PA, OH, MD and WV with varying levels of HWA infestation and management were assessed to help elucidate ecology and multipartite interactions. Five fungal taxa were frequently isolated from both hemlock needles and HWA at all sites including Colletotrichum fioriniae, Epicoccum nigrum, Pestalotiopsis microspora, Rhizosphaera macrospora and a novel Leotiomycete. Lecanicillium sp. was recovered from hemlock needles in Ohio, which confirms that hemlock tissues can serve as a viable, albeit rare, reservoir for L. muscarium. Identifying fungal species in eastern hemlock tissues and HWA provides baseline data on fungal community structure to allow studies of possible interactions between L. muscarium and ubiquitous fungal community members. Follow-up studies will include inoculation of asymptomatic hemlock foliage and healthy HWA with recovered fungi to test both phyto- and entomo-pathogenicity.

Examining the suppression of charcoal rot of soybean with secondary nutrients

T. WILKERSON (1), M. Tomaso-Peterson (2), B. Golden (3), S. Lu (4), A. Brown (5), T. Allen (6)

(1) Mississippi State Univ, Greenville, MS, U.S.A.; (2) Mississippi State University, Mississippi State, MS, U.S.A.; (3) Mississippi State UniversityDelta research and Extension Center, Stoneville, MS, U.S.A.; (4) Mississippi State Univ, Mississippi State, MS, U.S.A.; (5) Mississippi State University, Starkville, MS, U.S.A.; (6) Mississippi State University, Stoneville, MS, U.S.A.

Phytopathology 105(Suppl. 4):S4.148

Charcoal rot (CR) of soybean, caused by Macrophomina phaseolina (Tassi) Goid $(M p)$, occurs annually and can limit profitable soybean production. Approximately 39.2 million soybean bushels were lost to charcoal rot nationally in 2013. CR is typically more devastating in hot dry years. The objective of this research is to evaluate the suppression of CR using secondary nutrients, specifically calcium (Ca) and magnesium (Mg). In 2014 an inoculated greenhouse rate response trial using a CR-susceptible cultivar evaluated seven rates, $0,25,100,250,500,750$, and 1,000 lb/A, of $\mathrm{Ca}$ and $\mathrm{Mg}$ applied alone and in combination at three timings: at plant, R1, and at plant followed by (fb) R1. A significant increase in seed weight was observed with the $1,000 \mathrm{lb} / \mathrm{A}$ rate of $\mathrm{Ca}+\mathrm{Mg}$ at planting compared to the non-treated. In 2014, non-irrigated field trials were conducted with a susceptible and moderately resistant cultivar. Treatment applications consisted of $1,000 \mathrm{lb} / \mathrm{A}$ rate of $\mathrm{Ca}$ and $\mathrm{Mg}$ alone and in combination at plant, $\mathrm{R} 1$, and at plant $\mathrm{fb} \mathrm{R} 1$ compared with a non-treated. Field plots were inoculated with $M p$ in-furrow in addition to non-inoculated plots. Yield was collected from all plots. $\mathrm{Mg}$ applied at R1 provided the greatest yield benefit, albeit not significant, over the inoculated non-treated in both trials. Some treatment combinations may have negatively affected yield. In addition, environmental conditions during 2014 were not conducive for the development of CR.

Development of a predictive model for Sclerotinia sclerotiorum apothecial development to control white mold in soybean fields

J. F. WILLBUR (1), H. Lucas (1), M. Kabbage (1), D. L. Smith (1)

(1) University of Wisconsin, Madison, WI, U.S.A.

Phytopathology 105(Suppl. 4):S4.148

White mold, caused by Sclerotinia sclerotiorum, is one of the most important yield-limiting diseases of soybean worldwide, and is a significant concern for control in soybeans grown in the great lakes region of the USA. Phenologicalbased fungicide applications are often inefficient and sometimes unnecessary due to the complex array of factors surrounding successful disease development. Conducive conditions for apothecial formation and ascospore release must occur simultaneously with the development of soybean flowers for infection to occur. Risk assessment tools are often used to more accurately predict the timing of fungicide applications based on weather conditions, pathogen presence, and host architecture. White mold forecasting models such as those for carrot and lettuce, however, do not exist for soybean systems. In 2014, we collected site-specific weather data and detailed observations of 
disease progression and severity in Wisconsin soybean fields. Using 30-day moving averages of weather variables and logistic regression, we developed a predictive model for apothecial development with a high-risk action threshold at $>40 \%$ probability of apothecial formation. Our risk assessment tool uses temperature (negative correlation) and leaf wetness (positive correlation) variables leading up to the soybean flowering period to predict the probability of apothecial development. Model validation and replicated data collection will be conducted in the 2015 field season.

Detection of viable Acidovorax citrulli in cucurbit seeds by

R. WILLMANN (1), A. Beerepoot (1), B. Woudt (1)

(1) Syngenta Seeds, Enkhuizen, Netherlands

Phytopathology 105(Suppl. 4):S4.149

The seed-borne bacterium Acidovorax citrulli (Acit) is the causative agent of bacterial fruit blotch in cucurbits (Schaad et al. 1978). Currently, the industry screens melon and watermelon seeds by means of PCR in order to detect Acit and prevent seed-transmitted infections (NSHS 2014, Woudt et al. 2009). However, due to persistence of DNA the current PCR set-up does not differentiate between viable and non-viable Acit. Therefore, PCR screening has to be complemented by grow-out methods in order to determine viability of Acit in PCR-positive seed samples. Here, we present a PCR-based method detecting viable Acit cells by exploiting the integrity of their bacterial cell envelope as well as by their capability to propagate. The former feature is exploited by a DNase I digestion of DNA from compromised Acit while DNA in viable Acit remains protected; the latter by liquid culture enrichment. These two elements are combined in a sequential approach in which Acit DNA is quantified in DNase I treated seed extracts and after enrichment. The reduction of DNA background derived from non-viable Acit enhances the detectability of multiplied viable Acit. In the presented method Acit is extracted from homogenized seed samples using matrix solubilization facilitating detection of internally located Acit and removal of adverse matrix components.

\section{Identifying spatial distribution of seedling disease pressure in cotton fields}

K. D. WILSON (1), C. S. Rothrock (1), T. N. Spurlock (2)

(1) University of Arkansas, Fayetteville, AR, U.S.A.; (2) University of Arkansas Southeast Research and Extension Center, Monticello, AR, U.S.A. Phytopathology 105(Suppl. 4):S4.149

Seedling diseases affect the germination, emergence, and early season growth of cotton resulting in variable stands and reduced yields. With the increasing costs of seed from the products applied to the seed, precision planting utilizing site-specific management strategies are being implemented in order to reduce planting costs and improve stand uniformity. The objective of this study was to examine the spatial distribution of seedling diseases using seed treatment fungicides for the development of predictive models for seedling disease potential. Four row plots were established across a cotton field with each row having one of four seed treatments ipconazol + myclobutanil + metalaxyl + penflufen + prothiooconazole, penflufen, metalaxyl (2) metalaxyl, (3) PCNB, and (4) no fungicide for each plot, soil temperature and moisture was recorded 1 and 5 days after planting along with soil EC (texture). Spatial data analysis and spatial regression was used to determine distributions of stands and correlations with soil factors. Soil temperature was shown to be significantly aggregated in this field $(\mathrm{P}<0.001)$. The minimum soil temperature ranged from $20.0-21.4^{\circ} \mathrm{C}$. Stand improvement was spatially correlated $(\mathrm{P}<0.0001)$ with sites with higher temperatures for all seed treatments. The stand difference between treated and non-treated rows was less in the sites with higher temperature. These results suggest that predictive maps for seedling disease risk are possible.

\section{A histological study of Aspergillus flavus colonization of wound inoculated corn kernels \\ G. L. WINDHAM (1), W. P. Williams (1) \\ (1) USDA ARS, Mississippi State, MS, U.S.A. \\ Phytopathology 105(Suppl. 4):S4.149}

Corn hybrids resistant (Mp313E x Mp717) and susceptible (GA209 x T173) to aflatoxin accumulation were grown in the field at Mississippi State, MS, in 2012 and 2013. Plants were hand pollinated, and individual kernels were inoculated with a needle dipped in a suspension of Aspergillus flavus conidia 21 days after pollination. Kernels were harvested 1, 2, 3, 4, 5, 6, 7, 10, 12, 14, and 21 days after inoculation (DAI). Kernels were fixed in FAA, dehydrated, embedded in paraffin, sectioned, and stained. Fungal hyphae were observed among endosperm cells 2 DAI in the susceptible hybrid and only at the wound site of the resistant hybrid. By 3 DAI, fungal spread in the endosperm of the susceptible hybrid was much more extensive than in the resistant hybrid. The embryo of the susceptible hybrid was colonized by A. flavus by 5 DAI. Fungal growth was slower in the resistant hybrid compared to the susceptible hybrid. By 10 DAI, A. flavus had colonized a large section of the embryo in the susceptible hybrid; whereas in the resistant hybrid, approximately half of the endosperm had been colonized and very few cells in the embryo were colonized. Fungal colonization in some of the kernels of the resistant hybrid was slowed in the aleurone layer or at the endosperm-embryo interface.

\section{Assessment of soil-applied fungicides for control of onion pink root}

P. WIRIYAJITSOMBOON (1), M. K. Hausbeck (2)

(1) Michigan State Univ, East Lansing, MI, U.S.A.; (2) Michigan State University, East Lansing, MI, U.S.A.

Phytopathology 105(Suppl. 4):S4.149

Pink root, caused by Setophoma terrestris (H.N. Hansen) Gruyter, Aveskamp \& Verkley, is a soil-borne disease that causes economic losses to the Michigan onion industry. Yields can be reduced by as much as $96 \%$ depending on the plant's growth stage at the time of infection. A greenhouse study was conducted to evaluate fungicides for their efficacy against pink root. Nine fungicides were applied singly as a soil drench to 6-week old 'Highlander' seedlings. Applications were made either at 0 day post inoculation (dpi) or at 0 and $14 \mathrm{dpi}$. Root density was rated at $49 \mathrm{dpi}$ and plant fresh weight was measured 55 dpi. Significant differences were observed among fungicides and treatment application. The penthiopyrad treatment resulted in significantly higher root density and plant fresh weight compared to other treatments and the untreated inoculated control. Complete control was not achieved with the fungicides included in this study; root density and fresh weight of onions treated with penthiopyred were significantly smaller than untreated uninoculated plants. Drench applications of fungicides containing either azoxystrobin or cyprodinil resulted in phytotoxicity; plant injury was more severe when plants received two applications compared with one application.

Cassava Bacterial blight: A main biological constraint for cassava production in West-Africa

I. Wonni (1), D. Kone (2), O. Koita (3), K. Dagno (4), V. VERDIER (5)

(1) INERA Farako-Ba and LMI Pathobios, Bobo-Dioulasso, Burkina-faso, Bobo-Dioulasso, Burkina faso; (2) Université Félix Houphouët-Boigny, UFR Biosciences, Laboratoire de Physiologie Végétale, Abidjan, Ivory Coast; (3) Laboratoire de Biologie Moléculaire Appliquée, Université des Sciences Techniques et Technologiques de Bamako, Bamako, Mali; (4) Institut d'Economie Rurale, Programme Sorgho-CRRA de Sotuba, Bamako, Mali; (5) IRD, UMR Interactions Plantes Microorganismes Environnement, IRD-CiradUniversité de Montpellier, Montpellier, France

\section{Phytopathology 105(Suppl. 4):S4.149}

Cassava is among the most important sources of calories in developing countries. It is the first food crop in sub-Saharan countries with Africa being the world's largest producer of cassava. A major constraint to cassava yield worldwide is a disease caused by Xanthomonas axonopodis pv. manihotis (Xam), causal agent of cassava bacterial blight (CBB). CBB is present in most of the tropical and subtropical world. The use of cassava cultivars with good level of resistance to Xam is one of the most economically ways to control CBB. However not a single cassava resistance gene has been deployed to combat Xam so far. This foliar and vascular disease severely affects cassava production in Africa, with losses of both yield and planting material. A significant recurrence of the disease was reported in recent years in various regions in West-Africa. Using molecular tools we recently reported or confirmed the presence of CBB in Burkina-Faso, Mali and Ivory Coast. Although cassava is largely recognized as a resilient crop for global warming, climate change is likely to amplify biotic constraints such as CBB. Higher temperatures can favor the occurrence of new strains of the pathogen. We will describe the distribution and prevalence of CBB disease, the molecular tools used and results obtained on the characterization of Xam strains in WestAfrica. The need for a powerful surveillance and diagnostic network that can stop the spread of CBB disease in West-Africa will be discussed.

On site testing for Clavibacter michiganensis subsp. sepedonicus in potato and environmental samples using loop mediated isothermal amplification J. WOODHALL (1), F. Hetherington (1), K. Perkins (1), H. Lekuona (1), K. Fairchild (2), P. S. Wharton (2)

(1) Food and Environment Research Agency, York, United Kingdom; (2) University of Idaho, Aberdeen, ID, U.S.A.

Phytopathology 105(Suppl. 4):S4.149

Clavibacter michiganensis subsp. sepedonicus (CMS) is the causal agent of ring rot of potato, so called due to the way it rots the vascular tissue of tubers. Losses in US crops have been as high as 50\% and in the European Union the pathogen is considered a quarantine organism listed in the EC Plant Health 
Directive. Loop mediated isothermal amplification (LAMP) has shown great potential for rapid on site testing, allowing for rapid turnaround of results and the implementation of disease management decisions. In this study, a species specific LAMP assay for CMS was used with the Genie II instrument to detect CMS in samples originating from tubers, stems and potentially contaminated surfaces (as would typically be found in potato storages). Robust onsite DNA extraction materials were developed for each of these sample types. Using a PEG/sodium hydroxide DNA extraction buffer, on site detection was possible in naturally infected tuber and stem samples within 5 to 30 minutes depending on the levels of bacteria. A swabbing method was developed for testing for CMS on contaminated surfaces. This method was highly sensitive and worked across a wide variety of surfaces (wood, plastic, metal and brick). Results showed that CMS was highly stable and could be detected by LAMP on all surface types even after 90 days. In conclusion, on site detection using LAMP offers a rapid and sensitive way of testing for CMS on a range of sample types.

Reproduction of Meloidogyne incognita and Rotylenchulus reniformis on select cotton cultivars

J. E. WOODWARD (1), T. A. Wheeler (2)

(1) Texas A\&M AgriLife Extension Service, Lubbock, TX, U.S.A.; (2) Texas A\&M AgriLife Research, lubbock, TX, U.S.A.

Phytopathology 105(Suppl. 4):S4.150

Root-knot nematodes (Meloidogyne incognita) and reniform nematodes (Rotylenchulus reniformis) are among the most economically important pests of cotton (Gossypium hirsutum) in the United States. Recently, commercial cultivars possessing improved resistance to $M$. incognita have been developed; however, there are currently no cultivars with high levels of resistance to $R$. reniformis available. Final nematode populations were determined for various cultivars evaluated in with different irrigation levels $(M$. incognita) or planting dates ( $R$. reniformis). Differences in lint yield were not observed among cultivars under the three irrigation levels evaluated. When compared to susceptible cotton cultivars, populations of $M$. incognita were reduced by $60.8,90.2,92.0 \%$ for Deltapine 1454 NRB2RF, Phytogen (PHY) 417WRF and PHY 427WRF, respectively. Differences in R. reniformis populations were observed when averaged across three planting dates. Populations were greatest for PHY 499WRF (478 nematodes/500cc soil) and lowest for the cultivars PHY 427WRF (228 nematodes/500cc soil) and PHY 417WRF (134 nematodes/500cc soil). Yields were negatively correlated with $R$. reniformis populations and were lowest for PHY 499WRF (617 kg/ha) and greatest for PHY 417WRF (980 kg/ha) followed by PHY 427WRF (896 $\mathrm{kg} / \mathrm{ha}$ ). Results from these studies corroborate other reports that PHY 417WRF and PHY 427WRF possess increased resistance to $M$. incognita that can greatly reduce populations in the soil. Little information exists regarding the ability of $R$. reniformis to reproduce on cultivars, which possess improved resistance to $M$. incognita. Additional studies evaluating these and other cotton cultivars are warranted.

Effect of temperature on Wheat streak mosaic virus replication and movement in resistant and susceptible winter wheat varieties

E. N. WOSULA (1), S. Tatineni (2), S. N. Wegulo (1), G. L. Hein (1)

(1) University of Nebraska-Lincoln, Lincoln, NE, U.S.A.; (2) United States Department of Agriculture-Agricultural Research Service and University of Nebraska-Lincoln, Lincoln, NE, U.S.A.

Phytopathology 105(Suppl. 4):S4.150

Wheat streak mosaic virus (WSMV) is an economically important virus causing annual average yield losses of $\sim 2 \%$ in winter wheat across the Great Plains. Temperature is an important factor that influences disease development and severity. The objective of this study was to determine the extent of WSMV replication and movement in winter wheat varieties 'Mace' (resistant) and 'Tomahawk' (susceptible) at $10,15,20$ and $25^{\circ} \mathrm{C}$. The number of days to WSMV expression at the point of inoculation increased as temperatures decreased from 4 to 18 days, but differences were not seen between the two varieties. WSMV in plants held at $10^{\circ} \mathrm{C}$ failed to move systemically at 21 days post inoculation, but those at $25^{\circ} \mathrm{C}$ were systemically infected within 10 days. After 21 days at the treatment temperatures, plants were moved to $27^{\circ} \mathrm{C}$ for 14 days, and systemic infection in Mace $(70 \%)$ was slightly lower than in Tomahawk (97\%). However, even with systemic infection, Mace plants did not display symptoms, while Tomahawk plants showed typical WSMV symptoms. Regrowth, from cut back plants previously held at 10 and $15^{\circ} \mathrm{C}$ with no systemic infection, expressed WSMV in $20-85 \%$ of the plants within 14 days at $27^{\circ} \mathrm{C}$. This study demonstrates that WSMV moves at undetectable levels under suboptimal temperatures, but rapidly begins to replicate and induce symptoms in wheat plants under optimal temperatures. This helps explain the pattern of infection and the development of symptoms under field conditions.
Dissecting the molecular basis of basil-Peronospora belbahrii interactions D. Wu (1), J. Win (2), D. Shao (1), M. TIAN (1)

(1) University of Hawaii at Manoa, Honolulu, HI, U.S.A.; (2) The Sainsbury Laboratory, Norwich, United Kingdom

Phytopathology 105(Suppl. 4):S4.150

Basil is an important herb crop widely used in Mediterranean cuisine, traditional medicine and cosmetics. Basil downy mildew, a disease caused by the obligate biotrophic oomycete pathogen Peronospora belbahrii, is threatening basil production worldwide. Understanding the molecular basis specifying pathogen virulence and host resistance is key to developing novel effective and environmentally friendly disease control strategies. To this end, we performed multiplexing mRNA-seq analysis with samples from $P$. belbahrii sporangia, and infected leaves of both susceptible and resistant basil lines during the infection time course. The reads generated from sporangia were assembled into transcripts by de novo assembly using Trinity. The secretome, putative effectors and their expression profiles during infection were analyzed to identify putative effectors that likely play key roles in $P$. belbahrii pathogenesis. In addition, the expression patterns of basil genes during susceptible and resistant interactions were analyzed to gain insight into the mechanisms of host resistance. In parallel, we were optimizing an agroinfiltration-mediated transient expression system in basil for functional genomic studies of genes from both the host and the pathogen. These resources are expected to better our understanding of the molecular basis of basil- $P$. belbahrii interactions and in turn help design novel disease control strategies.

Citrus root morphological changes caused by Phytophthora spp. and Candidatus Liberibacter spp.

J. WU (1), E. G. Johnson (1), D. Bright (1), K. Gerberich (1), J. H. Graham (1)

(1) University of Florida, Lake Alfred, FL, U.S.A.

Phytopathology 105(Suppl. 4):S4.150

Phytophthora nicotianae (P.n.) causes root rot of citrus, damaging the fibrous roots, depleting carbohydrate reserves, and reducing water and nutrient uptake capacity. Huanglongbing (HLB) is a systemic citrus disease, caused by phloem-limited Candidatus Liberibacter asiaticus (Las), that damages fibrous roots and disrupts photosynthate production and transport. To investigate how Las and P.n. cause root decline, Swingle citrumelo rootstock seedlings were inoculated with Las or P.n. Root systems of all seedlings were trimmed at the time of P.n. inoculation to induce root growth. Roots were separated into diameter classes, and biomass and root length were measured. Las caused more total root loss than P.n. by 11 weeks post inoculation (wpi), $32 \%$ and $12 \%$, respectively. P.n. caused greater damage to fibrous roots from 0 to $1.2 \mathrm{~mm}$ diameter by $11 \mathrm{wpi}$. Compared to healthy seedlings, Las- or P.n.infected seedlings at 5 wpi produced more or less length of new roots, respectively, suggesting that after the early stage of infection Las stimulated and P.n. inhibited root growth. Las induced root loss was evident at 11 wpi. Lower total leaf area by 11 wpi indicated that canopy decline followed root loss. In conclusion, Las stimulated root growth at the early stage of HLB development, which reduced carbohydrate status and canopy production at a more advanced stage of disease.

The profile of GHF5 and GHF45 cellulases in 14 Aphelenchoides besseyi isolates orginated from different hosts

G. WU (1), P. J. Chen (1)

(1) Natl Chung Hsing Univ, Taichung, Taiwan

Phytopathology 105(Suppl. 4):S4.150

Cellulase ( $\beta$-1,4-endoglucanase) and pectate lyase of plant parasitic nematodes are important effectors for disease development. Nematode $\beta-1,4-$ endoglucanases have two different topological structures, glycosyl hydrolase family 5 and 45 (GHF5 and GHF45) whose coding genes have been speculated to be originated from bacteria and fungi, respectively by horizontal gene transfer. GHF5 and GHF45 have not been reported to co-exist in a single nematode species. The presence of GHF5 or GHF45 were evaluated in fourteen Aphelenchoides besseyi isolates, of which 6 were collected from bird's nest fern, 7 from rice and 1 from strawberry. The isolates collected from rice and strawberry did not cause disease symptoms when infiltrated into the leaves of bird's nest fern. PCR amplification with the GHF5 degenerate primer set produced a single $491 \mathrm{bp}$ band from the bird's nest fern isolates, whereas no products were detected from the rice or strawberry isolates. GHF45 degenerate primer set amplified two bands of 548 and 439 bp in sizes from the bird's nest fern isolates and a single smaller band ( $387 \mathrm{bp}$ ) from the rice and strawberry isolates. The results suggested that the $A$. besseyi isolates collected from bird's nest fern are unique in which they likely have two different cellulases. 
Development of non-pesticide cultivated technique to control Colletotrichum spp. associate with anthracnose on coffee plants in Taiwan Z. B. WU (1), F. L. Chi (2), J. S. Tsay (1)

(1) Department of Horticulture, National Taitung Jr. College, Taitung County, Taiwan; (2) Department of Horticulture, National Chung Hsing University, Taichung County, Taiwan

Phytopathology 105(Suppl. 4):S4.151

Coffee production is a newly agricultural industry in eastern Taiwan. Anthracnose is one of the economic limitations in coffee production. Seven Colletotrichum isolates, designed as $\mathrm{C} 1 \sim \mathrm{C} 7$, were isolated from diseased Coffea arabica plants in the orchards. The cDNA fragments corresponding to $480 \mathrm{bps}$ were amplified by PCR technique using the universal primers designed for amplifying the internal transcribed spacer regions. Sequence analysis of these amplicons are carried out to evaluate their phylogenetic relationships. Back inoculation of the isolates $\mathrm{C} 1$ and $\mathrm{C} 2$ to non-anthracnose coffee seedlings induced similar symptoms as observed in the fields. Three plant essential oils derived from basil (Ocimum basilicum), garlic (Allium sativum) and red pepper (Capsicum annuиm) plants by steam distillation were tested for the inhibition of mycelial growth and suppression of spore germination of the seven isolates on PDA plates. The basil and garlic essential oils were found effective at suppressing spore germination of five isolates, and showed high inhibiting ratio of $>98 \%$. The results of mycelial-inhibition showed no effect on C4 C6 isolates by using basil or garlic essential oils but the inhibiting rates of $26 \% \sim 66 \%$ were presented on other isolates. There were no significant effects in the results of each test using red pepper essential oils. Our results provide useful information in pest management of anthracnose on coffee plants conform to organic agriculture in Taiwan.

Profiling and functional characterization of the secondary metabolites produced by plant-growth-promoting Bacillus pumilus PMB102

J. J. Wu (1), W. L. DENG (2), J. Y. Tzeng (2), J. W. Huang (2)

(1) National Chung Hsing University and Academia Sinica, Ph.D. Program in Microbial Genomics, Taichung, Taiwan; (2) National Chung Hsing University, Department of Plant Pathology, Taichung, Taiwan Phytopathology 105(Suppl. 4):S4.151

Plant-growth-promoting Bacillus pumilus PMB102 is a Gram-positive bacterium belonging to $B$. subtilis group. The draft genome of PMB102, determined by Illumina shotgun sequencing technology, contains 4,183 predicted genes with a genome size of $4,051,387 \mathrm{bp}$. RAST annotation and antiSMASH-assisted genome mining revealed that PMB102 harbors genes coding for hydrolytic enzymes, bacteriocins, antimicrobial peptides, siderophores, and genes for the biosynthesis of volatile organic compounds and IAA, whose gene products might be involved in promoting plant growth and antagonism. In preliminary assays, tomatoes treated with formulated PMB102 by root drenching or foliage dipping showed reduced disease severity of bacterial spot cause by Xanthomonas campestris pv. raphani cxsp3. Culture filtrates containing lipopeptides were fractionated by C18 SPE column, eluted by water-methanol gradient solvents, and tested for the antagonistic activity on the mycelial growth of Alternaria brassicicola ABA31 to show that the $100 \%$-methanol elutant has the antifungal activity, which can be further separated to 3 compounds with different $\mathrm{Rf}$ values by TLC using chloroform-methanol-water $(65: 25: 4)$ as the mobile phase. The crude extracts of siderophores were also found to inhibit the growth of fungi (ABA31 and Fol-04) and bacterium (cxsp3) in vitro. Further purification and antimicrobial activity assays are underway to identify the bioactive compounds involving in antagonism.

Cryptic sex in the laurel wilt pathogen, Raffaelea lauricola, and sexual states in three other closely related ambrosia beetle symbionts

C. E. WUEST (1), T. C. Harrington (2), D. L. McNew (1), S. W. Fraedrich (3) (1) Iowa State University, Ames, IA, U.S.A.; (2) Department of Plant Pathology and Microbiology, Ames, IA, U.S.A.; (3) USDA Forest Service, Athens, GA, U.S.A.

Phytopathology 105(Suppl. 4):S4.151

Previously, it had been thought that the mycangial symbionts of ambrosia beetles are strictly asexual. The laurel wilt fungus, Raffaelea lauricola, along with its ambrosia beetle vector, Xyleborus glabratus, was introduced to the southeastern USA from Asia. Isolates of the fungus collected from $X$. glabratus adults in the USA, Taiwan, and Japan were tested for the presence of mating type genes using PCR and primers developed for the MAT1-1-3 or MAT1-2 genes, corresponding to the MAT1 or MAT2 phenotypes, respectively. Of the 26 isolates tested from Taiwan, 14 possessed only the MAT1-1-3 gene, and 12 possessed only the MATI-2 gene. Of the seven tested isolates tested from Japan, six possessed only the MAT1-1-3 gene, and one possessed only the MAT1-2 gene. The finding of both mating types in Taiwan and Japan suggests that the laurel wilt pathogen is capable of sexual reproduction within its native range. All USA isolates tested thus far possessed only the MAT1-2 gene (MAT2), consistent with the hypothesis that there had been a single strain of $R$. lauricola introduced from Asia. Sexual states were found in three undescribed Raffaelea species that are closely related to $R$. lauricola and also symbionts of ambrosia beetles. Based on these observations, it appears that sexual reproduction is common among ambrosia beetle symbionts and likely occurs in R. lauricola populations in Asia.

Development of SP-SNP markers and use them to characterize populations of the stripe rust pathogen and identify markers associated to avirulence genes

C. XIA (1), M. Wang (1), A. Wan (1), X. Chen (2)

(1) Washington State University, Pullman, WA, U.S.A.; (2) Washington State University; USDA-ARS, Wheat Genetics, Quality, Physiology, and Disease Research Unit, Pullman, WA, U.S.A.

Phytopathology 105(Suppl. 4):S4.151

Puccinia striiformis f. sp. tritici is a basidiomycete fungus causing of stripe rust, one of the most important diseases of wheat, but not many markers are available for determining the population structures and studying virulence. We developed 92 secreted protein derived-single nucleotide polymorphism (SPSNP) markers and used them, together with virulence tests, to characterize the pathogen populations in various stripe rust epidemiological regions in the US. The SP-SNP data had a moderate, but significant correlation $(r=0.43 ; P=0.01)$ with the virulence data. Bayesian clustering algorithm, neighbor-joining method, and principal component analysis clustered the 242 multi-locus genotypes obtained from 352 isolates into two genetic lineages and an admix group with different levels of heterozygosity. Analysis of molecular variance detected significant differences between eastern and western US populations and among some populations of the epidemiological regions. The western US isolates were more diverse than the eastern US isolates, and the isolates from southwestern US were more related those in the eastern than to those in the other western regions. Significantly associated SP-SNPs were detected for nine avirulence genes. The results indicated that SP-SNPs are useful in characterizing the pathogen populations, determining evolutionary mechanisms, and identifying markers associated to avirulence genes.

Detection and characterization of viruses in strawberry plants showing decline symptoms from eastern Canada

Y. XIANG (1), M. Bernardy (1), B. Bhagwat (1), R. DeYoung (1), M. Bouthillier (1)

(1) Agriculture and Agri-Food Canada, Pacific Agri-Food Research Centre, Summerland, BC, Canada

Phytopathology 105(Suppl. 4):S4.151

Strawberry decline (SD) is a disease probably caused by the synergistic effects of mixed virus infections. It has been an increasing problem in strawberry production in North America. The nursery industry in the east coast region of Canada was severely affected by a recent outbreak of the disease, resulting in an urgent effort to identify the viruses associated with SD symptoms. Using deep sequencing and reverse transcription PCR, four different RNA viruses, namely strawberry mild yellow edge virus (SMYEV), strawberry mottle virus $(\mathrm{SMoV})$, strawberry pallidosis associated virus (SPaV) and strawberry polerovirus 1 (SPV1) were identified in strawberry plants sampled from Prince Edward Island and Nova Scotia, Canada. Sequence analysis suggested a diversified SMYEV population including an emerging subgroup of the virus. Three different $\mathrm{SMoV}$ genome sequences were assembled from the deep sequencing data and sequence comparisons indicated a reassortment had occurred between the two RNA segments of the virus. SPaV was detected at a low incidence among the samples. SPV1 is a previously unreported virus discovered as a result of this study. Genome sequence analysis reveals that SPV1 is a new virus species belonging to the genus Polerovirus. A literature study suggests that SPV1 may not be a newly emerging virus but likely a newly discovered virus that is potentially widely distributed in North America and may act as a helper virus for field transmission of SMYEV by aphids.

Plant growth promotion of PGPR on soybean and cotton with and without Heterodera glycines or Meloidogyne incognita

N. XIANG (1), K. S. Lawrence (2), J. W. Kloepper (2), J. A. McInroy (3)

(1) Auburn University, Auburn, AL, U.S.A.; (2) Auburn University, Auburn, AL, U.S.A.; (3) Auburn Univ, Auburn, AL, U.S.A.

Phytopathology 105(Suppl. 4):S4.151

The objective of this research was to select potential PGPR strains that can promote plant growth in soybean and cotton with and without soybean cyst nematode $(\mathrm{SCN})$ or root-knot nematode $(\mathrm{RKN})$. Nineteen PGPR strains including 18 Bacillus strains and a strain of Lysinibacillus xylanilyticus, were evaluated. The experiments were RCBD with five replications and were 
repeated twice. Data were analyzed in SAS 9.2 using PROC GLIMMIX. Results of soybean trials indicated that soybean plant height, shoot and root fresh/dry weights, were significantly higher when no SCN were present than with $\mathrm{SCN}$ present $(P \leq 0.1)$. Strain AP-305 increased soybean plant height without SCN $(P \leq 0.1)$. Soybean shoot and root fresh/dry weights were similar among all the PGPR strains $(P \leq 0.1)$. Cotton plant height and shoot fresh/dry weight were higher when treated without RKN $(P \leq 0.1)$. However, root fresh weight was significantly higher when RKN were present $(P \leq 0.1)$ than plants without RKN. Strain AP-301 significantly increased shoot and root fresh/dry weights, AP-18 increased shoot fresh/dry weights, AP-3 increased root fresh weight compared with aldicarb standard without RKN $(P \leq 0.1)$. Of all those strains, AP-301 and AP-52 reduced soybean cysts populations in soybean, and AP-283 reduced eggs/gram of roots of RKN in cotton in previous studies. These results showed that some PGPR strains promoted growth both with and without nematodes and reduce nematode populations.

Whole-genome expression analysis of Rice black-streaked dwarf virus in different plant hosts and small brown planthopper

Q. XU (1), Y. Zhou (1)

(1) Jiangsu Academy of Agricultural Sciences, Nanjing, China

Phytopathology 105(Suppl. 4):S4.152

Rice black-streaked dwarf virus (RBSDV) can infect a number of gramineous plants and cause severe crop yield losses in southeast Asian countries. The virus is transmitted by the small brown planthopper (SBPH) in a persistent circulative manner. The interactions between RBSDV and its different hosts remain unknown. Besides, how the virus adjusts itself to infect different hosts is unclear. In the present study, the relative RNA levels of the thirteen RBSDV proteins in rice, maize, wheat and SBPH were measured by real-time quantitative PCR. P7-1 and P10 genes were predominantly expressed whereas P8 and P7-2 genes were expressed at relatively low levels in plant hosts. Similar to the expression in rice, P7-1 was the most abundantly expressed gene and P8 was expressed at the lowest level in SBPH, indicating that RBSDV adopts the same strategy to infect distinct hosts. The high expression levels of the P7-1 gene in both plants and insect suggest that it can be used as the target gene for disease diagnostics. However, the expression levels of other genes varied from host to host. The components of the RBSDV viroplasm, including P5-1, P6 and P9-1, are differentially expressed in different hosts. Moreover, western blot analysis showed that the viral P9-1 protein was expressed more abundantly in SBPH than in plant host. These data indicate that virus may adjust their own gene expression to replicate in different hosts. Analysis of real-time gene expression revealed that P7-1 stands out as the only gene highly expressed at the earliest time point and its expression precedes all others throughout infection from 8 to 24 days post inoculation. On the other hand, P4 appeared as the least abundantly expressed gene consistently from 8 to 24 days post inoculation, while the relative expression levels of other genes fluctuated through this time period. The high expression levels of the P7-1 gene suggest that it plays a significant role in RBSDV-host interaction. Those viral genes that were differentially expressed in distinct hosts may be involved in the interactions between the virus and specific hosts.

Sclerotial development in Sclerotinia sclerotiorum depends on low pH, independent of oxalic acid production

L. XU (1), M. Xiang (1), W. Chen (2)

(1) Department of Plant Pathology, Washington State University, Pullman, WA, U.S.A.; (2) USDA ARS Washington State University, Pullman, WA, U.S.A.

Phytopathology 105(Suppl. 4):S4.152

Sclerotinia sclerotiorum, a devastating plant pathogen of over 400 plant species, produces melanized sclerotia upon which survival, sexual process and spore production of the fungus depend. It was previously assumed that sclerotial development in $S$. sclerotiorum is connected with oxalic acid production simply based on UV-induced mutants that concomitantly lost oxalate production and sclerotial development, along with lost pathogenicity. However, in this study three T-DNA insertion mutants selected from a TDNA mutant library of about 800 transformants cannot produce oxalic acid, and a single T-DNA insertion was found in the ORF of the oxaloacetate acetylhydrolase (oah) gene. The oah gene was also specifically deleted. Both the T-DNA insertion and the oah-deletion mutants completely lost oxalic acid production, but accumulated fumaric acid and were capable of producing functional sclerotia (able to produce apothecia and viable ascospores) when the medium $\mathrm{pH}$ was low $(\mathrm{pH}<4)$, and also retained pathogenicity on faba bean and pea. When cultured on PDA buffered at high pHs (5 to 6.8), the oxalic acid-minus mutants developed abnormal sclerotia or cannot develop sclerotia at all, while wild-type strain can develop normal sclerotia on the pH-buffered media. Our results showed sclerotial development in S. sclerotiorum depends on low $\mathrm{pH}$, independent of oxalic acid production, suggesting those UVinduced mutants harbor a developmental defect, which could impair pathogenicity.

Development and validation of multiplex RT-PCR protocols for viruses indexing scheme in Canadian seed potato certification program

H. XU (1), S. Cody (2), D. L. Hammill (2)

(1) Canadian Food Inspection Agency, Charlottetown Laboratory, Charlettetown, PE, Canada; (2) Canadian Food Inspection Agency, Charlottetown Laboratory, Charlottetown, PE, Canada

Phytopathology 105(Suppl. 4):S4.152

Ten viruses are known to be present in seed potato lots in Canada including potato viruses A, M, S, X, Y, Potato leafroll virus, Potato mop-top virus, Potato latent virus, Tobacco rattle virus and Alfalfa mosaic virus. There is a requirement for indexing these viruses in potato nuclear stocks and in seed potato lots in Canada. Primers specific to these viruses were designed based on sequence analysis in this study or obtained from published data and evaluated for their sensitivity and specificity for detecting these viruses in potato samples of leaves, sprouts, microplantlets and tubers. Characterized and identified virus isolates were used for the evaluation tests. Serial dilutions of RNA extracts and composite samples (w/w, v/v) were used to assess the sensitivity of RT-PCR. Duplex and multiplex formats of RT-PCR using various primer combinations were developed. Field samples of tubers and imported microplants were screened using the protocol(s) developed in this study and testing results were validated by testing the grown out plants using RT-PCR, ELISA and bioassay. Repeatability and reproducibility were also assessed. Virus was readily detected in composite samples of 100-400 tubers, leaves or microplants. Compared to ELISA, multiplex RT-PCR can be 300500 times more sensitive. The standard protocols of duplex and multiplex RTPCR have been introduced for detecting viruses in seed potatoes for export.

Introducing molecular diagnostic technologies for detecting viruses in potato quarantine testing

H. XU (1), S. Cody (2), D. L. Hammill (2), X. Li (2)

(1) Canadian Food Inspection Agency, Charlottetown Laboratory, Charlettetown, PE, Canada; (2) Canadian Food Inspection Agency, Charlottetown Laboratory, Charlottetown, PE, Canada

Phytopathology 105(Suppl. 4):S4.152

In Canada, Solanum germplasm approved for entry under a permit to import must be subjected to a process of quarantine testing in the Potato Post Entry Quarantine (PPEQ) program. The quarantine testing lab in Charlottetown, PE, receives approx. 40 accessions each year. The quarantine testing scheme consists of 3 streams, pre-greenhouse (microplants), greenhouse (grown out plants) and pre-release (microplants) testing. The entire process of quarantine testing takes 8-12 months. Some conventional diagnostic methods, such as bioassay using indicator plants, electron microscopy, ELISA and reverse polyacrylamide gel electrophoresis (R-PAGE) have been employed for many years and still play critical roles in plant quarantine testing. For modernizing this program and improving the efficacy of potato quarantine testing, some molecular diagnostic methods, real-time quantitative PCR (qPCR), RT-PCR, RT-qPCR and LAMP were evaluated and validated in this lab. Known virus isolates were used for evaluating the specificity and sensitivity of the primers and probes. Serial dilutions of RNA extracts and composite samples (w/w, $\mathrm{v} / \mathrm{v}$ ) were used. RT-PCR, LAMP and qPCR were shown to be superior to the conventional technologies in several aspects, such as sensitivity, rapidity and multiplicity. They are at least 128-512 times more sensitive than ELISA and R-PAGE for detecting potato pathogens (several viruses, a viroid) and consume much less time than the bioassay does.

A distal stem loop is required for efficient readthrough of PLRV coat protein gene stop codon

Y. XU (1), S. M. Gray (2)

(1) Cornell University, Ithaca, NY, U.S.A.; (2) USDA-Agricultural Research Service; Department of Plant Pathology and Plant-Microbe Biology, Cornell University, Ithaca, NY, U.S.A.

Phytopathology 105(Suppl. 4):S4.152

Translational read-through of the capsid protein UAG stop codon is an evolutionarily conserved feature that members of the Luteoviridae have adopted as a strategy to produce the minor capsid protein referred to as the readthrough protein (RTP). Distinct domains of the RTP of Potato leafroll virus (PLRV) have been associated with the regulation of virus movement and tissue tropism in plant hosts, and in aphid transmission. The elements regulating PLRV RTP expression are not well understood. Deletion mutagenesis of the RTP-encoding sequences beyond the CP stop codon revealed a distal element located 640 nucleotides downstream of the $\mathrm{CP}$ stop codon that is required for readthrough translation. This region forms a 
complex stem loop structure. Site-directed mutational analyses of this region confirmed that conserved nucleotides in a loop and in paired regions of the stem are critical for readthrough translation. Further deletion mutagenesis suggests that distance between the stop codon and the distal element is not critical, but may influence the efficiency of readthrough translation.

\section{Plant-parasitic nematodes on field pea in North Dakota}

G. P. YAN (1), J. S. Pasche (1), K. Zitnick-Anderson (1), S. Pederson (2) (1) North Dakota State University, Department of Plant Pathology, Fargo, ND, U.S.A.; (2) North Dakota State University, North Central Research Extension Center, Minot, ND, U.S.A.

Phytopathology 105(Suppl. 4):S4.153

Plant-parasitic nematodes are an important group of pests that restrict productivity of field pea. Limited information exists on the occurrence of nematodes on field pea in North Dakota. Nematode surveys in 2004 and 2005 indicated that stunt and pin nematodes were the predominant genera in 87 fields sampled from 12 counties. Stunt and pin nematodes were found in 76 and $33 \%$ of the fields, respectively. The population densities of stunt and pin nematodes were as high as 22,180 and 7,370 per $\mathrm{kg}$ of soil, respectively. Root-lesion, dagger, root-knot, lance and spiral nematodes were found in low densities and low frequency of occurrence. A nematode survey was also conducted in 2014 and 57 soil samples were collected from 20 fields in nine counties. Stunt and pin nematodes were again found to be predominant. Pin and stunt nematodes were present in 81 and $40 \%$ of the samples, respectively, followed by lesion (11\%), dagger (9\%), spiral $(9 \%)$, and root-knot $(4 \%)$ nematodes. The densities of pin and stunt nematodes were as high as 15,048 and 4,356 per kg of soil, respectively. Compared with the survey results in 2004, stunt nematode populations in 2014 were lower in both occurrence frequency and highest density but pin nematode populations were substantially higher. More soil samples will be collected in 2015 to confirm the occurrence and abundance of nematodes in pea fields across the state. The key nematode species will be determined using molecular and morphological methods.

Molecular technology as a useful tool for nematode diagnostics: A case study of root-lesion nematode and cereal cyst nematode

G. P. YAN (1)

(1) North Dakota State University, Department of Plant Pathology, Fargo, ND, U.S.A.

Phytopathology 105(Suppl. 4):S4.153

Plant-parasitic nematodes are an important group of pests that affect agricultural production. Accurate identification of nematode species and awareness of population density are critical for designing effective control measures. Morphological identification of nematode species is difficult due to morphological similarity, overlapping morphometric measurements, and great variability of individual specimens. Molecular techniques offer opportunities for identifying and quantifying species of nematodes to an extent that could not be achieved with morphological diagnostics. PCR-RFLP was optimized to identify cereal cyst nematodes affecting wheat in the Pacific Northwest (PNW), which led to the first detection of the cyst nematode Heterodera filipjevi in Oregon. Discovery of H. filipjevi in Oregon represented a new record for the occurrence of that species in North America. Less expensive species-specific PCR was developed for identifying field samples infested with cyst nematodes, which led to the first discovery of $H$. filipjevi in Washington. Real-time PCR assays were developed to detect and quantify the root-lesion nematodes Pratylenchus neglectus and P. thornei restricting wheat production in non-irrigated PNW fields. Real-time PCR provides a useful platform for efficient detection and quantification of nematodes directly from soil and plants, which has the potential to allow us to avoid traditional nematode extraction, microscopic identification and counting steps.

Plant-parasitic nematodes on field crops in Southeastern and Northeastern North Dakota

G. P. YAN (1), A. Plaisance (1), W. Ye (2)

(1) North Dakota State University, Department of Plant Pathology, Fargo, ND, U.S.A.; (2) North Carolina Department of Agriculture \& Consumer Service, Agronomic Division, Nematode Assay Section, Raleigh, NC, U.S.A. Phytopathology 105(Suppl. 4):S4.153

Plant-parasitic nematodes are an important group of pests that affect productivity of field crops. Limited information exits on the occurrence of nematodes on field crops in North Dakota. A nematode survey was conducted in 2014 on five field crops and 117 soil samples were collected from seven Northeastern counties and five Southeastern counties. Spiral (prevalence: $61 \%$; highest density: 7,260 per $\mathrm{kg}$ of soil), root-lesion (44\%; 2,800$)$, stunt $(44 \% ; 2,600)$, pin $(28 \% ; 1,320)$ and dagger $(3 \% ; 375)$ nematodes were detected in corn fields. Spiral $(58 \% ; 4,285)$, stunt $(42 \% ; 2,250)$, root-lesion
$(23 \% ; 2,552)$, dagger $(26 \% ; 220)$, pin $(10 \% ; 1,408)$ and lance $(6 \% ; 44)$ nematodes were present in wheat fields. Spiral $(68 \% ; 18,315)$, root-lesion $(41 \% ; 875)$, stunt $(41 \% ; 2,475)$, pin $(34 \% ; 3,608)$, dagger $(10 \% ; 375)$, stubby root $(7 \% ; 495)$ and lance $(5 \% ; 100)$ nematodes were found from soybean fields. Spiral $(75 \% ; 13,950)$, pin $(50 \% ; 750)$, root-lesion $(25 \% ; 550)$, stubby root $(25 \% ; 22)$ and stunt $(25 \% ; 290)$ nematodes were present in barley fields. Root-lesion $(100 \% ; 1,628)$, spiral $(80 \% ; 750)$, pin $(60 \% ; 88)$, stunt $(40 \%$; $300)$, stubby root $(20 \% ; 44)$, dagger $(20 \% ; 220)$ and ring $(20 \% ; 250)$ nematodes were detected in a potato field. The nematode species and population level revealed the potential damage to these crops in North Dakota. More field soil samples will be collected and assayed in 2015 to confirm the occurrence and abundance of nematodes on these field crops across the state.

Bacillus spp. evaluation to control anthracnose infection on Andean lupin seed (Lupinus mutabilis Sweet)

V. YÁNEZ-MENDIZÁBAL (1), C. E. Falconí (2), A. C. Grijalva (1)

(1) Universidad de las Américas, Quito, Ecuador; (2) Universidad de las Fuerzas Armadas -ESPE, Quito, Ecuador

Phytopathology 105(Suppl. 4):S4.153

Anthracnose, caused by Colletotrichum acutatum, is the mayor fungal disease of lupin worldwide. The main infection way is contaminated seed. Bacillus spp. efficacy, based on antifungal compounds production, has been demonstrated on a wide range of plant fungal pathogens. The objective of this study was to evaluate the potential of Bacillus spp. to control anthracnose on Andean lupin seed (Lupinus mutabilis). Antifungal activity of living cells and cell free compounds from several Bacillus strains against $C$. acutatum was evaluated. Strains that showed the highest $C$. acutatum inhibition were selected to evaluate for their lipopeptide antifungal activity by TLCbioautography analysis and efficacy to control anthracnose artificial infection on seed. Bacterial treatments were prepared from 72-h-old cultures at $2 \times 10^{8}$ CFU mL $\mathrm{m}^{-1}$ and pathogen at $2 \times 10^{6}$ conidia $\mathrm{mL}^{-1}$. Antifungal activity assay showed that living cells and cell free compounds from four Bacillus spp. strains inhibited C. acutatum micelial growth up to $78.0 \%$ and $100 \%$, respectively. TLC-bioautography analysis showed that lipopeptide fractions corresponding to fengycins and iturins from each Bacillus strain have strongly antifungal activity against $C$. acutatum. Bacteria treatments significantly reduced anthracnose incidence from $94 \%$ (untreated control) to $0 \%$. These results suggest that Bacillus spp. strains are potential agents to control anthracnose infection on Andean lupin seed.

Optimization and characterization of the oil in water $(\mathrm{O} / \mathrm{W})$ nano emulsion in control of citrus huanglongbing

C. Yang (1), M. Zhang (2), Y. Duna (3), R. G. Shatter (3), C. A. POWELL (1) (1) IRREC-IFAS, University of Florida, Fort Pierce, FL, U.S.A.; (2) Univ of Florida, Fort Pierce, FL, U.S.A.; (3) USHRL, USDA-ARS, Fort Pierce, FL, U.S.A.

Phytopathology 105(Suppl. 4):S4.153

Nanoemulsion is a promising delivery system for the control of plant disease that reduces pesticide use and lessens the risk of environmental damage in agricultural practice. Huanglongbing is a serious disease for the citrus industry. Based on various physiochemical characteristics of oils, surfactants, and organic solvents, a novel oil in water $(\mathrm{O} / \mathrm{W})$ nanoemulsion was optimized to combat citrus huanglongbing disease. This nanoemulsion was produced using a spontaneous emulsification method for the efficient delivery of the antimicrobial compound ampicillin into citrus phloem by bark painting. The nanoemulsion prepared from Cremophor ${ }^{\circledR}$ EL (viscous oil), acetone (water miscibility organic solvent) and Span 80/Tween 80 (surfactant) exhibited small droplet size $(13.68 \pm 0.26 \mathrm{~nm})$. This nanoemulsion had an improved absorption rate (time of peak concentration $\left(\mathrm{t}_{\max }\right)=2 \mathrm{~d}$, peak of maximum concentration $\left(\mathrm{C}_{\max }\right)=71.9 \mathrm{ng} / \mathrm{g}$ ) and bioavailability of ampicillin (Relative Bioavailability $=274.63 \%$ ) in HLB-affected citrus, as compared with Amp solution alone ( $\mathrm{t}_{\max }=6 \mathrm{~d}, \mathrm{C}_{\max }=56.4 \mathrm{ng} / \mathrm{g}$ and $\left.\mathrm{RBA}=100 \%\right)$. Furthermore, $\mathrm{Ca}$. L. asiaticus in HLB-affected citrus was eliminated 2 to 6 months after the initial treatments. This study thus provides a novel, efficient and eco-friendly self-nanoemulsifying delivery system to control citrus huanglongbing disease.

Glutathione peroxidase is required for oxidative stress resistance and virulence in the citrus fungal pathogen Alternaria alternata

S. L. Yang (1), K. R. CHUNG (2)

(1) University of Florida, Gainesville, FL, U.S.A.; (2) National Chung-Hsing Univ, Taichung Taiwan, Taiwan

Phytopathology 105(Suppl. 4):S4.153

We determined a glutathione peroxidase (GPx) coding gene involved in NADPH oxidase (NOX) signaling network that is essential for the detoxification of cellular stresses induced by reactive oxygen species (ROS) and for A. alternata pathogenesis in citrus. Mutant strains carrying defective 
GPx displayed an increased sensitivity to a wide range of ROS-generating compounds. Moreover, the A. alternata GPx is required for correct fungal development. The GPx mutant strains showed a severe reduction in conidiation, and accumulated higher chitin content than the wild type. Virulence assays revealed that $A$. alternata GPx is required for full virulence. The expression of GPx gene was down-regulated in fungal strains carrying defective NOX, YAP1 and HOG1, suggesting the involvement of GPx among these regulatory pathways. These results further support the important role of ROS detoxification during A. alternata pathogenesis in citrus. Our study provides genetic evidence to define the central role of A. alternata GPx in the development of conidia, resistances to oxidative stress and virulence.

\section{Dissecting the various responsive modes to drought stress responses in} corn with contrasting tolerance to drought

L. YANG (1), J. Fountain (1), T. Jiang (2), D. Lee (3), R. Kemerait (1), B. Scully (4), S. Chen (5), B. Guo (6)

(1) University of Georgia, Department of Plant Pathology, Tifton, GA, U.S.A.; (2) Northeast Forestry Universit, Harbin, China; (3) University of Georgia, Department of Crop and Soil Sciences, Tifton, GA, U.S.A.; (4) USDA-ARS, U.S. Horticultural Research Laboratory, Fort Pierce, FL, U.S.A.; (5) University of Florida, Department of Biology, Genetics Institute, and Plant Molecular \& Cellular Biology Program, Gainesville, FL, U.S.A.; (6) USDA ARS CPMRU, Tifton, GA, U.S.A.

Phytopathology 105(Suppl. 4):S4.154

Drought decreases yield and exacerbates aflatoxin levels. Deciphering crop tolerance can contribute to drought through breeding. In order to investigate the responsive modes to drought, corn lines with contrasting drought tolerance were used with treatments of stressed and well-watered control. Proteomics revealed that sensitive line exhibited a vigorous induced response to stress, while tolerant line displayed stable, constitutive expression, including hormone regulation and redox homeostasis. Further physiological/biochemical assays found that drought-sensitive lines presented a sharp rise of ABA and IAA contents after stress induction and a significant decrease as drought prolong in contrast to control. The contents of ABA and IAA displayed a progressive stable increase for tolerant lines in response to continued drought. Sensitive lines was found to accumulate more reactive oxygen species (ROS) and nitric oxide, and rapid increase in enzymatic activities involved in ROS and reactive nitrogen species (RNS) metabolism compared to tolerant lines. Observed antioxidant activities of superoxide dismutase and catalase were constitutively higher in tolerant lines than sensitive lines, which showed a rapid induction following drought. The results suggest that drought sensitivity was associated with diverse metabolic pathways in corn, and tolerant lines may be able to better sequester excessive accumulation of harmful metabolites, like ROS and RNS, than sensitive lines.

\section{Microbiome study of potato taste defect in coffee beans from Rwanda}

J.-I. YANG (1), P. Ruegger (2), T. Miller (2), P. Philippe Rolshausen (2), J. Borneman (2)

(1) National Taiwan University, Taipei, Taiwan; (2) University of California, Riverside, Riverside, CA, U.S.A.

Phytopathology 105(Suppl. 4):S4.154

The purpose of this research is to study the microbiome associated with the rotten potato taste of roasted coffee beans from Rwanda. In the past decade, a significant portion of the coffee beans from Rwanda and Burundi have expressed a bad odor and flavor reminiscent of rotten potatoes after processing, commonly refer to as "potato taste defect (PTD)." Coffee beans eared the tasting score at 60-70 are usually considered bad coffee, and the ones scoring above 80 are considered good quality beans. Raw coffee beans of various tasting scores (disease severities) from Rwanda were analyzed by high-throughput sequencing of the 16S rRNA gene (bacteria) and rRNA ITS regions (fungi) on an Illumina HiSeq 2500. The abundance of several microbes positively correlated with the disease severities, indicating that they may be the causal agents of this phenomenon; Microbes including two Leuconostocaceae bacteria, Leuconostoc sp. and Weissella sp. and one fungus, Aspergillus sp. This discovery provides a lead for studies to determine if these microorganisms can cause PTD and fiuther reseach for the remedies to this problem.

Phytophthora intercalaris, a novel species from streams and irrigation water in eastern United States

X. YANG (1), N. Brazee (2), A. Loyd (3), C. Hong (4)

(1) Virginia Tech, Virginia Beach, VA, U.S.A.; (2) University of Massachusetts, Amherst, MA, U.S.A.; (3) Bartlett Tree Diagnostic Laboratories, Stamford, CT, U.S.A.; (4) Virginia Polytechnic Institute and State University, Virginia Beach, VA, U.S.A.

Phytopathology 105(Suppl. 4):S4.154
The genus Phytophthora includes a number of destructive plant pathogens. Recent surveys for Phytophthora species in streams and nursery irrigation systems in the eastern United States have recovered a novel Phytophthora species. This novel species is heterothallic and all examined isolates are $\mathrm{A}^{1}$ mating type. It produces ornamented and plerotic oogonia and amphigynous antheridia when being paired with $\mathrm{A}^{2}$ mating type testers of $P$. cinnamomi. Sporangia of this new species are non-papillate and mostly non-caducous. Phylogenetic analyses based on sequences of the internal transcribed spacer region and beta-tubulin gene placed this novel species close to, but not grouped in clade 10 of the genus Phytophthora. This new species is here described as Phytophthora intercalaris sp. nov. The potential implications of this new discovery and description are discussed.

Evaluation of different substrates for inducing the volatile-antibiotic compounds from Nodulisporium sp. PDL-005 and control of citrus green mold C. C. YEH (1), W. H. Chung (1)

(1) National Chung Hsing University, Taichung, Taiwan

Phytopathology 105(Suppl. 4):S4.154

One endophytic isolate, Nodulisporium sp. PDL-005, from Peperomia dindygulensis could produce antifungal volatile organic compounds (VOCs) and inhibit several postharvest pathogens after 3 days cultured on potato sucrose agar (PSA). Moreover, PDL-005 could reduce more than $60.0 \%$ disease severity of gray mold in strawberry. However, the PDL-005 cultured on PSA did not show a stable efficacy on controlling Penicillium digitatum $(P d)$ growth. In this study, different substrates were be examined the efficiency for VOCs enrichment and evaluated the control efficacy of green mold. The carbon and nitrogen sources test indicated that the single carbon or nitrogen source could not enhance the PDL-005 ability against $P d$. On the other hand, PDL-005 cultured on malt extract agar (MEA) could inhibit $P d$. GC-MS analysis indicated that the major antifungal VOCs of PDL-005 were associated with different substances or media, such as 3-Methoxy-2-napthol (in PSA) and 2-ethyl-2-hexenal (in MEA). Nine different substrates, sesame meal, tea seed pomace, soybean meal, wheat grain, wheat bran, oatmeal, rice bran, rice hulls and bagasse, were tested the efficiency for VOCs enrichment of PDL-005 and the inhibition of $P d$. Result showed that the PDL-005 cultured on bagasse could increase the inhibition rate of $P d$ to $90.6-100.0 \%$. The in vivo test showed that PDL-005 on bagasse had significant efficacy on control of citrus green mold. Thus, PDL-005 has potential on controlling postharvest disease.

Bacterial communities on wheat grown under long-term conventional tillage and no-till in the Pacific Northwest of the U.S.

C. YIN (1), K. L. Schroeder (2), N. Mueth (1), S. H. Hulbert (1), T. Paulitz (3)

(1) Washington State University, Pullman, WA, U.S.A.; (2) University of Idaho, Pullman, WA, U.S.A.; (3) USDA ARS, Pullman, WA, U.S.A. Phytopathology 105(Suppl. 4):S4.154

Soil microbial diversity can be used to assess the impact of agricultural practices such as no-till, which is being adopted by more wheat growers in the dryland areas of the inland Pacific Northwest. Bacterial communities in bulk and rhizosphere soil of wheat in two locations near Albion, WA and Genesee, ID, were assessed with pyrosequencing for two years, comparing long-term no-till plots to adjacent plots under conventional tillage. Long-term grassland was also sampled at the WA site. The most abundant phyla were Proteobacteria, Actinobacteria, Bacteroidetes, Gemmatimonadetes and Acidobacteria. Communities were more diverse in bulk soil compared to the rhizosphere. Proteobacteria were more frequent in rhizosphere soil, while Acidobacteria and Gemmatimonadetes were more abundant in bulk soils. Bacteroidetes were more frequent under conventional tillage. Actinobacteria were more abundant in perennial grass soils compared to cropped soil. In general, families were more affected by the position of the sample (rhizosphere vs bulk soil) than by tillage practices. Only three families were influenced by tillage, and all were more predominant in bulk soil. Rhodospirillaceae and Solirubrobacteraceae were higher in no-tilled compared to conventional tilled soils, but Chitinophagaceae were higher in conventionally tilled soils. The results suggest that bacterial communities in soil were more influenced by plant effects (rhizosphere vs bulk soil) than by tillage practices.

Survey of Citrus tristeza virus populations in Central California that react with MCA13 monoclonal antibody

R. K. YOKOMI (1), S. Hajeri (2), J. Barnier (2)

(1) USDA ARS PWA, Parlier, CA, U.S.A.; (2) Citrus Pest Detection Program of the Central California Tristeza Eradication Agency, Tulare, CA, U.S.A. Phytopathology 105(Suppl. 4):S4.154

The Citrus Pest Detection Program (CPDP) of the Central California Tristeza Eradication Agency monitors Citrus tristeza virus (CTV) in Central Cali- 
fornia. MCA13 is a severe strain discriminating monoclonal antibody used to screen for potentially virulent CTV isolates. MCA13-reactive CTV isolates are rare in Central California and are considered nonindigenous. Under the Effective Plan implemented in 2009, the CPDP surveys for only MCA13reactive CTV isolates. For decades, participating pest control districts practiced mandatory CTV suppression. In these districts, from 2009 to 2013, $100 \%$ of citrus acreage (over 162,000) was subsampled using the hierarchical subsampling (HS) method. Of 985,797 samples, 25 were suspect positive for MCA13 CTV. Follow-up surveys confirmed 32 individual trees positive for MCA13 CTV, and these trees were removed. In an adjacent non-participating district that ceased mandatory removal in 1996 , about $16 \%$ of over 100,000 citrus acres was subsampled under contract during the 2009 to 2013 period. Of 130,359 HS samples, 84 were suspect MCA13-positive. In two localized sites, follow-up surveys revealed 366 MCA13-positive trees. Some of these trees were not removed. These data suggest that non-removal of CTV-infected trees led to increased genetic diversity of CTV and more potentially virulent CTV isolates. Results of RT-qPCR assays using genotype-specific TaqManbased probes support this observation.

\section{Genetic variation and population structure of CChMVd using deep sequencing \\ J. Y. YOON (1), S. K. Choi (2), M. Park (3), G. S. Choi (2), P. Palukaitis (3)}

(1) Natonal Institute of Horticultural and Herbal Science, RDA, Jeon-Ju, South Korea; (2) National Institute of Horticultural and Herbal Science, RDA, Jeonju, South Korea; (3) Seoul Women's University, Seoul, South Korea Phytopathology 105(Suppl. 4):S4.155

Viroids, small single-stranded circular RNAs and non-protein encoding are unique infectious agents. Chrysanthemum chlorotic mottle viroid (CChMVd), a member of the family Avsunviridae, was identified from chrysanthemum plants showing chlorotic leaf symptoms. Pyrosequencing is a powerful tool to determine rapidly sequences of viroid quasi-species without cloning procedures. Thus, we investigated the population structure and the sequence variation of two CChMVd isolates using deep sequencing (pyrosequencing) and we assessed the effectiveness of pyrosequencing technology compared with the conventional method for analyzing quasi-species population structures and the sequence variation of CChMVd isolates. A total of 93,701 and 99,055 from two CChMVd isolates (I and II) were identified by 454pyrosequencing. High variation of nucleotide sequences of CChMVd-I was observed at positions $93,136,156,292,335$ and 362, and CChMVd-II at positions $93,134,154,292,335,362$, all around the secondary structure of CChMVds. These results were verified by sequence analyses of the same RTPCR products using the conventional Sanger-sequencing method. These data indicate that 454-pyrosequencing is a useful technology to access rapidly the quasi-species population of CChMVd variants being generated from infected chrysanthemum species. The detailed relationship between the population structures of the two CChMVds and sequence variations of their genomes is discussed.

Fusaruim graminearum gene FgHal2 which is down-regulated by Fusaruim graminearum virus 1 (FgV1) infection affects host response to FgV1

J. YU (1), K. M. Lee (1), M. Son (1), K. H. Kim (1)

(1) Seoul Natl Univ, Seoul, Korea

Phytopathology 105(Suppl. 4):S4.155

The Fusarium graminearum virus 1 (FgV1) infects Fusarium graminearum and is associated with hypovirulence traits such as reduced mycelial growth, increase pigmentation, and reduced pathogenicity in infected host. Transcriptomic and proteomic expression profiling have shown that many $F$. graminearum genes are differentially expressed upon $\mathrm{FgV1}$ infection. One of the host gene $\mathrm{FgHal2}$, bifunctional gene which has a highly conserved 3'phosphoadenosine 5'-phosphatase domain or inositol monophosphatase domain, shows reduced expression in response to $\mathrm{FgV1}$ infection. The FgHal2 deletion mutant $(\triangle \mathrm{FgHal2})$ showed retarded growth, defect in aerial mycelia formation, and reduced pigmentation whereas over-expression mutant (FGHAL2 OE) had normal morphological phenotype compared to the wild type (WT). Additionally, the conidia production of $\triangle F g H a l 2$ was reduced significantly and abnormal conidia was observed frequently. Comparing to the wild-type, FgV1 RNA accumulation was not increased in the $\triangle F g H a l 2-\mathrm{VI}$ from 48 to 120 hpi. However, viral RNA accumulation in the FGHAL2 OE-VI was reduced at 120 hpi relative to the WT but was similar to that of the WT at $72 \mathrm{hpi}$. The decrease in vertical transmission and low stability after successive subcultures on agar for the $\Delta \mathrm{FgHal} 2$ suggest that $\mathrm{FgHal2}$ might be required for maintenance of $\mathrm{FgV1}$ in host cell. Overall, these results indicate that FgHal2 plays diverse biological functions and might facilitate the maintenance of $\mathrm{FgV} 1$ in the host fungus.
Diversity and recombination of DNA-U3 of Banana bunchy top virus

N. Yu (1), P. Zhou (1), J. Wang (1), Z. Liu (1), Z. XIONG (2)

(1) Institute of Tropical Bioscience and Biotechnology, Chinese Academy of Tropical Agricultural Sciences, Haikou, China; (2) University of Arizona, Tucson, AZ, U.S.A.

Phytopathology 105(Suppl. 4):S4.155

Banana bunchy top virus (BBTV) causes a devastating disease of banana. It has a single-stranded DNA genome consisting of 6 components. While the functions of nearly all the BBTV DNA components are known, the coding product and function of DNA-U3 is unknown. In order to understand the DNA-U3 better, we obtained over 90 complete DNA-U3 sequences from 43 BBTV-infected samples collected in Hainan, China. Sequencing analysis showed large diversity among the DNA-U3 sequences, ranging from $81 \%$ to nearly $100 \%$ in sequence identity. All the sequenced DNA-U3 components belonged to the Southeast Asia group and were further clustered to two distinct clades. Furthermore, some samples contained distinct DNA-U3 components from both clades. Among nineteen full-length DNA-U3 clones randomly selected for sequencing in one BBTV-infected sample, eleven belonged to clade I, four belonged to clade II, and four were recombinants between the two clades. The frequent recombination between two clades of DNA-U3 was further confirmed by clade-specific restriction digestion analysis. Among 100 clones analyzed, 48 belonged to clade I, 25 belonged to clade II, and 17 were recombinants. Our study revealed the co-presence of two distinct species of DNA-U3 in single infected individuals and indicated a high rate of recombination among DNA-U3.

A high-density genetic map in Fusarium constructed with Genotyping-bySequencing markers

W. YUE (1), N. M. I. Mohamed Nor (1), J. F. Leslie (1), C. Toomajian (1)

(1) Kansas State University, Manhattan, KS, U.S.A.

Phytopathology 105(Suppl. 4):S4.155

The Fusarium fujikuroi species complex contains many agronomically important plant pathogens noted for the production of secondary metabolites such as mycotoxins and gibberellic acids (GAs) that are involved in pathogen virulence. Two members of this species complex, F. proliferatum and F. fujikuroi, are closely related phylogenetically but differ for pathogen traits. Our longterm aim is to explore the genetic bases behind these differences, and we have begun by conducting QTL analyses of progeny from an interspecific cross between these species. We adapted a Genotyping-by-Sequencing (GBS) protocol to generate high-density genotype data from 85 progeny from the cross. Illumina reads from our GBS library were mapped to the $F$. fujikuroi reference sequence to produce $>26,000$ GBS loci. We constructed a genetic map containing 2685 segregating GBS markers across the 12 chromosomes from these species by using the R/qtl package. To demonstrate the utility of the map, we performed QTL mapping on GA levels of these 85 progeny. We identified multiple significant QTL related to GA production, including one corresponding to the known GA biosynthetic gene cluster on chromosome 5 . This high-density genetic map provides valuable information for understanding the genetic bases underlying differences between these two closely related Fusarium species. The adapted GBS protocol and analysis pipeline provide powerful tools for additional Fusarium genetic and genomic studies.

Developing and testing an undergraduate level case study on cassava viral diseases

S. Yusuf (1), S. MALlOWA (2), S. Ruongo (3), G. Abucheli (4), N. Korir (5), D. W. Miano (6), J. P. Legg (7), A. E. Robertson (8)

(1) Makerere University, Kampala, Uganda; (2) Iowa State Univ, Ames, IA, U.S.A.; (3) Independent communications consultant, Bondo, Kenya; (4) Chuka University, Meru, Kenya; (5) Kenyatta University, Nairobi, Kenya; (6) University of Nairobi, College of Agriculture and Veterinary Sciences, Department of Plant Sciences and Crop Protection, Kangemi, Nairobi, Kenya; (7) International Institute of Tropical Agriculture, Dar es Salaam, Tanzania; (8) Iowa State University, Ames, IA, U.S.A.

Phytopathology 105(Suppl. 4):S4.155

Cassava is an important food security crop and the New Partnership for Africa's Development (NEPAD's) crop of choice to fight poverty in Africa. Two viral diseases, cassava mosaic disease (CMD) and cassava brown streak disease (CBSD) are important biotic constraints to its production. The goal of this study is to develop an undergraduate level teaching resource (case study) that acquaints students with diagnosis of CBSD, presents facts on the disease and discusses principles related to its management. Case studies are useful tools to create awareness and encourage critical thinking of important disease issues. Students take on the roles of professionals, growers or consumers of cassava, process factual knowledge on cassava disease management (including resistant varieties) and apply it to solve a real-world problem. The case study will be tested at University of Nairobi, Kenyatta University, Chuka 
University and Makerere University in East Africa. This poster will present highlights of the case study, data regarding the students' appreciation of the learning approach and how it influenced their comprehension on the diagnosis and management of these important diseases.

\section{Association of Phomopsis longicolla and Macrophomina phaseolina with} zone lines in soybean roots at maturity

M. L. ZACCARON (1), R. T. Holland (1), T. J. Hughes (2), C. R. Grau (3), J. C. Rupe (1)

(1) University of Arkansas, Fayetteville, AR, U.S.A.; (2) Monsanto Co, Chesterfield, MO, U.S.A.; (3) University of Wisconsin, Madison, WI, U.S.A. Phytopathology 105(Suppl. 4):S4.156

Zone lines were observed in upper taproots and lower stems of senesced soybean plants. This phenomenon causal agent is not known, but it appears to restrict the presence of Macrophomina phaseolina. To assess the prevalence and the causal agent of this phenomenon, a set of 18 cultivars was grown at two locations (Rohwer and Stuttgart, AR) and a second set of 26 cultivars was grown at Marianna, AR in fields artificially infested with M. phaseolina. Zone lines incidence significantly varied among cultivars at Stuttgart $(P$ 0.002) and at Rohwer $(P<0001)$, but not at Marianna $(P 0.519)$. Zone lines incidence was significantly higher at Rohwer than at Stuttgart $(P<.0001)$ with a significant interaction between location and cultivar $(P<0.001)$. Isolations were made from tissues 1) enclosed by the zone lines, 2) within the lines, and 3) outside the zone lines. Phomopsis longicolla was isolated at frequencies of $95.4 \%$, $91.3 \%$ and $31.5 \%$, while $M$. phaseolina frequencies were $2.5 \%, 15.2 \%$, and $50 \%$, from sites 1,2 , and 3 , respectively $(\mathrm{n}=368)$. M. phaseolina was recovered from $4.8 \%$ of sites with no microsclerotia $(n=748)$. No microsclerotia were observed in tissues enclosed by zone lines. In senesced soybean plants, $P$. longicolla-associated zone lines seem to be common and affected by cultivar and location. Zone lines seem to restrict the colonization of the enclosed root tissues by M. phaseolina, which could be a confounding factor when screening cultivars for resistance.

\section{Molecular mechanisms of Cercospora pathogenicity revealed through comparative genomics}

A. Zaccaron (1), J. Ridenour (2), J. Smith (2), S. Sharma (2), N. Lawson (2), M. L. Zaccaron (2), A. Fakhoury (3), B. BLUHM (4)

(1) University of Arkansas, Fayetteville, AR, U.S.A.; (2) University of Arkansas, Fayetteville, AR, U.S.A.; (3) Southern Illinois University, Carbondale, IL, U.S.A.; (4) Univ of Arkansas, Fayetteville, AR, U.S.A.

Phytopathology 105(Suppl. 4):S4.156

Cercospora species cause devastating foliar diseases of crops such as soybean (Glycine max) and maize (Zea mays). To date, few genomic resources are available for Cercospora. The goals of this project were to 1) perform de novo genome sequencing for key members of Cercospora, and 2) characterize mechanisms of pathogenicity through comparative and functional genomics. We utilized next-generation DNA sequencing technologies (Roche-454, Illumina, and Pacific Biosciences) to obtain genome sequences for C. sojina and C. cf. flagellaris (pathogens of soybean) and C. zeina (pathogen of maize). Genomes were assembled with a hybrid assembly strategy utilizing SPAdes along with LoRDEC, SSPACE-Standard, AHA scaffolder, GapFiller, and PBJelly. The final C. sojina, C. cf. flagellaris, and C. zeina assemblies resulted in $31.1 \mathrm{Mb}, 33.2 \mathrm{Mb}$, and 45.2 Mb genomes with L50s of 76, 5, and 63 , respectively. Genes were predicted with support from RNA sequence data (RNA-Seq) and proteins from closely related species. Comparative analyses have focused on genes related to pathogenicity, secondary metabolism, small secreted proteins, carbon-active enzymes, genes potentially undergoing diversifying selection, and exclusive/shared genes among Cercospora spp. This work provides novel genomic resources for key Cercospora pathogens and illuminates mechanisms of pathogenesis potentially shared among diverse members of the Cercospora.

Phylogenetically identified Fusarium fujikuroi associated with fusariosis of pineapple in peninsular Malaysia

L. ZAKARIA (1), N. F. Ibrahim (2), M. Mohd (2), N. M. Mohamed Nor (2)

(1) Universiti Sains Malaysia, Minden, Penang, Malaysia; (2) Universiti Sains Malaysia, Minden, Malaysia

Phytopathology 105(Suppl. 4):S4.156

Fusariosis symptoms were detected in pineapple plantation in the states of Kedah, Penang, Selangor and Johor in Peninsular Malaysia. From fusariosis symptoms of pineapple fruits and leaves, eight Fusarium isolates morphologically similar to $F$. fujikuroi and $F$. proliferatum were recovered. BLAST results of translation elongation factor 1-alpha (TEF-1 $\alpha$ ) sequences, against Fusarium-ID showed $98-100 \%$ similarity to $F$. fujikuroi NRRL43610. Phylogenetic analysis of combined TEF- $1 \alpha$ and $\beta$-tubulin sequences showed that the eight isolates from pineapple fusariosis clustered with $F$. fujikuroi
NRRL13566 which showed the isolates from pineapple fusariosis were phylogenetically similar to $F$. fujikuroi. Selected fusariosis isolates were able to produce fumonisin $\mathrm{B}_{1}(0.47-28.81 \mu \mathrm{g} / \mathrm{g})$ and moniliformin $(3.84-47.78$ $\mu \mathrm{g} / \mathrm{g}$ ) which indicated there are possibility of fumonisin $B_{1}$ and moniliformin contamination in pineapple tissues. From pathogenicity test, all tested pineapple fruits and leaves produced fusariosis symptoms similar to those observed on infected pineapple plant in the field. The Fusarium isolates were re-isolated and Koch's postulate was fulfilled. The present study indicated that phylogenetically identified $F$. fujikuroi isolates are the causal pathogen of pineapple fusariosis in Peninsular Malaysia. To our knowledge, this is the first report of phylogenetically identified $F$. fujikuroi associated with pineapple fusariosis in Peninsular Malaysia.

Coat protein gene diversity of Papaya ringspot virus in Puerto Rico C. ZAMBRANA-ECHEVARRIA (1), L. De Jesus-Kim (1), R. G. MarquezKarry (1), D. A. Jenkins (2), D. Siritunga (1)

(1) University of Puerto Rico, Mayaguez campus, Mayaguez, U.S.A.; (2) Tropical Agriculture Research Station, USDA-ARS, Mayaguez, U.S.A. Phytopathology 105(Suppl. 4):S4.156

Plant viruses are responsible for production losses in many agronomically important crops. In papaya, Papaya ringspot virus (PRSV) is the most damaging virus that affects the plant's production worldwide leading to lower yield, quality, marketability and sugar content. In Puerto Rico, commercial papaya plantations can be nearly $100 \%$ infected within 9 months of planting. In this study, we aim to assess the genetic diversity of PRSV for the first time in Puerto Rico using the coat protein gene (CP). Samples with PRSV-like symptoms were collected from different municipalities in the island and tested with a DAS-ELISA. Total RNA was extracted from symptomatic and ELISApositive leaves and converted into cDNA. RT-PCR with gene specific primers was used to amplify the $\mathrm{CP}$ and, subsequently, the resulting amplicons were Sanger sequenced. Phylogenetic analysis of CP nucleotide sequences is ongoing in order to determine the genetic variability of the viral population in the island. Also, the $\mathrm{CP}$ homology and relationship between the Puerto Rican isolate and isolates from the rest of the world are currently being incorporated in this study. This project will aid in the establishment of a control strategy for PRSV in papaya, such as the use of transgenics, and to determine the origin and evolution of PRSV in Puerto Rico. Our results will contribute to the global understanding of PRSV, including it's spread and evolution in different geographical locations.

Field evaluate tomato varieties for resistance to Tomato chlorotic spot virus, an emerging threat to tomato production in South Florida S. ZHANG (1), E. McAvoy (2), Q. Wang (3), D. R. Seal (1)

(1) TREC - Univ of Florida, Homestead, FL, U.S.A.; (2) UF/IFAS Hendry County Extension, LaBelle, FL, U.S.A.; (3) UF/IFAS Miami-Dade Extension, Homestead, FL, U.S.A.

Phytopathology 105(Suppl. 4):S4.156

Tomato chlorotic spot virus (TCSV), a new tospovirus was first detected in tomato from South Florida in 2012. A severe outbreak of TCSV has primarily occurred in Homestead, FL since the fall of 2014. The occurrence of TCSV has caused significant losses to tomato growers with up to $30 \%$ plants infected in some tomato fields. In the spring of 2015, a field trial is being conducted in Homestead, FL to evaluate host resistance in tomato varieties for the potential to manage TCSV. Fourteen varieties of tomato resistant to Tomato spotted wilt virus (TSWV), are evaluated and the tomato var. FL47 was included as a susceptible commercial variety. Tomato seedlings were transplanted into the beds covered with metalized plastic mulch. The trial was designed as a randomized complete block (RCB) with four replications for each variety, and fourteen plants were planted in each plot. First symptoms appeared on a plant of var. FL47 three weeks after transplanting. Five and a half weeks after transplanting, two varieties did not show any symptoms of TCSV, four varieties had lower than $5 \%$ plants infected, and eight varieties had a disease incidence of $5-10 \%$. However, $62.5 \%$ plants of var. FL 47 , the susceptible control, were infected with TCSV. Results from this field study indicate that tomato varieties resistant to TSWV are also resistant or highly tolerant to TCSV, which will provide a practical and cost-effective measure for tomato growers to manage TCSV in South Florida.

An outbreak of Tomato chlorotic spot virus, an emerging tospovirus threatening tomato production in the United States

S. ZHANG (1), D. R. Seal (1), Q. Wang (2), E. McAvoy (3), J. E. Polston (4)

(1) TREC - Univ of Florida, Homestead, FL, U.S.A.; (2) UF/IFAS MiamiDade County Extension, Homestead, FL, U.S.A.; (3) UF/IFAS Hendry County Extension, LaBelle, FL, U.S.A.; (4) Department of Plant Pathology, Univ of Florida, Gainesville, FL, U.S.A.

Phytopathology 105(Suppl. 4):S4.156 
A severe outbreak of Tomato chlorotic spot virus (TCSV) has occurred in Homestead, FL since the fall of 2014, causing significant losses to tomato growers. Up to $30 \%$ plants were infected in some tomato fields. Initial symptoms appeared three weeks after transplanting, primarily on top leaves showing tiny necrotic lesions and chlorotic spots. Within one week, symptoms quickly expanded to cause terminal stem and leaf death, wilting, bronzing, necrosis and deformation of leaves. TCSV resulted in severe stunting and eventually death of the plant if tomato plants were infected at an early stage. Fruit from infected plants, if any, showed necrotic rings, and as such was unmarketable. TCSV was first reported in tomato from South Florida in 2012, and is transmitted by thrips species, i.e. western flower thrips (Frankliniella occidentalis), common blossom thrips ( $F$. schultzei) and possibly other species. The known hosts of TCSV include tomato, pepper, eggplant, tomatillo, lettuce, tobacco, petunia, impatiens, and solanaceous weeds. In response to the crisis, a team consisting of the University of Florida research and extension faculty quickly promoted awareness of TCSV via workshops, field days, pest alerts, factsheets, and newsletters. Field trials are being conducted in Homestead, FL to assess genetic resistance in tomato varieties and to evaluate insecticides for their potential to reduce disease incidence. Results from these trials will be presented.

\section{Multiple detection of four Banana viruses by reverse transcription Loop- mediated isothermal amplification}

J. Zhang (1), W. B. Borth (2), B. Lin (3), M. J. Melzer (2), H. Shen (3), X. Pu (3), J. HU (2)

(1) Univ of Hawaii At Manoa; Key Laboratory of New Technique for Plant Protection in Guangdong, Institute of Plant Protection, Guangdong Academy of Agricultural Sciences, Honolulu, HI, U.S.A.; (2) Univ of Hawaii At Manoa, Honolulu, HI, U.S.A.; (3) Key Laboratory of New Technique for Plant Protection in Guangdong, Institute of Plant Protection, Guangdong Academy of Agricultural Sciences, Guangzhou, China

Phytopathology 105(Suppl. 4):S4.157

BBTV, BSV, CMV and BBrMV are the most devastating viruses of banana worldwide. Effective control relies on robust virus detection, virus-free propagation, elimination of infected plants, and quarantine. LAMP is a powerful tool for virus detection due to its sensitivity, specificity, efficiency, and rapidity. Conserved sequences obtained by aligning 42 coat protein sequences from $\mathrm{BBTV}, 70$ RNase $\mathrm{H}$ sequences from BSV, 44 coat protein sequences from $\mathrm{CMV}$, and 47 coat protein sequences from BBrMV, were used to design LAMP primers. RNAs were isolated from symptomatic plants whose virus infections were confirmed by PCR, and also from virus-free plants. Using RT-LAMP, all BBTV, BSV and CMV infected plants showed measurable amplification within $60 \mathrm{~min}$, with high sensitivity. Furthermore, plants infected with only one of these three viruses could be detected in single reactions containing three primers. RNA based RT-LAMP eliminates false positives arising from banana genomes with integrated virus genomes, such as the integrated BSV genome. Compared to RT-LAMP, only a few DNA viruses (BBTV and BSV) could be detected in infected plants by RT-PCR, possibly because of low levels of virus transcripts in vivo. We also developed an immuno-capture RT-LAMP assay for BBrMV detection that was more sensitive than either RT-PCR or single RT-LAMP. These assays will be useful to detect multiple viruses from plants and to study virus distributions and locations in the field.

\section{Characterization of the promoter of Grapevine vein clearing virus} Y. ZHANG (1), C. A. Angel (2), S. Valdes (3), W. Qiu (4), J. Schoelz (1)

(1) University of Missouri-Columbia, Columbia, MO, U.S.A.; (2) Colombian Sugarcane Research Center, Cali, Colombia; (3) International Center for Tropical Agriculture, Cali, Colombia; (4) Missouri State University, Mountain Grove, MO, U.S.A.

Phytopathology 105(Suppl. 4):S4.157

Grapevine vein clearing virus (GVCV) is the first DNA virus discovered in grapevine and it has been proven to be closely associated with a grapevine vein clearing and vine decline syndrome observed in Missouri and surrounding states. However, Koch's postulates have not been completed because of the difficulties with purification of GVCV virions and reinoculation of purified virions to grapevines. To address these issues, we have sought to create an infectious clone of GVCV. As a first step towards making an infectious clone, we characterized the GVCV promoter, as well as the 5' and 3' ends of the transcript. The minimal boundaries of the promoter were defined by cloning portions of the GVCV large intergenic region into the binary vector pKYLX7 and fusing the putative promoter with GFP. Constructs were then electroporated into Agrobacterium tumefaciens. ELISA was performed to assess GFP expression level three days after infiltration of agrobacteria into Nicotiana benthamiana leaves. The GVCV segment between nucleotides 7,332 and 7,672 was proven to be sufficient for strong GFP expression. In addition, 5' and 3' RACE showed that GVCV RNA transcripts start predominantly at nucleotide 7,571 and end at nucleotide 7,676. This information has guided the development of a GVCV infectious clone. The assembled terminally redundant GVCV sequence was cloned into the binary vector pKYLX7 and agroinfiltration will be used to test its infectivity.

Lonely peninsula: The mating-type and population of Phyllosticta citricarpa in Florida

K. ZHANG (1), N. Y. Wang (2), M. M. Dewdney (2), J. A. Rollins (1)

(1) University of Florida, Gainesville, FL, U.S.A.; (2) University of Florida, Lake Alfred, FL, U.S.A.

Phytopathology 105(Suppl. 4):S4.157

Phyllosticta citricarpa is the causal agent of citrus black spot (CBS), a disease first reported in Australia in 1895. In 2010, CBS was found in Southwest Florida and has continued to spread in this region. We have previously determined by sequence analysis that individual isolates of $P$. citricarpa contain either the MAT1-1 or MAT1-2 mating-type idiomorph. From this we surmise that $P$. citricarpa is a heterothallic fungus requiring compatible mating-types to generate airborne ascospores. In this project, locus specific primers for MAT1-1-1 and MAT1-2-1 were designed to screen the matingtype of 113 isolates from Florida and 26 from Australia. Only MAT1-2-1 isolates were found in Florida while stains from Australia display a 1:1 ratio of both the mating-types. Based on this screen, we hypothesized that the Floridian population is clonal. To test this hypothesis, seven polymorphic microsatellite/simple sequence repeat (SSR) markers were designed to establish multi-locus genotypes (MLGs) for Floridian and Australian populations. With SSR genotyping, 11 MLGs were revealed in 24 Australian isolates, but only one MLG was present in 72 Floridian isolates representing isolates from multiple groves collected over four years. This data suggests that the $P$. citricarpa population in Florida is a clonal asexually reproducing population derived from a limited introduction and the disease management strategies should be focused on controlling production and movement of conidia.

Intimate interactions between two fungal RNA viruses: A capsidless ssRNA virophage hosted by an unrelated novel dsRNA virus

R. Zhang (1), S. Hisano (1), A. Tani (1), H. Kondo (1), S. Kanematsu (2), N. SUZUKI (1)

(1) IPSR, Okayama University, Kurashiki, Japan; (2) NARO Institute of Fruit Tree Science, Morioka, Japan

Phytopathology 105(Suppl. 4):S4.157

We show unique mutualistic interactions between an as-yet-undescribed positive-strand (+) RNA virus, with properties similar to but distinct from a virophage, and a novel, hosting double-stranded (ds) RNA virus. We found a mixed viral infection in a hypovirulent strain of the plant pathogenic fungus, Rosellinia necatrix. Co-infecting viruses are tentatively termed yado-kari virus 1 (YkV1) with a (+) RNA genome of approximately $6 \mathrm{~kb}$ and yadonushi virus 1 (YnV1) with a dsRNA genome of approximately $9 \mathrm{~kb}$. YkV1 possesses one single open reading frame (ORF) encoding RNA-dependent RNA polymerase (RdRp), while YnV1 has two ORFs, each encoding capsid protein $(\mathrm{CP})$ and RdRp. Immunological and molecular analyses revealed trans-encapsidation of not only YkV1 RNA but also RdRp by the major CP of the other virus, YnV1 and trans-enhancement of YnV1 by YkV1. Virion transfection assay and previous epidemiological data strongly suggest that YkV1 depends on YnV1 for viability, although it probably encodes functional RdRp. This hypothesis was confirmed by establishing infectious full-length cDNA of YkV1. We propose the term "RNA virophage" for the capsidless (+) RNA virus, YkV1, which highjacks CP of the dsRNA virus, YnV1, for the trans-encapsidation of its genome and RNA polymerase at the replication site.

Comparative genomics analysis suggests positive selection is the main driving force for evolution of citrus canker causing Xanthomonas

Y. ZHANG (1), N. Jalan (1), X. Zhou (1), E. M. Goss (2), J. B. Jones (2), J. C. Setubal (3), X. Deng (4), N. Wang (1)

(1) Citrus Research and Education Center, Department of Microbiology and Cell Science, University of Florida, Lake Alfred, FL, U.S.A.; (2) Department of Plant Pathology, University of Florida, Gainesville, FL, U.S.A.; (3) Departamento de Bioquímica, Instituto de Química, Universidade de São Paulo, São Paulo, Brazil; (4) Department of Plant Pathology, South China Agricultural University, Guangzhou, China

Phytopathology 105(Suppl. 4):S4.157

Citrus canker caused by Xanthomonas citri pv. citri (Xac) is one of the most devastating diseases on citrus. Xac consists of multiple pathotypes including 
$\mathrm{A}, \mathrm{A}^{*}$ and $\mathrm{A}^{\mathrm{w}}$. XacA has wider host range and geographic distribution than the other two pathotypes. However, the genetic and evolutionary basis underlying the host range and virulence difference between these pathotypes is not well determined. Here we sequenced 21 Xac strains (14 A, $3 \mathrm{~A}^{*}$ and $\left.4 \mathrm{~A}^{\mathrm{w}}\right)$ with different host ranges and conducted comparative genomic and evolutionary analyses. The results suggested that acquisition of beneficial genes and loss of detrimental genes most likely endowed XacA to infect a broader range of hosts. Mutation is more frequent than recombination in the evolution history of Xac. Besides Xac, X. fuscans pv. aurantifolii (Xau) can also cause citrus canker (canker B and C). We added Xau strains into analysis to reveal the evolutionary mechanisms underlying their specialization process on citrus via convergent evolution. Positive selection was found to have affected $14 \%$ $(395 / 2822)$ of core genes. The genes affected are enriched in 'carbohydrate transport and metabolism' and 'DNA replication, recombination and repair' $(\mathrm{P}<0.05)$. Many genes related to virulence, especially genes involved in the type III secretion system and effectors, are affected by positive selection. Our analysis advances our understanding of the genomic basis of specialization by positive selection in bacterial evolution.

Development of a novel isothermal AmplifyRP method combining both real-time and endpoint assays in single tubes for rapid detection of plant pathogens

S. ZHANG (1), P. Russell (1), N. McOwen (2), S. Bohannon (2), B. Davenport (2)

(1) Agdia, Inc, Elkhart, IN, U.S.A.; (2) Agdia Inc., Elkhart, IN, U.S.A. Phytopathology 105(Suppl. 4):S4.158

Recombinase polymerase amplification, a leading isothermal amplification technology, has been increasingly used in detection of nucleic acids. Agdia Inc. utilized this technology and developed an AmplifyRP ${ }^{\circledR}$ platform for the detection of plant pathogens. Currently available are pathogen-specific AmplifyRP $^{\circledR}$ tests, either in a qualitative endpoint assay (Acceler8) or in a quantitative real-time assay (XRT), and Discovery kits applicable to any pathogens. In either of Acceler8 or XRT assays, it uses two target-specific primers and one internal probe and all specific recombination and amplification occur rapidly at a single constant temperature of $39^{\circ} \mathrm{C}$. In this study, we report further development of a novel isothermal AmplifyRP ${ }^{\circledR}$ method in which both XRT and Acceler8 assays were combined into a single reaction assay and both quantitative real-time fluorescence data and qualitative visual endpoint results were achieved through a single reaction. Using plum pox virus as an example, the virus was detected with crude plant extracts or purified RNA. The detection sensitivity and specificity obtained using portable fluorometer, real-time PCR machine or lateral flow strip were compared and shown that this combined assay method is comparable to the regular Acceler8 or XRT assays. In addition, it preserves the simplicity and rapidity of both Acceler8 and XRT, and opens up a great opportunity for rapid high throughput screening for plant pathogens through isothermal amplification.

\section{Analysis of the core genome of Streptomyces species reveals novel and robust genetic markers for phylogenetic reconstruction \\ Y. ZHANG (1), J. Huguet-Tapia (1), R. Loria (1) \\ (1) Department of Plant Pathology, University of Florida, Gainesville, FL, U.S.A. \\ Phytopathology 105(Suppl. 4):S4.158}

Streptomyces is the largest bacterial genus, comprising $>600$ species, with about 10 plant pathogenic species. Species are often poorly defined and $16 \mathrm{~S}$ rDNA or other markers are not able to provide sufficient resolution to discriminate closely related species. Our long-term goals are to understand the evolutionary relationships of Streptomyces pathogenic species and define the genetic attributes that allow some species to acquire and utilize virulence genes. The increase in availability of Streptomyces genomes provides genes that could be used for building phylogenies. This study presents a comprehensive analysis of 120 Streptomyces genomes. The core genome of Streptomyces reaches a plateau at 728 orthologs, but the pan-genome appears to be open. Using single-copy genes from core genome, we reconstructed Streptomyces branch tree of life. The Streptomyces species tree based on the single-copy orthologs has strong bootstrap support, indicating whole genome sequences are valuable resources for the investigation of Streptomyces evolution. This study also shows that single gene trees could not derive a reliable species tree. We then explored the possibility of using combined sets of markers to resolve the Streptomyces species tree. Five marker genes are shown to be able to accurately recover the Streptomyces phylogeny, demonstrating the feasibility to apply this combined set of five markers to Streptomyces systematics.
Analysis of full length infectious genomic cDNA clones of SPFMV

C. ZHANG (1), T. Benjain (2), G. Smith (2), Y. Meng (2), V. Njiti (2), C. A. Clark (3)

(1) Alcorn State University, Clinton, MS, U.S.A.; (2) Alcorn State University, Lorman, MS, U.S.A.; (3) Louisiana State University, Baton Rouge, LA, U.S.A. Phytopathology 105(Suppl. 4):S4.158

There are two major viruses infecting sweet potato worldwide, Sweet potato leaf curl virus (SPLCV) and Sweet potato feathery mottle virus (SPFMV). SPFMV, a potyviruse in the family of Potyviridae has a single stranded positive sense RNA genome. SPFMV infection reduces yield as well as quality of sweet potato. A field isolate of SPFMV was collected from Alcorn State, MS and genomic cDNAs were cloned and molecularly characterized. Based on the information, full length genomic cDNA clones were constructed and sequenced. Whole genome sequencing of the SPFMV-AS isolate indicates it has a $11.5 \mathrm{~kb}$ genome that encodes a open reading frame of 3481 amino acids. To test infectivity of these whole genome clones, the SPFMV cDNA were placed between CaMV $35 \mathrm{~S}$ promoter and Nos terminator, and biolistically introduced into Ipomoea spp. plants. Large scale screening of SPFMV cDNA clones was done using tissue culture sweet potato plants. Visual inspection and RT-PCR were used to evaluate SPFMV cDNA clone infection. Recently, plant viral vectors have been developed as valuable tools for heterologous gene expression in plants for gene function studies. The SPFMV full length infectious cDNA clones were engineered using multiple designs for foreign gene expression in sweet potato to explore the potential of using SPFMV as virus-based gene expression technology for sweet potato functional genomics studies.

\section{Genome dissection of pathogens that cause Fusarium wilt of banana} Y. ZHANG (1), C. Li (2), L. J. Ma (3)

(1) University of Massachusetts Amherst, Amherst, MA, U.S.A.; (2) Guangdong Academy of Agricultural Science, Fruit Tree Research Institute, GUANGZHOU, China; (3) University of Massachusetts Amherst, AMHERST, MA, U.S.A.

Phytopathology 105(Suppl. 4):S4.158

Panama disease of banana caused by $F$. oxysporum f. sp. cubense (Foc) hampers agricultural production and ravages the wellbeing of a society. Recently, this disease has spread from South-east Asia to Mozambique and Jordan, further illustrated its devastating effects. Here, we have sequenced the genomes of 33 isolates of Foc, including 18 race1 isolates, 6 tropical race 4 (TR4) isolates, 5 subtropical race 4 (STR4) isolates, 2 VCG 121 isolates and 2 non-pathogens. Our genomic dissection analysis of TR4 strain II5 confirmed the existence of lineage-specific (LS) chromosomes. Meanwhile, we provide solid phylogenetic analysis of re-sequencing strains and confirmed the monophyletic origin of all TR4 isolates. The monophyletic origin of TR4 strains inspired the search of functional genes that may contribute to TR4 virulence but not in race 1 and STR4. We found candidate genes that may contribute to Foc specific pathogenicity including chitin binding domain containing genes and candidate effectors (FOIG_16547 and FOIG_ 15370). Real-time PCR revealed that candidate effector (FOIG_16547) was induced in planta, confirming its importance in host-specific pathogenicity.

\section{Optimization of Bacillus subtilis electric transformation scheme}

X. Y. ZHAO (1), S. Gao (1), Z. G. Ren (1), Z. P. Liu (1), Y. M. Wei (1), Q. X. Shang (1)

(1) Beijing University of Agriculture, Beijing, China

Phytopathology 105(Suppl. 4):S4.158

Experimental genetic manipulation has been an essential tool for studying of bacterial metabolism, physiology and pathogenesis and developing microbial biotechnology. In order to improve the transformation efficiency and build the genetic transformation system of Bacillus subtilis, electric transformation was adapted in this study. The results showed that preparation of competent cells was significant for the transformation efficiency. The optimizing scheme of competent cells preparation was like below. B. subtilis cells were cultured overnight in LB broth and transferred in $1 \%$ volume to a new flask with LB and $0.5 \mathrm{M}$ sorbitol, with 10 times of dissolved oxygen. Bacteria were shaking cultured to $\mathrm{OD}_{600}=0.5$, then added $2 \%$ glycine and $1 \%$ threonine and kept on shaking until the $\mathrm{OD}_{600}$ turn downward, and put the flask on ice immediately. Bacteria cells was collected with centrifuge and washed with electric shock buffer (0.5Msorbitol, $0.5 \mathrm{Mmannitol}$ and $10 \%$ glycerol) in equal volume, $1 / 2$ volume and $1 / 4$ volume in turn, then resuspended and aliquoted into100 $\mu$ l. Keeping the cells chilling and treating the cells gently were essential for competent. Transformation efficiency of B. subtilis cells with the system would be $1.6 \times 10^{3} \mathrm{cfu} / \mu \mathrm{g}$ DNA. B. subtilis is familiar in plant disease biocontrol. Improvement of the transformation efficiency is helpful for the research of biocontrol mechanism and development of biocontrol agent. Thanks to Natural Science Foundation of China (31201559). 
Enhancing detection sensitivity and accuracy on "Candidatus Liberibacter asiaticus" through Next Generation Sequencing technology

Z. Zheng (1), X. Deng (1), J. CHEN (2)

(1) Department of Plant Pathology, South China Agricultural University, Guangzhou, China; (2) USDA ARS PWA, Parlier, CA, U.S.A.

Phytopathology 105(Suppl. 4):S4.159

"Candidatus Liberibacter asiaticus" (CLas) is associated with citrus Huanglongbing (HLB, yellow shoot disease). Detection of this unculturable bacterium exclusively depends on polymerase chain reaction (PCR). Appropriate primer design is key to assure detection sensitivity and accuracy, which depend on quality of available bacterial sequences. Next Generation Sequencing (NGS) is a term describing several recent sequencing technologies with high throughput capacity. For example, the Illumina MiSeq platform can generate a Giga-base level sequence data set in one instrumental run. Therefore, NGS increases nucleotide coverage (high sequence accuracy) and allows thorough collection of all DNA species (deep sequencing) in a sample. This study utilized the Illumina MiSeq data sets from three HLB samples to enhance CLas detection. Three whole genome sequences of CLas strains were assembled. Genome comparison revealed a gene with a copy number of five. Primer sets were designed to increase PCR detection sensitivity; By comparing the 16S rRNA gene from whole genome sequences with those previously published. A missing $G$ base was found and new primers were design to correct the deficiency; Analyses on the MiSeq sequence data from California clarified possible presence of " $\mathrm{Ca}$. L. africanus" by revealing a previous error in primer design. In summary, NGS technology has a high potential for enhancing HLB diagnosis.

Genome-wide binding sites identification of a GntR transcriptional regulator required for virulence in Xanthomonas citri subsp. citri

X. ZHOU (1), Q. Yan (2), Y. Zhang (3), N. Wang (3)

(1) Univ of Florida, Lake Alfred, FL, U.S.A.; (2) Oregon State University, Corvallis, OR, U.S.A.; (3) University of Florida, Lake Alfred, FL, U.S.A. Phytopathology 105(Suppl. 4):S4.159

Citrus canker, caused by Xanthomonas citri subsp. citri (Xac), is one of the most severe diseases affecting citrus industry worldwide. GntR is a helix-turn helix transcriptional regulator family that regulates metabolism as well as virulence in bacteria. Herein, we showed that a GntR regulator YtrA1, which belongs to YtrA subfamily, plays a crucial role in Xac virulence. In order to identify YtrA1 regulon, we performed microarray-based transcriptional analysis and ChIP-exo, an exonuclease-treated chromatinimmunoprecipitation assay identifying protein-DNA interaction at nearly single-nucleotide resolution. Our results revealed that YtrA1 regulates three individual operons by binding to a conserved palindromic motif "GGTG-N ${ }_{16}$ CACC" at the promoter region. GUS reporter assay demonstrated that YtrA1 is a repressor regulator under conditions including Nutrient Broth, XVM2 medium and in planta. Site-directed mutagenesis of conserved residues in DNA binding domain suggested an indispensable role of YtrA1 DNA binding activity in regulating bacterial virulence. However, the deletion of individual genes in YtrA1 regulon fails to generate abolished virulence as ytrA1 mutant, indicating that further study is needed to better elucidate the regulation mechanism of YtrA1 in Xac virulence.

Organic rice disease management using genetic resistance, cover crop and organic fertilizer

X. G. ZHOU (1), F. Dou (1), A. M. McClung (2)

(1) Texas A\&M AgriLife Research, Beaumont, TX, U.S.A.; (2) USDA ARS, Dale Bumpers National Rice Research Center, Stuttgart, AR, U.S.A.

Phytopathology 105(Suppl. 4):S4.159

The strong market demand for organic rice has driven the continued increase of organic rice production in the US. However, growers still lack effective tools to manage narrow brown leaf spot (NBLS) caused by Cercospora janseana and brown spot caused by Cochliobolus miyabeanus, two common diseases affecting organic rice production. An experiment was conducted in a field under organic management at Beaumont, TX in 2012, 2013 and 2014 to evaluate the impact of cultivar resistance, cover crop, and organic fertilizer on NBLS and brown spot severity and yield potential. Tesanai 2 had the highest resistance to both diseases followed by the hybrids XL723 and XL753 as compared to the susceptible cultivar Presidio. Production following the incorporation of clover winter cover crop resulted in a consistent reduction in NBLS and brown spot than following winter fallow. Production following ryegrass cover crop incorporation reduced NBLS in 1 of 3 years and brown spot in 2 of 3 years. An application of the soil amendment NatureSafe or Rhizogen at 90,150 or $210 \mathrm{~kg} \mathrm{~N} /$ ha significantly reduced NBLS and brown spot compared to the nonamended control. Combined use of cultivar resistance (Tesanai 2), clover cover crop, and soil amendment at $150 \mathrm{~kg} \mathrm{~N} / \mathrm{ha}$ provided the greatest reduction in both diseases and resulted in the highest rice yield. Cultivar, cover crop, and fertility rate are important factors for management of NBLS and brown spot diseases in organic rice production.

Field efficacy of new fungicides for management of narrow brown leaf spot of rice

X. G. Zhou (1), S. S. UPPALA (2)

(1) Texas A\&M AgriLife Research, College Station, TX, U.S.A.; (2) Texas A\&M AgriLife Research, Beaumont, TX, U.S.A.

Phytopathology 105(Suppl. 4):S4.159

Narrow brown leaf spot (NBLS) caused by Cercospora janseana is one of the major foliar diseases of rice with yield losses up to $40 \%$. Host plant resistance is effective in managing NBLS. However, host resistance might not be durable due to evolution of new races of the pathogen. NBLS is managed primarily through application of fungicides. In this study, field experiments were conducted in fields naturally infested with NBLS from 2011 to 2014 to evaluate the efficacy of new and unlabelled fungicides for control of NBLS. The fungicides containing azoxystrobin (Quadris @ $0.69 \mathrm{~L} / \mathrm{ha}$ ), propiconazole (Tilt @ 0.35 1/ha or 0.73 L/ha), azoxystrobin plus propiconazole (Quilt @ $1.63 \mathrm{~L} / \mathrm{ha}$, QuiltXcel @1.2 L/ha), trifloxystrobin plus propiconazole (Stratego (a) 1.39 L/ha), fluxapyroxad (Sercadis @ 0.5 L/ha), pryraclostrobin (Headline (a) $0.66 \mathrm{~L} / \mathrm{ha}$ ), antibiotics originated from Steptomyces spp. (validamycin @ 1 L/ha and kasugamycin@1 L/ha), and Silicon (1 L/ha) were sprayed at the booting stage. NBLS severity was visually rated at one or two weeks prior to harvest. The fluxapyroxad and propiconazole fungicides provided greatest reductions in NBLS severity, resulting in significant increases in yield. Fluxapyroxad (new) and propiconazole fungicides can be an effective tool in the management of rice NBLS in the US.

Differential transcriptional regulation of defense-associated genes among apple rootstock genotypes in response to Pythium ultimum

Y. ZHU (1), M. Mazzola (1), G. Fazio (2)

(1) USDA ARS Tree Fruit Research Lab, Wenatchee, WA, U.S.A.; (2) USDA ARS Plant Genetic Resources Unit, Geneva, NY, U.S.A.

Phytopathology 105(Suppl. 4):S4.159

Apple replant disease (ARD) poses a substantial threat to orchard establishment on replant sites. Deployment of resistant apple rootstocks is an ecologically desired, cost effective and durable disease control strategy particular for perennial production system. Candidate genes which function in pathogen recognition, hormone mediated defense activation and generation of antimicrobial metabolites were identified based on RNA-Seq based transcriptome profiling in response to Pythium ultimum infection. Regulation patterns of selected genes were examined among ARD tolerant (G41, G935) and susceptible (B9, M26) rootstocks. Expressed as a ratio of the quantified expression values in mock inoculated and $P$. ultimum infected roots, significant differences in expression patterns were observed between tolerant and susceptible varieties. Genes functioning in the biosynthesis of and signaling pathways regulated by the defense hormones ethylene and jasmonate (ACS, AOS, LOX1 and ERF) demonstrated either long-lasting activation, 3 days post-inoculation in $\mathrm{G} 41$, or the presence of a secondary peak at 14 dpi in G935, while the activation of these genes was transient in susceptible M26 and B9 rootstocks. Genes with annotated functions for production of antimicrobial secondary metabolites, such as those encoding beta-chitinase, chalcone synthase and biphenyl synthase, exhibited stronger and prolonged activation in tolerant G41 and G935, compared with susceptible M26 and B9 rootstocks.

Invasion of the dicot-infecting mastrevirus Chickpea chlorotic dwarf virus in solanaceous hosts in Pakistan

M. Zia-Ur-Rehman (1), U. Hameed (1), H. W. Herrmann (2), M. J. Iqbal (1), M. S. Haider (1), J. K. BROWN (2)

(1) Institute of Agricultural Sciences, University of Punjab, Punjab, Pakistan; (2) University of Arizona/School of Plant Sciences, Tucson, AZ, U.S.A. Phytopathology 105(Suppl. 4):S4.159

Most mastreviruses (Geminiviridae) infect monocotyledonous plants, however some infect dicots instead. Chickpea chlorotic dwarf virus (CpCDV) is a dicot-infecting mastrevirus first identified infecting chickpea in India, and since then it has been reported throughout the Indian subcontinent, North Africa, and Arabian Peninsula. Currently, twelve strains A-L are recognized based on $>94 \%$ nt identity. In 2014, CpCDV strain L was reported infecting upland cotton in Pakistan, and strain D and F from pepper (Capsicum аппиит) in India and Oman, respectively. During 2012 tomato plants showing geminivirus-like symptoms were observed in Vehari, Pakistan. DNA was isolated from symptomatic leaves and subjected to rolling circle amplification, followed by cloning and DNA sequencing. An apparently full-length mastrevirus genome of $2.6 \mathrm{~kb}$ was determined. It shared the greatest $\mathrm{nt}$ identity, at 99\%, with $\mathrm{CpCDV}$ (KM377673) strain C, which was previously 
identified infecting lentil crops in Pakistan. Based on the criteria for species and strain demarcation for the genus, Mastrevirus, at $<78 \%$ and $<94 \%$, respectively, this is the first discovery of strain $\mathrm{C}$ infecting tomato plants. The broader-than-expected host range of $\mathrm{CpCDV}$, which now includes legumes, cotton, and solanaceous species, has widespread economic implications, and is suggestive of a host shift that possibly enabled CpCDV to spread within and beyond where it previously (apparently) infected only legume hosts.

Population diversity of Gaeumannomyces graminis var. graminis from St. Augustinegrass in Texas

M. Zidek (1), Y. K. JO (1)

(1) Texas A\&M University, College Station, TX, U.S.A

Phytopathology 105(Suppl. 4):S4.160

Gaeumannomyces graminis var. graminis (Ggg) is an ectotrophic root infecting fungus that is the causal agent of take-all root rot of St. Augustinegrass (Stenotaphrum secundatum) and root decline of other warmseason turfgrasses. Ggg is also the etiological agent of crown sheath rot of rice (Oryza sativa). Colony morphologies of $76 \mathrm{Ggg}$ isolates from St. Augustinegrass throughout Texas were examined on potato dextrose agar. Three distinctive phenotypic groups were found, where 10.5, 80.3, and 9.2\% of the isolates were highly melanized with a round colony formation (termed $\mathrm{M}$ group), non to slightly melanized with a round colony formation (termed $\mathrm{L}$ group), and slow-growing and highly melanized exhibiting an irregular or filamentous colony formation (termed $\mathrm{H}$ group), respectively. The $\mathrm{M}$ group grew faster at $30^{\circ} \mathrm{C}$ and was significantly more aggressive than $\mathrm{L}$ and $\mathrm{H}$ groups based on a rice seedling pathogenicity assay. A maximum likelihood phylogenetic analysis based on partial sequences of internal transcribed spacer and large subunit of the ribosomal DNA, supported that M, L, and $\mathrm{H}$ groups were separated into different clades and were associated with other Ggg sequences retrieved from the NCBI GenBank database. M, L, and H groups were also distinct from $G$. graminis varieties tritici and avenae. To our knowledge, this is the first study to describe the population diversity of Ggg with regards to morphological and biological characterization and genetic background.

\section{A novel approach to using PCR-GBS in survey data}

K. ZITNICK-ANDERSON (1), K. Simons (2), J. Pasche (2)

(1) North Dakota State Univ, Moorhead, MN, U.S.A.; (2) North Dakota State Univ, Fargo, ND, U.S.A.

Phytopathology 105(Suppl. 4):S4.160

Genotyping by sequencing (GBS) uses next generation technology traditionally for association studies and marker assisted breeding. A new application for GBS is the identification and quantification of plant pathogens. In 2014, a survey was performed in North Dakota to identify root rot pathogens causing damage on field peas. The genus Fusarium represented the majority of the isolated organisms. The seven Fusarium species identified were $F$. acuminatum, $F$. avenaceaum, $F$. culmorum, $F$. graminearum, $F$. oxysporum, $F$. redolens, $F$. solani and $F$. sporotrichioides. Current methods of isolating and identifying organisms directly from diseased plant roots are time consuming, laborious, and expensive, and it is difficult to accurately quantify organisms using traditional methods. Also, given the numerous organisms associated with pea roots, qPCR would be complicated to perform. Therefore, the objective of our research was to develop a novel approach that would use GBS in place of current plating methods. The strategy developed is a modification of highly multiplexed PCR-GBS. A small panel of primers was designed from conserved regions of DNA spanning SNPs or small indels in various genes. Sequencing allowed the identification of each Fusarium species present and the proportion of the reads determined the relative quantity. Preliminary results indicate this strategy can be used to directly identify and quantify root rot inhabiting organisms effectively and efficiently.

Evaluation of rotation and tank-mixture programs for gray mold management in strawberry

A. ZUNIGA (1), A. Amiri (2), N. A. Peres (2)

(1) University of Florida, Wimauma, FL, U.S.A.; (2) University of Florida, Wimauma, FL, U.S.A.

Phytopathology 105(Suppl. 4):S4.160

The efficacy of several fungicides registered to control Botrytis cinerea, the causal agent of gray mold on strawberry, has been reduced because of widespread resistance development in Florida and worldwide. In this study, we evaluated the efficacy of different rotation and tank-mixing strategies to improve gray mold management. Weekly rotation or tank-mixtures of the single-site fungicides fludioxonil+cyprodinil (Switch), fenhexamid (Elevate), penthiopyrad (Fontelis), fluopyram (Luna Privilege) and the multi-site fungicides captan or thiram were applied for two seasons in two strawberry fields. These fungicides were selected because resistant populations have not yet built to high levels or they were newly registered. Fruit were harvested twice weekly to determine gray mold incidence and yield. Although some disparities were observed between locations and years, some treatments such as fludioxonil+cyprodinil rotated with fluopyram or penthiopyrad have significantly improved yield. Beside rotating fludioxonil+cyprodinil with fluopyram and penthiopyrad, tank-mixing the two latter fungicides with either captan or thiram reduced gray mold incidence from $30 \%$ to less than $10 \%$ and from $10 \%$ to less than $3 \%$ at locations 1 and 2 , respectively. These results indicate that rotating newly registered single-site fungicides with a "low risk" fungicide such as fludioxonil or tank-mixing with the multi-site fungicides should improve gray mold management in strawberry fields.

New approaches to phenotyping early time points of wheat stem rust infection process on barley

J. D. ZURN (1), S. Dugyala (1), P. Borowicz (1), R. Brueggeman (1), M. Acevedo (1)

(1) North Dakota State University, Fargo, ND, U.S.A.

Phytopathology 105(Suppl. 4):S4.160

Basal defense is an important component for mitigating disease at early stages of infection. The ability to phenotype at these early stages can provide valuable insights for both pathogen infection and host defense response. Relative qPCR and fluorescence microscopy approaches were utilized to phenotype barley for seedling resistance to wheat stem rust (Puccinia graminis f. sp. tritici) race MCCFC at multiple time points. The susceptible cultivar Steptoe, resistant cultivars containing only Rpg1 (Beacon, Morex, and Chevron), and the resistant line Q21861 containing Rpg1 and rpg4/Rpg5 stem rust resistance genes were evaluated at 24 hours post inoculation (HPI), 48 HPI, 6 days post inoculation (DPI), and 12 DPI. At 12 DPI the seedlings were evaluated using a Stakman scale. Statistical differences $(P<0.05)$ were found between cultivars as early as 24 HPI using the qPCR assay and displayed similar hierarchal ordering to microscopy observations. Additionally, the fungal development observed at 24 and $48 \mathrm{HPI}$ was different than what was observed at 12 DPI. At early stages, the susceptible cultivar Steptoe had less fungal DNA (more resistant) than the barley lines containing the Rpgl and rpg4/Rpg5 resistance genes suggesting potential early pre-haustorial resistance contributions. Temporal variation in resistance ranking suggests the qPCR assay may be a valuable method for dissecting pre- and post-haustorial resistance mechanisms in the barley-wheat stem rust pathosystem. 